\title{
AC-DC Difference Calibrations
}
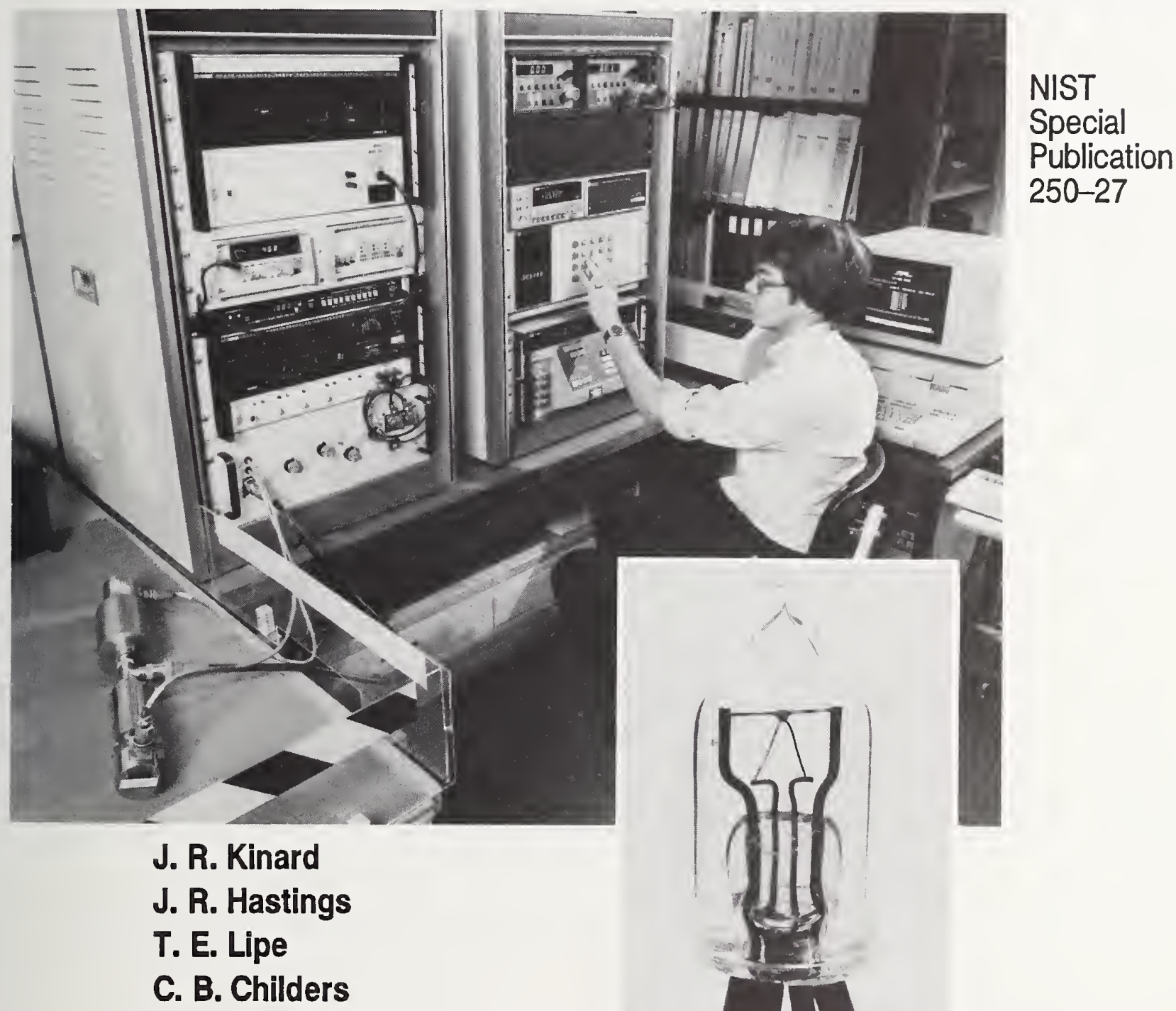

U.S. Department of Commerce

National Institute of Standards and Technology 


\section{Center for Basic Standards}

The Center for Basic Standards develops and maintains the scientific competencies and laboratory facilities necessary to preserve and continue to refine the base physical quantities upon which the Nation's measurement system is constructed. The Center is responsible for the standards of voltage, current, resistance and impedance, temperature, vacuum, leak rate, pressure, mass, length, time and frequency. The Center collaborates closely with the standardizing laboratories of other countries to ensure that measures used in international trade are fully compatible. The Center also performs research at the frontiers of physics in order to improve measurement capability and quantitative understanding of basic physical processes that underlie measurement science.

\section{Electricity Division}

Maintains and improves the national standards of electrical measurements; conducts experiments to realize the electrical units in terms of the mechanical units; develops stable electrical standards and improved methods for the dissemination of the units of electrical measurements; determines fundamental physical constants related to electrical units; develops instrumentation for precise electrical measurements directly referenced to the national standards; implements techniques for their use and puts them into practical applications; assists in applying scientific and technological development to measurement problems, especially in other areas of NIST research.

\section{Temperature and Pressure Division}

Maintains and improves the national standards of temperature and pressure; conducts research towards the development of new concepts for these standards; calibrates practical standards for the U.S. scientific and technical community in terms of the primary standards; develops methods and devices to assist user groups in the assessment and enhancement of the accuracy of their measurements of temperature and pressure; prepares and promulgates evaluations and descriptions of temperature and pressure measurement processes; coordinates these standards and measurement methods nationally and internationally; and determines the accuracy of the national standards of temperature and pressure with respect to fundamental thermodynamic relations.

\section{Length and Mass Division}

Develops and uses the competence necessary to implement a more accurate measurement system for length and mass; applies research, utilizing the most advanced technology available, to the improvement of our present standards and devises new standards and new methods for their transfer.

\section{Time and Frequency Division}

Maintains, develops, and improves the national standards for time (which are coordinated with the United States Naval Observatory) and frequency, and the time scales based on these standards; carries out research in areas of importance to the further fundamental improvement of frequency standards and their applications focusing on microwave and laser devices, atomic and molecular resonances, and the measurement of fundamental physical phenomena and constants; adapts time and frequency standard devices and concepts to special scientific and technological demands; develops time and frequency measurement methods in the radio frequency, microwave, infrared, and visible radiation regions; coordinates the national time and frequency standards, time scales, and measurement methods nationally and internationally in conjunction with the United States Naval Observatory; operates time and frequency dissemination services, such as radio stations and broadcasts, for the purpose of traceability to the national standards of time and frequency.

\section{Quantum Physics Division}

Engages in research in atomic and molecular physics at the forefront of the field, and performs basic, highly accurate measurements and theoretical analyses that help establish a reliable foundation for scientific and technological measurement and data.

\section{Quantum Metrology Division}

Engages in forefront research in quantum metrology; contributes to new determinations of the fundamental physical constants, and the extension and refinement of the electromagnetic scale and devises, where possible, tests of basic symmetries and invariances. 


\section{NIST MEASUREMENT SERVICES: AC-DC Difference Calibrations}

J. R. Kinard, J. R. Hastings, T. E. Lipe, and C. B. Childers

Center for Basic Standards

National Measurement Laboratory

National Institute of Standards and Technology

Gaithersburg, MD 20899

May 1989

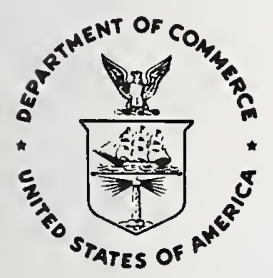

NOTE: As of 23 August 1988, the Natlonal Bureau of Standards (NBS) became the National Institute of Standards and Technology (NIST) when President Reagan signed Into law the Omnlbus Trade and Competitiveness Act.

U.S. DEPARTMENT OF COMMERCE, Robert A. Mosbacher, Secretary NATIONAL INSTITUTE OF STANDARDS AND TECHNOLOGY

Raymond G. Kammer, Acting Director 
Library of Congress Catalog Card Number: 89-600736

National Institute of Standards and Technology Special Publication 250-27

Natl. Inst. Stand. Technol. Spec. Publ. 250-27, 308 pages (May 1989)

CODEN: NSPUE2

Certain commercial equipment, instruments, or materials are identified in this paper in order to adequately specify the experimental procedure. Such identification does not imply recommendation or endorsement by the National Institute of Standards and Technology, nor does it imply that the materials or equipment identified are necessarily the best available for the purpose.

\section{U.S. GOVERNMENT PRINTING OFFICE}

WASHINGTON: 1989

For sale by the Superintendent of Documents, U.S. Government Printing Office, Washington, DC 20402-9325 


\section{PREFACE}

Calibrations and related measurement services of the National Institute of Standards and Technology provide the means for makers and users of measuring tools to achieve levels of measurement accuracy that are necessary to attain quality, productivity, and competitiveness. These requirements include the highest levels of accuracy that are possible on the basis of the most modern advances in science and technology as well as the levels of accuracy that are necessary in the routine production of goods and services. More than 300 different calibrations, measurement assurance services, and special tests are available from NIST to support the activities of public and private organizations. These services enable users to link their measurements to the reference standards maintained by NIST and, thereby, to the measurement systems of other countries throughout the world. NIST Special Publication 250, NIST Calibration Services Users Guide, describes the calibrations and related services that are offered, provides essential information for placing orders for these services, and identifies expert persons to be contacted for technical assistance.

NIST Special Publication 250 has recently been expanded by the addition of supplementary publications that provide detailed technical descriptions of specific NIST calibration services and, together with the NIST Calibration Services Users Guide, they constitute a topical series. Each technical supplement on a particular calibration service includes:

specifications for the service

design philosophy and theory

description of the NIST measurement system

NIST operational procedures

measurement uncertainty assessment.

error budget

systematic errors

random errors

NIST internal quality control procedures

The new publications will present more technical detail than the information that can be included in NIST Reports of Calibration. In general they will also provide more detail than past publications in the scientific and technical literature; such publications, when they exist, tend to focus upon a particular element of the topic and related elements may have been published in different places at different times. The new series will integrate the description of NIST calibration technologies in a form that is more readily accessible and more useful to the technical user. 
The present publication, SP 250-27, NIST Measurement Services: AC-DC Difference Calibrations, by Joseph R. Kinard, John R. Hastings, Thomas E. Lipe, and Clifton B. Childers, is one of approximately 20 documents in the new series published or in preparation by the Center for Basic Standards. It describes calibration technology and procedures utilized in connection with NIST Service Identification Numbers from 53310 to 53343 listed in the NIST Calibration Services Users Guide. Inquiries concerning the contents of these documents may be directed to the authors or to one of the technical contact persons identified in the Users Guide (SP 250).

Suggestions for improving the effectiveness and usefulness of the new series would be very much appreciated at NIST. Likewise, suggestions concerning the need for new calibration services, special tests, and measurement assurance programs are always welcome.

Joe Simmons, Acting Chief

Office of Physical Measurement Services

Katharine B. Gebbie, Acting Director

Center for Basic Standards 
The primary purpose of this publication is to collect and summarize the specialized information needed to operate the ac-dc difference laboratory and calibration service at NIST (Gaithersburg). This document should be used in conjunction with the computer programs stored on discs and listed in Room A144 Metrology Building and the laboratory notebooks located in the same room.

An additional purpose, however, is to provide the users of this calibration service and other interested people with a convenient reference source. The service and its underlying background are documented here in considerable detail.

Contained in this publication are:

an annotated table of contents, a topical index, and a glossary of common ac-dc acronyms

an overview of the service

selected published papers

instructions for the operation of the comparator systems

a schedule for the recalibration and periodic checks of the NIST thermal converters

a sample report of calibration

The annotated table of contents and the topical index serve as a "road map" to the different sections of this document, which consists largely of previous writings. Each section is numbered consecutively, and the section numbers are shown on the first page of each one. The glossary lists and defines some frequently used acronyms. A good starting place in learning about ac-dc difference calibrations at NIST is the overview presented as section 1 , to supplement the brief account given in NIST Special Publication 250, NIST Calibration Services Users Guide.

In recent decades many papers or reports have discussed various aspects of acdc difference measurements, especially as they are made at NIST. Twelve of these publications from 1952 to 1987, including one by a non-NIST author, are presented as sections 2 through 13. Collectively they document both the theoretical and the practical basis of the NIST calibration service for ac-dc differences. Other publications could have been added, but these 12 cover the subject. Hence, the aids mentioned above for finding the discussions of different topics have been prepared instead of newly written sections that would have duplicated what already existed. 
Sections 14, 15, and 16 are instructions for operating the comparator systems. They were prepared for use by the NIST staff and have not been reviewed as thoroughly as the preceding sections. Nevertheless, they can be helpful to non-NIST readers interested in operational details. They are revised from time to time, of course, as changes are made in the equipment and procedures.

A schedule for the recalibration and periodic checks of the NIST thermal converters follows as section 17. These quality control procedures grew out of recommendations given in section 4. Finally, section 18 is a sample report of calibration. 
(1) "AC-DC Difference Calibrations at NBS," by J. R. Kinard, in Proc. Meas. Sci. Conf., Irvine, CA, Jan. 1986, pp. 3-8. An overview of the NBS calibration service for thermal converters, including descriptions of the primary, reference, and working standards. The upper voltage and current limits and the uncertainties of the ac-dc difference calibrations are given as a function of frequency.

(2) "AC-DC Comparators for Audio-Frequency Current and Voltage Measurements of High Accuracy," by F. L. Hermach, IEEE Trans. Instrum. Meas., IM-25, pp. 489494, Dec. 1976. A survey of ac-dc transfer instruments and basic standards.

(3) "AC-DC Transfer Instruments for Current and Voltage Measurements," by F. L. Hermach, IRE Trans. Instrum., I-7, pp. 235-240, Dec. 1958. Describes different types of instruments for the measurement of ac current and voltage and outlines the advantages of the electrothermal type.

(4) "An Investigation of the Uncertainties of the NBS Thermal Voltage and Current Converters," by F. L. Hermach, Natl. Bur. Stand. (U.S.), Rep. NBSIR 842903, Apr. 1985. This report discusses: the establishment of a new set of NBS primary ac-dc standards; the construction of a precise comparator for intercomparing these standards; the NBS techniques for frequency, voltage, and current extension; and the study and evaluation of random and systematic errors for the NBS standards and the calibration service.

(5) Multijunction Thermal Converters as the NBS Primary AC-DC Transfer Standards for AC Current and Voltage Measurements," by F. L. Hermach, J. R. Kinard, and J. R. Hastings, IEEE Trans. Instrum. Meas., IM-36, pp. 300-306, June 1987. A conference report based on the work reported in (4) and including later results.

(6) "An Investigation of Multijunction Thermal Converters," by F. L. Hermach and D. R. Flach, IEEE Trans. Instrum. Meas., IM-25, pp. 524-528, Dec. 1976. A study of a group of multijunction thermal converters (MJTC's) which later became NBS primary ac-dc standards. 
(7) "Theoretical Analysis of the AC/DC Transfer Difference of the NPL Multijunction Thermal Convertor Over the Frequency Range DC to $100 \mathrm{kHz}$," by F. J. Wilkins, IEEE Trans. Instrum. Meas., IM-21, pp. 334-340, Nov. 1972. Provides a theoretical analysis of the thermoelectric and reactive errors of MJTC's and supports the expectation of very small ac-dc differences for the NBS primary standards.

(8) "Thermal Converters for Audio-Frequency Voltage Measurements of High Accuracy," by F. L. Hermach and E. S. Williams, IEEF Trans. Instrum. Meas., IM-15, pp. 260-268, Dec. 1966. Describes the construction of the NBS Model F-1 reference set of thermal voltage converters (TVC's). Gives the results of a study of the F-1 TVC set and special NBS thermoelements (TE's) used as thermal current converters ( $\left.\mathrm{TCC}^{\prime} \mathrm{s}\right)$.

(9) "Thermal Voltage Converters and Comparator for Very Accurate AC Voltage Measurements," by E. S. Williams, J. Res. Natl. Bur. Stand. (U.S.), 75C, pp. 145-154, July-Dec. 1971. Describes the construction of the NBS Model F-7 TVC set of working standards and the NBS manual comparator.

(10) "Thermal Voltage Converters for Accurate Voltage Measurements to 30 Megacycles Per Second," by F. L. Hermach and E. S. Williams, Trans. AIEE (Communication and Electronics), 79, pt. I, pp. 200206, July 1960. Describes the construction and characteristics of the NBS Model-C TVC set used from $100 \mathrm{kHz}$ to $1 \mathrm{MHz}$.

(11) "Thermal Converters as AC-DC Transfer Standards for Current and Voltage Measurements at Audio Frequencies," by F. L. Hermach, J.Res. Natl. Bur. Stand. (U.S.), 48, pp. 121-138, Feb. 1952. An experimental study of thermal converters as well as a theoretical discussion of thermal transfer errors.

(12) "Thermal Current Converters for Accurate AC Current Measurement," by E. S. Williams, IEEE Trans. Instrum. Meas., IM-25, pp. 519-523, Dec. 1976. Describes TCC's and intercomparison techniques. 
(13) "A Dual-Channel Automated Comparator for AC-DC Difference Measurements," by E. S. Williams and J. R. Kinard, IEEE Trans. Instrum. Meas., IM-34, pp. 290-294, June 1985. Describes the NBS automated comparator systems used for ac-dc difference calibrations.

(14) "General Instructions for the Operation of NBS Automated AC-DC Comparator System II," by T. E. Lipe. Provides a general description and setup instructions and tells how to: use the system status utility and amplifier gain checking utility, create and modify files containing calibration procedures for the test instruments and a correction table for the standards, calibrate various types of thermal converters, and prepare ac-dc test reports.

(15) "Instructions for Using the Manual AC-DC Difference Comparator System," by E. S. Williams and C. B. Childers. Provides a general introduction and describes the power supplies and controls, console, TE comparator, and galvanometer circuits and shields. Also discusses ac-dc difference tests, grounding, the calculation of $\mathrm{nE}$ (where $\mathrm{n}$ is the exponent relating the input current $I$ to the output $E M F E=k I^{n}$ ), and low-frequency tests.

(16) "Instructions for Using the MJTC Comparison Equipment," by F. L. Hermach. Discusses the ac-dc standards, comparison equipment, n-compensation, and the basic equation for this comparator. Describes in detail the operation of the equipment under the headings: connections, preliminary adjustments, $n-$ measurement, n-compensation, dc reversal compensation, ac setting, ac-dc determinations, recording and computing data, and shutting down. Also discusses the maintenance of the equipment through inspections and periodic tests.

(17) "Schedule for Recalibration and Periodic Checks of NBS Thermal Converters." Specifies regular tests of the reference and working standards. 
(18) "Report of Calibration." A sample report.

NOTE: Section (4) in its original form included reprints of three papers to make it easy to refer to them. Since these papers are also included in the present compilation as sections (6), (8), and (9), they are omitted from section (4) to avoid duplication. 


\section{Topical Index}

$\underline{\text { Topic }}$

$\underline{\text { Sections }}$

Specifications for the Service

1,18

An overview of the calibration service, including the frequency dependence of the upper voltage and current limits and the uncertainties of the calibrations, is given in (1). Section (18) is a sample calibration report.

Design Philosophy and Theory

$2,3,4,5,6,7,8,9,10,11,12,13$

A survey of ac-dc transfer instruments and basic standards is presented in Section (2). The advantages of electrothermal instruments are discussed in Section (3). MJTC's are introduced in Sections (4), (5), and (6). Section (4) is a internal report that serves as a basis for much of the calibration service. A theoretical error analysis for MJTC's is given in Section (7). The NBS reference and working sets of TVC's are described in sections (8), (9), and (10). Section (11) is noteworthy for its theoretical discussion of thermal transfer errors. A set of TCC's is described in Section (12), and the NBS automated comparators are discussed in Section (13).

Description of the NBS Measurement

$$
1,4,5,6,8,9,10,13,14,15,16
$$

Most of these sections have been cited earlier, but they are relevant to this topic also. The primary, reference, and working standards are described in Section (1). The most precise of the NBS comparators is discussed in Sections (4), (5), and (6). Special NBS TE's used as TCC's are described in Section (8), and the NBS manual comparator is discussed in Section (9). Section (10) deals with the high-frequency TVC's. Section (13) is about the automated comparators. Detailed descriptions of the different comparator systems are given in Sections (14), (15), and (16).

NBS Operational Procedures $14,15,16$

These references explain how to use the different comparator systems.

Measurement Uncertainty Assessment

A detailed analysis of the random and systematic uncertainties for the NBS standards and the calibration service is presented in this basic reference.

NBS Internal Quality Control Procedures

Recommendations for maintaining the quality of the standards and the calibration process are given in Section (4). A schedule for recalibration and periodic checks of the standards is presented in Section (17). 


\section{Glossary of Common AC-DC Acronyms}

AC: alternating current

$\mathrm{AV}$ : alternating voltage

DC: direct current

DV: direct voltage

TE: thermoelement

TC: thermal converter

TCC: thermal current converter

TVC: thermal voltage converter

SJTE: single-junction thermoelement

SJTC: single-junction thermal converter

MJTC: multijunction thermal converter 


\section{AC-DC DIFFERENCE CALIBRATIONS AT NBS* \\ Joseph R. Kinard \\ Electricity Division \\ National Bureau of Standards}

\section{ABSTRACT}

The NBS (Gaithersburg) calibration service for thermal voltage and current converters relies on a group of primary multijunction thermal converters and sets of reference and working standards for extending their ranges and frequencies. The converter sets that constitute the NBS standards -- primary, reference, and working -- as well as the build-up and bootstrap techniques used in their characterization over the full ranges of voltage, current, and frequency are described briefly. The upper voltage and current limits and the uncertainties of the ac-dc difference calibrations are given as a function of frequency.

\section{INTRODUCTION}

Alternating voltage or current is commonly measured by using a thermal converter to compare the heat produced by the alternating signal to the heat produced by a dc signal of nearly equal magnitude. NBS (Gaithersburg) provides a calibration service for the ac-dc differences of such thermal voltage converters (TVC's) and thermal current converters (TCC's). (TVC calibrations at frequencies above $1 \mathrm{MHz}$ are performed at the NBS branch in Boulder.) The ac-dc difference is conventionally defined as

$$
\delta=\left(\mathrm{Q}_{\mathrm{a}}-\mathrm{Q}_{\mathrm{d}}\right) / \mathrm{Q}_{\mathrm{d}}
$$

where $Q_{d}$ is the average of the two polarities of dc input that produce the same output from the thermal converter as that produced by the ac input $Q_{a}$. Thermal converters are calibrated at NBS by comparing them with standards whose ac-dc differences have been carefully determined. The NBS primary ac-dc transfer standards are a group of multijunction thermal converters (MJTC's) from several sources, manufactured at different times and in different ways. Voltage, current, and frequency ranges have been extended beyond those of the MJTC's by using reference and working sets of TVC's and TCC's.

*An earlier version of this paper was presented at the Measurement Science Conference in January 1986. This revised version was prepared in December 1987. 
Over the past several years a series of comparisons has led to the establishment of a group of eight MJTC's as the NBS primary standards for acdc difference [a]-[c]. These converters are constructed with bifilar heaters and as many as 200 or more thermocouples to sample the heater temperature essentially along its entire length. Thermoelectric errors, such as Thomson and Peltier heating and cooling, as well as other errors have been shown theoretically to be below the $1 \mathrm{ppm}$ level [d]. Dielectric loss and other defects do exist, however, but compensation techniques have been found to greatly reduce their effect [a]. The fact that the MJTC's were obtained over a period of years from different manufacturers who used different designs and fabrication techniques considerably improves the confidence in this group as primary standards, since the likelihood that a systematic error will be discovered is much greater for intercomparisons of such varied artifacts than for repeat measurements on one standard or for comparisons of like standards. The MJTC's have heater ratings ranging from $5 \mathrm{~mA}$ to $50 \mathrm{~mA}$ with voltage drops from $2 \mathrm{~V}$ to $10 \mathrm{~V}$ and output EMF's of $30 \mathrm{mV}$ to $120 \mathrm{mV}$. This high output EMF and a carefully constructed comparator enable a pooled standard error (standard deviation of the mean) for MJTC intercomparisons of about $0.2 \mathrm{ppm}$ to be obtained. Based on these comparisons and the above mentioned experimental and theoretical considerations, the average ac-dc difference of this group is less than $0.5 \mathrm{ppm}$ from $30 \mathrm{~Hz}$ to $10 \mathrm{kHz}$.

The extension of the voltage, current, and frequency ranges beyond those of the MJTC's has been achieved by the use of TVC's and TCC's whose ac-dc differences have been shown, as described below, to be nearly independent of voltage or current level and only slightly dependent on frequency.

\section{THERMAL VOLTAGE CONVERTER STANDARDS}

The NBS reference and working TVC standards are of the coaxial single-range type. They include special thermoelements ( $T E^{\prime} s$ ) with Evanohm heaters and temperature compensation [e] as well as some conventional standard pattern and UHF type TE's. The reference and working sets of TVC's were constructed so that adjacent ranges could be compared to step up or step down on the voltage scale. The reference TVC set (NBS F-1) consists of two $5 \mathrm{~mA}$ TE's and 13 plugin coaxially mounted resistors [f]. Each resistor, in conjunction with one of the TE's, is dedicated to a particular voltage range. The extension from the base $10 \mathrm{~V}$ range up to $1000 \mathrm{~V}$ and down to $0.5 \mathrm{~V}$ requires 14 adjacent-range comparisons at each frequency. The working TVC set (NBS F-7) consists of a $2.5 \mathrm{~mA} \mathrm{TE}$, a $5 \mathrm{~mA} \mathrm{TE}$, and six plug-in coaxially mounted resistors [e]. Each resistor may be used for two voltage ranges, since the ac-dc differences of the resistors for both of these well constructed sets (F-1 and $F-7$ ) are nearly independent of voltage level. For the working TVC set (F-7) only a sevenstep voltage build-up and build-down comparison process is required to characterize all the ranges from $1 \mathrm{~V}$ to $1000 \mathrm{~V}$ at each frequency. 
The extension of the frequency range above $100 \mathrm{kHz}$ was achieved by the use of converters (NBS $\mathrm{C}$-set) made with cylindrical, deposited-carbon resistors mounted coaxially in metal cylinders with $5 \mathrm{~mA}$ or $10 \mathrm{~mA}$ UHF type TE's [g]. Two techniques were used to establish the frequency variation of ac-dc difference up to $1 \mathrm{MHz}$. Firstly, the converters were made according to designs whose residual reactance effects were nearly zero based on theoretical calculations. The intercomparisons of adjacent voltage ranges, whose resistors have very different resistances but nearly equal reactances, showed hardly any relative ac-dc difference variations with frequency. This process and other special tests strongly supported the theoretical calculations indicating that the frequency coefficients were very small. Secondly, the frequency coefficients were verified by the use of a bolometer bridge to measure the performance of the TVC's in the 30-40 $\mathrm{MHz}$ region. If present, the residual reactance error would be expected to be quite large at these high frequencies. The bolometer bridge had sufficient accuracy to detect a reactance error equivalent to less than $100 \mathrm{ppm}$ transferred down to $1 \mathrm{MHz}$. No such error was found.

\section{THERMAL CURRENT CONVERTER STANDARDS}

The NBS reference and working standard sets of TCC's are comprised of two types of TE's [f]. In the $5 \mathrm{~mA}$ to $1000 \mathrm{~mA}$ range the TCC's are vacuum TE's with Evanohm heaters, some of special design. From 1 A to 20 A the current transfer standards are air-cooled TE's made a number of years ago to NBS specifications. Current build-up and build-down in the region up to 1 is accomplished for the reference standards by successively comparing two TE's of a lower range to each other and then, after connecting them together in parallel, to each of two TE's of a higher range. Pairs of almost identical low-reactance resistors are connected in series with the heaters to assure that the paralleled currents are sufficiently in phase. Above the $1 \mathrm{~A}$ level, adjacent-range TCC's are compared in series at the lower rated current [h]. Current dependence is checked by comparing selected ranges with shunted lowrange TE's at frequencies low enough so that shunt reactance errors are negligible. All of these TE's have Evanohm heaters with small, frequencyindependent thermoelectric effects, so the measured current dependence can be extrapolated to $50 \mathrm{kHz}$. To check for the possibility of errors from loss in the bead insulating the thermocouple from the heater, some measurements are taken with the grounded end interchanged.

To establish the frequency dependence of the ac-dc difference for TCC's, two 5 $\mathrm{mA}$ TE's are compared by using them with two nearly identical $30 \mathrm{~V}$ series resistors to form TVC's. By interchanging the resistors and averaging the results, the difference in the reactances of the resistors is essentially eliminated and the TE's are evaluated as current converters. This method avoids bead loss errors since both TE outputs are grounded. By using this method to compare their frequency dependence [g], reference TE's of different design and construction are characterized up to $50 \mathrm{kHz}$. The frequency independence of their relative ac-dc difference strongly indicates the absence of reactive and other effects. 
The calibration of customer's TVC's and TCC's is performed by comparing the test unit to NBS standards whose ac-dc differences have been characterized as described above. In the case of voltage, the test and standard TVC's are connected in parallel to the same voltages in a sequence such as ac, dct, dc-, ac, etc. In the case of current, the test and standard TCC's carry the same current in series. The heater and thermocouple of the test converter each have one end connected to ground. For both voltage and current, the TE outputs are monitored by comparator circuits that enable the difference in response to $\mathrm{ac}$ and $\mathrm{dc}$ to be recorded. Three types of comparators are used in NBS calibrations. In the first circuit, the output of the test TE is monitored and held constant for applied ac and dc [e]. Simultaneously, the difference between test and standard outputs is determined by resistively dividing down the larger for comparison to the smaller. The second comparator, used for the highest precision work, is a modified version of the first [a]-[c]. Important modifications include a compensation circuit, which relaxes the need to maintain a constant output from one $T E$, and an analog integrator on the output of the null detector. The third comparator is a dual-channel type used in the NBS automated systems [i]. In this comparator, two separate voltage nulling and monitoring circuits permit the measurement of the variations in the output of each $T E$ when the input is switched back and forth from ac to dct or dc-. Though difficult to operate manually, this method lends itself to automation.

Table I summarizes the frequency dependence of the upper voltage and current limits as well as the uncertainties of the ac-dc difference calibrations at NBS (Gaithersburg) as they exist in December 1987. These uncertainties were recently reduced, as noted at several points in the table, and further reductions are anticipated as a result of research that is presently under way. Figs. 1-6 show these uncertainties pictorially. Fig. 1 is for multirange TVC's and Figs. 2-4 are for coaxial single-range TVC's. The upper voltage limit is $1000 \mathrm{~V}$ from $20 \mathrm{~Hz}$ to $100 \mathrm{kHz}$ and $50 \mathrm{~V}$ or $100 \mathrm{~V}$ at lower and higher frequencies (between $2 \mathrm{~Hz}$ and $1 \mathrm{MHz}$ ). Figs. 5 and 6 are for TCC's, for which the upper current limit is $18 \mathrm{~A}$ from $100 \mathrm{~Hz}$ to $5 \mathrm{kHz}$ and less outside this range. 
[a] Hermach, F. L. and Flach, D. R., "An Investigation of Multijunction Thermal Converters," IEEE Trans. Instrum. Meas., Vol. IM-25, Dec. 1976, pp. 524-528.

[b] Hermach, F. L., "An Investigation of the Uncertainties of the NBS Thermal Voltage and Current Converters," NBS Internal Report 84-2903, April 1985.

[c] Hermach, F. L., Kinard, J. R., and Hastings, J. R., "Multijunction Thermal Converters as the NBS Primary AC-DC Transfer Standards for AC Current and Voltage Measurements," IEEE Trans. Instrum. Meas., Vol. IM36, June 1987, pp. 300-306.

[d] Wilkins, F. J., "Theoretical Analysis of the AC/DC Transfer Difference of the NPL Multijunction Thermal Convertor Over the Frequency Range DC to $100 \mathrm{kHz}, "$ IEEE Trans. Instrum. Meas., Vol. IM-21, Nov. 1972, pp. 334-340.

[e] Williams, E. S., "Thermal Voltage Converters and Comparator for Very Accurate AC Voltage Measurements," J.Res. Natl. Bur. Stand., Vol. 75C, July-Dec. 1971, pp. 145-154.

[f] Hermach, F. L. and Williams, E. S., "Thermal Converters for AudioFrequency Voltage Measurements of High Accuracy," IEEE Trans. Instrum. Meas., Vol. IM-15, Dec. 1966, pp. 260-268.

[g] Hermach, F. L. and Williams, E. S., "Thermal Voltage Converters for Accurate Voltage Measurements to 30 Megacycles Per Second," Trans. AIEE (Communication and Electronics), Vol. 79, Pt. I, July 1960, pp. 200-206.

[h] Hermach, F. L., "Thermal Converters as AC-DC Transfer Standards for Current and Voltage Measurements at Audio Frequencies," J. Res. Natl. Bur. Stand., Vol. 48, Feb. 1952, pp. 121-138.

[i] Williams, E. S. and Kinard, J. R., "A Dual-Channel Automated Comparator for AC-DC Difference Measurements," IEEE Trans. Instrum. Meas., Vol. IM34, June 1985, pp. 290-294.

NOTE: These references are designated by letter rather than number to avoid confusion with the section numbers of the compilation. This paper is section (1). The following table shows the correspondence between references and sections.

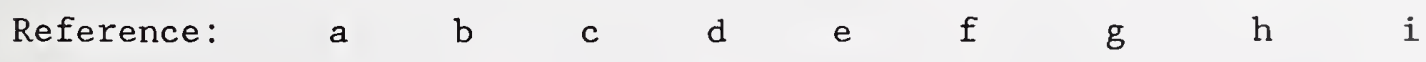

$\begin{array}{llllllllll}\text { Section: } & 6 & 4 & 5 & 7 & 9 & 8 & 10 & 11 & 13\end{array}$




\begin{tabular}{|c|c|c|c|c|c|c|c|}
\hline Erequency: & $\begin{array}{r}2-5 \\
\mathrm{~Hz} \\
\end{array}$ & $\begin{array}{c}5-20 \\
\mathrm{~Hz} \\
\end{array}$ & $\begin{array}{c}20-20 \mathrm{k} \\
\mathrm{Hz} \\
\end{array}$ & $\begin{array}{c}20-50 \\
\mathrm{kHz} \\
\end{array}$ & $\begin{array}{c}50-100 \\
\mathrm{kHz} \\
\end{array}$ & $\begin{array}{c}0.1-0.5 \\
\mathrm{MHz} \\
\end{array}$ & $\begin{array}{l}0.5-1 \\
M \mathrm{~Hz} \\
\end{array}$ \\
\hline Voltage Limits(V) & 50 & 100 & $1000 *$ & 1000 & $1000 *$ & 100 & 100 \\
\hline Current Limits $(A)$ & 0.05 & 0.05 & $18[a]$ & 16 & & & \\
\hline & \multicolumn{5}{|c|}{ UNCERTAINTY (parts per million) [b] } & & \\
\hline $\begin{array}{l}\text { Multirange TVC's } \\
>100 \mathrm{~V}\end{array}$ & & & 50 & 70 & 100 & & \\
\hline$\leqq 100 \mathrm{~V}$ & 200 & 100 & $30^{*}$ & $50 *$ & $70^{*}$ & $100^{*}$ & $200^{*}$ \\
\hline $\begin{array}{c}\text { Coax Single Range } \\
\text { TV'S }>100 \mathrm{~V} \text {. }\end{array}$ & & & 20 & 30 & 50 & & \\
\hline $5 \mathrm{~V} \leqq x \leqq 100 \mathrm{~V}$ & 200 & 100 & $15[c] *$ & $25^{*}$ & $40 *$ & $100^{*}$ & $200 *$ \\
\hline$<5 \mathrm{~V}$ & 200 & 100 & 20 & 30 & 50 & $100 *$ & $200 *$ \\
\hline $\begin{array}{l}\text { Special[d] } \\
5 \mathrm{~V} \leqq \mathrm{x} \leqq 100 \mathrm{~V}\end{array}$ & & & 10 & & & & \\
\hline $\begin{array}{l}\text { TCC'S } \\
>5 \mathrm{~A}\end{array}$ & & & 100 & $150^{*}$ & & & \\
\hline $50 \mathrm{~mA}<\mathrm{x} \leqq 5 \mathrm{~A}$ & & & 50 & $70^{*}$ & & & \\
\hline$\leqq 50 \mathrm{~mA}$ & 200 & 100 & 50 & $70^{*}$ & & & \\
\hline
\end{tabular}

* Indicates reduced uncertainty or extended voltage range, compared to the previous NBS calibration service.

[a]5 A at $20 \mathrm{~Hz}$, increasing to $18 \mathrm{~A}$ from $100 \mathrm{~Hz}$ to $5 \mathrm{kHz} ; 16 \mathrm{~A}$ above $5 \mathrm{kHz}$. 20 A shunts are calibrated at less than rated current.

[b]The lower uncertainty applies at the crossover frequencies. Uncertainties may be increased if the ac-dc differences are large or affected by selfheating or other instability.

$[\mathrm{c}]_{20} \mathrm{ppm}$ from $20 \mathrm{~Hz}$ to $100 \mathrm{~Hz}, 15 \mathrm{ppm}$ at $100 \mathrm{~Hz}$ and above.

[d] Vormally available by prearrangement for coaxial, single range TVC's between $100 \mathrm{~Hz}$ and $20 \mathrm{kHz}$. An additional cost and a longer turnaround time at NBS are required. 


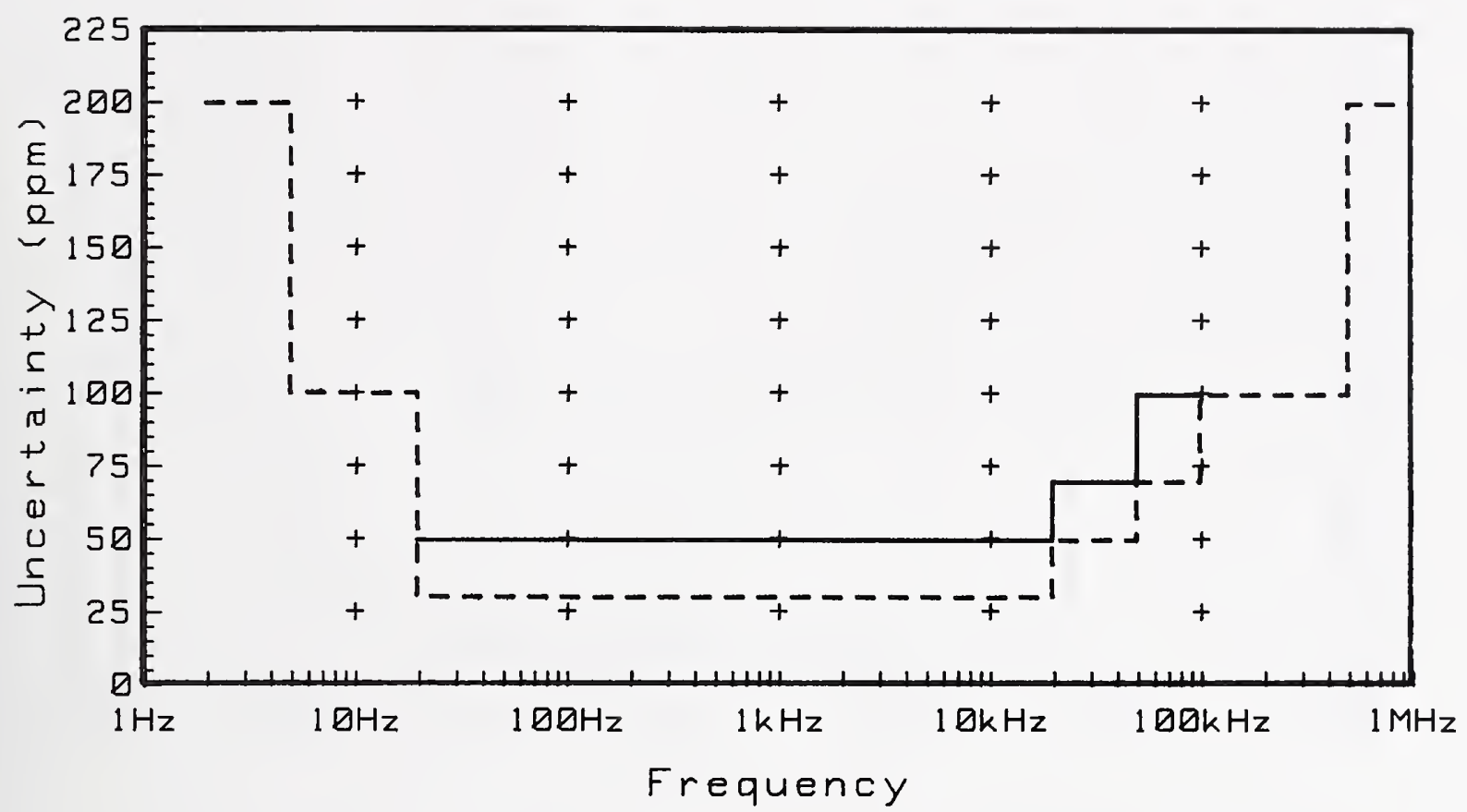

Figure 1. Uncertainties for Multirange Thermal Voltage Converters
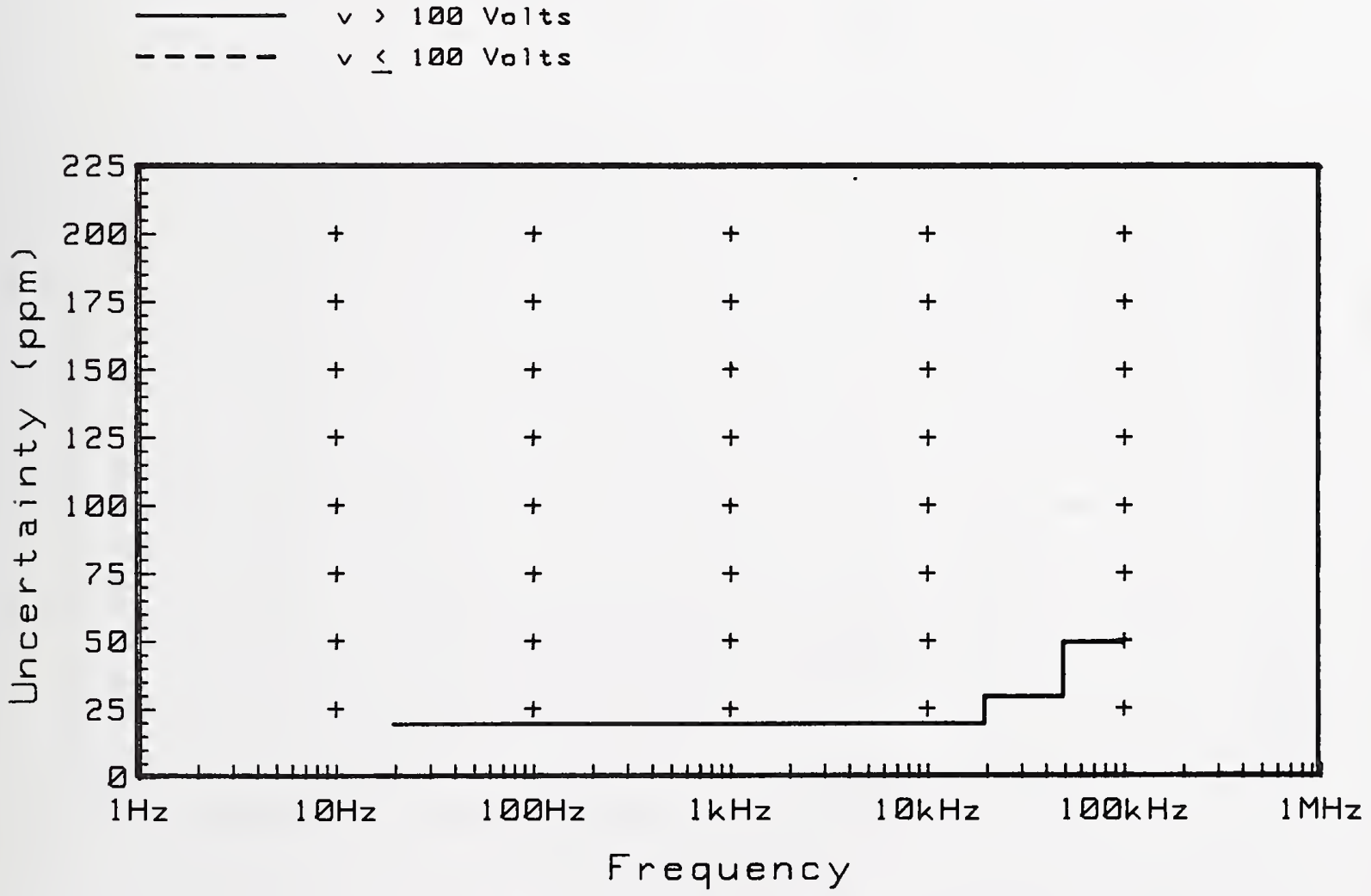

Figure 2. Uncertainties for Coaxial Thermal Voltage Converters 


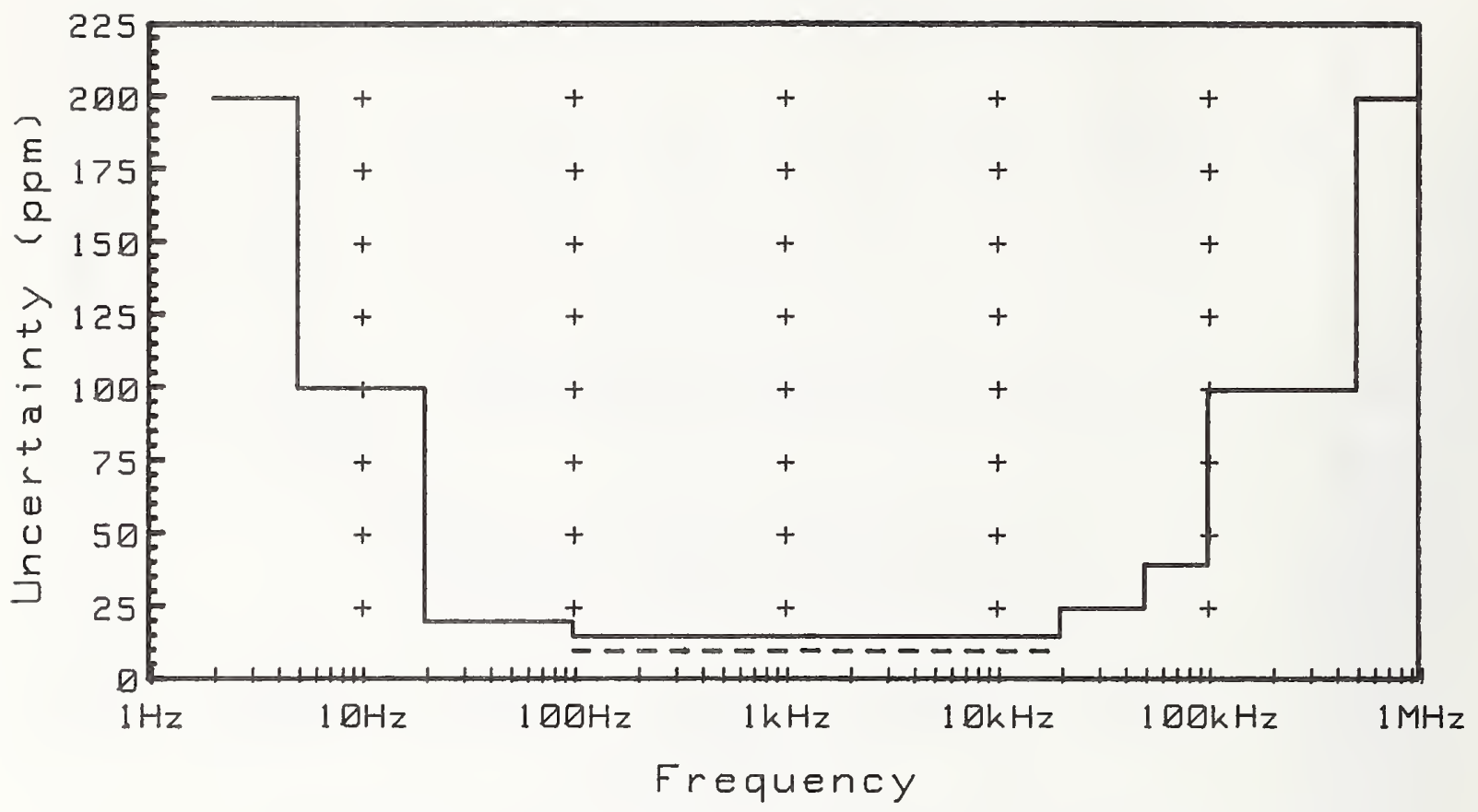

Figure 3. Uncertainties for Coaxial

Thermal Voltage Converters
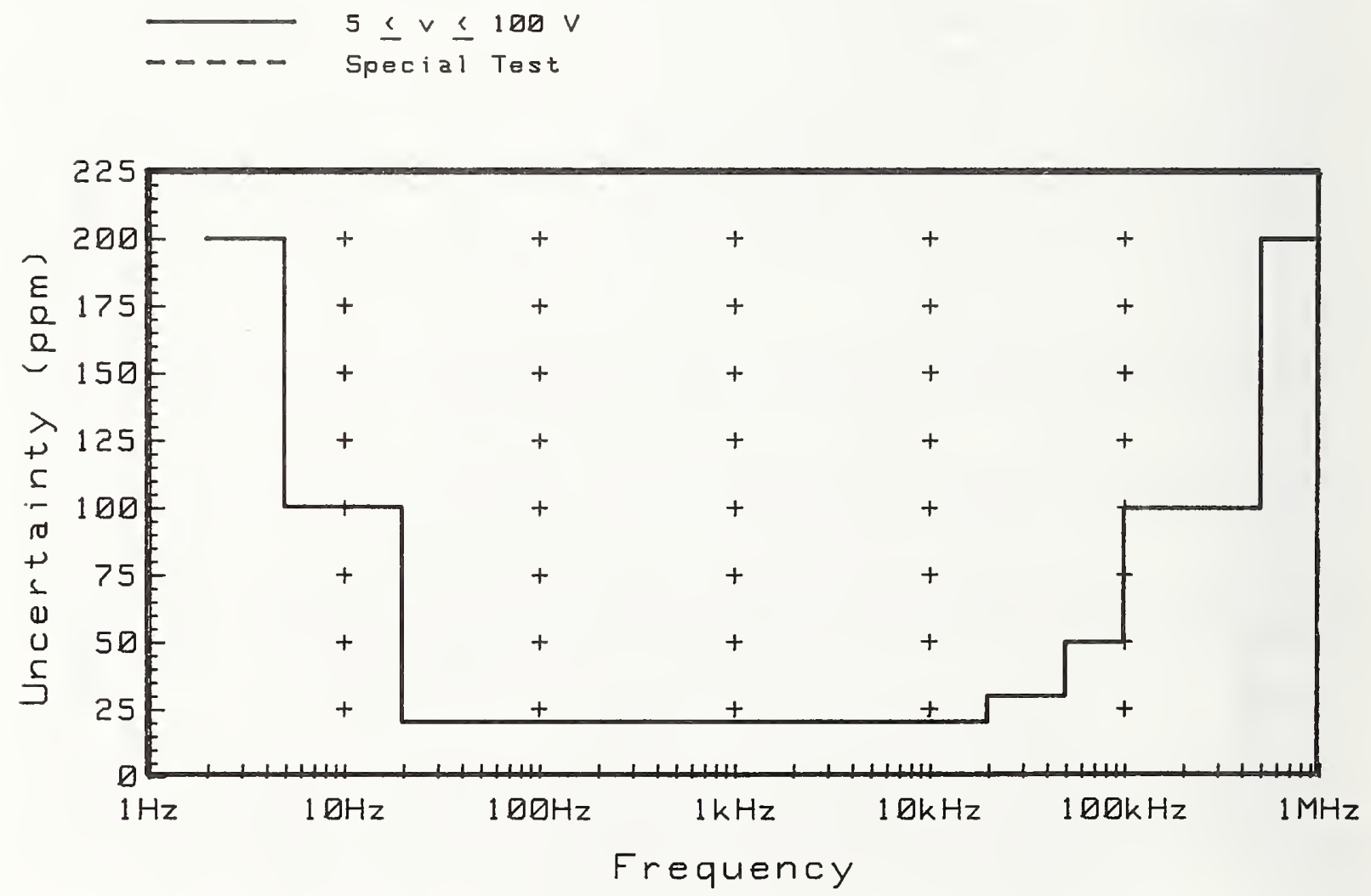

Figure 4. Uncertainties for Coaxial Thermal Voltage Converters 


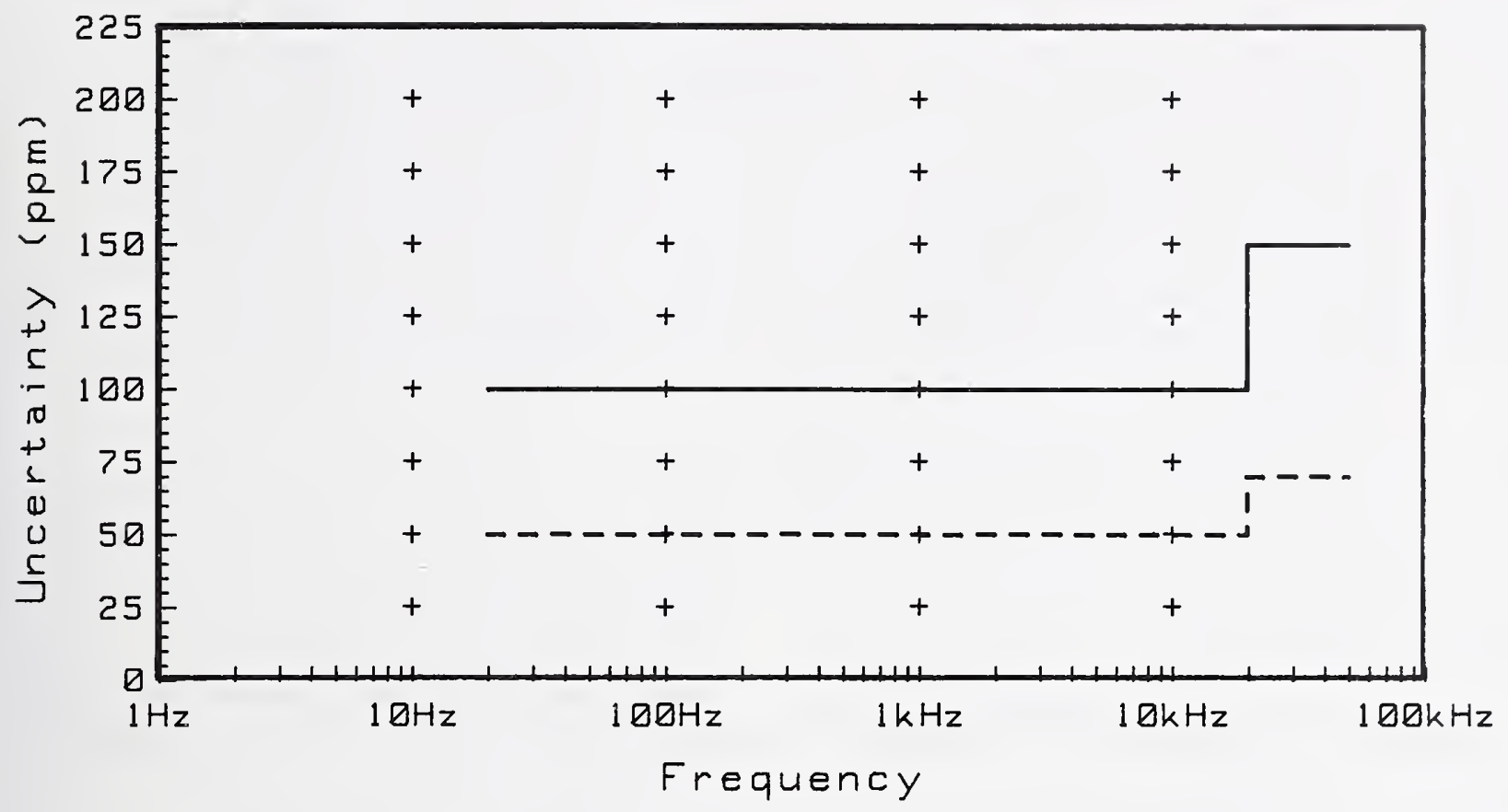

Figure 5. Uncertainties for Thermal Current Converters
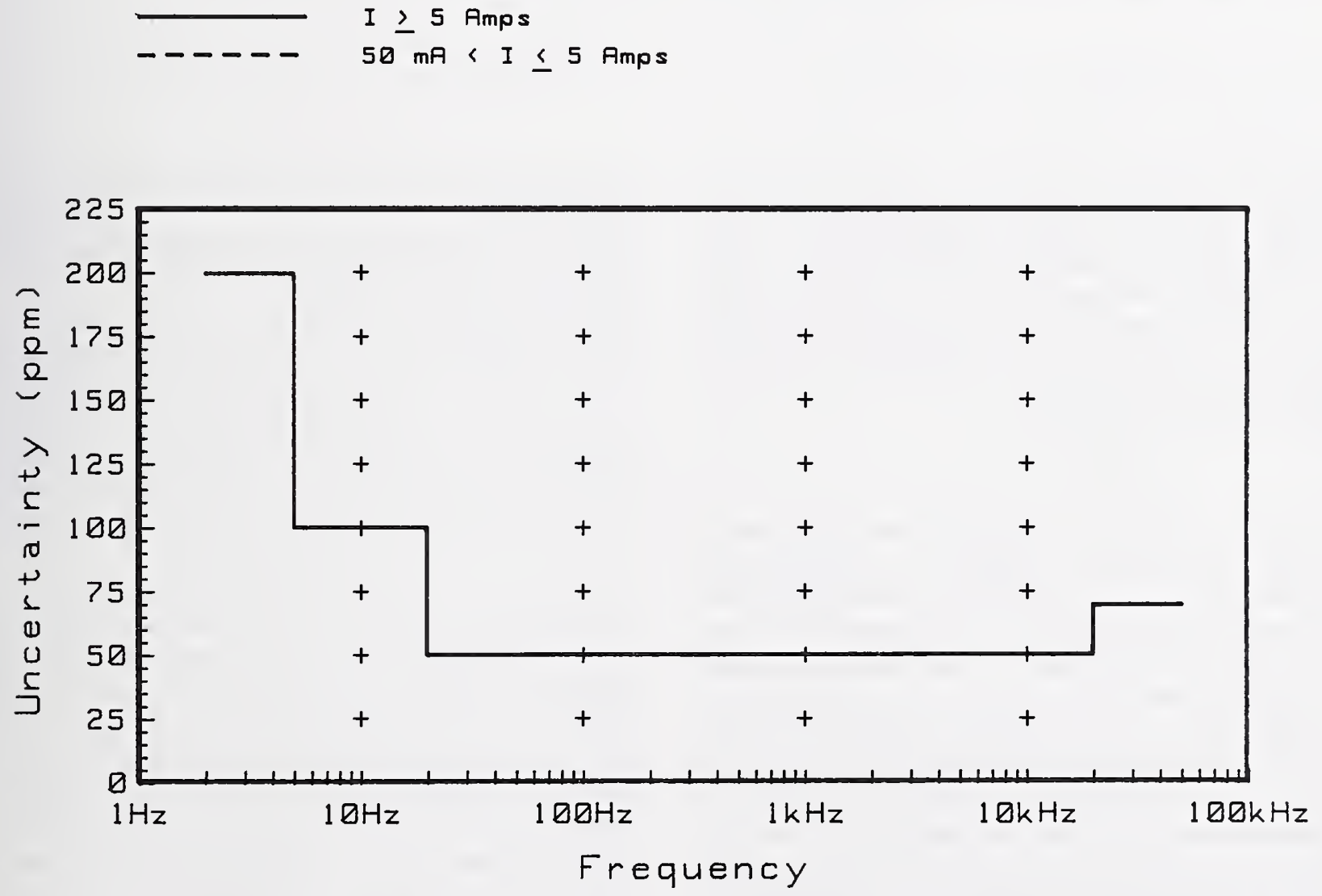

Figure 6. Uncertainties for Thermal Current Converters $I \leq 50$ mAmps 
Section 2

\title{
AC-DC Comparators for Audio-Frequency Current and Voltage Measurements of High Accuracy
}

\author{
FRANCIS L. HERMACH, FELLOW, IEEE
}

\begin{abstract}
This paper surveys recent developments in highaccuracy ac-de comparators, the basic standards for ac current and voltage measurements at audio and higher frequencies. These instruments compare the unknown ac quantity with a de reference. The ac-dc transfer characteristics of thermal converters, the most widely used comparators, can be evaluated to about $10 \mathrm{ppm}$ at audio frequencies in national metrology laboratories. With recent developments even better accuracies, and greater convenience with automatic comparisons, are feasible.
\end{abstract}

\section{Basic PRINCIPLES}

T IS NOW FAIRLY well known that all measurements of ac current and voltage depend fundamentally upon precise ac-dc comparators (ac-dc transfer standards), which either simultaneously or sequentially compare the unknown ac quantity with a known equal or nearly equal dc counterpart. Such comparators are designed and constructed for high precision and small stable ac-dc differences, ${ }^{1}$ but need not have the long term stability of reading required in other instruments. They are used with basic dc standards to evaluate, for example, calibrated ac voltage or current sources. These in turn are used to evaluate instruments such as digital voltmeters,

Manuscript received June 29, 1976.

The author is with the Electricity Division, National Bureau of Standards, Washington, DC 20234.

${ }^{1}$ The ac-dc difference is defined as $\delta=\left(Q_{\mathrm{ac}}-Q_{\mathrm{dc}}\right) / Q_{\mathrm{dc}}$, where $Q_{\mathrm{ac}}$ and $Q_{\mathrm{dc}}$ are the ac and dc quantities (current or voltage) required to produce the same response (output) of the comparator. or ac-dc converters for such instruments. Since accuracies of commercially available sources and instruments now approach 0.01 percent $(100 \mathrm{ppm})$, very high accuracies are required in the ac-dc transfer.

The determination of the ac-dc differences of such comparators has been, and continues to be, an important task of national metrology laboratories. The ampere or the volt is established with direct current or voltage in these laboratories to a few ppm by electrodynamic or electrostatic instruments, which in effect weigh the electrical unit by balancing an electrical force against the force of gravity. The pertinent constants of the instruments must be determined to the full accuracy of the measurement. While in principle this could be done with alternating current or voltage, the difficulties would be greater. The most reproducible or stable references are based on the Josephson effect and the cadmium standard cell, which provide dc standards. For these reasons ac measurements are referred to these dc standards by ac-dc transfer standards whose constants must simply be equal at the two frequencies.

Either the rms, crest, or average value of an ac wave may be compared with a dc value. The rms value is most often desired or required, because it governs the interchange of electrical with other forms of energy. However one of the others may sometimes be more suitable, as in iron-loss or dielectric strength testing.

One of these values cannot be deduced readily from another. This is not academic. As accuracies increase, the 
common error of measuring a voltage or current of unknown waveform with an average- or peak-responding instrument scaled in terms of the rms value of a sine wave can become very serious. Because of their simplicity such instruments are now widely used. To avoid compounding the error they should be calibrated with corresponding peak-to-dc or average-to-dc comparators, or with an ac source having a crest factor or form factor (as needed) equal to that of a sine wave to the required accuracy. Highly accurate peak and average ac-dc comparators have been designed, but only over limited ranges to date.

\section{RMS-DC COMPARATORS}

Classical methods of ac-dc comparison are based on electrodynamic, electrostatic, or electrothermic instruments, and have been described in earlier survey papers [1]-[4]. Each of these comparators responds inherently to the rms value of the voltage or current because of its operating principle and its mechanical or thermal inertia.

For example, the equation governing a conductor heated by a current $i$ with all of the conductor at the same temperature is, at each instant, $i^{2} R=k \theta+M d \theta / d t$, where $R$, $k$, and $M$ are parameters which in general depend upon the temperature rise $\theta$. If the thermal inertia is high enough so that $\theta$ is essentially constant during the cycle then, by termwise integration over one cycle, the steady-state temperature rise is simply $\theta_{a}=I^{2} R / k$, where $I$ is the rms current.

The temperature rise is thus a true measure of the rms current $I$ even if $R$ and $k$ are temperature dependent. This inherent rms response holds under much more general conditions as long as the cyclic variations of $\theta$ are negligible. Note that as an ac-dc comparator, $\theta_{a}$ does not have to be an exact quadratic function of $I$. However, at low frequencies the average value of the cyclically varying $\theta$ is then not correctly a measure of $I$.

Similar relationships hold for electrodynamic and electrostatic instruments, but have not yet been synthesized with high accuracy by electronic means.

\section{ThERMAl CONVERTERS}

The temperature rise of a thin straight conductor carrying a given current is nearly independent of frequency over a wide range because of the low reactance of the wire, and can easily be sensed with high precision by one or more thermocouples. The ac-dc differences of such thermal converters (TC's) are quite small and have been studied extensively in national laboratories over the past few decades. TC's with appropriate electrical "readouts" have almost entirely replaced the other forms of ac-dc comparators, which are at present more limited in accuracy by frequency-dependent effects or in ranges of voltage and current.

A conventional simple TC (in ranges from about 2 to 500 $\mathrm{mA}$ ), consists of a small evacuated glass bulb containing a heater wire $1 / 2$ to $1 \mathrm{~cm}$ long, with the hot junction of a single thermocouple thermally connected to its midpoint by a tiny insulating bead. Thomson and Peltier heating and cooling of the heater can affect the temperature on direct current. ${ }^{2}$ Even when the average of the two directions is taken a small error remains. Because of thermal inertia they do not affect it on alternating current (except at low frequencies), leading to a frequency-independent ac-dc difference [5], [6]. Fortunately this can be reduced to a few ppm or less by proper design and selection of materials. However, exact calculation is questionable, even though appropriate formulas have been developed, because of possible effects of heat treatment on the thermoelectric constants during fabrication.

Many commercial TC's have lead-in wires of magnetic alloys to match the temperature coefficient of the glass bulb. Skin effect in these leads can cause additional ac heating. Even more serious ac-dc differences can occur in voltage measurements because of the increased ac resistance. They are minimized with nonmagnetic leads, which, however, must have low thermal EMF's versus the heater alloy and yet provide a good seal.

Dielectric loss in the insulating bead generally varies with frequency and temperature, and can thus cause significant ac-dc differences. The differences are dependent on current, frequency, and the voltage between the heater and thermocouple.

Thermoelectric errors are negligible in multijunction thermal converters (MJTC's) in which many series-connected thermocouples are spread along the heater. Very ingenious designs have been developed, some with as many as 200 junctions and with bifilar heaters which further reduce these errors to theoretical values far below $1 \mathrm{ppm}$ [7]. They have other advantages as well, such as higher rated output (up to $100 \mathrm{mV}$ instead of 10 ). Investigations have shown, however, that some constructions are subject to dielectric loss and other errors [36].

Series resistors are used with TC's to form thermal voltage converters (TVC's). These may be wire-wound or film-type resistors, switch-selected for convenience in obtaining multiple ranges, typically from 1 to $1000 \mathrm{~V}$. Cylindrical metal-film resistors mounted coaxially in metal cylinders make it possible to compute the effects of residual reactances to $10 \mathrm{MHz}$ or more, at least at low voltages. Intercomparisons of adjacent ranges of such sets of TVC's provide valuable confirmation and also assurance against other sources of ac-dc differences (which are not likely to be the same for all ranges). Such tests can be made to a few ppm at audio frequencies [8].

With two TC's of different current ratings (for example, 2.5 and $5 \mathrm{~mA}$ ), a single series resistor can serve for two voltage ranges. With only 6 resistors in a typical set, ranges from 1 to $1000 \mathrm{~V}$ can be obtained. If they are properly designed and constructed, only 7 intercomparisons are necessary to determine their relative ac-dc differences [9]. Similarly, shunt resistors can be used with two TC's of different voltage ratings to make convenient thermal

\footnotetext{
2 They also cause de reversal differences when the bead is not at the exact thermal midpoint.
} 


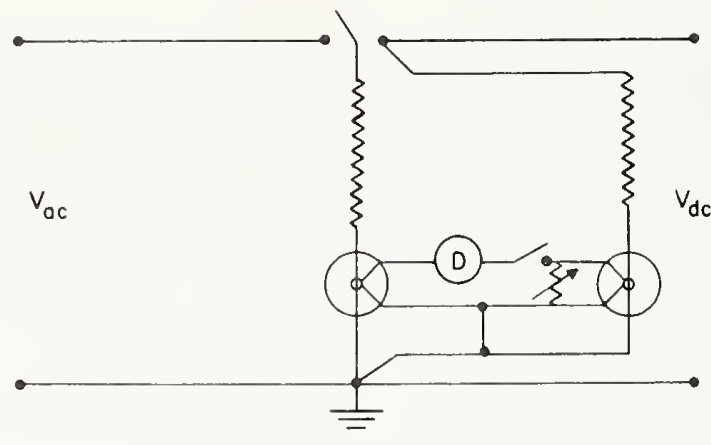

Fig. 1. Simultaneous ac-de comparator.

current converters (TCC's), with ranges up to $20 \mathrm{~A}$ or more.

Transformers can also be used to provide voltage ranges. Only the heater of the TC needs to be switched to the dc reference. Excellent accuracy is possible at midaudio frequencies, but the design of suitable transformers for wide voltage and frequency ranges is difficult.

Simultaneous ac-dc comparators have been developed, to reduce drifts from ambient and self-heating teinperature changes, which can sometimes be serious. These usually have dual circuits, as shown for one such arrangement in Fig. 1 [10]. One TVC is supplied by the ac voltage and the other by a known dc voltage with the TVC outputs connected in opposition to a null detector. Unfortunately the input-output characteristics of TC's are not equal, (do not "track") even when equalized at one level. ${ }^{3}$ Thus for highest accuracy, a preliminary adjustment to null the detector with both converters connected to the same current or voltage is generally required. This is accomplished with the adjustable resistor shown in Fig. 1, which forms a voltage divider with the internal resistance of the thermocouple (not indicated).

Alternatively, the null-balance adjustment can be made at a fixed dc current level, which is supplied to both heaters by a Zener-diode reference. The heater of one TC is then switched to a decade range resistor (or an amplifier with gain-control resistors) for the ac measurement. With proper choice of components the resistor can be direct reading in volts when it is adjusted to obtain the same output balance [11], [12].

Similar arrangements can be used with indirectly heated thermistors, each consisting of a small thermistor bead in thermal contact with the heater wire. Appropriate bridge circuits have been developed for the output balances. High sensitivity is obtainable because of the high negative temperature coefficients of the thermistors [13], [14].

Such simultaneous comparators, with TC's or thermistors, can provide known fixed ac reference currents or voltages for ac potentiometers (and are sometimes called "ac standard cells"). In a true potentiometer an unknown voltage (or a definite fraction of it) is balanced against an adjustable known voltage through an appropriate null detector. For ac measurements the two voltages must be of exactly the same frequency, magnitude and phase. Thus the known voltage must be phase-locked to the unknown and must be adjustable with high accuracy without affecting the stability of the reference. In addition, with the usual tuned detector, only the fundamental component of the unknown is measured. For these reasons ac potentiometers have been used in the USA chiefly for special measurements, when both phase and magnitude information are required.

The heater of a TC is easily burned out by overloads. This serious disadvantage can now be overcome, however, by rapidly acting relays or electronic circuits, which are available with some comparators [15]. This is very desirable, provided that normal changes in the components do not affect the ac-dc difference.

The continuing development of ac-dc comparators makes standardization of accuracy classes, voltage and frequency ranges, etc, impracticable. However, standardization and agreement on terminology (including accuracy), general requirements, and test methods is very desirable, and has been accomplished in American $\mathrm{Na}$ tional Standard C100.4 [16].

\section{USE OF THERMAL CONVERTERS}

For the measurement of an ac voltage, or the calibration of an ac voltmeter, the TVC is connected as shown in Fig. 2. With $V_{\text {ac }}$ applied, the output EMF is balanced against a stable dc source with a null detector (not shown). The TVC is switched to a known dc voltage, which is adjusted to give the same output EMF. The dc voltage is reversed and readjusted. The detector null should be checked with the ac voltage applied to guard against drifts. Then $V_{\mathrm{ac}}=$ $V_{\mathrm{dc}}(1+\delta)$, where $\delta$ is the fractional ac-dc difference of the TVC and $V_{\mathrm{dc}}$ is the average for the two directions.

Alternatively, if the detector is linear, its scale can be calibrated by adjusting the dc voltage by a small known amount $\Delta V$ and noting the detector change $\Delta D$. A fixed dc voltage (nearly equal to the ac voltage) can be set as a convenient reference, and the detector reading can be recorded at each step in a sequence such as $V_{\mathrm{dc}}+, V_{\mathrm{ac}}, V_{\mathrm{dc}}-$. Then $V_{\mathrm{ac}}=V_{\mathrm{dc}}\left[1+\delta+s\left(D_{\mathrm{ac}}-D_{\mathrm{dc}}\right)\right]$, where $D_{\mathrm{ac}}$ and $D_{\mathrm{dc}}$ are the detector readings with $V_{\text {ac }}$ applied and the average of the readings with the two directions of $V_{\mathrm{dc}}$ applied, respectively, and $s=\Delta V / V \Delta D$. Errors from drifts are minimized if the steps are taken at equal time intervals.

The switches and output circuit should be shielded to avoid interaction. Coaxial and shielded leads should be used as shown, to minimize induced voltages, particularly at the test frequency. The voltage drops in the leads between the ac-dc switch and $V_{\mathrm{ac}}$ and $V_{\mathrm{dc}}$ should be equal, and errors from ground currents must be evaluated. ${ }^{4}$

Similar techniques are used for ac current measurements, but careful analysis of ground-current errors is

${ }^{4}$ If a grounded instrument is used to measure $V_{d c}$ it should be connected between the two switches. 


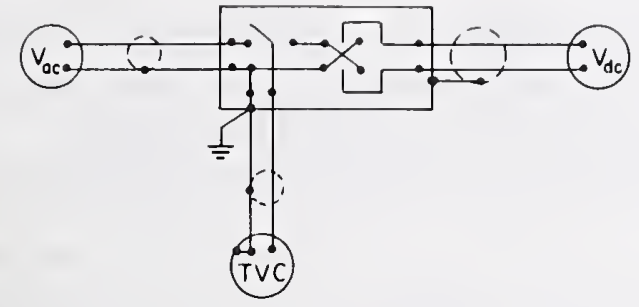

Fig. 2. Sequential ac-dc transfer.

needed, particularly in the test of another instrument which must itself be grounded.

A typical commercially available comparator has a built-in ac-dc transfer switch, and an adjustable stable dc source in series with a detector to balance the output EMF. Most of them do not have dc reversal switches, however, generally relying on TC's with low dc-reversal differences. This should be checked periodically at two or more heater currents, for the error in the voltage measurement is $1 / 2$ this difference, and can be much larger than the ac-dc difference.

Standard C100.4 describes some simple yet practical ways to detect and guard against ground-current errors, radio-frequency interference and other errors in the intercomparison of two current or voltage comparators. These techniques can also be applied when ac-dc comparators are used for ac measurements or for the calibration of ac instruments. Comparators with 3- or 4-terminal inputs (with properly designed shields and driven guards) greatly reduce circuit-connection errors, but are not widely used.

If the ac-dc difference of the comparator is known and applied as a correction along with corrections for the dc standards, and if proper techniques are used to made valid measurements, it is not at all necessary that the "accuracy of the standard shall be ten times that of the test instrument" as is so often stated. The uncertainty in the ac measurement is simply the sum of the residual errors in the standards and the random error in the test (caused by both the standard and test instrument). In this way maximum use is made of the inherent accuracy of the ac-dc comparator and the dc standards. The application of such corrections to remove determined errors avoids this $10 / 1$ noose, which often strangles echelons of laboratories with unnecessary accuracy requirements.

\section{AUTOMATIC COMPARATORS AND CONVERTERS}

All of these manual procedures are time consuming and tedious. Automatically-switched comparators and ac-dc converters (which generate a dc voltage accurately proportional to the input) have been developed to simplify the measurements. In some automatic comparators, essentially the same procedure is followed as in manually operated instruments (with TC's of low dc reversal difference and drift to simplify the ac-dc sequence). In others, the switching is done quite rapidly (at about $1 \mathrm{~Hz}$, for example)

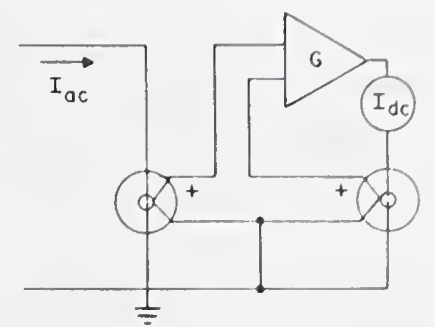

Fig. 3. Basic TC feedback circuit

to overcome drifts. The ac component at the switching frequency in the detector is a measure of the difference between the ac and dc voltages, either directly or through a feedback circuit [17]. Gain-stabilized amplifiers provide wide voltage ranges conveniently, with minimum loading.

For rms measurements many ac-dc converters are based on the feedback circuit shown in simplified form in Fig. 3 [18]. When $I_{\mathrm{ac}}$ is applied, $I_{\mathrm{dc}}$ increases until the outputs of the two matched TC's are nearly equal. If the transconductance of the amplifier is $G$ and $E=k I^{2}$ for each TC, $I_{\mathrm{dc}}=G k\left(I_{\mathrm{ac}}^{2}-I_{\mathrm{dc}}^{2}\right)$. Then if $a=1 / G k I_{\mathrm{dc}} \ll 1, I_{\mathrm{dc}} \approx I_{\mathrm{ac}}$ $\sqrt{1-a} \approx I_{\mathrm{ac}}$. Interestingly, this is an rms instrument with a linear scale (except near zero).

To avoid tracking errors, decade input resistors, or amplifiers controlled by decade feedback resistors, can provide appropriate voltage ranges at a fixed current. Alternatively a dual-heater MJTC can be used with fixed resistors for tracking to about 0.05 percent over a range of currents.

Other types of converters have also been developed and used in this general feedback circuit. One uses dual monolithic thermoelements, with two resistors and two temperature-sensing transistors on a single chip [19]. In another, two indirectly heated thermistors, each with dual heaters, are used with dual feedback so that each thermistor is always at the same temperature, to avoid tracking errors [20].

These feedback circuits greatly reduce drifts and the effective response time of the TC's (which is about $1 \mathrm{~s}$ ). However the ac-dc differences of the input resistors and amplifiers in these instruments must be small and stable. In addition, the design of the feedback amplifier is complicated by stability problems and by the double-frequency ripple in the TC output at low frequencies.

A number of electronic techniques for $\mathrm{rms}$ to dc converters have been developed, based on approximating a square-law function with straight-line segments or with an appropriate transistor characteristic. Feedback circuits have made these more practical [21]. They have shorter response times and wider ranges than thermal converters.

\section{OTHER AC-DC COMPARATORS}

Electrodynamic and electrostatic instruments have been used as ac-dc comparators. The frequency range of an electrodynamic instrument, however, is limited by the 
reactances of the coils. The range can be much greater for an electrostatic instrument, but the torque/weight ratio is low at moderate voltages, and surface films can cause ac-dc differences that are difficult to evaluate. With both types the double-frequency periodic variations in the ac torque can cause vibrations in parts of the moving system, with resultant errors. It is difficult, also, to obtain a resolution of a few ppm even with light-beam pointers. For all of these reasons these instruments find their place mainly in national metrology laboratories, where these problems can be studied at length and overcome [22]-[24].

Putting a rectifier in the feedback loop of a suitable operational amplifier greatly reduces the effects of the rectifier's imperfections. Refinements of this arrangement have made average-responding ac-dc comparators and converters of $20 \mathrm{ppm}$ accuracy possible [25]. Instruments with electronic or electromagnetic relays which compare the crest (peak) value with a dc voltage have also been refined to better than $50 \mathrm{ppm}$, at least at low audio frequencies [26], [27]. Rapid electronic sampling and computing techniques, which measure the instantaneous value of the wave at selected intervals, now make it possible to compute the rms, crest and average values, and thus to determine crest- and form-factors with high accuracy as well [28], [29].

\section{Evaluation OF AC-DC COMPARATORS}

The ac-dc differences of well-designed and well-constructed comparators are small and quite stable. In many cases they need not be redetermined, once known, if all adjacent ranges (such as 10 and $20 \mathrm{~V}$ ) can be compared to detect changes. The evaluation of ac-dc differences in an absolute sense is difficult and time consuming. It has generally been attemptè only at national metrology laboratories, which can carefully establish and study ac-dc transfer standards, develop the necessary precise intercomparison apparatus, and then use this equipment to calibrate the comparators of many other laboratories. In this way the standards receive wide use in establishing compatability and accuracy in these measurements. This is most economical, too, because the results of the research are then available to all users. Such services are important functions of national laboratories.

These "absolute" ac-dc standards must be investigated both theoretically and experimentally. This usually involves 1) theoretical analyses of sources of ac-dc errors, 2) design and construction of standards that minimize these errors, 3 ) comparisons and other tests of these standards to evaluate the errors, and 4) comparison with another type of transfer standard which has also been carefully studied.

The last step is very desirable to guard against errors from unknown sources, but it implies the development of different types of standards of nearly equal precision and ac-dc'accuracy. At the present time other types cannot match thermal converters in ranges or accuracy. Thus comparisons of different kinds, constructions and ranges of 'TC's, which are free of known sources of error, are vital. If these converters agree in ac-dc difference it is unlikely that they have a common source of error, because of the differences in their design and construction.

Remarkably stable ac and dc power supplies are now commercially available, with rms fluctuations of only a few ppm in a minute or so. Extraordinary stabilities $(0.5$ $\mathrm{ppm} / \mathrm{h}$ ) have been achieved in recent special laboratory designs [30], [31]. These, together with techniques which largely cancel the effects of drifts and of small supply variations, have made possible high precision in the intercomparison of TC's and TVC's.

Careful evaluations of TVC's from 1 to $1000 \mathrm{~V}$ and TCCs from $1 \mathrm{~mA}$ to $1 \mathrm{~A}$ or more have been made in national laboratories to about $10 \mathrm{ppm}$ at audio frequencies, with reduced accuracies at higher frequencies [8], [32], [33], [34]. Comparisons with electrostatic and crest-responding instruments in these laboratories have provided valuable confirmation of the absence of large errors. At the Electrotechnical Laboratory in Japan an electrostatic voltmeter is planned, to provide the basic ac-dc transfer above $1 \mathrm{kV}$ [35].

The development of MJTC's now makes even better accuracy possible. At NBS MJTC's of different manufacture and range have been compared, with a standard deviation of less then $0.2 \mathrm{ppm}$. A number of sources of error which cause MJTCs to depart from the ideal have been uncovered, and either overcome in new designs or compensated. The ac-dc difference of a group of 8 TVC's ( 6 to $9 . \mathrm{V}$ ) is believed to be less than $0.3 \mathrm{ppm}$ at $160 \mathrm{~Hz}$, the frequency of an important absolute volt determination, and 0.5 ppm from 30 to $10,000 \mathrm{~Hz}$ [36].

An intercomparison among several national laboratories of 4 sets of TC's and TVC's is now almost finished. It should provide a better basis for international agreement on the ac-dc transfer of current and voltage from $40 \mathrm{~Hz}$ to $50 \mathrm{kHz}$.

\section{Conclusions}

At the present time research and development have provided ac-dc comparators which have the accuracy needed (about $10 \mathrm{ppm}$ ) to support commercial developments in ac current and voltage measurements at audio frequencies. With electronic aids, more rapid and convenient automated comparators are now feasible and are beginning to appear. There is no slackening in the importance or development of this field of measurements.

A cataloging of all these developments would be neither feasible nor digestible in a survey paper such as this. The basic principles of a number of them have been highlighted, instead, to illustrate the present status and recent trends in high-accuracy ac measurements.

\section{REFERENCES}

The following references in the open (published) literature were selected because of their general nature or extensive bibliographies, or because they illustrate recent 
developments. A list of references published between 1945 and 1975 on high accuracy audio-frequency ac-dc current and voltage comparators is available on request from the Electrical Instruments Section, National Bureau of Standards, Washington, DC 20234.

[1] M. Iwamura and T. Yamazaki, "General review of maintenance of alternating current standards," Circular No. 147, ETL, Dec. 1957. (In Japanese with English abstract, 81 references.)

[2] F. L. Hermach, "Ac-dc transfer instruments for current and voltage measurements," IRE Trans. Instrum., vol. I-7, pp. 235-240, Dec. 1958.

[3] T. B. Rozhdestvenskaya, "Electrical comparators for measurements of current, voltage and power," JPRS 29373, 187 pp., 1965, National Technical Information Service, Springfield, VA 22161. (English translation of a Russian Monograph with 112 references.)

[4] H. Helke, "Ac-dc converters and comparators for alternating current," Arch. Tech. Messen, no. J942-2, pp. 227-232, Oct. 1967; no. J942-3, pp. 255-260, Nov. 1967; no. J942-4, pp. 11-13, Jan. 1968. (In German, 137 references.)

[5] F. L. Hermach, "Thermal converters as ac-dc transfer standards for current and voltage measurements at audio frequencies," J. Res. Nat. Bur. Stand., vol. 48, pp. 121-138, Feb. 1952.

[6] F. C. Widdis, "Theory of Peltier- and Thomson-effect errors in thermal ac-dc transfer devices," Proc. Inst. Elec. Eng., vol. 109, pt. C (Monograph 497 M) pp. 328-334, 1962.

[7] F. J. Wilkins, "Theoretical analysis of the ac/dc transfer difference of the NPL multijunction thermal converter over the frequency range DC to $100 \mathrm{kHz}$," IEEE Trans. Instrum. Meas., vol. IM-21, pp. 334-340, 1972.

[8] F. L. Hermach and E. S. Williams, "Thermal converters for audio frequency voltage measurements of high accuracy," IEEE Trans. Instrum. Meas., vol. IM-15, pp. 260-268, 1966.

[9] E. S. Williams, "Thermal voltage converters and comparators for very accurate ac voltage measurements," J. Res. Nat. Bur. Stand., vol. $75 \mathrm{C}$, pp. 145-154, 1971.

[10] F. L. Hermach, J. E. Griffin, and E.S.Williams, "A system for accurate direct and alternating voltage measurements," IEEE Trans. Instrum. Meas., vol. IM-14, pp. 215-224, 1965.

[11] W. Rump, "On the exact absolute measurement of a-c voltages and a potentiometer for calibrating a-c instruments," Elektrotecknik, vol. 5, pp. 64-67, 1951 (in German).

[12] J. E. Griffin and F. L. Hermach, "A differential thermocouple voltmeter," AIEE Trans., vol. 81-I, pp. 339-345, 1962.

[13] F. C. Widdis, "The indirectly heated thermistor as a precise ac-dc transfer device," Proc. Inst. Elec. Eng., vol. 103B, pp. 693-703, 1956.

[14] H. J. Schrader, "A-c current measurements with indirectly heated NTC resistors," ETZ, vol. 73A, p. 547, 1952 (in German).

[15] "IR sensor protects transfer standard," Electronics, vol. 48, pp.
36-37 Oct 16,1975

[16] "AC-DC transfer instruments and converters," American National Standard, C100.4, 1973. ANSI, 1430 Broadway, New York, NY 10018 .

[17] L. G. Cox and N. L. Kusters, "An automatic rms/dc comparator," IEEE Trans. Instrum. Meas., vol. IM-23, pp. 322-325, 1974

[18] R. Gilbert and J. Miller, "Thermal converter," U.S. Patent 2857 569,1958

[19] W. E. Ott, "A new technique of thermal rms measurement," IEEE $J$ Solid State Circuits, vol. SC-9, pp. 374-380, 1974

[20] P. Richman, "A new wideband true rms-dc converter," IEEE Trans. Instrum. Meas., vol. IM-16, pp. 129-134, 1967.

[21] P. Richman and W. Walker, "A new fast-computing rms/dc conversion," IEEE Trans. Instrum. Meas., vol. IM-20, pp. 313-319, 1971.

[22] W. Smith and W. Clothier, "Determination of the dc-ac transfer error of an electrostatic voltmeter," J. Inst. Elec. Eng., vol. 101, pp. $465-469,1954$.

[23] T. Yamazaki, S. Iwamoto and T. Ando, "Estimation of the ac/dc differences of electrostatic voltage comparator," Bull ETL, vol. 32 pp. 1085-1099, 1968.

[24] G. Schuster, "A high resolution electrodynamic ac-dc power transfer instrument," IEEE Trans. Instrum. Meas., vol. IM-23, pp. 330-333, 1974.

[25] L. A. Marzetta and D. R. Flach, "Design features of a precision AC-DC converter," J. Res. Nat. Bur. Stand., vol. 73C, pp. 47-55, 1969.

[26] P. Richman, "A new peak-to-dc comparator for audio frequencies," IRE Trans. Instrum., vol. I-11, pp. 115-122, 1962.

[27] D. R. Flach and Louis A. Marzetta, "Calibration of peak ac to dc comparators," Proc. ISA, paper no. 14.2-3-65, 1965.

[28] F. Deist and R. Kitai, "Digital transfer voltmeters," Proc. Inst. Elec. Eng., vol. 110, pp. 1887-1904, 1963

[29] R. S. Turgel, "Digital wattmeter using a sampling method," IEEE Trans. Instrum. Meas., vol. IM-23, pp. 337-341, 1974.

[30] H. Schoenwetter, "An ultra stable power supply for an absolute volt determination," Metrologia, vol. 10, pp. 11-15, 1974.

[31] G. Schuster, "A precision stabilized sine wave source for AC power measurements," IEEE Trans. Instrum. Meas., vol. IM-22, pp. 391-394, 1973.

[32] S. Iwamoto and H. Hirayama, "Ac/dc thermal converters of the ETL," IEEE Trans. Instrum. Meas., vol. IM-23, pp. 326-329, 1974.

[33] C. H. Dix, "Electrical standards of measurement, Pt 1, dc and low frequency standards," Proc. Inst. Elec. Eng., vol. 122, no. 10R, pp. 1018-1036, 1975

[34] O. P. Galakhova and T. B. Rozhdestvenskaya, "State of the standardization of references and methods of $\mathrm{dc}$ and ac voltage measurements," Meas. Tech., vol. 18, pp. 1339-1341, Sept. 1975.

[35] K. Shida, "Ac-dc high voltage comparator with balance," Bull. ETL, vol. 39, pp. 415-420, 1975 (in Japanese).

[36] F. L. Hermach and D. R. Flach, "An investigation of multijunction thermal converters," to be published. 


\section{AC-DC Transfer Instruments for Current and Voltage Measurements ${ }^{*}$}

FRANCIS L. HERMACH $\dagger$

\section{INTRODUCTION}

T HE basic electrical units are main tained by groups of standard cells and resistors. With these standards and a dc potentiometer, measurements of direct voltage and current are readily made with an accuracy of 0.01 per cent or better. Corresponding measurements of alternating voltage and current at power and audio frequencies depend, at the present time, upon ac-dc transfer instruments which have nearly equal ac and $\mathrm{dc}$ response. Such instruments may be calibrated on direct current and then used on alternating current, or alternatively, may be used directly to make ac-dc difference measurements, as in the certification of other instruments.

In addition to the instantaneous values as determined by an oscilloscope or point-by-point methods, there are at least seven other quantities that may be determined for a periodic ac wave in which all values separated by the periodic time, $T$, are equal. These are: 1) the rms or effective value, $I$, defined by

$$
I^{2}=\frac{1}{T} \int_{0}^{T} i^{2} d t
$$

where $i$ is the instantaneous value;2) and 3 ) the positive and negative average values, i.e., the average of all the positive or all of the negative values during one cycle, defined by

$I_{a+}=\frac{1}{2 T} \int_{0}^{T}(|i|+i) d t$ and $I_{a-}=\frac{1}{2 T} \int_{0}^{T}(|i|-i) d t$

where $|i|$ is the magnitude of $i ; 4)$ the rectified fullwave average, defined by

$$
I_{a r}=\frac{1}{T} \int_{0}^{T}|i| d t
$$

5) and 6) the positive and negative crest or peak values; and 7) the crest-to-crest or peak-to-peak value.

Instruments for measuring each of these values of an ac voltage or current wave are commercially available (with widely varying accuracies). Ordinarily, however, only one of these values is really required in a measurement. Unfortunately, it is difficult to deduce accurately one value from the measurement of another, except for a few waveforms (which, of course, must be known). In most cases the rms value is the one really desired, since the rate of transformation of electrical to other forms of energy (which is governed by the heating produced by a current and by the electromagnetic force produced by currents acting on each other) is a function of the square of this value.

The three general types of ac-dc transfer instruments which are at present suitable for rms measurements of better than 0.1 per cent accuracy are dependent upon these same laws of the interchange of energy. They are: 1) electrodynamic instruments, which depend upon the force between current-carrying conductors; 2) electrostatic instruments, which depend upon the force between charged conductors; and 3) electrothermic instruments, which depend upon some effect produced by the heating of a current-carrying conductor. The first and third respond essentially to current and the second to vol tage, but series or shunt resistors make all three types suitable for both measurements, while other circuit arrangements make power measurements almost equally feasible.

\section{REsponse Equations}

The instantaneous torque of an electrodynamic instrument in which two sets of conductors, one fixed and one rotatable about an axis, carry the same current, $i$, is $^{1}$

$$
\tau=i^{2} \frac{d M}{d \theta}
$$

where $M$ is the mutual inductance and $\theta$ some welldefined angle between the two sets of conductors.

In its simplest form such as instrument has an opposing torque, $U \theta$, so that, with direct current applied, the rest position, $\theta_{f}$, is defined by

$$
\left.U \theta_{f}=B_{1} I^{2} \quad \text { where } \quad B_{1}=\frac{d M}{d \theta}\right]_{\theta=\theta_{f}} .
$$

The differential equation governing the angular deflection is ${ }^{1}$

$$
P \theta^{\prime \prime}+A \theta^{\prime}+U \theta=B i^{2}
$$

where $P$ and $A$ are the inertial and damping constants, respectively. If the mechanical inertia is sufficiently great that with alternating current applied the periodic fluctuations of $\theta$ are negligible compared with the average value, termwise integration over an integral number of cycles gives

${ }^{1}$ F. K. Harris, "Electrical Measurements," John Wiley and Sons, Inc., New York, N. Y., pp. 47 and 413; 1952. 


$$
U \theta_{f}=\frac{B_{1}}{T} \int_{0}^{T} i^{2} d t=B_{1} I^{2} .
$$

Thus if $U$ and $B$ each have the same value on direct as on alternating current, the instrument has the same response, $\theta_{f}$.

The instantaneous torque of the usual two-element electrostatic instrument with an applied voltage, $v$, is

$$
\tau=\frac{v^{2}}{2} \frac{d C}{d \theta}
$$

where $C$ is the capacitance between the elements. Similar considerations lead again to identical expressions for the rest position with direct or alternating voltage applied, viz.,

$$
\left.U \theta_{f}=B_{2} V^{2} \quad \text { where } \quad B_{2}=\frac{1}{2} \frac{d C}{d \theta}\right]_{\theta=\theta j} .
$$

With reasonable restrictions, the temperature rise, $\theta$, of a thin homogeneous conductor carrying a current $i$ in the $x$ direction is governed by the differential equation

$$
\frac{\partial^{2} \theta}{\partial x^{2}}-\frac{H \theta}{K}+\frac{i^{2} R}{K}=\frac{1}{D} \frac{\partial \theta}{d t}
$$

where $D$ is the thermal diffusivity and $R, K$, and $H$ are the electrical resistance, thermal conductance, and rate of surface heat loss per degree rise of a unit length of the material. Again, if the "thermal inertia" is sufficiently great that periodic fluctuations in temperature are negligible, the average temperatures are equal with equal direct and rms alternating currents.

In each instrument the equality holds even if the "constants" such as $U, B, R, D, H$, and $K$ in the equations are dependent upon the response, $\theta$. This important advantage stems from the property that each instrument combines in a single measuring element a function proportional to the square of the instantaneous current or voltage, a restraining function, and an inertial function or "flywheel effect" to enable it to integrate so that the time-average value of the response is proportional to the square of the rms current or voltage. For this reason high accuracy may be more easily attained with these instruments than with other squaring devices or circuits, which must synthesize exact and equal square-law responses in two quadrants (or in one quadrant with an accurate rectifier) and provide a separate integrator. However, each of these three types of instruments has a lower limit of frequency below which the average value of the response is incorrect if the constants are dependent upon $\theta$.

\section{TRANSFER INSTRUMENTS}

It is easier in an actual instrument to insure that the constants in the equations are indeed equal on direct and alternating current than it is to measure them separately and compute the response of the instrument. Thus with few exceptions ${ }^{2}$ absolute instruments, such as the electrodynamic current balance, ${ }^{3}$ are used solcly for dc measurements, and most ac instruments of high accuracy are calibrated in terms of dc standards. In the usual instruments, this calibration is preserved on a scale, and the instrument is tested or standardized periodically to guard against changes in the factors which affect the response. However, if the instrument is calibrated at the time of each use, long-time stability, extremely low temperature coefficients, and other normally desirable characteristics become of secondary importance, and the instrument may be designed and constructed to have high resolution and the best. possible frequency characteristic. Indeed, in a standardizing laboratory in which the standard is used primarily to test other instruments, a dc calibration may not be necessary because most instruments which are submitted to such laboratories can also be used on direct as well as alternating current. The scale calibration of such instruments may be verified on direct current with a potentiometer (taking the mean of the values required to obtain the same deflection for the two directions of applied current or voltage). The ac-dc difference or frequency influence may then be determined by connecting the instrument under test and the transfer instrument so that they respond to the same quantity (current, voltage, or power) and successively switching both to alternating-, direct-, reversed-direct, and alternating current. In each case the measured quantity is adjusted to obtain the same deflection of the test instrument and the differences in deflection of the standard instrument are observed. From these differences and the scale factor of the standard instrument (per cent change per division) the ac-dc difference of the test instrument may be determined.

This procedure has been used for over 40 years in the certification of instruments at the National Bureau of Standards, because it separates several sources of errors and can be carried out with great accuracy. Since the ac-dc differences of a well-designed instrument are small within its working frequency range and are relatively permanent, they need not ordinarily be redetermined, so that the periodic tests of the instrument need be made only with direct current.

For either a direct ac measurement or an ac-dc difference determination, however, the ac-dc difference of the standard instrument must be known to the full accuracy of the measurement. The determination of this difference has been recognized as a major problem in

${ }^{2}$ H. B. Brooks, F. M. Defandorf, and F. B. Silsbee, "An absolute electrometer for the measurement of high alternating voltages," J. Res. NBS, vol. 20, pp. 253-316; 1938.

${ }^{3}$ R. L. Driscoll and R. D. Cutkosky, "Measurement of current with the NBS current balance," J. Res. NBS, vol. 60, pp. 297-305; 1958. 
most national standardizing laboratories. In general, there are three rather distinct steps in a complete determination. These are: 1 ) choice of two different types of instruments that are inherently suitable as ac-dc transfer instruments, and a theoretical study of all known effects that can cause ac-dc differences in each type; 2) construction of instruments of each type in such a way that each of these effects by computations and tests can be evaluated over the required ranges; and 3) comparison of the actual transfer performance of the two instruments to guard against unknown sources of error. The tests of step 2) will of ten take three forms. These are: a) tests of components of the instrument for the effects of known factors (such as magnetic susceptibility, for example; b) intercomparisons of instruments of the same type but of different ranges, in which some of the effects (such as residual reactance) may be expected to be very different; and c) repeated intercomparisons, in which each known source of error in one instrument is accentuated by definite amounts (such as by the deliberate introduction of known reactance). Normally the comparisons of step 3) can be made over only part of the useful ranges of frequency, voltage, etc., and the performance must also be judged by the results of steps 1) and 2). Normally also one of the two instruments will have better-known characteristics, wider or more suitable ranges, or better-behaved ac-dc differences than the other, and thereafter is used as the principal transfer instrument for that laboratory, but both are important.

The accuracy of ac measurements is of ten limited by the stability of the source rather than the instrument. For the calibration of instruments with a transfer instrument, stabilized sources free from short-time fluctuations are required, but long-time stability and good load regulation are of less importance..$^{4,5}$ Repeated sets of readings make it possible to determine the ac-dc difference to a precision considerably better than the fluctuations in the source, and the procedure described tends to eliminate drifts in both the test and standard instruments. If both instruments inherently respond to the same values (rms for example), low-order harmonics up to several per cent ordinarily cause the test to be in error by much less than 0.1 per cent.

\section{ELECTRODYNAMIC INSTRUMENTS}

The electromagnetic force between two conductors with reasonable geometry and currents is so small that to obtain satisfactory torque the effect is magnified by coiling the conductors. The torque-weight ratios thus obtained are still considerably less than those enjoyed by dc instruments with permanent magnets, but rea-

A. H. M. Arnold, "Alternating-current instrument testing equipment," Proc. IEE, vol. 101, pp. 121-133; 1954.

'F. L. Hermach, "Power supplies for 60-cycle tests of instruments and meters," Proc. ISA, vol. 11, paper 56-21-3; 1956. sonably rugged and portable pointer-and-scale instruments of the 0.1 per cent accuracy class, based on Weston's designs, have been available for a number of decades. For increased resolution and accuracy, recently developed commercial instruments, following Silsbee's composite-coil design, ${ }^{6}$ now use a taut-suspension system with the moving coil of a second instrument rigidly fastened to it to provide an electromagnetic restoring torque. ${ }^{7-9}$ This torque can be precisely determined by measuring the current through the second instrument with a resistor and dc potentiometer.

As the torque equations indicate, an electrodynamic instrument responds to the square of the current, the scale being marked to indicate current directly. As an unshunted ammeter (limited to about 0.1 ampere because of the springs or ligaments which must carry the current to the moving coil), it will be useful as a transfer instrument over the range of frequencies for which the average torque, $T=f(I)$, is the same as with direct current. The chief factors which affect this in an actual instrument are: 1) capacitances between turns and between coils, which alter the currents; 2) eddy currents in neighboring metals, which alter the field that links with the moving coil; and 3 ) electrostatic torque, caused by differences in potential between the fixed and moving coils. In general, these set an upper limit of about 1 $\mathrm{kc}$ for such instruments. The frequency ranges of ammeters, which use shunts across the moving coils, and voltmeters, which use series resistors to limit the current, are more sharply limited by the inductances of the coils. The manufacturer must insure that the ratio of the impedances of the two parts of the divided circuit of the ammeter is equal to the ratio of resistances, to the full accuracy of the measurement over the frequency range of interest. Similarly he must insure that the magnitude of the impedance of the voltmeter (defined as the ratio of the applied voltage to the current through the coils) is equal to its dc resistance. By connecting resistors in series with the coils of the ammeter and capacitors across portions of the voltmeter, the upper frequency limit for 0.1 per cent error in commercial instruments can be raised from $100 \mathrm{cps}$ to about $1000 \mathrm{cps}$, and for 0.25 per cent error to about $2500 \mathrm{cps}^{10}$

Because the mutual inductance between the coils changes with scale position (the operating torque depends upon this) complete compensation cannot be attained at all scale positions. Mutual inductance intro-

${ }^{6}$ F. B. Silsbee, "Composite coil electrodynamic instruments," J. Res. NBS, vol. 8, pp. 217-264; 1932.

7 G. F. Shotter and H. D. Hawkes, "A precision ac/dc comparator for power and voltage measurements," Proc. IEE, vol. 93, pp. 314$324 ; 1946$.

' J. Sorge, "A new precision instrument for ac power measurements," VDE Fachberichte, vol. 17, pp. 27-30; 1953.

${ }^{9}$ R. F. Estoppey, "Inductronic Electrodynamometer," Conference on Electronic Standards and Measurements, paper 35; August, 1958.

${ }_{10} \mathrm{~J}$. H. Miller, "Frequency compensation of ac instruments," Trans. AIEE, vol. 70, pp. 217-221; 1951. 
duces other errors as well, so that the electrodynamic ac-dc transfer instruments designed and constructed at the U.S. National Bureau of Standards ${ }^{11}$ and the National Physical Laboratory of South Africa ${ }^{12}$ are normally operated only over a narrow range of deflections about the position of zero mutual inductance, by the use of a continuously adjustable external multiplier for the first instrument and a torsion-head for the second. In the NBS instrument (see Fig. 1), two sets of coils are astatically arranged to eliminate the effect of uniform external fields, and a strip suspension and light-beam pointer with a scale 2 meters from the instrument insure definite readings and permit a scale factor at 0.01 per cent $/ \mathrm{mm}$ at currents from 0.1 to 20 amperes. The instrument is normally used as a wattmeter or ammeter, and a similar but older companion instrument as a voltmeter (10 to 600 volts). ${ }^{13}$ The NPLSA instrument is quite similar, but may be connected as a voltmeter as well ( 0.05 to 5 amperes and 25 to 500 volts). The ac-dc difference of each of these instruments is believed to be known to better than 0.01 per cent at power frequencies and to perhaps 0.1 per cent up to $3 \mathrm{kc}$ (above 50 volts), verified by careful study and by comparison with electrothermic and electrostatic instruments.

\section{Electrostatic Instruments}

The electrostatic force between two conductors at reasonable spacings is very low at normal line voltages. The resultant torque can be multiplied, as in the Kelvin electrostatic voltmeter, by interleaving the fixed and moving conductors (plates), but unfortunately only on a one-to-one basis, so that torque-weight ratio remains low. Thus electrostatic instruments, as distinct from electrodynamic instruments where the fixed coil may be designed to have more ampere-turns than the moving coil, seem rather delicate except at voltages above about $1 \mathrm{kv}$, and are rarely available commercially for precision measurements. However, taut-suspension, light-beampointer constructions, using improved optical systems and modern materials, have begun to appear commercially in Europe and may presage a renaissance of this type of instrument.

Electrostatic instruments inherently respond to voltage but may be adapted for current measurements by measuring the voltage across a resistor carrying the current. However, the voltage must be rather high ( 50 to 100 volts) for adequate accuracy and the shunt must then dissipate considerable power at high currents, or an instrument transformer must be used (either a current transformer with a resistor in its secondary, or a voltage transformer between a resistor and the instrument).

${ }^{11}$ J. H. Park and A. B. Lewis, "Standard electrodynamic wattmeter and ac-dc transfer instrument," J. Res. NBS, vol. 25, pp. 545$579 ; 1940$.

i2 J.W.Whittaker, "A precision electrodynamometer standard and ac/dc transfer instrument," Proc. IEE, vol. 101, pp. 11-20; 1954.

${ }_{18} \mathrm{~F}$. K. Harris, "A suppressed zero electrodynamic voltmeter," J. Res. NBS, vol. 3, pp. 445-457; 1929.

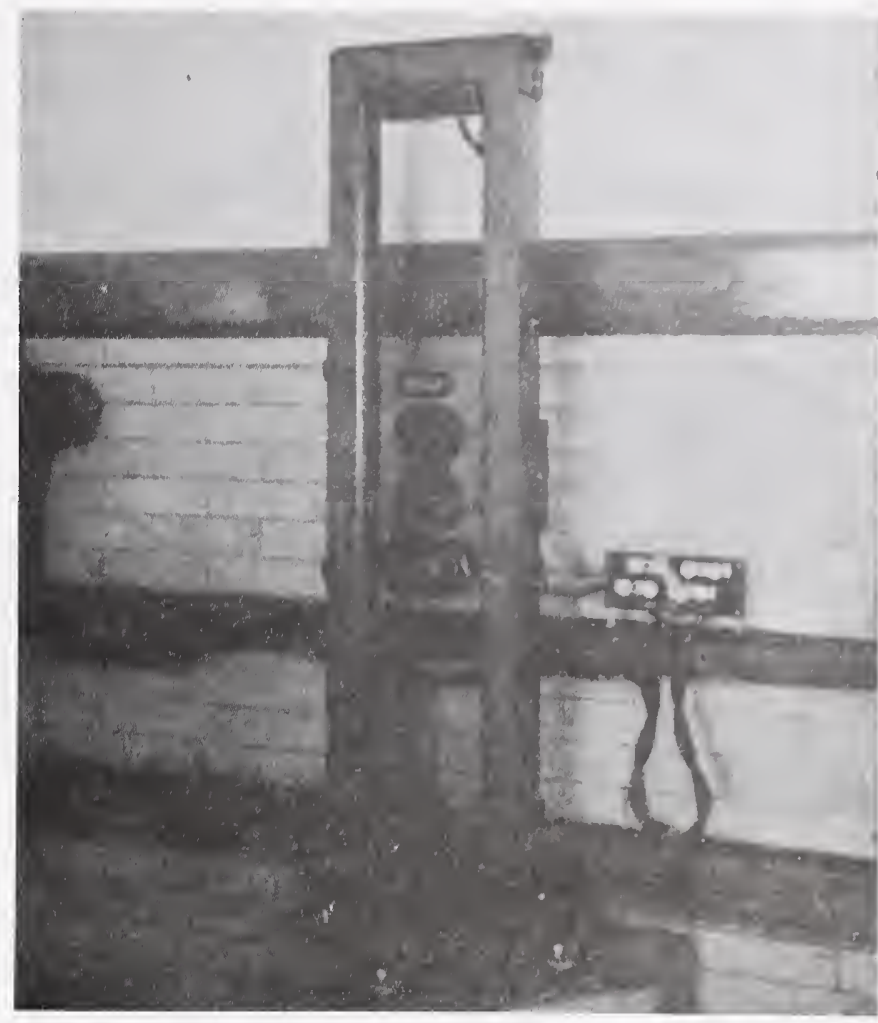

Fig. 1-NBS electrodynamic ac-dc transfer instrument (external multiplier and scale not shown).

Electrostatic instruments also have a number of sources of ac-dc differences. Among these are: 1) the series impedance of the suspension or springs, 2) surface charges on insulation in the electric field, which can modify the field with direct voltage applied, and 3) imperfectly conducting films on the plates which can cause the field strength between the plates to differ on direct and alternating voltage. ${ }^{14}$ None of these limit the frequency range as sharply as does the inductance of electrodynamic instruments, and electrostatic voltmeters may be accurate to perhaps $1 \mathrm{mc}$. However, when an electrostatic instrument is used as an ammeter the impedance of the 4 -terminal resistor must be equal to its $\mathrm{dc}$ resistance. (If a transformer is used, it is no longer an ac-dc transfer instrument.) This is difficult to achieve above about $20 \mathrm{kc}$.

Electrostatic voltmeters with ranges from 50 to 160 volts have been the principal ac instruments for over 40 years at the National Physical Laboratory in England ${ }^{14}$ and somewhat modified instruments of the same basic design are similarly used at the National Standards Laboratory in Australia. The ac-dc differences of the NPLE instruments are known to 0.01 per cent at power frequencies and to better than 0.05 per cent up to $100 \mathrm{kc}$ (verified by calculations of the known sources of error and by comparison with electrodynamic and electrothermic instruments and with other electrostatic

${ }^{14}$ R. S. J. Spilsbury and A. Felton, "The electrostatic voltmeter as a dc/ac transfer instrument," J. IEE, vol. 89, pp. 129-137; 1942. 
instruments having noble-metal conductors). Special transformers and resistors have been constructed and tested to permit the measurement of current and of voltage below 20 volts, and resistance voltage dividers have been evaluated for voltage measurements to 1000 volts (at audio frequencies). ${ }^{4}$

\section{ELECTROTHERMiC INSTRUMENTS}

Easily measurable effects are produced by the heating of short straight metallic conductors or very small beads of semiconductors, having very low electrical timeconstants. These effects can thus be independent of frequency up to $100 \mathrm{mc}$ or more. In addition, the effects can be measured electrically, rather than mechanically, with high precision and freedom from mechanical resonances. These are the chief advantages of electrothermic instruments.

Because their output is electrical, and because of their low reactance, thermocouple instruments and bolometer bridges are the only two forms now generally used. In commercial thermocouple instruments the temperature rise of a conductor (heater) is measured with a thermocouple and millivoltmeter. The combination of the heater and thermocouple is called a thermal converter. Many commercial bolometer bridges now contain bead thermistors (temperature-sensitive resistors) and are unbalanced by the temperature rise caused by the heating of the applied current. The unbalance voltage is measured or balance is restored by additional currents of a different frequency, which are measured. Thermocouple instruments of the $\frac{1}{2}$ to 2 per cent class have long been commercially available for current and voltage measurements, while most commercial bolometers are used for power measurements (absorbing all of the measured power). In either case undesired thermal effects, such as high ambient temperature coefficients, accelerated aging of the heater at the elevated temperature, and transfer of heat to other parts of the measuring element have limited the sustained accuracy obtainable. However, by using such instruments solely as ac-dc transfer instruments, calibrating them before and after each reading, these effects can largely be eliminated. Studies at the National Bureau of Standards ${ }^{15}$ have shown that properly designed thermal converters may be used as ac-dc transfer instruments at currents from 1 ma to 50 amperes with an accuracy approaching 0.01 per cent at power and audio frequencies and very probably up to $200 \mathrm{kc}$. Their low reactance also makes possible transfer voltmeters from 0.2 to 600 volts (at 133 ohms per volt) with wire-wound series resistors that have ac-dc differences less than 0.03 per cent at audio frequencies. Voltmeter elements with film resistors in coaxial lines terminated by thermal converters have been constructed and intercompared to 0.05 per cent to

1s F. L. Hermach, "Thermal converters as ac-dc transfer standards for current and voltage measurements at audio frequencies," J. Res. NBS, vol. 48, pp. 121-138; February, 1952.
$10 \mathrm{mc}^{16}{ }^{16}$ The calculated ac-dc differences of the converters and of the voltmeter elements were verified by intercomparisons of different ranges and by comparisons (over limited ranges) with electrodynamic and electrostatic instruments. A Lindeck deflection potentiometer is used at NBS with these converters and voltmeter elements to provide high resolution $(0.005$ per cent $/ \mathrm{mm}$ at rated output emf) and rapid reading.

Similarly it has been shown that a properly selected and mounted pair of indirectly heated thermistors may be used in a bridge as an ac-dc transfer instrument for an audio-frequency potentiometer to better than 0.02 per cent at frequencies from 0.2 to $20,000 \mathrm{cps}^{17}$ Thermistors are also used for ac measurements at the Physikalisch-Technische Bundesanstalt in Germany. ${ }^{18}$ Their rather long thermal time constants (about 10 seconds) provide good low-frequency response but make highly stable sources necessary.

The small ac-dc differences of thermal converters and thermistor bridges at audio frequencies are caused almost solely by: 1) thermoelectric effects in the heater, which affect the temperature rise on direct current, but because of thermal inertia do not affect it on alternating current; 2) integration errors at low frequencies, which are inversely proportional to the square of the frequency; and 3) small reactance effects in the voltmeter elements, such as capacitance currents between parts of the multiplier, and from the multiplier to its surrounding shield. Fortunately, it is possible to obtain thermal converters with heaters of manganin or other alloys of low thermoelectric effects and of sufficient length to make the ac-dc differences 0.01 per cent or less from 20 cps to $200 \mathrm{kc}$.

A single 10-ma thermal converter is used with multirange shunts and series resistors for ac measurements in the U.S.S.R., both in a central standardizing laboratory and in convenient consoles at branch laboratories. ${ }^{19}$ Series and shunt resistors are also used in the laboratories of the Germany Authority for Weights and Measures, but with two converters in a differential circuit. ${ }^{20}$ As a further modification of the transfer principle, the heater of the thermal converter alone (without the shunt or series resistors) can be switched to direct current for the dc calibration. ${ }^{21}$ Only a small dc source is

${ }^{16} \mathrm{~F}$. L. Hermach, "Electrothermic instruments for the measurement of alternating current and voltage," Proc. NPL Symposium on Precision Electrical Measurements, HMS Stationary Office, London, Eng., paper No. 15; 1955.

${ }_{17}$ F. C. Widdis, "The indirectly heated thermistor as a precise ac-dc transfer device," Proc. IEE, vol. 103, pt. B, pp. 693-703; 1956.

${ }_{18} \mathrm{H}$. J. Schrader, "A-C potentiometer measurements with indirectly heated NTC' resistors," Electrotech. Z., vol. 73-A, pp. 547$549 ; 1952$.

ig K. P. Shirokov, "An installation for calibrating ammeters and voltmeters at high frequencies," Proc. Russ. Inst. Meteorology, vol. 24, p. 24,1954 .

${ }_{20} \mathrm{~W}$. Rump, "On the exact absolute measurement of ac voltages and a potentiometer for testing ac instruments," Elektrotechnik, vol. 5 , pp. 64-67; 1951.

${ }^{21} \mathrm{~F}$. L. Hermach and E. S. Williams, "Multirange audio-frequency thermocouple instruments of high accuracy," J. Res. NBS, vol. 52, pp. 227-234; 1954 . 
required, making possible a convenient portable accessory to a dc potentiometer, which, in a recently improved model (see Fig. 2) provides ranges of 7.5 ma to 20 amperes and 0.5 to 600 volts with an accuracy of 0.05 per cent at frequencies from 5 to $50,000 \mathrm{cps}$.

\section{Other INSTRUMENTS}

A transfer instrument is an essential part of any accurate ac potentiometer. Such potentiometers have been used to some extent in Europe but rarely in the United States, except for special measurements. They require phase as well as magnitude balance, and with the usual tuned detector, measure only the fundamental component rather than the rms value. An electrodynamic milliammeter is most commonly used with a standard cell to standardize the current through the resistance network, but a thermal converter or thermistor bridge could serve equally well and over a wider range of frequencies. ${ }^{15,17}$ Recent studies ${ }^{22}$ have shown that some converters have excellent long-time stability if they are maintained at a constant ambient temperature.

A method of determining the equality of a dc voltage and the crest-to-crest value of an ac voltage to better than 0.005 per cent has been developed at the National Standards Laboratory of Australia ${ }^{23}$ as well as a method of generating an ac voltage wave with no harmonic greater than 0.001 per cent of the fundamental. The equipment has been used to verify the ac-dc performance of the NSL electrostatic voltmeter to better than

$22 \mathrm{~J}$. J. Hill, "A precision thermo-electric wattmeter for power and audio frequencies," Proc. IEE, vol. 105, pt. B, pp. 61-68; January, 1958.

${ }^{23}$ W. E. Smith and W. K. Clothier, "Determination of the dc/ac transfer error of an electrostatic voltmeter," Proc. IEE, vol. 101, pp. $465-469 ; 1954$.

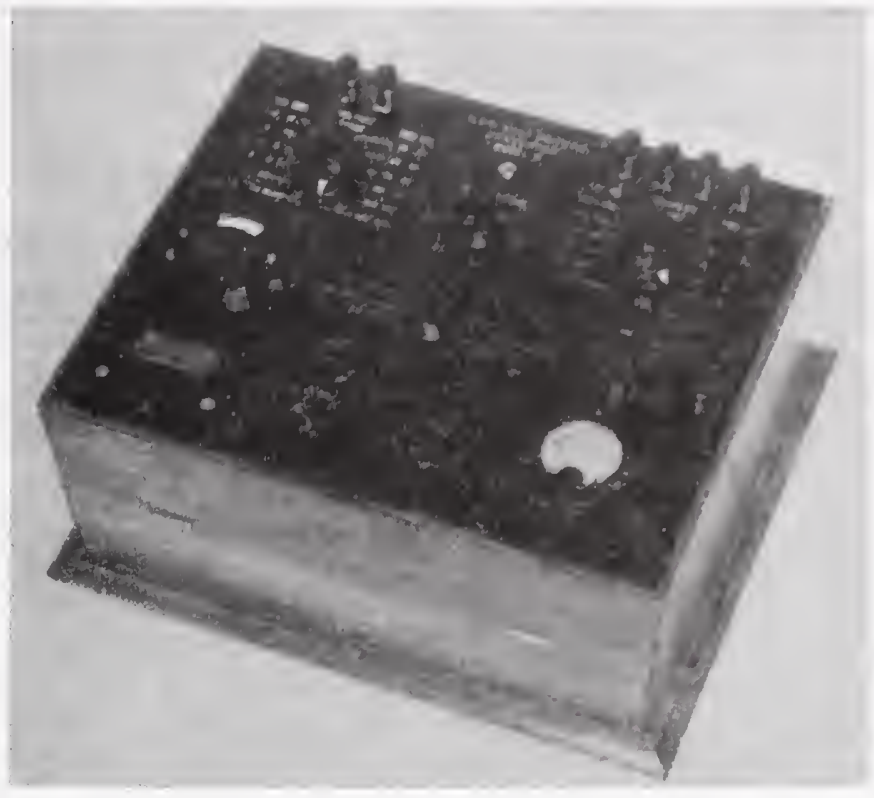

Fig. 2-Volt-ampere converter for ac current and voltage measurements.

0.01 per cent at $50 \mathrm{cps}$ and may be considered to be a crest-value ac-dc transfer instrument.

High-gain amplifiers with a rectifier in a feedback loop, and driven rectifiers based on the ring modulator, may be used with a potentiometer to measure the average value of an ac voltage wave to 0.1 per cent or better, provided the galvanometer or other detector properly integrates the rectified output. Their accuracy must also be verified by the procedure outlined for ac-dc transfer instruments. At present this is most easily done by comparison with rms instruments in circuits with voltages of known waveforms.

Reprinted from IRE TRANSACTIONS

ON INSTRUMENTATION

Volume I-7, Numbers $3 \& 4$, December, 1958

PRINTED IN THE U.S.A. 
NBSIR 84-2903

\section{AN INVESTIGATION OF THE \\ UNCERTAINTIES OF THE NBS THERMAL VOLTAGE AND CURRENT CONVERTERS}

F. L. Hermach

U.S. DEPARTMENT OF COMMERCE

National Bureau of Standards

Center for Basic Standards

Electricity Division

Gaithersburg, MD 20899

April 1985

Final Report, NBS Contract NB81SBCA0711

Submitted February 28, 1983

Revised August 1984

This report and the work described in it were supported by the Calibration Coordination Group of the U.S. Department of Defense.

U.S. DEPARTMENT OF COMMERCE, Malcolm Baldrigo, Secretary NATIONAL BUREAU OF STANDARDS. Emeet Ambler. Diroctor 
Summary . . . . . . . . . . . . . . . . . . 1

I. INTRODUCTION . . . . . . . . . . . . . . . . . . . . . . . . . 3

A. Scope of Contract and Reports.............. 3

B. Present NBS Standards . . . . . . . . . . . . . . 3

C. Ranges and Uncertainties of Calibrations . . . . . . . . 4

II. SYNOPSIS OF THE AC-DC STUDY . . . . . . . . . . . . . . 5

III. MJTC's AND COMPARATOR . . . . . . . . . . . . . . . . . . . . . 7

A. Comparator . . . . . . . . . . . . . . . 7

B. Comparison of MJTC's ............... 10

IV. REFERENCE TE'S FOR F, TVC'S TO $10 \mathrm{kHz} \ldots \ldots$. . . . . . . . 12

V. EXTENSION OF FREQUENCY RANGE . . . . . . . . . . . . . . . 13

A. Thermoelements . . . . . . . . . . . . . . . 13

B. Thermal Voltage Converters . . . . . . . . . . 15

VI. EXTENSION OF CURRENT RANGE . . . . . . . . . . . . . . . 16

VII. EXTENSION OF VOLTAGE RANGE . . . . . . . . . . . . . . . 19

VIII. WORKING STANDARDS . . . . . . . . . . . . . . . . 23

A. Current Standards . . . . . . . . . . . . . . . . 23

B. Voltage Standards . . . . . . . . . . . . . 23

IX. THERMOELEMENT REPLACEMENT . . . . . . . . . . . . . . . . . 24

X. ERROR ANALYSIS - GENERAL REMARKS . . . . . . . . . . . . 25

XI. RANDOM (STATISTICALLY EVALUATED) ERRORS . . . . . . . . . . 29

A. MJTC Comparator .................. . . . . 29

B. Other Comparators ................ 30

C. Statistical Parameters and Tests . . . . . . . 30

D. Pooled Standard Deviations . . . . . . . . . . . . 31

E. Experimental Designs and Tests . . . . . . . . . 31

XII. ESTIMATES OF RESIDUAL SYSTEMATIC ERRORS (Not Statistically

Evaluated) ....................... 32

XIII. COMBINATION OF UNCERTAINTIES . . . . . . . . . . . . . . 33

XIV. CONCLUSIONS . . . . . . . . . . . . . . . . . . 35

A. Uncertainties of the NBS Standards . . . . . . . . . 35

B. Calibration Uncertainties . . . . . . . . . . 36

XV. RECOMMENDATIONS . . . . . . . . . . . . . . . . . . . 37

A. Maintenance of AC-DC Standards . . . . . . . . . . 37

B. Quality Control of the Calibration Process . . . . . . 38

C. Other Recommendations . . . . . . . . . . . . 39 
Table of Contents (Continued)

Page

XVI. ACKNOWLEDGEMENTS ............................ 40

REFERENCES . . . . . . . . . . . . . . . . 4 41

TABLES:

1 Comparison of Four MJTC's . . . . . . . . . . . . . 43

2 Differences Between 1981 \& 1976 Evaluations of MJTC's . . . 44

3 Results of Comparisons of FX and FY with MJTC's . . . . . 45

4 Results of TVC Tests with Twin Resistors . . . . . . . . 46

5 Ampere-Range Comparisons . . . . . . . . . . . 47

6 AC-DC Differences of 1 A to $20 \mathrm{~A}$ TE's . . . . . . . . 48

7 Results of Evaluation of $\mathrm{FX}_{2}$ and $\mathrm{FY}_{2}$. . . . . . . . . 49

8 Comparisons of $E_{1}$ TVC's . . . . . . . . . . . . . 50

$9 \quad A C-D C$ Differences of $F_{1}$ TVC's with $F X_{2}$ and $F Y_{2} \ldots \ldots$

10 Comparisons of Working and Reference Standard TE's . . . . . 52

11 AC-DC Differences of Working Standard TE's . . . . . . . 53

12 Comparisons of $\mathrm{F}_{7}$ TVC's . . . . . . . . . . . . . . 54

13 AC-DC Differences of $E_{7}$ TVC's . . . . . . . . . . . 55

14 Pooled Standard Deviations, $\mathrm{s}_{\mathrm{pa}}$. . . . . . . . . . . 56

15 Estimated Limits of Residual Systematic Uncertainties . . . . 57

16 Calculation of Uncertainties . . . . . . . . . . 61

17 Summary of Uncertainties of NBS AC-DC Transfer Standards . . 64

18 Comparison of Estimated Calibration Uncertainties with

Present Allowances ........................... 65

19 Estimated Uncertainties for Special Tests . . . . . . 
Table of Contents (Continued)

FIGURES:

Page

Road Map . . . . . . . . . . . . . . . . . .

2 Comparisons of MJTC's . . . . . . . . . . . . . .

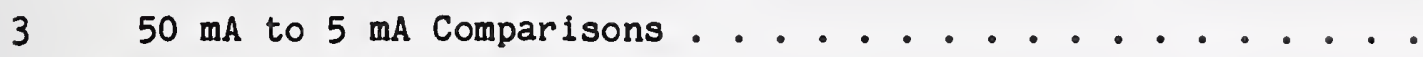

$450 \mathrm{~mA}$ to $1 \mathrm{~A}$ Comparisons ................... . . 70

5 Comparator with n-Compensation . . . . . . . . . . . 71

6 Dlagram of EMF Comparator . . . . . . . . . . . . . 72

\section{APPENDICES :}

1 n-Compensation and n-Measurements .............

2 EMI, Harmonics and DC Offset ............. 2-1

3 Effect of Relay off-Time . . . . . . . . . . . 3-1

4 Effects of Bead Heating . . . . . . . . . . . 4 4-1

5 Impedances and Substitution Corrections . . . . . . . . 5-1

6 Recommendation of the Working Group on the Statement of Uncertainties presented to Comite International des Poids et Mesures ................... 


\section{AN INVESTIGATION OF THE UNCERTAINTIES OF THE \\ NBS THERMAL VOLTAGE AND CURREIT CONVERTERS}

\section{SUMAARY}

This investigation required a complete redetermination of the ac-dc differences of the NBS ac-dc transfer standards, from $20 \mathrm{~Hz}$ to $100 \mathrm{kHz}$. It involved the following steps:

1. Setting up a new and considerably more accurate set of primary ac-dc standards, based on multijunction thermal converters (MJTC's) and a precise emf comparator for comparing pairs of standards,

2. Using them to evaluate 5-mA single junction thermoelements (TE's) and a $10-\mathrm{V}$ thermal voltage converter (TVC), up to $10 \mathrm{kHz}$,

3. Extending the frequency range by complex "bootstrap" techniques that were developed for this, and justifying these techniques,

4. Extending the current and voltage ranges of sets of reference-standard TE's and TVC's by appropriate build-up methods, up to 20 A and from 1 to $500 \mathrm{~V}$,

5. Using these reference standards to evaluate the NBS working standards that serve for calibrating clients' standards,

6. Determining the random errors of each of the above steps,

7. Studying and evaluating residual systematic errors (after known corrections were applied) by special tests and experimental designs, then estimating their individual limits (magnitudes), 
8. Combining these estimates and random errors in a $1081 \mathrm{cal}$ way to arrive at estimates of the uncertalnties of each of the many reference and working standards (following BIPM Committee guldelines),

9. Carrying out a number of largely mathematical studies relating to the investigation and these sources of ac-dc differences, and, finally,

10. Recommending steps to be taken to improve and insure the continued accuracy of the NBS callbration program for ac-dc differences.

As a result of this work 1 it is belleved that the accuracy of the NBS standards and the output of 1ts callbration process for ac-dc transfer are on a much firmer basis than heretofore, with much better documentation.

The studies showed that in certain ranges of voltage, current and Prequency considerable improvement in the calibration accuracy can be attained -- factors of 2 to 5 for high-quality single-range TE's and TVC's. Considerable improvement should also be possible for commercial multirange instruments after further study of their stab1lity and other characteristics.

For very special tests, such as international comparisons, accuracies approaching 1 ppm should be possible. 


\section{INTRODUCTION}

\section{A. Scope of Contract and Reports}

In brief, this investigation, performed under contract with NBS, required a theoretical and experimental analysis of the uncertainties in maintaining and using the NBS ac-dc transfer standards for current and voltage measurements at frequencies up to $50 \mathrm{kHz}$, plus suitable documentation of the study. In effect, the contract required a redetermination of the NBS ac-dc transfer standards. This was carried out to $100 \mathrm{kHz}$ for voltage measurements because of frequent calibration requests at the higher erequency.

The contract was completed and the final report was submitted to NBS in February 1983. The report was revised and the appendices were added in August 1984 for publication as an NBS Internal Report.

\section{B. Present NBS Standards}

The basic or primary ac-dc transfer standards at NBS at these frequencies are thermal current and voltage converters, described in [1] and [2]. The working standards used in the calibration of client thermal voltage converters (TVC's) are described in [3]. Papers [2] and [3] are appended to this report for ready reference.

Extensive research was carried out at NBS to establish these standards and verify their accuracy. The basic principles and brief descriptions of the investigations and of the many special tests required for these pioneering developments are given in the references. The work described in [1] (1952) established the ac-dc transfer to $0.01 \%$ (100 ppm) for current measurements from $1 \mathrm{~mA}$ to $20 \mathrm{~A}$ and voltage measurements from 0.2 to $750 \mathrm{~V}$ at audio frequencies. In the work described in [2] (1966) a basic set of 5 to 20-mA single-junction thermoelements (TE's) was evaluated to a few ppm, and 
two selected TE's were used with speclal serles resistors as thermal voltage converters up to $500 \mathrm{~V}$ to $10 \mathrm{ppm}$. An ems comparator was developed to compare the ac-de differences of pairs of converters to $\pm 2 \mathrm{ppm}$.

Fifteen comparisons of adjacent or similar ranges are required at each frequency with this primary set of TVC's (called $F_{1}$ ), to verify the build-up (step-up) from 0.5 to 500V. In [3], Williams described a set of TVC's which required only 7 comparisons, thus considerably shortening the time required for routine verification of accuracy. His set (called $F_{7}$ ) is now the working standard for the determination of ac-dc differences of TVC's sent in to NBS for calibration. In a later paper [6] he described a similar set of shunts with two thermoelements (TE's) for evaluating thermal current converters (TCC's). Because of grounding and capacitance difficulties with the shunts, however, TE's are still used as the working as well as the reference standards for TCC calibrations. The contact-type TE's formerly used at $1 \mathrm{~A}$ and above were replaced with insulating-bead types; to eliminate the troublesome direct coupling between the heater and thermocouple circuits. The reference TE's from 25 to $500 \mathrm{~mA}$ were also made to NBS special specifications a number of years ago. Each has a Evanohm ${ }^{1}$ heater, with Evanohm leads from it to the glass press, to minimize ac-dc differences from Thomson and Peltier effects, and from the skin effect of the magnetic leads normally used.

\section{Ranges and Uncertalntles of Callbrations}

The following table shows (for ready reference) the stated uncertainties actually avallable in the calibration of client ac-dc converters at the start of this investigation. It is considered to be very unlikely that the errors would exceed these values.

\footnotetext{
Certain commercial products or materials are identified in this paper to specify the experimental procedure adequately. Such identification does not imply recommendation or endorsement by NBS, nor does it imply that they are necessarily the best available for the purpose.
} 


\begin{tabular}{|c|c|c|c|c|c|c|c|}
\hline Frequency & $\begin{array}{l}2-5 \\
\mathrm{~Hz} \\
\end{array}$ & $\begin{array}{c}5-20 \\
\mathrm{~Hz} \\
\end{array}$ & $\begin{array}{c}20-20 \mathrm{~K} \\
\mathrm{~Hz} \\
\end{array}$ & $\begin{array}{c}20-50 \\
\mathrm{kHz} \\
\end{array}$ & $\begin{array}{l}50-100 \\
\mathrm{kHz} \\
\end{array}$ & $\begin{array}{l}0.1-0.5 \\
\mathrm{MHz} \\
\end{array}$ & $\begin{array}{l}0.5-1 \\
\mathrm{MHz} \\
\end{array}$ \\
\hline Voltage limits (V) & 50 & 100 & $1000^{1}$ & 1000 & 600 & 100 & 100 \\
\hline Current limits (A) & 0.05 & 0.05 & $20^{2}$ & 15 & & & \\
\hline & \multicolumn{7}{|c|}{ Uncertainty (percent) 3} \\
\hline Multirange TVCs & 0.02 & 0.01 & 0.005 & 0.007 & 0.01 & 0.02 & 0.03 \\
\hline $\begin{array}{l}\text { Coax single range } \\
\text { TVCs }\end{array}$ & 0.02 & 0.01 & 0.002 & 0.003 & 0.005 & 0.02 & 0.03 \\
\hline $\operatorname{TCC}(.005$ to $5 \mathrm{~A})$ & 0.02 & 0.01 & 0.005 & 0.01 & & & \\
\hline $\operatorname{TCC}(>5$ to $20 \mathrm{~A})$ & & & 0.01 & 0.02 & & & \\
\hline
\end{tabular}

${ }_{2}^{1} 200$ volts at $20 \mathrm{~Hz}$, increasing to $1000 \mathrm{~V}$ at $200 \mathrm{~Hz}$. 25 amps at $20 \mathrm{~Hz}$, increasing to $20 \mathrm{~A}$ at $200 \mathrm{~Hz}$.

3 The lower uncertainty applies at the crossover frequencies. Uncertainties may be increased if the ac-dc differences are large or affected by selfheating.

The larger uncertainties of the multirange converters reflect, in part, the poorer precision and stability of these more complex but very convenient instruments.

\section{SXYPSIS OF THE AC-DC STUDY}

Quite a number of years have elapsed since the comparisons were made which formed the basis for the present accuracy estimates. NBS now has new types of TE's. A series of comparisons of the NBS current converters was started a few years ago, but has not been completed. For these reasons a meaningful study of uncertainties required setting up a new base (primary) group of TE's, as well as carrying out new complete series of interrange comparisons of both the current and voltage standards (reference and working) for extending the ranges from the base (build-up). 
The same emf comparator and the same or new types of TE's could have been used for the base and for these comparisons, as before. However, it was very desirable to use, instead, a group of the newer multijunction thermal converters (MJTC's) and a new comparator, described in [4], (1976), which is also attached to this report. At that time the ac-dc differences (d's) of these 5- to $12-\mathrm{V}$ standards were evaluated to $0.5 \mathrm{ppm}$ up to $10 \mathrm{kHz}$ for the NBS Absolute-Volt Program. However, the converters have not been used since then. For this study the apparatus was set up again with the MJTC's to form an excellent base for directly evaluating the corresponding ranges of the reference $F_{1}$ set of thermal voltage converters (TVC's), and the TE's used in them as current converters. A considerably better accuracy was thereby attained. Thus this study was based on the new MJTC's as primary standards, with subsequent experimental comparisons with TE's, which were then used for the extensions in frequency and range.

Special studies and tests were made to extend the frequency range from 10 to $100 \mathrm{kHz}$ for voltage measurements and to $50 \mathrm{kHz}$ for current measurements. The d's of the 5-mA reference TE's from all known causes except bead heating should theoretically be independent of frequency from $1 \mathrm{kHz}$ up to at least $100 \mathrm{kHz}$. To buttress the theory they were compared with other TE's of different types and construction, which should also be independent of frequency in this range. As described later in this report, the agreement was excellent.

Resistors of calculable reactance are used with a pair of the reference $T E$ 's to form the voltage ranges of the $F_{1}$ reference set. In the 1966 evaluation [2], agreement of the 5 to $50 \mathrm{~V}$ ranges, where the computed reactance errors were negligible, formed the basis for assigning d's. Since then evidence of dielectric loss effects has been found. To evaluate them the 10-V range was compared with TVC's containing resistors of measured ac-dc characteristics, and with two of the NBS 30-MHz TVC's [5]. These rf TVC's have carbon-film resistors, with agreement in d's to $0.1 \%$ out to $40 \mathrm{MHz}$. The results showed that there was indeed an ac-dc difference in the 10-V $F_{1}$ resistor, but that it was less than $6 \mathrm{ppm}$, even at $100 \mathrm{kHz}$. 
A block diagram of these major steps in the evaluation of the NBS ac-dc reference and working standards is shown in figure 1.

Analyses and tests were also made of the important problem of accurately deducing the d's of the many ranges of the $F_{1}$ and $F_{7}$ TVC sets when the TE's are replaced, without retesting all of the ranges. Many other analyses were carried out to evaluate systematic errors from known sources in $T E ' s$ and in the comparison of TE's. Suitable statistical analyses of random errors were also carried out, and some additional sources of systematic error were discovered.

\section{MJTC's AND COMPARATOR}

\section{A. Conparator}

For this study the precision MJTC comparator and associated equipment were set up in the NBS AC-DC Laboratory, room A144, MET, which has excellent temperature stability, with a typical range throughout the day of $\pm 0.6{ }^{\circ} \mathrm{C}$. A simplified diagram of the comparison circuits and a description of the procedure for comparing two MJTC's are given in [4]. Briefly, with a resistance divider a fraction of the higher emf of one of the MJTC's is opposed to the full emf of the other. The small differences are read on a nanovoltmeter (nVM), with ac, dc direct, dc reversed, and ac, successively applied to the two heaters at equal time intervals (to minimize the effects of drifts). From these the difference between the two d's is calculated.

Several important changes and improvements were made so that the comparator could also be used for TE's. Brief descriptions follow.

(1) In place of oil immersion for the standards, the metal tank was thermally lagged. This was found to be adequate. 011 immersion would have been impracticable for many of the NBS standards. 
(2) When the two TE's have equal $n ' s,(n-I \Delta E / E \Delta I$, where I is the heater current and $E$ the output emf), the steady-state balance is independent of small changes in the ac and do supplies, greatly reducing the uncertainty In the measurement and the need to keep the emf of one of the TE's constant during a determination. However, $n$ may range from 1.6 to 2.0 for TE's, so that some of these advantages are lost. A simple method of compensating the comparator for different $n$ 's was developed. It is described in appendix 1. With this modification the basic equation for comparing two TE's is $\Delta=d_{T}-d_{S}=\left(N_{d}-N_{a}\right) / n_{s} E_{l}$, where $T$ refers to the $T E$ with the higher $n$, to which the Lindeck potentiometer shown in figure 6 is connected, and $S$ to the other. $N_{d}$ and $N_{a}$ are the average readings of the nVM with dc and ac applied to the heaters, respectively, and $E_{\ell}$ is the lowest emf of the two TE's. With $\mathrm{N}$ in $\mathrm{nV}$ and $\mathrm{E}$ in $\mathrm{mV}, \Delta$ is in $\mathrm{ppm}$.

(3) An operational-amplifier integrator and timer (called an "averager") was designed and built to integrate the $1-V$ output of the nVM for $10 \mathrm{~s}$, and display the average on a DVM. This significantly reduced the uncertainty from Johnson and nVM noise, and greatly aided the operator. Typically, repeated 10-s averages fluctuated by less than $1 / 10$ th of the peak-to-peak nolse seen in $10 \mathrm{~s}$ on the nVM.

(4) The 11-k $\Omega$ input resistance of the 3-stage divider was reduced to $1 \mathrm{k} \Omega$, by switching out the first stage when two single-junction $T E^{\prime} \mathrm{s}$, with their low output resistance, were compared. This improved the performance of the nVM and reduced the Johnson noise of the circuit. The peak-to-peak noise in $10 \mathrm{~s}$ was about $15 \mathrm{nV}$, about 3.5 times the calculated Johnson noise. This is $1.5 \mathrm{ppm}$ of the typical $10 \mathrm{mV}$ rated output of a TE, and $5 \mathrm{ppm}$ with the $3-\mathrm{mV}$ output encountered in interrange comparisons.

(5) Adequate shielding and grounding are very important. All ground leads were connected to the grounded metal tank in which the TE's were placed. A bypass capacitor was connected between each lead and the tank, to reduce any lead-borne interference. Although there was no direct evidence 
that it was significant, electromagnetic interference (EMI) can be particularly troublesome at IV frequencies, where leads are near a quarter wavelength and so pick up electromagnetic energy efficlently.

A simple direct test was devised to see if EMI caused significant errors. With the TE's and supplies connected, but with all power supply switches of , a "phantom" ac-dc test is performed, using the formula for $\Delta$ given in IIIA(2) above with the value of $n_{s} E_{l}$ to be used in the actual test. The result should be zero within the random-error limits. As shown in appendix 2, this test is sensitive enough to detect significant errors.

(6) If there are ac components in the input, chopper-type dc electronic nanovoltmeters (such as the one normally used) may be subject to intermodulation effects which can result in false zero-order (dc) outputs. These normal-mode errors were noted in the MJTC study [4], and were reduced by filtering. Careful measurements showed that at the commonly-used frequencies the ac output of a TE is typically less than $1 \mu \mathrm{V}$, and that this is too small to cause a significant error from this insiduous effect.

(7) About midway in this study, a zener-diode protective circuit, discussed in section VII, was installed at the input to the TVC's, to reduce the possibility of burnout from over-voltages.

A detailed diagram of the comparator, with these changes, is shown in figure 6. It is quite similar to the comparator shown in figure 8 of [3], attached, and the same precautions in 1 ts construction, described in [3], were taken. The first stage of the divider is set at zero when the Read 2 position of the switch is used. Shielding, polarity markings, and the fine controls of the Lindeck potentiometer, are not shown. Correct polarity is assured if the nVm goes upscale (more positive) with the set key down when the input to the TVC'S or TE's is Increased.

The conventional definition of ac-dc difference of a TE is $d=\left(I_{a}-I_{d}\right) / I_{d}$, where $I_{d}$ is the arithmetic average of the two directions of the applied dc current required to give the same output emf as the rms 
current, $I_{a}$. From this $I_{a}=I_{d}(1+d)$, so that an unknown ac current can be measured directly, if $d$ is known, by observing the emf with $I_{a}$ applied and measuring the two polarities of dc current which give the same emf. The same formulas apply for TVC's, with V replacing I.

In a recent paper, however, [7] Inglis has shown that this definition is In error if the TE has a proportional de reversal difference, $p=\left|I_{1}-I_{2}\right| / I_{d}$, with $E_{1}=E_{2}$, where the subscripts, 1 and 2, refer to the two polarities. He has proposed a new definition, $d_{1}=\left(I_{a}-I_{d}\right) / I_{d}$, where $I_{d}=I_{1}=I_{2}$ and $\left(E_{1}+E_{2}\right) / 2=E_{a}$. It is free of this error, but can be awkward to use directly, for ac measurements. The difference between the two definitions is dependent upon a number of factors. However, for a square-law TE in which $p$ is well behaved (approximately independent of $E$ ), the difference between the two definitions is only about $3 p^{2} / 8$. If $p=5 \times 10^{-4}$ (rather large for a precision TE intended for ac-dc measurements) the difference is less than $0.1 \mathrm{ppm}$.

At NBS the primary standards are the MJTC's, with p's much less than $1 \times 10^{-4}$. The other TE's are evaluated by the steps shown in flgure 1 , with the conventional definition of $d$.

Additional analysis shows that, with the conventional definition, there are small error terms which should be added to the basic formula for the NBS emf comparators when TE's having large de reversal differences are compared. However, they are about the same magnitude as the $3 p^{2} / 8$ term just $g 1$ ven, and are therefore also negligible for the NBS TE's, which have small and well behaved p's.

\section{B. Comparison of MJTC's}

The ac-dc difference of 4 MJTC's were carefully compared at 6 to 10 volts (limited by voltage ratings) at $30 \mathrm{~Hz}, 1 \mathrm{kHz}$ and $10 \mathrm{kHz}$. These are 4 of the 8 that formed the MJTC base described in [4]. The 4 are of different design and manufacture. This diversity greatly strengthens the confidence in 
standards, because sources of systematic error are more likely to be discovered in such comparisons than by simply repeating measurements on one standard, or comparing like standards.

The results of the evaluations (including repeat tests on different days) are given in table 1 , as $\mathrm{d}_{\mathrm{T}}-\mathrm{d}_{\mathrm{S}}$ in $\mathrm{ppm}$ for the MJTC's identified as $\mathrm{T}$ and S. The supplementary designations of the MJTC's shown in parenthesis in the figure, are those given in the 1976 paper.

The pooled standard error, $s_{a}$, (standard deviation of the average) for these measurements was $0.16 \mathrm{ppm}$. If the difference between two averages in repeat tests is less than $\sqrt{2} t_{a}$ ( $t$ is Student's $t$ ) we have no evidence (at a given confidence level) that the difference is statistically significant. At a $95 \%$ confidence level for $k(n-1)$ degrees of freedom with $k=19$ (the number of averages in table 1 ), and $n=4$ (the number of determinations in each), $\sqrt{2} \mathrm{ts}_{\mathrm{a}}=1.4 \times 2.0 \times 0.16=0.45 \mathrm{ppm}$. None of the differences between averages was as large as this.

The results of these measurements at each of the 3 frequencles are shown more vividly in figure 2. Here the number near the head of each arrow is $d_{T}-d_{S}$, where $T$ is the MJTC at the arrowhead. The best estimate for $d_{G r 25-1}-d_{G 44}=M$ is obtained from the paths between them, giving double weight to the direct path. The best estimate for $d_{G 5 C}$ is obtained by assigning to each pertinent arrow half of the discrepancy between $M$ and the sum of the two arrows involving G5C. Similarly d $d_{\text {NPL } 14}$ is determined. (NPL14 could not be used at $10 \mathrm{kHz}$ because of its magnetic lead wires.)

The value assigned to each MJTC, on the assumption that the average of all 4 is zero, is shown to the nearest $0.1 \mathrm{ppm}$ in each circle. GiOC was not included in this group because it was compared only with G5C, in early measurements, which were not repeated. 
The same MJTC's were evaluated in 1976 [4] as part of a larger group. Table 2 shows the differences between the present assignments and those of 1976. They are gratifyingly small, indicating the high stability as well as the low ac-dc differences of these thermal converters of different deslgn and construction.

\section{REPERENCE TE'S FOR F TVC's TO $10 \mathrm{kHz}$}

The first comparisons of the 10-V reference TVC with one of the MJTC's, and subsequent intercomparisons of TE's, disclosed several problems. The TVC was unstable because of variable contact resistance in its sliding connectors. Some TE's had a peculiar dependence of ac-dc difference upon the ac-dc sequence used, differing by as much as $3 \mathrm{ppm}$ with ac, dct, dc-, ac applied than with ac, dc-, dct, ac applied. Several of the TE's required 15 to $20 \mathrm{~s}$ to come within $1 \mathrm{ppm}$ of the final value after the input was switched, even though the off-time of the relay was less than $0.5 \mathrm{~ms}$.

To overcome these problems simple modifications were made in the TVC connectors and new 5-mA TE's were chosen after extensive screening tests. Each consisted of two special TE's with Evanohm heaters, welded to the copper skin of the CuNi lead-in wires to reduce thermoelectric errors. 2 The two heaters were connected in series, as were the two thermocouples, to obtain a rated output of $12 \mathrm{mV}$. They were temperature compensated by the method used by Williams [3].

Two of them, labeled FX and FY, were compared as both current and voltage converters with two of the MJTC's at $30 \mathrm{~Hz}$, and 1 and $10 \mathrm{kHz}$, using the 5- and 10-V F, resistors for the TVC tests. The results are shown in table 3. The third TE, FZ, was compared with FX to $100 \mathrm{kHz}$.

${ }^{2}$ Later analysis indicated that welding to the copper instead of the core might not greatly reduce the Peltier error. 
The measured d's were gratifyingly small. There was no appreciable d from Thomson and Peltier effects in the heaters of any of the three TE'S. There were small low-frequency errors at $30 \mathrm{~Hz}$, and evidence of impedance errors (about $1 \mathrm{ppm}$ at $10 \mathrm{kHz}$ ) in the TVC resistors.

To check on possible current-dependent d's at high frequencies, $F X$ and FY were paralleled and compared with FZ at $5 \mathrm{~mA}$, with excellent agreement.

Puzzling drifts of some single-junction TE's led to a study of transients caused by switching the input voltage during an ac-dc determination. As shown in appendix 3, if the response of a TE to a step-change in input is a simple exponential with a time constant, $t_{c}$, and the relay off-time in transit is $t_{0},\left(t_{0}<t_{c}\right)$ then the proportional change in output emf at a time, $t$, after switching (caused by the off time) is $p=t_{o} / t_{c} e^{a}$, where $a=t / t_{c}$. For the high-speed miniature relays now used, $t_{0}$ is only $0.4 \mathrm{~ms}$. For a TE with a $t_{c}$ of $2 \mathrm{~s}$ (fairly typical) a time $t=10 \mathrm{~s}$ is required to make $\mathrm{p}<1 \mathrm{ppm}$. This is comfortably below the $30 \mathrm{~s}$ interval (time before reading nVm) now used.

The present relays can be used only up to $200 \mathrm{~V}$ in the circuit, and high-voltage relays have much longer off times. Longer intervals might then be required. Surprisingly, for $t_{0}=4 \times 10^{-3} \mathrm{~s}, t=15 \mathrm{~s}$, only a modest increase. Thus the excessively long drifts of some TE's are evidence of other problems, of unknown causes.

\section{EXTEUSION OF FREQUEICY RAHGE}

\section{A. Thermoelements}

Because of reactances in the complex structure of the 5- and $10-\mathrm{mA}$ MJTC's, these direct comparisons could not be extended reliably in frequency. The simpler TE's, with their smaller reactance errors, should have wider frequency ranges. To verify this, several 5-mA TE's of different construction and/or manufacture were compared with FX and FY as current converters from $30 \mathrm{~Hz}$ to $50 \mathrm{kHz}$. Two of them were in the original 1966 
primary set. All of them have Evanohm heaters and should have low thermoelectric errors. These were determined from the 1-kHz MJTC tests, and were 1ndependent of frequency. The 1mportant criterion is that the measured differences between each palr of TE's should be the same at higher frequencles, g1ving strong evidence that reactance and other errors were negl1g1ble.

Unfortunately the measurements showed no such agreement. At $50 \mathrm{kHz}$ discrepancles of up to $4 \mathrm{ppm}$ from the $1-\mathrm{kHz}$ values were observed. It seemed 11 kely that some of the differences could be caused by leakage current through the insulating bead whlch fastens the hot junction of the thermocouple of a TE to the center of the heater.[1,2] The bead resistance is a complex function of frequency and of bead temperature and voltage. This was verifled by making additional measurements with added resistance between the heaters of the two TE'S, whlch changed the bead voltage of the ungrounded one. (The thermocouple clrcult is grounded at the emf comparator.) Apparently the bead resistance of these TE's was less at $50 \mathrm{kHz}$ than at $1.6 \mathrm{kHz}$, where bridge measurements were made earlier (as described in [2]).

Detalled analysis of bead resistance errors, given in appendix 4, led to some surprising conclusions. They showed (1) that bead heat ing (caused by leakage current from the heater to the thermocouple through the bead) is twlce as effective in ralsing the thermocouple temperature as heating from the heater wire, (2) that if one end of the heater is grounded, no error would be caused by bead heating, and (3) this would be true even if a palr of equal TE's, with heaters in series, is used, as In FX and FY. (In the comparisons with the MJTC's descrlbed earlier, FX and FY were at the ground end.)

With this background, the comparisons of pairs of TE's were made by following a method used by williams. They were compared as IVC's with two nearly 1dentical $30-\mathrm{V}$ series resistors. One end of each of the heaters of the two TE's under test could then be at ground. Appendix $5 \mathrm{C}$ shows that by repeating the measurements with the resistors interchanged and averaging the 
results, errors from small differences in the reactances of the resistors could be largely eliminated, and the TE's could be evaluated as current converters.

The results of these measurements are shown in table 4 . Except at $30 \mathrm{~Hz}$, there was no significant frequency dependence of the average of the 6 $T E^{\prime}$ 's up to $50 \mathrm{kHz}$, with FX as the reference. Thus there is good evidence that the corrections assigned to FX and FY up to $10 \mathrm{kHz}$ by comparisons with the MJTC's could be extended without change to $50 \mathrm{kHz}$.

TE's are subject to low-frequency errors (1) caused by failure to integrate the heating effect of the ac current wave correctly. For FX, $\mathrm{d}=+0.9 \mathrm{ppm}$ at $5 \mathrm{~mA}$ and $30 \mathrm{~Hz}$. From these tests, $30 \mathrm{~Hz}$ values could be assigned to all of the other TE's.

\section{B. Therval Voltage Converters}

As shown in [2], the ac-dc difference, $d_{v}$, of a TVC is $d_{v}=d_{t}+d_{c}$, where $d_{t}=\left(Z_{t}-R_{t}\right) / R_{t}$ and $d_{c}=\left(I_{a}-I_{d}\right) / I_{d}$ for the same output emf. In these equations, $z_{t}$ is the magnitude of the ac impedance, defined as the ratio of the input voltage to the current, I, through the ungrounded input of the TE heater, $R_{t}$ is the dc resistance, and $I_{d}$ is the dc current (average for the two directions) required to give the same output emf as $I_{a}$. Note that $Z_{t}$ includes the impedance of both the TE and the serles resistor, and that the equation for $d_{t}$ is a perfectly general one.

Preliminary comparisons of the mid-ranges of the TVC's to evaluate high frequency effects disclosed some disturbingly large differences at 50 and 100 $\mathrm{kHz}$. Calculations showed that the d's caused by the reactances of the series resistors should be much less than $1 \mathrm{ppm}$, and should vary as $\mathrm{f}^{2}$. The observed differences were roughly proportional to $f$, suggesting dielectric losses as the cause. This supposition was strengthened by the $-1.0 \mathrm{ppm}$ average of $F_{1} 10-F Y$ and $F_{1} 5-F X$ in the comparisons at $10 \mathrm{kHz}$ with the MJTC's 
(table 3). The reactance errors should be completely negligible at this frequency.

To investigate this further, F, 10-FY was compared with a special TVC that Haddad had studied in 1969 for his GWU Master's thesis under Cutkosky[18]. He had determined the change in impedance (resistive and reactive components) between 1.6 and $16 \mathrm{kHz}$. The comparisons indicated that F $10-F Y$ had a $\Delta d$ of about $-1.5 \mathrm{ppm}$.

To carry this to higher frequencies, $F_{1} 10-F Y$ and $F_{1} 20-F X$ were compared with the corresponding ranges of the $r f$ Model C TVC's, which had been evaluated to $40 \mathrm{MHz}[5]$. These TVC's have unspiraled carbon film resistors on cylindrical ceramic bases, as distinct from the spiraled metal-film resistors, of the $F_{1}$ set, with much smaller possibility of dielectric effects.

The results of these several tests were not completely concordant, but all of them pointed strongly to a small but significant $d_{t}$ in F/10-FY. Later tests showed that the $d_{s}$ of the resistor alone was about $-5 \mathrm{ppm}$ at $100 \mathrm{kHz}$.

\section{EXTENSION OF CURRENT RNWGE}

Range extension (build-up) is based on comparisons of adjacent ranges, so that all are determined in terms of a base or reference range. Evaluating the validity of the method is, of course, important, and is often very difficult. For these current ranges, the extension was carried out by two methods, as follows:

(a) From 5 to $1000 \mathrm{~mA}$ two almost 1dentical TE's of one range were first compared. They were then paralleled and compared with each of two TE's of the next higher range, and so on. This is the method used in [2] to compare the 5- to 50-mA TE's. Like that work, pairs of almost 1dentical low-reactance resistors were connected in series with the heaters to assure that the paralleled currents were sufficiently in phase. The analysis of 
appendix 5E shows that the phase angles of the two paths must be equal to better than one mrad. This is readily measured on a commercial impedance comparator, to $100 \mathrm{kHz}$. When the phase angles are negligible, the basic formula for this method, taking into account small differences in the characteristics of the paralleled TE's, is given in appendix II of [2]. This method has the strong advantage that each TE is used at or near rated current, so that the results are not affected by current-dependent errors.

(b) Above $1 \mathrm{~A}$ the heater resistances are too low to be measured on the bridge, and mutual inductances can cause serious errors, so a more complex approach following [1] was necessary. Adjacent-range TE's were compared at the rated current of the lower range. To investigate current-dependent d's, selected ranges were compared with shunted low-range TE's at frequencies low enough so that the reactance errors of the shunts were negligible. Each such comparison was made at two currents. All of the TE's had Evanohm heaters with small thermoelectric effects independent of frequency, so that the extrapolation of current-dependence could safely be made to $50 \mathrm{kHz}$.

The 5 to 50-mA build-up was made with the MJTC comparator in method (a). It was convenient to start with a 50-mA (OF-19) MJTC of Wilkin's later, and very different design[8]. His calculations showed that this MJTC should have less than $1 \mathrm{ppm}$ error up to $100 \mathrm{kHz}$. It agreed with the NPL-14 50-mA MJTC at $30 \mathrm{~Hz}$ and $1 \mathrm{kHz}$ to $0.1 \mathrm{ppm}$, verifying low-frequency concordance. (NPL-14 is frequency-limited because of skin effect in its magnetic leads.)

The results of these 5- to 50-mA comparisons are shown in figure 3 . The numbers near each arrow are $d_{T}-d_{S}$ in $p p m$, at the four frequencles shown in the key, where the subscript T designates the TE at the arrowhead. The columns headed "Average" are the averages of the two adjacent TE's, as determined by the two comparisons of that paralleled pair with the two TE's above them in the figure. The numbers in each block are the d's determined from the intercomparisons (starting with MJTC OF-19), with forced closure of each triangle. (One third of the closure error is applied to each leg.) The 
closure errors were very small, requiring adjustments of only $0.3 \mathrm{ppm}$ or less.

The pooled standard deviation $s_{p a}$ of the average of four determinations of $\mathrm{d}_{\mathrm{T}} \mathrm{d}_{\mathrm{S}}$ was only $0.28 \mathrm{ppm}$. These high precision tests showed excellent performance in these Evanohm-heater TE's. At the low end of this rather long chain of measurements, the values obtained for FZ and FY as current converters agreed with those previousiy determined from the 5mA MJTC's to $1 \mathrm{ppm}$ at $20 \mathrm{~Hz}^{3}$ and $50 \mathrm{kHz}$, and to $0.5 \mathrm{ppm}$ at intermediate frequencies. Thus systematic errors in the chain were very small indeed.

of particular interest is the excellent agreement at $1 \mathrm{kHz}$ (better than $0.6 \mathrm{ppm}$ ) between the MJTC's and the average of the two $50 \mathrm{~mA}$ and two $25 \mathrm{~mA}$ TE's. ${ }^{4}$ Each of these four single-junction converters has an Evanohm heater and Evanohm stems (lead-1n wires) from the heater to the glass press (which serves as a good heat sink). Thus the Peltier as well as Thomson errors of each TE should be very small. Any reslduals would not likely be the same in all of them, nor the same as those in the MJTC's. Thus the agreement provides valuable additional confirmation that the ac-de differences of the NBS standards caused by thermoelectric effects are considerably less than 1 ppm.

The fast-acting relays controlling the inputs to the heaters limit the high precision MJTC comparison equipment to $50 \mathrm{~mA}$ or $100 \mathrm{~V}$ (not simultaneously). For comparisons at higher voltages and currents, the 1966 comparator [2] was used. The results of the paralleling measurements, method (a), from $50 \mathrm{~mA}$ to $1 \mathrm{~A}$ are shown in flgure 4, along w1th a comparison of one of the 1-A TE's w1th FG-1, a shunted 10-mA TE described in [6]. The build-up is based on a 0 ppm assignment to the average of the two 50-mA TE's, together with forced closure of each triangle of tests in the four loops up to $1 \mathrm{~A}$.

3 Extrapolated from the $30 \mathrm{~Hz}$ measurements.

${ }^{4} \mathrm{At} 1 \mathrm{kHz}$ reactance errors and low frequency errors should be negligible. 
For these measurements, $s_{\text {pa }}$ was about $1.7 \mathrm{ppm}$, practically independent of frequency. This is not as good as the precision of the MJTC comparator, but is low enough to meet the needs for high-current calibrations. The evaluation of FG-1 with the 1-A TE agreed with Williams' earlier tests [6] to $5 \mathrm{ppm}$, which is well within the limits of random error for the number of stages involved.

The results of the comparisons of the 1- to 20-A TE's by method (b) are shown in table 5. These air-cooled TE's were made by the Weston Company a number of years ago to NBS speciflcations. Each has an Evanohm heater, with an insulating bead at the hot junction of the thermocouple. To guard against current-dependent errors, some of them were compared with Williams' shunted TE's. Each comparison was made at two currents, with no consistent evidence of such dependence, so that the results of table 5 could be combined with the values assigned to the 1-A TE to determine the d's of all of the ranges, as shown in table 6 .

For these comparisons $s_{p a}$ was about $1.7 \mathrm{ppm}$, again not greatly dependent on frequency. The TE's showed rather large d's at $50 \mathrm{kHz}$, probably because of skin effect in the lower ranges, and a peculiar "proximity effect" in the 5 to 20 A ranges. These TE's have heaters of $\mathrm{C}$-shaped cross section rather than tubular. For these ranges $d$ was markedly dependent upon the position of the return lead.[6]

\section{EXTENSION OF VOLTAGE RANGE}

The extension from the base $10-V$ range of the $F_{1}$ reference set of TVC's up to the $1000-\mathrm{V}$ and down to the $0.5-\mathrm{V}$ ranges required 14 adjacent-range comparisons at each frequency, using the FX and FY TE's with 13 plug-in resistors [2]. Unfortunately, shortly after it was completed a major fault in the ac amplifier burned out both of these excellent TE's. (A lower-range TE connected in series with them as a fuse was more rugged.) subsequently, 
two unusual sources of error in the ac-dc comparisons were discovered, casting some doubts on the accuracy of the TVC bulld-up.

The first of these was an unexpectedly large error caused by a dc offset voltage, $V_{\text {os }}$, in the output of the same direct-coupled ac amplifier. Because this was only one direction of $d c$, it would be integrated incorrectly if the TE had a dc-reversal difference, $8=\Delta E / E$, for the two directions of the same dc voltage. As shown in appendix $2 \mathrm{C}$, the error can be as much as $\mathrm{e}=\mathrm{gu} / 2$, where $u=v_{o s} / V_{a}$. Thus if $u=1 \times 10^{-2}$ and $g=5 \times 10^{-4}$, e can be $2.5 \mathrm{ppm}$. As shown in $2 B$, this is much larger than the output error $e^{\prime}=q^{2} e_{h}$ caused by $a$ small proportional error, $e_{h}$, in measuring a small harmonic, $v_{h}$, in an ac wave, $v_{a}$, where $q=v_{h} / v_{a}$. If $q=1 \times 10^{-2}$ and the error of an rms instrument in measuring the harmonic is $e_{h}=5 \times 10^{-4}$, the error in measuring the combined wave is only $0.05 \mathrm{ppm}$.

The second source of error was an unexpectedly large skin effect in the brass center conductor of the coaxlal connectors. At $100 \mathrm{kHz}$, a 6-ppm error was caused by an L-shaped connector sometimes used in front of one of the $0.5-V$ TE's in a comparison. The error decreased, of course, for higher ranges with their higher resistances.

The effects of the dc offset and troublesome dc translents in the amplifler were eliminated by using a wide-range output transformer. The reference plane in each TVC-comparison was taken as the center of a coaxial "T" connector to whlch each of the two TVC's was thereafter directly connected.

Two new 5-mA TE's, $F X_{2}$ and $F Y_{2}$ were assembled to replace $F X$ and $F Y$. They were evaluated by comparisons with MJTC 644 to $10 \mathrm{kHz}$ and with FZ from 2.5 to $5 \mathrm{~mA}$ up to $100 \mathrm{kHz}$. $\mathrm{F}_{1} 10-\mathrm{FY}_{2}$ was evaluated at $20 \mathrm{~Hz}, 1$ and $10 \mathrm{kHz}$ with 644 as a TVC. Because of the importance of the frequency extension, the tests described in section $V-B$ were repeated with $F Y_{2}$. Results of these extensive measurements are shown in table 7 . 
The results of the interrange comparisons of the $F_{1}$ set are shown in table 8, and the values assigned to each range, with d of $F_{1} 10-F_{2}$ from table 7 as the basis, are shown in table 9. In the comparisons each TE is used at two currents, with a series resistor of the given range. If its $d_{c}$ is current dependent, a correction must be applied at each step. Since $d_{v}=d_{t}+d_{c}$ (see section $\left.v-B\right), d_{v 2}-d_{v 1}=-d_{c 2}-d_{c 1}$, at the two currents corresponding to subscripts 2 and 1 , on the assumption that $d_{t}$ is not voltage dependent.

Unfortunately each of these new TE's has a significant $d_{c}$, which is also somewhat current-dependent, as shown by the results in table 7 . It was difficult to evaluate the current dependence accurately enough, because of the low emf's at the low currents. Any inaccuracy is multiplied by the number of steps from the $10-\mathrm{V}$ base range to the extreme ranges. To reduce the uncertainty, an average value of $d_{c}=d_{c 2}-d_{c 1}$ was calculated from all of the values for both TE's from 1 to $100 \mathrm{kHz}$. Subscript 2 applies at $5 \mathrm{~mA}$ and subscrlpt 1 at elther 3.3 or $2.5 \mathrm{~mA}$. This value, $+0.8 \mathrm{ppm}$, was applied uniformly in calculating the d's in table 9 from the comparisons of table 8. The tests with FZ showed that it was independent of frequency from 1 to $100 \mathrm{kHz}$. At $20 \mathrm{~Hz}$ the values of $d_{c}$ were taken directly from table 7 .

As a check on the accuracy of this average value for $d_{c}$, the ac-dc difference, $d_{S}$, of the serles resistor of each range was calculated at $1 \mathrm{kHz}$ by formulas given in appendix 5D, starting with the values of $d_{c}$ of the $T E$ 's as given in table 7 , and $d_{v}$ as shown at $0.6 \mathrm{~V}$ in table 9 . At this frequency each $d_{s}$ should be negligible. The computed values ranged from +0.4 to -0.9 ppm, and the average for all voltage ranges, with regard to sign, was only $-0.1 \mathrm{ppm}$. This is an excellent check indeed.

Both $F X_{2}$ and $F Y_{2}$ have $d_{c}$ 's which are positive, and so cannot be caused by Thomson effects in their heaters [1]. Unfortunately they also have unusually large negative $d_{h}$ 's for their heater impedances $(-10$ and $-12 \mathrm{ppm}$, respectively), calculated from the measurements at $1 \mathrm{kHz}$. These values of $d_{c}$ 
and $d_{h}$ are indicative of large Peltier effects at the heater to lead-in junctions.

At higher frequencies the even larger $d_{h}$ for the $0.6-V$ ranges (TE's alone) are caused by skin effect, which increases the resistance of the magnetic heater leads, causing positive ac-dc differences.

With the $F_{1}$ TVC set these factors require troublesome corrections, but do not invalidate the results, as the correlation at $1 \mathrm{kHz}$ shows. Indeed, they provide excellent checks on the correctness of the baslc bulld-up formulas.

It is to be noted that these TE's cannot be used interchangeably; $1 . e$. $\mathrm{F}_{1} 10-\mathrm{FX}_{2}$ is not known from table 9. Substitution corrections are discussed in section $I X$.

The TVC comparisons below $200 \mathrm{~V}$ were made with the MJTC comparator. Because the thermocouple emf's of the higher-range TE in each pair were low ( $3 \mathrm{mV}$ or less), the precision was poorer than with $10 \mathrm{mV}$ outputs. The pooled standard deviation, spa, was $0.52 \mathrm{ppm}$. The 1966 comparator was used for the higher voltage ranges, with the original TE'S, FX and FY. To avoid repeating these measurements the substitution formula given in section IX was used to calculate the $d_{v}^{\prime} s$ with $F_{2}$ and $F Y_{2}$.

A study of protective circults to guard TVC's from burnout, sparked by the loss of FX and FY, showed that a simple combination of a pair of back-to-back zener dlodes directly in parallel with the TVC's and a 10-mA expendable $\mathrm{TE}$ in series with the combination should provide adequate protection against reasonable kinds of over voltages. The zener voltage should be near, but not less than, twice the supply voltage, and there are limits on the zener resistance and the time constant of the fuse (which is why a TE was chosen). The method does work well, and TE's of large dc reversal difference, which are relatively inexpensive, can serve well as fuses, but a CRO across the TVC's is advised, to monitor the wave form. 


\section{WORKING STANDARDS}

\section{A. Current Standards}

The working standards which are used in calibrating thermal converters sent to NBS were evaluated by direct comparison (heaters in series) with the corresponding ranges of the reference converters, in the calibration console normally used for such measurements. This had the advantage of giving direct information on the precision of calibrating TE's as current converters (TCC's). Usually the input line to the working standard was grounded. To check on possible bead resistance errors, some measurements were repeated with the reference standard at ground. (The ground should never be placed on the line connecting the two heaters, because capacitance and leakage currents from the ac and dc sources to ground could cause the two heater currents to differ.)

The results of these comparisons are shown in table 10, and the values assigned to the working standards are shown in table 11. The overall spa was 2.4 ppm.

\section{B. Voltage Standards}

The $F_{7}$ set of TVC's for calibrating TVC's sent to NBS was evaluated by adjacent-range comparisons, as in [3], with the calibration console. The results are shown in table 12 .

The 10-V range was compared with $F_{1} 10-F Y_{2}$, as the basis for the build-up. However, insuring the validity of the build-up process is somewhat more complicated than with the $F_{1}$ set. Each series resistor is used with both TE's, $F_{7}$ and $F_{7}$, to form two ranges (such as 3 and $6 \mathrm{~V}$ with the $800-\Omega$ resistor). Then, since $d_{v}=d_{t}+d_{c}$, the difference between those two ranges is $d_{v 2}-d_{v 1}=d_{t 2}-d_{t 1}+d_{c 2}-d_{c 1}$. Thus $d_{c}$ of each of the two TE's must be evaluated (at the comparison currents if it is current dependent). Since $d_{t}$ depends on the heater of the TE as well as the resistor, any difference between the two $d_{t}$ 's should also be evaluated to determine $d_{v 2}-d_{v 1}$, the correction to be 
applied at each step of the build-up comparisons. This is quite complicated unless the reactance terms are negligible as shown in append $1 x$ 5B and discussed in the next section.

Fortunately the $d_{t}$ variations caused by TE's decrease with increasing range. For this build-up of the $F_{7}$ set, $d_{c}$ corrections were evaluated from the comparison of the $6-\mathrm{V}$ and higher ranges at $20 \mathrm{~Hz}$ and $1 \mathrm{kHz}$ (where the efect of $d_{h}$ of each heater is negligible). Since the $d_{s}$ 's of the series resistors are also negligible at these relatively low frequencies, the same $d_{c 2}-d_{c 1}=d_{v 2}-d_{v 1}$ is evaluated in each of these comparisons, where $d_{c 2}$ refers to $E_{7} 1$ at 1.25 or $1.67 \mathrm{~mA}$, and $d_{c 1}$ to $E_{7}$ at $5 \mathrm{~mA}$. The average value is less subject to random errors. For these TVC's each $d_{V 2}-d_{v 1}$ was very small. At $20 \mathrm{~Hz}$ and at $1 \mathrm{kHz}$ the average of all of them at $6 \mathrm{~V}$ or above was 0 ppm.

Thus the $d_{c 2} d_{c 1}$ corrections were negligible at these frequencies. They were taken to be the same at higher frequencies. This assumption was checked to a few ppm by special tests involving the known $F_{1}$ TVC's and the $F_{7}$ and $F_{7} 2$ TE's. Similarly, the $d_{h}$ corrections caused by heater impedances were very small.

The results of the $E_{7}$ build up are given in table 13. They were checked by comparing the $1-V$ and $100-V$ ranges with the same ranges of the $F_{q}$ set. The differences ranged from $2 \mathrm{ppm}$ or less at $1 \mathrm{kHz}$ to $6 \mathrm{ppm}$ at $100 \mathrm{kHz}$, and are generally within the random error limits imposed by $s_{p a}$, which were $1.2 \mathrm{ppm}$ at $1 \mathrm{kHz}$ and $2.8 \mathrm{ppm}$ at $100 \mathrm{kHz}$.

\section{THERMOELEMENT REPLACEMENT}

It is highly desirable to be able to replace a TE in a TVC set without recalibrating all of the ranges. The analysis in appendix $5 B$ of the substitution error which may result shows that the ac-dc differences as current converters, $d_{c}$, and as voltage converters, $d_{v h}$ of both the old and new TE's must be known, and the phase angles of the 1mpedances must be less 
than certain specifled limiting values. At NBS, $d_{c}$ and $d_{v h}$ can be measured by comparison with the reference standards, and the phase angles can be determined closely enough with a commercial impedance comparator to $100 \mathrm{kHz}$.

If the phase angles are negligible, the change in $d_{v}$ of a given range of a TVC set when $T E_{1}$ is replaced by $T E_{2}$ (of the same nominal range and input resistance) is given by

$$
d_{v 2}-d_{v 1}=\frac{R_{h}}{R_{t}}\left(d_{v h 2}-d_{v h 1}\right)+\frac{R_{s}}{R_{t}}\left(d_{c 2}-d_{c 1}\right)
$$

where $R_{h}$ and $R_{S}$ are the nominal values of the heater and series resistances, $R_{t}=R_{h}+R_{g}$, and $d_{v h}$ applies to the heater alone.

It is to be noted that as the voltage range is increased, the first term becomes negligible and the second approaches $d_{c 2}-d_{c 1}$.

If the phase angles are not negligible or cannot be determined, it is best to repeat the lower inter-range comparisons. Although the phase-angle term is complicated, the analysis shows that the error in neglecting it will be less than $1 \mathrm{ppm}$ if the angles of the two TE's are equal to within 0.1 mrad and the angles of the resistors and the TE's are less than 5 mrad.

\section{ERROR ANALYSIS - GENERAL REMARKS}

These are personal views on varlous phases of error analysis.

(1) There are many sources of systematic errors in these standards and in the comparison process. Practically all are independent. Our estimates of their individual limits, after all known corrections are applied, should then be added in quadrature as the square root of the sum of their squares (rss), following Youden [10]. 
(2) Youden recommends adding the estimate of the random errors (such as $2 s_{a}$ or $3 s_{a}$ ) to the rss of (1) to obtain the total uncertainty.

(3) However, it is evidently desirable to follow the much more recent guidelines of the BIPM Working Group on the Statement of Uncertainties, in accordance with B. N. Taylor's memorandum of August 21, 1981 [11]. This involves adding all of the components in quadrature, including $s_{a}$. They are to be estimated as the equivalent of "standard deviation". For convenience, the BIPM recommendations are reproduced in appendix 6 .

(4) It is generally not feasible to determine a probability distribution for each of these error estimates, even though one feels that the true value may be nearer the center than at the estimated limits. Thus it is safer (more conservative) to assume a rectangular distribution, with limits $\pm b$. The standard deviation, $\sigma$, of such a distribution is $\mathrm{b} / \sqrt{3}$.

C. F. Dietrich, of the British Calibration Service, has shown that if rectangular distributions are combined with each other and with a normal (Gaussian) distribution, the standard deviation of the combination is

$$
\sigma_{c}=\left(\sum_{1}^{n} \sigma_{1}^{2}\right)^{1 / 2}
$$

As a result of his extensive analysis of such combinations he states that [12]

"4.69 Let us look at this another way round. If we know the standard deviations of the Gaussian uncertainties of an item of calibration, and thus the total Gaussian standard deviation, and if we assess the maximum value of each of the estimated uncertainties, and assume that these have a rectangular distribution, we can at once find the total standard deviation of the measurements. We can then state that the probability of an uncertainty lying outside $\pm 2 \sigma$ is less than 0.0455 or the probability of an uncertainty lying outside \pm 30 is less than 0.0027 , without having to go to all the trouble of calculating the combined distribution and calculating the required probabilities. The correct 
probabilities will always be less than the corresponding Gaussian ones having the same tolerance limits and standard deviation, but usually by only a small amount."

This very important conclusion provides a simple yet generally realistic method of combining error estimates. It is on the conservative side (1.e., does not underestimate the total).

(5) A very important factor in error analysis is to gain enough knowledge of, and experience with, the measurement process to be able to find the (generally hidden) causes of residual uncorrected errors, and to get reasonable estimates of the error limits. This inevitably takes time.

(6) Because of the dificulty of finding error sources by simply repeating measurements in the same way, it is very desirable, as is well known, to vary the conditions of the measurement and note the effects on the results. The imposed variations should be well beyond the normal limits.

(7) Thus in research to establish the accuracy of physical standards, every effort should be made to vary or change the associated measuring equipment. In contrast, as Churchill Eisenhart has emphasized [13], the use of standards in calibrating others should be considered as a measurement process which must be kept under statistical control, with only specified ranges of the variables allowed. The measurement process should be well documented to insure this through the years.

(8) It is also very desirable in the research phase to make measurements with different standards and different comparison apparatus, whenever possible. We know that having different TE's made in different ways by different manufacturers can give a lot of confidence in the results if all of them agree, and can help disclose sources of error if they do not. 
(9) "Round-Robin" tests of three or more TE's or TVC's of the same range, and other more complex experimental designs, can also be very valuable means of disclosing systematic errors, as is well known.

(10) As figure 1 shows, there are several steps in the determination of the accuracy of the final or working standards of ac-dc difference. It is well to note that, if corrections are applied at each step for the known (determinate) errors, the estimates of remaining residual errors (these are estimates not errors) are combined as the square root of the sum of their squares (rss), not added directly. There is no need for the crippling "accuracy ratios" or factors of $3 / 1$ to $10 / 1$ in each step, as so often specified.

(11) Operator mistakes and certain equipment malfunctions are not amenable to analysis and inference, but can be major sources of error. For established calibration programs, where the volume of work justifies it, they can be reduced by computer control and computer verification of important parameters. They can also be reduced by repeating critical calibrations with a second operator and a second calibration console, and by the calibration of check standards in an adequate qual1ty-control program. Having a second comparator and equipment avallable has been very helpful in the ac-dc program, in both the research and calibration phase.

(12) Incorrect procedures, generally stemming from incomplete understanding, can also be major sources of error. As F. B. Silsbee has emphasized [14], the person in charge of a standardizing laboratory must have a high degree of technical knowledge and competence. This is especially true for ac-dc difference measurements, which are deceptively simple in principle but require a person with a sound knowledge of ac circuit theory to detect and overcome problems.

(13) It is to be expected that the ranges of the reference standards closest to those of the primary standards will be known with the best accuracy, and that the accuracy will decrease as both range and frequency are extended. Thus a realistic view of the uncertainty, $u$, will be that of a 
rather flat-topped cone in a 3-dimensional space, with the range and frequency as $\mathrm{x}$ and $\mathrm{y}$ coordinates and $1 / \mathrm{u}$ as the $\mathrm{z}$ (vertical) coordinate. The views for the working standards will, of course, be similar.

\section{RANDOM (STATISTICALLY EVALUATED) ERRORS}

\section{A. MJTC Comparator}

A limiting factor in the precision of TE comparisons was the noise level of the circuit and nanovoltmeter. With the 10-s averager for each reading and with $120 \mathrm{~s}$ required for an ac-dc determination, the pass band of interest extended from about 0.01 to $0.1 \mathrm{~Hz}$. Both the noise level and the response time of the nVM were dependent on the source resistance. With these factors in mind, a number of tests were made to evaluate the effects of noise and zero drift of the comparator and its nVM on ac-dc measurements, with the two thermocouple inputs shorted.

The first of these consisted of eyeballing the peak-to-peak excursions of the nVM for $10 \mathrm{~s}$, while at the same time taking 10-s averages of the nVM output (expressed in nanovolts of input). The average difference between these 10-s averages was generally $1 / 8 \mathrm{th}$ to $1 / 20$ th of the average of the peak-to-peak excursions, a marked improvement. However a better measure of the effect of noise on the integrated output was obtained by calculating a phantom $\Delta^{\prime}=d_{T}-d_{S}$, by the formula given in section III, using groups of successive 10-s readings and assuming $n E=20 \mathrm{mV}$. For both the 300 and 1000-nV ranges of the nVM usually used, the average $\Delta^{\prime}$ (without regard to sign) was less than $0.1 \mathrm{ppm}$.

Similar (although fewer) measurements were made with maximum source

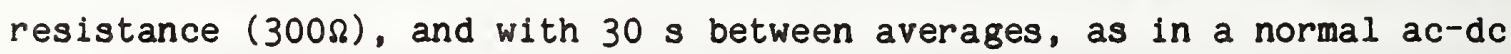
comparison. The values of $\Delta^{\prime}$ ranged up to $0.15 \mathrm{ppm}$. They would be expected to be 3.3 times as great with emf's of $3 \mathrm{mV}$, as in the TVC build-ups. These 
are the true limiting minimums of random errors imposed by the comparison process.

\section{B. Other Comparators}

Similar tests were not made for the 1966 comparator and for the calibration console. Both have been in use for long periods of time. Instead the pooled standard deviations of each were evaluated under the conditions of the present investigation, for TE and TVC comparisons, as discussed in the next sections.

\section{Statistical Parameters and Tests}

Four independent determinations were normally made at each frequency in each comparison of the TE's, and the average, $\Delta_{a}$, and its standard deviation, $s_{a}$, were computed. Since the same measurement process was involved in many such tests, pooled standard deviations were computed to increase the precision of estimating the true $\sigma$. If each of $\mathrm{m}$ comparisons has the same number of determinations, the formula for the pooled standard deviation of the average is simply

$$
s_{\mathrm{pa}}=\left(\frac{1}{\mathrm{~m}} \sum_{1}^{\mathrm{m}} \mathrm{s}_{\mathrm{a} 1}{ }^{2}\right)^{1 / 2}
$$

Preliminary calculations were needed to determine which tests to pool. Obviously comparisons at low emf's were likely to have larger $s_{a}$ 's than those at $10 \mathrm{mV}$. On the other hand tests at different frequencies rarely had significantly different $s_{a}$ 's.

The pooled "within-day" $s_{a}$ 's were used to more sharply determine the significance of differences between averages of measurements repeated on different days. For two measurements the "t" test, with the formula given in section III, was simplest. For more measurements, appropriate tests were used [15]. 
The round-robin triads of figures 3 and 4, the bulld-up comparisons for the TVC's, and the comparisons of ampere-range TE's with shunted TE's at low frequencies were all examined for evidence of systematic errors.

\section{Pooled Standard Deviations}

Table 14 shows the values of the pooled standard deviations, $\mathbf{s}_{\mathrm{pa}}$, for the several paths in the Road Map of figure 1. Each was grouped by parameters, such as type of comparator, frequency, and emf level, that were found to be significant.

The table shows the wide range of $s_{p a}$ 's from the MJTC's to the working standards. These values are combined in section XIII, with the systematic error estimates of section XII.

\section{E. Experimental Designs and Tests}

The sequence of readings in a determination (ac, dct, dc-, ac, equally spaced in time) is an example of a simple experimental design that eliminates the error from linear drifts in the TE's and comparator. Knight and his coworkers at NPL have used a least-squares fit to the readings in multiple ac-dc sequences to correct for 2 nd and $3 r d$ order drifts as well. However the excellent temperature stability in MET A144 at NBS, and the long thermal time-constant afforded by the thermally-insulated tank in which the TE's are placed, have made this unnecessary for this investigation. Erratic changes, chiefly from the nVM, would generally mask small non-linearities.

Experimental designs based on left-right interchanges of two standards to detect offsets could not be used directly, because of the often large differences in the emf's of the TE's (as great as 3/1). With the MJTC comparator, the higher emf must be connected to the divider, and the output of the TE with the higher $n$ must be connected to the potentiometer. 
Trlads of comparisons (round robins) are shown in figures 3 and 4 . In each of these there are two paths from one $T E$ to a second; one direct and the other via the third $T E$. If the $d_{2}-d_{1}$ paths differ by more than $(2 \sqrt{3}) s_{p a}$, there is evidence of systematic error at about the $95 \%$ confidence level. None of these 28 triads showed such evidence. Other more complicated round robins, used to evaluate TE's after the loss of FX and FY, also showed small closure errors.

In the 50 to $5 \mathrm{~mA}$ comparisons of figure 3 , the values of $\mathrm{d}$ for $F Y$ and EZ, obtained after 4 stages from OF 19, were within 1 ppm of those obtained from the direct MJTC tests at $1 \mathrm{kHz}$ and the subsequent frequency extensions.

\section{ESTIMATES OF RESIDUAL SYSTEMATIC ERRORS (NOT STATISTICALLY EVALUATED)}

There are many known sources of residual errors in the TE's, TVC's and comparator. A listing of those which are slgniflcant and of the estimates of their limits (bounds) is given in ppm (and percent of $\Delta=d_{T}-d_{S}$ where applicable) in table 15 for each of the blocks in the Road Map of figure 1. The estimates of limits are given for $1 \mathrm{kHz}$, where many errors are at a minimum, and for the highest frequency. Unless otherwise noted, equal bounds are implied; $1 . e ., 0.5$ signifies that it is considered to be very unlikely that the residual error will be outside the range -0.5 to $+0.5 \mathrm{ppm}$.

Many of the bounds were determined from actual measurements or specifications. Others were difficult to estimate, and a few are simply educated guesses; based, however, on extensive experience in ac-dc measurements. Some pertinent notes are given at the end of the table. Almost all of the sources of error are independent (not correlated). The few correlated sources are discussed in the next section. 


\section{COMBINATION OR UNCERTAIMTIES}

From the basic dimensionless equation given in section III-A $\mathrm{d}_{\mathrm{T}}=\mathrm{d}_{\mathrm{S}}+\left(\mathrm{N}_{\mathrm{d}}-\mathrm{N}_{\mathrm{a}}\right) / \mathrm{n}_{\mathrm{S}} \mathrm{E}_{\ell}$. Here the exponent of each variable is +1 or -1 . From the theory of errors [16], the propagation-of-error formula for the fractional standard deviation, $s_{r}$, of $d_{T}$, with uncorrelated variables having small random errors, reduces to $\left(\sum s_{1}^{2}\right)^{1 / 2}$, where each of the $s_{i}$ 's is the fractional standard deviation of an individual factor (considering $\left(N_{d^{-}} N_{a}\right)$ as a factor). In accordance with Dietrich [12], the same formula will apply safely enough for combinations of estimated residual uncertainties ${ }^{5}$, each taken to be uniformly distributed between estimated bounds, $\pm b$, with the standard deviation of each taken as $s_{1}= \pm b_{1} / \sqrt{3}$. These values of $s_{1}$ are the "equivalent standard deviations" of the BIPM report (appendix 6). Twice the overall standard deviation, $2 s_{0}$, is taken as a reasonable measure of the overall uncertainty. According to Dietrich the probability of a value lying outside this will be less than 0.05 .

This use of $2 s_{0}$ for the overall uncertainty, $U_{O}$, is strongly recommended by Dunn of the National Research Council of Canada [17], who also recommends the approach taken by Dietrich to the combination of uncertainties. It seems more appropriate than $3 s_{0}$ when many of the sources of error are considered to be of uniform probability density, since the long tails of the normal distribution are truncated.

Because of the several successive steps and the three different comparators used in evaluating the NBS reference and working standards, and because of the many steps in the extensions of the frequency and other ranges, the rather straightforward procedure of combining uncertainties from tables 14 and 15 becomes very involved. The detalled calculations are shown in table 16. They are generally self-explanatory. The results are the same as those obtained by adding variances and finding the overall uncertainty as twice the square root of the overall variance. However, no definite confidence limit is associated with the uncertainty, $U$.

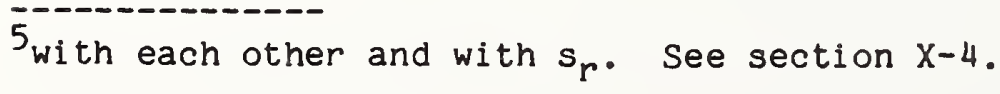


Extreme rigor in making these calculations did not seem warranted, especlally for the smaller U's, whlch do not effect the rss results very much.

In the $F_{1}$ TVC build-up the errors in determining the changes in the $d_{c} s$ of the TE's $\left(F X_{2}\right.$ and $\left.F Y_{2}\right)$ with current level are correlated; $1 . e .$, they add in each step of the build-up. Simllarly in the $F_{7}$ TVC build-up, the errors in determining the difference between the $d_{c}$ 's of $F_{7} 1$ and $F_{7} 2$ at the particular currents used add at each step. These correlated uncertainties were separately evaluated in the calculations of table 16.

It is to be noted that in each bulld-up involving chains of successive measurements the uncertainty at the end of each chain is given in table 16.

It is difficult to estimate the effects of the uncertainties which depend on the magnitude of $\Delta$, particularly for these chalns of measurements. These range from 2.8\% of A for the MJTC comparator to $3.6 \%$ for the other comparators. A prudent allowance for them is given in B of table 17 . Fortunately the values of d for the NBS standards are small, so that this is a signiflcant factor in only a few cases.

The values of $d$ and $U$ apply at the currents and voltages shown in the various tables. At other voltages and currents additional corrections must be applied to the d's, and the uncertainties will be somewhat greater. Appropriate currents and voltages were used in evaluating the reference standards. Fortunately the working standards are rarely used at other than rated inputs. 


\section{CONCLUSIOAS}

\section{A. Uncertainties of the NBS Standards}

The overall results of the calculations of the uncertainties are given in the important summary table 17. They apply at rated current and voltage, with the exceptions shown.

Several conclusions can be drawn from this table and the other results of this study, as follows:

(1) The long-term stability of the d's of the MJTC's is excellent. This, together with their low d's and $0.5 \mathrm{ppm}$ uncertainty, amply fulfilled the hope that they would be superior primary standards of ac-dc difference at the base frequency of $q \mathrm{kHz}$, and over the range from $30 \mathrm{~Hz}$ to $10 \mathrm{kHz}$.

(2) Their accuracy can be transferred to the single-junction TE's at $5 \mathrm{~mA}$ and 5 to $10 \mathrm{~V}$ to $9 \mathrm{ppm}$.

(3) The accuracy of these base ranges can be extended to $100 \mathrm{kHz}$ to better than 2 ppm.

(4) As expected, (see section $\mathrm{x}-13$ ) the uncertainty of the reference standards increases with increasing $V$ or I range. The most precise comparator can only be used to $50 \mathrm{~mA}$ or $100 \mathrm{~V}$, so the uncertainty cone has discontinuities in slope.

(5) As expected, the difficulties of extending the range increase at higher frequencies, and so do the uncertainties; generally by a factor of 1.5 to 2 times those at $1 \mathrm{kHz}$.

(6) As shown in table 16, the random-error component is significant in almost all comparisons. There is generally a reasonable balance between 
random and systematic components. However, better precision would be the first step to improving accuracies.

\section{B. Calibration Uncertainties}

(1) If TE's and TVC's of the same type and quality as the working standards are calibrated with the calibration console, it would be expected that the overall uncertainty would be the rss of the working standards from table 17 and the single step U's of $16-\mathrm{H}$ (minus the $\mathrm{s}_{\mathrm{e}}$ of the reference standard) or 16-I of table 16. The results of such calculations are given in table 18, along with the present calibration allowances, from section $I-C$.

(2) As the table shows, little improvement may be possible for the coaxlal TVC calibrations. For TCC calibrations the differences between the rss uncertainties and the allowances are deceptive. Individual TE's of the same quality as our standards are no longer avallable in the ampere ranges. The multirange TVC's with shunts that are commerclally avallable are much more complex. Experience has indlcated that they have larger and less stable d's than do the single-range TE's and coaxial TVC's.

(3) If NBS is pressed for better accuracy, particularly for TVC's, the needs might be met by using the uncertainty-cone concept to specify smaller uncertainties at and near the apex. In this way the basic accuracy at NBS could be transported via sultable ac-dc transfer standards to the client's standards laboratory.

(4) Very high accuracies are attainable over limited ranges for very special tests (such as international comparisons) in which such accuracies are really needed. They would be made by directly comparing single-range coaxial TVC's up to $100 \mathrm{~V}$ with the $F_{1}$ resistors and reserve $T E ' s$ (FA and FB), or single-junction TE's up to $50 \mathrm{~mA}$ with one of the pairs of reference TE's, using the MJTC comparator in each case. The results of calculations (similar to those described in B-1 of this section) are shown in table 19, to substantiate this. 
(5) A comparison was made of the d's of the working standards shown in tables 11 and 13 with the values previously assigned from earlier tests and presently used in calibration work. For the $F_{7}$ TVC's, the largest difference between the d's was $23 \mathrm{ppm}$, on the 600-V range at $100 \mathrm{kHz}$ (the highest voltage available at this frequency). It was $10 \mathrm{ppm}$ on the $1000-\mathrm{V}$ range at $50 \mathrm{kHz}$, and much less at most other ranges and frequencles. The average difference for all ranges at all frequencies (with regard to sign) was only $2 \mathrm{ppm}$. For the current-measuring TE's the largest difference between the d's was $36 \mathrm{ppm}$. The average for all ranges at all frequencles was only $8 \mathrm{ppm}$. These low differences attest to both the quality of the earlier measurements and the stability of the NBS ac-dc transfer standards. They are comfortably below the uncertainties of calibrations shown in section I-C of this report.

\section{RECOMENDATIONS}

\section{A. Maintenance of AC-DC Standards}

(1) The NBS standards are belleved, with reason, to be very stable. However if better accuracy than heretofore is to be maintained, it would be prudent to compare the working standard TE's with the reference standards every three years, and to compare adjacent ranges of the $F_{7}$ TVC set at similar intervals. This could be modified by experience -- see recommendation $\mathrm{B}-6$.

(2) The difficult and very time-consuming recalibrations of the reference standards might only be necessary every 9 years, if the 3-year tests show no significant changes.

(3) Spare $F_{7} 1$ and $F_{7}{ }^{2}$ modules should be avallable in case of burnout, with values $d_{v}$ and $d_{c}$ known by prior comparison with the present ones. FA and FB, an extra pair of 5-mA TE's with known $d_{v}$ and $d_{c}$, are already available for the $F_{1}$ reference set. The substitution formulas of section IX 
should make it possible to calculate the $d_{v}$ 's for the other TVC ranges, without recalibrating each one, when a TE is replaced.

(4) AC-DC differences as large as $9 \mathrm{ppm}$ have been observed even in Evanohm-heater TE's, so replacements should never be used without recalibration.

\section{B. Quality Control of the Calibration Process}

(1) The principles of quality control given by Eisenhart [13] should be followed. Check standards should be evaluated periodically in the calibration console. They should be of the same types as the major kinds of ac-dc standards normally calibrated. In particular the multirange standard, which was purchased in part for this purpose, should be put in service, as well as the spare Model F TVC's on which a partial history has already been garnered.

(2) NBS statisticans should be consulted and should be of major help in setting up these programs. Careful selection of a few ranges and frequencies should not make these very Important quality-control tests too time-consuming or onerous.

(3) The waveform and frequency of the applied ac should be observed on a callbrated CRO in each test, and the frequency setting of the oscillator should be double-checked to avoid mistakes.

(4) The very simple EMI test described in section III-A should be made occaslonally, especlally for low ranges.

(5) A second comparator and associated equipment should always be avallable and ready for use as a check against unsuspected (or suspected) errors. This is particularly valuable when new or modifled ac-dc standards are first tested. 
(6) As experience with the check standards and the bienniel calibrations of the working standards is accumulated, the recommended calibration periods of $\mathrm{A}^{-1}$ for the working and reference standards may safety be revised.

\section{Other Recommendations}

(1) Almost all of the TE's from $5 \mathrm{~mA}$ to $20 \mathrm{~A}$ were made to our special order by American manufacturers who no longer make TE's. The MJTC's are not commercially available at all. All of these now form an unique collection of well-evaluated reference standards, and should be reserved solely for evaluating the NBS working standards and for comparisons with other national laboratories, or for other special test in which the highest accuracy is really required. The MJTC comparator, with its high precision, should be avallable for use when this is really needed.

(2) The new types of solid-state thermoelements (SSTE's) have much higher emf's than the conventional TE's, but are subject to the same basic limitations on ac-dc difference from Thomson, Peltier and other effects. If Wilkins'-type MJTC's can not be obtained commercially in the near future, serious consideration should be given to designing and making thin-film MJTC's by cooperation with other divisions at NBS.

(3) The new values of d for the working standards, from tables 11 and 13. should be used in place of those now in the computer. However any stated improvement in the calibration uncertainties should be withheld until some checks on the error analyses are made by comparing the NBS working and reference standard TE's and TVC's in both the calibration and 1966 consoles. In addition any improvement in the stated uncertainty of the more complex multirange TVC's and their shunts should await the installation of a quality-control program and further studies of these instruments. 


\section{ACWOULEDGEHENTS}

Sincere thanks are expressed to J. R. Hastings, E. S. Will1ams, C. B. Chllders, and K. B. Krishnamurthy, who assisted in this invest1gation, to $N$. Beleckl for his encouragement and support, to $C$. Reeve for his helpful suggestions and review of the statistical procedures, and to $T$. Lipe for his review of the entire report. 


\section{REFERENCES}

1. Thermal Converters as AC-DC Transfer Standards for Current \& Voltage Measurements at Audio Frequencies, F. L. Hermach, Jour. Res. NBS, v. 28, no. 2, p. 121 , Feb. 1952.

2. Thermal Converters for Audio-Frequency Voltage Measurements of High Accuracy, F. L. Hermach and E. S. Williams, IEEE Trans. on Instrum. \& Measmt., v. IM-15, p. 260, Dec. 1966.

3. Thermal Voltage Converters \& Comparator for Very Accurate AC Voltage Measurements, E. S. Williams, Jour. Res. NBS, v. 75C, p. 145, July-Dec. 1971.

4. An Investigation of Multijunction Thermal Converters, F. L. Hermach and D. R. Flach, IEEE Trans. on Instrum. \& Measmt., v. IM-25, p. 524, Dec. 1976.

5. Thermal Voltage Converters for Accurate Voltage Measurements to 30 Megacycles per Second, F. L. Hermach and E. S. Williams, AIEE Trans., v. 79-I, p. 200, July 1960.

6. Thermal Current Converters for Accurate AC Current Measurement, E. S. Williams, IEEE Trans. on Instrum. \& Measmt., v. IM-25, p. 519, Dec. 1976.

7. Errors in AC-DC Transfer Arising from a DC Reversal Difference, B. D. Inglis, Metrologia, v. 17, p. 111, 1981.

8. Theoretical Analysis of the AC/DC Transfer Difference of the NPL Multijunction Thermal Converter Over the Frequency Range DC to $100 \mathrm{kHz}$, F. J. Wilkins, IEEE Trans. on Instrum. \& Measmt., v. IM-21, p. 334, Nov. 1972. 
9. Evaluation of AC-DC Transfer Errors for Thermal Converter-Mult1plier Combinations, B. D. Ingl1s, Metrolog1a, v. 16, p. 177, 1980.

10. Uncertainties In Calibrations, W. Youden, IRE Trans. Instrum., V. I-11, p. 133, Dec. 1962.

11. Uncertainty Ass1gnment, B. Taylor, Memo for Technical Staff of the NBS Electricity Division, Aug. 21, 1981.

12. Uncertainty, Calibration, and Probability, C. F. Dietrich, Halsted Press, 1973.

13. Realistic Evaluation of the Precision and Accuracy of Instrument Calibration Systems, C. Eisenhart, J. Res. NBS, v. 67C, p. 161, Apr-June 1963.

14. Suggested Practices for Electrical Standardizing Laboratories, F. B. Silsbee, NBS C1rcular 578, 1956.

15. Experimental Stat1st1cs, M. G. Natrella, NBS Handbook 91, 1966 (Chapters 3 and 15).

16. Notes on the Use of Propagation of Error Formulas, H. H. Ku, J. Res. NBS, v. 70C, p. 263, Oct-Dec 1966.

17. Measurement Assurance, A. F. Dunn, NRC, Canada, Report 18843, 1980.

18. A Resistor Calculable from $A C$ to $w=10^{5} \mathrm{rad} / \mathrm{s}$, R. J. Haddad, Thesis, GWU School of Engineering and Applied Science, April 1969.

19. The Theory of Peltier \& Thomson Errors in Thermal AC-DC Transfer Devices, F. C. Widdis, IEE Monograph 497M, Jan 1962. 
Table 1

Comparisons of Four MTC's

\begin{tabular}{|c|c|c|c|c|c|c|}
\hline \multirow[b]{2}{*}{$\mathbf{v}$} & \multirow[b]{2}{*}{$\mathbf{I}$} & \multirow[b]{2}{*}{$\mathbf{s}$} & \multicolumn{3}{|c|}{$-d_{T}-d_{S}(p p m)$} & \multirow[b]{2}{*}{ Date } \\
\hline & & & $30 \mathrm{~Hz}$ & $1 \mathrm{kHz}$ & $10 \mathrm{kHz}$ & \\
\hline \multirow[t]{2}{*}{10.0} & $\begin{array}{c}G 44 \\
(B 10-44)\end{array}$ & $\begin{array}{c}\text { G5C } \\
(B 5-2)\end{array}$ & +0.47 & +0.15 & -0.44 & $12 / 08 / 81$ \\
\hline & & & & -0.10 & & $12 / 11 / 81$ \\
\hline 6.0 & & & & -0.02 & & $12 / 11 / 81$ \\
\hline \multirow[t]{2}{*}{6.0} & $\begin{array}{l}\text { Gr25-1 } \\
\text { (C10-1) }\end{array}$ & $\begin{array}{c}\text { G5C } \\
(B 5-2)\end{array}$ & +0.32 & & -0.29 & $12 / 11 / 81$ \\
\hline & & & & -0.03 & & $12 / 14 / 81$ \\
\hline \multirow[t]{2}{*}{6.0} & $\begin{array}{l}\text { Gr25-1 } \\
\text { (c10-1) }\end{array}$ & $\begin{array}{c}G 44 \\
(B 10-44)\end{array}$ & +0.17 & -0.01 & -0.05 & $12 / 14 / 81$ \\
\hline & & & & +0.06 & & $12 / 18 / 81$ \\
\hline \multirow[t]{3}{*}{8.0} & $\begin{array}{c}\text { NPL } 14 \\
(A 50-14)\end{array}$ & $\begin{array}{c}\text { G44 } \\
\text { (B10-44) }\end{array}$ & & +0.34 & & $12 / 18 / 81$ \\
\hline & & & & +0.36 & & $12 / 18 / 81$ \\
\hline & & & -0.40 & +0.27 & & $12 / 18 / 81$ \\
\hline 6.0 & $\begin{array}{l}\text { Gr25-1 } \\
(\mathrm{C} 10-1)\end{array}$ & $\begin{array}{c}\text { NPL } 14 \\
(A 50-14)\end{array}$ & +0.52 & -0.22 & & $12 / 18 / 81$ \\
\hline
\end{tabular}


Table 2

Differences Between 1981 and 1976 Evaluations of MJT's

\begin{tabular}{cccc} 
& \multicolumn{3}{c}{$\mathrm{d}_{81}-\mathrm{d}_{76}(\mathrm{ppm})$} \\
\cline { 2 - 3 } & $\underline{30 \mathrm{~Hz}}$ & $\underline{1 \mathrm{kHz}}$ & $\underline{10 \mathrm{kHz}}$ \\
G44 & +0.2 & 0.0 & +0.1 \\
Gr25-1 & 0.0 & 0.0 & -0.4 \\
G5C & +0.3 & -0.1 & +0.1 \\
G10C & -0.3 & 0.0 & 0.0 \\
NPL 14 & -0.2 & 0.0 & -
\end{tabular}




\section{Table 3}

Results of Comparisons of FX and FY with MTC's

\begin{tabular}{|c|c|c|c|c|}
\hline \multirow{2}{*}{$\begin{array}{l}\text { TE } \\
\text { or } \\
\text { TVC } \\
\end{array}$} & \multirow{2}{*}{$\begin{array}{r}I \\
\text { or } \\
V \\
\end{array}$} & \multicolumn{3}{|c|}{$d(p p m)$} \\
\hline & & $30 \mathrm{~Hz}$ & $1 \mathrm{kHz}$ & $10 \mathrm{kHz}$ \\
\hline FX & $5 \mathrm{~mA}$ & - & -0.3 & -0.2 \\
\hline$E X$ & $2.5 \mathrm{~mA}$ & - & 0.0 & -0.1 \\
\hline$E_{15}-E X^{1}$ & $5 \mathrm{~V}$ & +0.9 & -0.4 & -1.2 \\
\hline$F_{15-E X}$ & $3 v$ & -0.1 & -0.7 & -1.0 \\
\hline FY & $5 \mathrm{~mA}$ & - & +0.2 & 0.0 \\
\hline EY & $3 \mathrm{~mA}$ & - & +0.4 & -0.2 \\
\hline$F_{1} 10-F Y$ & $10 \mathrm{~V}$ & +0.8 & -0.3 & -0.8 \\
\hline$F_{1} 10-F Y$ & $5 \mathrm{~V}$ & +0.2 & +0.1 & -1.2 \\
\hline
\end{tabular}

${ }^{1} F_{15-F X}$ consists of the 5-V resistor of the F TVC set, in series with TE FX. 
Table 4

Results of TVC Tests with Twin Resistors

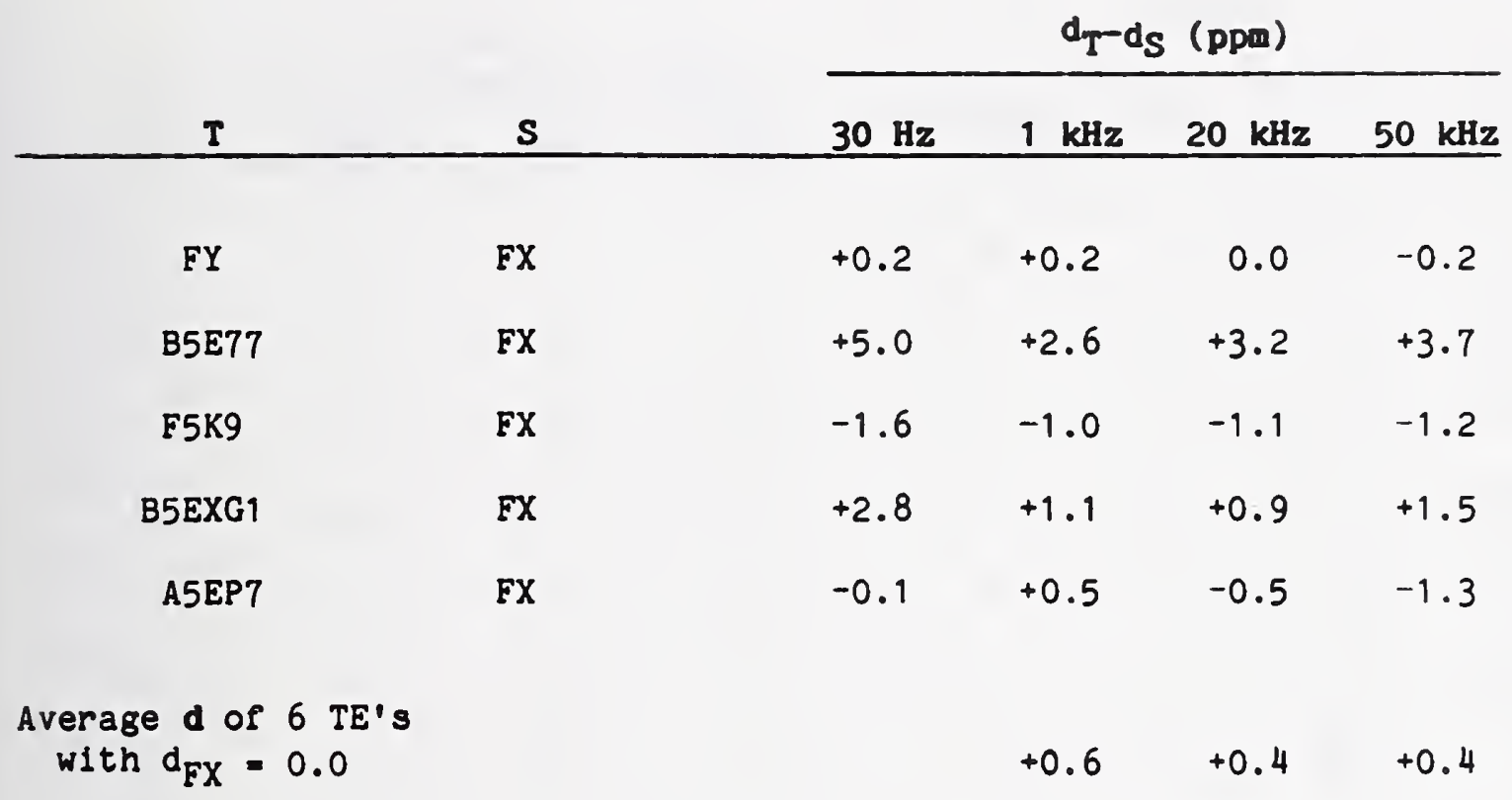

NOTE: All tests at $5 \mathrm{~mA}$.

$30 \mathrm{~Hz}$ results not averaged. See text. 
Table 5

Appere-Range Comparisons

\begin{tabular}{|c|c|c|c|c|c|c|c|}
\hline \multirow[b]{2}{*}{$I$} & & & \multirow{2}{*}{$\begin{array}{c}I \\
(A)\end{array}$} & \multicolumn{4}{|c|}{$d_{T} d_{S}$} \\
\hline & \multicolumn{2}{|c|}{$s$} & & $20 \mathrm{~Hz}$ & $1 \mathrm{kHz}$ & $20 \mathrm{kHz}$ & $50 \mathrm{kHz}$ \\
\hline W20A $7-1$ & WIOA & $\# 0-1$ & 10 & +1 & +11 & +17 & +10 \\
\hline W1OA:0-1 & W5A & $\# 5-2$ & 5 & -5 & -2 & 0 & +16 \\
\hline W1OA:0-1 & W5A & $\# 5-1$ & 5 & -6 & -6 & -6 & +17 \\
\hline W5A $5-2$ & W3A & $\# 4-1$ & 3 & +1 & -1 & +4 & +16 \\
\hline W3A $4-1$ & W2A & $\# 3-2$ & 2 & +3 & 0 & -7 & -11 \\
\hline W2A $: 3-2$ & WIA & $\# 7-2$ & 1 & -4 & -2 & -5 & -13 \\
\hline $\begin{array}{l}\text { FG3A } \\
\text { FG3A }\end{array}$ & $\begin{array}{l}\text { W3A } \\
\text { W3A }\end{array}$ & $\begin{array}{l}* 4-1 \\
\# 4-1\end{array}$ & $\begin{array}{l}3 \\
2\end{array}$ & $\begin{array}{l}-2 \\
-4\end{array}$ & $\begin{array}{r}0 \\
+1\end{array}$ & $\begin{array}{r}+15 \\
+15\end{array}$ & $\begin{array}{l}+43 \\
+46\end{array}$ \\
\hline $\begin{array}{l}\text { FG1A } \\
\text { FG1A }\end{array}$ & $\begin{array}{l}\text { W1A } \\
\text { W1A }\end{array}$ & $\begin{array}{l}\# 7-2 \\
\# 7-2\end{array}$ & $\begin{array}{c}1 \\
0.6\end{array}$ & $\begin{array}{l}-3 \\
+1\end{array}$ & $\begin{array}{r}-2 \\
0\end{array}$ & $\begin{array}{l}+11 \\
+15\end{array}$ & $\begin{array}{l}+29 \\
+36\end{array}$ \\
\hline $\begin{array}{l}\text { FH2A } \\
\text { FH2A }\end{array}$ & $\begin{array}{l}\text { W2A } \\
\text { W2A }\end{array}$ & $\begin{array}{l}* 3-2 \\
\# 3-2\end{array}$ & $\begin{array}{l}1.2 \\
2.0\end{array}$ & -2 & $\begin{array}{r}-2 \\
0\end{array}$ & $\begin{array}{l}-12 \\
-14\end{array}$ & $\begin{array}{l}-40 \\
-33\end{array}$ \\
\hline $\begin{array}{l}\text { FH2OA } \\
\text { FH2OA }\end{array}$ & $\begin{array}{l}\text { W2OA } \\
\text { W2OA }\end{array}$ & $\begin{array}{l}\not 7-1 \\
\# 7-1\end{array}$ & $\begin{array}{l}10 \\
16\end{array}$ & & & $\begin{array}{l}-21 \\
-22\end{array}$ & $\begin{array}{l}-49 \\
-59\end{array}$ \\
\hline
\end{tabular}

HOTB: FG1A, FH2A, FG3A, and FH2OA are Williams' shunts with low-range TE's, $F G$ and $F H$. 
Table 6

AC-DC Differences of $1 \mathrm{~A}$ to $20 \mathrm{~A} \mathrm{TE}^{\prime} \mathrm{s}$

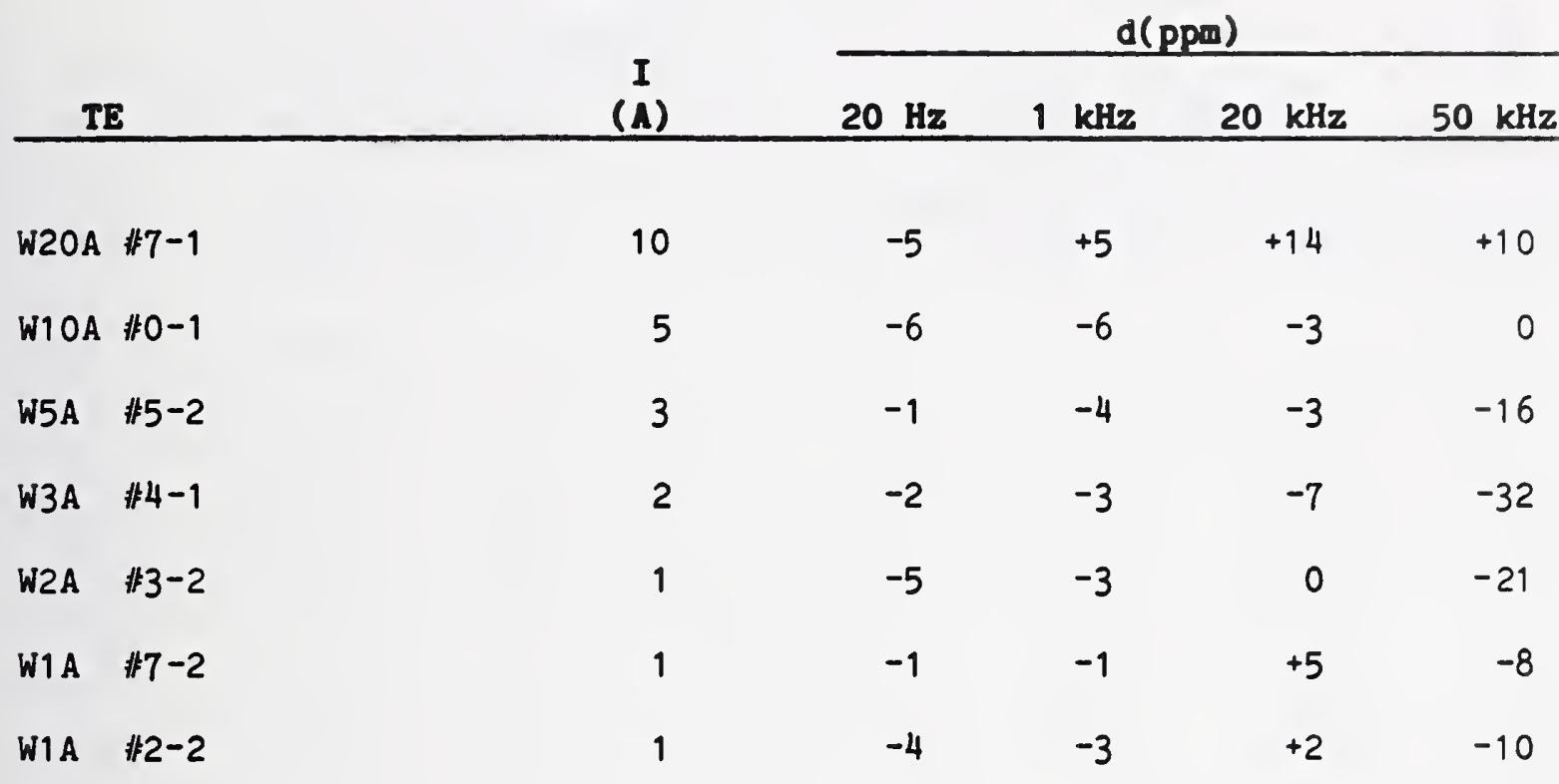


Table 7

Results of Evaluation of $\mathrm{FX}_{2}$ and $\mathrm{FY}_{2}$

\begin{tabular}{|c|c|c|c|c|c|c|c|}
\hline \multirow{2}{*}{$\begin{array}{c}\text { TE } \\
\text { or } \\
\text { TVC } \\
\end{array}$} & \multirow{2}{*}{$\begin{array}{r}I \\
\text { or } \\
\text { V } \\
\end{array}$} & \multicolumn{6}{|c|}{$d(p p$ m $)$} \\
\hline & & $20 \mathrm{~Hz}$ & $1 \mathrm{kHz}$ & $10 \mathrm{kHz}$ & $20 \mathrm{kHz}$ & $50 \mathrm{kHz}$ & $100 \mathrm{kHz}$ \\
\hline \multirow{3}{*}{$F_{1} 10-F Y_{2}$} & $10 \mathrm{~V}$ & +3.9 & +2.4 & +1.3 & +1.0 & +0.6 & +0.3 \\
\hline & 7 & +2.0 & +1.4 & +0.6 & & & \\
\hline & 5 & +1.6 & \pm 1.4 & 0.0 & & & \\
\hline \multirow[t]{3}{*}{$\mathrm{FY}_{2}$} & $5 \mathrm{~mA}$ & +4.7 & +3.2 & & +3.6 & +3.5 & +3.9 \\
\hline & 3.3 & +2.8 & +2.4 & & & & +2.9 \\
\hline & 2.5 & +2.2 & +2.3 & & & & \\
\hline \multirow[t]{3}{*}{$\mathrm{FX}_{2}$} & $5 \mathrm{~mA}$ & +3.5 & +2.2 & & +2.4 & +2.1 & +2.2 \\
\hline & 3.3 & +2.3 & +1.3 & & & & +1.3 \\
\hline & 2.5 & +1.6 & +1.9 & & & & \\
\hline
\end{tabular}


Tabl• 8

Comparisons of FitVC's

$\left(F X_{2}\right.$ and $\left.F Y_{2} \mathrm{TE}^{\prime} \mathrm{s}\right)$

$\Delta=d_{T}-d_{S}(p p a)$

T

s

$20 \mathrm{~Hz}, 1 \mathrm{kHz}$

$20 \mathrm{kHz}$

$50 \mathrm{kHz}$

$100 \mathrm{kHz}$

$\begin{array}{lllccccc}F 200-F X_{2} & F 100-F Y_{2} & 100 & - & -1.1 & +0.2 & +1.4 & +17.8 \\ F 100-F Y_{2} & F 50-F X_{2} & 50 & - & +0.6 & -0.8 & -2.8 & -6.2 \\ F 50-F X_{2} & F 30-F Y_{2} & 30 & - & -2.5 & -1.6 & -2.0 & -1.5 \\ F 30-F Y_{2} & F 20-F X_{2} & 20 & -1.3 & +0.7 & -0.3 & 0.0 & -2.0 \\ F 20-F X_{2} & F 10-F Y_{2} & 10 & -3.8 & -1.7 & -2.1 & -2.9 & -6.0 \\ F 10-F Y_{2} & F 5-F X_{2} & 5 & -0.6 & +0.2 & -0.3 & -1.3 & -2.5 \\ F 5-F X_{2} & F 3-F Y_{2} & 3 & -1.6 & -0.6 & -0.8 & -3.1 & -6.3 \\ F 3-F Y_{2} & F 2-F X_{2} & 2 & 0.0 & +1.4 & +0.7 & -1.1 & -4.1 \\ F 2-F X_{2} & F 1-F Y_{2} & 1 & +0.4 & +1.9 & +0.7 & -3.9 & -13.1 \\ F 1-F Y_{2} & F X_{2} & 0.5 & +1.0 & +3.4 & +4.2 & -0.7 & -6.1 \\ F X_{2} & F Y_{2} & 0.5 & +1.2 & +1.1 & +1.9 & +2.2 & +2.5\end{array}$

Comparisons with $F_{7} 1, E X$, and $F Y$ in place of $F X_{2}$ and $F Y_{2}$

$\begin{array}{llllllll}\text { F1000-F } 71 & \text { F500-FX } & 500 & - & -2.0 & -15.1 & -1.7 & - \\ \text { F500-FX } & \text { F300-FY } & 300 & - & -0.8 & -0.3 & -4.1 & -33 \\ \text { F300-FY } & \text { F200-FX } & 200 & - & -0.7 & -0.5 & -2.3 & +6.7\end{array}$


Table 9

AC-DC Differences of $F_{1}$ TVC's with $F X_{2}$ and $F Y_{2}$

$d(\mathrm{ppm})$

\begin{tabular}{|c|c|c|c|c|c|c|}
\hline$E_{1000 E-E_{7}}$ & 500 & - & +2.7 & $-11 \cdot 5$ & -6.1 & - \\
\hline $\mathrm{F} 500-\mathrm{FX}_{2}$ & $\begin{array}{l}500 \\
300\end{array}$ & - & $\begin{array}{l}+2.5 \\
+1.7\end{array}$ & $\begin{array}{l}+1.2 \\
+0.4\end{array}$ & $\begin{array}{l}-6.5 \\
-7.3\end{array}$ & $\begin{array}{l}-18.6 \\
-19.4\end{array}$ \\
\hline$F 300-F_{2}$ & $\begin{array}{l}300 \\
200\end{array}$ & - & $\begin{array}{l}+3.5 \\
+2.7\end{array}$ & $\begin{array}{l}+1.9 \\
+1.1\end{array}$ & $\begin{array}{l}-1.8 \\
-2.6\end{array}$ & $\begin{array}{l}+15.6 \\
+14.8\end{array}$ \\
\hline $\mathrm{F} 200-\mathrm{FX}_{2}$ & $\begin{array}{l}200 \\
100\end{array}$ & - & $\begin{array}{l}+2.4 \\
+1.6\end{array}$ & $\begin{array}{l}+0.4 \\
-0.4\end{array}$ & $\begin{array}{l}-1.7 \\
-2.5\end{array}$ & $\begin{array}{l}+6.4 \\
+5.6\end{array}$ \\
\hline$F_{100-F Y_{2}}$ & $\begin{array}{l}100 \\
50\end{array}$ & - & $\begin{array}{l}+2.7 \\
+1.9\end{array}$ & $\begin{array}{l}-0.6 \\
-1.4\end{array}$ & $\begin{array}{l}-3.9 \\
-4.7\end{array}$ & $\begin{array}{l}-12.2 \\
-13.0\end{array}$ \\
\hline $\mathrm{F} 50-\mathrm{FX}_{2}$ & $\begin{array}{l}50 \\
30\end{array}$ & - & $\begin{array}{l}+1.3 \\
+0.5\end{array}$ & $\begin{array}{l}-0.6 \\
-1.4\end{array}$ & $\begin{array}{l}-1.9 \\
-2.7\end{array}$ & $\begin{array}{l}-6.8 \\
-7.6\end{array}$ \\
\hline$F 30-F Y_{2}$ & $\begin{array}{l}30 \\
20\end{array}$ & $\begin{array}{l}+2.6 \\
+0.7\end{array}$ & $\begin{array}{l}+3.0 \\
+2.2\end{array}$ & $\begin{array}{l}+0.2 \\
-0.6\end{array}$ & $\begin{array}{l}-0.7 \\
-1.5\end{array}$ & $\begin{array}{l}-6.1 \\
-6.9\end{array}$ \\
\hline $\mathrm{F} 20-\mathrm{FX}_{2}$ & $\begin{array}{l}20 \\
10\end{array}$ & $\begin{array}{l}+2.0 \\
+0.1\end{array}$ & $\begin{array}{l}+1.5 \\
+0.7\end{array}$ & $\begin{array}{l}-0.3 \\
-1.1\end{array}$ & $\begin{array}{l}-1.5 \\
-2.3\end{array}$ & $\begin{array}{l}-4.9 \\
-5.7\end{array}$ \\
\hline $\mathrm{F}_{10} \mathrm{O}-\mathrm{FY}_{2}$ & $\begin{array}{r}10 \\
5\end{array}$ & $\begin{array}{l}+3.9 \\
+1.4\end{array}$ & $\begin{array}{l}+2.4 \\
+1.6\end{array}$ & $\begin{array}{l}+1.0 \\
+0.2\end{array}$ & $\begin{array}{l}+0.6 \\
-0.2\end{array}$ & $\begin{array}{l}+0.3 \\
-0.5\end{array}$ \\
\hline $\mathrm{F} 5-\mathrm{FX}_{2}$ & $\begin{array}{l}5 \\
3\end{array}$ & $\begin{array}{l}+2.0 \\
+0.7\end{array}$ & $\begin{array}{l}+1.4 \\
+0.6\end{array}$ & $\begin{array}{l}+0.5 \\
-0.3\end{array}$ & $\begin{array}{l}+1.1 \\
+0.3\end{array}$ & $\begin{array}{l}+2.0 \\
+1.2\end{array}$ \\
\hline $\mathrm{F}_{3}-\mathrm{FY}_{2}$ & $\begin{array}{l}3 \\
2\end{array}$ & $\begin{array}{l}+2.3 \\
+0.4\end{array}$ & $\begin{array}{l}+1.2 \\
+0.4\end{array}$ & $\begin{array}{l}+0.5 \\
-0.3\end{array}$ & $\begin{array}{l}+3.4 \\
+2.6\end{array}$ & $\begin{array}{r}+7.5 \\
+6.7\end{array}$ \\
\hline$F 2-F X_{2}$ & $\begin{array}{l}2 \\
1\end{array}$ & $\begin{array}{l}+0.4 \\
-1.5\end{array}$ & $\begin{array}{l}-1.0 \\
-1.8\end{array}$ & $\begin{array}{l}-1.0 \\
-1.3\end{array}$ & $\begin{array}{r}+3.7 \\
+3.9\end{array}$ & $\begin{array}{l}+10.8 \\
+10.0\end{array}$ \\
\hline $\mathrm{F}_{1}-\mathrm{FY}_{2}$ & $\begin{array}{c}1 \\
0.5\end{array}$ & $\begin{array}{l}-1.9 \\
-4.4\end{array}$ & $\begin{array}{l}-3.7 \\
-4.5\end{array}$ & $\begin{array}{l}-2.5 \\
-3.3\end{array}$ & $\begin{array}{l}+6.8 \\
+6.0\end{array}$ & $\begin{array}{r}+23.1 \\
+22.3\end{array}$ \\
\hline $\mathrm{FX}_{2}$ & 0.6 & -5.4 & -7.9 & -7.5 & +6.7 & +28.4 \\
\hline $\mathrm{FY}_{2}$ & 0.6 & -6.6 & -8.9 & -9.4 & +4.5 & +25.9 \\
\hline
\end{tabular}


Table 10

Comparisons of Horking and Reference Standard TE's

\begin{tabular}{|c|c|c|c|c|c|c|}
\hline \multirow[b]{2}{*}{ Test } & \multirow[b]{2}{*}{ Standard } & \multirow{2}{*}{$\begin{array}{c}I \\
(m A)\end{array}$} & \multicolumn{4}{|c|}{$\Delta(p p m)$} \\
\hline & & & $20 \mathrm{~Hz}$ & $1 \mathrm{kHz}$ & $20 \mathrm{kHz}$ & $50 \mathrm{kHz}$ \\
\hline A3 非95 & $E A$ & 5 & +5 & +2 & +9 & +18 \\
\hline G10 非57 & B1 OEP2 & 10 & -5 & -5 & -4 & -3 \\
\hline F20 非1 & W25E1 & 20 & -9 & -7 & -5 & +1 \\
\hline W30 \#1 & W25E1 & 25 & -12 & -11 & -12 & -12 \\
\hline F50 非2 & W50E1 & 50 & -37 & 0 & +3 & -2 \\
\hline B100 非3 & W1OOEI & 100 & -18 & -20 & -20 & -24 \\
\hline A250 非 4 & W250E1 & 250 & -19 & -22 & -28 & -44 \\
\hline
\end{tabular}

(A)

W0.5A झ0-2 W0.5A 非-1

W1A 非7-1 N1A 非-2

W2A 非8-3 W2A 非-2

W3A 非-2 W3A 非-1

W5A 非-1 W5A 非-2

W1OA 非-3 W1OA 非-1

W20A 非-2 W2OA 非-1
0.5

1

2

3

5

$-5$

$25 \mathrm{~Hz}$

10

$-6$

$-5$

$-13$

$-28$

$100 \mathrm{~Hz}$

16

$-2$

$-2$

$+1$

$-1$

$-7$

$-11$

$-13$

\section{0 tes}

$+3$

$-10$

$-18$

$-14$ 
Table 11

AC-DC DIfferences or Horking Standard TE's

\begin{tabular}{|c|c|c|c|c|c|}
\hline \multirow[b]{2}{*}{ TE } & \multirow{2}{*}{$\begin{array}{c}I \\
(m)\end{array}$} & \multicolumn{4}{|c|}{ AC-DC Difference (ppm) } \\
\hline & & $20 \mathrm{~Hz}$ & $1 \mathrm{kHz}$ & $20 \mathrm{kHz}$ & $50 \mathrm{kHz}$ \\
\hline A5 \#95 & 5 & +2 & 0 & +8 & +17 \\
\hline G10 非7 & 10 & -6 & -6 & -5 & -4 \\
\hline F20\#1 & 20 & -10 & -8 & -6 & 0 \\
\hline W30 \#1 & 30 & -13 & -12 & -13 & -13 \\
\hline F50 \#2 & 50 & -36 & -1 & +2 & -3 \\
\hline B100 \#3 & 100 & -20 & -21 & -20 & -23 \\
\hline A250 \#4 & 250 & -24 & -22 & -28 & -43 \\
\hline
\end{tabular}

(A)

W0.5A \#0-2

0.5

0

$-2$

$-3$

$-7$

W1A $\# 7-1$

$-4$

$-5$

$-14$

W2A \#8-3

2

$-2$

$+9$

$-23$

W3A \#9-2

3

0

$-2$

$+1$

$-35$

W5A \#5- 9

5

$-6$

$-4$

$-8$

$-35$

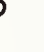

$25 \mathrm{~Hz}$

W1OA \#0-3

10

$-12$

$-11$

$-16$

$-28$

$100 \mathrm{~Hz}$

W20A \#1-2

16

$+8$

$-5$

$-4$

$-4$ 
Table 12

Comparisons of $\mathrm{F}_{7}$ TVC's

T

$\mathrm{F}_{7} 1000-10 \mathrm{E}-\mathrm{F}_{7} 1$

$\mathrm{F}_{7} 500-10 \mathrm{C}-\mathrm{F}_{7} 1$

$E_{7500-10 D-F_{7}}$

$E_{7300-F_{7}}$

$\mathrm{F}_{7} 100-\mathrm{F}_{7} 1$

$\mathrm{F}_{7} 30-\mathrm{F}_{7} 1$

$F_{7}^{10-F_{7}}$

$\mathrm{F}_{7} 3-\mathrm{F}_{7} 1$

$F_{71}$ s

$$
\mathrm{F}_{7} 600-\mathrm{F}_{7}{ }^{2}
$$$$
\mathrm{F}_{7} 600-\mathrm{F}_{7}{ }^{2}
$$

$\mathrm{F}_{7} 600-\mathrm{F}_{7}{ }^{2}$

$\mathrm{F}_{7} 200-\mathrm{F}_{7} 2$

$\mathrm{F}_{7} 60-\mathrm{F}_{7} 2$

$\mathrm{F}_{7} 20-\mathrm{F}_{7}{ }^{2}$

$F_{7} 6-F_{7} 2$

$\mathrm{F}_{7}{ }^{2}$

$\mathrm{F}_{7}{ }^{2}$ v $20 \mathrm{~Hz}, 1 \mathrm{kHz}$

$\Delta=d_{T}-d_{S}(p p m)$

$20 \mathrm{kHz} \quad 50 \mathrm{kHz} \quad 100 \mathrm{kHz}$

600

$\begin{array}{ll}0 & -13\end{array}$

$-7$

500

500

$200-1$

$60-1$

$20+1$

$6+2$

$2+2$

$1+1$
0

0

$-1$

$+1$

0

0

$-2$

$-4$
$-7$

0

0

0

$+12$

$-6$

$-13$

$+1$

$+3$

$-1$

$-1$

$-1$

$-1$

$-1$
$-2$

$-3$

$-3$ 
Tab1e 13

AC-DC Differences of $\mathrm{P}_{\boldsymbol{7}}$ TVC's

$d(p p a)$

v

$20 \mathrm{~Hz}, 1 \mathrm{kHz} 20 \mathrm{kHz}$

$50 \mathrm{kHz}$

$100 \mathrm{kHz}$

$\mathrm{F}_{7} 1000-10 \mathrm{E}-\mathrm{F}_{7}$

600

$-2$

$-15$

$-2$

$\mathrm{F}_{7} 500-10 \mathrm{C}-\mathrm{F}_{7} 1$

500

$-2$

$-9$

$+16$

$\mathrm{F}_{7} 500-10 \mathrm{D}-\mathrm{F}_{7} 1$

500

$\mathrm{F}_{7} 600-\mathrm{F}_{7}{ }^{2}$

500

$\mathrm{F}_{7} 300-\mathrm{F}_{7} 1$

300

$\mathrm{F}_{7} 200-\mathrm{F}_{7}{ }^{2}$

200

$\mathrm{F}_{7} 100-\mathrm{F}_{7} 1$

100

$\mathrm{F}_{7} 60-\mathrm{F}_{7} 2$

60

$\mathrm{F}_{7} 30-\mathrm{F}_{7} 1$

30

$\mathrm{F}_{7} 20-\mathrm{F}_{7}{ }^{2}$

20

$\mathrm{F}_{7}^{10-\mathrm{F}_{7}}$

10

$\mathrm{F}_{7} 6-\mathrm{F}_{7}{ }^{2}$

6

$\mathrm{F}_{7} 3-\mathrm{F}_{7}$

3

$\mathrm{F}_{7}{ }^{2}$

2

$\mathrm{F}_{7} 1$ 
Table 14

Pooled Standard Deviations, $S_{p a}$ $S_{p a}$ (ppe of Input $V$ or I)

Type of Test

1. Comparison of MJTC's

2. Comparison of MJTC's vs.

Reference TE's and $F_{1} 10 F Y_{2}$

3. Extension of of frequency range

4. Build up-

Reference TE's

a. 5 to $50 \mathrm{~mA}$

b. $50 \mathrm{~mA}$ to $1 \mathrm{~A}$

c. $1 \mathrm{~A}$ to $20 \mathrm{~A}$

5. Build up-

$$
F_{1} \text { TVC's }
$$

a. 0.5 to $100 \mathrm{~V}$

3

$0.49 \quad 0.47$

$0.26 \quad 0.23$

(11)

(13)

0.35

(11)

10

5

$1.80 \quad 1.68$

(11)

(16)

1.57

(15)

$1.77 \quad 1.56$

(13)

(12)

1.48

(12)

1.74

(16)

$0.24 \quad 0.25$

(5)

0.52

0.57

$100 \mathrm{kHz}$ Average

0.14

0.25

0.24

b. 200 to $1000 \mathrm{~V}$

(7)

(12)

$-\quad 1.71$

(4)

1.41

(4)

1.06

(3)

10

\subsection{6}

2.77

2.47

(11)

1.72

(17)

Reference and

Working TE's

$5 \mathrm{~mA}-20 \mathrm{~A}$

7. Build up

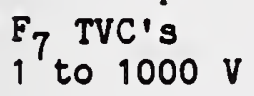

3

$1.58 \quad 1.20$

2.16

2.54

(6) (9)

(9)

(9)

0.54

0.52

1.55

2.79

2.05

(9)

0.28

1.76

1.64

(2)

Values in parentheses are the number of tests. 


\section{Estimated Limits or Residual Systematic Uncertainties \\ (not evaluated by statist 1 cal means) \\ (See notes at end of table)}

$\frac{\text { Linits, } \pm b}{\frac{1 \mathrm{kHz}}{\mathrm{ppm} \text { s of } \Delta}}$

A. MJTC Comparator \& Equipment

1. nVm \& averager (2\% of indication)

2. Determination of $n_{s}$

3. n-Compensation

4. DC-reversal difference definitions

5. AC effects

$$
b_{0}=\left(\Sigma b^{2}\right)^{1 / 2}
$$

B. 1966 Comparator \& Equipment

1. 1966 evaluation

2. Detector accuracy

3. Determination of $n_{3}$

4. AC effects

$\begin{array}{cccc}- & 2 & - & 2 \\ - & 2 & - & 2 \\ 0.1 & & 0.1 & \\ 0.1 & & 0.1 & \\ 0.1 & - & 0.2 & - \\ 0.17 & 2.8 & 0.25 & 2.8\end{array}$

$$
\text { bo }
$$

C. Calibration-Console

1. Basic accuracy

2. Detector accuracy

3. Determination of $n_{s}$

4. AC effects

$\begin{array}{llll}1.0 & - & 1.0 & - \\ - & 3 & - & 3 \\ - & 2 & - & 2 \\ 1.0 & 3.6 & & 2.0\end{array}$

\begin{tabular}{|c|c|c|c|}
\hline 1.0 & - & 1.0 & - \\
\hline 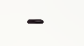 & 3 & - & 3 \\
\hline - & 2 & - & 2 \\
\hline- & - & 2.0 & \\
\hline 1.0 & 3.6 & 2.2 & \\
\hline
\end{tabular}

$$
b_{0}
$$

D. MJTC's

1. 1976 assignment of group average

2. Possible changes 1976-1982

3. Assignment of $\mathrm{G}-44$
0.3
0.1
0.1
0.33

$b_{0}$ 


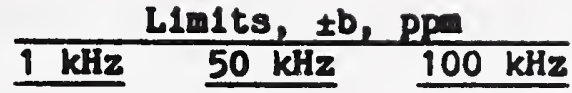

E. $5 \mathrm{~mA}$ Reference TE's

1. Residual thermoelectric effects

$0.2 \quad 0.2$

2. High frequency evaluation

$\underline{0.0} \quad \underline{0.5}$

$b_{0}$

$0.20 \quad 0.54$

F. $\mathrm{F}_{1} 1 \mathrm{YY}_{2}$, Base TVC

1. $1 \mathrm{kHz}$ base

0.2

0.2

2. High frequency extension

0.0

1.0

bo

0.2

1.0

G. Reference TE's, build-up

to $50 \mathrm{~mA}$ (3 stages)

1. Bead error (each stage)

$0.2 \quad 0.3$

2. Paralleling error (each stage)

$\underline{0.1} \quad \underline{0.3}$

\section{each stage $b_{0}$}

0.22

0.42

H. Reference TE's, bulld-up from

$50 \mathrm{~mA}$ to $1 \mathrm{~A}$ ( 4 stages)

1. Bead error (each stage)

$0.2 \quad 1.0$

2. Paralleling error (each stage)

$0.3 \quad 1.0$

3. AC effects (each stage)

$0.0 \quad 1.0$

\section{each stage $b_{0}$}

$0.36 \quad 1.73$

I. Reference TE's, bulld-up

$1 A$ - 20A (5 steps)

1. Dependence of $d_{c}$ on $I$ (each step)

$2.0 \quad 2.0$

2. AC effects (induced emf's, etc.) (each step)

3. Proximity effect (each step)

$1.0 \quad 3.0$

$\underline{0.0} \quad \underline{2.0}$

$$
\text { each step } b_{0}
$$

2.24 .1

J. Extension of Voltage Range

10 to $0.5 \mathrm{~V}, 10$ to $200 \mathrm{~V}$

ranges (5 steps each)

1. Residual dependence of $d_{c}$

on I (correlated)

$\underline{0.2}$

$\underline{0.5}$

each step $b_{0}$

0.2

0.5 
Table 15 (Continued)

Linits, $\pm b, p p a$

$1 \mathrm{kHz} \quad 50 \mathrm{kHz} \quad 100 \mathrm{kHz}$

K. Extension of $\mathrm{V}$ range from 200

to $1000 \mathrm{~V}$ range (at $500 \mathrm{~V}$ )

(3 steps)

1. Dependence of $d_{v}$ on $v$

(Avg. for 3 steps)

0.5

2.0

2. Dependence of $d_{f}$ on $I$

0.2

0.5

3. Transfer FX, FY\& to $\mathrm{FX}_{2}$, $\mathrm{FY}_{2}$

0.5

$\underline{2.0}$

each step $b_{0}$

0.73

2.9

L. Working standard TE's $5 \mathrm{~mA}$ to 20 A range (by comparison with reference stds, same range)

1. Bead resistance and other ac effects

3.0

6.0

5.0

10.0

$5 \mathrm{~mA}$

2. Dependence of $d_{c}$ on $I$

$\underline{2.0}$

$\underline{2.0}$

each step bo

$\begin{array}{rrr}3.5 & 5.4 & 5 \mathrm{~mA} \\ 6.3 & 10.1 & 20 \mathrm{~A}\end{array}$

M. Working Standards TVC's ( $F_{7}$ set)

Build-up $10 \mathrm{~V}$ to $1000 \mathrm{~V}$ range

(4 steps) and build-down $10 \mathrm{~V}$

to $1 \mathrm{~V}$ range (3 steps)

1. $d_{c}$ of each TE (correlated)

2. Dependence of $d_{r}$ on $V$ (high ranges), build-up

1.0

3.0

0.0 to

0.0 to

3. $\Delta d_{r}$ error (low ranges),

0.5

2.0

build-down

0.0 to

0.0 to

$\underline{0.5}$

$\underline{2.0}$

average, each step $b_{0}$

1.1

3.3 


\section{Table 15 (Continued)}

\section{N O T B S :}

\section{Sections}

$A, B, \& C$ \& of $\Delta$ errors depend on magnitude of $\Delta=d_{T}-d_{S}$.

$n_{s}$ determined once, but error affects every ac-dc determination. AC effects are estimated residuals or allowances for $\mathrm{rf} 1$, induced voltages, etc.

E-1 \& F-1 Main effects evaluated by comparison with MJTC. These are residuals.

G-1 \& H-1 Estimate of error from finite bead resistance.

G-2 \& H-2 Estimate of $\Delta \theta$ error, of paralleled TE's, etc.

I-1 Variation of $d_{c}$ with I was not applled in bulld-up.

I-3 Proximity of return lead on current distribution in TE heater.

J-1 The error in measuring $\Delta d_{c}$ adds at each step.

K-1 Self-heating effect on $d_{r}$.

K-3 Residual substitution error.

M-2 See $\mathrm{K}-1$ note.

M-3 $\Delta \theta$ of $F_{7}$ TE's. See section VIII-B. 
Calculation of Uncertainties

$$
\begin{array}{r}
s_{e}=s_{p e} \text { or } b / \sqrt{3}, s_{0}=\left(\Sigma s_{e}^{2}\right)^{1 / 2}, U_{0}=2 s_{0} \\
s \text { or } U(p p m)
\end{array}
$$

$1 \mathrm{kHz} \quad 50 \mathrm{kHz} \quad 100 \mathrm{kHz}$

A. MJTC $\mathrm{G4} 4$

$$
\begin{array}{ccc}
S_{p a} \text { from table 14-1 } & S_{e}=S_{p a} & 0.16 \\
S_{e} \text { for comparator, } & S_{e}=b / \sqrt{3} & 0.09 \\
\text { table 15-A } & S_{e}=b / \sqrt{3} & 0.19 \\
S_{e} \text { MJTC'S (15-D) } & & \\
& U_{A}=2 S_{0}=2\left(\sum S_{e}{ }^{2}\right)^{1 / 2} & 0.53
\end{array}
$$

\begin{tabular}{|c|c|c|c|}
\hline $\begin{array}{l}S_{p a}(14-2 \& 3) \\
S_{e} \text { comparator }(15-A) \\
S_{e} T E^{\prime} s(15-E \text { or } F)\end{array}$ & $\begin{array}{l}0.29 \\
0.09 \\
0.12 \\
\end{array}$ & $\begin{array}{l}0.29 \\
0.14 \\
0.31 \\
\end{array}$ & $\begin{array}{l}0.29 \\
0.14 \\
0.58 \\
\end{array}$ \\
\hline$=2 \mathbf{S}_{0}$ & 0.65 & 0.89 & 1.33 \\
\hline$u_{B}=\left(u_{0}^{2}+u_{A}^{2}\right)^{1 / 2}$ & 0.84 & 1.04 & 1 \\
\hline
\end{tabular}

B. Reference $5 \mathrm{~mA} T E^{\prime} \mathrm{s} \& \mathrm{~F}_{1} 10 \mathrm{Y}_{2}$

$$
\text { TVC to } 100 \mathrm{kHz}
$$

C. Reference TE's 10 to $50 \mathrm{~mA}$ (3 stages)

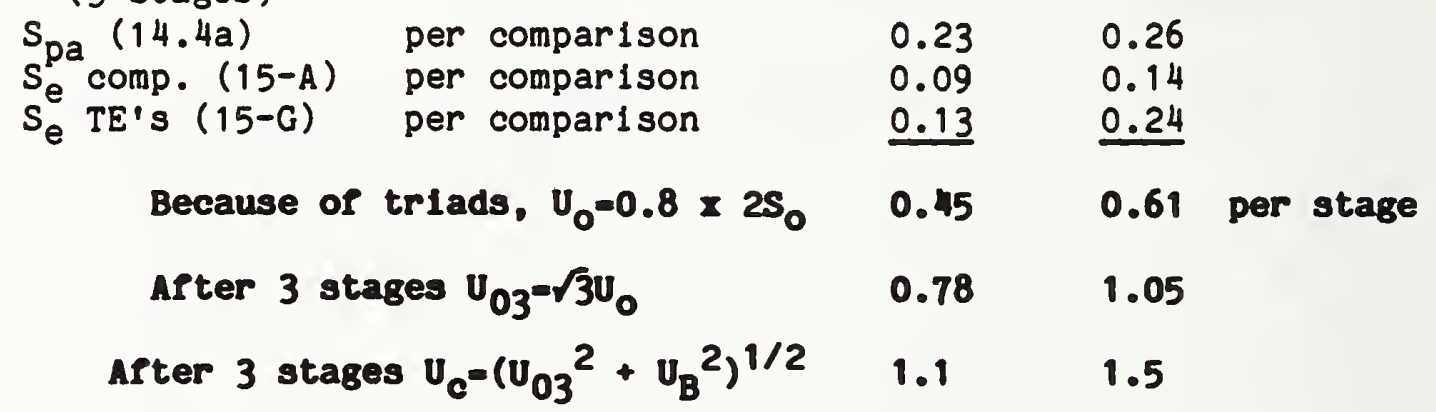

D. Reference TE's $100 \mathrm{~mA}$ to $1 \mathrm{~A}$ (4 stages)

$\begin{array}{rlll}S_{p a}(14-4 b) & \text { per comparison } & 1.7 & 2.0 \\ S_{e} \text { comp. }(15-B) & \text { per comparison } & 0.6 & 1.3 \\ S_{e} T E^{\prime} s(15-H) & \text { per comparison } & \underline{0.2} & 1.0 \\ \text { After } 1 \text { stage } U_{0}=0.8 \times 2 S_{0} & 2.9 & 4.1 \\ \text { After } 4 \text { stages } U_{04}=\sqrt{4 U_{0}} & 5.8 & 8.2 \\ U_{D}=\left(U_{04}{ }^{2}+U_{C}{ }^{2}\right)^{1 / 2} & 5.9 & 8.3\end{array}$




$\mathrm{S}$ or $\mathrm{U}(\mathrm{ppm})$
$1 \mathrm{kHz} \quad 50 \mathrm{kHz} \quad 100 \mathrm{kHz}$

E. Reference TE's 2A to 20A (at 10A)

$$
\text { (5 steps) }
$$

$\begin{array}{cccc}S_{p a}(14-4 C) & \text { per step } & 1.6 & 1.7 \\ S_{e} \text { comp. }(15-B) & \text { per step } & 0.6 & 1.3 \\ S_{e} \text { TE's (15-I) } & \text { per step } & \underline{1.3} & \underline{2.4} \\ \text { After } 1 \text { step } U_{0}=2 S_{0} & 4.3 & 6.4 \\ \text { After } 5 \text { steps } U_{05}=\sqrt{5} U_{0} & 9.6 & 14 \\ U_{E}=\left(U_{05}{ }^{2}+U_{D}{ }^{2}\right)^{1 / 2} & 11 & 1.6\end{array}$

F. F TVC's 10 to $0.5 \mathrm{~V}$ and 10 to $100 \mathrm{~V}$ ( 6 and 4 steps)
$S_{\mathrm{pa}}(14-5 \mathrm{a})$
$s_{e}^{p a}$ comp. (15-A) each step
each step
each step
each step
a
0.47
0.54
0.09
0.14
$\mathrm{S}_{e}$ (correlated)
b
$\underline{0.91}$
$\underline{0.29}$
After 1 step $U_{0}=2 S_{0}$
0.98
1.26
After 5 steps $U_{05^{-2}}=2\left((5 c)^{2}+5(a+b)^{2}\right)^{1 / 2}$
2.7
4.2$$
u_{\mathrm{P}}=\left(\mathrm{U}_{05}{ }^{2}+\mathrm{U}_{\mathrm{B}}^{2}\right)^{1 / 2}
$$
2.8
4.4

G. F TVC's 200 to $1000 \mathrm{~V}$ range (at 500V) (3 steps)

\begin{tabular}{|c|c|c|c|}
\hline $\begin{array}{l}S_{p a}(14-5 b) \\
s_{e} \text { comp. }(15-B) \\
s_{c} \text { TVC's }(15-K)\end{array}$ & $\begin{array}{l}\text { each step } \\
\text { each step } \\
\text { each step }\end{array}$ & $\begin{array}{l}1.7 \\
0.6 \\
0.4 \\
\end{array}$ & $\begin{array}{l}1.6 \\
1.3 \\
1.7 \\
\end{array}$ \\
\hline After 1 step U & $b_{0}=2 s_{0}$ & 3.7 & 5.3 \\
\hline After 3 steps & $U_{03}=\sqrt{3} U_{0}$ & 6.4 & 9.2 \\
\hline & $U_{G}=\left(U_{03}{ }^{2}+U_{F}^{2}\right)^{1 / 2}$ & 7.0 & 10 \\
\hline
\end{tabular}


Table 16 (Continued)

$\frac{\mathrm{s} \text { or U (pp) }}{1 \mathrm{kHz} \quad 100 \mathrm{kHz}}$

H. Working TE's $5 \mathrm{~mA}$ to $20 \mathrm{~A}$ range $S_{\text {pa }}(14-6)$ $\mathrm{S}_{\mathrm{e}}^{\mathrm{pa}}$ comp. (15-C)

$S_{e}$ TE's (15-L)

$$
\begin{aligned}
& 5 \mathrm{~mA} \\
& S_{e} \text { Reference TE's (16-B) 5mA } \\
& (16-E) 20 \mathrm{~A}
\end{aligned}
$$

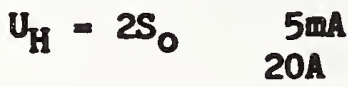

\begin{tabular}{ll}
2.8 & 1.7 \\
0.6 & 1.2 \\
2.9 & 3.1 \\
3.6 & 5.8 \\
0.4 & 0.5 \\
5.6 & 8.2 \\
\hline 7.1 & 7.5 \\
15 & 21
\end{tabular}

I. $\mathrm{F}_{7}$ Working Standard TVC's

10 to $1000 \mathrm{~V}$ range (5 steps);

10 to $1 \mathrm{~V}$ range (3 steps)

$S_{\mathrm{pa}}(14-7)$ each step

$\mathrm{s}_{e}$ comp. (15-C) each step

$S_{e}$ TVC's (15-M)

each step

a $\quad 1.2$

2.8

0.6

1.2

(correlated)

c

0.6

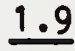

After 1 step $v_{0}=2 s_{0}$

2.9

7.2

After 3 steps $U_{03}=2\left((3 c)^{2}+3(a+b)^{2}\right)^{1 / 2}$

7.2

18

(at 1V)

$$
U_{I}=\left(U_{03}^{2}+U_{B}^{2}\right)^{1 / 2}
$$

7.2

18

After 5 steps $U_{05}=2\left((5 c)^{2}+5(a+b)^{2}\right)^{1 / 2}$

26

$$
\text { (at 500V) } \quad U_{I}=\left(U_{05}^{2}+U_{B}^{2}\right)^{1 / 2}
$$

10

26 
Table 17

Summary of Uncertalntles of NBS AC-DC Transfer Standards

I. Uncertainties Independent of Magnitude of $d$

$\mathrm{U}_{\mathrm{A}} \mathrm{Ppm}$

Standard

$1 \mathrm{kHz}$

$50 \mathrm{kHz}$

$100 \mathrm{kHz}$

$A^{1}$ MJTC (one of group)

B Reference base, 5 mA TE's \& $10 \mathrm{~V}$ TVC 0.53

B Reference base, $5 \mathrm{~mA}$ TE's \& $10 \mathrm{~V}$ TVC 0.84

C Reference TE: $50 \mathrm{~mA}$ range

D 1 A range

E $20 \mathrm{~A}$ range

F Reference TVC: $0.5 \& 100 \mathrm{~V}$ ranges

1.1

5.9

11

$\begin{array}{lll}\text { G Working TE's: } 5 \mathrm{~mA} \text { range } & \text { at 500V) } & 7.0 \\ \mathrm{H} & 7.1\end{array}$

$\mathrm{H}$ Working TE's: $5 \mathrm{~mA}$ range

$20 \mathrm{~A}$ range

I Working TVC's: $10 \mathrm{~V}$ range

15

IV range

$500 \mathrm{~V}$ range

2.9

$1.0 \quad 1.4$

1.5

8.3

16

2.8

4.4

10

7.5

21

7.2

10

II. Uncertainties dependent on magnitude of $d$ :

For all standards $U_{B}=0.05 \mathrm{~d}$ should be added to $U_{A}$.

$U_{B}<1$ ppm with few exceptions.

III. Uncertainties at $20 \mathrm{~Hz}$ are somewhat, but not signiflcantly, greater than at $1 \mathrm{kHz}$.

IV. Uncertainties at less than rated $V$ or I require further study. However, the working standards are almost always used at or near rated $V$ or $I$.

V. Self-heating effects at $1000 \mathrm{~V}$ were evaluated in [3].

$1_{\text {Letters refer to table } 16 .}$ 
Coaparison of Estimated Calibration Uncertainties with Present Allowances

$\frac{U(p p m)}{\frac{1 \mathrm{kHz}}{5 \mathrm{~mA}} \frac{50 \mathrm{kHz}}{5 \mathrm{Am} \quad 20 \mathrm{~A}}}$

Working standard TE's (16-H)

Comparison (16- $\mathrm{H}$ without $\mathrm{S}_{e}$ of ref. stds.)

$$
\text { U (rss) }
$$

Present allowance $5 \mathrm{~mA}-5 \mathrm{~A}$

Present allowance 5A - 20A

Working standard TVC's (16-I)

Comparison (16-I, one step)

$$
\text { U (rss) }
$$

Present allowance (for coaxial TVC's)

Present allowance (for multirange TVC's)

$\begin{array}{ll}7.1 & 15.0 \\ 7.1 & \frac{9.2}{10}\end{array}$

50

100

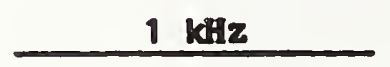

\section{IV $10 \mathrm{~V} \quad 500 \mathrm{~V}$}

$\begin{array}{lll}7.2 & 3.0 \quad 10\end{array}$

2.9

$2.9 \quad 2.9$

7.8

4.2

10

20

20

20

50

50

50

7.5

21

$7.5 \quad 12$

11

24

100

200 


\section{Table 19}

Estimated Uncertaint1es for Special Teats

(Calibration of a TE or TVC against a reference standard, with MJTC comparator)

Reference standard TE 5-50 mA

Comparison (16B \& 16C without $S_{e}$ of $T E^{\prime} \mathrm{S}$ )

rss

\begin{tabular}{|c|c|c|c|}
\hline \multicolumn{2}{|c|}{$1 \mathrm{kHz}$} & \multicolumn{2}{|c|}{$50 \mathrm{kHz}$} \\
\hline 5 모 & $50 \square$ & $5 \mathrm{aA}$ & $50 \mathrm{~mA}$ \\
\hline 0.9 & 1.1 & 1.0 & 1.5 \\
\hline 0.6 & 0.6 & 0.6 & 0.6 \\
\hline 1.1 & 1.2 & 1.2 & 1.6 \\
\hline
\end{tabular}

$100 \mathrm{kHz}$

1Y 10V 100V

$\begin{array}{lll}2.8 & 0.9 \quad 2.8\end{array}$

$0.6 \quad 0.6 \quad 0.6$

$0.5 \quad 0.5 \quad 0.5$

$2.9 \quad 1.2 \quad 2.9$

\begin{tabular}{lll}
\multicolumn{3}{c}{$100 \mathrm{kHz}$} \\
\hline IV & 10V & 100V \\
4.4 & 1.4 & 4.4 \\
0.6 & 0.6 & 0.6 \\
$\frac{2.0}{4.9}$ & $\frac{1.0}{1.8}$ & $\underline{2.0}$ \\
4.9 & 1.8
\end{tabular}




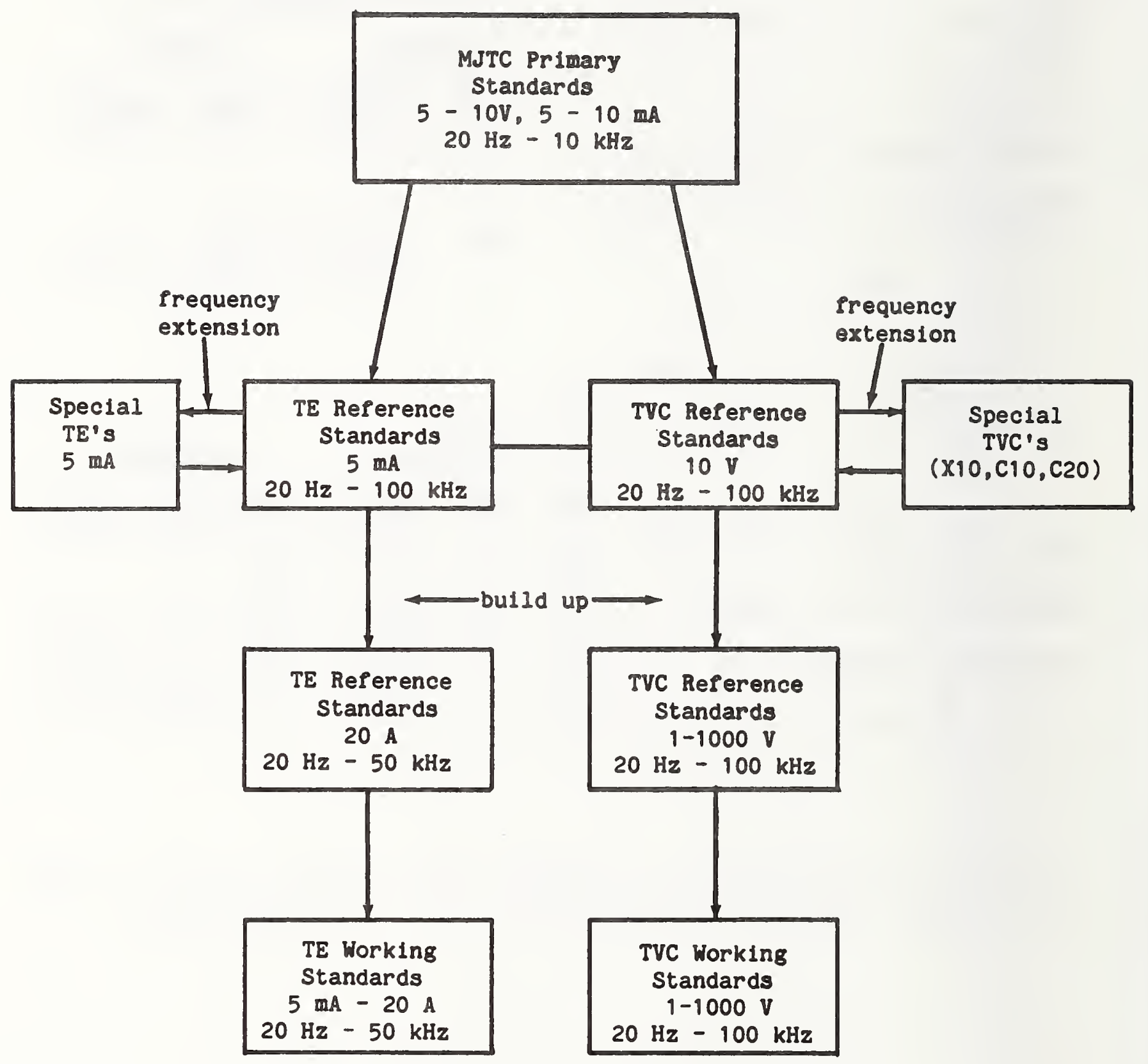

Flgure 1. "Road Map" 
$30 \mathrm{~Hz}$

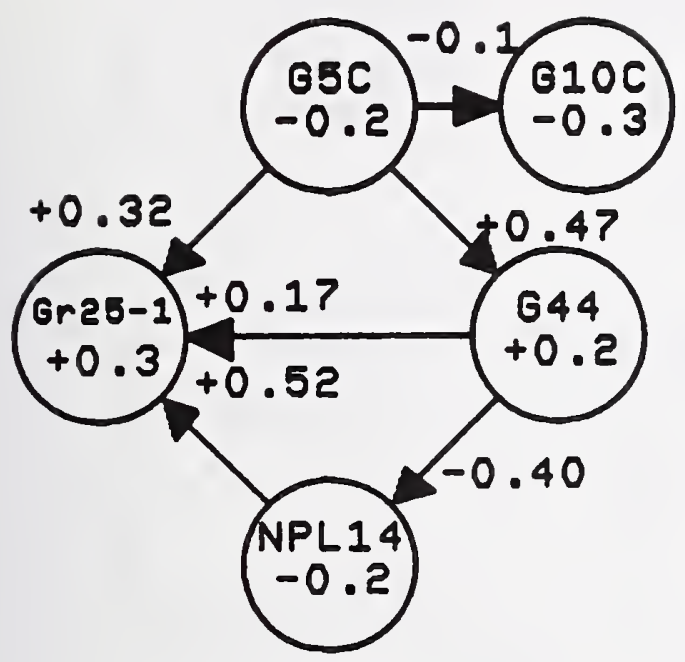

$1 \mathrm{kHz}$

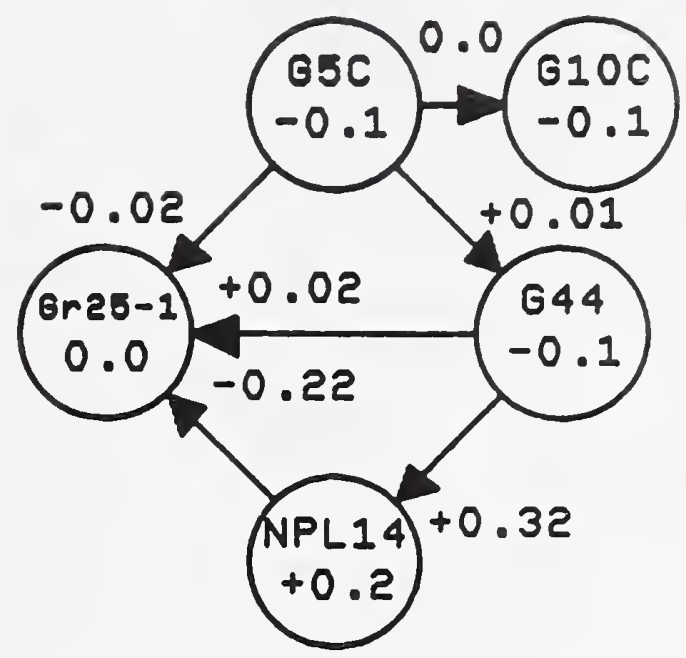

$10 \mathrm{kHz}$

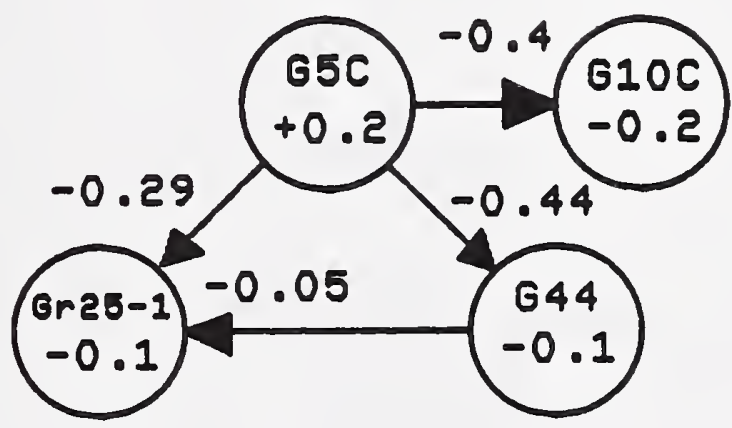

Figure 2. Comparisons of MJTC's 


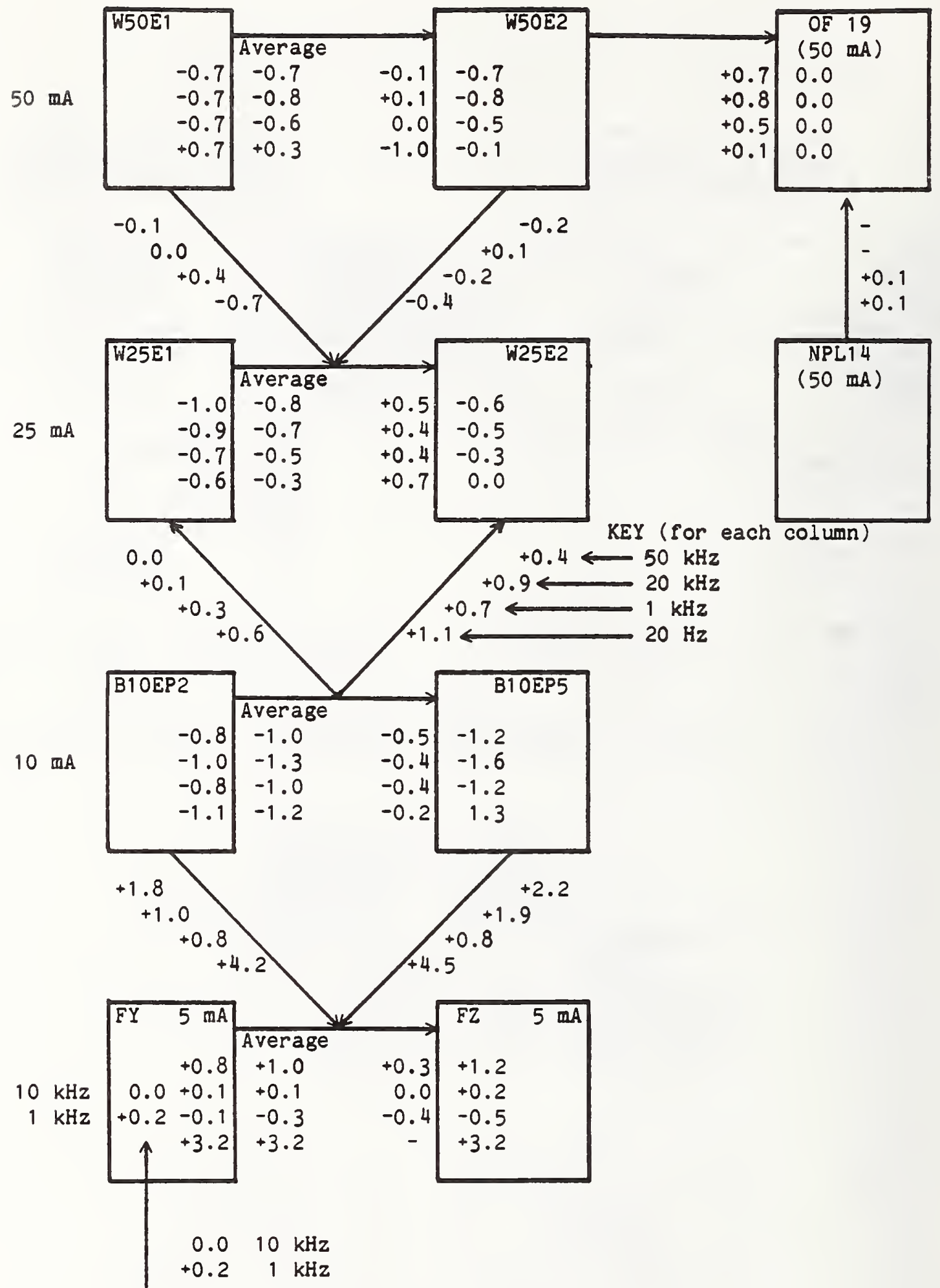




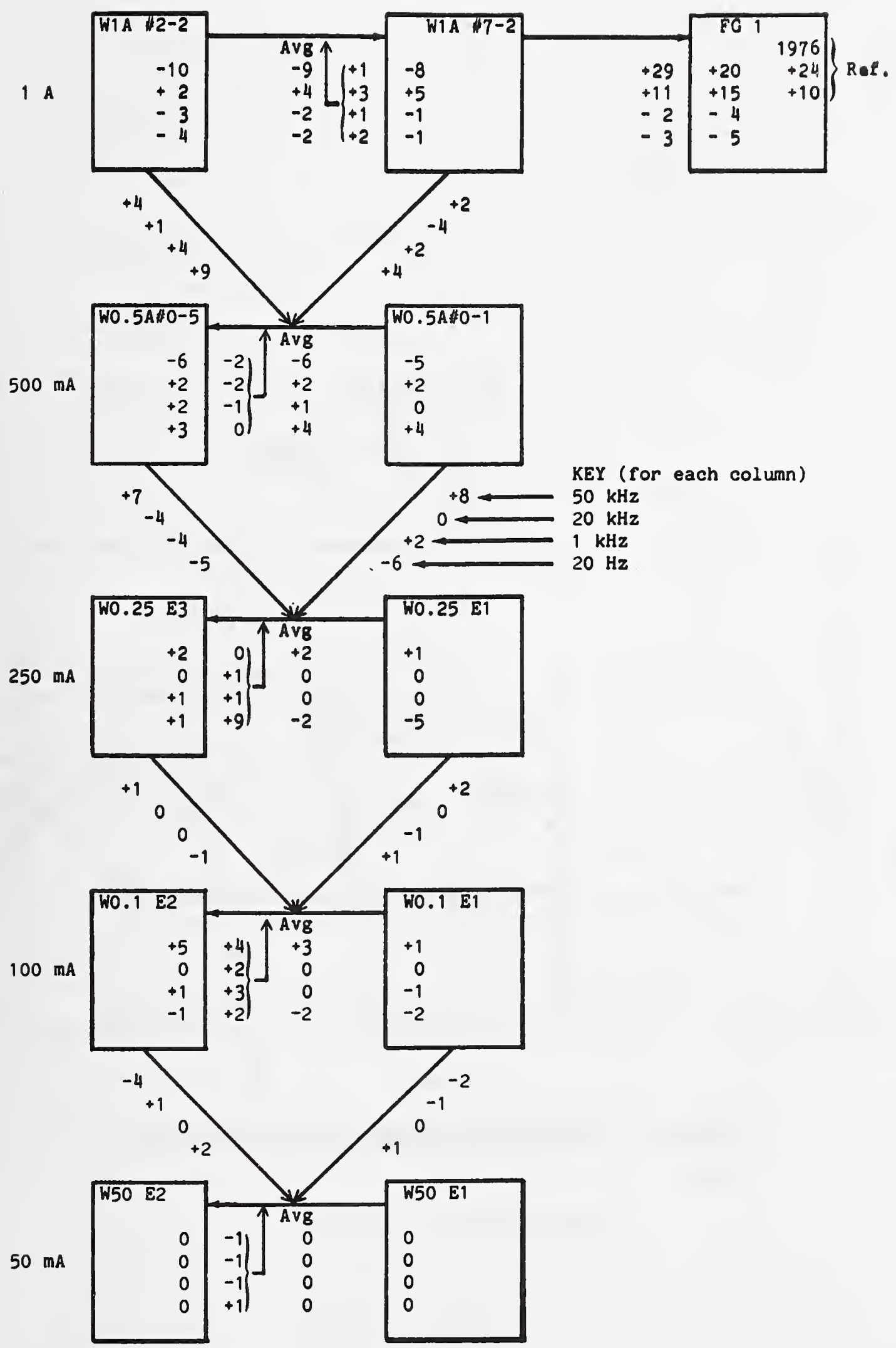

Figure. 4: $50 \mathrm{~mA}$ to 1 A Comparisons 


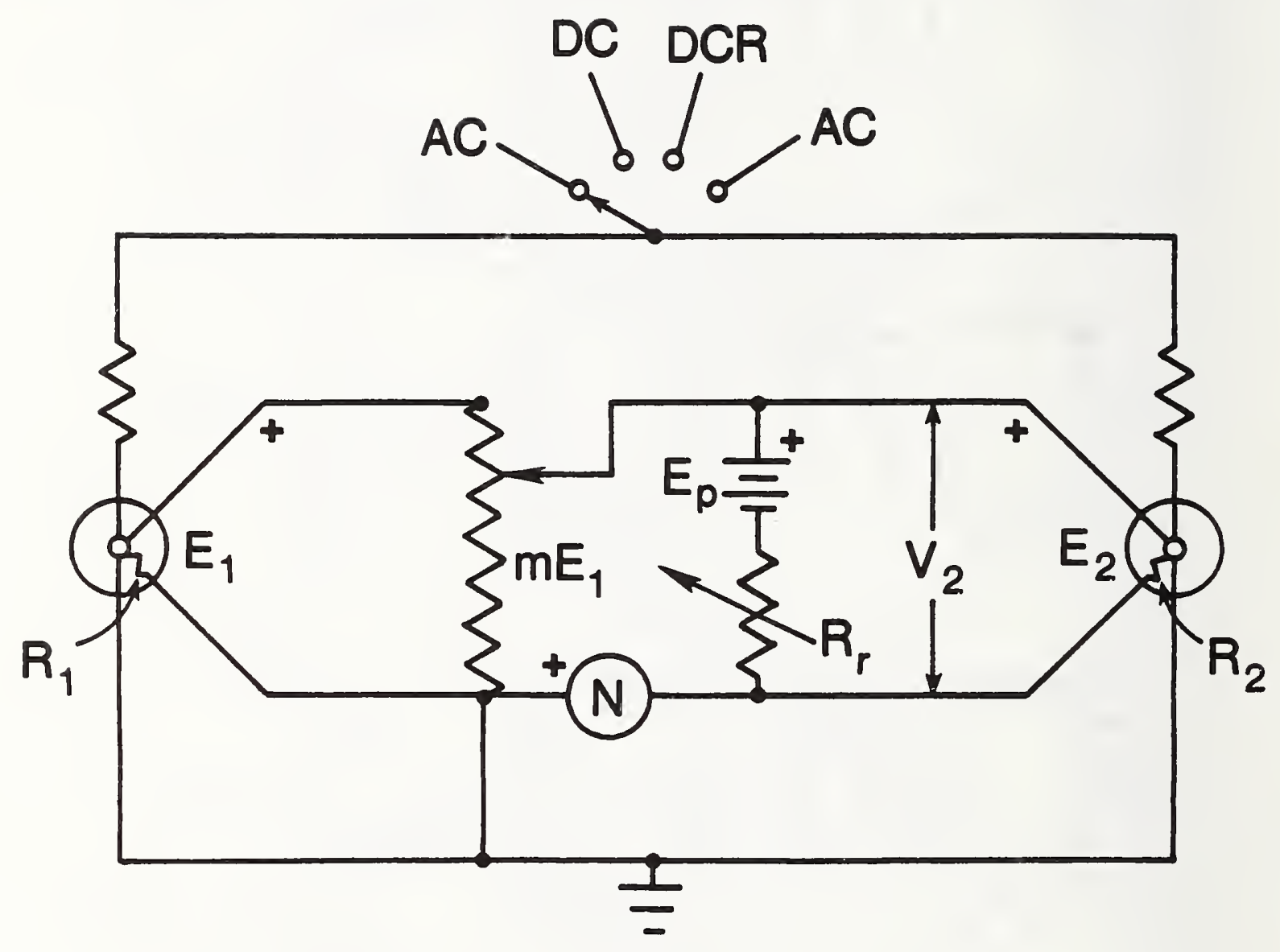

Figure 5. Comparator With n-Compensation 


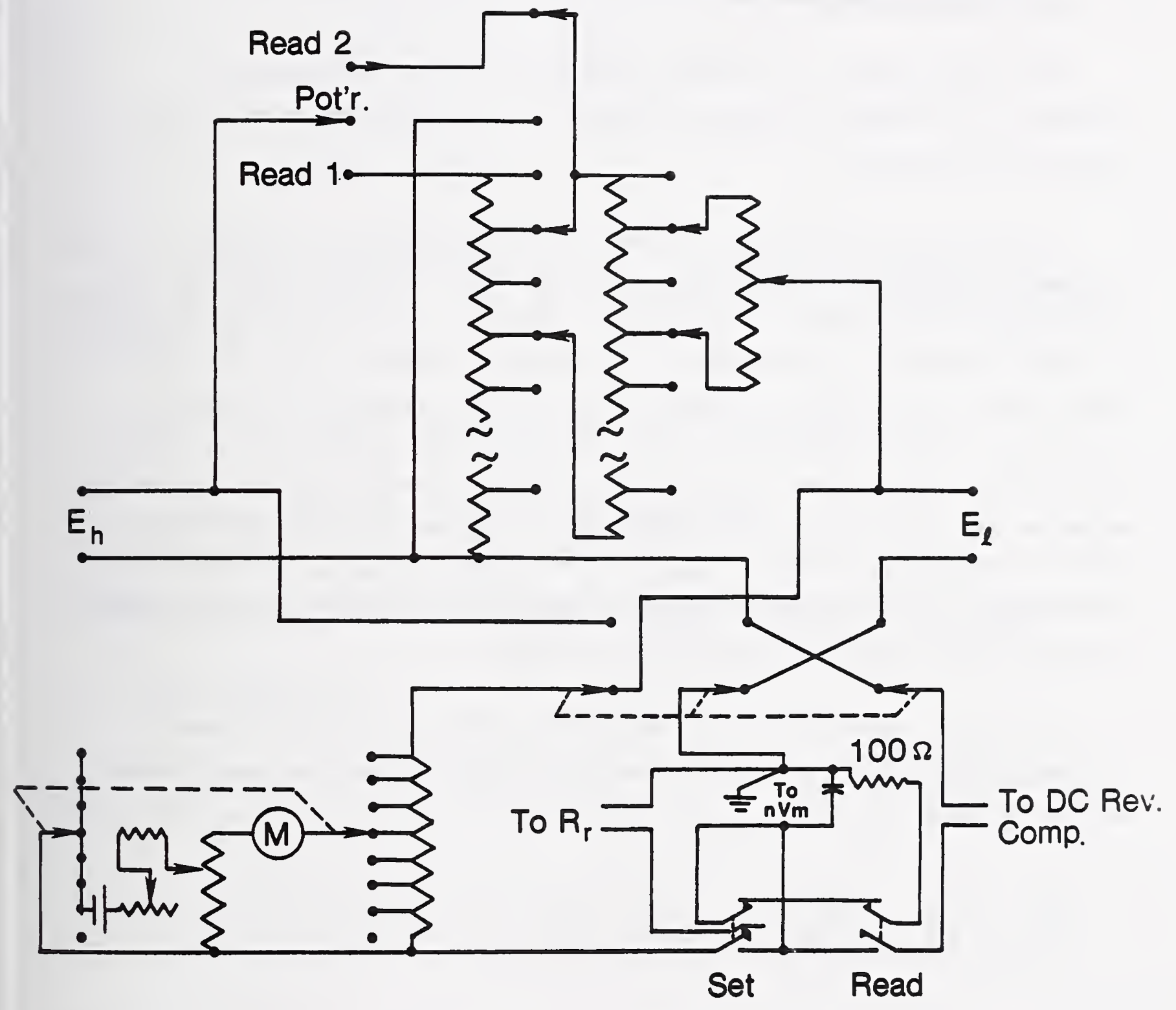

Figure 6. Dlagram of EMF Comparator 


\section{APPENDIX 1}

\section{n-COMPENSATION and n-MEASUREMATS}

\section{A. Principle of Compensation}

The basic circuit of the MJTC comparator with the $n$-compensation resistor, $R_{r}$, included is shown in figure $5 . E_{p}$ represents an adjustable Lindeck potentiometer.

At the initial balances, with $N=0$, ( $N$ is the reading of the high impedance nanovoltmeter, $n V m$ ), $m E_{1}=E_{2}=E_{p}=V_{2}$. (The $E_{p}$ setting is made by switching $n V m$ in place of $R_{r}$ ) Now if the input voltage $V_{1}$, is increased by a small amount, $\Delta V_{1}$, then $\Delta E_{1} / E_{1}=n_{1} \Delta V_{1} / V_{1}$ and $\Delta E_{2} / E_{2}=n_{2} \Delta V_{1} / V_{1}$ (from the definition of $n$ given in section IIIA with $V_{1}$ replacing I). A small current then flows in the $E_{2}-E_{p}$ loop. Because of the voltage divider formed by $R_{2}$ and $R_{r}, \Delta V_{2}$ is less than $\Delta E_{2}$. Thus if $n_{2}$ is greater than $n_{1}, R_{r}$ can be adjusted unt1l $m \Delta E_{1}=\Delta V_{2}$, maintaining $N=0$. This makes the steady-state balance insensitive to small changes in the supply voltages, and makes it unnecessary to adjust these voltages to keep one of the emf's constant during an ac-dc determination, as heretofore required.

From the figure and with the output resistance of the potentiometer included in $R_{r}, \Delta V_{2}=q \Delta E_{2}$, where $q=R_{r} /\left(R_{r}+R_{2}\right)$. Since the same $\Delta V / V$ is applied to both TVC's

$$
\frac{\Delta E_{1}}{n_{1} E_{1}}=\frac{\Delta E_{2}}{n_{2} E_{2}}=\frac{m \Delta E_{1}}{q n_{2} m E_{1}} \text {, so that } q=\frac{n_{1}}{n_{2}}
$$




\section{B. Basio TE Equation}

Since nelther TE has a constant output during a comparison of two TVC's (or TE's) the development of the comparator equation given in appendix 2 of [3] is not directly applicable. It will be convenlent to use a general equation for a TVC with slightly different ac and dc input voltages, $V_{a}$ and $v_{d}$, applied. We may write, as an identity,

$$
v_{a}=v_{d}\left(1+\frac{v_{d}^{\prime}-v_{d}}{v_{d}}+\frac{v_{a}-v_{d}^{\prime}}{v_{d}}\right)
$$

where $V_{d}$ ' is the average of the two directions of dc voltage required to produce the same emf as $V_{a}$, and $v_{d}$ is the average of the two directions which produce an emf, $E_{d}$.

Then, from the definitions of $n$ and $d$, and, since $E_{a}=E_{d}$.

$$
v_{a}=v_{d}\left(1+\frac{E_{a}-E_{d}}{n E_{d}}+d\right) \text {, to 1st-order terms. }
$$

A similar equation applies for current measurements, with I replacing $V$.

\section{AC-DC Comparisons}

From the above basic TVC equation, and with the same voltage applied to both TVC's, as in $\mathrm{fig} .5$.

$$
v_{a}=v_{d}\left(1+\frac{E_{1 a}-E_{1 d}}{n_{1} E_{1 d}}+d_{1}\right)=v_{d}\left(1+\frac{E_{2 a}-E_{2 d}}{n_{2} E_{2 d}}+d_{2}\right)
$$

Thus

$$
d_{1}-d_{2}=\frac{E_{2 a}-E_{2 d}}{n_{2} E_{2}}-\frac{E_{1 a}-E_{1 d}}{n_{1} E_{1}}
$$


But $N_{a}=V_{2 a}-m E_{1 a}$ and $N_{d}-V_{2 d}-m E_{1 d}$, with the polarity shown, so that $N_{a}-N_{d}=V_{2 a}-V_{2 d}-m\left(E_{1 a}-E_{1 d}\right)=q\left(E_{2 a}-E_{2 d}\right)-m\left(E_{1 a}-E_{1 d}\right)$. Combining these equations, and noting that $q=n_{1} / n_{2}$ and $m E_{1}-E_{2}$, gives, closely enough,

$$
d_{1}-d_{2}=\frac{\left(E_{2 a}-E_{2 d}\right)}{n_{1} E_{2}}-\frac{m\left(E_{1 a}-E_{1 d}\right)}{n_{1} E_{2}}=\frac{N_{a}-N_{d}}{n_{1} E_{2}}
$$

If $n_{1}>n_{2}$, the potentiometer and $R_{r}$ are connected across $E_{1}$, and the polarity of the nanovoltmeter is reversed. $R_{r}$ is adjusted as before, so that there is no change in $N$ when $V_{1}$ is increased. Because of the input resistance, $R_{d}$, of the divider, analysis shows that

$$
\frac{n_{2}}{n_{1}}=\frac{R_{p}}{R_{p}+R_{1}} \quad \text { where } R_{p}=\frac{R_{r} R_{d}}{R_{r}+R_{d}}
$$

The comparator formula then becomes

$$
d_{2}-d_{1}=\frac{N_{a}-N_{d}}{n_{2} E_{2}}
$$

Equations $A 1 B-1$ and $A 1 B-2$ may be written as

$$
d_{T}-d_{S}=\frac{N_{d}-N_{a}}{n_{S} E_{\ell}}
$$

where the subscript $\mathrm{T}$ refers to the TE to which the potentiometer is connected, and $E_{\ell}$ refers to the TE with the lowest emf. 


\section{Precautions in n-Measurement}

The characteristic, $n$, is convenlentiy measured, after the initial balance is obtained with the set key down, by changing the input quantity, $Q$, by about $0.1 \%$, noting the change in output emf $E$, then calculating $n=Q \Delta E / E \Delta Q$. The usual precautions in measuring small changes should be observed, as in an ac-dc difference test, to avoid errors from drifts etc.

A more insiduous error can occur, however, if the heater alloy of a TE has even a small temperature coefficient of resistance, $\alpha$, so that $R=R_{0}(1+\alpha \theta)$, where $\theta$ is the temperature rise above ambient. Then, since $V=I R$ for the heater, and $\theta=k I^{2}$

$$
\begin{aligned}
\frac{d V}{V} & =\frac{d I}{I}+\frac{d R}{R}=\frac{d I}{I}+\frac{d R}{d I} \frac{d I}{R}=\frac{d I}{I}+\frac{2 R_{0} \alpha k I d I}{R} \\
& =\frac{d I}{I}(1+2 \alpha \theta) \text { since } R_{0}=R
\end{aligned}
$$

From the definition of $\mathrm{n}$ and the above equation

$$
n_{1}=n_{v}(1+2 \alpha \theta) \text {. }
$$

For the NiCr alloys normally used in low-range TEs, $\alpha$ can be $-1 \times 10^{-4}$ to $-2 \times 10^{-4}$. $\theta$ is usually about $1.5 \times 10^{2}$ at rated $I$. Thus $n_{v}$ and $n_{1}$ can differ by 3 to $6 \%$. For alloys like Evanohm the difference is much smaller.

Thus the approprlate $\mathrm{n}$ should be measured and used. Since it enters only in a difference term with this comparator, small errors in determining it are not slgniflcant unless $d_{T}-d_{S}$ is large. 


\section{E. Square Law Output and RMS Response}

It is worth emphasizing that even if $n * 2$, so that $E * K I^{2}$ with $K a$ constant, a TE inherently responds to the true rms current, I. This can be shown easily for the simplest case in whlch all of the heater is at the same constant temperature, $\theta$. Then, equating heat input and output at any instant, $1^{2} R=K_{1} \theta=K_{2} E . R, K_{1}$ and $K_{2}$ are generally somewhat dependent on $\theta$, but if the frequency of the heater current, 1 , is high enough, thermal Inertia keeps $\theta$, and hence $E$ constant. Then term by term integration with respect to time, $t$, over one period, $T$, and division by $T$, gives, directly, $I^{2} R=K_{2} E$. Because of the temperature dependence of $R$ and $K_{2}$ the output is not exactly proportional to $I^{2}$, but it is st1ll a measure of $I$, the rms current. This can also be shown to be true under far more general conditions (see appendix 3 of [1]). 


\section{Appendix 2}

\section{EMI. Harmonics and DC Oefset}

\section{A. Test for Line-Borne Electromagnetic Interference (EMI)}

Only about $1 \mu \mathrm{W}$ of heater power, over a wide range of frequency, can produce $0.1 \%$ of rated emf in a typical 5-mA TE. Thus TE's are sensitive to EMI, particularly at IV and FM frequencies. (They were the detectors in early field-strength meters.) Laboratory leads at these frequencies can transfer radiated energy fairly efficiently to the heaters. Of course if the leads are twisted or shielded they make very poor antennas. However shielding is not completely effective, and in areas of high field strength (up to $1 \mathrm{~V} / \mathrm{m}$ in some areas) errors can result if the $\mathrm{IE}$ responds to currents at both the main and interfering frequency.

However, because of their square-law characteristics ( $E=K I^{2}$ where $I$ is the rms current) a small heater current, $I_{e}$, at a different frequency than the main current, $I_{m}$, does not cause as large an error as the ratio $a=I_{e} / I_{m}$ might indicate.

$$
I=\left(I_{m}^{2}+I_{e}^{2}\right)^{1 / 2}=I_{m}\left(1+a^{2}\right)^{1 / 2}
$$

If the response sensitivity is the same at both frequencies

$$
E=K I^{2}=K I_{m}^{2}\left(1+a^{2}\right)
$$

Without $I_{e}, E_{m}=K I_{m}^{2}$. Thus $\left(E-E_{m}\right) / E_{m}=\Delta E / E_{m}=a^{2}$. With only $I_{e}$ present, $E_{e}=K I_{e}^{2}$ so that $E_{e} / E_{m}=a^{2}$.

If a measurement of $I_{m}$ is desired, $\Delta E / E_{m}$ is an error term. It can be evaluated to see if it is significant by making a measurement with all of the connections in place but with the power supplies producing $I_{m}$ turned off. 
In the more general case $\mathrm{K}$ is somewhat dependent in $\mathrm{E}$, so that the sensitivity of this test is affected slightly. Also, if both TE's in an ac-dc comparison respond equally well to currents at both frequencies, there w11 be no EMI error. However the EMI test will determine this also.

In the MJTC comparator, RF bypass capacitors (of very low inductance) are connected from each ungrounded lead to the metal tank to reduce the possibility of EMI errors. The tank itself is an excellent shield.

\section{B. EPfect of Harmonics (see Section VII)}

Small amounts of low-order harmonics in the input voltage generally cause insignificant errors in the measurement of the rms value by a TVC, or in the comparison of two TVC's. This is a great advantage for true rms-measuring instruments. This can be shown rather simply for a wave having a fundamental voltage, $v_{1}$, and a single harmonlc, $v_{h}$. Then $v=\left(v_{1}{ }^{2}+v_{h}{ }^{2}\right)^{1 / 2}$. Because of reactance, skin effect, etc., the response of the TVC may be different at the two frequencies, so that $E=k V^{2}=k_{1} v_{1}^{2}+k_{h} V_{h}^{2}$. Let $v_{h} / V_{q}=q$ and $K_{h}=K_{1}\left(1+e_{h}\right)$. Then $E=k_{1} V^{2}\left(1+q^{2} e_{h}\right)$ so that the proportional error is only $q^{2} e_{h}$.

For several harmonics this becomes $\sum q_{m}{ }^{2} e_{m}$. where $m$ refers to the order of the harmonic.

\section{DC orrset}

The above equations should apply even if the harmonic is of zero order (dc). However if the TE has a dc reversal difference (which can be caused by Thomson or Peltier heating) there is an added output, $E=\mathrm{K}_{0} \mathrm{~V}_{\text {os }}$, proportional to the first power of any dc offset voltage, $v_{o s}$, in the ac wave, $v_{a}$. Then

$$
E=k\left(v_{a}^{2}+v_{o s}^{2}\right)^{1 / 2}+k_{o} v_{o s}=k v^{2}\left(1+\frac{K_{o} v_{o s}}{E}\right)
$$


With a de voltage, $v_{d}$, equal to $V$ applied and then reversed, the dc reversal difference in output is $\Delta E=2 K_{0} V$. Thus

$$
E=k v^{2}\left(1+\frac{g u}{2}\right)
$$

where

$$
g=\frac{\Delta E}{E} \text { and } u=\frac{V_{\text {OS }}}{V}
$$

Note that this is proportional to the ist power of $u$, and that, for a square law TE, $g$ is twice the value of the proportional de reversal difference as normally defined $(\Delta V / V$ for constant $E)$. 


\section{Appendix 3}

\section{EPfect of Relay OPI-Time}

Let the response, $E$, of a TE be approximated by a simple exponential with a time constant, $t_{c}$. Then after the steady-state heater current is turned off for a time $t_{0}<t_{c}$ (the of p-time of the relay when switched), we have

$$
E=E_{f} e^{-t_{0} / t_{c}}=E_{f}\left(1-t_{0} / t_{c}\right) .
$$

Thus $\Delta E=E_{f}-E=E_{f} t_{o} / t_{C}$.

When the relay is closed (with the same heater current), E recovers to $E_{f}$ exponentially, so that

$$
E=E_{f}-\Delta E e^{-t_{s} / t_{c}}
$$

where $t_{s}=t-t_{0}$. Then $p=\left(E_{f}-E\right) / E_{f}=b / e^{a}$ where $b=\Delta E / E_{f}=t_{o} / t_{c}$ and $a=t_{s} / t_{c}=t / t_{c}$ when $t_{s}>t_{c}$. If $p=1 \times 10^{-6}$ (recovery to $1 \mathrm{ppm}$ ), $t_{0}=$ $4 \times 10^{-4} \mathrm{~s}$, and $t_{c}=2 \mathrm{~s}$, then $b=2 \times 10^{-4}$ and $a=5.3$. Thus $t=a t_{c}=10.4 \mathrm{~s}$.

Interestingly, when $t_{0}=4 \times 10^{-3} \mathrm{~s}, t=15.2 \mathrm{~s}$ to recover to $1 \mathrm{ppm}$, only a modest increase for this 10-fold increase in off time. 


\section{Appendix 4}

\section{Effects of Bead Heating}

\section{A. Relative Effectiveness of Bead and Heater Power}

Widdis has shown (equation 17 of [19]) that the midpoint temperature rise, $\theta_{m}$, of the heater of a TE carrying a current, I, taking into account the heat loss through the thermocouple wires as well as the heater, to heat sinks at the ends $($ all at $\theta=0)$ is $\theta_{m}=D l^{2} / 2 M$. Here the very small Peltier and Thomson heating is neglected, $D=I^{2} \rho / A^{2} K, \rho$ and $K$ are the electrical resistivity and thermal conductivity of the heater, $A$ is the cross sectional area and $2 l$ the length of the heater, and $M=1+K_{1} / K_{0}$, where $K_{1}$ is the sum of the thermal conductances of the two thermocouple wires in parallel and $K_{0}$ is the sum of the conductances of the two halves of the heater.

Since $k_{0}=2 K A / l$ and $2 p l / A=R_{h}$, the heater resistance, we find that

$$
\theta_{m}=\frac{P_{h}}{2\left(K_{0}+K_{1}\right)}
$$

where

$$
P_{h}=I^{2} R_{h}
$$

If the bead between the heater and the hot function of the thermocuple has a resistance $R_{b}$, its power dissipation is $P_{b}=I_{b}{ }^{2} R_{b}$. The added temperature rise from this small heating is $\Delta \theta=P_{b} /\left(K_{1}+K_{0}\right)$. Thus bead power is twice as effective as heater power in raising the temperature of the hot junction. 


\section{B. AC-DC Difference Caused by Bead Heating}

One end of the heater of a TVC is grounded, and the thermocouple circuit is usually grounded also for safety and to keep the bead voltage low. Thus half the heater voltage is across the bead. From the formula in section $V-B$ the significant current is $I_{1}$ through the ungrounded heater terminal. The heater power is, to first order terms,

$$
P_{h}=\frac{I_{1}{ }^{2} R_{h}}{2}+\frac{I_{1}{ }^{2} R_{h}}{2}(1-a)^{2}=I_{1}{ }^{2} R_{h}(1-a)
$$

where

$$
a=\frac{I_{b}}{I_{1}}=\frac{R_{h}}{2 R_{b}} \ll 1
$$

The temperature rise, with the effect of bead power included, is

$$
\theta=U P_{h}+2 U P_{b}=U I_{1}{ }^{2} R_{h}(1-a)+2 U a^{2} I_{1}{ }^{2} R_{b}
$$

where

$$
U=1 / 2\left(k_{0}+k_{1}\right)
$$

Thus

$$
\theta=U I_{1}{ }^{2} R_{h}\left(1-a+2 a^{2} R_{b} / R_{h}\right)=U I_{1}{ }^{2} R_{h}
$$

with no error from the bead current, to first order terms.

This will, of course, not be true if neither heater terminal is at ground. 


\section{c. Two TE's in Series}

A similar but more involved analysis shows that if two equal series-connected TE's are used in a grounded TVC, the resultant output will also be unaffected by the bead currents. 


\section{Appendix 5}

\section{Inpedances and Substitution Corrections}

Ingles has studied the general impedance relationships in TV's and the effects of changing TE's.[9] However the following alternative approach, with its explicit treatment of phase defect angles, was developed at NBS. It has been used very effectively for several purposes, and so is given here. It is analogous to a much earlier unpublished NBS treatment of shunted TE's for current measurements.

\section{A. Impedance of a TVC}

A TVC set with well-defined ac-dc characteristics (independent of the surroundings) generally consists of a shielded TE in series with one of a group of three-terminal shielded resistors, as shown in fig. 1 of [2]. As shown in section VI of [2], the ac-dc difference of a combination as a TVC is, in the present notation, $d_{v}=d_{t}+d_{c}$, where $d_{t}=\left(z_{t}-R_{t}\right) / R_{t}$ and $d_{c}=\left(I_{h a}-I_{h d}\right) / I_{h d}$. In these expressions, $Z_{t}=V_{1} / I_{h}$, and is the magnitude of the impedance of the combination, with an applied rms voltage $v_{i}$ and a resultant heater current $I_{h}$. $I_{h a}$ and $I_{h d}$ are the magnitudes of the ac and dc currents required to produce the same output emf.

This is a very general equation, applicable for any series resistor and TE combination. To evaluate the component impedances we may write as an identity

$$
\bar{z}_{t}=\left(\bar{v}_{1}-\bar{v}_{h}\right) / \bar{I}_{h}+\bar{v}_{h} / \bar{I}_{h}=\bar{z}_{s}+\bar{z}_{h}
$$

where $\bar{v}_{h}$ is the voltage across the heater. The bars signify either phasors or complex numbers, and their absence signifles magnitudes. 
We may also write

$$
\bar{z}_{s} / R_{s}=1+a+j b \text { and } \quad \bar{z}_{h} / R_{h}=1+c+j e
$$

with $a, b, c$ and e each much less than unity for a useful TVC. $R_{s}$ and $R_{h}$ are the do values. Then

$$
\frac{\bar{z}_{t}}{R_{t}}=\frac{1+a+j b+p(1+c+j e)}{1+p}=1+g+j h
$$

where $p=R_{h} / R_{s}, g=(a+p c) /(1+p)$ and $h=(b+p e) /(1+p)$. Note that $h=\tan \alpha_{t}=\alpha_{t}$ the phase defect angle in radians, closely enough, since $g$ and $h$ are very small. Then to a sufficient approximation

$$
\frac{Z_{t}}{R_{t}}=\left[1+2 g+n^{2}\right]^{1 / 2}=1+g+\frac{n^{2}}{2}
$$

and

$$
d_{t}=\frac{z_{t}}{R_{t}}-1=g+\frac{h^{2}}{2}
$$

When $\mathrm{p} \leq 1, \mathrm{~h}^{2} / 2<1 \times 10^{-6}$ if $\mathrm{b}$ and $e$ are each less than 1 mrad, so that the phase angle term is negligible. In this case

$$
d_{t}=g, d_{s}=a, \text { and } d_{h}=c
$$

where $d_{s}=\left(z_{s} / R_{s}\right)-1, d_{h}=\left(z_{h} / R_{h}\right)-1$. 


\section{B. Substitution Corrections (See Section IX)}

Consider two different $T E^{\prime} s$ of the same nominal $R_{h}$, each with a known $d_{c}$ as a current converter, and a known $d_{\text {vh }}$ as a voltage converter (with no series resistor). The difference in $d_{v}$ of a TVC range with the same series resistor, is, if all phase angles are negligible,

$$
d_{v 2}-d_{v 1}=82-81+d_{c 2}-d_{c 1}=p\left(c_{2}-c_{1}\right) /(1+p)+d_{c 2}-d_{c 1} \text {. }
$$

But

$$
p /(1+p)=R_{h} / R_{t} \text { and } c_{2}-c_{1}=d_{h 2}-d_{h 1}=\left(d_{v h 2}-d_{c 2}\right)-\left(d_{v h 1}-d_{c 1}\right)
$$

Then

$$
d_{v 2}-d_{v 1}=\frac{R_{h}}{R_{t}}\left(d_{v h 2}-d_{v h 1} j+\frac{R_{s}}{R_{t}}\left(d_{c 2}-d_{c 1}\right) .\right.
$$

Thus the substitution correction for any range is expressed in terms of the directly measurable d's of the new and old TE's as current and voltage converters.

If the phase angles are not negligible a term, $h_{1}{ }^{2}-h_{2}{ }^{2}$, must be added. This reduces to

$$
h_{1}^{2}-h_{2}^{2}=\frac{p b\left(e_{2}-e_{1}\right)+2 p^{2}\left(e_{2}^{2}-e_{1}^{2}\right)}{(1+p)^{2}}
$$

Thus it is zero if $e_{2}=e_{1}$.

If $b=1 \times 10^{-3}=e_{1}$ and $e_{2}=2 \times 10^{-3}$ (as rather extreme examples) this added phase angle correction is less than $1 \mathrm{ppm}$ even at $\mathrm{p}=1$ (low voliage 
range). It is much smaller for higher ranges, even if $b$ is larger (as it may be because of capacitive currents).

At NBS a commercial impedance comparator has been very convenient for comparing phase angles to better than $1 \mathrm{mrad}$ up to $100 \mathrm{kHz}$. It is used to screen the series resistors and TE heaters to make sure that their phase angles are negligible.

\section{Interchanged Series Resistors}

As explained in section $\mathrm{V}$, two nearly identical $30 \mathrm{~V}$ 3-terminal resistors, $R_{1}$ and $R_{2}$, of negligible phase angles, in separate coaxial shields, are connected in serles with two nearly identical TE's. The resulting TVC's are compared, to evaluate $d_{c 1}-d_{c 2}$ of the TE's as current converters. Then the resistors are interchanged and the comparison is repeated to essentially eliminate inequalities in the magnitudes of the reactances of the resistors. For the first measurement, with $R_{1}$ in series with $T E_{1}$ and $R_{2}$ in serles with $T E_{2}$,

$$
a_{T}-a_{S}=\Delta_{1}=\frac{a_{1}+p c_{1}-\left(a_{2}+p c_{2}\right)}{1+p}+d_{c 1}-a_{c 2}
$$

For the second measurement. with $R_{1}$ and $R_{2}$ interchanged

$$
\begin{aligned}
& \Delta_{2}=\frac{a_{2}+p c_{1}-\left(a_{1}+p c_{2}\right)}{1+p}+d_{c 1}-d_{c 2} \\
& \frac{\Delta_{1}+\Delta_{2}}{2}=\frac{R_{h}}{R_{t}}\left(c_{1}-c_{2}\right)+d_{c 1}-d_{c 2}=d_{c 1}-d_{c 2} \text {, closely enough, }
\end{aligned}
$$

since

$$
\frac{p}{1+p}=\frac{R_{h}}{R_{t}}=\frac{1}{60} \quad \text { for the usual } 0.5 V T E^{\prime} s
$$


A more extended treatment, including the effects of phase angles, leads to added terms in $b_{1}, b_{2}, e_{1}$ and $e_{2}$, similar to those given in appendix $5 B$. They are negligible if they do not exceed the limits given there.

\section{AC-DC Difference of Serles Resistor}

For a given range of a TVC, from the equations of appendix 5A, with all phase angles negliglble,

$$
d_{v}=d_{t}+d_{c}=\frac{d_{s}+p d_{h}}{1+p}+d_{c}
$$

Thus

$$
d_{s}=(1+p)\left(d_{v}-d_{c}\right)-p d_{h}=\frac{R_{t}}{R_{s}}\left(d_{v}-d_{c}\right)-\frac{R_{h}}{R_{s}} d_{h}
$$

since $d_{h}=d_{v h}-d_{c}$, where $d_{v h}$ is the ac-dc difference of the TE as a TVC.

\section{E. Effect of a Small Phase Difference Between the Paralleled Impedances in the Current Build Up}

The current through the single higher-range $T E$ in the build-up is the phasor sum of the currents through the two paralleled lower-range TEs. It will differ from the sum of the magnitudes of the two currents, $I_{1}$ and $I_{2}$, if the two are not in phase.

For simplicity let $\bar{I}_{1}=I_{1} \angle 0, \bar{I}_{2}=I_{2} \angle \theta=I_{1} \angle \theta$ with $\theta<<1$ (in radians). The bar signifies a phasor quantity, and its absence a scalar. Since $I_{1}$ is the reference,

$$
\begin{aligned}
\bar{I} & =\bar{I}_{1}+\bar{I}_{2}=I_{1} \angle 0+I_{1} \angle \theta=I_{1}+I_{1}(\cos \theta+j \sin \theta) \\
& \left.=I_{1}\left[\left(2+\theta^{2} / 2\right)+j \theta\right] \text { (neglecting higher order terms in } \theta\right)
\end{aligned}
$$


From this and by continuing to neglect terms in $\theta^{4}$ and higher

$$
I=2 I_{1}\left(1-\theta^{2} / 8\right)
$$

The proportional difference is

$$
\varepsilon=\left(2 I_{1}-I\right) / I=\theta^{2} / 8
$$

If $\varepsilon=3 \times 10^{-7}, \theta=0.1 \times 10^{-3}$ radians. Since the two 1mpedances, $z$, and $z_{2}$, are in parallel,

$$
\bar{I}_{1} \bar{z}_{1}=I_{1} \angle 0 \quad z_{1} \angle 0=\bar{I}_{2} \bar{z}_{2}
$$

Th1s w1ll be true only if $z_{2}=z_{1} L-\theta$. Thus the phase difference between $z_{1}$ and $z_{2}$ must be less than $1 \times 10^{-3}$ rad to keep the phase error less than about $0.3 \mathrm{ppm}$. 


\section{Appendix 6}

\section{RECOMMENDATION}

of the Working Group on the Statement of Uncertainties presented to Comite International des Poids et Mesures

Assignment of experimental uncertainties

\section{RECOMMENDATION INC-1 (1980)}

1. The uncertainty in the result of a measurement generally consists of several components which may be grouped into two categories according to the way in which their numerical value is estimated:

A - those which are evaluated by statistical methods,

B - those which are evaluated by other means.

There is not always a simple correspondence between the classification into categories A or B and the previously used classification into "random" and "systematic" uncertainties. The term "systematic uncertainty" can be misleading and should be avoided.

Any detailed report of the uncertainty should consist of a complete list of the components, specifying for each the method used to obtain its numerical value.

2. The components in category $A$ are characterized by the estimated variances, $s_{i}{ }^{2}$, (or the estimated "standard deviations" $s_{1}$ ) and the number of degrees of freedom, $v_{i}$. Where appropriate, the estimated covariances should be given.

3. The components in category $B$ should be characterized by quantities $u_{j}{ }^{2}$, which may be considered as approximations to the corresponding variances, the existence of which is assumed. The quantities $u_{j}{ }^{2}$ may be treated like 
variances and the quantities $u_{j}$ like standard deviations. Where appropriate, the covariances should be treated in a similar way.

4. The comblned uncertainty should be characterlzed by the numerical value obtalned by applying the usual method for the combination of variances. The comblned uncertalnty and 1 ts components should be expressed in the form of "standard dev1ations".

5. If, for particular applications, it is necessary to multiply the combined uncertainty by a factor to obtain an overall uncertainty, the multiplying factor used must always be stated. 


\title{
Multijunction Thermal Converters as the NBS Primary AC-DC Transfer Standards for AC Current and Voltage Measurements
}

\author{
FRANCIS L. HERMACH, LIFE FELLOW, IEEE, JOSEPH R. KINARD, SENIOR MEMBER, IEEE, \\ AND JOHN R. HASTINGS, MEMBER, IEEE
}

\begin{abstract}
A set of multijunction thermal converters (MJTC's) has been established as the NBS primary ac-dc transfer standard for ac current and voltage measurements. This choice is based upon the advantages of the MJTC's, the development of an improved EMF comparator, an extensive series of intercomparisons, and numerous supporting investigations.
\end{abstract}

\section{INTRODUCTION}

$\mathrm{T}$ HE USE OF ac-dc transfer standards enables ac currents and voltages to be accurately measured in terms of their dc equivalents and the underlying basic electrical units. Although electrostatic and electrodynamic instruments can be used for ac-dc transfer, electrothermal instruments such as thermal converters are generally used because of their greater accuracy and wider current, voltage, and frequency ranges. The ac-dc difference of a transfer standard is conventionally defined as $\delta=\left(Q_{a}-\right.$ $\left.Q_{d}\right) / Q_{d}$, where $Q_{d}$ is the average of the two polarities of the dc input quantity (current or voltage) required to produce the same output as that produced by the ac input $Q_{a}$. Hence $Q_{a}=Q_{d}(1+\delta)$.

Thermal voltage converters (TVC's) and thermal current converters (TCC's) are calibrated at NBS by comparing them with transfer standards whose ac-dc differences have been carefully determined. Most thermal converters contain a single-junction thermoelement (SJTE), consisting essentially of a wire heated by the ac or dc current and a thermocouple to sense the temperature rise. A multijunction thermal converter (MJTC) can have up to 200 or more thermocouple junctions uniformly spaced along a bifilar heater to increase the output EMF and reduce thermoelectric errors [1]. An investigation of MJTC's at NBS several years ago revealed a number of shortcomings which were overcome by changes in design or by compensation. The average ac-dc difference of a set of eight MJTC's of different construction and current ranges and from different manufacturers was shown to be very probably $0.5 \mathrm{ppm}$ or less from $30 \mathrm{~Hz}$ to $10 \mathrm{kHz}$ [2]. These MJTC's have recently been reevaluated in a study

Manuscript received June 23, 1986. This work was supported in part by the Calibration Coordination Group of the U.S. Department of Defense.

The authors are with the Electricity Division, National Bureau of Standards, Gaithersburg, MD 20899.

IEEE Log Number 8613427. of the uncertainties of the NBS standard TVC's and TCC's [3], which was undertaken partly in response to the need for better ac-dc transfer standards to support improved ac calibrators and voltmeters. Four additional MJTC's of two new designs have also been compared to the NBS set.

\section{Precision Comparator System}

The comparator system, consisting of an air bath, an EMF comparator, ac and dc supplies, and a protective circuit, is shown in simplified form in Fig. 1, with an MJTC and an SJTE connected in parallel as voltage converters. A more detailed circuit diagram of the comparator itself is given in [3]. The thermal converters are located in the air bath, which is a thermally lagged metal tank. The comparator basically works as follows. With a resistive divider a fraction of the higher output EMF of one converter is opposed to the full output EMF of the other. The three-stage divider $m V_{1}$ and the Lindeck potentiometer $E_{p}$ are first adjusted to bring the detector, a commercial chopper-type nanovoltmeter (nVM), near zero for both positions of the key with dc applied and are then left undisturbed during an ac-dc determination. Changes in the balance are measured sequentially as ac, each polarity of dc, and then ac are applied at 40-s time intervals. An operational-amplifier integrator and timer are used to integrate the $1-\mathrm{V}$ output of the $\mathrm{nVM}$ for $10 \mathrm{~s}$ and display the average on a digital voltmeter. This significantly reduces the uncertainty from Johnson and $\mathrm{nVM}$ noise and simplifies the data collection. Typically, repeated 10-s averages fluctuate by less than one-tenth of the peak-topeak noise seen in $10 \mathrm{~s}$ on the $\mathrm{nVM}$, which is about 35 $\mathrm{nV}$ in the comparison of two MJTC's, approximately 3.5 times the calculated Johnson noise. To provide the best compromise between loading and noise level, the input resistance of the divider is switched from $10 \mathrm{k} \Omega$ in the comparison of two MJTC's or an MJTC versus an SJTE to only $1 \mathrm{k} \Omega$ (with one stage bypassed) in the comparison of two SJTE's. A reversal compensator (RC), consisting of an adjustable $0-13-\mu \mathrm{V}$ dc source with negligible change on reversal [2], is used when the dc reversal difference is larger than the $\mathrm{nVM}$ range.

A study of protective circuits to guard the converter heaters against burnout showed that a simple arrangement 


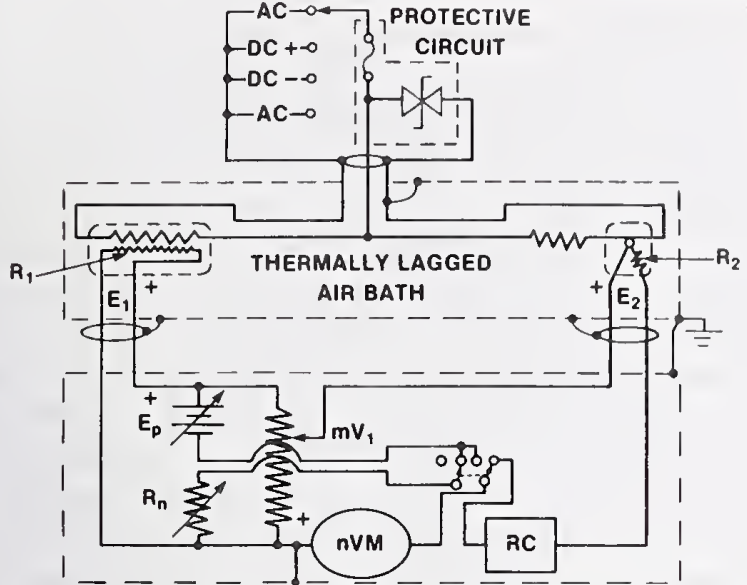

Fig. 1. Precision comparator system with an MJTC and an SJTE connected as voltage converters.

of a pair of back-to-back Zener diodes directly in parallel with the heaters and an expendable thermoelement (TE) in series with the combination provides adequate protection against reasonable kinds of overvoltages. The Zener voltages are chosen to be about twice the supply voltages. TE's with large dc reversal differences, which are relatively inexpensive, serve well as fuses.

The converters require time to recover following any interruption in the heater current. The resulting moderate restrictions on the ofF times of the input relays, so that the converters can recover sufficiently within $30 \mathrm{~s}$ after switching, are discussed in Appendix C. Relays with an OFF time of less than $0.5 \mathrm{~ms}$ are used to switch between the ac and dc inputs.

When the two converters being compared have equal values of $n$, where $n=I \Delta E / E \Delta I, I$ is the heater current, and $E$ is the output EMF, the steady-state balance is independent of small changes in the output of the ac and dc supplies. This reduces the uncertainty in the measurement and the need to keep the EMF of one TE constant during a determination. Although $n$ is very nearly 2.0 for MJTC's, it ranges from about 1.6 to 2.0 for the SJTE's in the reference standards against which the MJTC's must be compared, and thus some of these advantages are lost. To compensate for the difference in $n$ values, an adjustable resistor $R_{n}$ was added to the comparator circuit, as shown in Fig. 1. If the input voltage changes slightly, a small current will flow in the $E_{1}-E_{p}$ loop. Because $R_{1}$ has a finite resistance and forms a divider with $R_{n}$, the change in voltage across the three-stage divider will be less than the change in $E_{1}$. The compensating adjustment of $R_{n}$ is discussed in Appendix $\mathrm{A}$ as part of the derivation of the basic equation for comparing two TE's:

$$
\Delta=\delta_{t}-\delta_{s}=\frac{N_{a}-N_{d}}{n_{s} E_{l}}
$$

where subscript $t$ refers to the TE with the higher $n$, to which the Lindeck potentiometer is connected, and subscript $s$ to the other. $N_{a}$ and $N_{d}$ are the average readings of the nVM with ac and dc applied, respectively, and $E_{l}$ is the lower of the two TE EMF's. If the TE on the right side of Fig. 1 has the higher $n$, both $E_{p}$ and $R_{n}$ are switched across $E_{2}$ and the polarity of the $n V M$ is reversed. The basic equation then remains the same. If $N$ is in nanovolts and $E$ in millivolts, $\Delta$ is in parts per million.

In a recent paper [4] Inglis has shown that the conventional definition of ac-dc difference is in error if the converter has a dc reversal difference $q=\left|Q_{1}-Q_{2}\right| / Q_{d}$, with $E_{1}=E_{2}$. Here subscripts 1 and 2 refer to the two polarities. He has proposed a new definition, $\delta^{\prime}=\left(Q_{a}-\right.$ $\left.Q_{d}^{\prime}\right) / Q_{d}^{\prime}$, where $Q_{d}^{\prime}$ is the dc input that, when applied with the same magnitude in both positive and negative polarities, produces a mean output $\left(E_{1}+E_{2}\right) / 2$ equal to the ac output $E_{a}$. It is free of this error but can be awkward to use directly. The difference between the two definitions depends on a number of factors. However, for a squarelaw TE in which $q$ is approximately independent of $E$, the difference is only about $3 q^{2} / 8$. If $q=5 \times 10^{-4}$ (rather large for a TE intended for precision measurements), the difference is less than $0.1 \mathrm{ppm}$. For the NBS primary MJTC's the values of $q$ are less than $1 \times 10^{-4}$, and that makes the difference between definitions insignificantly small. The results given in this paper were evaluated with the conventional definition of $\delta$.

To test for possible errors caused by electromagnetic interference (EMI), "phantom" measurements were made with the supplies connected to the TE's but switched off, as discussed in Appendix B. No such errors were found. As a precaution all ground leads and shields are connected to the grounded metal tank, which is an excellent shield, and all ungrounded leads are bypassed to the tank with capacitors (of very low inductance). The effects of harmonics and dc offset in the ac input are also discussed in Appendix B.

\section{RESUlts}

The improved EMF comparator has been used to intercompare very thoroughly four of the eight MJTC's that were investigated earlier [2]. These four are labeled A50$14, \mathrm{~B} 10-44, \mathrm{~B} 5-2$, and $\mathrm{C} 10-1$ and will be called the basic subset. Five other MJTC's (labeled D-55, D-60, E-72, 1, $\mathrm{E}-73,1$, and B10-2) have also been studied. The results of the intercomparisons as voltage converters at $30 \mathrm{~Hz}, 1$ $\mathrm{kHz}$, and $10 \mathrm{kHz}$ are shown in Fig. 2(a)-(c), respectively. The number near the head of each arrow is the measured $\delta_{t}-\delta_{s}$ in parts per million, where $\delta_{t}$ and $\delta_{s}$ are the ac-dc differences of the MJTC's at the head and tail of each arrow, respectively. The measurements within the basic subset had a pooled standard deviation of the mean of $\mathrm{C} .16$ ppm. There was no evidence that the differences between averages in repeat tests were statistically significant.

To determine the values assigned to each MJTC in Fig. 2 , the best estimate of $\delta_{t}-\delta_{s}$ for $\mathrm{C} 10-1$ and $\mathrm{B} 10-44$ was first obtained from the three paths between them; double weight was given to the direct path. The best estimates of $\delta$ for the four basic MJTC's, on the basis that their average is zero at each frequency, were then easily determined and are shown to the nearest $0.1 \mathrm{ppm}$ in each ellipse. A50- 


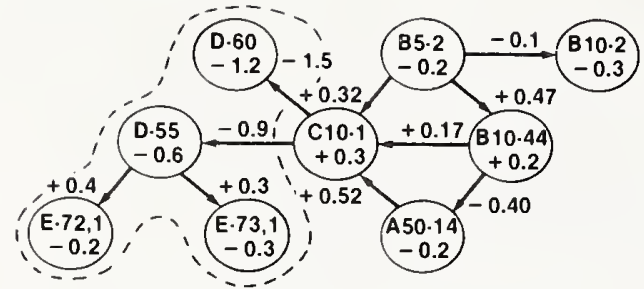

(a)

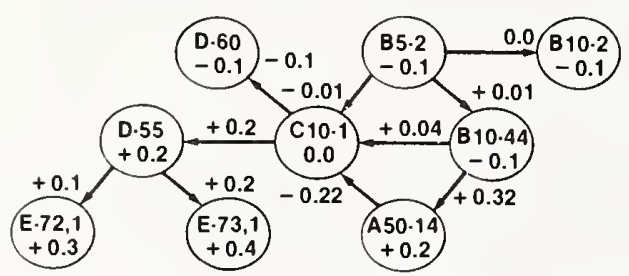

(b)

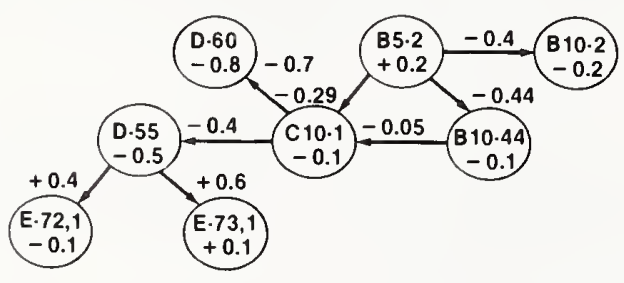

(c)

Fig. 2. Results of the intercomparisons of MJTC's as voltage converters at (a) $30 \mathrm{~Hz}$, (b) $1 \mathrm{kHz}$, and (c) $10 \mathrm{kHz}$. At $30 \mathrm{~Hz}$ the MJTC's outside the dashed line are compensated; those inside the dashed line are uncompensated

14 is not useful at $10 \mathrm{kHz}$ because it has magnetic leads. B10-2 was not included in the basic subset because it was compared only to B5-2 in early measurements which were not repeated. D-55, D-60, E-72,1, and E-73,1 are newer MJTC's which have been compared more recently.

The values of $\delta$ for the four basic MJTC's and for B 102 differ from the corresponding values obtained in the previous investigation by no more than $0.4 \mathrm{ppm}$. At $1 \mathrm{kHz}$, where the previous values for all eight were no larger than $\pm 0.2 \mathrm{ppm}$, four of the five remeasured MJTC's have the same value as before and the other (B5-2) is $0.1 \mathrm{ppm}$ lower. This excellent agreement demonstrates that these MJTC's of different design and construction are very stable.

Comparisons as current converters have also been made between primary MJTC's and specially constructed reference-standard SJTE's. The results of the intercomparisons between a MJTC of one design, F50-19, at $50 \mathrm{~mA}$ and another MJTC of a different design, C10-1, at $5 \mathrm{~mA}$, through intervening steps of SJTE's, are shown in Fig. 3. Two almost identical TE's of one range were first compared in series at rated current (for example, $F Z$ and $F Y$ at $5 \mathrm{~mA}$ ). They were then connected in parallel to form a unit that was compared in series with each of two TE's of the next higher range (B10EP2 and B10EP5 at $10 \mathrm{~mA}$ ), and so on. This is the method used in [5]. As in that work, pairs of almost identical low-reactance resistors were connected in series with the heaters to ensure that the paralleled currents were sufficiently in phase. The phase angles of the two paths must be equal to better than $1 \mathrm{mrad}$, and the two paralleled TE's must be well matched [3], [5].

This method has the advantage that each TE is used at or near rated current, so the results are not affected by current-dependent errors. The 50-mA MJTC was assigned midfrequency $\delta$ values of zero, based on theoretical [1] and experimental [2] analyses. The numbers to the right inside the squares are the $\delta$ values determined from the intercomparisons at $\mathrm{l}$ and $20 \mathrm{kHz}$ with forced closure of each measurement triangle. The closure adjustments of the sides of the triangles averaged only $0.1 \mathrm{ppm}$. Results of the individual comparisons and at other frequencies (20 $\mathrm{Hz}$ and $50 \mathrm{kHz}$ ) have been omitted for simplicity. Assignment began at the $50-\mathrm{mA}$ level and progressed down to the 5-mA $F Z$ and $F Y$ TE's. At $5 \mathrm{~mA}, F Y$ was compared to another MJTC, C10-1, with the results shown on the left of the square. The values from the build-down process and those by direct measurement at $5 \mathrm{~mA}$ agree to within $0.3 \mathrm{ppm}$. Thus the systematic errors in the chain are very small. The pooled standard deviation of the mean of four determinations in an evaluation of $\delta_{t}-\delta_{s}$ was 0.28 ppm.

Of particular interest is the excellent agreement at $1 \mathrm{kHz}$ (within $0.6 \mathrm{ppm}$ ) between the MJTC's and the averages of the two 50-mA and the two 25-mA TE's. Each of these four single-junction converters has an Evanohm heater and Evanohm stems (lead-in wires) between the heater and the glass press (which serves as a good heat sink). Thus the Peltier as well as the Thomson errors of each TE should be very small. Any residuals would probably not be the same in all of them, nor the same as those in the MJTC's. This agreement provides valuable additional confirmation that the ac-dc differences due to thermoelectric effects in the NBS standards are considerably less than $1 \mathrm{ppm}$.

\section{Significance of Midfrequency Evaluation}

Low-frequency ac-dc difference effects, due to doublefrequency ripple, and high-frequency effects, due to reactance and dielectric loss, are generally negligible over a rather broad midfrequency range. This leaves only what have been called "intrinsic errors," caused chiefly by thermoelectric effects in the heaters themselves. Wilkins showed theoretically [1] that these can be less than 0.1 ppm with attainable constructions of MJTC's, which is much less than for SJTE's. Nevertheless, imperfections in materials and heat treatment can cause actual MJTC's to differ substantially from theoretical models, so that the experimental verification outlined above is very important [2].

The agreement shown in Figs. 2 and 3 is thus highly significant. It is very improbable that any errors would be the same for such a diverse set of thermal converters. Supplemental studies showed no significant effects from known sources of ac-dc difference at $1 \mathrm{kHz}$.

Zhang and Zhang have shown [6] that MJTC's have an ac-dc difference caused by the Thomson effect in the regions near the ends of the heater. For an experimental MJTC with a constantan heater and 44 thermocouples they 


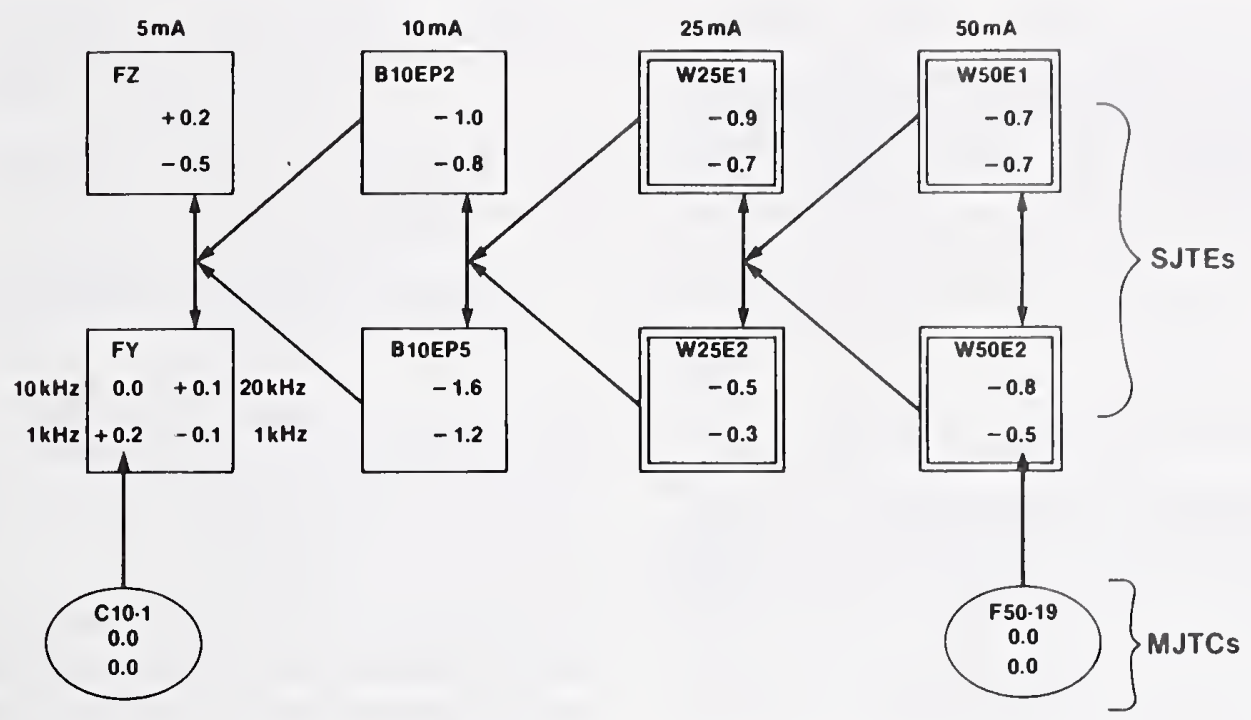

Fig. 3. Results of the intercomparisons of MJTC's and SJTE's as current converters between 5 and $50 \mathrm{~mA}$. The numbers to the right inside the squares are the $\delta$ values determined at 1 and $20 \mathrm{kHz}$.

found that the ac-dc difference $\delta_{e}$ resulting from the end effects was about $10 \mathrm{ppm}$ at three times the normal heater current. Since $\delta_{e}$ is a second-order effect, it would be expected [7] to be proportional to the square of the Thomson coefficient $\sigma$, and hence less than $0.1 \mathrm{ppm}$ for similar MJTC's with Evanohm or comparable heaters for which $\sigma$ is only about 5 percent as large as it is for constantan. In addition, $\delta_{e}$ would be expected to become smaller as the number of couples is increased and as the temperature rise is reduced. The NBS MJTC's have Evanohm or comparable heaters with approximately 50-200 couples and operate at temperature rises of only $15-40^{\circ} \mathrm{C}$ at rated current. The excellent agreement among them is good evidence that end effects are negligible.

\section{Low-Frequency Evaluation}

As the frequency is reduced and the heater temperature begins to follow the double-frequency variation in instantaneous power, ac-dc difference effects are caused by the temperature dependence of the electrical resistance and thermal conductance. Measurements have shown [2] that these effects are much more complex in MJTC's than in SJTE's, and no satisfactory theory is known to us. Effects of $3 \mathrm{ppm}$ or more have been observed at $30 \mathrm{~Hz}$ in otherwise satisfactory 5-mA MJTC's. The major source is apparently a change in the effective heater resistance [2]. Fortunately, MJTC's can be compensated by adding a matched resistor (in series for voltage, in parallel for current) whose resistance is approximately equal to the heater resistance [2]. In Fig. 2 at $30 \mathrm{~Hz}$ the MJTC's with the letters $A, B$, or $C$ in their label are compensaced in this way, and their values of $\delta$ agree with the average of the subset to within $0.3 \mathrm{ppm}$. The newer $D$ and $E$ MJTC's are uncompensated, but even so, their $\delta$ values are between 0 and $-1.2 \mathrm{ppm}$ at this frequency.

\section{Vi. High-Frequency Evaluation}

Theoretically, MJTC's can have ac-dc differences that are smaller than $2 \mathrm{ppm}$ up to $100 \mathrm{kHz}$ [1], but TVC intercomparisons have shown much larger differences in the NBS primary set, whose use is presently limited to no more than $10 \mathrm{kHz}$. This limit has been extended to 100 $\mathrm{kHz}$ (with poorer accuracy) by complex intercomparisons of reference TVC's, each consisting of an SJTE in series with a resistor [3]. Additional measurements of newer types of TVC's and MJTC's are in progress, as are further studies of the causes of ac-dc difference.

\section{SUMMARY}

The accuracy and the stability of the NBS set of MJTC's have been verified. The average ac-dc difference of this set is still $0.5 \mathrm{ppm}$ or less from $30 \mathrm{~Hz}$ to $10 \mathrm{kHz}$. An improved comparator system has been used to make precise intercomparisons of these MJTC's and the SJTE's in the NBS reference standards. As a result of this work these MJTC's now serve as the NBS primary ac-dc transfer standards for ac current and voltage measurements.

\section{APPENDIX A}

\section{Comparator Equations and $n$-Measurements}

\section{A. Comparator Equations}

Since neither TE has a constant output during a comparison of two TVC's (or TE's), the development of the comparator equations given in [8, Appendix II] is not directly applicable to the improved comparator. It will be convenient to use a general equation for a TVC with slightly different ac and dc input voltages, $V_{a}$ and $V_{d}$, applied. We may write, as an identity

$$
V_{a}=V_{d}\left(1+\frac{V_{d}^{\prime}-V_{d}}{V_{d}}+\frac{V_{a}-V_{d}^{\prime}}{V_{d}}\right)
$$


where $V_{d}^{\prime}$ is the average of the two polarities of dc voltage required to produce the same EMF as $V_{a}$, and $V_{d}$ is the average of the two polarities that produce an EMF, $E_{d}$. Then from the definitions of $n$ and $\delta$ given in the text and the fact that $E_{a}=E_{d}^{\prime}$ :

$$
V_{a}=V_{d}\left(1+\frac{E_{a}-E_{d}}{n E_{d}}+\delta\right) \text { to first-order terms. }
$$

A similar equation with $I$ replacing $V$ applies for current measurements.

The basic circuit of the comparator, with the $n$-compensation resistor $R_{n}$ included, is shown in Fig. 1. At the initial balances, with $N=0$ ( $N$ is the reading of the highimpedance $\mathrm{nVM}), m V_{1}=E_{2}$ and $V_{1}=E_{p}$, where $V_{1}$ is the voltage at the input of the divider and $E_{p}$ refers to the Lindeck potentiometer. Now if the TVC input voltage $V_{i}$ changes by a small amount $\Delta V_{i}$, then

$$
\frac{\Delta E_{1}}{E_{1}}=n_{1} \frac{\Delta V_{i}}{V_{i}}
$$

and

$$
\frac{\Delta E_{2}}{E_{2}}=n_{2} \frac{\Delta V_{i}}{V_{i}}
$$

from the definition of $n$. Note that second-order terms are neglected in this approximation.

The small change in the input voltage causes a small change $\Delta I_{1}$ in the output current from the MJTC, so that $\Delta V_{1}=\Delta E_{1}-R_{1} \Delta I_{1}$. By the superposition theorem

$$
\Delta V_{1}=\Delta E_{1}\left(1-\frac{R_{1}}{R_{1}+R_{p}}\right)=p \Delta E_{1}
$$

where $p=R_{p} /\left(R_{1}+R_{p}\right), R_{p}=R_{n} R_{d} /\left(R_{n}+R_{d}\right)$, and $R_{d}$ is the input resistance of the divider. If $R_{n}$ (which includes the output resistance of the potentiometer) is adjusted so that $\Delta N=0$

$$
\Delta E_{2}=m \Delta V_{1}=m p \Delta E_{1} .
$$

Then from (A3) and (A5)

$$
\frac{\Delta E_{2}}{n_{2} E_{2}}=\frac{\Delta E_{1}}{n_{1} E_{1}}=\frac{\Delta E_{2}}{m p n_{1} E_{1}}=\frac{s \Delta E_{2}}{p n_{1} E_{2}}
$$

since $E_{2}=m s E_{1}$ at balance, where $s=R_{d} /\left(R_{1}+R_{d}\right)$. Thus

$$
p=\frac{s n_{2}}{n_{1}} .
$$

Note that $s n_{2}$ must be less than $n_{1}$ in this configuration.

In an ac-dc test the same voltages are applied to both TVC's, so that from (A2)

$$
\delta_{1}-\delta_{2}=\frac{E_{2 a}-E_{2 d}}{n_{2} E_{2 d}}-\frac{E_{1 a}-E_{1 d}}{n_{1} E_{1 d}} .
$$

Since (A4) is a linear equation

$$
\begin{aligned}
N_{a}-N_{d} & =E_{2 a}-E_{2 d}-m\left(V_{1 a}-V_{1 d}\right) \\
& =E_{2 a}-E_{2 d}-m p\left(E_{1 a}-E_{1 d}\right)
\end{aligned}
$$

where the subscripts $a$ and $d$ correspond to the ac and $\mathrm{dc}$ input voltages. Thus

$$
\begin{aligned}
\delta_{1}-\delta_{2} & =\frac{E_{2 a}-E_{2 d}}{n_{2} E_{2 d}}-\frac{m s\left(E_{1 a}-E_{1 d}\right)}{n_{1} E_{2 d}} \\
& =\frac{\left(E_{2 a}-E_{2 d}\right)-m p\left(E_{1 a}-E_{1 d}\right)}{n_{2} E_{2 d}} \\
& =\frac{N_{a}-N_{d}}{n_{2} E_{2 d}} .
\end{aligned}
$$

If $n_{2}>n_{1}$, both $E_{p}$ and $R_{n}$ are switched across $E_{2}$ instead of $E_{1}$ and the polarity of the $\mathrm{nVM}$ is reversed. $R_{n}$ is then adjusted as discussed above, so the $N$ remains the same when $V_{i}$ changes. In this case a similar (and simpler) analysis shows that

$$
\delta_{1}-\delta_{2}=\frac{N_{d}-N_{a}}{n_{1} E_{2 d}} .
$$

More generally, (A10) and (A11) may be written as

$$
\delta_{t}-\delta_{s}=\frac{N_{a}-N_{d}}{n_{s} E_{l}}
$$

where $\delta_{t}$ refers to the TE or MJTC to which the Lindeck potentiometer is connected and $E_{l}$ is the lower of the two EMF's.

\section{B. Precautions in n-Measurement}

The characteristic $n$ is conveniently measured, after balance has been obtained, by changing the input quantity $Q$ by about 0.1 percent, noting the change in $E$, and then calculating $n=Q \Delta E / E \Delta Q$. The usual precautions in measuring small changes should be observed, as in an acdc difference test, to avoid errors from drifts, etc.

A more insidious error can occur, however, if the heater alloy of a TE has even a small temperature coefficient of resistance $\alpha$, so that $R=R_{0}(1+\alpha \theta)$, where $\theta$ is the temperature rise above ambient. Then since $V=I R$ for the heater and $\theta=\kappa I^{2}$

$$
\begin{aligned}
\frac{d V}{V} & =\frac{d I}{I}+\frac{d R}{R}=\frac{d I}{I}+\frac{d R}{d I} \frac{d I}{R}=\frac{d I}{I}+\frac{2 R_{0} \alpha \kappa I d I}{R} \\
& =\frac{d I}{I}(1+2 \alpha \theta) \text { since } R_{0} \approx R .
\end{aligned}
$$

From the definition of $n$ and the above equation

$$
n_{i}=n_{v}(1+2 \alpha \theta) \text {. }
$$

For the NiCr alloys generally used in low-range TE's, $\alpha$ is typically around $-1 \times 10^{-4}$ to $-2 \times 10^{-4} . \theta$ is usually about $1.5 \times 10^{2}$ at rated current. Thus $n_{t^{\prime}}$ and $n_{i}$ can differ by 3-6 percent. For alloys like Evanohm the difference is much smaller.

The appropriate $n$ should be measured and used. Since 
it enters only in a difference term for this comparator, small errors in determining it are not significant unless $\delta_{t}$ $-\delta_{s}$ is large.

\section{APPENDIX B}

EMI, HaRmonics, and DC OFFSET

\section{A. Test for Line-Borne Electromagnetic Interference (EMI)}

As little as $1 \mu \mathrm{W}$ of heater power, over a wide frequency range, can produce about 0.1 percent of rated EMF in a typical 5-mA TE. Hence TE's are sensitive to EMI, particularly at TV and FM frequencies (they were the detectors in early field-strength meters). Laboratory leads at these frequencies can transfer radiated energy fairly efficiently to the heaters unless, of course, the leads are twisted or shielded. Shielding is not completely effective though, and in areas of high field strength (up to 1 $\mathrm{V} / \mathrm{m}$ ) errors can result if the TE responds to currents at both the interfering and the measurement frequencies.

However, because of their square-law characteristics ( $E$ $=K I^{2}$, where $I$ is the rms current $)$, a small heater current $I_{e}$ at a frequency different from that of the measurement current $I_{m}$ does not cause as large an error as the ratio $w$ $=I_{e} / I_{m}$ might suggest. Here

$$
I=\left(I_{m}^{2}+I_{e}^{2}\right)^{1 / 2}=I_{m}\left(1+w^{2}\right)^{1 / 2} .
$$

If the response sensitivity is the same at both frequencies

$$
E=K I^{2}=K I_{m}^{2}\left(1+w^{2}\right) \text {. }
$$

Without $I_{e}$, the output is simply $E_{m}=K I_{m}^{2}$. Thus ( $E-$ $\left.E_{m}\right) / E_{m}=\Delta E / E_{m}=w^{2}$. With only $I_{e}$ present, the output is $E_{e}=K I_{e}^{2}$ and $E_{e} / E_{m}=w^{2}$.

If a measurement of $I_{m}$ is desired, $\Delta E / E_{m}$ is an error term. It can be evaluated to see if it is significantly by making a test measurement with all of the connections in place but with the power supplies that produce $I_{m}$ turned off. In general, $K$ is somewhat current dependent, which slightly affects the sensitivity of this test. There will be no EMI error if the TE's in an ac-dc comparison respond equally well to the currents at both frequencies. The EMI test will determine this too.

\section{B. Effect of Harmonics}

Small amounts of low-order harmonics in the input voltage generally cause insignificant errors in the measurement of the rms value by a TVC or in the comparison of two TVC's. This is a great advantage for true rmsmeasuring instruments. It can be shown rather simply for a wave having a fundamental voltage $V_{1}$ and a single harmonic $V_{h}$. Then $V=\left(V_{1}^{2}+V_{h}^{2}\right)^{1 / 2}$. Because of reactance, skin effect, etc., the response of the TVC may be different at the two frequencies, so that $E=k V^{2}=$ $k_{1} V_{1}^{2}+k_{h} V_{h}^{2}$. Let $V_{h} / V_{1}=r$ and $k_{h}=k_{1}\left(1+e_{h}\right)$. Then $E=k_{1} V^{2}\left(1+r^{2} e_{h}\right)$, and the proportional error is only $r^{2} e_{h}$. For several harmonics this becomes $\Sigma r_{m}^{2} e_{m}$, where $m$ refers to the order of the harmonic.

\section{DC Offset}

The above equations should apply even if the harmonic is of zero order (dc). However, if the TE has a dc reversal difference caused by Thomson or Peltier heating, there is an added output $E_{o}=K_{o} V_{o s}$, proportional to the first power of any dc offset voltage $V_{o s}$ in the ac wave $V_{a}$. Then if $E_{o} \ll E$

$$
E=k\left(V_{a}^{2}+V_{o s}^{2}\right)+K_{o} V_{o s} \approx k V^{2}\left(1+\frac{K_{o} V_{o s}}{E}\right) .
$$

With a dc voltage equal to $V$ initially applied and then reversed, the dc reversal difference in the output is $\Delta E=$ $2 K_{o} V$. Thus

$$
E=k V^{2}\left(1+\frac{g u}{2}\right)
$$

where

$$
g=\frac{\Delta E}{E}
$$

and

$$
u=\frac{V_{o s}}{V}
$$

Note that this is proportional to the first power of $u$, and that, for a square-law TE, $g$ is twice the value of the $\mathrm{dc}$ reversal difference as usually defined $(\Delta V / V$ for constant $E$ ).

\section{APPENDIX C \\ Effect of Relay OfF Time}

Let the response of a TE, whose final value is $E_{f}$, be approximated by a simple exponential with a time constant $t_{c}$. When the steady-state heater current is interrupted for a short time $t_{o} \ll t_{c}$, where $t_{o}$ is the ofF time of the relay when it is switched, we have

$$
E=E_{f} e^{-t_{o} / t_{c}} \approx E_{f}\left(1-t_{o} / t_{c}\right) .
$$

Thus $\Delta E=E_{f}-E=E_{f} t_{o} / t_{c}$.

After the relay is closed again (and the same heater current is restored), $E$ recovers to $E_{f}$ exponentially, so that

$$
E=E_{f}-\Delta E e^{-t_{s} / t_{r}}
$$

where $t_{s}=t-t_{o}$. Then $h=\left(E_{f}-E\right) / E_{f}=b / e^{a}, b=$ $\Delta E / E_{f}=t_{o} / t_{c}$, and $a=t_{s} / t_{c}$, which approaches $t / t_{c}$. when $t_{s} \gg t_{o}$. If we assume that $h=1 \times 10^{-7}$ (recovery to $0.1 \mathrm{ppm}), t_{o}=4 \times 10^{-4} \mathrm{~s}$, and $t_{c}=2 \mathrm{~s}$, then $b=2$ $\times 10^{-4}$ and $a=7.6$. Thus $t=a t_{c}=15.2 \mathrm{~s}$.

If, instead, $t_{o}=4 \times 10^{-3} \mathrm{~s}$, then $t=19.8 \mathrm{~s}$ for recovery to $0.1 \mathrm{ppm}$, an increase of only $4.6 \mathrm{~s}$ for this tenfold increase in OFF time. This interesting result (a simple consequence of the fact that $\ln 10=2.3$ ) shows the relative insensitivity of the recovery time to changes in the relay OFF time whenever $t_{o} \ll t_{c}$. 


\section{REFERENCES}

[1] F. J. Wilkins, "Theoretical analysis of the ac/dc transfer difference of the NPL multijunction thermal convertor over the frequency range $\mathrm{dc}$ to $100 \mathrm{kHz}$," IEEE Trans. Instrum. Meas., vol. 1M-21, pp. 334-340, Nov. 1972.

[2] F. L. Hermach and D. R. Flach, "An investigation of multijunction thermal converters," IEEE Trans. Instrum. Meas., vol. IM-25, pp. 524-528, Dec. 1976.

[3] F. L. Hermach, "An investigation of the uncertainties of the NBS thermal voltage and current converters," Nat. Bur. Stand. (US), Rep. NBSIR 84-2903, Apr. 1985.

[4] B. D. Inglis, "Errors in ac-dc transfer arising from a dc reversal dif- ference," Metrologia, vol. 17, pp. 111-117, Nov. 1981.

[5] F. L. Hermach and E. S. Williams, "Thermal converters for audiofrequency voltage measurements of high accuracy," IEEE Trans. Instrum. Meas., vol. 1M-15, pp. 260-268, Dec. 1966

[6] D. Zhang and Z. Zhang, "Method for reduction of ac-dc transfer error caused by the Thomson effect for the multijunction thermal converter," IEEE Trans. Instrum. Meas., vol. IM-29, pp. 412-415, Dec. 1980.

[7] F. L. Hermach, "Thermal converters as ac-de transfer standards for current and voltage measurements at audio frequencies," J. Res. Nat. Bur. Stand., vol. 48, pp. 121-138, Feb. 1952.

[8] E. S. Williams, "Thermal voltage converters and comparator for very accurate ac voltage measurements," J. Res. Nat. Bur. Stand., vol. 75C, pp. 145-154, July-Dec. 1971 


\title{
An Investigation of Multijunction Thermal Converters
}

\author{
FRANCIS L. HERMACH, FELLOW, IEEE, AND DONALD R. FLACH
}

\begin{abstract}
The relative ac-de differences of a group of multijunction thermal converters (MJTC's) have been determined over the frequency range $30 \mathrm{~Hz}-10 \mathrm{kHz}$. These MJTC's are of different ranges and were obtained from several sources. Differences were observed at low frequencies when converters of various ranges were intercompared. For voltage measurements, the use of matched resistors in series with the MJTC heater resistors greatly reduced these errors and contributed to the reduction of other errors as well. It is believed that the average ac-dc difference of this group is less than $0.3 \mathrm{ppm}$ at $160 \mathrm{~Hz}$ and $0.5 \mathrm{ppm}$ up to $10 \mathrm{kHz}$.
\end{abstract}

\section{INTRODUCTION}

$\mathbf{M}$ EASUREMENTS of rms current and voltage have been based on a technique which converts electrical energy into heat and compares this with the heat produced by a dc signal. Single-junction thermal converters, each consisting of a wire heated by an electric current with a thermocouple to sense the temperature-rise, have been in use for almost 3 decades at NBS for accurate rms ac-dc transfer measurements. Recently, a potentially more accurate form of the resistor-thermocouple combination, the multijunction thermal converter (MJTC), was developed at NPL by Wilkins [1]. Verification of these MJTC's, to $0.5 \mathrm{ppm}$ at $9 \mathrm{~V}$ and $159 \mathrm{~Hz}$, was initially needed at NBS for an absolute volt experiment, now well underway. Later, a goal of $1 \mathrm{ppm}$ from $30 \mathrm{~Hz}$ to $10 \mathrm{kHz}$ was established as a marked and useful advance over the present $10 \mathrm{ppm}$.

Each MJTC has a bifilar heater wire with from 50 to 200 series-connected thermocouples to sense the heater current, overcoming fundamental limitations in single-junction thermal converters [2]. Since actual converters may depart from the ideal values, experimental comparisons of converters of different construction, range, etc are desirable to buttress the theoretical accuracy. Unfortunately there are at present no other suitable kinds of ac-dc transfer standards to provide additional verification [3].

MJTC's with heaters ranging from 5 to $25 \mathrm{~mA}$ and with rated output EMF's of 30 to $120 \mathrm{mV}$ were obtained from commercial manufacturers for this investigation, and 50-mA MJTC's were donated by NPL. Techniques were developed for intercomparing MJTC's with high precision, and a number of ac-dc errors were uncovered and overcome, to attain the desired goals.

\section{EMF COMPARATOR}

The ac-dc difference is defined as $\delta=\left(Q_{a}-Q_{d}\right) / Q_{d}$ where $Q_{a}$ and $Q_{d}$ are the ac and dc inputs which produce

Manuscript received June 29, 1976.

The authors are with the Electricity Division, National Bureau of Standards, Washington, DC 20234. the same output of a thermal converter. A modified Williams EMF comparator [4] was used with a commercial chopper-type nanovoltmeter to determine $\Delta=\delta_{1}-\delta_{2}$, for two MJTC's whose heaters were connected either in series as thermal current converters (TCC's) or in parallel as thermal voltage converters (T'VC's). A simplified schematic of the measuring circuit is shown in Fig. 1. The 11-k $\Omega$ three-stage divider $D$, (adjustable to $1 \mathrm{ppm}$ ) and the Lindeck potentiometer $P$, were first adjusted to bring the nanovoltmeter $N$, near zero for both positions of the key $K$ with dc applied, and were thereafter not disturbed during an ac-dc determination. $S$ was switched to ac, dc-D (direct), dc-R (reversed), and AC in succession. In each case the supply voltage was adjusted to bring $N$ to zero ${ }^{1}$ in the $A$ position of $K$ and the reading of $N$ in the $B$ position was recorded. As shown in [4], $\Delta=\delta_{1}-\delta_{2}=\left(N_{d}-\right.$ $\left.N_{a}\right) / n_{2} E_{2}$, where $N_{a}$ and $N_{d}$ are the average voltage readings with ac and the two directions of dc applied, respectively, and $n_{2}$ is 2 for these square-law MJTC's.

When the dc reversal difference was larger than the range of $N$, the reversal compensator ( $\mathrm{RC}$ ) was used. It consisted of an adjustable, $0-13-\mu \mathrm{V} \mathrm{dc}$ source, with negligible change on reversal. It was switched on for one direction of the applied dc current and reversed for the other, so that there was no net change in the average.

Carefully timed sequences of ac-dc readings, with 30 -s intervals and less than $10-\mathrm{ms}$ off times were necessary to overcome serious limitations from drifts and fluctuations in the MJTC's as well as the power supplies. In some cases Johnson noise was $0.3 \mathrm{ppm}$, and detector noise was 0.6 ppm, peak to peak, in the pass band of the detector. The MJTC's were placed in a $0.02 \mathrm{~m}^{3}$ passive oil bath in a temperature controlled room. Even so, some had to be discarded because of excessive drifts or large switching transients.

The detector had a 20-dB narrow-band rejection filter centered at $60 \mathrm{~Hz}$, and was sensitive to common-mode voltage at and near harmonics of its chopper frequency of $94 \mathrm{~Hz}$. Significant ac outputs at twice the input frequency ${ }^{2}$ were observed in MJTC's below $100 \mathrm{~Hz}$ (approximately proportional to $1 / f^{1.5}$ ), and at the input frequency above $1 \mathrm{kHz}$. Errors from these were reduced by modifying the filter and connecting a $2-\mu \mathrm{F}$ capacitor across the instrument.

To verify the accuracy of the nanovoltmeter, a number of ac-dc difference measurements were also made with a

\footnotetext{
${ }^{1}$ Close settings were not necessary for square law MJTC's. Like the prototype the potentiometer could be connected across $E_{2}$, but this was rarely done, except to measure $E_{2}$.

2 Peak-to-peak ac outputs of up to 0.5 percent, over 1000 times the normally used $0.3-\mu \mathrm{V}$ range of the detector, were observed at an input frequency of $30 \mathrm{~Hz}$.
} 


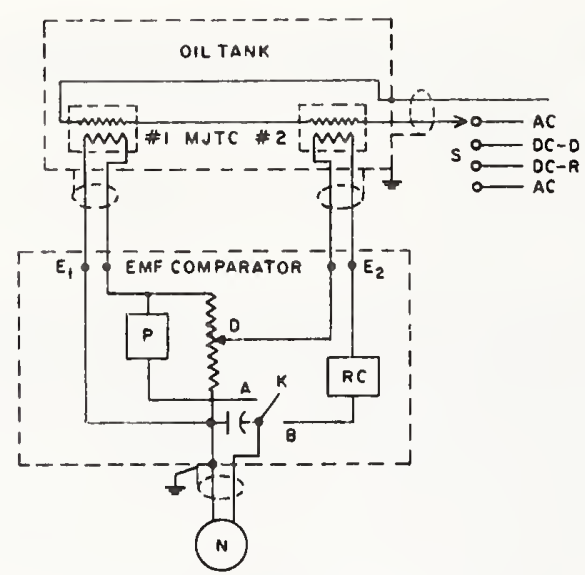

Fig. 1. Comparison circuit.

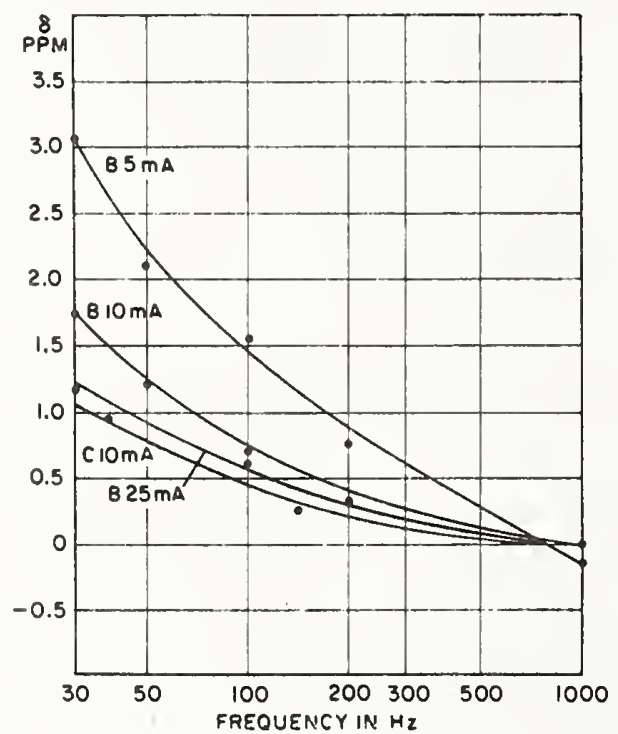

Fig. 2. Ac-de differences of TCC's.

photocell galvanometer amplifier as a null detector, inserting small known voltages in the detector circuit with the reversal compensator. The results with the two detectors were in agreement to $0.2 \mathrm{ppm}$ or better.

\section{LOW-FREQUENCY ERRORS}

Initial tests were made from 30 to $1000 \mathrm{~Hz}$ (a range near $5 / 1$ about $160 \mathrm{~Hz}$ ). Stepup comparisons as thermal current converters (TCC's) were made by connecting, for example, two 5-mA MJTC's in parallel (outputs in series) and connecting the pair in series with a $10-\mathrm{mA} \mathrm{MJTC}$. The two 5-mA MJTC's were also compared, with their heaters in series. These tests disclosed alarming differences between ranges, roughly inversely proportional to frequency, as shown (at rated currents) in Fig. 2, with the $50 \mathrm{~mA}$ MJTC's taken as a base. The cause of these differences is as yet unknown. The error was affected by shunting the heater and was opposite in sign for TVC's. Thus it was caused by a change in the effective resistance of the heater at low frequencies.

As the frequency of the applied current decreases, the heater temperature of a thermal converter begins to follow the double-frequency variation of the instantaneous input power. This can cause low-frequency errors if the resistance is dependent on the temperature. However, the MJTC heaters are quaternary alloys of mainly $\mathrm{NiCr}$, which have low temperature coefficients $\left(0 \pm 20 \mathrm{ppm} /{ }^{\circ} \mathrm{C}\right)$. One pair of MJTC's of the same range had nearly equal lowfrequency errors but opposite temperature coefficients. The error was generally opposite in sign from that deduced mathematically by treating all of the heater as a mass at uniform temperature, with all of the heat lost by temperature-independent lateral cooling. The thermal situation must be much more complex than this, as evidenced by the measured $1 / f^{1.5}$ variation of ac output with heater frequency, instead of $1 / f$ as predicted by this and other simple models when the ratio of ac to dc outputs is small.

A prototype MJTC with a NiCr heater from a third manufacturer had much larger ac dc differences; about $-100 \mathrm{ppm}$ as a TCC and $+120 \mathrm{ppm}$ as a TVC at $30 \mathrm{~Hz}$. This shows rather strikingly the necessity for experimentally verifying new designs. These errors agreed with the sign predicted by the model, but varied roughly as $1 / f$ instead of $1 / f^{2}$ as predicted for low ac outputs.

Ac bridge measurements showed that the low-frequency insulation resistance between the halves of a heater that had been cut in the middle was too high to account for the low-frequency error. Thus the low-frequency error is apparently not caused by the temperature coefficient of resistance of the heater or dielectric loss between the halves of the heater.

A well-known theorem states that the power in a resistor $R_{1}$ fed by a constant voltage source having a fixed internal resistance $R_{2}$ is a maximum when $R_{1}=R_{2}$. From this, as shown in the appendix, the power in the heater of an MJTC used to measure voltage would to the first order be independent of small changes in the heater resistance if a matched (equal) fixed resistor were connected in series with the heater. Analogously, for measuring current the power would be independent of small changes with a matched resistor across the heater. Since the thermocouples respond to the power dissipated in the heater, these interesting applications of the maximum-power-transfer theorem make possible compensated MJTC's, in which the first-order errors from changes in effective resistance are eliminated, at the cost of doubling the current and voltage ranges. Calculations and tests showed that matching to 10 percent is adequate for the desired accuracy.

\section{OTHER ERRORS}

Other errors in voltage measurements arise from Peltier effects at the junctions between the heater and its lead-in wires, and skin effect in magnetic leads used in some MJTC's [1], [2]. For a given construction the errors from Peltier effects are independent of frequency (except at low frequencies) and current level, and are inversely proportional to the resistance, so that they could in principle be 
TABLE I

TVC Comparisons

\begin{tabular}{|c|c|c|c|c|c|c|c|}
\hline \multirow{2}{*}{\multicolumn{2}{|c|}{$\begin{array}{c}\text { MJTC } \\
01\end{array}$}} & \multirow{2}{*}{ 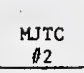 } & \multirow[b]{2}{*}{$v$} & \multicolumn{4}{|c|}{$\Delta=\delta_{1}-\delta_{2} \quad(\mathrm{ppm})$} \\
\hline & & & & $30 \mathrm{~Hz}$ & $160 \mathrm{~Hz}$ & $1 \mathrm{kHz}$ & $10 \mathrm{kHz}$ \\
\hline & $810^{\prime \prime 2}$ & B5 2 & 9 & +0.4 & -0.2 & -0.2 & -0.4 \\
\hline & $810 " 44$ & $B 5 \# 2$ & 9 & +0.6 & -0.1 & 0.0 & -0.2 \\
\hline & $810^{\# 2}$ & $\mathrm{~B} 10^{\#} 44$ & 6 & +0.1 & - & 0.0 & +0.1 \\
\hline & $"$ & $"$ & 9 & 0.0 & 0.0 & -0.2 & +0.2 \\
\hline & $\mathrm{c}_{10}{ }^{\prime \prime}$ & $"$ & 6 & +0.4 & +0.1 & +0.1 & +0.2 \\
\hline & $c 10^{\# 1}$ & $"$ & 6 & +0.4 & +0.1 & +0.1 & +0.6 \\
\hline & $210 " 2$ & $810^{\# 45}$ & 6 & +0.4 & -0.1 & -0.3 & -0.1 \\
\hline & $=10^{\# 1}$ & $"$ & 6 & +0.3 & 0.0 & +0.3 & +0.2 \\
\hline & $"$ & $\mathrm{~B} 10^{\#} 2$ & 6 & +0.1 & +0.2 & 0.0 & +0.4 \\
\hline & $"$ & A50\#14 & 6 & +0.4 & 0.0 & -0.2 & - \\
\hline & $150 \# 14$ & A $50 \# 15$ & 8 & +0.2 & 0.0 & -0.1 & - \\
\hline & $50 \# 15$ & $\mathrm{~B} 10^{\#} 2$ & 9 & 0.0 & -0.1 & +0.2 & - \\
\hline NOTE: & $\begin{array}{l}\mathrm{B1O}^{\# 2} \\
\mathrm{~B}^{\#} 2 \\
\mathrm{~A} 5 \mathrm{\#}^{\mathrm{\#}} 1 \\
\mathrm{~A} 11 \text {. }\end{array}$ & $\begin{array}{l}\text { nifies } \\
\text { B10 } 2\end{array}$ & & ser & $\begin{array}{l}\text { mber } 2 \\
\text { ted } \mathrm{g} 1\end{array}$ & manu & $\begin{array}{l}\text { urer B. } \\
\text { NBS. } \\
\text { leads. }\end{array}$ \\
\hline
\end{tabular}

evaluated by making TVC tests with different series resistors. However the low-frequency variation can be complicated, and the evaluation is difficult in the presence of other errors. Instead these and skin-effect errors were eliminated by specifying MJTC's with copper leads, which have low thermal emfs against the quaternary heater alloys.

At higher frequencies (up to $10 \mathrm{kHz}$ ), tests disclosed errors in some of the MJTC's from dielectric losses between the heater and the thermocouples. ${ }^{3}$ For example, differences of up to several ppm at $10 \mathrm{kHz}$ could be obtained by changing the ground from one line to the other in tests of two 5-mA TCC's in series. Dielectric errors were also greatly reduced by the matched resistors. (See Appendix.)

\section{TVC TESTS}

Since the needs were for voltage measurements, a group of $8 \mathrm{MJTC}$ 's of 4 different constructions and 3 current ranges, and with copper leads, were compensated with matched series resistors of quaternary alloys wound on thin mica cards. ${ }^{4}$ Each MJTC with its resistor was mounted in an aluminum box (through which oil could circulate), with input binding posts and a 2-pin output connector of low thermal EMF's. They were compared as TVC's at 6 to $9 \mathrm{~V}$, at $30,160,1000$, and $10000 \mathrm{~Hz}$. The results are shown in Table I.

At the important frequency of $160 \mathrm{~Hz}$ the results are shown more vividly in Fig. 3. The circles identify the MJTC's and the number adjacent to each arrow gives $\delta_{h}$ $-\delta_{t}$ in ppm, where $\delta_{h}$ and $\delta_{t}$ are, respectively, the ac-dc

\footnotetext{
${ }^{3}$ Dielectric losses in the oil were negligible.

${ }^{4}$ Two MJTC's, with 50-mA ranges, have magnetic leads and were not used above $1 \mathrm{kHz}$. A series resistor of $200 \Omega$ was used with each, to give a 10 -V range. Tests with other resistors showed that resistance-dependent low-frequency errors of these MJTC's were less than $0.5 \mathrm{ppm}$. Series resistors for the other MJTC's were within 10 percent of the heater resistances.
}

TABLE II

Assigned AC-DC Differences

\begin{tabular}{|c|c|c|c|c|}
\hline \multirow[b]{2}{*}{ MJTC } & \multicolumn{4}{|c|}{$\delta(\mathrm{ppm})$} \\
\hline & $30 \mathrm{~Hz}$ & $16 n \mathrm{~Hz}$ & $1 \mathrm{kHz}$ & $10 \mathrm{kHz}$ \\
\hline B $10^{\# 44}$ & 0.0 & -0.1 & -0.1 & -0.2 \\
\hline$B 10^{\#} 45$ & 0.0 & +0.1 & -0.1 & 0.0 \\
\hline $\mathrm{C} 10^{\#}$ & +0.3 & +0.1 & 0.0 & +0.3 \\
\hline $\mathrm{C} 0^{\mathrm{\#}} 2$ & +0.4 & 0.0 & -0.2 & 0.0 \\
\hline$B 10 \% 2$ & 0.0 & -0.1 & -0.1 & -0.2 \\
\hline $\mathrm{BS}^{\# 2} 2$ & -0.5 & +0.1 & 0.0 & +0.1 \\
\hline $\mathrm{A} 50^{\#} 14$ & 0.0 & 0.0 & +0.2 & - \\
\hline A $50^{\#} 15$ & -0.1 & -0.1 & +0.2 & - \\
\hline
\end{tabular}

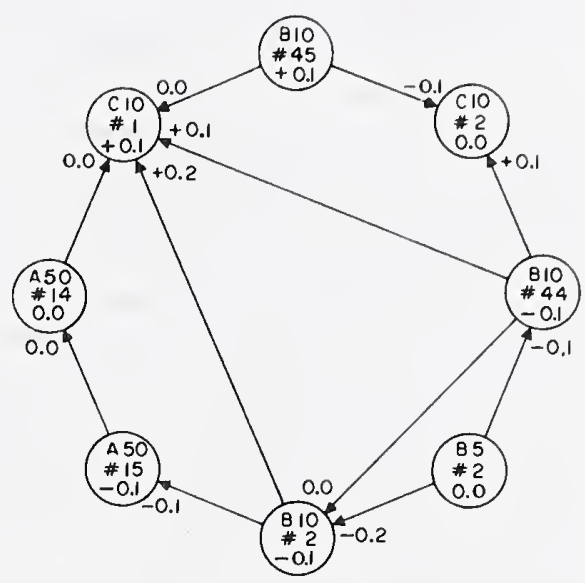

Fig. 3. TVC's at $160 \mathrm{~Hz}$.

differences of the MJTC's at the head and tail of the arrow. In the absence of systematic errors the arrows around the loops should sum to zero within the limits imposed by the random errors of the comparisons. Alternatively failure to close properly is an indication of systematic errors. Techniques described by Youden [5] can be used to evaluate networks such as this.

The values of $\delta$ assigned in this way to each MJTC at 160 $\mathrm{Hz}$ are shown in each circle, on the assumption that the average of all is zero. Each value is less than $0.2 \mathrm{ppm}$, and the closure errors are very small.

Similar analyses were carried out for the measurements at other frequencies. The assigned ac-dc differences are shown in the columns marked $\delta_{v}$ in Table II. The discrepancies are somewhat larger than at $160 \mathrm{~Hz}$ but there is no evidence of systematic error, or of significant residual low-frequency errors in these compensated MJTC's.

The standard error $s$ (standard deviation of the average of 4 determinations) in a comparison of $2 \mathrm{MJTC}$ 's was 0.12 ppm. Because of the network of comparisons the standard deviation of an MJTC with respect to the average should be somewhat less than this.

\section{SERIEs REsistors}

The high-quality wire-wound series resistors used with these MJTC's have excellent ac-dc characteristics at audio frequencies. The phase angles are small and cause only a 


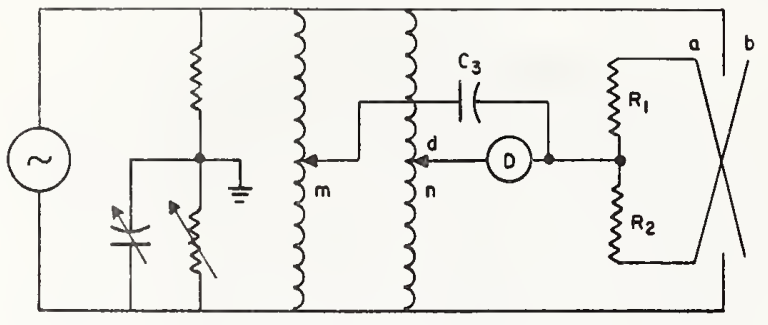

Fig. 4. Reversing bridge.

second-order effect on impedance magnitude. However there is a possibility of dielectric loss in the insulation at the higher audio frequencies. (The maximum powertransfer theorem does not help here.) To determine this, the $1 / 1$ inductive-voltage-divider bridge shown in Fig. 4 was set up to compare two nearly equal resistors $R_{1}$ and $R_{2}$. A balance was made for each switch position of this "reversing bridge" by adjusting $\mathrm{m}$ and $\mathrm{n}$ with the detector lead $d$, as shown, after adjusting the Wagner arms with $d$ grounded. Most of the bridge errors were eliminated in this way, leaving as the difference equations, $\left(R_{2}-R_{1}\right) / R_{1}=$ $2\left(n_{a}-n_{b}\right)$ and $\alpha_{2}-\alpha_{1}=\gamma\left(m_{a}-m_{b}\right)$ where $R$ and $\alpha$ are the resistive components and the phase angle, respectively, of the parallel equivalent circuit for each resistor, $\gamma=$ $\omega C_{3} R$, and the subscripts $a$ and $b$ refer to the switch positions shown. These simple equations hold to $0.1 \mathrm{ppm}$ if the phase angles of $C_{3}$ and $m$ are each less than $0.1 \mathrm{mrad}$ and $\left(\alpha_{2}-\alpha_{1}\right)$ is less than 1 mrad. ${ }^{5}$

A $1-\mathrm{k} \Omega$ resistor of the same type as those used with the MJTC's was mounted in a metal box, for four-terminalpair ac-dc measurements on Cutkosky's bridges [6] from 160 to $16000 \mathrm{~Hz}$. This served as a standard for evaluating two $1-\mathrm{k} \Omega$ unmounted resistors. These were paralleled to evaluate a 5 -section $500-\Omega$ card. The 5 sections were intercompared and then used to evaluate the 300,400 , and $500-\Omega$ cards for the MJTC's. The $1300-\Omega$ card for the $5-\mathrm{mA}$ MJTC was compared with the $1-\mathrm{k} \Omega$ card in series with 3 $100-\Omega$ sections.

An upper limit of $10 \mathrm{kHz}$ was imposed by the effects of lead inductance and of stray capacitance from the cards to the grounded aluminum boxes in which they were tested and used. Below this, changes in the parallel resistances were less than $0.1 \mathrm{ppm}$ for drastic changes in the positions of the resistors. The cases of the inductive voltage dividers and the shields of all leads of the reversing bridge were grounded. $C_{3}$ was a low-loss 3 -terminal air capacitor, and $D$ was a tuned null detector. All bridge components were commercially available.

Measurements were made at $0.1,1$, and $10 \mathrm{kHz}$ with 8 $\mathrm{V}$ applied. The results are shown in Table III as $\delta_{e}$ and $\alpha$, where $\delta_{e}=\left(R_{f}-R_{p}\right) / R_{p}$ and $\alpha$ is the phase angle. $R_{f}$ and $R_{p}$ are the parallel-equivalent-circuit resistances at the frequency $f$ and the base frequency $(0.1 \mathrm{kHz})$, respectively. For each resistor, $\alpha$ was closely proportional to $f$, and so is not shown at $1 \mathrm{kHz}$ in the table.

${ }^{5}$ The dividers were rated up to $10 \mathrm{kHz}$. Only the lower dials were changed on reversal, with only moderate accuracy required.
TABLE III

Resistance Measurements

\begin{tabular}{|c|c|c|c|c|c|c|}
\hline \multirow[b]{2}{*}{ MJTC } & \multirow{2}{*}{$\begin{array}{l}\mathrm{R}_{\mathrm{s}} \\
\Omega\end{array}$} & \multirow{2}{*}{$\frac{1 \mathrm{kHz}}{\delta_{\mathrm{e}}}$} & \multicolumn{4}{|c|}{$10 \mathrm{kHz}$} \\
\hline & & & $\delta_{e}$ & $\alpha$ & $\delta_{r}$ & ${ }^{\delta_{c}}$ \\
\hline $\mathrm{BiO}^{\#} 44$ & 500 & -0.1 & -0.7 & -0.55 & -0.5 & +0.3 \\
\hline$B 10^{*} 45$ & " & +0.1 & +0.3 & -0.41 & +0.1 & -0.1 \\
\hline $\mathrm{C} 10^{\#} 1$ & 300 & 0.0 & +0.5 & -0.60 & +0.1 & +0.2 \\
\hline $\mathrm{C} 10^{\#} 2$ & " & 0.0 & 0.0 & -0.41 & -0.1 & +0.1 \\
\hline $\mathrm{B} 10^{\#} 2$ & 400 & 0.0 & +0.4 & -0.47 & +0.1 & -0.3 \\
\hline $85^{\# 2} 2$ & 1300 & 0.0 & 0.0 & -0.26 & 0.0 & +0.1 \\
\hline
\end{tabular}

NOTE: All values of $\delta$ in $\mathrm{ppm}$, a in mrad. See text.

The effect of these small errors on the magnitude of the current through the MJTC is approximately $\delta_{r}=\delta_{e} / 2-$ $3 \alpha^{2} / 8$. This was negligible at $1 \mathrm{kHz}$ and, except for one resistor, even at $10 \mathrm{kHz}$. The average for all of the resistors was less than $0.1 \mathrm{ppm}$ at each frequency, so that no corrections need be applied to Table II.

The ac-dc differences of a TVC is $\delta_{v}=\delta_{r}+\delta_{c}$ [2] where $\delta_{c}$ is the ac-dc difference of the thermal converter. Since $\delta_{v}$ and $\delta_{r}$ were evaluated as indicated above, $\delta_{c}$ could be determined for each MJTC. As shown in Table III, each was $0.3 \mathrm{ppm}$ or less at $10-\mathrm{kHz}$.

\section{CONCLUSIONS}

This work reaffirms the value of both experimental and theoretical investigations to evaluate ac-dc differences in an absolute sense, and to determine how well actual ac-dc transfer standards meet the theoretical ideals. A number of sources of ac-dc differences were uncovered, and were eliminated by changes in design, or were compensated for. The agreement between a group of 8 MJTCs which met these requirements was excellent. Because of this, because of the significant differences in construction and ranges of these MJTC's, and because of our long investigation, we believe it is very unlikely that the average ac-dc difference of this group exceeds $0.3 \mathrm{ppm}$ at $160 \mathrm{~Hz}$ or $0.5 \mathrm{ppm}$ at 30 and $10000 \mathrm{~Hz}$. This belief is supported by an analysis based on estimates of the limits of error from such factors as mismatch of series resistors, Peltier-heat exchanges, ac-dc differences of resistors, systematic errors in the comparator, and random errors. For the latter we took as the 95 percent confidence limit $2 s / \sqrt{n}$, where $n$ is the number of MJTC's in the group.

A few measurements over several months indicate a repeatability of $0.3 \mathrm{ppm}$ or better. The long-term stability of ac-dc difference is as yet unknown, but there is no reason to expect drifts greater than this.

Extensions to higher voltages and frequencies seem feasible. Small ac-dc differences in the effective heater resistance could be compensated with combined shunt and series resistors, as indicated in the Appendix. A wide range of film resistors could be mounted in a geometry that would allow the reactance to be calculated, so that the powerful comparison technique of [2] could then be applied. 


\section{APPENDIX}

\section{COMPENSATION FOR CHANGES IN RESISTANCE}

When a resistor $m R$ is in series with MJTC heater, $R(1$ $+a), a \ll 1$, the current (with no reactance) is

$$
I=\frac{V}{(m+1) R(1+a /(m+1))}
$$

$$
\simeq \frac{V}{(m+1) R}\left(1-\frac{a}{m+1}\right)
$$

neglecting higher order terms in $a$. The power in the heater is

$$
P=I^{2} R(1+a) \simeq \frac{V^{2}}{(m+1)^{2} R}\left(1-\frac{2 a}{m+1}+a\right) .
$$

For a fixed $V$ and $m$, the power is independent of $a$ if $m$ $=1$ (neglecting higher order terms). Thus since the thermocouples respond to the power dissipated in the heater, a small difference between the effective $\mathrm{dc}$ and ac heater resistances will cause no first-order ac-dc difference in a TVC with a matched series resistor. Matching to 10 percent will reduce a $2 \mathrm{ppm}$ ac-dc difference (from this cause) to $0.1 \mathrm{ppm}$.

Similarly for a TCC with $m R$ in parallel with $R(1+a)$ the power in the heater is independent of $a$ if $m=1$, so that there will be no first-order ac-dc difference with a matched shunt.

This compensation will be effective, however, only if the heater is a two-terminal resistor in the measuring circuit, and if the thermocouples are insensitive to any change in the distribution of the power. For example, an error would occur with dielectric leakage from the heater to the ther- mocouple of an MJTC unless the thermocouple output and one end of the heater were connected together, and the thermocouple resistance was much less than the insulation resistance.

Matching provides only a single compensated voltage or current range. However, for higher voltage ranges a similar analysis shows that if a resistor $n R$ is connected across the heater and $m R$ in series with the combination, compensation is achieved if $n=m /(m-1)$.

\section{ACKNOWLEDGMENT}

The authors are very grateful to F. Wilkins for the NPL MJTC's, to R. Cutkosky for the ac-dc measurements of the mounted resistor, and to J. Sutcliffe and I. Malcolm of Guildline Instruments Ltd. for their extended cooperation.

\section{REFERENCES}

[1] F. J. Wilkins, "Theoretical analysis of the ac/dc transfer differences of the NPL thermal converter over the frequency range dc-100 kHz," IEEE Trans. Instrum. Meas., vol. IM-21, pp. 334-340, 1972.

[2] F. L. Hermach and E. S. Williams, "Thermal converters for audiofrequency voltage measurements of high-accuracy," IEEE Trans. Instrum. Meas., vol. IM-15, pp. 260-268, 1966.

[3] F. L. Hermach, "Ac-dc comparators for audio-frequency current and voltage measurements," to be published.

[4] E. S. Williams, "Thermal voltage converters and comparators for very accurate ac voltage measurements," J. Res. Nat. Bur. Stand., 75C, pp. 145-154, July-Dec. 1971.

[5] W. J. Youden, "Measurement agreement comparisons," in Proc. 1962 Standards Lab. Conf., NBS Misc. Publ. 248, pp. 147-152, Aug. 1963.

[6] R. Cutkosky, "New NBS measurements of the absolute Farad and Ohm," IEEE Trans. Instrum. Meas., vol. IM-23, pp. 305-309, 1974. 


\section{Theoretical Analysis of the AC/DC Transfer Difference of the NPL Multijunction Thermal Convertor Over the Frequency Range $\mathrm{DC}$ to $100 \mathrm{kHz}$}

\section{F. J. WILKINS}

\begin{abstract}
Measurements of the rms values of alternating currents are frequently made by means of thermal transfer instruments. At low and midfrequencies Thomson heating can have an important influence on the ac/dc transfer difference of these devices, whereas at higher frequencies the variation with frequency of the effective resistance of their heaters is the most decisive factor. The present paper examines the influence of both Thomson heating and the reactive components of the heater on the performance of the NPL multijunction convertor, and sets out the theoretical analysis that supports the claim that its ac/dc transfer difference does not exceed 1 or 2 ppm up to a frequency of $100 \mathrm{kHz}$.
\end{abstract}

\section{NOMENCLATURE}

$\theta$

$\theta_{J}$

$\lambda$

$A$

$\alpha_{T}$

$i$

$2 l$

$N$

$2 L=2 L N$

$P$

$P_{2 L}$

$P_{E}$

$r_{1}$

$R_{1}$

$\rho$

$V_{2 L}$

$V_{x}$

$l_{1}$

$C_{2(P)}$

$R_{2(P)}$

$\tan \delta$
Temperature rise of heater above ambient temperature.

Temperature rise of point at which a thermocouple crosses the heater.

Thermal conductivity of heater material.

Cross-sectional area of heater.

Thomson coefficient of the heater material.

Heater current.

Length of heater between adjacent thermojunctions.

Number of thermojunctions on heater.

Total length of heater.

Power loss for unit temperature rise from unit length of heater by convection and radiation.

Total power loss by a radiation and convection from the full length of the heater.

Total power conducted through an element.

Resistance of unit length of heater.

Total heater resistance.

Resistivity of heater material.

Voltage drop across heater.

Voltage at point $x$ along heater.

Inductance/unit length of heater.

Capacitance between heater and inner housing.

Parallel loss resistance between heater and in-

ner housing.

Manuscript received June 9, 1972; revised July 13, 1972.

The author is with the Division of Electrical Science, National Physical Laboratory, Teddington, Middlesex, England.

1972 IEEE. Reprinted, with permission, from IEEE Transactions on

Instrumentation \& Measurement, Vol. IM-21, pp. 334-340, November 1972.

\begin{tabular}{|c|c|}
\hline$C_{3(P)}$ & $\begin{array}{l}\text { Capacitance between one half of heater and } \\
\text { the other half. }\end{array}$ \\
\hline$R_{3(P)}$ & $\begin{array}{l}\text { Parallel loss resistance between one half of } \\
\text { heater and the other half. }\end{array}$ \\
\hline $\tan \delta_{2}$ & $\begin{array}{l}\text { Loss angle between one half of heater and } \\
\text { the other half. }\end{array}$ \\
\hline$y_{2}=r_{2}+j w c_{2}$ & Admittance/unit length heater to housing. \\
\hline$y_{3}=r_{3}+j w c_{3}$ & $\begin{array}{l}\text { Admittance/unit length one half heater to } \\
\text { the other. }\end{array}$ \\
\hline 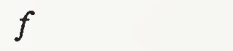 & Frequency of alternating current. \\
\hline 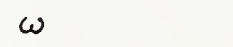 & Radian frequency of alternating $\mathrm{cl}$ \\
\hline & Relative permeability of heater material. \\
\hline
\end{tabular}

\section{INTRODUCTION}

7 HERMAL transfer methods are in common use for the intercomparison of alternating and direct currents. A fundamental study of the limitations of the single junction convertor was first given in 1952 by Hermach [1] and subsequently the ac/dc transfer difference of the earliest form of the NPL multijunction thermal convertor at power and the lower audio frequencies, due almost entirely to the effects of Thomson heating, was estimated to be much less than $1 \mathrm{ppm}$ [2]. This determination was based on the solution of the equations describing the heat-flow pattern along the heater and the accuracy claimed seemed more than adequate for all ac measurements. Occasionally, however, as in absolute determinations, a more detailed knowledge of ac/dc transfer performance is required, and moreover in the intervening years the design of the NPL convertor has been modified so as to improve its ac/dc characteristics. The present analysis therefore not only supplements the previous considerations by taking into account the factors affecting the performance of the convertor at both lower and higher frequencies but also gives a more accurate appraisal of the influence of Thomson heating on the latest design, which has an increased number of thermoelements.

\section{BRIEF DESCRIPTION OF CONVERTOR}

The element or functional part of the multijunction convertor is a twisted bifilar heater $26 \mathrm{~mm}$ long on which are supported 220 pairs of series-connected thermojunctions having common cold junction temperatures. The combined out- 
put from the thermojunctions thus gives an accurate indication of the mean temperature of the heater with respect to that of the main structural part of the convertor, and being large may be measured with ease to a few parts in $10^{7}$.

In the earlier form of the convertor the thermocouples were formed as a helix of triangular section with the heater at one corner of the triangle, the support or return copper lead at another, and a glass rod at the third corner as shown in Fig. 1(a). The later elements are rectangular in section, as illustrated in Fig. 2(a), and formed on, and thus attached to, a copper rod insulated with ML enamel. The heaters are twisted bifilar lengths of ML insulated manganin or quaternary alloys; both alloys have low Peltier coefficients against copper and low Thomson coefficients. Buffer leads of manganin are interposed between the element and thermocouple leads from each element and the pins in the glass and alumina leadthrough assemblies. These buffer leads are taken close to the structural members in order to make good thermal contact with them and so to prevent the direct flow of heat between the pins and the element from influencing the temperature rise of the heater. In the early design the through leads were of nickel-iron, having a high permeability, whereas in the final arrangement they are of copper.

Each form of element is mounted in an inner housing as shown in Figs. 1(b) and 2(b). The glass rod of the first design is located in a groove cut between the two blocks of copper and thus serves to anchor the element securely, whereas in the second type the copper rod is placed in a channel in the surface of the square-section rod. Each form of mounting is such that the element is surrounded by a copper shield having the same temperature as that of the thermocouple cold junctions.

Both elements have been included in the preceding description for almost all the numerical values that are to be quoted subsequently apply to the early design whereas it is now thought most future convertors will be made to the second. However the distributed capacitance of each heater to screen and its inductance do not differ appreciably in the two designs and thus their ac-dc characteristics should be the same at the higher frequencies.

\section{Principle of Operation}

The convertor is normally operated to give an output voltage of $100 \mathrm{mV}$ when the temperature rise of the heater is about $15^{\circ} \mathrm{C}$. Little heat is therefore lost by radiation, more especially as the element is surrounded by an aluminized radiation shield and thus almost all the heat developed in the heater wire is conducted to the copper base through the thermocouple wires. The thermocouple wires therefore sense the coldest parts of the heater and in presenting the sole thermal impedance to the heat flow determine its temperature rise. Hence the length of the thermocouple wires, the thermal con. ductivity, and cross-sectional areas of both the copper and constantan all combine to determine the temperature rise of the heater, and the cold junction temperatures and relative resistances of the copper and constantan paths control the variation of the output voltage $/{ }^{\circ} \mathrm{C}$ rise of the heater.

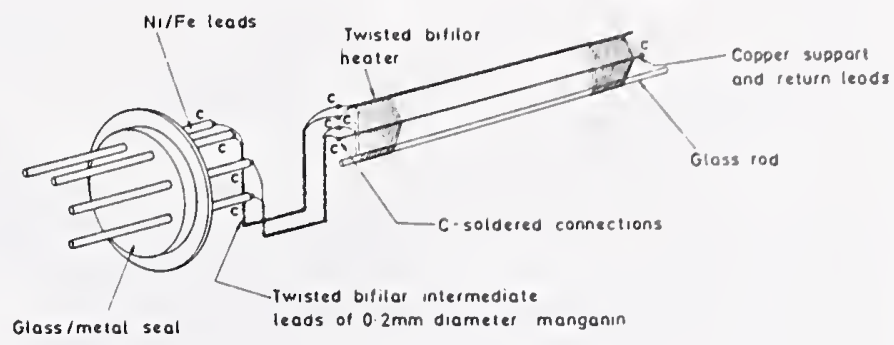

(a)

Twisted bifilar heater

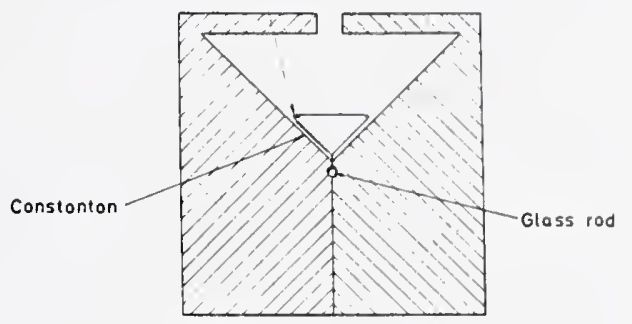

(b)

Fig. 1. (a) Triangular element and connecting leads. (b) Triangular element mounted in housing.

\section{Factors Influencing AC/DC Transfer Characteristics}

\section{A. Midfrequency Response}

The lowest frequency in this band is that at which the heater attains a steady and constant temperature for any constant power input and the resulting thermocouple output voltage is almost free from double frequency ripple. Within the wide range set by this frequency and that at which the reactive components of the heater affect the current distribution along its length Thomson heating is the main cause for the convertor responding differently to alternating and direct currents.

The difference between the two responses may be studied, and an upper limit assigned to its magnitude, by considering the heat-flow pattern along a single length of heater wire bounded by two thermal elements as indicated in Fig. 3. When thermal equilibrium is established the resultant flow across an element $\delta x$ is zero and thus

$$
\lambda A \frac{d^{2} \theta}{d x^{2}}-i \alpha_{T} \frac{d \theta}{d x}-P \theta+i^{2} r_{1}=0
$$

The solution of this equation [1], when the appropriate boundary conditions are imposed, shows that the heat conducted from the heater through each pair of thermocouple wires $P_{E}$ is

$$
P_{E}=2 \lambda A\left\{\frac{i^{2} r_{1}}{P}-\theta_{J}\right\} a\left\{\frac{\cosh 2 a l-\cosh 2 n l}{\sinh 2 a l}\right\}
$$

and it may be convenient at this stage to restate that

$$
2 n=\frac{i \alpha_{T}}{\lambda A} \quad m^{2}=\frac{P}{\lambda A} \quad k=\frac{i^{2} r_{1}}{\lambda A} \quad a^{2}=m^{2}+n^{2} .
$$




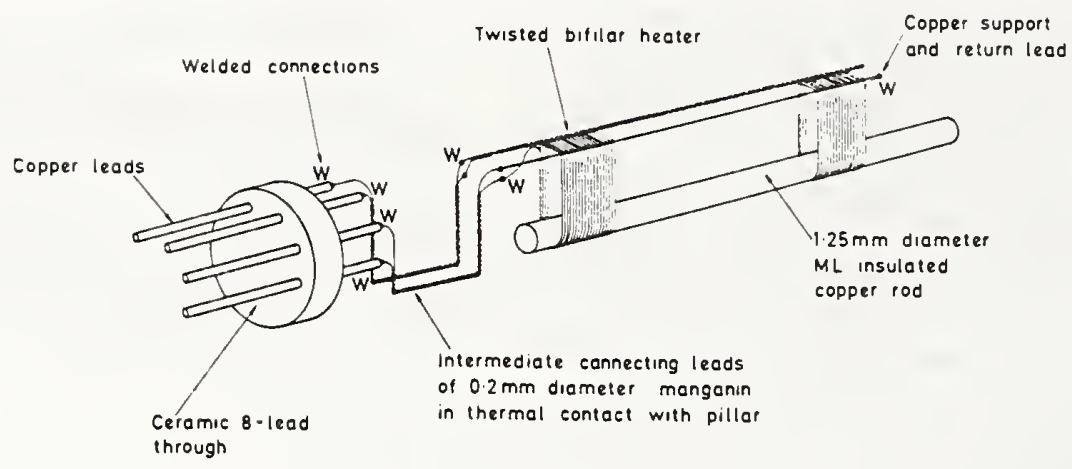

(a)

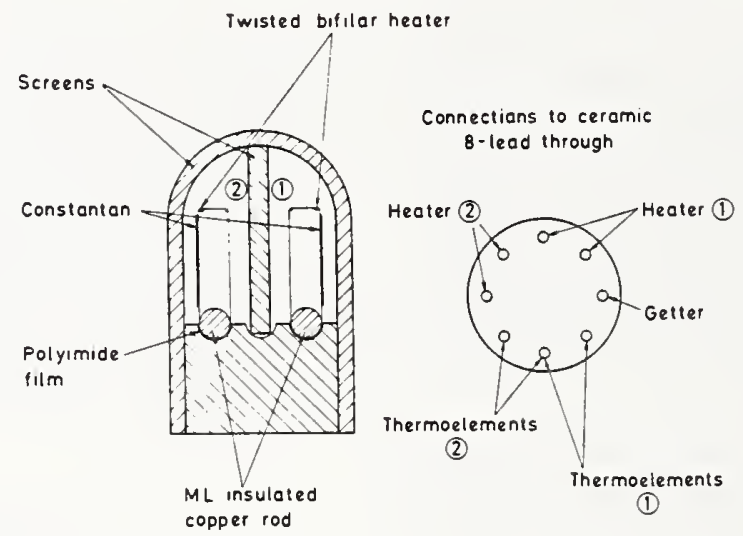

(b)

Fig. 2. (a) Rectangular element and connecting leads. (b) Rectangular elements mounted in housing.

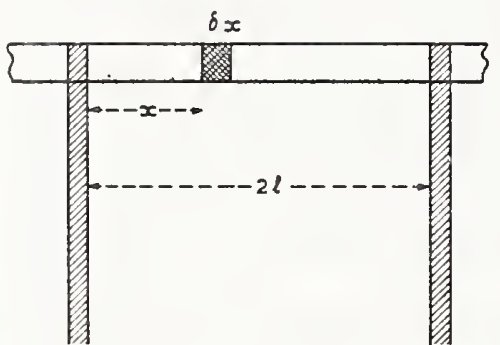

Fig. 3. Section of heater between two thermocouple wires.

Now $m^{2}$ is much greater than $n^{2}$ and as a consequence in the previous consideration a convenient, nevertheless justifiable, approximation was made by substituting $m$ for $a$ and it was then argued that the ac/dc transfer of the multijunction convertor was less than 1 part in $10^{6}$.

An alternative and more precise simplification is derived if each of the hyperbolic functions in the term $a\{(\cosh 2 a l-$ $\cosh 2 n l) / \sinh 2 a l\}$ is expanded in terms of its appropriate series. Subsequent adjustment, with the retention of all terms up to and including $l^{5}$, then reduces it to the form

$a\left\{\frac{\cosh 2 a l-\cosh 2 n l}{\sinh 2 a l}\right\}=l m^{2}\left\{1-\frac{1}{3} l^{2} m^{2}\right.$

$$
\left.+\frac{2}{9} l^{4} m^{4}+\frac{4}{45} m^{2} n^{2} l^{4}\right\} \text {. }
$$

This expression includes one term only, namely $\frac{4}{45} m^{2} n^{2} l^{4}$, that is dependent on the Thomson coefficient and thus the additional heat conducted through the elements when the heater is carrying direct current is

$$
2 \lambda A\left\{\frac{i^{2} r_{1}}{P}-\theta_{J}\right\}\left\{l m^{2}\right\}\left\{\frac{4}{45} m^{2} n^{2} l^{4}\right\} .
$$

The temperature of the hot junctions will then be increased over that when the heater is supplied with alternating current in the ratio

$$
\frac{\frac{4}{45} m^{2} n^{2} l^{4}}{1-\frac{1}{3} l^{2} m^{2}+\frac{2}{9} l^{4} m^{4}+\frac{4}{45} m^{2} n^{2} l^{4}} \simeq \frac{4}{45} m^{2} n^{2} l^{4}
$$

and thus the percent ac/dc transfer difference $=9 m^{2} n^{2} l^{4}$. This is transposed into a more convenient form for evaluation when the appropriate substitutions are made for $m$ and $n$. The percent ac/dc transfer difference is then

$$
\frac{1}{7} \frac{P_{2 L} V_{2 L} R_{L} \alpha_{T}^{2}}{\lambda^{3} \rho^{3} N^{4}}
$$

For a $0.005-\mathrm{mm}$ heater (i.e., $50-\mathrm{mA}$ heater in NPL design) typical values for substitution in the preceding expression are as follows:

$$
\begin{aligned}
& P_{2 L}=2 \times 10^{-5} \mathrm{~W} /{ }^{\circ} \mathrm{C} \quad V_{2 L}=0.5 \mathrm{~V} \text { ) only half heater } \\
& \left.\alpha_{T}=2 \times 10^{-6} \mathrm{~V} /{ }^{\circ} \mathrm{C} \quad R=10 \Omega\right\}_{\text {considered }} \\
& N=220 \\
& \rho \lambda=1 \times 10^{-5} \mathrm{~W} /{ }^{\circ} \mathrm{C} \text { (constant most }
\end{aligned}
$$

The calculated percent ac/dc transfer difference is then 
$\simeq 10^{-11}$, which is an extremely small value and defines the original claim of an accuracy of better than 1 in $10^{-6}$ with greater certainty. Moreover this is an upper limit as there is an additional compensation, not allowed for in the calculation, due to the bifilar arrangement of the heater. The two wires are in close thermal contact with each other and each will therefore have the same temperature gradients along its length and yet the current flows will be in opposite senses. The net Thomson heating across any plane will thus tend to be zero, so that when allowance is made for this further factor the ac/dc transfer difference previously calculated is made even smaller.

\section{B. Low Frequency Response}

The temperature of a heater supplied with an alternating current of frequency lower than the midfrequencies of the previous section will vary continuously throughout each cycle and the corresponding thermocouple output voltage will therefore include a double frequency component. Nevertheless provided the power input and thermocouple output are directly proportional to each other the true rms value of the alternating current may be determined by equating the mean of the varying output voltage to the constant voltage developed by a direct current. Nonlinearities in the relation will, however, result in errors in the rms value and the greater the nonlinearities and temperature excursions the larger will be the errors.

The elements or functional part of the thermal convertor is mounted in an evacuated container so that the heater does not lose any heat by convection and moreover, because its tem. perature is only a few degrees above ambient, its loss by radiation is small and proportional to its temperature rise. Almost all the heat is therefore conducted through the thermal elements to the heat sink, which is the structural part of the convertor. The temperature rise of the heater for a given power input is therefore determined by the length and crosssectional areas of the copper and constantan, and the thermal conductivities of both copper and constantan. Published fig. ures indicate that the conductivity of constantan increases and that of copper decreases with increasing temperature. However, when due allowance is made for these variations and for the slight loss of heat by radiation, the temperature rise of the heater over its normal restricted working range can be shown to be directly proportional to the power input.

The thermal EMF of a copper-constantan thermocouple may be expressed in the form

$$
E=\alpha t+\beta t^{2} \quad(\alpha \text { and } \beta \text { both positive })
$$

and thus if there were no further compensating factor the performance of a convertor would tend to be oversquare, that is the ratio of voltage output/power input would increase with increasing power input. The thermal EMF of a thermocouple system formed by the electrochemical deposition of copper on discrete lengths of constantan is however also dependent on the resistance of the copper path and may be shown to be given by the expression

$$
\text { effective EMF }=\frac{R_{2}}{R_{2}+R_{1}} \quad \begin{aligned}
& (\text { copper-constantan } \\
& \text { thermal EMF }),
\end{aligned}
$$

where $R_{1}$ is the resistance of each length of copper and $R_{2}$ the resistance of the constantan lying underneath it. The cross-sectional area of the electrochemically deposited copper is therefore made such that the decrease of the first term with rising temperature, due to the large temperature coefficient of copper, will just compensate for the increase in the second and the output voltage is then directly proportional to power input. Under this condition it is claimed that the low frequency response of the convertor will be equally as accurate as at midfrequencies. Any slight departure from the direct proportionality between output voltage and power input will lead to the greatest errors when the temperature excursions of the heater are largest and will only have a slight effect on the ac/dc transfer difference when the double frequency ripple is small.

The responses previously described are dependent on conditions that must be realized while the element is being constructed. Thus precautions are taken to ensure that the copper is of a specified and uniform thickness, that the pressure inside the housing remains at constant low value, and that the loss of heat by radiation is well defined. These various design features will be described in greater detail in a further paper.

\section{High-Frequency Response}

In the determinations of the ac/dc transfer differences of the multijunction convertor at low and midfrequencies both the current paths and also the distribution of the current across all sections of the heater have been assumed to be the same for both direct and alternating currents. At much higher frequencies these assumptions are no longer valid for the reactive components of the heater and their associated loss resistances influence the pattern of flow of the alternating current to such an extent that it will deviate considerably from that of the direct current; moreover because of "skin" and "proximity" effects the alternating current is confined to surface regions of the heater and not distributed uniformly across it. The present theoretical investigation examines, from first principles, how the reactive components of the heater influence the power dissipation within it when it is carrying a highfrequency alternating current and also assesses, in terms of the standard formulas of Maxwell and Butterworth, its change of resistance with frequency due to the concentration of the current in its surface regions. The resultant of both effects determines the ac/dc transfer difference at high frequencies.

1) Theoretical Analysis of the Effects of the Reactive Components of the Bifilar Heater on the Power Dissipation Within It: Fig. 4 shows a bifilar heater having a distributed impedance $z_{1} /$ unit length due to its resistance and inductance, distributed admittance $y_{2} /$ unit length between the heater and the inner housing, and distributed admittance $y_{3} /$ unit length between one half heater wire and the other. The admittances in each case are due to the capacitances between the respective parts of the convertor and their corresponding dielectric losses. 


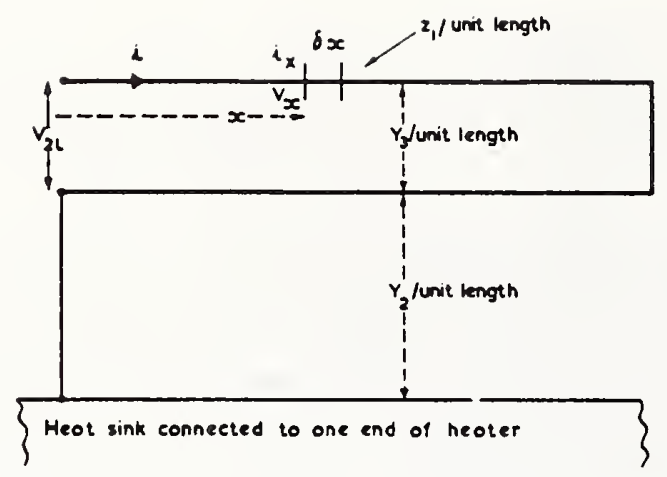

Fig. 4. Bifilar heater showing impedance $z_{1} /$ unit length and distributed admittances $y_{2}$ and $y_{3} /$ unit length.

Thus

$$
\begin{array}{ll}
z_{1}=r_{1}+j w l_{1} & \begin{array}{l}
\text { (effective impedance/unit length of heater) } \\
y_{2}=r_{2}+j w c_{2}
\end{array} \\
\begin{array}{ll}
\text { (effective admittance/unit length of } \\
\text { heater to case) }
\end{array} \\
y_{3}=r_{3}+j w c_{3} & \begin{array}{l}
\text { (effective admittance/unit length heater } \\
\text { to heater). }
\end{array}
\end{array}
$$

The impedance/unit length of the heater is determined solely by the nature, dimensions, and separation of the heater conductors, whereas the effective admittances between heater and case, and between one half heater and the other are dependent on the properties and quantity of the materials lying external to the conductors. Thus the enamel on the wire, the adhesive bonding the elements to the heater and also lying between the two heater wires, and the insulation between the thermal elements and base are all of importance in high-frequency considerations.

The rate of change of current and voltage with distance along heater are as follows:

$$
\frac{d i}{d x}=y_{2} V_{x}+y_{3}\left(V_{x}-V_{2 L-x}\right)
$$

and

$$
\frac{d V}{d x}=z_{1} i_{x}
$$

When these equations are expressed with respect to the center of the heater they appear as

$$
\begin{aligned}
& \frac{d i}{d y}=y_{2} V_{y}+y_{3}\left(V_{y}-V_{-y}\right) \\
& \frac{d V}{d y}=z_{1} i_{y}
\end{aligned}
$$

and the solution to these equations gives

$$
\begin{aligned}
& V_{y}=\frac{V_{2 L}}{2}\left\{\frac{\cosh b y}{\cosh b L}+\frac{\sinh c y}{\sinh c L}\right\} \\
& i_{y}=\frac{V_{2 L}}{2 z_{1}}\left\{\frac{b \sinh b y}{\cosh b L}+\frac{c \cosh c y}{\sinh c L}\right\}
\end{aligned}
$$

and

$$
\begin{aligned}
b^{2} & =z_{1} y_{2}=\left(r_{1}+j \omega l_{1}\right)\left(r_{2}+j \omega c_{2}\right) \\
& =r_{1} r_{2}-\omega^{2} l_{1} y_{2}+j \omega\left(r_{1} c_{2}+r_{2} l_{1}\right) \\
& =p_{1}+q_{1} \\
c^{2} & =z_{1}\left(y_{2}+y_{3}\right)=\left(r+j \omega l_{1}\right)\left\{r_{2}+r_{3}+j \omega\left(c_{2}+c_{3}\right)\right\} \\
& =r_{1}\left(r_{2}+r_{3}\right)-\omega^{2} l_{1}\left(c_{2}+c_{3}\right)+j \omega\left\{r_{1}\left(c_{2}+c_{3}\right)+r_{2} l_{1}\right\} \\
& =p_{2}+j q_{2} .
\end{aligned}
$$

The power dissipation within heater

$$
=\int_{-L}^{+L}\left|i_{y}\right|^{2} d y=F(b c L) .
$$

The integration is derived by expanding $i_{y}$ in powers of $b$ and $c$ and then, neglecting terms of the order $b^{5}$ and $c^{5}$ it is shown that

$$
\begin{aligned}
F(b c L) \fallingdotseq 2 L \rho\left|i_{0}^{2}\right|\left\{1-2 L^{2}\left(p_{1}+p_{2} / 3\right)+2 L^{4} / 3\right. \\
\left.\cdot\left[3\left(2 p_{1}^{2}+p_{1} p_{2}+p_{2}^{2} / 5\right)-\left(2 q_{1}^{2}+q_{1} q_{2}+q_{2}^{2} / 5\right)\right]\right\}
\end{aligned}
$$

and if we write

$$
\begin{array}{ll}
P_{1}=L^{2} p_{1} & P_{2}=L^{2} p_{2} \\
Q_{1}=L^{2} q_{1} & Q_{2}=L^{2} q_{2}
\end{array}
$$

then

$$
\begin{aligned}
F(b c L) \fallingdotseq 2 L \rho\left|i_{0}^{2}\right|\left\{1-\frac{2}{3}\left(3 P_{1}+P_{2}-\right.\right. & {\left[3\left(2 P_{1}^{2}+P_{1} P_{2}+P_{2}^{2} / 5\right)\right.} \\
- & \left.\left.\left.\left(2 Q_{1}^{2}+Q_{1} Q_{2}+Q_{2}^{2} / 5\right)\right]\right)\right\}
\end{aligned}
$$

and thus the ac/dc transfer difference expressed as a fraction of the " $\mathrm{dc}$ " power dissipation

$$
\begin{aligned}
\fallingdotseq \frac{2}{3}\left\{3 P_{1}+P_{2}-\left[3 \left(2 P_{1}^{2}+P_{1} P_{2}+\right.\right.\right. & \left.P_{2}^{2} / 5\right) \\
& \left.\left.-\left(2 Q_{1}^{2}+Q_{1} Q_{2}+Q_{2}^{2} / 5\right)\right]\right\} .
\end{aligned}
$$

When $p_{1}$ and $p_{2}$ are small the ac/dc transfer difference approximates to

$$
2 L^{2}\left(p_{1}+\frac{1}{3} p_{2}\right)=2 L^{2}\left(\frac{4}{3} r_{1} r_{2}+r_{1} r_{3} / 3+\frac{4}{3} \omega^{2} l_{1} c_{2}+\frac{1}{3} \omega^{2} l_{1} c_{3}\right)
$$

and this formula is adequate for considerations of the NPL convertor up to $100 \mathrm{kHz}$. However both $p_{1}$ and $p_{2}$ are frequency dependent and thus when the performance of the convertor is required at higher frequency levels the more exact formula is necessary; the values of both terms are also determined by the heater resistance. In the case of a typical $10 \mathrm{~mA} \mathrm{NPL}$ thermal convertor $p_{1}$ is approximately $5 \times 10^{-3}$ at $100 \mathrm{kHz}, p_{2}$ is $1 \times 10^{-2}$, and the ac/dc difference is then $1.5 \times 10^{-6}$ as shown in Table I.

2) Determination of Reactive Components of Heater:

a) Inductance/Unit Length: The inductance of the bifilar heater is small, difficult to measure, and is therefore calculated from the standard formula namely: inductance/unit length = $\left(\mu_{0} / 4\right)\left(2 \log _{e} D / a_{0}+\frac{1}{2}\right) H / m-D$ is the distance between 
TABLE I

\begin{tabular}{|c|c|c|c|c|c|c|c|c|c|}
\hline \multirow{3}{*}{$\begin{array}{l}\text { Frequency } \\
\quad(\mathrm{kHz})\end{array}$} & \multicolumn{3}{|c|}{ Heater-Base (Parallel) } & \multicolumn{3}{|c|}{ Half-Heater-Half-Heater (Parallel) } & \multirow{2}{*}{\multicolumn{3}{|c|}{ Calculated AC/DC Transfer Difference }} \\
\hline & & & $R_{2}(P)$ & & & $R_{3}(P)$ & & & \\
\hline & $C_{2(P)} \times 10^{-12}$ & $\tan \delta_{1}$ & $(\Omega)$ & $C_{3(P)} \times 10^{-12}$ & $\tan \delta_{2}$ & $(\Omega)$ & $50 \mathrm{~mA}$ & $35 \mathrm{~mA}$ & $10 \mathrm{~mA}$ \\
\hline 1 & 1.2 & 0.008 & $1.6 \times 10^{10}$ & 3.3 & 0.005 & $1 \times 10^{10}$ & & & \\
\hline 10 & 1.2 & 0.003 & $5 \times 10^{9}$ & 3.3 & 0.006 & $1 \times 10^{9}$ & $7 \times 10^{-9}$ & $1.5 \times 10^{-8}$ & $1.5 \times 10^{-7}$ \\
\hline 20 & 1.2 & 0.002 & $3 \times 10^{9}$ & 3.3 & 0.007 & $5 \times 10^{8}$ & $1.5 \times 10^{-8}$ & $4 \times 10^{-8}$ & $3 \times 10^{-7}$ \\
\hline 50 & 1.2 & 0.002 & $1 \times 10^{9}$ & 3.3 & 0.008 & $2 \times 10^{8}$ & $3 \times 10^{-8}$ & $8 \times 10^{-8}$ & $7 \times 10^{-7}$ \\
\hline 100 & 1.2 & 0.002 & $5 \times 10^{8}$ & 3.3 & 0.008 & $1 \times 10^{8}$ & $7 \times 10^{-8}$ & $1.5 \times 10^{-7}$ & $1.5 \times 10^{-6}$ \\
\hline
\end{tabular}

centers and $a_{0}$ is the radius of the conductors. A value of $D$ equal to $2 a_{0}$ increased by 5 percent to allow for the enamel coatings was assumed in all calculations and thus $z=r_{1}+$ $j \omega\left(2 \times 10^{-7}\right)$, where $r_{1}$ equals the resistance of unit length of heater.

b) Admittances of Heater to Case and of One Half-Heater to the Other: The admittances $y_{2}$ and $y_{3}$ were both calculated from measurements of the capacitance and loss angle made directly on elements of triangular section that were mounted in the normal manner in inner housings but without the added complication of the outer housing. The capacitance and $\tan \delta$ between the complete heater and the housing were first determined and then the looped end of the heater was cut and the capacitance and loss angel between the two halves of the heater measured independently. The measurements were repeated on each of three sizes of heater, at each of a number of frequencies up to $100 \mathrm{kHz}$, so that the variation of the ac/dc difference with frequency of each could be ascertained. The scatter in capacitance and $\tan \delta$ values between one heater and another was not large enough to affect the calculated ac/dc difference significantly so that only mean values are quoted in the following. All measurements gave parallel capacitance and resistance and these values were first transposed to give the required admittances; the results and conclusions are summarized in Table I and should also apply to the rectangular elements.

3) Calculated $A C / D C$ Transfer Difference Due to Reactive Components: The heater resistances are $50 \mathrm{~mA}-15 \Omega$, $35 \mathrm{~mA}-40 \Omega, 10 \mathrm{~mA}-360 \Omega$, and $l_{1}=2 \times 10^{-7} \mathrm{H} / \mathrm{m}$. The ac/dc difference is

$$
2 L^{2}\left\{\frac{4}{3} r_{1} r_{2}+\frac{1}{3} r_{1} r_{3}+\frac{4}{3} \omega^{2} l_{1} c_{2}+\frac{1}{3} \omega^{2} l_{1} c_{3}\right\}
$$

The preceding analysis, therefore, leads to the conclusion that the ac/dc differences due to the reactive components of the heater are small even at frequencies as high as $100 \mathrm{kHz}$. They are, nevertheless, of greater magnitude than any ac/dc differences caused by Thomson heating.

4) Determination of Change of Heater Resistance With Frequency: The change in resistance of a single heater wire with frequency, the skin effect, is determined from the standard Maxwell formula [3] namely

$$
\frac{R_{a c}-R_{d c}}{R_{d c}}=\frac{1}{12} B^{2}-\frac{1}{180} B^{4}
$$

where

$$
B=\frac{2 \pi^{2} a_{0}^{2} f \mu}{10^{7}}
$$

The proximity of the second current carrying wire, which will give rise to an asymmetric current distribution around the surface of the first wire, causes an additional increase in the effective resistance of the heater. This further increase is calculated from a development of Butterworth's equation by Arnold [4] in which the fractional eddy current losses in any conductor are expressed as a product of the skin effect and a factor $y^{2}$ that is dependent on the form of the conductor system. For a bifilar unit such as the convertor heater $y$ is shown to be 2 and thus the increase in resistance due to the skin effect must be doubled to allow for the proximity effect.

Insertion of the known constants of manganin in the preceding formula allowing for the proximity effect, shows that the resistance of a $0.05-\mathrm{mm}$ wire will be 6 parts in $10^{8}$ higher at $100 \mathrm{kHz}$ than with direct current, the corresponding changes for a $0.03-\mathrm{mm}$ diameter wire will be 6 parts in $10^{9}$, and for a $0.012-\mathrm{mm}$ connecting wire 2 parts in $10^{7}$. The latter, however, is only a small part of the total resistance and may be neglected.

5) Calculated Overall AC/DC Transfer Difference: The main source of ac/dc transfer difference at frequencies of the order of $100 \mathrm{kHz}$ is therefore that resulting from the reactive components, but even in the case of a $10-\mathrm{mA}$ convertor and the ac/dc transfer difference probably does not exceed more than 2 parts in $10^{6}$.

6) Other Sources of High Frequency AC/DC Transfer Difference That Could Affect Measurement of Voltage by Convertor: In the earlier type of convertor the leads through the matched glass seal were of a nickel-iron alloy having a relative permeability of about 10000 . The resistance of the leads was therefore very frequency dependent and even at $5 \mathrm{kHz}$ the change of resistance from the $\mathrm{dc}$ value was sufficient to cause appreciable errors in some voltage measurements. In the later design the corresponding leads are of copper and the calculated change of their resistance even at $100 \mathrm{kHz}$ is so small that it should not impair the overall accuracy of the convertor for any voltage measurement. The one serious limitation of the earlier form of the NPL convertor has thus been rectified.

Eddy currents induced in structural components might be another source of error for in order to define the temperature of the intermediate leads they are taken in close proximity to 
to the stout copper pillar on which the element is mounted. These leads are closely twinned and should not therefore have any extraneous magnetic field surrounding them to cause induced currents. Indeed observations indicate there is probably no ac/dc difference due to this effect; it is nevertheless an issue that could be affected by slight changes in constructional procedure.

Lead inductance is yet another possible cause of ac/dc transfer difference at high frequencies. Thus, while all leads to and from the welded joints are carefully disposed to reduce loops to the minimum possible, they cannot be eliminated completely and at some frequency level their inductance will begin to limit the accuracy of the convertor for voltage measurement.

\section{CONCLUSION}

The analysis set out in the paper has shown that the ac/dc transfer difference of the NPL multijunction thermal convertor is almost completely free from the effects of Thomson heating and does not exceed $2 \mathrm{ppm}$ at $100 \mathrm{kHz}$ due to the change with frequency of the effective heater resistance.

\section{ACKNOWLEDGMENT}

The author wishes to acknowledge the help of F. L Hermach of NBS who suggested the revised approach of Section IV-A, of G. F. Miller and B. T. Hinde of NPL for their solution to the equations developed in Section IV-C-1 and of G.H. Rayner of NPL for his capacitance measurements given in Section IV-C-3.

\section{REFERENCES}

[1] F. L. Hermach, "Thermal convertors as ac/dc transfer standards for current and voltage measurements at audio frequencies," J. Res. Nat. Bur. Stand., vol. 48, p. 121, 1952.

[2] F. J. Wilkins, T. A. Deacon, and R. S. Becker, "Multijunction thermal convertor-An accurate dc/ac transfer instrument," Proc. Inst. Elec. Eng. (London), vol. 112, p. 4, 1965.

[3] Golding and Widdis, Electrical Measurements and Measuring Instruments. London: Pitman and Sons Ltd., pp. 201-3.

[4] A. H. M. Arnold, "Alternating current resistance standards," Proc. Inst. Elec. Eng. (London), vol. 100, pt. 2, no. 75, 1953. 


\title{
Thermal Converters for Audio-Frequency Voltage Measurements of High Accuracy
}

\author{
F. L. HERMACH, FEllow, IEEE, AND E. S. WILlTAMS
}

\begin{abstract}
The ac-dc differences of a reference group of thermoelements have been evaluated at audio frequencies to a few parts per million (ppm) at currents from 5 to $20 \mathrm{~mA}$. A technique for comparing the ac-dc differences of two thermoelements with an uncertainty of about $2 \mathrm{ppm}$ has been developed. Two $5 \mathrm{~mA}$ thermoelements are used with a plug-in set of resistors of computable reactances to form thermal voltage converters for voltage measurements. With this same technique adjacent ranges of these converters can be compared to step up from 0.5 to $500 \mathrm{~V}$ to better than $10 \mathrm{ppm}$.
\end{abstract}

\section{INTRODUCTION}

II N PRINCIPLE, the current balances which have been developed to determine the ampere in absolute measure with direct current could also be used with alternating current. However, the most stable electrical standards are still the dc standard cell, and $1 \mathrm{ohm}$ resistors, which are not usually designed for ac use. Thus, ac measurements of current and voltage have, in practice, been based on rms ac-dc transfer standards or comparators. For many decades these were electrodynamic or electrostatic instruments, designed to have the same torque constant with either alternating or direct current (or voltage) applied, making it easier to obtain a wider frequency range than with instruments like the current balance which must have a computable torque constant. More recently, electrothermic instruments such as thermocouple transfer standards and bolometer or thermistor bridges have been used. All of these instruments have been highly developed in the national laboratories of several countries to make ac measurements at audio frequencies to about $100 \mathrm{ppm}$ in combination with dc standards. The combination possesses a number of advantages over ac potentiometers (which must also include a transfer instrument), and over electronic and other squaring circuits (which must have exact squarelaw characteristics in two quadrants).

Recent advances in operational amplifiers and inductive voltage dividers have made possible wide-range ac power sources with linearity and day-to-day stability better than $100 \mathrm{ppm}$. This implies a need for better accuracy in basic ac-dc transfer standards.

At this accuracy, conventional forms of electrodynamic transfer instruments are limited to power frequencies by the effects of inductive and capacitive re-

Manuscript received June 23, 1966. This paper was presented at the 1966 Conference on Precision Electromagnetic Measurements, Boulder, Colo. This work was supported in part by the Army Metrology and Calibration Center, Frankford Arsenal, and the Metrology Engineering Center, Bureau of Naval Weapons, Pomona, Calif.

The authors are with the Electrical Instruments Section, Electricity Division, National Bureau of Standards, Gaithersburg, Md. actances in their coils, and electrostatic instruments have a serious minimum-voltage limitation because of their low torque-weight ratio. Both would require new means of sensing deflections to obtain precisions of 10 ppm or better. This could be done by measuring the reactance, which is a function of the deflection of the instrument. However, a major difficulty would remain - that of vibrations from external forces or from the cyclic variations of the ac driving torque. Experience has shown that it is of ten difficult to control spurious torques caused by minor mechanical resonances. It is possible that floating the moving system in a liquid of the same density would minimize these difficulties. However, it has seemed to the authors that improved thermal converters (thermocouple instruments) offer better promise of obtaining an ac-dc transfer accuracy of $10 \mathrm{ppm}$ or better, and considerable effort has been concentrated on them over the past five years. This paper may be considered as a progress report describing the present group of reference thermoelements and coaxial thermal voltage converters, and the techniques and equipment which have been developed to compare them to a few ppm.

\section{Thermal Converters}

A thermoelement (TE), defined as the simplest type of thermal converter [1], consists of a heater and thermocouple. In its usual form, the heater is a short, straight wire suspended between two supporting leadin wires in an evacuated glass bulb. The hot junction of a thermocouple is fastened to the midpoint of the heater, and is electrically insulated from it with a small bead. The thermocouple EMF (about 5 to $10 \mathrm{mV}$ for a conventional $\mathrm{TE}$ at rated current $)^{1}$ is then a measure of the heater current. For voltage measurements, a resistor is connected in series with the heater to form a thermal vol tage converter [1].

At the National Bureau of Standards, fourteen TEs form the present reference group for ac-dc difference. Twelve of them (made by four different manufacturers) are of conventional design but with heaters of Evanohm or Karma (modified nickel-chromium alloys), which have low thermoelectric effects. ${ }^{2}$ They were chosen from

1 The international system (SI) of units with its symbols is used in this paper. Because of their frequent occurrence, the word thermoelement is abbreviated as TE and thermal voltage converter as TV C in this paper.

${ }^{2}$ Evanohm and Karma are registered trademarks of the Wilbur B. Driver Company and the Driver-Harris Company, respectively. (See Acknowledgments.) 
larger groups on the basis of low dc reversal differences and high bead resistances, and are all mounted in one aluminum enclosure lined with foamed plastic for thermal insulation. Each is supported by its heater leads, which are soldered directly to binding posts mounted on the low-loss phenolic top plate of the enclosure, and its thermocouple output leads, which are soldered to two-pin connectors. The other two thermal current converters are of a radically different design having many thermocouples ( 40 or more) attached to a bifilar (doubled-back) heater [2]. They were obtained from their inventor, F. J. Wilkins of the National Physical Laboratory in England.

The fourteen TEs are identified in Table I, along with certain pertinent characteristics, as well as the acdc differences assigned to them at audio frequencies as a result of the tests to be described.

\section{TABLE I}

Reference Thermoelements

\begin{tabular}{|c|c|c|c|c|c|c|c|}
\hline \multirow{3}{*}{$\begin{array}{l}\text { Identi- } \\
\text { fication }\end{array}$} & \multicolumn{2}{|c|}{ Rated } & \multirow{3}{*}{$\begin{array}{c}\text { Dc } \\
\text { re- } \\
\text { versal† } \\
\text { ppm }\end{array}$} & \multirow{3}{*}{$\begin{array}{l}\text { Approx. } \\
\text { heater } \\
\text { length } \\
\mathrm{mm}\end{array}$} & \multirow{3}{*}{$\begin{array}{c}\text { Bead } \\
\text { resis- } \\
\text { tance } \neq \\
\text { G } \Omega\end{array}$} & \multirow{2}{*}{\multicolumn{2}{|c|}{$\begin{array}{l}\text { Ad-dc differ- } \\
\text { ence ( } p \text { pm) }\end{array}$}} \\
\hline & \multirow{2}{*}{$\begin{array}{c}\text { Cur- } \\
\text { rent } \\
\mathrm{mA}\end{array}$} & \multirow{2}{*}{$\underset{\mathrm{mV}}{\mathrm{EMF}}$} & & & & & \\
\hline & & & & & & $20 \mathrm{~Hz}$ & $2 \mathrm{kHz} \|$ \\
\hline NPL $14 *$ & 20 & 25 & $<10$ & - & $>1$ & 0 & 0 \\
\hline NPL $15^{*}$ & 20 & 25 & $<10$ & - & $>1$ & 0 & 0 \\
\hline $\mathrm{A} 20 \mathrm{E} 3$ & 20 & 5 & 4 & 5 & $>1$ & 0 & 0 \\
\hline A20E6 & 20 & 5 & 60 & 5 & $>1$ & 0 & -1 \\
\hline A10E 18 & 10 & 8 & 130 & 5 & 0.3 & +6 & +1 \\
\hline E19 & 10 & 8 & 16 & 5 & 0 . & 0 & -1 \\
\hline W10E1 & 10 & 7 & 200 & 8 & 0 . & +1 & +3 \\
\hline $\mathrm{E} 2$ & 10 & 7 & $<10$ & 8 & 0.8 & -2 & 0 \\
\hline E45 & 10 & 8 & 160 & 4 & $>1$ & -8 & -2 \\
\hline $\mathrm{A} 5 \mathrm{E} 8$ & 5 & 7 & 10 & 5 & 0. & +1 & +1 \\
\hline & 5 & 6 & 40 & 5 & 0.6 & 0 & -1 \\
\hline & 5 & 4 & 20 & 5 & 0.5 & -3 & -2 \\
\hline F5K13 & 5 & 4 & 260 & 5 & 0.5 & -2 & 0 \\
\hline B5E77 & 5 & 8 & 110 & 6 & $>1$ & +6 & 0 \\
\hline
\end{tabular}

* These can be used at much higher currents.

$\dagger \Delta I / I$ for the same EMF.

$\ddagger$ At $2 \mathrm{kHz}$ and rated $I$.

|| Same value assigned at $20 \mathrm{kHz}$.

\section{Thermal Voltage Converters (TVCs)}

Each of a new set of TVCs developed at NBS for this work consists of one or more cylindrical metal-film resistors in a coaxial metal cylinder. The resistors can be connected in series with one of two $5 \mathrm{~mA}$ TEs mounted in separate cylinders so that the output EMF is then a measure of the input voltage. Like an earlier set developed at NBS [3], the effect of the reactance on the frequency response can be computed at least roughly from the simple geometry. Adjacent ranges of these TVCs may be compared with greater certainty than those of the older design, which had integrally mounted TEs.

As shown in Fig. 1, a single two-watt resistor is used for each range below $200 \mathrm{~V}$. The higher-voltage resistor units have two or more resistors in larger cylinders, with two adjustable inner shields to control capacitance currents, as shown in Fig. 1, and have ports and baffles to permit the controlled flow of cooling air from the laboratory compressed-air supply.
Pertinent data on the TVCs, and the ac-dc differences assigned to each range are given in Table II. The TVCs are shown in Fig. 2.

\section{EMF COMPARATOR}

The ac-dc differences of a transfer instrument may be defined as

$$
\delta=\left(X_{a}-X_{d}\right) / X_{d}
$$

where $X_{a}$ and $X_{d}$ are the magnitudes of the ac and dc quantities that are required to give the same response (such as deflection or output EMF) of the instrument. (Normally, the average for the two directions of the dc quantity gives the best measure of the dc reference.) This is a useful definition, for the ac quantity for a given response is then simply $X_{a}=X_{d}(1+\delta)$. As is well known, the instrument may be used as an ac-dc transfer standard simply by observing the response with the ac quantity applied, then measuring (with externai standards) the average for the two dc quantities required to obtain the same response, thus avoiding many of the limitations of an ordinary instrument.

At the National Bureau of Standards, TEs are evaluated in pairs with the EMF comparator shown in Fig. 3, to determine the differences in their ac-dc transfer performance, i.e., $\delta_{1}-\delta_{2}$ as defined previously. TVCs are connected in parallel and evaluated in the same way. With the desired current or voltage applied, $R_{\mathbf{1}}$ and $R_{\mathbf{2}}$ are adjusted in a preliminary balance to obtain a near null on the detector $D_{1}$. Then, in regular succession, alternating-, direct-, reversed direct-, and alternatingcurrents are applied to the TEs at nearly equal time intervals and without changing $R_{1}$ or $R_{2}$. In each case, the current is adjusted to obtain the same output EMF, $E_{1}$ of $T E_{1}$, as indicated by an auxiliary Lindeck potentiometer ( $P$ with its detector $D_{2}$ ), and the resulting deflections of $D_{1}$ are observed. Then, as shown in Appendix $\mathrm{I}$, the relative ac-dc difference is

$$
\delta_{1}-\delta_{2}=s\left(D_{a}-D_{d}\right)
$$

where $D_{a}$ and $D_{d}$ are the averages of $D_{1}$ with alternating and direct currents applied, respectively. The sensitivity factor $s$ can be determined in one of several ways, as indicated in the Appendix, such as by changing the current in the heater of $\mathrm{TE}_{2}$ by a small known amount and observing the resulting deflection of $D_{1}$.

The sequence of readings, nearly equally spaced in time, greatly reduces errors from drifts, temperature changes, etc., in either TE and from either constant or slowly changing thermal EMFs in the comparator. The comparator accommodates any reasonable range of thermocouple EMFs, which need not be equal, and the small currents (up to $20 \mu \mathrm{A}$ ) in the thermocouple circuit do not affect the measured values.

With this circuit, small fluctuations in the supply (heater) current do not appreciably change the deflection if the two TEs have reasonably equal proportional changes in $\operatorname{EMF}(\Delta E / E)$, with a given change in heater current and reasonably equal time constants. Although 

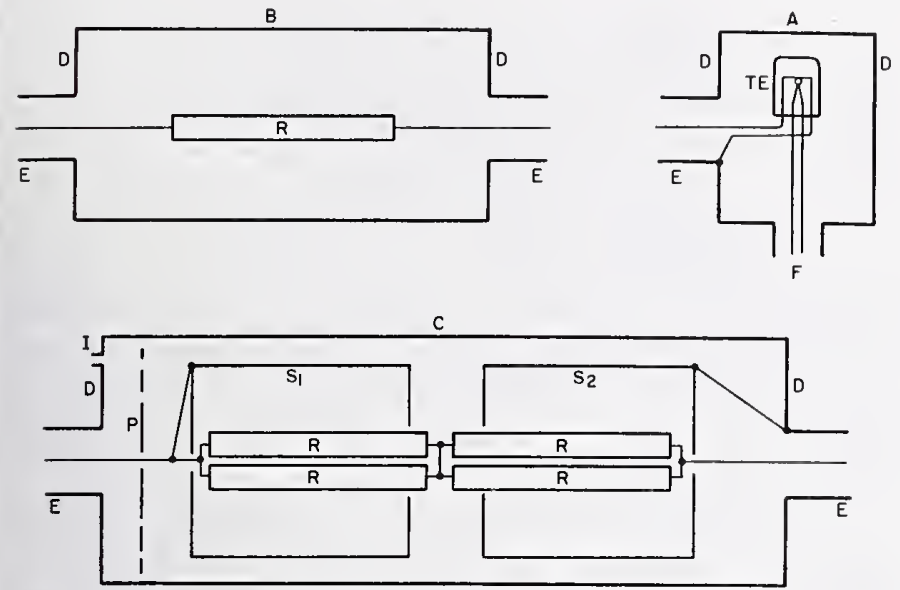

Fig. 1. Essentials of thermal voltage converters (construction details not shown).

A Thermoelement unit, with $5 \mathrm{~mA} 100 \Omega \mathrm{TE}$, for $0.5 \mathrm{~V}$, in brass cylinder, $4 \mathrm{~cm}$ long and $5.1 \mathrm{~cm}$ diameter, with brass end plate D.

B Low-voltage resistor unit ( 1 to $100 \mathrm{~V}$ ) with one 2-watt metal film resistor $\mathrm{R}$ in brass cylinder $11 \mathrm{~cm}$ long and $5.1 \mathrm{~cm}$ diameter.

C High-voltage resistor unit (200-500 V) with multiple 2-watt metal-film resistors, in brass cylinder $18 \mathrm{~cm}$ long and $6.4 \mathrm{~cm}$ diameter with brass end plate D. Air inlet at I, outlet holes at other end (not shown).

$\mathrm{S}_{1}, \mathrm{~S}_{2}$ Inner brass shields $5.5 \mathrm{~cm}$ long, $5.1 \mathrm{~cm}$ diameter, connected as shown, with no end plates. $S_{2}$ can be moved parallel to axis and locked in place.

$\mathrm{P}$ Non-conducting air-baffle plate with holes.

E Coaxial connector GR874.

F Two-pin output connector AN10SL.

it would be difficult to calculate the resultant "stabilization factor" of the circuit, in practice, fluctuations are much less than those observed when the output EMF of each $\mathrm{TE}$ is balanced against a constant voltage source, as in earlier comparators [4]. Thus, with this circuit and technique, TEs may be intercompared with an imprecision which is much less than the fluctuations of the supplies used.

$R_{1}$ consists of ten $100 \Omega$ resistors mounted on an enclosed-contact silver-alloy switch whose thermal EMFs are much less than $0.1 \mu \mathrm{V}$ when the wiper is stationary. $R_{2}$ is a $100 \Omega$, 10-turn helical resistor of manganin, with a manganin wiper to minimize thermal EMFs. Detector $D_{1}$ consists of a commercially available primary galvanometer, incorporating photocells in an adjustable negative-feedback circuit, which serves as a current amplifier to a secondary galvanometer. The scale factor of the combination is about $10 \mathrm{pA} / \mathrm{mm}$, and the input resistance (which is dependent on the negative feedback) ranges from 200 to $3000 \Omega$. The primary galvanometer is liquid filled to reduce disturbances from mechanical shocks and vibrations and, with its period of two seconds, is an excellent mechanical lowpass filter. A similar pair of galvanometers is used for $D_{2}$. Both secondary light beams appear on one scale so that the observer may quickly read the deflection of $D_{1}$ if it is stationary and if $D_{2}$ is in the desired range. (Residual
TABLE II

NBS "Model F" Thermal Voltage Converters

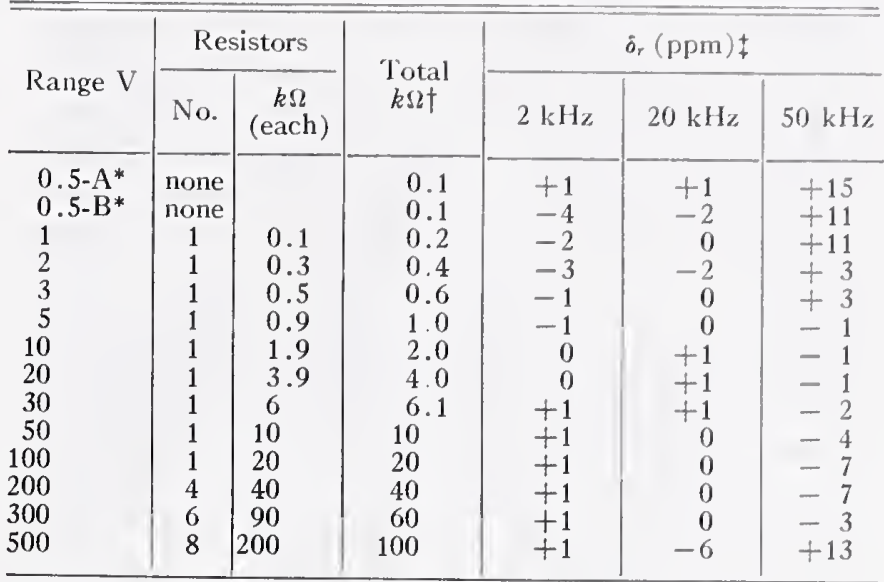

* TEs A and B as TVCs, without series resistors.

$\dagger$ Including 0.5 V TE. Resistors connected in series-parallel for higher ranges.

$\ddagger \delta_{\mathbf{r}}$ is the impedance error only (see text) computed from Table III with either TE of pair \#1. A similar table with the \#2 pair differs from it by not more than $1 \mathrm{ppm}$ at 2 and $20 \mathrm{kHz}$ except at $0.5 \mathrm{~V}$ range.

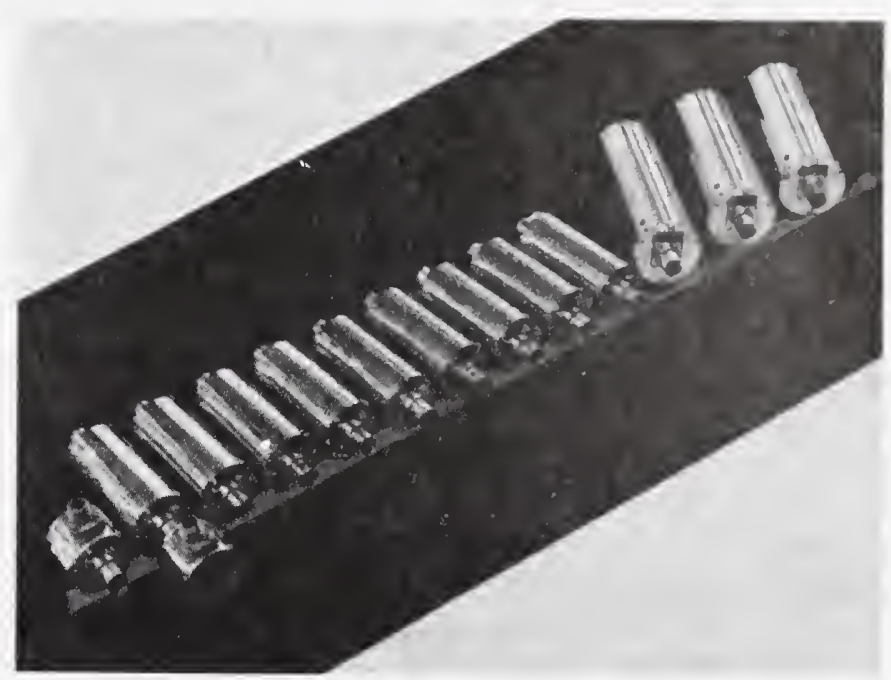

Fig. 2. Thermal voltage converters.

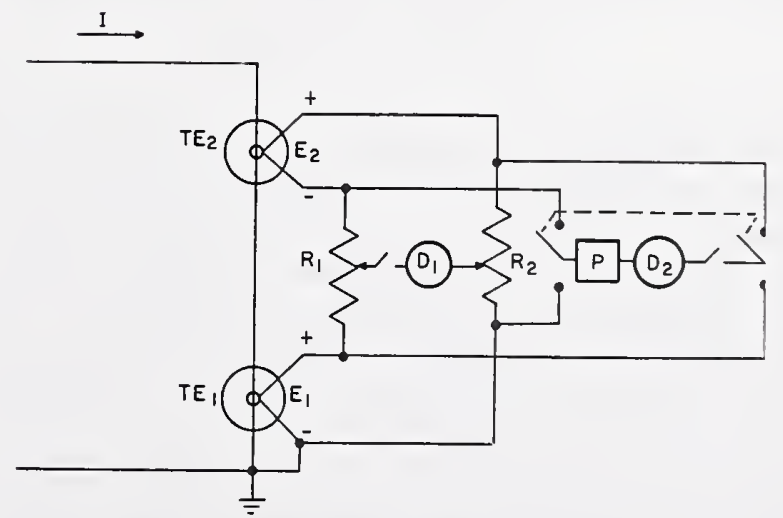

Fig. 3. Essentials of EMF comparator. 
deflections of $D_{2}$ are less critical if the TEs are well matched.)

All of the components of the comparator except the detectors are mounted in a metal box behind a metal panel to provide electrostatic shielding, and the box is lined with foamed plastic to reduce temperature fluctuations. The metal cases of the primary galvanometers are connected to this box by the shields of two-conductor cables.

With $R_{1}$ and $R_{2}$ at their midpoints and each of the thermocouple inputs to the EMF comparator shortcircuited, peak-to-peak fluctuations in the secondary galvanometer of $D_{1}$ in periods of 10 seconds amount to 1 $\mathrm{mm}$ at the gain setting normally used. With $7 \mathrm{mV}$ at each thermocouple input, this corresponds to a value of $\delta_{1}-\delta_{2}$ of $2 \mathrm{ppm}$. This is only about four times the calculated Johnson-noise value for this circuit.

A comparison of two TEs (or TVCs) usually consists of four or more determinations of $\delta_{1}-\delta_{2}$ by the method described. Three times the standard error (standard deviation of the average) computed from a number of typical series of such measurements is about $2 \mathrm{ppm}$. This is a reasonable measure of the uncertainty in the comparator. Comparisons of various pairs taken from three TEs, with the TEs interchanged in the comparator and with the EMF of first one TE and then the other held constant in each sequence, have disclosed no systematic error as large as $1 \mathrm{ppm}^{3}$ These measurements were made with commercially available $\mathrm{dc}$ and ac voltage sources, having peak-to-peak instabilities, observed with a TVC in 10-second intervals, of about 10 and $20 \mathrm{ppm}$, respectively, at $2 \mathrm{kHz}$.

A TVC can be used for ac measurements with appropriate dc standards by balancing its output EMF against an adjustable stable voltage source such as the potentiometer $P$. To reduce errors from drifts, a similar sequence of ac and dc readings can be used, with the setting of $P$ fixed, and the direct voltage $V_{d}$ preset and accurately known. Then

$$
V_{a}=V_{d}\left[1+\delta+s\left(D_{a}-D_{d}\right)\right]
$$

where $s$, the sensitivity factor, is determined as before.

\section{Comparisons of TEs}

The fourteen reference TEs were compared, using sinusoidal currents at frequencies of $20 \mathrm{~Hz}$, and 2 and $20 \mathrm{kHz}$. All of these measurements (as well as the comparisons of TVCs) were made in a shielded room to reduce electromagnetic interference, which is particularly troublesome with TEs because of their high sensitivity and very wide frequency range. The step down from one current to the next was made by connecting

\footnotetext{
${ }^{3}$ If the net dc reversal difference of the two TEs is more than 300 $\mathrm{ppm}$, an error is introduced by nonlinearities in $D_{1}$. This can be minimized by taking determinations with first one and then the other TE EMF held constant.
}

the heaters of two low-current TEs, $a$ and $b$, in parallel and their thermocouples in series aiding, and considering the combination as a single $\mathrm{TE}$ connected in the $\mathrm{TE}_{1}$ position of Fig. $3 .^{4}$ As shown in Appendix II, if the TEs are reasonably well matched, the ac-dc difference of the parallel combination is simply $\delta_{p}=\left(\delta_{a}+\delta_{b}\right) / 2$ to better than $1 \mathrm{ppm}$. From the test, $\delta_{p}-\delta_{2}=K_{1}$ where $\delta_{2}$ is the ac-dc difference of the high range TE in position 2 , and $K_{1}=s\left(D_{a}-D_{d}\right)$. The two low-current TEs are then connected in series and compared to determine $K_{2}=\delta_{a}-\delta_{b}$. Thus, $\delta_{a}=K_{1}+\delta_{2}+K_{2} / 2$ and $\delta_{b}=K_{1}+\delta_{2}$ $-K_{2} / 2$.

The network of comparisons at $2 \mathrm{kHz}$ and the results are shown in Fig. 4. Here the circles identify individual TEs and the rectangles represent a pair in parallel. The arrows indicate comparisons, and the numbers on them are the values of the relative ac-dc differences in $\mathrm{ppm}$; i.e., the ac-dc difference of the $\mathrm{TE}$ at the point of the arrow is greater or less than that at the tail by the amount shown. These tests have extended over a period of three years during the development of this project; in many cases, each number is the average of two or more tests, each consisting of four or more sequences of ac-dc readings already described. ${ }^{5}$

As indicated by Wilkins, the ac-dc difference of the two NPL TEs should, theoretically, be less than $1 \mathrm{ppm}$. In tests at NBS at $20 \mathrm{~mA}$, the two agreed to better than $0.2 \mathrm{ppm}$ with a $3 \sigma$ imprecision of $0.3 \mathrm{ppm} .^{6}$ Thus, it is reasonable as well as convenient to start with an assignment of zero ppm to the ac-dc differences of each. Then, following principles given by Youden [5] in weighting the various paths in a network, it is possible to calculate the ac-dc differences of the other TEs. The resulting values are given to the nearest $\mathrm{ppm}$ in each circle and in Table I.

The average for all of the conventional TEs is only $-0.3 \mathrm{ppm}$. Thus, these simple TEs agree with the more complicated multijunction converters very closely, and essentially the same values would have been obtained for each TE on the alternative basis of assigning zero to the average of the entire group.

A complete but less redundant chain of measurements was carried out at $20 \mathrm{~Hz}$ to evaluate low-frequency errors of each TE. The precision was less at that frequency because of much larger instability in the ac source. The results of the values assigned on the basis of zero error for the NPL TEs are given in Table I.

Not enough TEs were intercompared at $20 \mathrm{kHz}$ to assign independent values to each, because ac-dc dif-

4 To minimize certain aberrations discussed in Section IX, resistors of at least five times the heater resistance were added to each of the heaters before paralleling them.

${ }_{5}^{5}$ Because measurements were made over an extended time in a developing program, the network is not symmetrical.

6 The EMF was higher and a higher detector sensitivity could be used than with other TEs since the two TEs were well matched, so that an extraordinarily high precision was attained. 


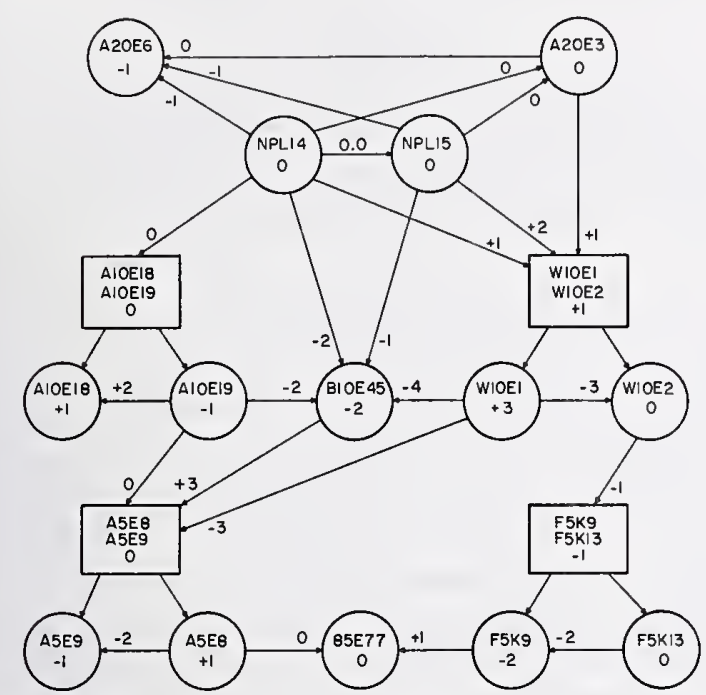

Fig. 4. Intercomparisons of NBS TEs, and assigned ac-dc differences in ppm at $2 \mathrm{kHz}$.

ferences from all known sources [3] should be the same as at $2 \mathrm{kHz}$ within $1 \mathrm{ppm}$. No measured values at the two frequencies differed by more than $2 \mathrm{ppm}$. Thus, the same values were assigned to each TE at the higher frequency.

\section{CoMparisons of TVCs}

The TVCs were designed so that adjacent ranges could be compared to step up and down the voltage scale. Their nearly identical $5 \mathrm{~mA}$ TEs were each first compared with one of the reference group of TEs as current converters, i.e., with the heaters connected in series. They were then paralleled through a " $T$ " connector and compared with each other as yoltage converters (basic range $0.5 \mathrm{~V}$ ). The lowest resistor (see Table 2) was then connected in series with one $\mathrm{TE}$, say $\mathrm{TE}_{a}$, to make a one-volt TVC. This was connected in parallel with the other, $\mathrm{TE}_{b}$, and compared at 0.5 volt. A twovolt TVC was then formed with the next resistor and $\mathrm{TE}_{b}$, and compared with the one-volt TVC at $1 \mathrm{~V}$. This process was continued until the highest range was reached. If the ac-dc difference of each combination is independent of the voltage level, the ac-dc difference of any range may be found in terms of one as a reference. This requirement is easily met if the TEs have no significant errors, for the reactance errors of each resistor unit should be independent of voltage. The validity of this assumption itself can easily be verified by making each comparison at two vol tage levels.

If we define $Z_{i}=V_{i} / I_{0}$ for a TVC, where $V_{i}$ is the magnitude of the applied voltage and $I_{0}$ is the magnitude of the current through the TE, then, from the definition for ac-dc difference, applied to a TVC,

$$
\delta_{v}=\frac{V_{i a}-V_{i d}}{V_{i d}}=\frac{I_{o a} Z_{\imath}-I_{o d} R_{t}}{I_{o d} R_{t}}
$$

where the subscripts $a$ and $d$ refer to alternating and direct current (or voltage). Since $I_{o a}$ and $I_{o d}$ produce equal output EMFs, $I_{o a}=I_{o d}\left(1+\delta_{c}\right)$. If we let $Z_{t}$ $=R_{\ell}\left(1+\delta_{r}\right)$, we get, if second-order terms are negligible, $\delta_{v}=\delta_{r}+\delta_{c}$. Thus, $\delta_{v}$ can be determined if $\delta_{r}$ can be deduced from the comparisons of adjacent ranges, since $\delta_{c}$ can be evaluated in terms of the reference group of TEs.

Two complete sets of adjacent-range comparisons from 0.5 to $500 \mathrm{~V}$ were carried out at frequencies of 2 , 20 , and $50 \mathrm{kHz}$, with two different pairs of $5 \mathrm{~mA}$ TEs. These TEs had $80 \mathrm{Ni} 20 \mathrm{Cr}$ heaters but were otherwise similar to those in the reference group. Measured values of $\delta$ as current converters were -1 or $-2 \mathrm{ppm}$, except at $20 \mathrm{~Hz}$ where they ranged from -6 to $-9 \mathrm{ppm}^{7}$ The results of the adjacent-range tests given in Table III showed excellent precision. In addition, the inter-range differences were less than $2 \mathrm{ppm}$ through $20 \mathrm{kHz}$, except for the lowest and highest ranges. The differences of a few ppm observed on the lowest ranges were rather puzzling and will be discussed in Section IX. They were essentially independent of frequency through 20 $\mathrm{kHz}$ but were negligible above the 1 volt range.

TABLE III

Comparisons of Thermal Voltage Converters

\begin{tabular}{|c|c|c|c|c|c|c|c|c|}
\hline \multirow{3}{*}{ TVC 1} & \multirow{3}{*}{ TVC 2} & \multirow{3}{*}{$\underset{\mathrm{V}}{\text { Voltage }}$} & \multicolumn{6}{|c|}{$\delta_{1}-\delta_{2}$ in $\mathrm{ppm}$} \\
\hline & & & \multicolumn{2}{|c|}{$2 \mathrm{kHz}$} & \multicolumn{2}{|c|}{$20 \mathrm{kHz}$} & \multicolumn{2}{|c|}{$50 \mathrm{kHz}$} \\
\hline & & & $\# 1 *$ & $\# 2 \dagger$ & $\# 1$ & $\# 2$ & $\# 1$ & $\# 2$ \\
\hline A & B & 0.5 & & & & +2 & & \\
\hline B & A & 0.5 & -3 & -3 & -4 & -3 & -4 & -4 \\
\hline $1-B$ & A & 0.5 & +2 & & 0 & & -2 & \\
\hline $1-A$ & B & 0.5 & +2 & & +2 & +1 & 0 & +3 \\
\hline $2-B$ & $1-\mathrm{A}$ & 1 & -1 & & -2 & -1 & -8 & -4 \\
\hline $3-A$ & $2-B$ & 2 & +2 & & +2 & +1 & 0 & -1 \\
\hline $5-B$ & $3-A$ & 3 & 0 & & 0 & 0 & -4 & $\mathrm{U}$ \\
\hline $10-\mathrm{A}$ & $5-B$ & 5 & +1 & +1 & +1 & 0 & 0 & -2 \\
\hline $20-B$ & $10-\mathrm{A}$ & 10 & 0 & & 0 & +1 & 0 & -1 \\
\hline $30-A$ & $20-B$ & 20 & +1 & & 0 & 0 & -1 & 0 \\
\hline $50-\mathrm{B}$ & $30-A$ & 30 & 0 & & -1 & 0 & -2 & 0 \\
\hline $100-\mathrm{A}$ & $50-\mathrm{B}$ & 50 & 0 & & 0 & 0 & -3 & -2 \\
\hline $200-B$ & $100-\mathrm{A}$ & 100 & 0 & +1 & 0 & -1 & 0 & +4 \\
\hline $300-A$ & $200-B$ & 200 & 0 & & 0 & 0 & +4 & +6 \\
\hline $500-\mathrm{B}$ & $300-A$ & 300 & 0 & & -6 & -5 & +16 & +8 \\
\hline
\end{tabular}

* Test \#1--With one pair of $5 \mathrm{~mA}, 100 \mathrm{ohm}$ TEs.

$\dagger$ Test \#2-With another pair of $5 \mathrm{~mA}, 100 \mathrm{ohm}$ TEs. These tests were made three months after those with pair \#1.

Except for this, the results of the comparisons are in excellent agreement with computed values (see Section VIII), which show that $\delta_{r}$ should be less than $1 \mathrm{ppm}$ through the $100 \mathrm{~V}$ range. It is extremely unlikely that any single source or combination of sources of ac-dc difference should produce the same $\delta_{r}$ in each of these ranges. Thus, the authors believe that, from the concordance of theory and experiment, it is safe to assign zero $\mathrm{ppm}$ to the average of the values of $\delta_{r}$ from the $2 \mathrm{~V}$

\footnotetext{
7 These TEs, which were the only ones available at the time with the correct heater resistance, will be replaced by others.
} 
through the $100 \mathrm{~V}$ ranges in establishing the values given in Table II from the comparisons of Table III.

The comparisons at $50 \mathrm{kHz}$ (which were less precise) were made to verify that ac-dc differences were not sharply dependent on frequency at $20 \mathrm{kHz}$. They indicate that excellent accuracy should be expected for most ranges at even higher frequencies.

A complete set of measurements at 75 percent of rated voltage was also made at $2 \mathrm{kHz}$ with TE pair $\# 1$ in order to investigate voltage coefficients. These results agreed with the rated-voltage measurements to $1 \mathrm{ppm}$, with no systematic difference.

A completely independent verification of the accuracy of these reference TVCs has come from some careful comparisons by Flach and Marzetta [8] of a "time-gate" peak ac-to-dc voltage comparator (designed by Marzetta) with an rms comparator which incorporated a TVC that had been calibrated with the reference set. In a long series of tests at 400 and $1000 \mathrm{~Hz}$, where the error in the comparison introduced by distortion of the ac source was less than $10 \mathrm{ppm}$, the two comparators agreed on the average to 4 and $8 \mathrm{ppm}$, respectively, at the two frequencies.

\section{Thermoelectric Errors}

Hermach's treatment of the ac-dc errors arising from Thomson effects in the heater of a conventional TE [4] has been extended by Widdis [6] to include the effects of Peltier heating, and to correct for the heat abstracted by the thermocouple. Widdis' expression for the Thomson ac-dc difference, $\delta_{t}=\alpha^{2} \theta / 12 \rho k$, is the same as that found by Hermach for high-range TEs, where $\alpha, \rho$, and $k$ are, respectively, the Thomson coefficient, electrical resistivity, and thermal conductivity of the heater alloy, and $\theta$ is the midpoint temperature rise $\left(100^{\circ} \mathrm{C}\right.$ to $150^{\circ} \mathrm{C}$ in a conventional TE). The error can thus be computed reasonably well if $\alpha$ can be determined. The Thomson heating of copper is reasonably well known $[7]$, and is not much affected by moderate temperature elevations or by the cold working of ordinary handling. Thus, this metal can serve as a practical reference. The authors have calculated the Seebeck and the absolute Thomson coefficients of a number of pertinent alloys from careful measurements of the EMF vs. temperature of thermocouples formed from each of these alloys and copper. The results are shown in Table IV, along with the calculated ac-dc difference $\delta_{t}$ (using Widdis' formula) in a TE having midpoint heater temperature rise of $150^{\circ} \mathrm{C}$ (giving a $10 \mathrm{mV}$ output with a typical $\mathrm{NiCr}$ constantan thermocouple). With Evanohm or Karma, $\delta_{t}$ should not exceed $1 \mathrm{ppm}$. At an output of $5 \mathrm{mV}$ it should be less than $1 \mathrm{ppm}$, since both $\theta$ and $\alpha$ are smaller.

Additional measurements indicated that the thermoelectric coefficients may be sensitive to cold working and may be changed by exposure to high temperature. For Evanohm and Karma significant changes were ob-
TABLE IV

ThermoeleCtric DATA AND ERrors

\begin{tabular}{|c|c|c|c|c|}
\hline \multirow[b]{2}{*}{$\begin{array}{l}\text { Metal } \\
\text { or Alloy }\end{array}$} & \multirow[b]{2}{*}{$\begin{array}{c}\alpha, \mu V /{ }^{\circ} \mathrm{C}^{*} \\
\text { at } 400^{\circ} \mathrm{K}\end{array}$} & \multirow[b]{2}{*}{$\stackrel{S}{\mu \mathrm{V} /{ }^{\circ} \mathrm{C}}$} & \multicolumn{2}{|c|}{ Calculated Error in a TE } \\
\hline & & & $\begin{array}{c}\text { Dc } \\
\text { reversal** } \\
\Delta l / l, \text { ppm }\end{array}$ & $\begin{array}{c}\text { Ac-dc } \\
\text { difference } \\
\delta_{\ell}, \text { ppm }\end{array}$ \\
\hline 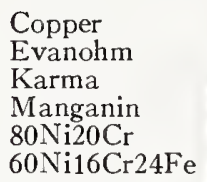 & $\begin{array}{l}-2.0 \\
-1.5 \\
-1.0 \\
-3.0 \ddagger \\
-3.9 \\
-2.3\end{array}$ & $\begin{array}{l}-\overline{+0.3 \dagger} \\
+1.8 \\
-1.8 \ddagger \\
+4.4 \\
+0.8\end{array}$ & $\begin{array}{l}-200 \\
150 \\
400 \\
500 \\
300\end{array}$ & $\begin{array}{l}- \\
-1 \\
<1 \\
-4 \\
-7 \\
-2\end{array}$ \\
\hline
\end{tabular}

* $\alpha$ (Thomson coefficient) is proportional to the absolute temperature.

$\dagger S$ is Seebeck coefficient vs. copper.

+ This was quite dependent on heat treatment.

I| From Thomson heating, with $\theta=150^{\circ} \mathrm{C}$ and $\rho k=25 \times$ $10^{-6} \mathrm{~V}^{2} /{ }^{\circ} \mathrm{C}$ at $\not 000^{\circ} \mathrm{K}$, the weighted average temperature of the heater.

** With the bead off center (see text).

served at $800^{\circ} \mathrm{C}$, but not at $400^{\circ} \mathrm{C}$. Thus, care must be taken in controlling the temperature of the wire near welded join ts and when out-gassing during the construction of TEs. Here the use of TEs in the reference group by four different manufacturers having differing techniques provided powerful assurance against unknown errors.

As indicated by Wilkins, the thermoelectric errors are greatly reduced in the NPL TEs because of the bifilar heater and the low temperature rise (about $10^{\circ} \mathrm{C}$ at 20 $\mathrm{mA}$ in the present TEs). The resulting error should be much less than $1 \mathrm{ppm}$, even with nickel-chromium heaters.

Rather unfortunately, commercial TEs have lead-in wires (see Fig. 1), through the glass bulb to the heater, of a copper-coated nickel alloy to match the thermal expansion coefficient of the glass. The combination has a rather uncertain but fairly large Seebeck coefficient vs. the heater alloys used. With direct current applied, Peltier heating and cooling of the junctions of these leadin wires and the heater can cause a temperature difference ( + at one junction and - at the other). Most of this heat is conducted by the lead-in to the thick glass press or disk which forms the base of the glass bulb. This can be considered as a "heat sink" so that the first-order junction temperature change is $\Delta \theta=T I S / K$, where $T$ is the absolute temperature, $I$ is the current, $S$ is the Seebeck coefficient, and $K$ is the thermal conductance of the lead-in. Widdis showed that this leads to an ac-dc difference term $\delta_{t p}=\alpha \Delta \theta / V_{h}$ where $\alpha$ is the Thomson coefficient and $V_{h}$ is the heater voltage. There may, in addition, be a second-order term at the midpoint because the Peltier heat itself depends on the junction temperature. This can be calculated by carrying out the second-order approximation mentioned by Hermach in Appendix 2 of reference [4] to give, with reasonable accuracy, $\delta_{p}=+\Delta \theta^{2} / T_{0} \theta$, where $T_{0}$ is the heat-sink temperature. Of course, $\Delta \theta$ can be calculated only very roughly, but this is sufficient. For a typical $10 \mathrm{~mA}$ TE 
it is less than $0.01^{\circ} \mathrm{C}$, and the Peltier errors, $\delta_{\iota p}$ and $\delta_{p}$ (independent of frequency), are less than $1 \mathrm{ppm}$. This has been verified by comparisons with Evanohm-heater TEs which had copper lead-in wires butt-welded to the nickel alloy wires at the glass press. However, $\delta_{p}$ in particular could be significant in higher-range TEs.

\section{Low- and High-Frequency Errors}

As the frequency is lowered, the temperature of a TE begins to follow the cyclic fluctuations of the square of the heater current. If the TE is not a linear device, the average temperature rise may no longer be the same as at higher frequencies. Hermach's solution of the resulting nonlinear differential equation for the temperature rise has been verified experimentally [4], at least for high-range TEs having large heaters in which the heat abstracted by the thermocouple is negligible. However, for the low range TEs we are concerned with now, these formulas may not apply. The solutions for such cases would be even more difficult, and have not been attempted, particularly since the thermal mass of the bead should then also be considered. In the simpler case, Hermach showed that the ac-dc difference is $\delta_{l}=h \theta d^{2} / \omega^{2} l^{4}$, where $d$ is the diffusivity of the heater alloy, $2 l$ is the heater length, $\omega=2 \pi f$, where $f$ is the frequency, and $h$ is a factor expressing the nonlinearities. The same relationship might be expected for the more complicated case, but with a different numerical factor. Thus, the agreement to better than $10 \mathrm{ppm}$ among the TEs at $20 \mathrm{~Hz}$, shown in Table I, with their varying lengths, gives strong verification to the absence of significant low-frequency errors down to this frequency.

These reference TEs have heaters less than 1 by $10^{-3}$ $\mathrm{cm}$ in diameter, with resistivities of $120 \mu \Omega \mathrm{cm}$. The computed errors from skin effect are much less than 1 ppm even at $50 \mathrm{kHz}$. The ac and dc conductances and the capacitance between binding posts on the phenolic top of the box in which the TEs are mounted are much less than the limiting values of $0.01 \mu \mathrm{A} / \mathrm{V}$ and $100 \mathrm{pF}$, respectively, which might cause $1 \mathrm{ppm}$ errors at audio frequencies.

Reactance and skin-effect errors in the resistor units of the TVCs, computed according to formulas developed by the authors [3], are also less than 1 ppm through 50 $\mathrm{kHz}$ up through the 100 volt range, and the comparisons verify this. They cannot easily be calculated for the higher ranges, which have several resistors and adjustable inner shields. Additional reactance errors can arise from the lumped capacitance $C$ of the connector between the resistor and the TE. This shunts the heater, which at these frequencies can be considered as a lumped resistor $R$ in series with an equivalent lumped inductance $L$. It can be shown that the resultant effect of this capacitance on the magnitude of the heater current approaches $\alpha^{2} / 2-\alpha \beta$ as the series resistance (voltage range) is increased, where $\alpha=\omega C R$ and $\beta=\omega L / R$ $(\alpha$ and $\beta \ll 1$ ). The computed error of less than $1 \mathrm{ppm}$ at $50 \mathrm{kHz}$ has been verified by deliberately doubling the capacitance.

\section{Other Errors}

Peltier effects at the heater and lead-in junctions can also cause a second-order change in the effective dc resistance of a TE. The calculation is difficult and the results uncertain, but the change should be much less than one ppm for the 5 to $20 \mathrm{~mA}$ TEs in the NBS reference group. Thus, this cannot account for the somewhat larger changes in effective dc resistance implied by the voltage comparisons of the TEs alone (without series resistors) in the TVCs. Even larger and more puzzling changes have been observed in some other TEs. There is good evidence that they may be caused by rectification at oxide films, which may persist in spite of the welded joints, but there is as yet no direct proof of this.

Dc reversal errors $(\Delta I / I$ for the same EMF) provide a good clue in studying these secondary effects. As shown in Table IV, $\Delta I / I$ calculated by Widdis' formulas should not exceed, perhaps, $200 \mathrm{ppm}$ with Evanohm or Karma, and $500 \mathrm{ppm}$ with nickel-chromium heaters, even if the thermocouple is mounted off the midpoint by 5 percent of the length of the heater (which is easily noticeable). Larger differences are, thus, a sign of other difficulties.

As Hermach showed [4], the current from the heater to ground through the bead of an ungrounded TE can cause heating of the bead and, thus, an error. This is difficult to compute because power dissipated in the bead is somewhat more effective in raising the thermocouple temperature than that in the heater, and the currents in the two halves of the heater are no longer iden tical. However, rough calculations indica te that with one volt across the bead of $\mathrm{TE}_{2}$ in the circuit of Fig. 3, the bead resistance of a $10 \mathrm{~mA}$ TE must be greater than 500 megohms if the error is to be less than $1 \mathrm{ppm}$. For the bead materials commonly used, the resistance is a sharp function of temperature and is much less at audio frequencies than with direct current. At NBS, bead resistances are normally measured with a $2 \mathrm{kHz}$ ac bridge with rated direct current through the heater of a TE. The results have been verified (within a factor of 2) by ac-dc comparisons of pairs of TEs with added resistance between the two heaters to significantly increase the bead voltage.

\section{Conclusions}

A reference group of two very different kinds of TEs has been established. The authors believe that it is very unlikely that the average ac-dc difference of the group differs from zero by more than $2 \mathrm{ppm}$ at audio frequencies. This is based on the agreement to better than $0.5 \mathrm{ppm}$ of the averages of the two kinds of TEs, and on allowance of $1 \mathrm{ppm}$ for the uncertainty of the average in the network of comparisons. The results are in excellent agreement with calculations, which show that the ac-dc differences should not exceed $1 \mathrm{ppm}$. With the precise techniques that have been developed, pairs of TEs of the group may be compared with a $3 \sigma$ uncertainty of about 2 ppm without any evidence to date (in over three years of measurements) of significant 
systematic errors. This is somewhat remarkable, since fluctuations in the best available wide-range ac and dc sources greatly exceed this, and most of these TEs have rather large residual thermal EMFs and have temperature coefficients of EMF exceeding $1000 \mathrm{ppm} /{ }^{\circ} \mathrm{C}$.

Adjacent ranges of the TVCs may also be compared with the same precision to step up and down the voltage scale. Their ac-dc differences can be determined to better than $10 \mathrm{ppm}$ when used with TEs of known ac-dc differences, again without evidence of systematic error. Alternating voltage measurements can be made to 20 ppm or better by using the TVCs or ac-dc transfer standards with appropriate dc standards.

This accuracy and precision in the ac-dc transfer, more than ten times better than heretofore available, are good indications of the virtue of simplicity in reference standards. Properly designed and constructed TEs are rather simple devices that correspond to the desired idealized mathematical models remarkably closely, thus providing the agreement between theory and experiment which is so desirable in a development such as this.

The agreement to better than $1 \mathrm{ppm}$ between the conventional TEs and the new very different multijunction NPL TEs (which should theoretically have smaller errors), in addition to providing semi-independent verification of the accuracy of both, indicates that even higher accuracy may be possible in the future with these new designs. Conventional single-junction TEs could be improved by the use of bifilar heaters and closely spaced lead-in wires with good thermal conductance between the two halves of the heater and between the two lead-ins. In this way, other heater alloys could be used.

Well-designed and well-constructed ac-dc transfer standards can be remarkably stable over most of their frequency range. Most factors which may change the response, such as temperature and dimensional changes, have little or no effect on the ac-dc difference. There is already some evidence that long-time stabilities of a few ppm can be attained in properly designed and constructed TEs and TVCs. However, we have noted an apparently abrupt change of +5 ppm in one TE, accompanied by a change of over $100 \mathrm{ppm}$ in its dc reversal difference. Thus, TEs should be compared frequently if the highest accuracy is desired.

Comparisons of adjacent ranges, with a step-up procedure such as the one described, can be used to guard against unexpected changes in TVCs. This has already been verified in the design and construction of two somewhat similar sets of TVCs by W. Scott, K. Ballard, and D. Bailey of the NBS Electronic Calibration Center, Boulder, Colo. These units incorporate large wirewound resistors of controllable reactances and low temperature coefficients, so that they do not require troublesome forced-air cooling. They will be described elsewhere.

The step-up procedure is also feasible with simpler sets of TVCs incorporating fewer plug-in resistor units and two TEs of different current ranges. Two such sets have been built but have yet to be completely evaluated.

\section{ApPENDix I}

\section{EMF COMPARATOR}

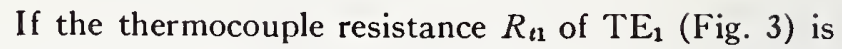
much less than $R_{1}+R_{2}$, the EMF $E_{1}$ is substantially the same with alternating and direct currents applied during the test (the procedure involves holding the voltage at the output terminals of $\mathrm{TE}_{1}$ constant). Then, from the definition of ac-dc difference, $\left(I_{a}-I_{d}\right) / I_{d}=\delta_{1}$. Similarly, for $\mathrm{TE}_{2}\left(I_{a}-I_{d}{ }^{\prime}\right) / I_{d}{ }^{\prime}=\delta_{2}$, where $I_{d}{ }^{\prime}$ is the direct current that would be required to produce the same $\mathrm{EMF}$ of $\mathrm{TE}_{2}$ as $I_{a}$. The change $D_{a}-D_{d}$ in detector $D_{1}$ which occurs when $I$ is changed from $I_{a}$ to $I_{d}$ ( $I_{d}$ being adjusted to keep $E_{1}$ constant) results from the change in $E_{2} . D_{a}-D_{d}$ may then be interpreted as a measure of the departure of $I_{d}$ from the value $I_{d}{ }^{\prime}$ which would have produced the same $E_{2}$ as would $I_{a}$. Thus, combining the above two equations and neglecting second-order terms, we have $\left(I_{d}^{\prime}-I_{d}\right) / I_{d}=\delta_{1}-\delta_{2}$.

The sensitivity of the detector to small current differences in $\mathrm{TE}_{2}$ can be determined by shunting its heater $R_{h}$ with a resistor $R_{b} \gg R_{h}$ to cause a small change in its heater current $\Delta I$, noting the change in deflection $\Delta D$ of $D_{1}$ and computing $s=\Delta I / I \Delta D\left(\Delta I / I=R_{h} / R_{s}\right.$ if the heater current of $\mathrm{TE}_{1}$ is unchanged). Then, if $s$ is constant over the range of deflections involved, we have $\delta_{1}-\delta_{2}=s\left(D_{a}-D_{d}\right)$, as in the text. Similarly, in the comparison of two voltage converters, a resistor $R_{s} \ll R_{m}$ can be added in series with TVC $_{2}$ to give proportional change in applied voltage $\Delta V / V=R_{s} / R_{m}$, where $R_{m}$ is the TVC resistance. Then $s=\Delta V / V \Delta D$.

These two calibration methods must leave the current in $\mathrm{TE}_{1}$ unchanged. The change $\Delta I$ can of ten be introduced and measured more conveniently in some way (as by changing the supply voltage) that changes the current in both TEs by a small known amount. We have found it convenient to disconnect $E_{1}$, set the voltage of $P$ to the same value, short $D_{2}$, observe the resulting $\Delta D$ when $I$ is changed, and compute $s$ as before.

If only a few TEs are ordinarily used in the "standard" position ( $\mathrm{TE}_{2}$ in Fig. 3), it is convenient to make use of the approximate output-input relation (at a fixed frequency) for a TE, $\Delta E / E=n \Delta I / I$, where $n$ is dependent on $I$ but not $\Delta I$ if $\Delta I \ll I$, and is close to 2 . For each such TE, $n$ can be determined in advance at several values of $E$ and plotted vs. $E$, since it is quite stable. Then the sensitivity of the detector in the circuit can be determined by inserting a small known voltage $\Delta E_{p} \ll E$ (with a Lindeck potentiometer, not shown in Fig. 3) in series with and opposing $E_{2}$, observing the change $\Delta D$ in $D_{1}$ and computing the factor $k=\Delta D / \Delta E_{p}$. Then, from this and the previous equations $\delta_{1}-\delta_{2}=\left(I_{d}^{\prime}-I_{d}\right) / I_{d}$ $=\left(E_{d}{ }^{\prime}-E_{d}\right) / n_{2} E_{2}=\left(D_{a}-D_{d}\right) / k n_{2} E_{2} . \quad E_{2}$ is easily measured with potentiometer $P$.

If $R_{\imath 1}$ is not negligible, $E_{1}$ is not constant during the test, since the currents in $R_{1}$ and $R_{2}$ depend on both $E_{1}$ and $E_{2}$. The corresponding equations for determining $\delta_{1}-\delta_{2}$ by means of deflections of $D_{1}$ then contain additional correction terms involving the resistances of the several parts of the EMF comparator, and are too com- 
plex to be useful for routine measurements. They show, however, that if $R_{t 1} \leq\left(R_{1}+R_{2}\right) / 100$, the simplified equations already derived are valid to a few percent of the computed $\delta_{1}-\delta_{2}$. They show that $R_{t 2}$ is not critical. For our comparator, $R_{1}+R_{2}=1100 \Omega$ and $R_{t 1} \leq 10 \Omega$ for the usual TEs.

For each NPL converter, $R_{t} \approx 1000 \Omega$. It is always placed in the $\mathrm{TE}_{2}$ position, except when the two NPL converters are intercompared, requiring the use of the more complicated formula.

\section{APPENDIX II}

\section{TES in Parallel}

By using the definitions for $n$ and $\delta$ of a TE (see Appendix I) we have, closely enough for small changes in $E$,

$$
\frac{E_{a}-E_{d}}{n E_{d}}=\frac{I_{a}-I_{d}}{I_{d}}-\delta .
$$

In the ac-dc test, nominally equal resistors are connected in series with $\mathrm{TE}_{a}$ and $\mathrm{TE}_{b}$, and the two branches are connected in parallel. The sum of the two EMFs, $E_{a}+E_{b}$, is held constant. Then, with second subscripts to identity the two TEs, we have

$$
E_{a a}+E_{a b}-E_{d a}-E_{d b}=0
$$

and

$$
\begin{aligned}
& n_{a} E_{d a}\left(\frac{I_{a a}-I_{d a}}{I_{d a}}\right)+n_{b} E_{d b}\left(\frac{I_{a b}-I_{d b}}{I_{d b}}\right) \\
&=n_{a} E_{a} \delta_{a}+n_{b} E_{b} \delta_{b}
\end{aligned}
$$

If the reactances of the two paths are equal

$$
\frac{I_{a a}-I_{d a}}{I_{d a}}=\frac{I_{a b}-I_{d b}}{I_{d b}}=\frac{I_{a}-I_{d}}{I_{d}}=\delta_{p}
$$

where $I_{a}$ and $I_{d}$ are the sums of the alternating and direct currents in the two branches.

If $E_{a}=m E$ and $E_{b}=(1-m) E$,

$$
\left[n_{a} m+n_{b}(1-m)\right]\left[\frac{I_{a}-I_{d}}{I_{d}}\right]=n_{a} m \delta_{a}+n_{b}(1-m) \delta_{b}
$$

If the reactances of the two paths are not equal, additional terms will be introduced which are difficult to calculate. However, they are zero if $n_{a} m=n_{\iota}(1-m)$, i.e., if the TEs are matched in response. Then $\delta_{p}$ $=\left(\delta_{a}+\delta_{b}\right) / 2$. Even if the reactances are unequal, the added terms are essentially eliminated if a second test is made with the two resistors interchanged and the results averaged.

\section{ACKNOWLEDGMENT}

The authors express their appreciation and thanks to F. J. Wilkins for the two multijunction TEs, to L. Julie of the Julie Research Laboratories for suggesting the basic idea of the EMF comparator, and to the following for their cooperation in the manufacture of other TEs used in this group: the Levy brothers of the American Thermoelectric Company, L. R. Graham of Graham Associates, F. Gay of the Best Products Company, M. Rosenfield of the former Field Electrical Instrument Company, and L. W. Pignolet of Weston Instruments Incorporated.

The use of these particular TEs with heaters of Evanohm and Karma should not in any way be construed as constituting an exclusive endorsement by NBS of these products or alloys. Other TEs, made to the same specifications with the same or other alloys of equally low thermoelectric effects, should be equally suitable.

\section{ReFERENCES}

[1] "American standard requirements for electrical indicating instruments." New York: American Standards Association, C.39.1, 1964.

[2] F. J. Wilkins et al., "Multijunction thermal converter," Proc. IEE (London), vol. 112, pp. 794-806, April 1965.

[3] F. L. Hermach and E. S. Williams, "Thermal voltage converters for accurate voltage measurements to 30 megacycles per second," Trans. AIEE (Communication and Electronics), vol. 79, pt. I, pp. 200-206, July 1960.

[4] F. L. Hermach, "Thermal converters as ac-dc transfer standards for current and voltage measurements at audio frequencies," $J$. Res. NBS, vol. 48, pp. 121-138, February 1952.

[5] W. J. Youden, "Measurement agreement comparisons," Proc. 1962 Standards Laboratories Conf. Washington, D. C.: NBS, Misc. Pub. 248, August 16, 1963, pp. 147-152.

[6] F. C. Widdis, "The theory of Peltier and Thomson effects in thermal ac-dc transfer devices," Proc. IEE (London), Monograph $497 \mathrm{M}$, January 1962.

[7] Landolt-Bornstein, "Zahlenwerte und Funktionen aus Physik, Chemie, Astronomie, Geophysik und Technik," 6. Auf., Band II/6. (6th ed. vol. 2, pt. 6). Berlin: Springer-Verlag, 1959.

[8] D. Flach and L. A. Marzetta, "Calibration of peak a-c to d-c comparators," Proc. 1965 ISA Conf., pt. 1, vol. 20, paper 14. 


\title{
Thermal Voltage Converters and Comparator for Very Accurate AC Voltage Measurements
}

\author{
E. S. Williams \\ Institute for Basic Standards, National Bureau of Standards, Washington, D.C. 20234
}

(July 22, 1971)

\begin{abstract}
A new fourteen-range set of thermal voltage converters and a thermoelement comparator are used to measure ac-dc difference, and a-c voltages relative to external d-c standards, with $20 \mathrm{ppm}$ (partsper-million) accuracy at audio frequencies. The imprecision is less than $2 \mathrm{ppm}$. Corrections relative to the very stable middle ranges can be redetermined for every range by a seven-step intercomparison of certain adjacent ranges.
\end{abstract}

Key words: AC-DC difference; comparator; thermoelement; transfer voltmeter; voltage measurements.

\section{Introduction}

An a-c voltage at audio frequencies is measured most accurately at the present time by using a thermal voltage converter (TVC) to compare it with a stable and accurately measured $\mathrm{d}-\mathrm{c}$ voltage, which is nominally equal to it. The basic d-c standards are then, in effect, "transferred" to the a-c measurement. The TVC may be simply a thermoelement (TE) in series with an appropriate multiplier resistor. The output emf of the TE is ordinarily monitored with a null detector and a balancing circuit, which may be a Lindeck potentiometer. A balancing circuit and a null detector are included in most commercial multirange models.

Before the TVC is used for a-c voltage measurements it must be tested for ac-dc difference or frequency influence so that corrections may be applied. The set of TVCs and the new TE comparator described in this paper were developed primarily for making these tests. However they may also be used for a-c voltage measurements as explained in section 9. For ac-dc difference measurements the TE comparator, whose read out instrument may be either a nanovoltmeter or a galvanometer, provides a considerable advantage over other methods in overcoming the difficulties caused by power supply instability and inexact voltage control.

The 14-range set of TVCs (designated No. 7) consists of six resistor units and two TEs, and extends from 1 to $1000 \mathrm{~V}$. The TEs are rated at 2.5 and $5.0 \mathrm{~mA}$, and each one may be attached to any one of the resistors by a coaxial connector (see fig. 1). This permits each resistor to be used for two voltage ranges, as shown in table 1. Certain adjacent ranges of the set can be intercompared, and the ac-dc differences of all the ranges can be determined relative to any one range. This set is otherwise similar to an earlier set $[1]^{1}$, which consisted of two $5-\mathrm{mA}$ TEs and 12 series resistors, and covered a range from 0.5 to $500 \mathrm{~V}$. The earlier set, designated No. 1, has been extended to $1000 \mathrm{~V}$ with one additional resistor as part of the present project. It is evaluated in a 14-step intercomparison in which each range is compared, at reduced voltage, with the next lower one.

In a well designed TVC the ac-dc difference is not affected by changes in the applied voltage. Therefore, an ac-dc difference determined for the $100-\mathrm{V}$ range at $60 \mathrm{~V}$ can be applied as a correction when this resistor is used at $200 \mathrm{~V}$ to test the nominal $300-\mathrm{V}$ range of the next higher resistor. Experience has shown this stepup procedure to be feasible. However if the higher volt. age ranges are not carefully built the ac-dc differences can change due to self-heating effects to be described later.

\section{Design and Construction}

The two TEs are mounted in 2 -in brass tubes (see figs. 1.and 2, upper right) with coaxial connectors for attaching to a resistor. The $2.5 \mathrm{~mA}$-TE has a $400 \Omega$ heater and is used alone as the $1 \cdot V$ range. The $5 \mathrm{~mA} \cdot \mathrm{TE}$ has a $125 \Omega$ heater and a $275 \Omega$ resistor is added inside its enclosure to make a $400 \Omega, 2 \cdot V$ range.

The middle ranges (six ranges, 3 to $60 \mathrm{~V}$ ) make use of three resistors $-0.8 \mathrm{k} \Omega, 3.6 \mathrm{ks}$, and $12 \mathrm{k} \Omega$ (see table 1). Each of these is a $2 \mathrm{~W}$ metal-film resistor mounted coaxially in a 3 -in brass cylinder $41 / 2$ in long with coaxial connectors at each end.

Figures in brachets indicate the literature references on page 153 

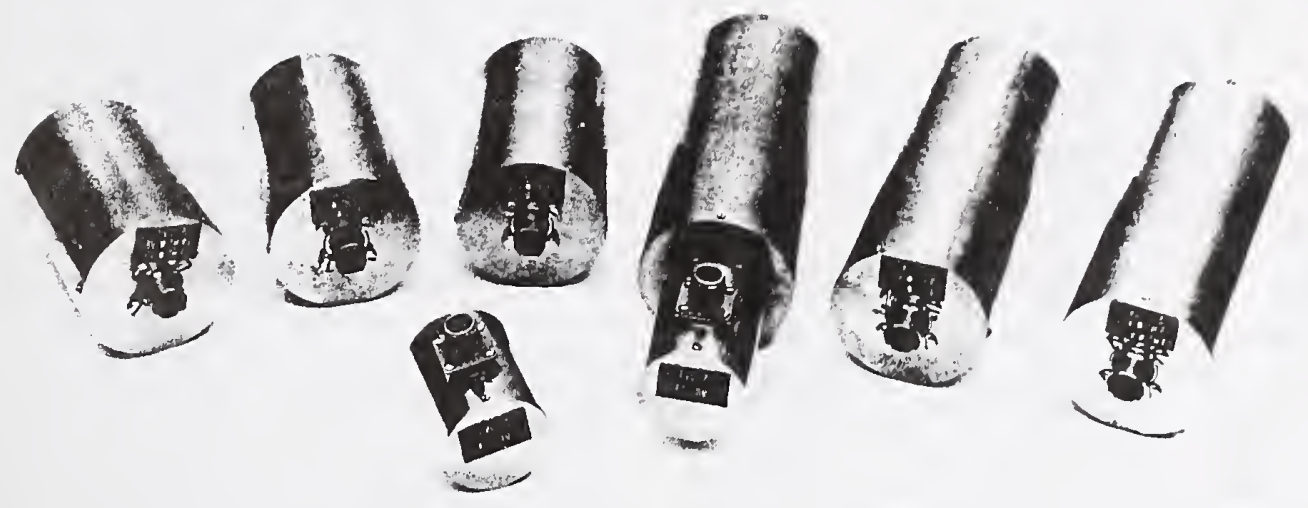

FIGURE 1. New set of thermal voltage converters.

The simple symmetrical geometry of these units permits approximate calculations of their reactances [2]. The calculations are inexact because of the necessary assumptions as to end-effects, and they neglect any small residual reactances of the resistors themselves. They do indicate however, that the frequency error of each resistor unit (without the TE) should be less than $1 \mathrm{ppm}$ at $50 \mathrm{kHz}$, even for tubes smaller than 2 in in diameter. (The 3 -in tube was chosen for the higher ranges where more space is necessary and was therefore used for the middle ranges also.)

The higher voltage ranges have larger resistor assemblies to avoid excessive temperature rise. The $100-200 \mathrm{~V}$ unit has a $40 \mathrm{k} \Omega$ spiraled metal-film resistor five inches long. ${ }^{2}$ The $300-600$ and $500-1000 \mathrm{~V}$ units have resistors of tin oxide deposited on a 0.81 -in glass tube 6 in long. A small voltage coefficient was anticipated in these resistors, but measurements have shown it to be negligible.

\footnotetext{
${ }^{2}$ This experimental resistor is not ordinarily available commercially. Four $40 \mathrm{k} \Omega$ meta film resistors in series parallel can also be satisfactory.
}

TABLE 1. NBS thermal voltage converter set No. 7

Fourteen voltage ranges (column 2) are formed with six series resistors and two TEs (F 1 and $F$ 2).

\begin{tabular}{|c|c|c|c|}
\hline $\begin{array}{l}\text { Series } \\
\text { resistor }\end{array}$ & $\begin{array}{l}\text { Voltage } \\
\text { range }\end{array}$ & $T E$ & $\begin{array}{c}\text { Total } \\
\text { resistance }\end{array}$ \\
\hline \multirow[t]{4}{*}{$k \Omega$} & & $m A$ & $k \Omega$ \\
\hline & 1 & $2.5(\mathrm{~F} 1)$ & 0.4 \\
\hline & 2 & $5.0(\mathrm{~F} 2)$ & 0.4 \\
\hline & 3 & 2.5 & 1.2 \\
\hline \multirow{2}{*}{$0.8 \ldots \ldots \ldots \ldots$} & 6 & 5.0 & 1.2 \\
\hline & 10 & 2.5 & 4.0 \\
\hline \multirow[t]{2}{*}{$3.6 \ldots \ldots \ldots \ldots$} & 20 & 5.0 & 4.0 \\
\hline & 30 & 2.5 & 12.4 \\
\hline \multirow{2}{*}{$12 \ldots \ldots \ldots \ldots$} & 60 & 5.0 & 12.4 \\
\hline & 100 & 2.5 & 40.4 \\
\hline \multirow[t]{2}{*}{40.} & 200 & 5.0 & 40.4 \\
\hline & 300 & 2.5 & 120.4 \\
\hline \multirow[t]{2}{*}{$120 \ldots \ldots \ldots$} & 600 & 5.0 & 120.4 \\
\hline & 500 & 2.5 & 200.4 \\
\hline $200 \ldots$ & 1000 & 5.0 & 200.4 \\
\hline
\end{tabular}

Errors in these ranges are caused mainly by capacitance between the resistor assembly and the outer casing, which permits alternating current to bypass the TE to ground. Therefore more a-c than $d \cdot c$ voltage is required for a given TE out put. Frequency compensation could be provided by placing relatively small capacitors in parallel with part of the resistance. However, such capacitors probably would not be sufficiently stable over a long period of time and might be affected by temperature changes which occur in the resistor enclosure.

Compensation was therefore provided (as in the earlier set No. 1) with an inner shield which is connected to the input and surrounds the high-or inputend of the resistor (see " $S$ " in fig. 2). The shield is positioned, relative to the resistor, to control the capac. itance currents and provide optimum high-frequency
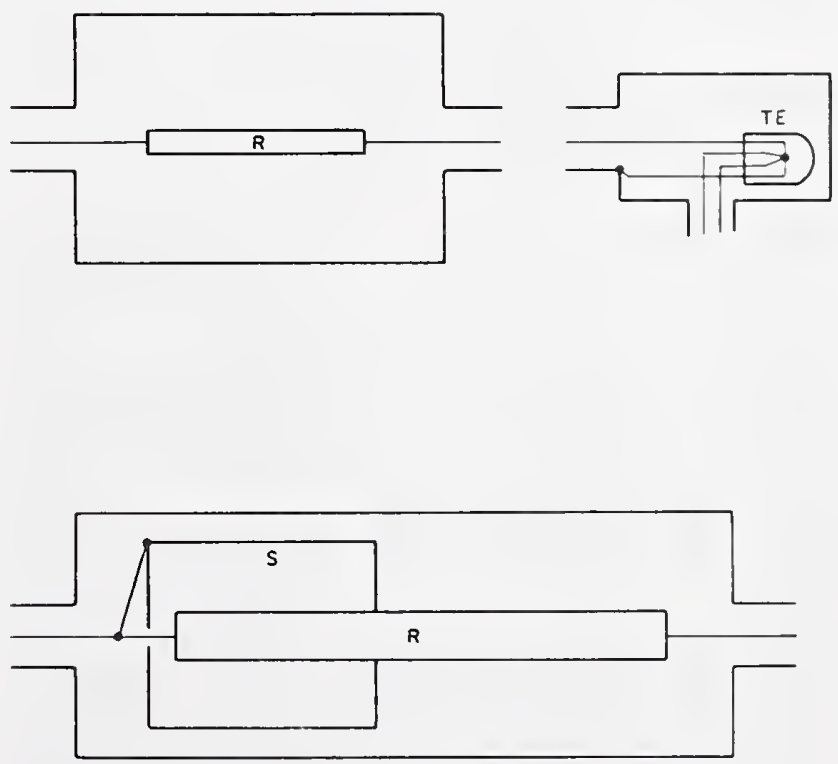

FIGURE 2. Essentials of thermal voltage converters.

Thermoelement (TE) enclosure. low voltage unit with resistor $(R)$. and hich voltage unit (100 to $1000 \mathrm{~V}$ ) with resistor $(\mathrm{K})$ and frequency compensation shield $(\mathrm{S}$ ). 
compensation; perfect compensation is possible only at one frequency.

\section{1000-V TVC}

As stated earlier the corrections for the high and low voltage ranges are determined relative to the middle range by intercomparison tests. It is necessary therefore that the ac-dc difference of all ranges be unaffected by voltage level. The voltage effect is rare in low and middle ranges, but it can be troublesome at higher ranges where the heat generated by the resistor is appreciable. All intercomparison tests were initially made at two voltage levels in order to evaluate this effect-or to prove its absence. If, for example, the nominal $600-\mathrm{V}$ range is compared with the $1000-\mathrm{V}$ range at both 400 and $600 \mathrm{~V}$, and the same results are obtained, it is unlikely that the $600-\mathrm{V}$ range is affected by the voltage increase (the $1000-\mathrm{V}$ range is very probably unaffected at these voltages). However both ranges are in question until we are assured that the $1000-\mathrm{V}$ range is also free of this effect.

The highest range, then presents a special problem. It is more likely to change due to increased voltage (heating) and there is no higher range to compare it with. If it is compared with another $1000-\mathrm{V}$ TVC at two or more voltages and no change is observed it is possible that both have the same voltage effect. However this is unlikely if TVCs are of somewhat different design. If intercomparisons of several units of difference design show no change between rated voltage and a lower voltage we may safely assume this effect is negligible in each one.

Five 1000-V TVCs (table 2) have therefore been built, each with differing components, and a network of intercomparison tests was made as described in the next section. The original set of TVCs [1] was included in these tests.

TABLE 2. Five 1000-V TVCs

\begin{tabular}{|c|c|c|c|c|}
\hline \multirow[t]{2}{*}{ Unit } & \multirow[t]{2}{*}{ Resistance } & \multicolumn{2}{|c|}{ Voltage range with $T E$} & \multirow[t]{2}{*}{ Resistor assembly } \\
\hline & & $F 1$ & $F 2$ & \\
\hline A & $\begin{array}{l}k \Omega \\
400\end{array}$ & 1000 & & $\begin{array}{l}\text { Four } 100 k \Omega \text { wire- } \\
\text { wound resistors in } \\
\text { series, each } 13 / 8 \text { in } \\
\text { long. }\end{array}$ \\
\hline B & 400 & 1000 & & $\begin{array}{l}\text { Four } 100 \mathrm{k} \Omega \text { wire- } \\
\text { wound resistors in } \\
\text { series, each } 13 / 8 \text { in } \\
\text { long. }\end{array}$ \\
\hline C & 200 & 500 & 1000 & $\begin{array}{l}\text { Four } 200 \mathrm{k} \Omega \text { metal- } \\
\text { film resistors in } \\
\text { series-parallel, each } \\
2 \text { in long. }\end{array}$ \\
\hline D & 200 & 500 & 1000 & $\begin{array}{l}\text { One } 200 \mathrm{k} \Omega \text { tin oxide } \\
\text { resistor } 6 \text { in long. }\end{array}$ \\
\hline $\mathrm{E}$ & 400 & 1000 & & $\begin{array}{l}\text { Four } 400 \mathrm{k} \Omega \text { metal- } \\
\text { film resistors in } \\
\text { series-parallel, each } \\
2 \text { in long. }\end{array}$ \\
\hline
\end{tabular}

The five 1000-V TVCs are designated alphabetically (A through $E)^{3}$ and all except " $A$ " have the inner shield, as described below, for frequency compensation. The resistor in unit " $A$ " is mounted concentrically in a set of six brass rings whose potentials are maintained by a capacitance divider. The whole assembly is mounted in a 4-in brass cylinder 7 in long.

Self-heating of a TVC may affect either the frequency compensation or the resistor itself. Apparently the dielectric losses in the insulation between conductors can be affected by heating and change the impedance of the resistor. This change occurred within a few minutes after a voltage increase or decrease in several resistor types, which were therefore discarded.

The frequency compensating shield may be moved, relative to the resistor, by thermal expansion of mounting parts and cause a change in ac-dc difference. This occurred in some of our early units and the change was slow, taking up to an hour. Later shields were therefore mounted very rigidly since a small displacement will have a large effect on the frequency influence.

The shield now in use is in two parts. A brass cylinder, with one end closed, is mounted firmly against the outer end piece (input end) with a poly. styrene insulator one-half inch thick (fig. 3). A movable tube fits tightly inside this cylinder and the shield length adjustment is made by pushing this piece forward with a small rod inserted through the end piece and the insulator. The rod is removed after each adjustment.

The shield is most effective at the leading edge where the voltage difference is greatest between the resistor and the shield. Therefore the movable part of the shield is cut at an angle, so that only part of it (see fig. 3) extends outside the fixed cylinder. Thereby the effect of small position changes is reduced. Even so, the compensation is affected by approximately 0.01 percent per millimeter of position change in some typical units.

The shield length adjustment is made in small steps, and tests are made after each adjustment. When optimum compensation is achiéved, the unit is opened and the shield parts are locked together with three screws through their overlapping portions.

\section{TE and TVC Tests}

As stated earlier the middle ranges are the most accurate, and they are the base from which higher and lower ranges are tested. However they are no more accurate than the TE used with them. Before being installed in a TVC the TEs are therefore compared with one of a group of carefully selected TE standards which were made according to NBS specifications. This group contains twelve elements made by four manufacturers, and are rated from 5 to $20 \mathrm{~mA}$. [1] They have either Karma or Evanohm ${ }^{4}$ heaters to reduce

\footnotetext{
"Unit " $D$ " is assigned to this set (No. 7).

- Certain commercial products and instruments are identified in this paper in order to apecify adequately the experimental procedure. In no case does such identification imply recommendation or endorsement by the National Bureau of Standards, nor does it imply that the products or equipment identified are necessarily the best available for the purpose.
} 


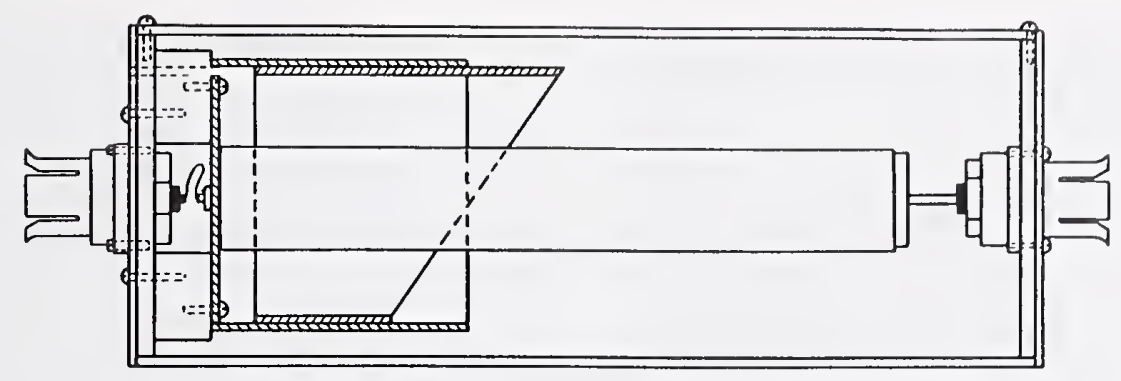

Figure 3. Cross section of high voltage TVC.

Input end is at the left. Fixed and adjustable shield parts are supported by a polystyrene insulator one-half inch thick.

thermoelectric effects in the heaters. They also have small reverse d-c differences (less than $200 \mathrm{ppm}$ ), high bead resistances (over $1000 \mathrm{M} \Omega$ ), and small ar-dc differences. An additional two are multi-junction elements of different design. All of these TEs have been intercompared as current converters with agreement to better than $3 \mathrm{ppm}$. Theoretical calculations, taking into consideration all known sources of error, also indicate that the error should not exceed a few ppm at audio frequencies. Because of this and because of the excellent agreement between these thermoelements of different design and construction, we may safely assume that the average ac-dc difference of the group is 0 to $\pm 2 \mathrm{ppm}$.

The low-frequency performance of a TVC depends on the TE since the reactance of the resistor is entirely negligible even at a few kilohertz. The accuracy of a TE at low frequency is mainly dependent on the length of the heater. A very short heater permits heat to flow more readily to the support stems and cooling to occur between current peaks. However most commercial TEs have errors less than $10 \mathrm{ppm}$ at $20 \mathrm{~Hz}$ and some have errors less than $10 \mathrm{ppm}$ even at $5 \mathrm{~Hz}$.

In testing selected commercial TEs for the TVCs at audio frequencies we often find a small ac-dc difference (up to $4 \mathrm{ppm}$ ) which is independent of frequency but dependent on heater current. It ordinarily decreases at lower heater currents. When these TEs are used in a TVC the ac-dc difference will therefore be slightly voltage dependent. In the step-up test process, where the TVC is alternately used at 50 and 100 percent of rated current. this relatively small thermoelement error is introduced at every step of the process. If the process is a long one, as in the 12-resistor set, there could be a significant accumulation of error. TVCs in the new set are intercompared by testing each resistor once with one TE at reduced current ( 60 or $67 \%)$, and again with rated current on the other TE. If these TEs differ in ac-dc difference at these currents, there will again be an accumulation of error.

This is one of the advantages of the set with only six resistors. If the $20 . \mathrm{V}$ range is the starting point (it is probably the most stable), we have only four steps up to the $1000-\mathrm{V}$ range, and three steps down to the $\mathrm{I} \cdot \mathrm{V}$ range. The other advantages are that less time is required for the intercomparisons and there are fewer units to construct.
Intercomparison data for this set of TVCs are listed in table 3 as well as determinations made using TVC set No. 1. (Test methods are discussed in later sections of the paper). The middle ranges differ by 2 ppm or less even though the resist ance varies from 1200 to $12000 \Omega$. Since the resistance enters into the theoretical calculation of ac-dc difference, this agreement is an additional indication of accuracy.

Step-down tests show that the two lowest voltage ranges usually have a positive correction (i.e., more a-c than d-c voltage is required for a given $\mathrm{TE}$ output) of $5 \mathrm{ppm}$ or less at $50 \mathrm{kHz}$. There is no resistor in series with the TE at the $1 \mathrm{~V}$ range, and at the $2-\mathrm{V}$ range the $T E$ is a large part of the total impedance. These ranges are therefore affected more by the small reactance of the TE. The correçtions for these ranges are more likely to change if a TE is replaced than for the higher ranges. A step-down test from the middle range is therefore advisable when $\mathrm{TE}$ replacements are made.

The five 1000-V TVCs were compared with the $300-$ and $500-\mathrm{V}$ ranges of TVC set No. 1 and the $600-\mathrm{V}$ range of the new set No. 7. The corrections are listed in table 4 for 20 and $50 \mathrm{kHz}$ and all values are averaged.

TABLE. 3. $A C-D C$ difference

\begin{tabular}{|c|c|c|c|c|c|c|c|c|}
\hline \multirow{3}{*}{ TVC-a } & \multirow{3}{*}{ TVC-b } & \multirow{3}{*}{$\begin{array}{l}\text { Applied } \\
\text { voltage }\end{array}$} & \multicolumn{6}{|c|}{ AC.DC difference (ppm) } \\
\hline & & & \multicolumn{3}{|c|}{$20 \mathrm{kHz}$} & \multicolumn{3}{|c|}{$50 \mathrm{kHz}$} \\
\hline & & & $\delta_{11}-\delta_{11}$ & $\delta_{s}$ & $\delta$ & $\delta_{\prime \prime}-\delta_{\prime \prime}$ & $\delta_{x}$ & $\delta$ \\
\hline $\begin{array}{l}\text { F1 } \\
\text { F2 } \\
\text { F2 } \\
\text { F1-3 } \\
\text { F1-3 } \\
\text { F2-6 } \\
\text { F1-10 } \\
\text { F1-10 } \\
\text { F2-20 } \\
\text { F1-30 } \\
\text { F1-30 } \\
\text { F2-60 } \\
\text { F1-100 } \\
\text { F1-100 } \\
\text { F2-200 } \\
\text { F1-300 } \\
\text { F1-300 } \\
\text { F2-600 } \\
\text { F1-500 } \\
\text { F1-500 } \\
\text { F2-1000 }\end{array}$ & $\begin{array}{l}F 1 \\
F 2 \\
F 2-6 \\
F 2-20 \\
F 2-60 \\
F 2-200 \\
F 2-600\end{array}$ & $\begin{array}{r}1 \\
1 \\
2 \\
2 \\
3 \\
6 \\
6 \\
10 \\
20 \\
20 \\
30 \\
60 \\
60 \\
100 \\
200 \\
200 \\
300 \\
600 \\
500 \\
500 \\
1000\end{array}$ & $\begin{array}{l}-1 \\
-1\end{array}$ & $\begin{array}{r}-3 \\
-2 \\
-1 \\
-1 \\
0 \\
0 \\
-1 \\
-1 \\
-2 \\
-2 \\
-4 \\
-4 \\
-6 \\
-6\end{array}$ & $\begin{array}{r}-3 \\
-2 \\
+1 \\
0 \\
+1 \\
0 \\
+1 \\
0 \\
0 \\
-1 \\
-3 \\
-4 \\
-3\end{array}$ & $\begin{array}{l}-2 \\
-4\end{array}$ & $\begin{array}{r}+1 \\
+1 \\
0 \\
0 \\
+1 \\
+1 \\
-1 \\
-1 \\
-5 \\
-5 \\
+1 \\
+1 \\
+27 \\
+27\end{array}$ & $\begin{array}{l}-3 \\
-2 \\
-2 \\
-3 \\
+1 \\
+1 \\
+2 \\
0 \\
-2 \\
-6 \\
+4 \\
+1 \\
+30\end{array}$ \\
\hline
\end{tabular}


Table 5 lists intercomparison data for the same frequencies for seven pairings of the five 1000-V TVCs. Values for 600 and $1000 \mathrm{~V}$ do not differ significantly and so they are all averaged together.

Corrections $(\delta)$ for each range of the new TVC set are listed as determined in comparison with TVC Set No. 1. Intercomparison data $\left(\delta_{a}-\delta_{b}\right)$ are also listed and a computed correction $\left(\delta_{s}\right)$ based on the intercomparison, starting with the average of the measured ac-dc differences of the 10 - and $20-\mathrm{V}$ ranges. The TVCs are identified as the combination of a TE (F1 or F2) with the appropriate resistor; i.e., F2-600 forms the $600-\mathrm{V}$ range.

Averages of the corrections for the five $1000-\mathrm{V}$ TVCs listed in table 4 are shown in the circles in figure 4 , and the connecting lines show the intercomparison values from table 5 . The agreement between the various units is illustrated by this figure.

Each of the values listed in tables 4 and 5 is the average of at least four separate determinations. An analysis of the data taken in a series of 26 intercomparison tests (an average of 5 determinations with each one), involving all ranges at two frequencies and usually two voltages, showed an imprecision ( $3 \sigma_{a}$, where $\sigma_{a}$ is the standard deviation of the average of five determinations) of only $1.4 \mathrm{ppm}$.

TABLE 4. Ac-dc difference corrections for five 1000-volt TVC (A-E) The standard instruments are the 300 . and $500 . \mathrm{V}$ units of Set No. $\mathrm{I}$ and the $600 . \mathrm{V}$ range of Set No. 7.

\begin{tabular}{l|c|c|c|c|c|c}
\hline \hline Standard & Volts & A & B & C & D & E \\
\hline \multicolumn{6}{c}{ AC-DC difference at $20 \mathrm{kHz}(\mathrm{ppm})$} \\
\hline $500-1$ & 500 & & -20 & -9 & $\begin{array}{l}-4 \\
-4\end{array}$ & $\begin{array}{l}-12 \\
-10\end{array}$ \\
$600-7$ & 300 & & & & $\begin{array}{l}-2 \\
-12,-10 \\
-10\end{array}$ \\
Ave. & 600 & -3 & -22 & & $\begin{array}{r}-3 \\
-11\end{array}$ \\
\hline
\end{tabular}

AC-DC difference at $50 \mathrm{kHz}(\mathrm{ppm})$

\begin{tabular}{l|l|l|l|l|l|l}
\hline $500-1$ & 500 & +21 & -6 & +11 & +30 & -3 \\
$300-1$ & 300 & & & & +30 & -4 \\
$600-7$ & 600 & +30 & -9 & & +30 & -6 \\
& & +23 & & & +32 & -6 \\
& +25 & -7 & +11 & +30 & -5 \\
\hline
\end{tabular}

TABLE 5. Relative ac-dc differences for seven pairings of members of the five $1000 . \mathrm{V} \mathrm{TVCs}$

\begin{tabular}{|c|c|c|c|c|c|c|c|}
\hline Volts & $\delta_{\mathrm{A}}-\delta_{\mathrm{B}}$ & $\delta_{\mathrm{B}}-\delta_{\mathrm{C}}$ & $\delta_{\mathrm{C}}-\delta_{\mathrm{D}}$ & $\delta_{\mathrm{D}}-\delta_{\mathrm{E}}$ & $\delta_{\mathrm{E}}-\delta_{\mathrm{A}}$ & $\delta_{\mathrm{A}}-\delta_{\mathrm{C}}$ & $\delta_{\mathrm{A}}-\delta_{\mathrm{D}}$ \\
\hline \multicolumn{8}{|c|}{ AC-DC difference at $20 \mathrm{kHz}(\mathrm{ppm})$} \\
\hline $\begin{array}{l}600 \\
1000 \\
600 \\
1000 \\
\text { Ave. }\end{array}$ & $\begin{array}{l}-11 \\
-17 \\
-16 \\
-17 \\
-15\end{array}$ & $\begin{array}{l}+14 \\
+13 \\
+17 \\
+15\end{array}$ & $\begin{array}{r}+2 \\
0\end{array}$ & $\begin{array}{r}-7 \\
-6 \\
-10 \\
-7\end{array}$ & $\begin{array}{r}+7 \\
0 \\
+4\end{array}$ & $\begin{array}{l}+3 \\
+9\end{array}$ & $\begin{array}{r}0 \\
+5 \\
-2 \\
+5 \\
+2\end{array}$ \\
\hline \multicolumn{8}{|c|}{ AC-DC difference al $50 \mathrm{kHz}(\mathrm{ppm})$} \\
\hline $\begin{array}{l}600 \\
1000 \\
600 \\
1000 \\
\text { Ave. }\end{array}$ & $\begin{array}{l}-20 \\
-22 \\
-25 \\
-24 \\
-23\end{array}$ & $\begin{array}{r}+14 \\
+6\end{array}$ & $\begin{array}{r}+10 \\
+2\end{array}$ & $\begin{array}{l}-33 \\
-33 \\
-37 \\
-34\end{array}$ & $\begin{array}{l}+28 \\
+35 \\
+32\end{array}$ & $\begin{array}{l}-15 \\
-14 \\
-15 \\
-15\end{array}$ & $\begin{array}{l}+3 \\
+4 \\
+2 \\
+1 \\
+2\end{array}$ \\
\hline
\end{tabular}

\section{Thermal Compensation}

The coaxial connector between the TE and the series resistor provides a low-reactance connection for minimum frequency error. Tests and calculations show the error is less than $1 \mathrm{ppm}$ at $50 \mathrm{kHz}$. However, it also permits heat to flow freely from the resistor, which dissipates up to five watts on the $1000-\mathrm{V}$ range, to the TE which has a temperature coefficient of emf of approximately 0.2 percent $/{ }^{\circ} \mathrm{C}$ (at constant input current).

Heat conduction through the center conductor of this connector was reduced, at $300 \mathrm{~V}$ and higher, by replacing the two polystyrene insulators in the coaxial connectors (one in the resistor output and one in the TE input) with two made of boron nitride. This material is a good electrical insulator and yet it conducts heat well enough to provide an effective heat sink for this conductor. It is used in both ends of the high volt age resistors to improve the heat flow from the resistor to the relatively heavy brass casing. This also helps to reduce the temperature rise in the resistor.

Heat flow through the outer part of these connectors will gradually raise the temperature of the TE casing, and this also causes a heating drift. The increase in temperature of the TE bulb is slowed by mounting it in a short section of brass tubing to increase the thermal mass and this tube is thermally insulated from the TE casing. Thermal compensation is also added by attaching a $10-\Omega$ thermistor to the tube containing the TE and connecting it in series with the TE output. A resistor appropriately chosen to complete the compensation circuit (see Fig. 7 and appendix I) will draw current through the thermistor sufficient to compensate for the temperature increase.

This resistor value may be computed for TEs with average temperature coefficients as in appendix $I$. However, temperature coefficients differ considerably and, for best results, it is usually necessary to match the resistor to the $\mathrm{TE}$ and thermistor combination. $\mathrm{A}$ suitable value can be found by substituting a variable resistor outside the $\mathrm{TE}$ casing and adjusting it for minimum warmup drift on a $1000-\mathrm{V}$ range. However, if the TVC is to be used with a TE comparator similar to the one to be described, it will frequently be connected
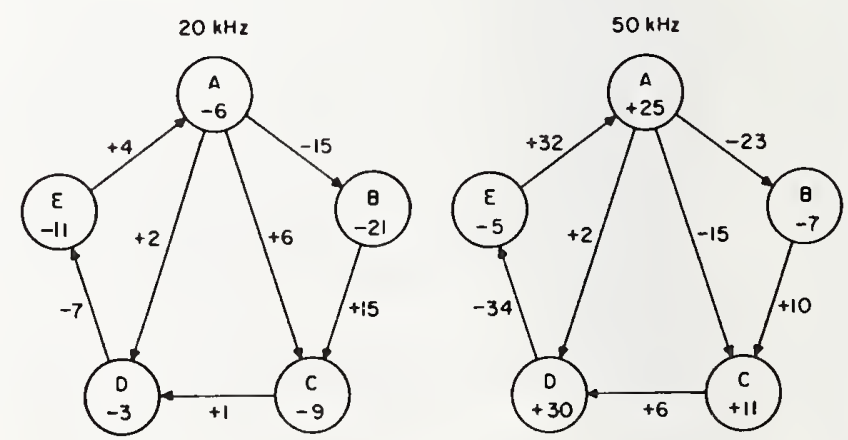

FigURE 4. Intercomparisons of 1000-volt TVCs.

Ac-de difference corrections (pI,m) from table 4 for five 1000-V TVCs are shown in the circles and the relative differences from table 5 are shown on the interconnecting lines. The algebraic difference between any pair shuuld be equal to the figure on the connecting line within experimental errur. 
across a $1000-\Omega$ divider, and this will affect the compensation by about 20 percent. A value about 10 percent higher than the one chosen experimentally should provide a satisfactory compromise and reduce the drift by a factor of five or more. With the compensation and the large thermal time constant of the TE in its casing, the drift can be reduced to less than $5 \mathrm{ppm} /$ minute under typical laboratory condition. The error from this is almost completely eliminated by taking a sequence of readings with a-c, d-c, reversed d-c, and a-c voltage applied, as described in the next section.

\section{TVC Test Methods}

A brief review of other comparison methods may be useful before describing the TE comparator. Figure 5 shows two TVCs connected in parallel. The separate balancing circuits (or Lindeck Potentiometers) (B) and null detectors (D) may or may not be built in. Stable a-c and $d-c$ voltage supplies are connected to the two TVCs by the ac-dc switch. The switching arrangement shown is typical and convenient, although others, such as reversing the d-c supply by a separate switch, are satisfactory. The d-c supply must have an ungrounded output and it should be possible to ground either terminal as the voltage is reversed. The reversed d-c differences of the TVC can rarely, if ever, be neglected in these tests.

For convenience and clarity, we shall call one TVC the "standard" and the other the "test." Normally the one with known ac-dc difference corrections is the standard, and the test TVC is usually treated differently in the test procedures, as explained below.

There are two procedures for this intercomparison. One might be called a null-balancing method. With a-c voltage applied to both TVCs, the balancing circuits of the test and standard instruments are adjusted to null the detectors. The d-c supply is then switched to the TVCs, and the voltage is adjusted to obtain a null balance first on the detector of the test instrument and then on the standard. Each d-c voltage is carefully measured, and the small difference between the two should equal the difference between the a-c voltage and

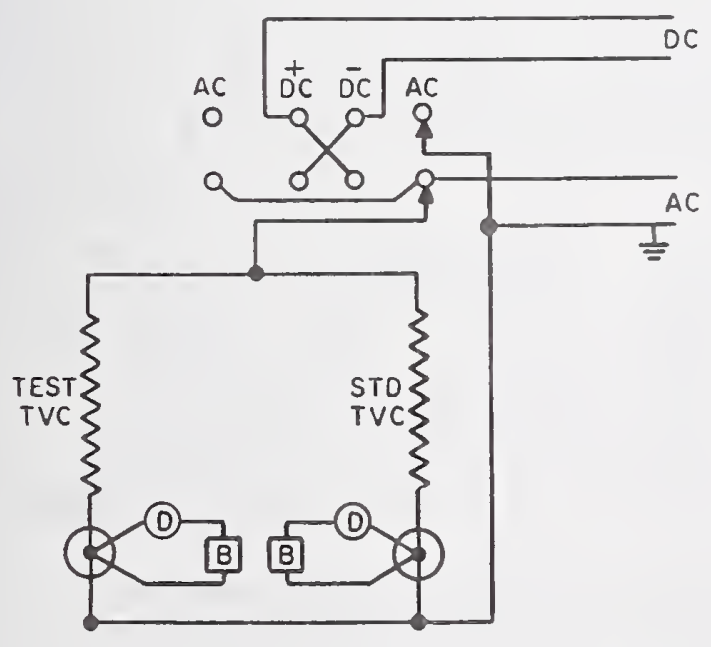

Ficure 5. Tuo-Potentiometer method for TVC comparisnns. one direction of $d-c$ voltage. The measurement is then repeated with reversed $d-c$ voltage, and an average is taken.

This method is tedious and time consuming, an accurate $d-c$ voltage standard is required, and if there is any drift in either instrument output due to warmup or other reasons, as there very often is, the dat a may lack both accuracy and precision.

A more satisfactory procedure and one that will minimize the effect of drift may be called a deflection method. Both balancing circuits are adjusted to near null, and then $\mathrm{a} \cdot \mathrm{c}$ and $\mathrm{d}-\mathrm{c}$ voltages are applied in the succession indicated in figure 5 (AC, DC+, DC-, AC). For each input the power supply is adjusted to set the test instrument detector to null. The deflection of the standard TVC detector is read for each setting, and the difference between the average reading on a-c voltage and d-c voltage is an indication of relative ac-dc difference. If the readings are made at nearly equal time intervals, and if the drift rate is reasonably constant (even though fairly large), the determinations can be made with satisfactory precision.

If the detector of the standard TVC is a galvanometer the reading may be in divisions or millimeters, but they can be converted to volts or percent ac-dc differences by calibrating the detector scale in one of several ways. A calibration factor can be determined simply and directly by making a small measured change in the d-c input and observing the detector response.

If the detector is a microvoltmeter and if the balancing circuit is a Lindeck potentiometer indicating the millivolt output of the thermoelement, the calibration step can be eliminated. With the four deflection readings on the detector the difference between the average reading on a-c voltage $\left(E_{11}\right)$ and those on d-c voltage $\left(E_{t}\right)$ is computed from the differences of the corresponding readings of the microvoltmeter. The ac-dc difference of the test instrument is

$$
\delta_{t}=\delta_{s}+\frac{E_{s a}-E_{s d}}{n_{s} E_{s d}}
$$

where the subscript $s$ indicates that these values come from the standard instrument, and $\delta_{s}$ is the ac-dc difference correction for the standard. ${ }^{5}$

The factor $n$ relates small changes in thermoelement heater current $(\Delta I)$ (at a fixed frequency) to corresponding changes in output emt $(\Delta E / E=n \Delta I / I$ approximately). For a TVC we may substitute $n \Delta V \mid V$ for the right hand expression, so that $n=V \Delta E / E \Delta V$. The value of $n$ is 2 if the thermoelement has a square law response. possibly at very low heater currents, but it is usually 1.7 to 1.9 at rated current. Determinations of $n$ should therefore be made in a $d \cdot c$ test at five or more current levels by measuring the values indicated. A plot of $n$ against $E$ can be made so that values corresponding to any emf can be found for substitution in the equation above (see sec. 8 ). 


\section{TE Comparator}

There are several variations of basic comparator circuits which are used to minimize the difficulty caused by power supply instability $[1,3]$. They employ a voltage divider circuit to which two emfs are connected. The divider is adjusted to null a detector, and at this point the divider setting corresponds to the ratio of the emfs. If one emf $\left(E_{t}\right)$ is held constant as a-c and then $d-c$ voltage is applied to the TVCs, the other emf $\left(E_{s}\right)$ will change if there is a relative ac-dc difference in the TVCs at the frequency $\left(\delta_{t} \neq \delta_{s}\right)$. The resulting unbalance in the divider will produce a change in the detector deflection proportional to the ac-dc difference. However, small fluctuations in the power supply will produce nearly equal proportional changes in the emfs and therefore the detector will not be affected appreciably. The stabilizing effect depends on how well matched the time constants and response characteristics of the TEs are, but the effect usually affords a significant advantage. Also, the monitored emf need not be held constant so exactly as with a simple balancing circuit.

In the new comparator (fig. 6) the higher of the two emfs is connected across a $1000-\Omega$ Kelvin-Varley divider, and the lower one is connected to the variable tap. The detector is brought to null by adjusting the divider. A Lindeck potentiometer and the same detector (with key $k_{1}$ ) is used to monitor the test TVC output $\left(E_{t}\right)$. The detector is labeled " $N$ " in the diagram because the use of a nanovoltmeter is suggested.

The test procedure is similar to that already mentioned. Preliminary settings of the potentiometer and divider are first made so the readings of the nanovoltmeter will fall near mid-scale. Then with a-c voltage applied, the key $k_{1}$ (labeled "SET" on the instrument, figs. 8 and 9) is depressed and the appro-

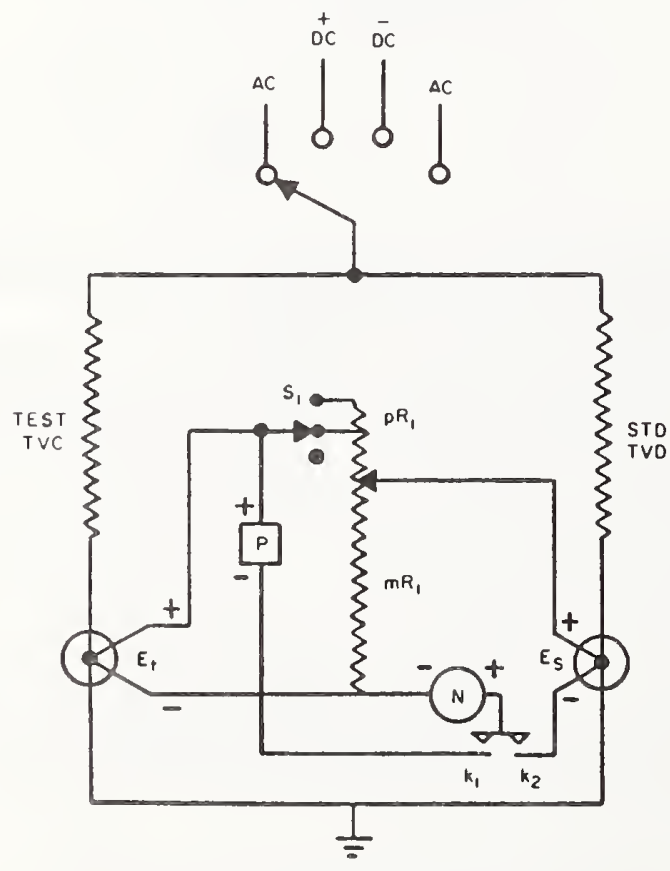

Figure 6. New TE comparator. priate power supply is adjusted for a null on the detector. Key $k_{2}$ (READ) is then depressed and the detector indication is read. The two-step procedure is repeated for two directions of $d \cdot c$ voltage and for a second a-c input. The average indication in nanovolts ${ }^{6}\left(N_{a}\right)$ for a-c input and the average for d-c input $\left(N_{d}\right)$ is computed and the ac-dc difference of the test instrument is

$$
\delta_{t}=\delta_{s}+\frac{N_{d}-N_{t}}{n_{s} E_{s}} \quad \text { (appendix II eq 5) }
$$

Figure 6 shows the test TVC output $\left(E_{\ell}\right)$ connected to the high emf terminal ( $\mathrm{E}_{H}$ in fig. 8). If $E_{t}$ is smaller than $E_{s}$ it will be necessary to interchange them, but this is a minor inconvenience. If $E_{l}$ is connected to the $E_{1}$. terminal the potentiometer is switched to that input with switch $S_{2}$ (fig. 8) and $E_{t}$ is monitored as before. The same switch also reverses the polarity of the detector, and therefore the sign of the indication. The slightly modified equation for $\delta_{t}$ is eq 11 in appendix II.

A galvanometer may also be used as a detector but since the circuit resistance is relatively high a photoelectric amplifier is necessary for sufficient sensitivity. The scale must be linear over the portion to be used and a scale calibration is also necessary to relate the readings in divisions or millimeters to a voltage change. The calibration can be made quite easily however by moving switch $S_{1}$ from READ to CAL with $k_{2}$ closed and observing the resulting deflection change. This increases the resistance of the $1000-\Omega$ divider by $0.5 \Omega$ and changes the divider current by 0.05 percent. The

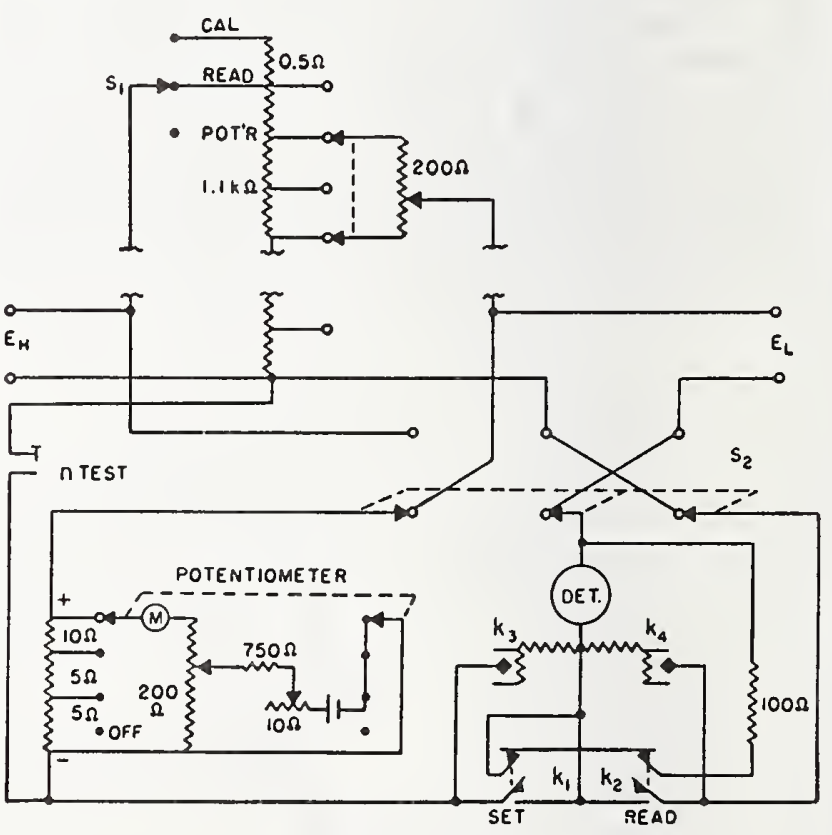

Figure 8. New TE comparator. 


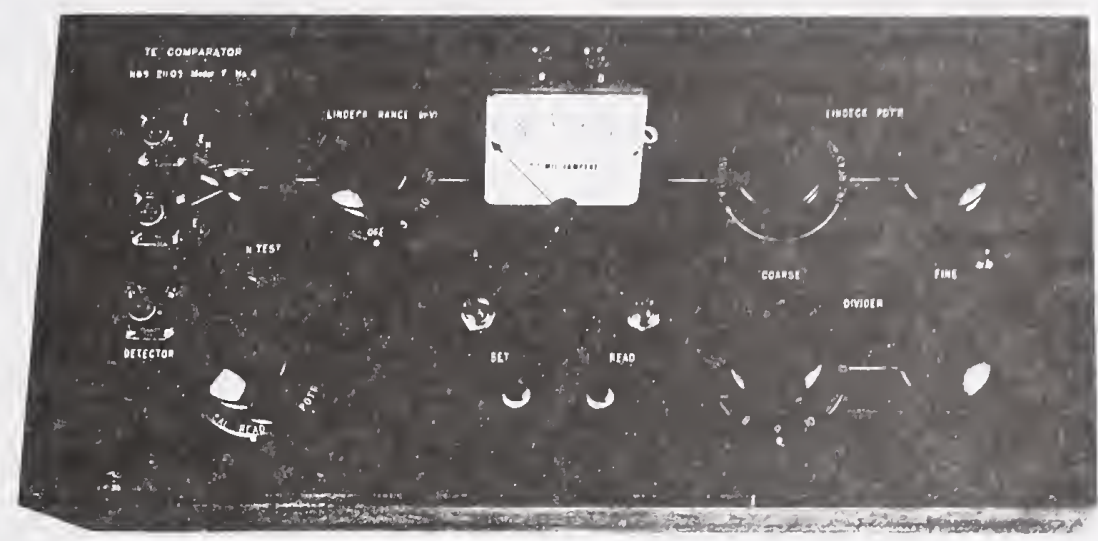

Figure 9. Photograph of TE comparator.

voltage applied to the galvanometer circuit is changed by the same amount and the deflection change $D_{1}$ is used in eq 10 and 12 in appendix 2 to determine $\delta_{t}$. A measurement of $E_{s}$ is made only to find the corresponding value of $n_{s}$ from a plot as before.

\section{8. $n$ Tests}

Measurements of $n$ are conveniently made with the comparator. The thermoelement to be tested is connected to the $E_{\mathrm{L}}$ input and the $\mathrm{E}_{\mathrm{H}}$ input is unused. $\mathrm{A}$ key labeled " $n$ TEST" (fig. 6) is locked down and switch $S_{2}$ is moved to $E_{H}$ connecting the potentiometer voltage across the divider. The divider is set arbitrarily but preferably near full range and the potentiometer is adjusted to null the detector. The input voltage is then changed by a small measured amount $\Delta V / V$, and the resulting change in TE output $(\Delta E)$ is measured by observing the change in indication of the detector (nanovolts or microvolts). ${ }^{7}$ Switch $S_{2}$ is then moved to $E_{\mathrm{I}}$ and a measurement of TE output $(E)$ is made with the potentiometer. The values for each factor of the equation given above $(n=V \Delta E$ ) $E \Delta V)$ are then known and $n$ is calculated from this equation.

If a galvanometer is used as the detector a measure. ment of $D_{1}$ is made as described above, and the deflection change resulting from the measured change in input voltage $(\Delta V)$ is designated $D_{2 .}$. Then $n=0.05$ $D_{2} / q D_{1}$, where $q$ is the percentage change in input voltage $(100 \Delta V / V)$.

Determinations of $n$ need be accurate only to a few percent, and, since they are quite stable, retesting is nut usually necessary.

\section{AC Voltage Measurements}

As suggested earlier, the comparator may be used with one TVC to measure an a-c voltage such as the

: A sntall change in the input to a TVC can be measured with a volt bux and potenti. ometer or by a setting with a calibrated $d \cdot c$ volteage supply. It is also convertient lo use resistor with a shorting switch in series with a TVC: to introduce a small currett rhange

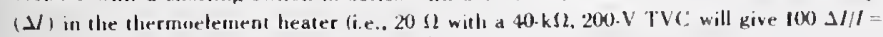
0.05 percent which is equivalent $11,100 \Delta V / V)$. input to a digital voltmeter or the output of a calibrated a-c voltage supply. Switch $\mathrm{S}_{1}$ has a third position marked "POT'R" which disconnects the divider from the $E_{H}$ input. If a TVC output is connected to $E_{H}$, and switch $\mathrm{S}_{2}$ is moved to that input, the potentiometer and detector can be used to measure the TVC output and changes in it. The circuit is as figure 5 without the test TVC. The d.c voltage is accurately measured and nom. inally equal to the a-c voltage.

It is suggested that $\mathrm{d}$-c voltage be applied to the TVC first and a detector reading taken. Then, at nearly equal time intervals, a second reading is taken with a-c volt. age and a third with reversed $d-c$ voltage. The differ. ence between the average of the readings with a $d-c$ voltage $\left(E_{d}\right)$ and the one with a-c voltage $\left(E_{a}\right)$ is related to the input voltage difference very much as before.

$$
V_{\mathrm{ac}}=V_{\mathrm{dc}}\left(1+\frac{E_{\mathrm{ad}}-E_{\mathrm{d}}}{n E}+\delta_{s}\right)
$$

With a nanovoltmeter the emfs are read directly, but if the detector is a galvanometer a scale sensitivity factor must be determined.

\section{Design Details}

The case of the TE comparator has thermal insulation and electrostatic shielding, and the comparator is carefully built to minimize thermal emfs and contact resistances. The switch decks of the Kelvin-Varley divider and $S_{1}$ and $S_{2}$ have enclosed sitver contacts and the keys $k_{1}$ and $k_{2}$ are of a low-thermal type. The back contacts on these keys connect a $100-\Omega$ shunt across the detector to reduce sensitivity and noise until one key or the other is depressed. The fine control for the divider is a ten-turn helical resistor with a special lowthermal sliding contact. The resolution of the uncatibrated divider is not quite sufficient for setting an exact null on the detector, but selting within a few divisions is adequate in the ordinary use of the instrument. 


\section{Conclusion}

The use of one resistor unit for two TVC voltage ranges has reduced by about half the time required, over previous TVC sets, to make a complete intercomparison test. Changes in any member of the set are readily detected since frequent intercomparison tests are more feasible.

The TE comparator combines the stabilizing feature of other comparators with much of the simplicity of the single Lindeck potentiometer or balancing circuit. It is relatively inexpensive to build and calculations are simplified and direct, especially where the detector is a nanovoltmeter. Repeated tests have shown that the imprecision is less than $2 \mathrm{ppm}$ and ac-dc transfer accuracies, with the TVC set, are $10 \mathrm{ppm}$ at audio frequencies and $20 \mathrm{ppm}$ up to $50 \mathrm{kHz}$.

This work was supported in part by the Army Metrology and Calibration Center, Redstone, Alabama. Their encouragement and support are gratefully acknowledged. The author also acknowledges the help of C. B. Childers and A. G. Perrey who made many of the exacting measurements described here.

\section{References}

[1] Hermach, F. L. and Williams, E. S., Thermal converters for audiofrequency voltage measurements of high accuracy, IEEE Transactions on Instrumentation and Measurement, IM-15, No. 4, 260-268, (Dec. 1966).

[2] Hermach, F. L. and Williams, E. S., Thermal voltage converters for accurate voltage measurements to 30 megacycles per second, Trans. AIEE (Communication and Electronics), 79, Pt. I, 200-206, (July 1960).

[3] Turgel, Raymond S., A comparator for thermal ac-de transfer standards, ISA Transactions, 6, No. 4, 286-292, (1967).

\section{Appendix I}

The temperature coefficient of emf, $\beta$, of the average $\mathrm{TE}$ is about -0.2 percent $/{ }^{\circ} \mathrm{C}$, with constant input current. Thus a corresponding decrease in the resistance, $R=R_{c}+R_{t}+R_{s}$ (see fig. 7) is required to maintain a constant voltage across the resistor $R_{s}$. For a 1 degree increase in temperature, the

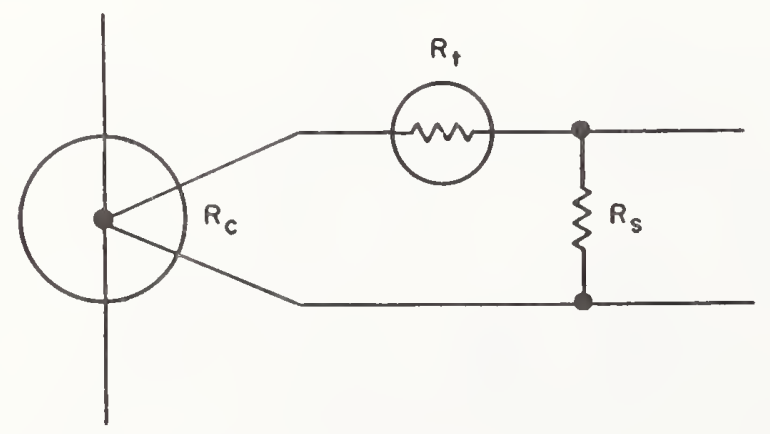

Figure 7. Thermal compensation for TE.

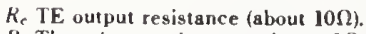

$R_{t}$ Thermistor resistance (about $10 \Omega$ )

$R$. Series resistor to complete compensation circuit. $\left(R_{e}\right.$ and $R$, have negligible temperature coefficients.) change in thermistor resistance, $\Delta R_{t}=\alpha R_{t} / 100$ where $\alpha$ is the temperature coefficient of the thermistor in percent $/{ }^{\circ} \mathrm{C}$-typically about -4 percent $/{ }^{\circ} \mathrm{C}$. Therefore, for thermal compensation,

$$
\begin{gathered}
\beta=100 \Delta R_{t} / R=\alpha R_{t} / R \\
\text { and } R=\alpha R_{t} / \beta
\end{gathered}
$$

With the typical values listed we find $R=200 \Omega$, and, subtracting $R_{c}$ and $R_{t}$, the value for $R_{s}$ is $180 \Omega$.

\section{Appendix II}

\section{Development of Equations ${ }^{8}$}

The ac-dc difference of a TVC is defined as

$$
\delta=\frac{V_{a}-V_{d}}{V_{d}}
$$

where $V_{a}$ is the a-c voltage and $V_{d}$ the average of the two directions of dc-voltage required to produce the same output emf. In the circuit of figure 6 the a-c and $\mathrm{d}$-c voltages are adjusted to give the same emf $E_{t}$, of the TVC under test, as indicated by a null on the detector $N$ with $\mathrm{k}_{1}$ closed. The same voltages are applied to the standard TVC. Then,

$$
V_{a}=V_{d}\left(1+\delta_{t}\right)=V_{d}^{\prime}\left(1+\delta_{s}\right)
$$

where $V_{d}^{\prime}$ is the d-c voltage required to produce the same output emf of the standard as $V_{a}$, and the subscripts, $t$ and $s$, refer to the test and standard instruments respectively. If $\frac{V_{d}^{\prime}-V_{d}}{V_{d}}<<1$, then, closely
enough

$$
\delta_{t}-\delta_{s}=\frac{V_{d}^{\prime}-V_{d}}{V_{d}} .
$$

From the definition of $n$ given in the paper

$$
\delta_{\ell}-\delta_{s}=\frac{E_{s a}-E_{s d}}{n_{s} E_{s d}}
$$

With the polarities as shown in figure 6 ,

$$
N=m E_{t}-E_{s}
$$

where $N$ is the detector voltage with $\mathrm{k}_{2}$ closed and $\mathrm{k}_{1}$ open, and $m$ is the divider ratio (with the detector resistance $R_{d}>>R_{1}$ ).

Thus.

$$
\delta_{t}=\delta_{s}+\frac{N_{d}-N_{a}}{n_{s} E_{s d}}
$$

where the subscript $a$ and $d$ have the same meaning as before.

- These equations were developed by F. L. Hermach. 
If a galvanometer is used as the detector, the current through it with $k_{2}$ closed is, by Thevenin's Theorem,

$$
I_{g b}=\frac{m E_{t}-E_{s}}{R_{e}}
$$

where $R_{e}$ is the resistance of the galvanometer circuit with $E_{t}$ and $E_{s}=0$. With $E_{\ell}$ and $E_{s}$ held constant and $p R_{1}$ inserted by means of $\mathrm{S}_{1}$,

$$
I_{g c}=\frac{m E_{t} /(1+p)-E_{s}}{R_{e}^{\prime}}
$$

where $R_{e}^{\prime}$ is the resistance of the galvanometer circuit.

Since $m E_{t}-E_{s}<<E_{s}$ and $p<<1$, we have to a sufficient degree of approximation,

$$
I_{g b}-I_{g c}=\frac{E_{s} p}{R_{e}}=\frac{\left(D_{b}-D_{c}\right)}{S}=\frac{D_{1}}{S}
$$

where $D_{b}$ and $D_{c}$ are the resulting galvanometer deflections and $S$ is the galvanometer current sensitivity.

In the ac-dc test, with $m E_{t}$ constant,

$$
I_{g a}-I_{g d}=\frac{E_{s d}-E_{s a}}{R_{e}}=\frac{D_{a}-D_{d}}{S}
$$

Thus, from (3), (8) and (9),

$$
\delta_{t}=\delta_{s}+\frac{p\left(D_{d}-D_{a}\right)}{n_{s} D_{1}}
$$

The test and standard TVCs may be interchanged if $E_{t}<E_{g}$. If $E_{t}$ is applied to the $\mathrm{E}_{\mathrm{L}}$ in put of figure 8 and held constant, with the detector and potentiometer connected as shown in the figure, a similar analysis leads to the following equations:

$$
\delta_{\ell}=\delta_{s}+\frac{N_{d}-N_{a}}{n_{8} E_{\ell}}
$$

and

$$
\delta_{t}=\delta_{s}+\frac{p\left(D_{d}-D_{a}\right)}{n_{s} D_{1}}
$$

The characteristic $n$ is determined from

$$
n=\frac{\Delta E / E}{\Delta V / V}
$$

by applying known changes in input voltage and observing the changes in output emf with a high resistance voltmeter, as described in the text. If a galvanometer is used as the detector instead of the voltmeter its sensitivity is determined by inserting $p R_{1}$ as described. From equations similar to (8) and (9)

$$
\frac{\Delta E_{s}}{E_{8}}=\frac{p \Delta D}{D_{1}}
$$

If the thermocouple resistance of the test TVC, $R_{t c}$, is significant, $p$ in equation (7) should be replaced by $p^{\prime}$, the fraction of $R_{1}+R_{t c}$ inserted by $\mathrm{S}_{1}$ * Similarly if the potentiometer resistance $R_{p}$ is significant, $p$ in equation (14) should be replaced by $p^{\prime \prime}$, the fraction of $R_{1}+R_{p}$ inserted by $\mathrm{S}_{1}$.

(Paper 75C3\&4-321)

* with a high impedance detector. With

a galvanometer the formula for $\delta_{t}$ is too complicated to be useful. 


\title{
Thermal Voltage Converters for Accurate Voltage Measurements to 30 Megacycles Per Second
}

\author{
F. L. HERMACH \\ MEMBER AIEE
}

Synopsis: Thermal voltage converters, each consisting of a resistor in series with a thermoelement in a coaxial line, have been developed for measurements of rms voltages of 1 to 200 volts at frequencies from $3 \mathrm{cps}$ (cycles per second) to $30 \mathrm{mc}$ (megacycles). An accuracy of $0.1 \%$ or better may be obtained by a-c-d-c transfer techniques up to at least $10 \mathrm{mc}$ and $0.2 \%$ at $30 \mathrm{mc}$.

\footnotetext{
A THERMAL VOLTAGE converter, according to ASA Standards, ${ }^{1}$ is a thermoelement of low current input rating with an associated series impedance or transformer, such that the emf (electromotive force) developed at the output terminals gives a measure of the voltage applied to the input terminals. Thermal voltage converters containing thermo-
}

elements in series with wire-wound re-

\author{
E. S. WILLIAMS \\ NONMEMBER AIEE
}

sistors can be used to make highly accurate voltage measurements at audio and ultrasonic frequencies..$^{2,8}$ The frequency range of such voltage converters is limited primarily by the residual reactances of their wire-wound resistors, but tests have indicated that for some of them good performance might be expected to frequencies approaching $1 \mathrm{mc}$.

This paper describes thermal voltage converters of low and computable reactance that have been developed at the National Bureau of Standards to meet the need for determining the frequency limit of such instruments. Single-range converters with depositedcarbon resistors have been constructed with ranges of 1 to 200 volts. Each has a frequency influence less than $0.1 \%$ to $10 \mathrm{mc}$ and less than $0.4 \%$ at $30 \mathrm{mc}$. These rms voltage converters may also be used to calibrate $1 / 2 \%$ r-f (radio-frequency) thermocouple voltmeters which are now commercially available, and, with sine wave generators, could be used to calibrate electronic voltmeters as well.

\section{Description and Construction of Converters}

The r-f thermal voltage converters make use of the transfer principle, ${ }^{2}$ in which a direct voltage is substituted for the alternating voltage to be measured. The direct voltage is adjusted to give the same output emf of the thermoelement that was obtained with the alternating

Paper 60-135, recommended by the AIEE Indicating and Integrating Instruments Committee and approved by the AIEE Tecbnical Operations Department for presentstion at the AIEE Winter General Meeting, New York, N. Y.o Jenuars 31February 5, 1960. Manuscript submitted October 30, 1959: made available for printing December 9 . 1958.

F. L. Hermact and E. S. Wrulaus are with tbe National Bureau of Standards, Weshington, D. C.

The authors misb to acknowledge the help of $R$. Richardson and J. Scofield, who cbecked the equations and carried out the computation wbich made possible Pig. 4 and the last column of Table II, and of J. B. Hill, who assisted with some of the measurements. Thanks are also extended to $M$ C. Selby and L. Behrent for their careful calibration of the 1-volt converter. 
$\mathrm{F}$

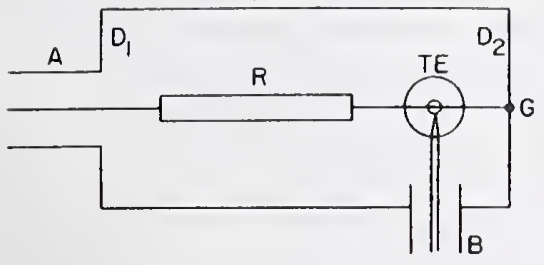

Fig. 1. Thermal voltage converter

$R=$ Film-type resistor $1 / 8$ in. diameter, 2 in. long

$\mathrm{TE}=$ Thermoelement

$A=$ Codxial input connector, GR874PB

$B=$ Two-pin output connector, AN10SL

$D_{1}, D_{2}=$ Circular brass disks, $3 / 16$ in. thick, secured to $F$ with 4 2-56 bross screws

$F=$ Cylindrical brass shield, 2 in. diameter, 4.25 in. lons

$G=$ Cross wire at center of $D_{2}$ (see text)

Note: Plane of thermocouple leads at right angles to axis of $F$

voltage applied, and is measured with a potentiometer or other suitable means. Thus only good short-time stability, high precision of reading, and small known frequency influence are required of the transfer standard.

Each converter consists of a cylindrical deposited-carbon resistor in series with a uhf (ultrahigh frequency) thermoelement having a short straight heater in line with its supports, coaxially mounted in a brass cylinder, as shown schematically in Fig. 1. The residual reactances are much smaller than those of wire-wound resistors, and can be computed at least approximately, so that the frequency errors can be estimated. The frequency errors of the uhf thermoelements as current converters are believed to be small to over $100 \mathrm{mc}$.

The dimensions of the converters and additional data are given in the caption of Fig. 1. Each converter is assembled by

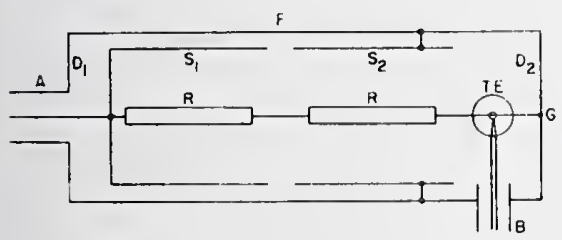

Fig. 2. Voltage converter with compensating shields

$\mathrm{S}_{1}=$ Cylindrical brass shield, 2 irı. diameter with one brass end-plate

$\mathrm{S}_{2}=$ Cylindrical brass shield, 2 in. diameter both ends open, axially adjustable (see text)

Other symbols same as Fig. 1 except that $F$ is $2.5 \mathrm{in}$. diameter and $7.5 \mathrm{in.}$ long

Note: Plone of thermocouple leads at right ongles to axis of $F$ first soldering the resistor, $R$, to the thermoelement, $T E$, and to the coaxial in put connector, $A$, which is mounted in disk, $D_{1}$. This disc is then fastened to the cylinder, $F$, and the two thermocouple leads are soldered to the 2-pin output connector, $B$. Disk $D_{2}$, which has a small hole with a cross wire at $G$, is then fastened to $F$ and the free end of the heater of the thermoelement is soldered to the cross wire. For clarity both thermocouple leads are shown in the figure. However the plane of the thermocouple leads and the pins of the output connector are actually at right angles to the axis of the cylinder so that minimum emf is induced in the output circuit. In use $F$ must be grounded through the input connector.

The 100- and 200-volt converters contain two resistors and two cylindrical inner shields, $S_{1}$ and $S_{2}$ as shown in Fig. 2 , to reduce the effect of the distributed capacitance from the resistors to the outer cylinder. This considerably extends their frequency range. Shield $S_{2}$ is centered in $F$ by a narrow ring, and is fastened to $F$ by three equally spaced screws. Slots in $F$ make axial adjustment of $S_{2}$ possible. The end-plate of $S_{1}$ is soldered to the input lead and to the resistor in assembly and this shield is fastened to $D_{1}$ by small insulators. The assembly is otherwise similar to that described for the other converters. The 50 -volt converter has a single resistor and a fixed inner shield like $S_{1}$ in a cylinder of the dimensions given in the caption of Fig. 1.

Additional information on the voltage converters is given in the first three columns of Table I. The commercially available deposited carbon resistors are $1 / 8$ inch in diameter and 2 inches long, and are vacuum sealed within $83 / 16$-inchdiameter glass cylinder. Some resistors of a different construction showed unaccountable a-c-d-c errors above $10 \mathrm{mc}$ when used in earlier converters. The 5and 10-ma uhf thermoelements, each with colinear supports and heater wire, are also commercially available, with an output voltage of about $7 \mathrm{mv}$ (millivolts) at rated current. Each is in an acornshaped evacuated glass bulb, and has a small bead between the heater and hot junction of the thermocouple to insulate them electrically but not thermally.

A Lindeck potentiometer rather than a millivoltmeter is connected to the output emf of the converter to provide high resolution. A circuit diagram is shown in Fig. 3. The potentiometer has ranges of $0.15,0.75,7.5$, and $15 \mathrm{mv}$. The two lowest ranges are used for sensitivity checks. With a galvanometer having a

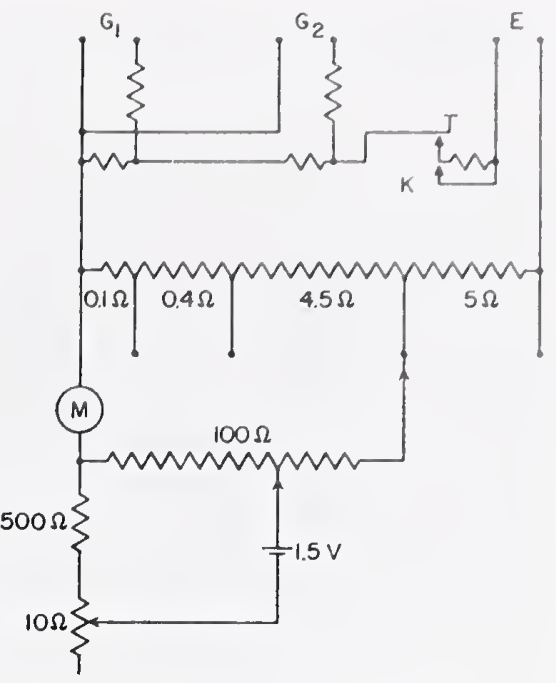

Fig. 3. Circuit of Lindeck potentiometer

$M=1.5-$ ma d-c milliammeter

$\mathrm{K}=$ Thermofree key

$E=E m f$ terminals, AN10SL

$\mathrm{G}_{1}, \mathrm{G}_{2}=$ Golvonometer terminals, AN10SL

Note: All fixed resistors of manganin, accuracy $+0.2 \%$

sensitivity of $200 \mathrm{~mm}$ (millimeter)/ $\mu \mathrm{a}$ (microampere) it provides a selected resolution of either 2 or $10 \mu \mathrm{v}$ (microvolts) per $\mathrm{mm}$ deflection. The potentiometer is completely shielded, and a 2-conductor shielded cable connects the output of the converter to the terminals marked $E$ in the figure. The advantages of this potentiometer are its low thermal emf (less than $1 \mu \mathrm{v}$ ) and its freedom from drift (much less than $0.01 \% /$ minute under ordinary laboratory conditions).

\section{Theory}

Because of the geometry, the resistor, $R$, in the cylinder of each low-voltage converter (without inner shields) may be represented as a transmission line of length, $\ell$, with a uniformly distributed series impedance of $z$ ohms per unit length and a uniformly distributed shunt ad-

Table I. Voltage Converters

\begin{tabular}{|c|c|c|c|}
\hline \multirow[b]{2}{*}{$\begin{array}{l}\text { Rated } \\
\text { Voltage, } \\
\text { Volts }\end{array}$} & \multicolumn{2}{|c|}{ Thermoelement } & \multirow[b]{2}{*}{$\begin{array}{l}\text { Series } \\
\text { Recistor, } \\
\text { Kilobms }\end{array}$} \\
\hline & $\begin{array}{c}\text { Rated } \\
\text { Curreat, } \\
\mathbf{M a}\end{array}$ & $\begin{array}{c}\text { Heater } \\
\text { Resistance, } \\
\text { Obms }\end{array}$ & \\
\hline $\begin{array}{r}1 \ldots \\
3 \ldots \\
5 \ldots \\
10 \ldots \\
20 \ldots \\
50 \ldots \\
100 \ldots \\
200 \ldots\end{array}$ & $\begin{array}{ll}\ldots & 5 \ldots \\
\ldots & 5 \ldots \\
\ldots & 5 \ldots \\
\ldots & 5 \ldots \\
\ldots & 10 \ldots \\
\ldots & 10 \ldots \\
\ldots & 10 \ldots \\
\ldots & 10 \ldots\end{array}$ & $\begin{array}{l}\ldots .90 \ldots \ldots \\
\ldots .90 \ldots \ldots \\
\ldots \ldots 90 \ldots \ldots \\
\ldots \ldots 90 \ldots \ldots \\
\ldots \ldots 25 \ldots \ldots \\
\ldots \ldots 25 \ldots \ldots \\
\ldots \ldots 25 \ldots\end{array}$ & $\begin{array}{ll}\ldots & 0.2 \\
\ldots & 0.5 \\
\ldots & 1 \\
\ldots & 2 \\
\ldots & 2 \\
\ldots & 5 \\
\ldots 10^{*} \\
\ldots & 20^{*}\end{array}$ \\
\hline
\end{tabular}

* Two equal resistors in configuration of Fig. 2. 


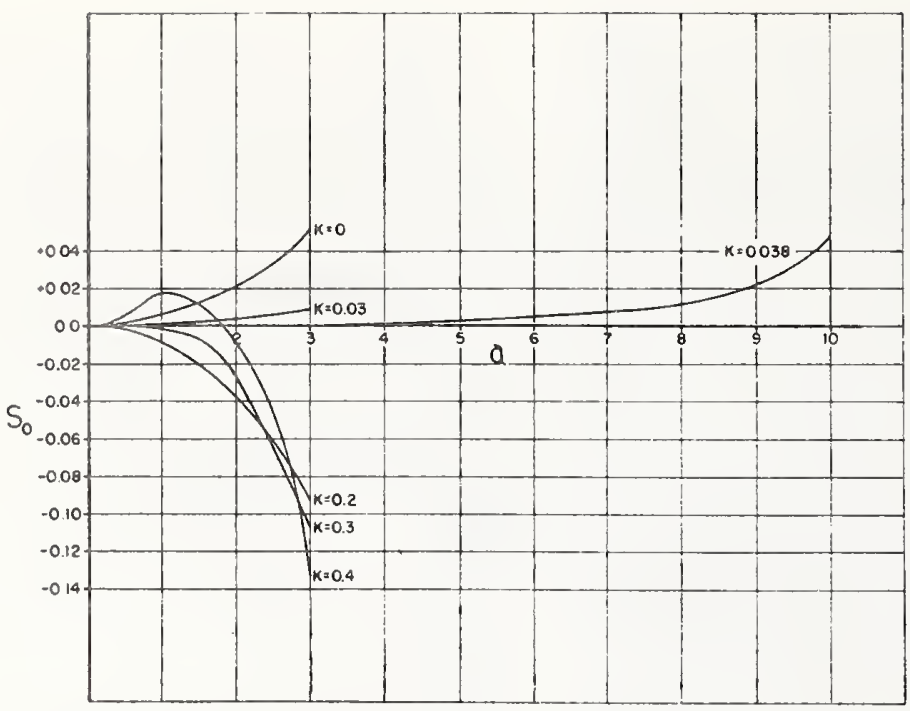

Fig. 4. Computed a-c-d-c difference as a function of the parameters and k

mittance of $y$ mhos per unit length. The line is terminated by the heater of the thermoelement. We are interested in the magnitude of the transimpedance $Z_{c}=V_{b} / I_{o}$ where $V_{1}$ is the input voltage being measured and $I_{0}$ is the current through the heater. If the heater resistance, $R_{h}$, is much less than that of the resistor, $R$, we may to a first approximation consider this a short-circuited line. It will be apparent later that this is not a drastic approximation at low frequencies since we are concerned only with the effect of the line reactance on $Z_{c}$. Then by ordinary steady-state transmission-line theory,

$Z_{c}=\frac{Z}{\sqrt{Z Y}} \sinh \sqrt{Z Y}$

where $Z=z \ell=R+j \omega L$, and $Y=y \ell=j \omega C$ and $R, L$, and $C$ are the total resistance, series inductance, and shunt capacitance of the line. We are interested in the lowfrequency range in which the effects of the reactances are small.

We may define the parameters $a=\omega C R$ and $b=\omega L / R$. If each of these is less than unity the hyperbolic function may be approximated by the first three terms of its series expansion, so that

$Z_{c}=Z\left(1+\frac{Z Y}{6}+\frac{(Z Y)^{2}}{120}+\ldots\right)$

Then if all terms in $a^{n} b^{m}$ for which $m+$ $n>3$ are discarded we find that

$Z_{c} \approx R(1+j b)\left(1-\frac{a b}{6}-\frac{a^{2}}{120}+\frac{j a}{6}\right)$

and, to the same order,

$$
\begin{aligned}
\left|Z_{c}\right| \approx R\left(1+\frac{a^{2}}{90}+b^{2}-\frac{a b}{3}\right)^{1 / 2} & \approx \\
R & \left(1+\frac{a^{2}}{180}+\frac{b^{2}}{2}-\frac{a b}{6}\right)
\end{aligned}
$$

The a-c-d-c difference of a voltage converter is defined as

$S=\frac{V_{a c}-V_{d c}}{V_{d c}}$

where $V_{a c}$ and $V_{d c}$ are the rms alternating and direct voltages required to obtain the same response (output emf) of the converter. If the thermoelement has no $\mathrm{a}-\mathrm{c}-\mathrm{d}-\mathrm{c}$ difference and if we define $k=b / a$, this becomes

$S_{0}=\frac{\left|Z_{c}\right|-R}{R} \approx \frac{a^{2}}{2}\left(k^{2}-\frac{k}{3}+\frac{1}{90}\right)$

where $S_{o}$ signifies that other possible causes of a-c-d-c differences are neglected.

The relation makes it possible to estimate $S_{0}$ to this approximation very simply. For the cylindrical construction of Fig. 1 with a cylindrical unspiraled resistor, $L=0.012 \ell M$ microhenry and $C=0.61 \ell / M$ picofarad, where $M=$ $\log _{10}(g / h)$ and $g$ and $h$ are the diameters of the cylinder and resistor, respectively, and $\ell$ is in inches. Thus numerically, $k=1.9 \times 10^{4}(M / R)^{2}$ and $a=3.8 \times 10^{-12}$ $f \ell R / M$, where $f$ is the frequency.
For example if $R=2,000$ ohms, $g$ and $h$ are 2 in (inches) and $1 / 8$ in., and $\ell=2.0$ in., $S_{o}=+0.001(+0.1 \%)$ at $40 \mathrm{mc}$.

It is apparent from equation 4 that to this approximation certain values of $k$ should make $S_{o}=0$. These are $k_{1}=0.038$ and $k_{2}=0.30$. The numerical values of resistance required are $R_{1}=710 M$ and $R_{2}=$ $250 M$. Unfortunately the logarithmic relationship for $M$ permits little range in $R$ for reasonable values of $g$ and $h$, for if $g$ is greater than the length of the resistor, end effects may become pronounced.

It is of interest to extend the analysis to higher values of the parameter, $a$. This has been done by solving equation 1 for $Z_{c}$ with the aid of Kennelley's tables and graphs of hyperbolic functions having complex arguments, ${ }^{4}$ and then computing $S_{o}=\left(\left|Z_{c}\right|-R\right) / R$. The results are shown in Fig. 4. It is rather striking that the first solution of equation $4, k_{1}=0.038$, results in reasonably small a-c-d-c differneces $(<5 \%)$ to $a=10$, a range much greater than that permitted by the initial assumptions.

For low-voltage converters the effect of the heater resistance $R_{h}$ cannot be neglected. To the next approximation the heater can be considered as a lumped resistance termination for the transmission line. Then from steady-state transmission-line equations,

$Z_{c}=R_{k} \cosh \sqrt{Z Y}+\frac{Z}{\sqrt{Z Y}} \sinh \sqrt{Z Y}$

By expanding the hyperbolic functions and discarding higher order terms as before (for $a<1$ and $b<1$ ) we find that if $R_{h}<R$

$S_{o}=\frac{\left|Z_{c}\right|-R_{\imath}}{R_{\ell}} \approx \frac{1}{2}\left(\frac{a}{1+m}\right)^{2}\left(k^{2}-A k+\frac{B}{6}\right)$

where $R_{t}=R+R_{h}, \quad R_{h} / R=m, A=m^{2}+$ $2 m / 3+1 / 3$ and $B=m^{2}+2 m / 5+1 / 15$.

Table II. Comperative A.C-D.C Differences of Voltage Converters

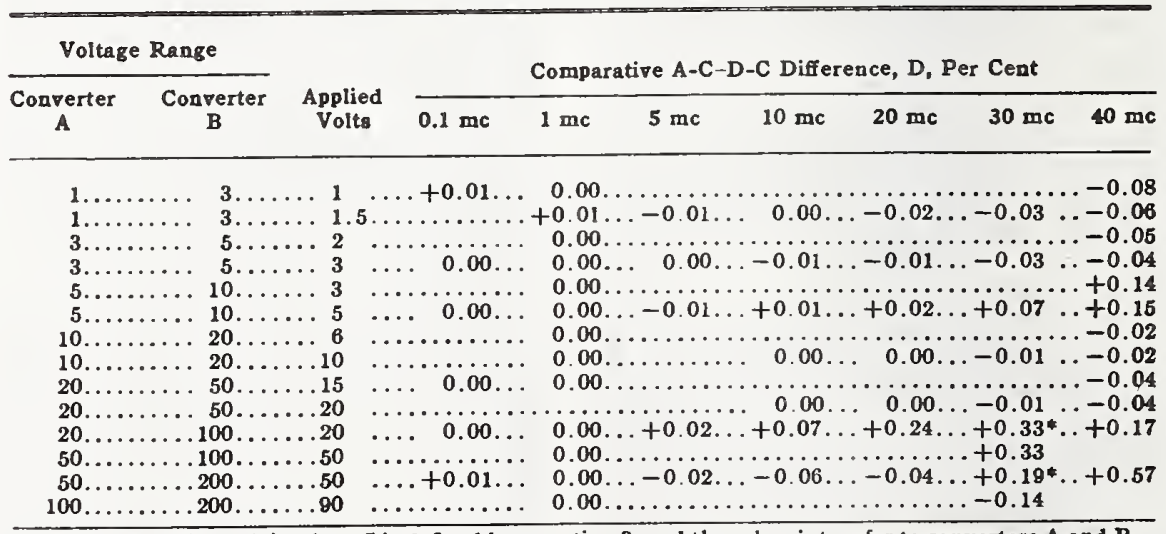

Note: $D=100(\mathrm{SB}-\mathrm{SA})$, where $\mathrm{S}$ is defined by equation 3 , and the subscripts refer to converters $\mathrm{A}$ and $\mathrm{B}$.

* Additional comparisons made at 25 and $35 \mathrm{mc}$. 
The values of $S_{0}$ for the 1 - to 20 -volt converters have been calculated by equation 6 at a frequency of $40 \mathrm{mc}$, at which $a$ and $b$ are still less than unity. The results are given in the last column of Table II.

When $k<0.038$, distributed capacitance from the resistor to the cylinder predominates. At a given frequency and geometry, the resultant error is proportional to the square of the resistance. It can be minimized by the construction shown in Fig. 2 in which two inner cylindrical shields are used, with one connected to the input lead. Thus there are now two transmission lines in series. If $R_{h}<<R$, application of transmission line equations, with careful attention to signs, gives for the desired transimpedance,

$$
\begin{array}{r}
Z_{c}=\sqrt{\frac{Z_{1}}{Y_{1}} \tanh \sqrt{Z_{1} Y_{1}} \cosh \sqrt{Z_{2} Y_{2}}+} \\
\sqrt{\frac{Z_{2}}{Y_{2}} \sinh \sqrt{Z_{2} Y_{2}}}
\end{array}
$$

where the subscripts 1 and 2 refer to the left and right hand lines (resistors and shields) of Fig. 2, and the resistance of the thermoelement is neglected.

$$
\text { If } Z_{1}=Z_{2}=Z \text { and } Y_{1}=Y_{2}=Y \text { we have }
$$

$$
Z_{c}=\frac{2 Z}{\sqrt{Z Y}} \sinh \sqrt{Z Y}
$$

Thus the transimpedance is twice that of either line, and the a-c-d-c difference, $S_{o}$, will be that of half the total line. The parameter, $a$, of equation 4 will then be one-fourth that for a line of the same dimensions without inner shields. Thus at a given frequency for which $a<1$ the use of the double shield rather than a single cylinder of the same diameter should reduce the frequency error, for resistors of the same value and dimensions, by a factor of 16 .

However, more detailed analysis indicates that even further improvement may be possible. For these converters $k<<0.038$, so that the series inductance of each line may be neglected. By expanding the hyperbolic functions of equation 7 and discarding higher order terms it can be shown by lengthy and rather tedious algebraic manipulation that $S_{0} \approx 0$ when $\ell_{1} / \ell_{2} \approx 1.032$, where $\ell_{1}$ and $\ell_{2}$ are the lengths of the left and right hand lines of Fig. 2, and $a<1$.

For the convenient dimensions shown in Fig. 1, the parameter, $a$, of each lowvoltage (up through 20 volts) converter now being used is low enough that it has not been necessary to proportion the converters for minimum error in accordance with equation 6 . The higher voltage elements are constructed with dual shields, with the grounded shield axially adjustable. This shield is set to give minimum! frequency error as explained in the next section.

A useful theoretical study of coaxial lines, terminated by resistors, was published by Crosby and Pennypacker. ${ }^{\circ}$ They show conditions for minimizing the input reactance.

\section{Tests}

Although long-time stability is not required of these converters, fluctuations or drifts in emf for the short time between the a-c use and the d-c calibration must be less than the desired accuracy. Such changes can arise from self-heating effects and ambient temperature changes, and from thermal emfs and other changes in the Lindeck potentiometer. Tests have shown that the self-heating of the carbon resistors, which have a load coefficient of about $1 \%$ per watt, is the largest source of drift. The change in resistance is very nearly exponential, with a time-constant of about 2 minutes. Since d-c calibrations can easily be made within 30 seconds of the a-c readings, this self-heating error is not significant in a-c tests if a short warm-up period is allowed. It is almost completely eliminated by the procedure used for a-c-d-c transfer tests. The effect of ambient temperature changes-the thermoelements have temperature coefficients up to $0.1 \% / \mathrm{C}$ (degrees centigrade) - should also be insignificant in a laboratory with reasonable temperature control.

A great advantage of thermoelements for $a-c-d-c$ transfer measurements is the almost complete electrical isolation of the input and output circuits. The uhf thermoelements have a small electrically insulating bead between the heater and hot junction of the thermocouple to eliminate conductive coupling. To minimize mutual inductance the plane of the thermocouple leads is at right angles to the heater and its colinear supports. Tests showed that at $4 \mathrm{mc}$ the induced a-c voltage in the output circuit of each thermoelement was less than $20 \mu \mathrm{v}$, the resolution of the detector used. Induced currents in the thermocouple circuit can cause errors by joule heating of the thermocouple, but calculations indicate this should not be significant if the induced voltage is less than a few millivolts.

The shield of the Lindeck potentiometer provides reasonable immunity from induced fields. In some tests at $10 \mathrm{mc}$ a current of $2 \mathrm{ma}$ from the shield to ground through the lead from the potentiomcter to the converter caused no significant error.

For transfer measurencuts the most important requirement is that the a-c-d c difference of each of these converters be known to the full accuracy desired. 'The general principles on which such dcterminations are based have bcen given. ${ }^{2}$ In the frcquency range studied, the major error of each of these converters is caused by the reactance. For a given converter this error should be independent of voltage level. Thus, it was feasible to evaluate the relative errors by intercomparing converters of adjacent voltage ranges to determine their differences in frequency response. A complete series of such comparisons was made at two voltage levels for each pair of converters, at frequencies up to $40 \mathrm{mc}$. In each comparison the two converters were connected in parallel to a coaxial lead through a tee fitting ( $G R$ 874), and a shielded potentiometer was connected to each output. Each potentiometer was adjusted for zero deflection at the test voltage. The converters were then supplied in succession with alternating, direct, reverseddirect, and alternating voltage. Each voltage was adjusted to produce the same emf of the higher range converter and the deflection of the galvanometer connected to the other converter was observed. From the differences in emf, directly determined from these differences in deflection, the difference in the frequency response of the two converters was determined. ${ }^{2}$ In these tests the movable inner shields of the 100 - and 200 -volt converters were adjusted for best performance over the desired frequency range, by comparison with the 20 - and 50 -volt converters. The errors of these adjustable converters were found to be complicated functions of the frequency and shield position.

The results of these intercomparisons from 0.1 to $40 \mathrm{mc}$ are given in Table II. They show that for each pair of converters the relative $a-c-d-c$ differences were independent of the applied voltage to $0.02 \%$ or less, and were less than $0.4 \%$ up to $30 \mathrm{mc}$. For the low-voltage converters they were less than $0.05 \%$ to $20 \mathrm{mc}$ without exception and without evidence of systematic errors.

The relative a-c-d-c differences of most of the thermoelements used in the voltage converters were also determined before the thermoelements were installed, by making similar a-c-d-c intercomparisons as current-measuring elements at $40 \mathrm{mc}$. For these tests the two thermoelements were connected in series along the axis of a brass cylinder having the same dimen. 
Table III. Resuits of Test of 1-Volt Voltmeter

\begin{tabular}{|c|c|}
\hline $\begin{array}{l}\text { Frequency. } \\
\text { Mc }\end{array}$ & $\begin{array}{c}\text { Applied Voltage, } \\
\text { Volts }\end{array}$ \\
\hline $1 \ldots$ & …..0.997 \\
\hline $10 \ldots$ & $\begin{array}{r}\ldots .0 .998 \\
\ldots .0 .998\end{array}$ \\
\hline $\begin{array}{r}30 \ldots \\
100 \ldots\end{array}$ & $\begin{array}{l}\text { …..0.988 } \\
\text {......0.998 }\end{array}$ \\
\hline $200 \ldots$ & $\ldots .0 .982$ \\
\hline $300 \ldots$ & $\ldots .0 .945$ \\
\hline $400 \ldots$ & ...0.883 \\
\hline
\end{tabular}

"For same reading of millivoltmeter at each frequency

sions as in Fig. 1 but with a coaxial input connector at each end and with two 2-pin output connectors. A split cylinder with one part readily removable facilitated changing thermoelements. The input was applied to one connector with the other short-circuited. Rough calculations indicated that at $40 \mathrm{mc}$ the current should change by about $0.5 \%$ along the transmission line formed by the heaters of the thermoelements in this cylinder, but that the distribution should not be greatly dependent on the resistance of the heaters, less than 100 ohms each. Therefore, two determinations of relative a-c-d-c differences were made with each pair of thermoelements, with first one end then the other of the cylinder short-circuited. For each pair the two determinations differed by about $0.4 \%$ at $40 \mathrm{mc}$, but in each case their algebraic average was less than $0.02 \%$.

These calculations and tests also provide assurance that even at $40 \mathrm{mc}$ the current along the heater of a single thermoelement terminating a voltage converter is well within $0.1 \%$ of the value at the mid-point of the heater.

The 1-volt converter was compared at rated voltage with a wire-wound thermal voltage converter of known a-c-d-c difference at $3 \mathrm{cps}$ and $20 \mathrm{kc}$, with observed differences of $+0.02 \%$ and $0.00 \%$ respectively.

The response of the 1 -volt converter, with a shielded d-c millivoltmeter connected to its output, was determined to better than $1 \%$ at frequencies from 1 to $400 \mathrm{mc}$ by the bolometer bridge of Selby and Behrent, ${ }^{6}$ with the results shown in Table III. It is evident that the frequency influence is very small to $100 \mathrm{mc}$. It increases rapidly at higher frequencies, becoming $-5.2 \%$ at $300 \mathrm{mc}$. The calculated a-c-d-c difference at $300 \mathrm{mc}$ by equation 6 is only $-0.9 \%$; this converter has a 1/4-in.-diameter unsealed resistor. Some of the discrepancy may be accounted for by the voltage rise in the connector. A type $N-U G 58 / U$ input connector was substituted for the type 874 for these tests.
As a check on equation 6 at larger a-cd-c differences, the 50 -volt converter was tested before its inner shield was installed. The measured a-c-d-c difference at 40 mc was $+1.3 \%$ and was accurately proportional to the square of the frequency. The computed values were $40 \%$ smaller, indicating that the effective length of the resistor, the end effect, was 1.2 times the actual length. At $40 \mathrm{mc}$ the measured $a-c-d-c$ difference was changed by less than $0.02 \%$ when the resistor was mounted $3 / 32$ inch off the axis of the cylinder, indicating that exact centering is not critical even when the errors are large.

The intercomparisons of Table II show that the a-c-d-c differences of the five low-voltage converters without inner shields, and with 1- to 20 -volt ranges, are all equal to better than $0.05 \%$ to more than $20 \mathrm{mc}$. At $40 \mathrm{mc}$ they agree to $0.1 \%$ with the values calculated by equation 6 . Values cannot readily be computed for the higher voltage elements with the inner shields. This unanimity between converters having such a wide range of resistors and different thermoelements gives good assurance against unknown sources of error. It is quite unlikely, but not impossible, that each converter would have the same a-c-d-c error. However the test of the 1-volt converter with the bolometer bridge provides most valuable additional assurance. The large errors of this converter above $100 \mathrm{mc}$ decrease rapidly with decreasing frequency, well within the stated accuracy of the bridge measurements. For converters without inner shields almost all known causes of such errors, such as the effect of reactance, equation 6, skin effects in the resistor or thermoelement, etc., should cause a-c-d-c differences approximately proportional to the square of the frequency, over the range for which the errors are small. Thus the authors believe that the large measured errors above $100 \mathrm{mc}$ can be extrapolated downward to lower frequencies to indicate with considerable confidence that the a-c-d-c difference of this converter is less than $0.2 \%$ at $40 \mathrm{mc}$ and less than $0.1 \%$ below $30 \mathrm{mc}$.

Based on the foregoing consideration the authors assigned a value of zero to the a-c-d-c difference of the 1-volt converter to $40 \mathrm{mc}$, and then determined the a-c$\mathrm{d}-\mathrm{c}$ differences of all the other converters from the intercomparison data in Table II. The results, rounded to the nearest $0.05 \%$ to $10 \mathrm{mc}$ and $0.1 \%$ to $40 \mathrm{mc}$, are given as observed values in Table IV.

\section{Use}

These converters are most conveniently used to measure the a-c-d-c difference or frequency influence of other rms instruments such as thermocouple voltmeters, which are now available with accuracies of $1 / 2 \%$ to $10 \mathrm{mc}$. A typical setup for this is shown in Fig. 5. The scale calibration of the voltmeter can then easily be checked on reversed direct current. The $a-c-d-c$ difference tests are similar to the intercomparisons already described, and d-c calibration of the converter is not necessary once the scale factor of the Lindeck potentiometer is determined for each converter. This is the per-cent change in input voltage per centimeter change of galvanometer deflection. ${ }^{2}$ Either direct or low-frequency alternating current may be used as the reference frequency and the test may be made rapidly and accurately. The results are only slightly affected by drifts in either instrument. At frequencies above about 20 mc a small lead correction may be necessary if the connectors between the junction plane and the two instruments are not electrically equal, but this is readily determined to the required accuracy.

For a-c measurements a d-c potentiometer of $0.1 \%$ accuracy or better is required to measure the $\mathrm{d}-\mathrm{c}$ reference voltage. A deffection potentiometer and volt box, or an automatic self-balancing potentiometer ("digital voltmeter") should be convenient for this. For testing electronic voltmeters frequency-response measurements (differences in reading for

Table IV. A-C-D-C Differences of Voltoge Converters

\begin{tabular}{|c|c|c|c|c|c|c|}
\hline \multirow{3}{*}{$\begin{array}{c}\text { Rated } \\
\text { Voltage, } \\
\text { Volts }\end{array}$} & \multicolumn{6}{|c|}{ Per-Cent A-C-D-C Differences, 100 S } \\
\hline & \multicolumn{5}{|c|}{ Observed } & \multirow{2}{*}{$\frac{\text { Calculated }}{40 \mathrm{mc}}$} \\
\hline & to $5 \mathrm{mc}$ & $10 \mathrm{mc}$ & $20 \mathrm{mc}$ & $30 \mathrm{mc}$ & $40 \mathrm{mc}$ & \\
\hline $\begin{array}{r}1 \ldots . \\
3 \ldots \\
5 \ldots \\
10 \ldots \\
20 \ldots \\
50 \ldots \\
100 . \ldots \\
200 \ldots\end{array}$ & $\begin{array}{l}<0.05 \\
<0.05 \\
<0.05 \\
<0.05 \\
<0.05 \\
<0.05 \\
<0.05 \\
<0.05\end{array}$ & $\begin{array}{l}<0.05 \\
<0.05 \\
<0.05 \\
<0.05 \\
<0.05 \\
<0.05 \\
+0.05 \\
-0.05\end{array}$ & $\begin{array}{r}0.0 \\
\cdot \quad 0.0 \\
\cdot \quad 0.0 \\
\cdot \quad 0.0 \\
\cdot \quad 0.0 \\
0.0 \\
+\quad 0.2 \\
\quad 0.0\end{array}$ & $\begin{array}{r}0.0 \\
\quad 0.0 \\
-0.1 \\
0.0 \\
0 \quad 0.0 \\
0.0 \\
+0.3 \\
+0.2\end{array}$ & $\begin{array}{r}0.0 \\
-0.1 \\
-0.1 \\
0.0 \\
0.0 \\
0.0 \\
+0.1 \\
+0.5\end{array}$ & $\begin{array}{l}\ldots \quad 0.0 \\
\ldots \quad 0.0 \\
\ldots \quad 0.0 \\
\ldots+0.1 \\
\ldots+0.1\end{array}$ \\
\hline
\end{tabular}




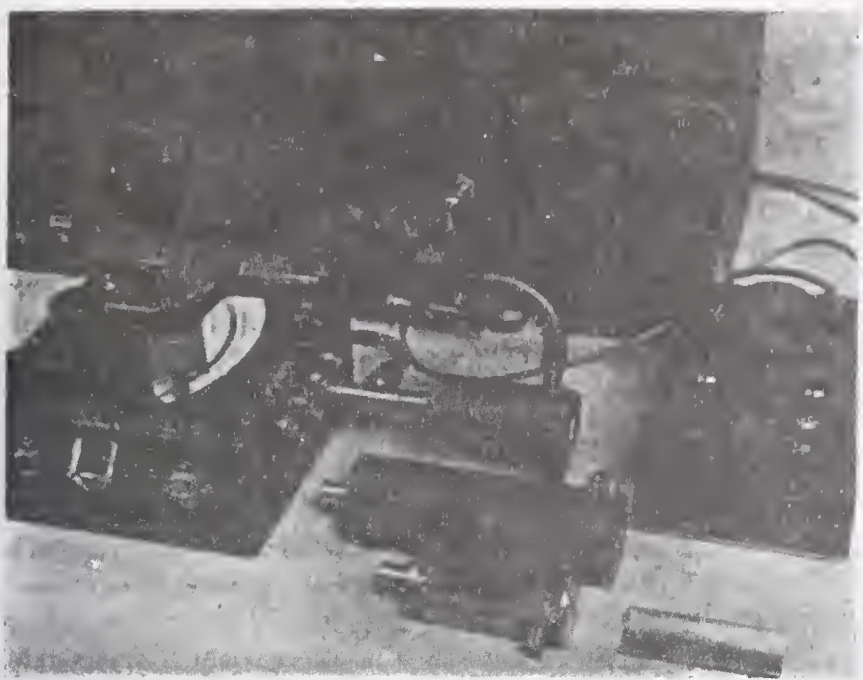

Fig. 5. 5-volt converter setup for a-cd-c test of experimental thermocouple voltmeter. Lindeck potentiometer at right, double-shield converter and experimental split-shield element in foreground, a-c-d-c switch not shown

the same voltage at the test and reference frequencies) should be particularly convenient. The form factor and crest factor of the a-c sources must be within $0.1 \%$ of the values for a sine wave, 1.111 and 1.414 respectively, since most electronic voltmeters respond essentially to the average or crest (peak) values. Since these factors depend upon the phase angle as well as the magnitude of each harmonic it would ordinarily be necessary to make sure that the ratio of the magnitude of the nth harmonic to the fundamental does not exceed $0.1 \times n \%$ when an averagereading instrument is tested and $0.1 \%$ when a crest-reading instrument is tested. The results should then differ from those obtained with a sine wave by less than $0.1 \%$.

An $\mathrm{r}-\mathrm{f}$ generator of at least 5 watts output and good voltage stability is required but only moderate frequency accuracy and stability are needed. It is very difficult to construct broad-band generators of good wave form at this power level. However, a simple adjustable L/C (inductance capacitance) tuned circuit, with the instruments connected across the capacitor, can be used simultaneously to improve the wave form, match the impedance of the instruments to that of usual 50-ohm source, eliminate the capacitance loading of the instruments on the source, and provide the higher voltages often required. For these combined purposes the values of inductance and capacitance should be chosen so that $\omega L=1 / \omega C \approx$ $\sqrt{R_{1} R_{2}}$, where $R_{1}$ and $R_{2}$ are respectively the resistances of the source, including the inductor, and the load (instruments). For low voltages a $50-0 h m$ resistor can be connected in series with the $\mathrm{L} / \mathrm{C}$ circuit and the instruments and attenuators as needed can be connected in parallel with this resistor for improved wave form.

\section{Conclusions}

Cylindrical film resistors in series with a thermoelement in a coaxial line makes possible single-range thermal voltage converters that are useful as $a-c-d-c$ transfer instruments over a very ride frequency range, $3 \mathrm{cps}$ to at least $30 \mathrm{mc}$, with unusually high accuracy. They are inexpensive and easy to construct. Their frequency influence may be estimated by reasonably simple equations, with results which agree well with the measured values up to $40 \mathrm{mc}$. The voltage converters may be useful to considerably higher frequencies. The higher range converters require considerable power, up to 2 watts, and have a marked but short warm-up drift. These disadvantages could be reduced by using inetal-film resistors of higher resistance with a 5-1na thermo. element, but this would very probably reduce the frequency range for the same attainable accuracy. The good performance of these converters was obtained with deposited-carbon resistors of a new type. Earlier converters with resistors of different construction showed discrepancies up to $0.5 \%$ at $40 \mathrm{mc}$. Further work is planned to determine the cause of these differences.

These voltage converters can be used quickly and easily to makc a-c-d-c diffcrence tests to determine the frequency influence of other rms instruments to $0.1 \%$ or better to at least $10 \mathrm{mc}$ and to $0.2 \%$ at $30 \mathrm{mc}$. Direct a-c measurements are made by the transfer technique, which in most cases can be arranged for reasonable simplicity. With sources of suitable waveform, average-reading and crest reading instruments could also be calibrated if desired. In all of these applications the applied frequency need not be closely determined or held because of the flat frequency response of these voltage converters.

\section{References}

1. Electrical Inaicating Instrdagnts. a $S A$ C39.1-1959, American Standards Association, Inc. New York, N. Y., sect. 3.11.3, May 1959.

2. Thermal Converters as aC-DC Transper Standardos por CURRENT ang VOLTAGB M BasUrz MBNTS at Aunio FrequBnci89, F. L. Hermach. Journal of Research, National Bureau of Standards, Washington, D. C., vol. 48, 1952, pp. 121-38.

3. A Wide-Range Volt-Ampere Converter For CURrent and Voltage Measurbrents, F. L. Hermach, E. S. Willinm AIEE Transac fions, pt I (Communicalion and Electronics), vol. 78 Sept. 1959, pp. 384-88.

4. Tables ant Chart atzas of Complex Hypergolic ann Circular Functions, A. B Kennelly. Harvard University Press, Cambridge, Mass., 1921.

5. RADIO-FREQUENCY RESISTORS AS UNTFORY Trangmission Lines, D. R. Crosby, C. H. Penaypacker. Proceedings, Institute of Radio Engineers, New York, N. Y., vol. 34,1946 , pp. $62-66$

6. A Bolometer Bridge por Standaguzino RADIO-FreqUency VOLtMeters, M. C. Selby, L. F. Behrent. Journal of Research. National Bureau of Standards, vol. 44, 1950, pp. 15-30.

\section{Discussion}

Morley J. Lush (Rawson Electrical Instrument Co., Cambridge, Mass.): I feel that the instruments described in this paper are a real contribution to the art of accurate voltage measurements at high frequencies. Although it is difficult to make resistors with low reactances, Mr. Hermach has shown that these can be obtained, and that roltmeters can be constructed which have a flat frequency response all the way from direct current out to the megacycle region. The frequency response will remain stable for long periods of time, so only d-c calibra. tions are needed, once the frequency response has been determined. I bave found from my own work that accuracies of $1 \%$ can be obtained at frequencies up to $100 \mathrm{mc}$ and now these instruments are available on the market. A precision of $0.1 \%$ can be obtained, but the absolute accuracy is limited by the lack of a primary standard. I have been told that the National Bureau of Standards expects to be able to offer $0.25 \%$ certifications by the end of 1960 .

Other workers in this field have used a fixed or variable capacitor as the attenuator element in series with the thermocouple. It is possible to make an excellent low-loss capacitor using a micrometer screw for adjustment. A wide frequency range and wide voltage range can be obtained on one instrument. However, the voltmeter cannot then be used for $d-c$ to a-c transfer meas. urements, since it has zero response at zero frequency. Indeed, the response is directly proportional to the frequency, and it is necessary to calibrate the voltmeter at each voltage and frequency for which it will be used.

It should be pointed out that connections to these voltmeters are \& problem, as they 
are in all high-frequency apparatus. The use of coaxial connectors can be misleading. as any appreciable length of 50-ohm coaxial line will cause errors due to standing waves, unless the voltmeter input impedance is made exactly equal to the impedance of the line. Careful matching of the line imped. ance will certainly be necessary if voltmeters of this type are to be used at frequencies above $100 \mathrm{mc}$.

F. L. Hermach and E. S. Williams: Mr. Lush's remarks are appreciated. We believe these thermal voltage converters, by taking advantage of the powerful $a-c-d-c$ transfer technique, should make possible a new order of accuracy in a-c voltage meas. urements up to at least $30 \mathrm{mc}$. Their usefulness will probably fall of rapidly at higher frequencies, except for the lower voltage ranges, up to perhaps 10 volts.

A major advantage of an a-c-d-c transfer standard is that its $a-c-d-c$ difference is relatively permanent so that once evaluated. it should not ordinarily need to be redetermined. Thus, elaborate repeated a-c calibrations are not required. This and the ease and simplicity of constructing these converters should offset the extra labor of making the a-c to $d-c$ transfer for each measurement.

IVe nicntioncd connection errors only bricfly. Each converter is a high-impedance load at the end of a short coaxial line from the junction plane to the converter.
If this line is a small fraction of a wavelength the voltage rise from the junction to the end of the line is approximately $50 h^{2} \%$, where $h=\omega C Z_{0}$ and $C$ and $Z_{0}$ are the total shunt capacitance and the characteristic impedance of the line. At $30 \mathrm{mc}$ for a 2-inch length of typical 50-ohm line the correction is only $0.1 \%$. It is offset by a similar correction if an cqual line is used betwecn the junction plane and the test instrument or lond to be measured. At higher frcquencies or for lunger lincs the correction can be large and this approximate formula nuy no longer be valid. In such cases, howcver, voltage measurcments probably have meaning only with lincs terminated in their claracteristic impedance, so that the standing-wave ratio is close to unity. 


\title{
Thermal Converters as AC-DC Transfer Standards for Current and Voltage Measurements at Audio Frequencies
}

\author{
Francis L. Hermach
}

\begin{abstract}
Thermal converters and associated equipment that are used as ac-de transfer standards at the National Bureau of Standards for the precise measurement of current and voltage at power and audio frequencies are described. The standards and the equipment are primarily used to standardize a-c ammeters and voltmeters submitted to the Bureau for certification. The ac-dc transfer may be made with these thermal converters at currents from 1 milliampere to 50 amperes, voltages of 0.2 to 750 volts, with an accuracy of 0.01 percent at frequencies from 25 to 20,000 cycles per second.

The special tests to insure the required accuracy of the transfer standards are described, and the results are presented. A number of factors that limit the transfer accuracy of thermal converters have been discovered, and the results of special tests and theoretical work to evaluate these factors are discussed. The solutions, by an approximation method, of certain pertinent nonlinear differential equations governing the heating of a conductor by an electric current are given.
\end{abstract}

\section{Introduction}

The increasing use of electric energy for aircraft, induction furnaces, and induction heating, and the greater accuracy required in measurements in electronics, have led to increasing demands for the accurate standardization of ammeters and voltmeters at frequencies extending upward from power frequencies through the entire audio-frequency range. To meet these demands, special instruments have been developed at the National Bureau of Standards for the measurement of current and voltage over rather wide ranges. They make use of thermal converters ${ }^{1}$ (often called thermoelements) like those incorporated in ordinary thermocouple instruments, but differ in the manner of reading and use. They may be used either directly to measure the ac-dc differences of ammeters and voltmeters, or with a suitable potentiometer and accessories to measure alternating currents and voltages. They were designed and are used primarily for testing electric instruments, at currents from $1 \mathrm{ma}$ to $50 \mathrm{amp}$ and voltages from 0.2 to $750 \mathrm{v}$, with an accuracy of 0.01 percent at frequencies from 25 to $20,000 \mathrm{c} / \mathrm{s}$.

\section{Transfer Principle}

The basic electrical units are defined in terms of a concordant system of mechanical units and are realized by absolute electrical measurements carried out at national standardizing laboratories to fix the value of groups of standard cells and resistors. These standards are used in conjunction with a potentiometer to make measurements of direct voltage, current, and power. It has been known for a long time, but not sufficiently realized, that the measurement of the corresponding alternating-

1 Proposed AIEE definition 30.89.040: A thermal converter is a device that consists of one or more thermojunctions in thermal contact with an electric beater or integral therewith, so that the electromotive force developed at its output terminals by thermoelectric action gives a measure of the input current in its heater. current quantities depends fundamentally on certain standard types of electric transfer instruments that ideally have the same response on direct and alternating current. If the instruments are calibrated on direct current at the time of each use and if the precision of reading is suitably increased, long-time stability, freedom from drift, small temperature influence, low losses, and other normally desirable characteristics become of secondary importance, and the instruments may be primarily designed and constructed to have the best possible frequency characteristic. For difference measurements such instruments need not have long scales in the ordinary sense, but rather may be designed for increased precision of reading by methods that would not be applicable to instruments intended for general service.

Special electrodynamic transfer instruments have long been used at the Bureau for accurate a-c measurements. These transfer standards are used largely to standardize (test) other instruments submitted to the Bureau for certification. There are two distinct types of such tests. For the "straight a-c test", the instrument under test and a transfer standard are connected to measure the same alternating electrical quantity (current, voltage, or power), which is adjusted to produce the desired deflection of the test instrument. The response of the standard instrument is observed, then the standard is transferred to direct current. The direct quantity is adjusted to give the same response of the standard and is then measured with a suitable potentiometer and accessories. For the so-called "ac-dc difference test", both instruments are connected to measure the same quantity first on alternating and then on direct current, which is in each case adjusted to give the same deflection of the test instrument. From the averaged difference in the response of the transfer standard, the ac-dc difference of the test instrument is computed. The second type of test can be made with somewhat higher accuracy than the first, and in conjunction 
with a test on direct eurrent gives more information about the performance of the test instrument. Because the ac-dc difference of an instrument depends upon geometrical factors that are relatively permanent, subsequent tests for checking the constancy of calibration need generally be made only on direct current. Occasionally transfer tests are made by using a selected low frequency (such as $60 \mathrm{c} / \mathrm{s}$ ) as the reference in place of direct current.

An example of the accurncy with which such tests are made, laboratory standard instruments, with scales 12 in. long, are regularly standardized and certified at the Bureau to 0.05 of a scale division. For a 150-division instrument this requires an accuracy of measurement of 0.03 percent or better.

\section{Development and Description of Electro- thermic Transfer Standards}

\subsection{Choice of Standards}

Several types of instruments were considered in choosing transfer standards to cover the full audiofrequency range. Electrodynamic instruments have been highly developed $[1,2,3]^{2}$ at the Bureau for measurements at the commercially important power frequencies and can be used with proper corrections, up to about $2,000 \mathrm{c} / \mathrm{s}$. However, unavoidable inductance and stray capacitance errors limit their useful frequency range and present little hope for the desired extension. Electrostatic instruments have been carefully studied $[4,5]$ at the National Physical Laboratory. They are useful over wide frequency ranges, but because of their low-torque-weight ratio at low voltage are not readily adaptable for either the low voltage or the current ranges desired in this application. Electrothermic instruments, which use an effect produced by the heating of a conductor carrying a current to be measured, seemed more promising. Of the several kinds, thermocouple instruments, in which the temperature rise of the conductor (heater) is measured by a thermocouple, seemed most feasible. Straight-wire heaters allow a wide frequency range, and the thermocouple measurements permit high precision of reading. The usual disadvantages of poor stability and large temperature influence are not important in their use as transfer instruments, and their low overload capacity is not a serious limitation in careful laboratory work. Fortunately, good thermal converters are commercially available. They have been used for measurements at frequencies up to about $65 \mathrm{Mc}$, with a d-c millivoltmeter as the indicator, and are generally considered in the $1 / 2-$ to 2-percent accuracy classes. So far as is known, they have not been previously studied for use as transfer instruments at the frequencies and the accuracies needed in this application.

For these reasons, commercial thermal converters were selected for study to determine their value as ac-de standards in a wide-range audio-frequency

\footnotetext{
2 Figures in brackets indicate the literature references at the end of this paper.
}

transfer voltmeter. A careful experimental and theoretical study of their performance led to the purchase of additional commercial thermal converters of suitable ranges and the design and construction of equipment for using them as transfer standards for voltage and current.

\subsection{Description of Transfer Standards}

Each thermal converter purchased in the milliampere ranges is of the vacuum type, mounted in an evacuated glass bulb with supports of copper wire embedded in the glass. 'The conductor, heated by the current to be measured, is a short straight wire generally less than $1 / 2 \mathrm{~cm}$ long and often less than $0.001 \mathrm{in}$. in diameter. The heater alloy and dimensions are chosen to give a temperature rise of about $200^{\circ} \mathrm{C}$ at the center where the hot junction of the thermocouple is fastened by a ceramic bead. This bead provides electrical but not thermal insulation between the heater and thermocouple circuits, with a coupling capacitance less than $1 \mu \mu \mathrm{f}$ and an insulation resistance greater than 50 megohms at $25^{\circ} \mathrm{C}$.

Because high current sensitivity is not required, the thermal converters in the 1 - to 50 -amp ranges are not evacuated. The hot junction of the thermocouple is welded directly to the midpoint of a thinwalled tubular heater. These thermal converters are temperature compensated [6].

For current measurements, thermal converters in the series $1,2,5$, 10, etc., with rated output electromotive forces of $10 \mathrm{mv}$ were purchased, with ranges from $1 \mathrm{ma}$ to 50 amp. Thermal converters of 7.5and 30-ma ratings, with appropriate series resistors, are used for voltage measurements. Figures 1 and 2 show some of the thermal converters and the model $A$ and model $B$ voltmeter elements. It should be emphasized that the caption NBS on the nameplate sbown in figure 2 signifies only that the equipment was assembled for use at the National Bureau of Standards. All the thermal converters were purchased commercially, as were the components of the voltmeters. It should also be emphasized that the thermal converters shown in these figures are the transfer elements only. An indicator (to be discussed in the next section) is necessary for all measurements, and a potentiometer and accessories are also necessary, except for transfer tests of other instruments.

The model A voltmeter was developed as a prototype instrument to meet an immediate need for voltage measurements. It consists of a $30-\mathrm{ma}$ thermal converter connected with its heater in series with a commercial decade resistance box, modified to have two 1,000-ohm-per-step decades, and a 100-, a 10- and a 1-ohm-per-step decade, all in series. As shown in figure 3 , one end of the heater of the thermal converter is connected to the low-side terminal of the box, with the shield of the box connected to the other end of the heater and to the shield of the coaxial cable connecting the instrument to the circuit. The center lead of this cable is connected directly to the high-side terminal of the box. 


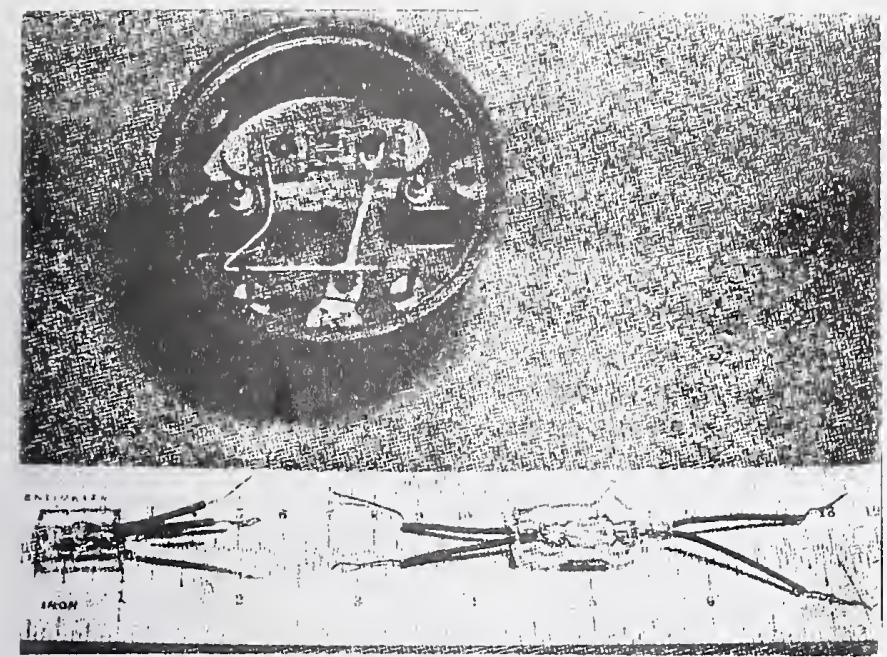

Fictik: 1. Typical thermal converters.

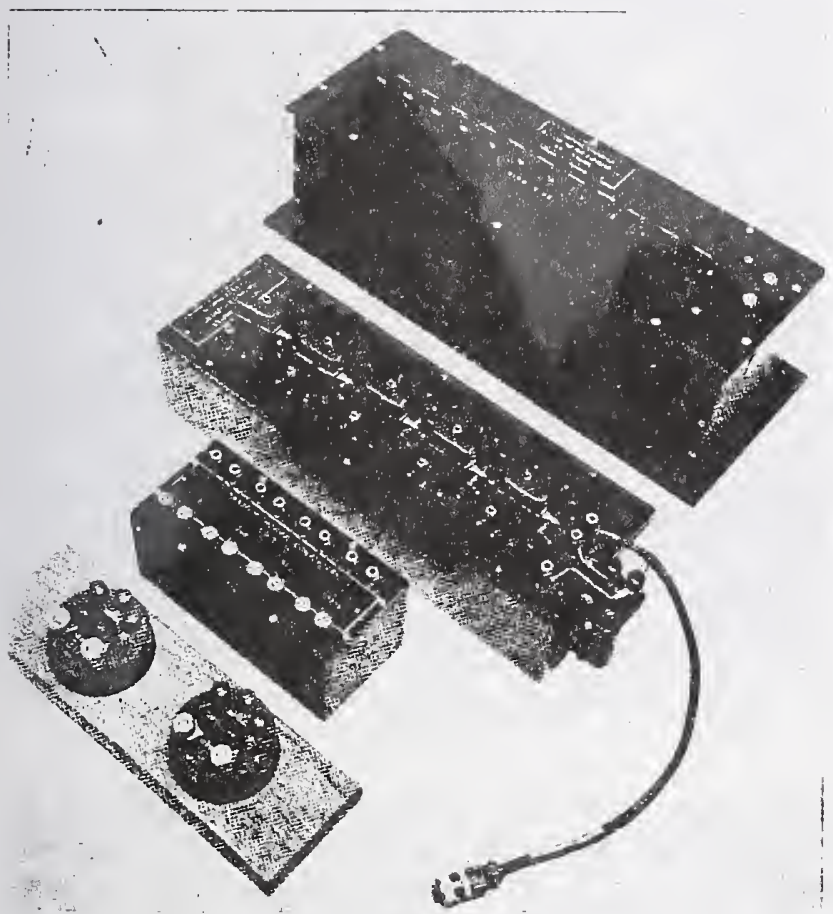

Figure 2. Mounted thermal converters and the model $A$ and model $B$ volt meter elements.

Like the electrodynamic transfer standards used at lower frequencies, the series resistor is adjusted in use to give the same nominal curient level for each measured voltage. This was chosen at $20 \mathrm{ma}$, resulting in a voltmeter having a constant $50 \mathrm{ohms} / \mathrm{v}$ and an upper range of $400 \mathrm{v}$. The model $\Lambda$ voltmeter, described in some detail in an earlier paper [7] is now no longer used for routine measurements.

For the model B voltmeter, a built-in 7.5-ma thermal converter was used, with a fixed resisiol having taps to give voltage rallges of $1.5,3,6,7.5$, $15,30,60,75,150,300,600$, and $750 v$. The resistance cards for this instrument were purchased commercially, and the cards and thermal converter were momnted in a suitable shicked box with the shield romected to the low-side terminal of the instrument, as shown in figure 3. For both instrumcits the rflects of capacitance currents and of the selfinductance of the resistors were carcfully considered; the limiting factors are cliscussed in another section of this paper.

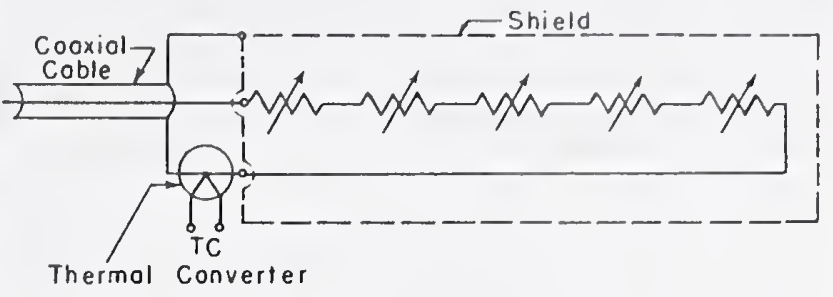

A

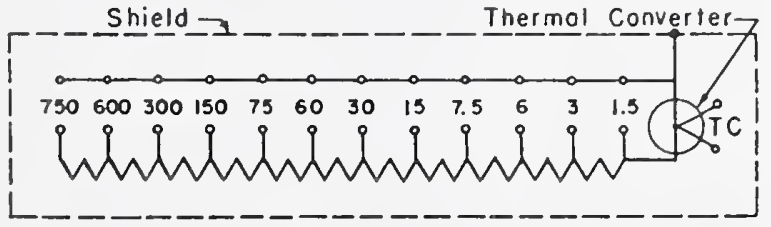

B

Figure 3. Circuit diagrams of the model $A$ and model $B$ voltmeter elements.

A, Model A voltmeter element; B, model B voltmeter element.

\subsection{Indicator}

Because of its convenience and portability, a millivoltmeter is generally used for measuring the emf in the usual thermocouple instrument. The precision of reading of the usual millivoltmeter is of course far too low for this application. For increased accuracy a potentiometer can be used to measure the thermocouple emf. However, if the thermal converter is used only as a transfer standard, the emf need not be measured in a "straight a-c" test; and in ac-dc difference tests it is only the small change in emf between the a-c and d-c settings that is significant. The change and the full emf with which it is compared need be measured with relatively low accuracy, provided the change is referred to a lighly stable base value. Thus high sensitivity and high stability but only moderate accuracy in the measurement of emf are required of the indicator. For these requirements, a lindeck potentiometer for providing the base value, used in conjunction with a suitable galvanometer, the deflection of which indicates the change, forms the ideal indicator. In a Lindeck potentiometer, based on Poggendorf's second principle [8], the emf to be measured is balanced by an adjustment of the current through a fixed resistor. At balance the voltage drop across the resistor, which is the product of the potentiometer current and the resistance, is equal to the measured emf. The current is ordinarily measured with a milliam- 
meter, which generally sets the limit on the accuracy obtainable with this form of potentiometer.

Such a potentiometer has been incorporated in a panel constructed for the audio-frequency testing. It was designed to have adequate stability, freedom from changing extraneous electromotive force and the required ranges, with sufficient precision of reading. Its circuit is shown in the central portion of figure 4. The resistor, $R$, is made of manganin, and a special thermofree key [8] and copper binding posts are used in the electrothermally sensitive emf eircuit. The components are mounted in the central part of the panel, as shown in figure 5 , and are enclosed by a

\subsection{Use of Instruments}

These transfer standards are used almost solely for standardizing otleer a-c instruments. The necessary switching and control eireuits for sucl tests have been incorporated in the panel shown in figure 5 . In addition to these circuits and the lindeck potentiometer, the panel contains the impedance-matching transformers for the high-voltage circuits used in testing voltmeters and the high-current circuits used in testing ammeters. The present transformers provide ranges up to $50 \mathrm{amp}$ and $800 \mathrm{v}$, with a nominal input voltage of $150 \mathrm{v}$ and a power level up to $100 \mathrm{w}$.

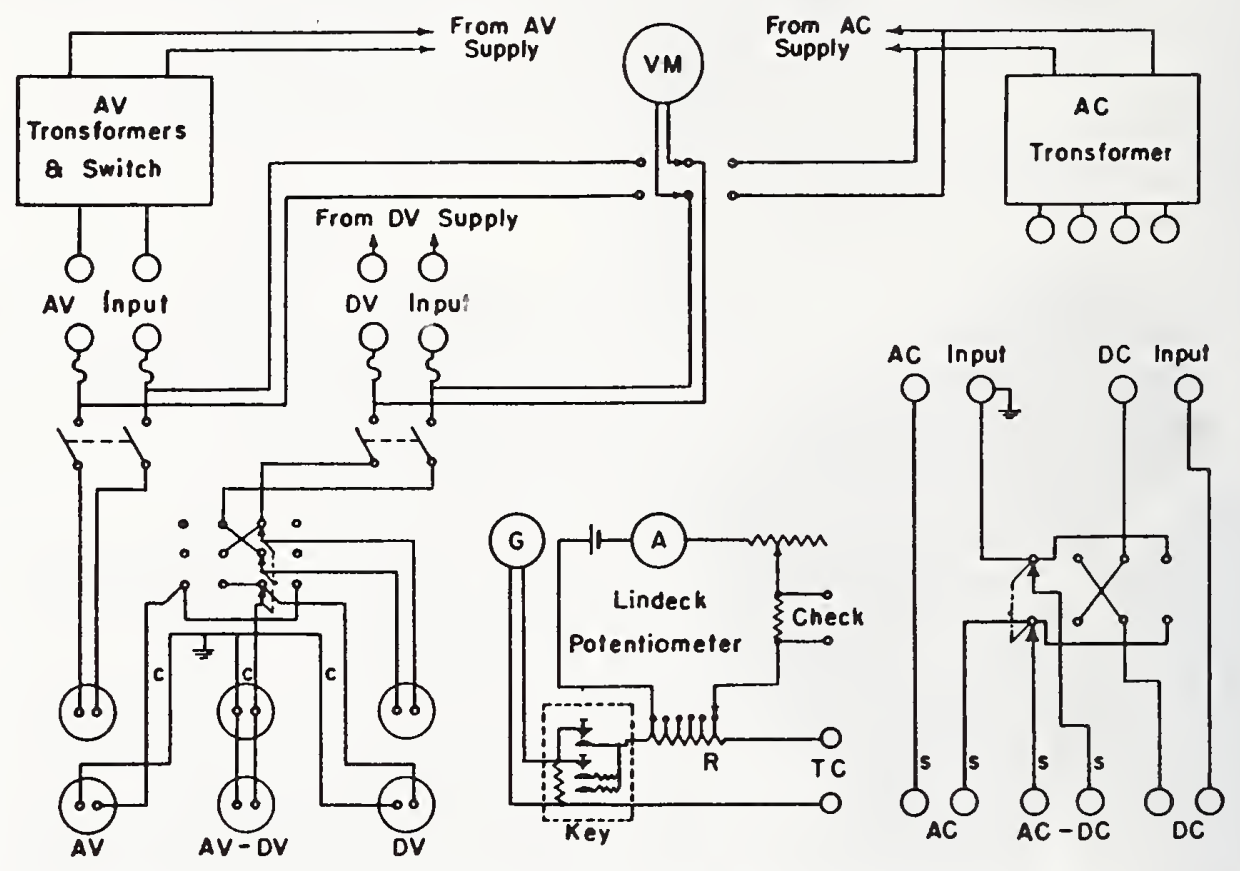

FIgURE 4. Schematic diagram of circuits used for testıng instruments.

C signifies coaxial lead with shield grounded; $S$ signifies shielded lead with shield grounded.

grounded metal shield. No other thermal precautions were found necessary. A reflecting wallmounted galvanometer is used, with a voltage sensitivity (in the circuit) of $1 \mathrm{~mm} / \mu \mathrm{v}$. A No. 6 dry cell supplies the potentiometer current, which is adjustable from 0.2 to $1.2 \mathrm{ma}$. At these current levels, repeated tests have shown that the steady drift in the voltage across the four terminal resistor is less than 0.02 percent per hour, and that superposed fluctuations are much less than 0.01 percent. The fourterminal manganin resistor has taps at $0.1,0.5,2.5$, 5,10 , and 25 olims, giving milivolt ranges, at a current of $1 \mathrm{ma}$, of these same 11umerical values. The lowest range is used in transfer tests as a quick check of the potentiometer. With the thermocouple of the thermal converter connected to the "TC" posts of the potentiometer, but with no current through its heater, a galvanometer deflection of 10.0 $\mathrm{cm}$ to the left on the scale with the Lindeck set for 0.1 $m v$ serves as a valuable partial eheck of the required accuracy and correctness of the Lindeck circuit.
Motor-generator sets and voltage stabilizers are available for tests at $60 \mathrm{c} / \mathrm{s}$ and audio-frequency oscillators and power amplifiers for tests at other frequencies. Batteries, controlled by suitable adjustable resistance voltage dividers and series resistors, are used for the necessary direct-current sources. The potentiometer, standard cells, volt boxes, and resistors are those normally used for instrument testing and are periodically standardized to insure a continued accuracy of considerably better than 0.01 percent.

A schematic diagram of the major circuits and equipinent on this panel is slown in figure 4. For an a-c test of an amineter, the transfer thermoelement is connected to the $\Lambda \mathrm{C}-\mathrm{DC}$ binding posts and its thermocouple to the TC posts on the panel. The test instrument is connected to the $\Lambda \mathrm{C}$ posts, and a suitable four-terminal standard resistor is connected to the DC posts fol use in measuring the $\mathrm{d}-\mathrm{c}$ current by means of a potentiometer. For low currents at the higher andio frequencies, shielded leads with the 
shields grounded are used to minimize errors due to stray capacitance currents. The alternating current is adjusted for the desired deflection of the test instrument, and the Iindeck potentiometer is adjusted for an "on-scale" doflection of the galvanometer. The standard instrument is then switched to direct current, which is adjusted to give the same galvanometer deflection and is then measured with the external potentiometer. The direct current through the heater of the thermoelement is then reversed and the d-c measurement repeated. ${ }^{3}$

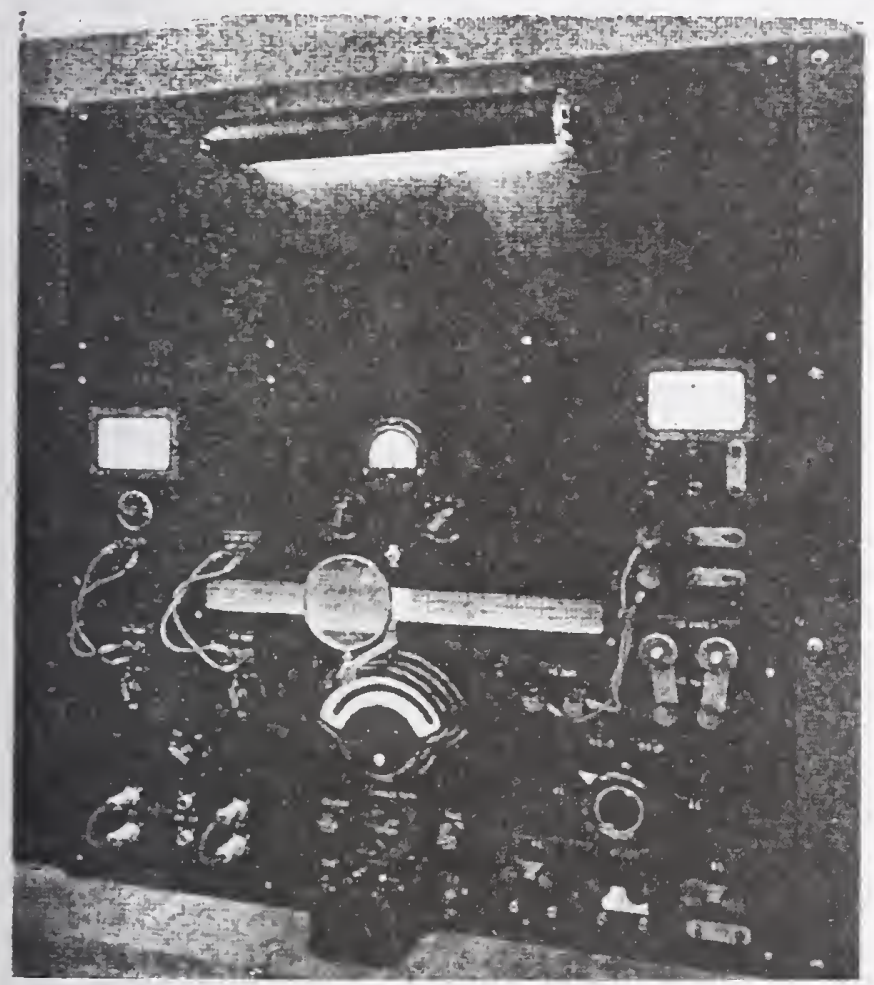

FIGURE 5. Instrument testing panel, including Lindeck element.

For an a-c test of a voltmeter, a similar procedure is used. 'The transfor standard is connected to the AV-DV receptacle on the panel with a coaxial lead, with due care to connect its thermal converter to the grounded shield of this lead, and the thermocouple is connected to the TC posts on the panel. 'The test instrument is connceted to the circuit with a special twin lead consisting of two coaxial lines permanently paralleled at the instrument and connecting to the two AV receptacles on the panel. Similar leads of the same length are used to connect the high side of a volt box for use with the external potentiometer to measure the direct voltage, to the two DV receptacles. The use of these leads eliminates the effect of any stray magnetic field or lead resistance on the measurements.

This simple procedure is reliable only if the drift of the response of the standard instrument is negligibly small in the time taken for a series of such

'Such reversed d-c readings are common practice for highest accuracy with all readings. This is fortunately true for each of the thermal converters used. After an initial warm-up period of a minute or so, the maximum observed drift under those laboratory conditions of any of the thermal converters tested was less than 0.5 percent per hour, and the average less than 0.05 pereent per hour. If it were necessary, the effect of significant drift could be practically eliminated by using a deflection method as outlined for transfer tests, calibrating the standard instrument on direct current at the nominally correct current or voltage before and after each a-c setting.

Fol ac-de difference or transfer tests, which are more frequent and usually more important than straight a-c tests, the test and standard instrurnents are connected in series to the $\mathrm{AC}-\mathrm{DC}$ posts ${ }^{4}$ in the case of ammeters, or in parallel with suitable coaxial leads to the AV-DV receptacles in the case of voltmeters. Suitable short leads are used to connect the two AV receptacles together and to connect the two DV receptacles together. 'The current or voltage is adjusted for the desired deflection of the test instrumont, and the Lindeck potentiometer is adjusted for a deflection near the null position at the center of the galvanometer scale. The two instruments are then in quick succession connected to alternating, direct, reversed direct, and then alternating current. 'The current is adjusted to give the same deflection of the test instrument in each case. 'The resulting deflections of the galvanometer of the Lindeck potentiometer are observed with the potentiometer controls undisturbed. 'This procedure practically eliminates any effect of drift in either instrument, and crrors in repeating the settings of the instrument under test can be made very small by using a lowpower microscope and setting the image of the instrument pointer in coincidence with a cross-hair in the eyepiece. The control circuits have been arranged to facilitate switching, and with two observers the procedure is rapid even with moderately fluctuating voltages as only one of the two instruments is set to a given deflection.

From the average of the deflections on alternating and on direct current, the ac-dc difference of the test instrument is readily computed by a simple formula. ${ }^{5}$

\section{Evaluation of Transfer Performance}

The ac-dc difference of each transfer standard must be known to the full accuracy desired in the measurements to be made. As, in a sense, there are no absolute transfer standards, such an evaluation essentially consists of four steps.

1. Choice of a type of instrument that theory indicates is suitable over the desired ranges.

2. Study of all known effects that can cause such an instrument to depart from this ideal.

3. Construction and use of an instrument in such a way that these effects, by computation or direct tests of the separate components, are negligible or known.

- One terminal of the beater is connected to the post thst is grounded on a)ternating current.

The formulas used in computing the results of the a-c and transler tests aro developed in appendix 1. 
4. Comparison of actual transfer performance of the stundard with that of some other instrument, preferably of a quite different type, whose performance has also been evaluater.

The evaluation of these electrothermic transfer standards was considered of primary importance because of the high oreler of aceuracy demanded in this application. The mnique facilities of the Bureau macle this quite feasible, particularly for step 4, which is necessary to gurard against crrors from unsuspected canses. Prototype instruments such as these, in which previously available ranges all'c greatly extended, cannot be elirectly compared with other types of standards over their full range. The evaluation therefore consisted of the study, computations, and special tests of steps 2 and 3 cluring the design and construction of these instruments and their pancl, the intercomparisons of thermal converters of adjacent current ranges in the series, the comparison at low frequencies of selected thermal converters with the electrodynamic transfer standards at the Bureau, and the comparison of these thermal converter's with certain portable hot-wire and electrostatic instruments also available.

A thermal converter can be used as a transfer standard for alternating-curcent measurements over the range of frequencies for which the response characteristic, $E=f(I)$, is the same as with direet current, where $E$ is the output emf, and $I$ the current through the heater of the thermal converter. For a voltmeter element consisting of a resistor in series with a thermal converter, the magnitude of the effective impedance $Z=\Gamma / I$ must also be cqual to the effective resistance on direct current, where $V$ is the applied roltage. In addition to these two requirements for instrument testing, the fundamental principle that the standard and the instrument under test "see" exactly the same quantity must be carefully observed.

The response characteristic of the types of thermal converters used were believed to be suitable at frequencies far higher than those used in this applica- cation, and their direet-current and low-frequency responses were verified hy sperial tests deseribed in other sections. 'The effective imperanee of each volumeter element was approximately computed from inductanece and capracitance datas supplied by the manufartule of the resistance cards, and was verified by special tests to be deseribed later. The eapacitance and resistance of the lindeck potentiometer to ground were measuresl and found to be $1,600 \mu \mu$ f and 10,000 monolums at an ambiont rolatiro humidity of 40 pereent. Thus the eomputed stray (arrents are suflieienty small so that no apporeciablo fraction of the currents measured will be diverted through the thermoeouple at audio frequencies when one end of the heater is grounded. The use of shielded leads where necessary, the avoidance of a ground between the test instrument and the standard in the current circuits, and the use of the special ('oaxial lines in the voltage circuits assure that the fundamental principle is observed even at the smallest current and voltage in the present ranges.

The comparison tests of step 4 were planned to give maximum possible assurance against elrors due to unsuspected causes. Each comparison consisted of repeated careful transfer (ac-de difference) tests, as described in section 3.4. The 1-, 2- and 5-amp thermal converters were compared with the NBS electrodynamic transfer ammeter at solceted frequencies from 25 to $200 \mathrm{c} / \mathrm{s}$. The model $\Lambda$ voltmeter was compared with the NBS suppressed-zero electrodynamic voltmeter at frequencies of 30 and $60 \mathrm{c} / \mathrm{s}$ and voltages from 20 to $240 \mathrm{v}$. Both of these electrodynamic instruments have been carefully studied $[1,2]$. They can be read with a precision of better than 0.005 pereent. The results of these comparisons, corrected for the small known transfer eirors of the electrodynamic instruments, are shown in table 1. They are estimated to be accurate to 0.005 percent or better. The results of these tests disclosed that the model A voltmeter and the electrodruamic voltmeter were in exeollent agreement at these frequencies.

TAHLE 1. Resulls of comparisons of thermal converters with other lypes of instruments

\begin{tabular}{|c|c|c|c|c|c|c|c|c|c|c|c|}
\hline \multirow{2}{*}{$\begin{array}{c}\text { Thermal converter } \\
\text { range }\end{array}$} & \multirow{2}{*}{ Other instrument } & \multirow{2}{*}{$\begin{array}{c}\text { Test } \\
\text { current } \\
\text { or } \\
\text { voltage }\end{array}$} & \multicolumn{9}{|c|}{ Comparative abe-de dilforence } \\
\hline & & & $\begin{array}{l}2.5 \\
\mathrm{c} / \mathrm{s}\end{array}$ & $\begin{array}{l}30 \\
\mathrm{c} / \mathrm{s}\end{array}$ & $\begin{array}{l}100 \\
\mathrm{c} / \mathrm{s}\end{array}$ & $\begin{array}{l}\operatorname{lik} 1 \\
(\%)\end{array}$ & $\begin{array}{l}2(x) \\
c / s\end{array}$ & $\begin{array}{l}\text { 1. } 1000 \\
c / s\end{array}$ & $\begin{array}{c}10,(1 \times k) \\
6 / 5\end{array}$ & $\begin{array}{l}15,10100 \\
1 \% / 5\end{array}$ & $\begin{array}{c}201,1000 \\
6 / 5\end{array}$ \\
\hline $\begin{array}{l}a m p \\
1 \\
1 \\
1 \\
2 \\
2 \\
5 \\
5 \\
5\end{array}$ & 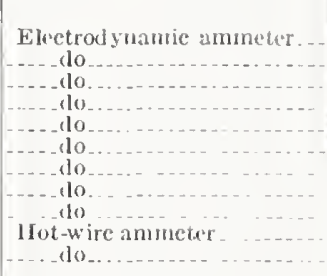 & $\begin{array}{c}a m p \\
0.4 \\
1 \\
1.2 \\
0.8 \\
2 \\
2.4 \\
1 \\
5 \\
6 \\
4 \\
5\end{array}$ & $\begin{array}{r}\% \\
-0.001 \\
-.053 \\
-.067 \\
-.022 \\
-.115 \\
-.154 \\
-.014 \\
-.010 \\
-.011 \\
-\end{array}$ & \begin{tabular}{c}
\multicolumn{2}{c}{$\%$} \\
$\%$ \\
\\
\\
\end{tabular} & $\begin{array}{r}\% \\
+0.001 \\
-.013 \\
-.1011 \\
-.1125 \\
-.1035 \\
-.1001 \\
-.018 \\
-\quad-.\end{array}$ & 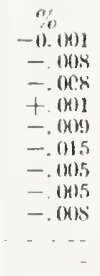 & $\begin{array}{r}\% \\
-0 .(1622 \\
-.1411 \\
-.1615 \\
+.11(1) \\
+.1611 \\
-.110 .2 \\
- \\
-\end{array}$ & 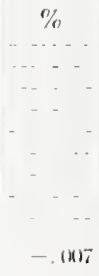 & $\begin{array}{l}\sigma_{j} \\
\cdots \\
\cdots \\
-0 .(1011\end{array}$ & $\begin{array}{c}r \\
-\quad \cdots \\
\cdots \\
\cdots\end{array}$ & 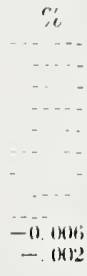 \\
\hline $\begin{array}{l}\text { Moded a voluneter. } \\
\text { Do } \\
\text { Do } 1 \text { Do.... } \\
\text { Do. } \\
\text { Jo } 1 \text {. } \\
\text { 1) }\end{array}$ & 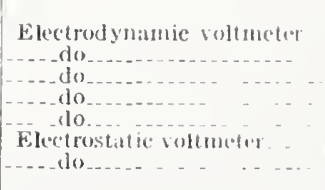 & $\begin{array}{r}v \\
201 \\
501 \\
1: 20 \\
1.00 \\
240 \\
150 \\
3110\end{array}$ & 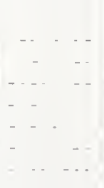 & $\begin{array}{l}-0.0102 \\
- \\
+\quad 1314 \\
+1414\end{array}$ & $\begin{array}{r}-140.1 \\
-.140 .5 \\
-.1413 \\
-.1412 \\
--\end{array}$ & $\begin{array}{l}2 \\
-1122 \\
-1114\end{array}$ & & $\begin{array}{r} \\
+0114 \\
+.011\end{array}$ & $-(K) 1$ & $-1)(1108$ & $\begin{array}{c}. \\
. \\
\cdots \\
-(111) 2 \\
+(14)\end{array}$ \\
\hline
\end{tabular}


They disclosed significant disagreements between the ampere-range thermal converters and the electrodynamic ammeter, which were considered to be duc to a low-frequeney error in the 1 - and 2-amp thermal converter's and a small error independent of frequency in the 5 -amp thernal converter.

'The model it voltmeter was also compared with two new high-grade portable pivoted electrostatic voltineters, having scales over $j$ in. long, at selected frequencies from 30 to $20,000 \mathrm{c} / \mathrm{s}$. For increased precision a low-power microscopc was focused on the pointer of the elcchostatic instrument under test. This instrument was always set to the same deflection and was tapped lightly before cach reading. Repeated sets of carcful readings were taken at each frequency, and all tests were at the full-scale deflection of the clectrostatic instrument. Because of these repeated readings and the favorable conditions, the averaged results are estimated to be accurate to 0.01 percent. The results, as shown in table 1 , verify the abscnec of capacitance errors in the thermal voltmeter. Dircet comparisons at the higher frequencies could not bc madc at lower voltages, but calculations indicate that skin effect and the cffect of inductance should be considerably less than 0.01 percent at $20,000 \mathrm{c} / \mathrm{s}$.

Similarly, the 5-amp thermal converter was compared with a high-quality portable 5-amp loot-wirc ammeter at frequencies from 25 to $20,000 \mathrm{c} / \mathrm{s}$. 'The results, as shown in table 1 , indicate cxcellent agreement at frequencies of $1,000 \mathrm{c} / \mathrm{s}$ and above. 'Tests at lower frequencies showed very puzzling discrepancies, which, in a separatc investigation, wele traced to vibration crrors in the hot-wire instrument. For that reason the results at frequencies less than $1,000 \mathrm{c} / \mathrm{s}$ are not shown in this table.

A plus sign in table 1 indicatcs that with equal response of thic thermal converter on direct and alternating currents the response of the other instrument was greatcr on alternating than on direct current.

Each thermal converter was compared with the next higher- and lower-range thermal converter in the series at two currents and at selected frequencies from 25 to $20,000 \mathrm{c} / \mathrm{s}$. Thic results of cach of these comparisons at the higher of the two currents at which tests were made are slown in table 2 . They are estimated to be accuratc to somewhat better than 0.01 percent, with very littlc possibility of systematic error. A plus sign in the table indicates that with equal response of the lower-range thermal converter on alternating and on direct currents, the response of the higher-lange thermal converter was greater on alternating than on direct currents. This series of tests was made to extend stepwise the results of low-frequency comparisons with the electrodynamic ammeter, and to indicate any highfrequency effects that might well be expected to differ in different thermal converters. ${ }^{7}$ The group

\footnotetext{
- Electromagnetic forces between the hot wire and its clusely adjacent iron mounting plate resulted in vibration of the $\mathrm{x}$-ire hecause the plate was magnetized by the damning magnet of the instrument. This vibration caused additional cooling and thus an error that was as large as 0.2 percent at certain frecinencies. The percentage errors due to any high-frequency effects would be expected to be independent of the current level and could thus be craluated by these tests.
}

TARIE 2. Resulls of intercomparisons of thermal convertcrs

\begin{tabular}{|c|c|c|c|c|c|c|c|}
\hline \multirow{2}{*}{$\begin{array}{c}\text { Thormal monvertur } \\
\text { ranges }\end{array}$} & \multirow{2}{*}{$\begin{array}{l}\text { Test } \\
\text { cosr- } \\
\text { rent }\end{array}$} & \multicolumn{6}{|c|}{ Comparative acedle differener } \\
\hline & & $\begin{array}{l}2.5 \\
\mathrm{e} / \mathrm{s}\end{array}$ & $\begin{array}{l}100 \\
c / 5\end{array}$ & $\begin{array}{l}400 \\
\mathrm{c} / \mathrm{s}\end{array}$ & $\begin{array}{c}1,000 \\
\mathbf{c}_{i}^{\prime} / \mathrm{s}\end{array}$ & $\begin{array}{c}10,0(0) \\
\varepsilon_{f}^{\prime} s\end{array}$ & $\begin{array}{c}20,(1 / n) \\
c / s\end{array}$ \\
\hline & $m a$ & $\%$ & $\%$ & 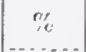 & $\because$ & $\%$ & $\frac{\pi}{6006}$ \\
\hline 2 i & 2.4 & $\begin{array}{r}1.060 \\
+.006\end{array}$ & & & $\begin{array}{r}0.1000 \\
+.1003 \\
\end{array}$ & & $-\begin{array}{l}T \\
-.003\end{array}$ \\
\hline & 6 & +.004 & & & .0000 & &.$(1) 0$ \\
\hline 10) $t$ & 12 & .000 & & & +.104 & & -.006 \\
\hline 20 to & 24 & +.001 & & $-0.00: 3]$ & -.001 & & $.0 \%$ \\
\hline $30=10.501$ & 36 & +.004 & & & .600 & & .000 \\
\hline 5) $t$ & 60 & +.024 & +0.038 & & +.1036 & & +.027 \\
\hline & 100 & -.046 & & & -.044 & -0.0 & -.049 \\
\hline & 240 & +.026 & & & & & +.039 \\
\hline 500 to $1,000 \mathrm{~m}$ & 600 & -.002 & & & -.002 & +.002 & +.000 \\
\hline & & & & & & & \\
\hline $1,090 \mathrm{ma}$ to $1 \mathrm{amp}$ & 1. 2 & +.034 & & & -.065 & $-.056 \mid$ & -.065 \\
\hline & $\frac{1}{2}$ & $\begin{array}{l}-.028 \\
-1123\end{array}$ & $\begin{array}{l}-.002 \\
-.011\end{array}$ & & $=.002$ & $\begin{array}{l}-.002 \\
+.004\end{array}$ & $\begin{array}{r}+.001 \\
+.009\end{array}$ \\
\hline 5 to & 5 & -.008 & $-.000 \mathrm{~s}$ & & -.006 & $\mid$\begin{tabular}{|c}
$T .007$ \\
-.007
\end{tabular} & -.004 \\
\hline & 10) & -.010 & -.010 & & -.008 & -.012 & -.000 \\
\hline 20 to $50 \mathrm{ill}$ & 20) & -.014 & -.015 & -.014 & -.018 & -.035 & \\
\hline
\end{tabular}

a This 30-ma thermal converter was the onc used in the model A voltmeter.

of tests disclosed small discrepancies independent of frequency in a few thermal converters, and verified the absence of any high-frequency errors in the thermal converters tested. However, they could not be used to assign numerical values to the crrors of individual thermal convertcrs in the milliampere range as the differences were found to depend upon thic current. The concluding series of tests to evaluate these crrors were comparisons of a shunted thermal converter with each of those pairs of thermal converters that showed discrepancies in the previous intercomparisons. 'The model A voltmeter was shunted with a bifilar resistor of computed skin effect and time constant for tests at currents from 1 to 40 amps, and with a suitable high-quality audio-frequency decade resistancc box for tests at lower currcnts. 'The results are shown in table 3 and are estimated to be accurate to better than 0.01 percent. A positive sign in this table indicates that with equal

TABLE 3. Results of comparisons of selected thermal converters with shunted model $A$ thermal voltmeter

\begin{tabular}{|c|c|c|c|c|}
\hline \multirow{2}{*}{$\begin{array}{l}\text { 'Therinal converter } \\
\text { range }\end{array}$} & \multirow{2}{*}{$\begin{array}{l}\text { Test } \\
\text { current }\end{array}$} & \multicolumn{3}{|c|}{ Conuparative ac-de difference } \\
\hline & & $25 \mathrm{c} / \mathrm{s}$ & $100 \mathrm{c} / \mathrm{s}$ & $400 \mathrm{c} / \mathrm{s}$ \\
\hline$m n$ & $m a$ & $\%$ & $\%$ & $\%$ \\
\hline $30 \ldots$ & $\begin{array}{r}60 \\
70\end{array}$ & $\begin{array}{l}-0.003 \\
\cdots\end{array}$ & $\begin{array}{r}-0.043 \\
-0.06\end{array}$ & $-\ldots \ldots$ \\
\hline 100 & $\begin{array}{l}100 \\
1 \neq 0\end{array}$ & -065 & $\begin{array}{r}-.062 \\
-. .--\end{array}$ & -0.065 \\
\hline $200 \ldots$ & 240 & -.010 & +.002 & +.002 \\
\hline $500 \ldots$ & 400 & -.056 & (n) & -.054 \\
\hline & 400 & $\begin{array}{l}-.048 \\
-.041\end{array}$ & & \\
\hline ـ & $\begin{array}{l}600 \\
800\end{array}$ & -.061 & $\begin{array}{l}-.060 \\
-.059\end{array}$ & (n....... \\
\hline$a n p$ & $a m p$ & -.004 & & -006 \\
\hline $10 \ldots$ & $\begin{array}{l}10 \\
12 \\
10\end{array}$ & $\begin{array}{r}.018 \\
-\quad 003\end{array}$ & -.014 & $\begin{array}{l}-.015 \\
-.020 \\
-.006\end{array}$ \\
\hline 20. & 20 & -023 & -.015 & -.017 \\
\hline $30 \ldots$ & $\begin{array}{l}24 \\
40\end{array}$ & $\begin{array}{l}-.008 \\
-.010\end{array}$ & - & $\begin{array}{l}-.010 \\
-.010\end{array}$ \\
\hline
\end{tabular}


response of the thermal converter on alternating and on direct current the response of the shunted model A voltmeter was greater on alternating than on direct current. The results of tests of the 1-,2-and 5 -amp thermal converters, duplicating those with the electrodynamic ammeter, are not shown in the table. The average difference between eight such duplicate results obtained with this ammeter and with the shunted model A voltmeter was 0.004 percent, and the largest difference was 0.013 percent. A few additional check tests of these thermal converters with the shunted voltmeter were made at other frequencies but are not tabulated.

The results of all these tests were combined to assign reliable values of ac-dc difference, $\delta$, to each thermal converter in the series from $1 \mathrm{ma}$ to $50 \mathrm{amp}$ and to the model $A$ voltmeter. These values were less than 0.01 percent for the model $\mathrm{A}$ voltmeter and for all the thermal converters of ranges less than 100 ma. The values for the other converters (rounded off to the nearest $0.005 \%$ ) are shown in table 4 .

TABLE 4. Observed percentage ac-dc differences of thermal converters (to the nearest 0.005 percent)

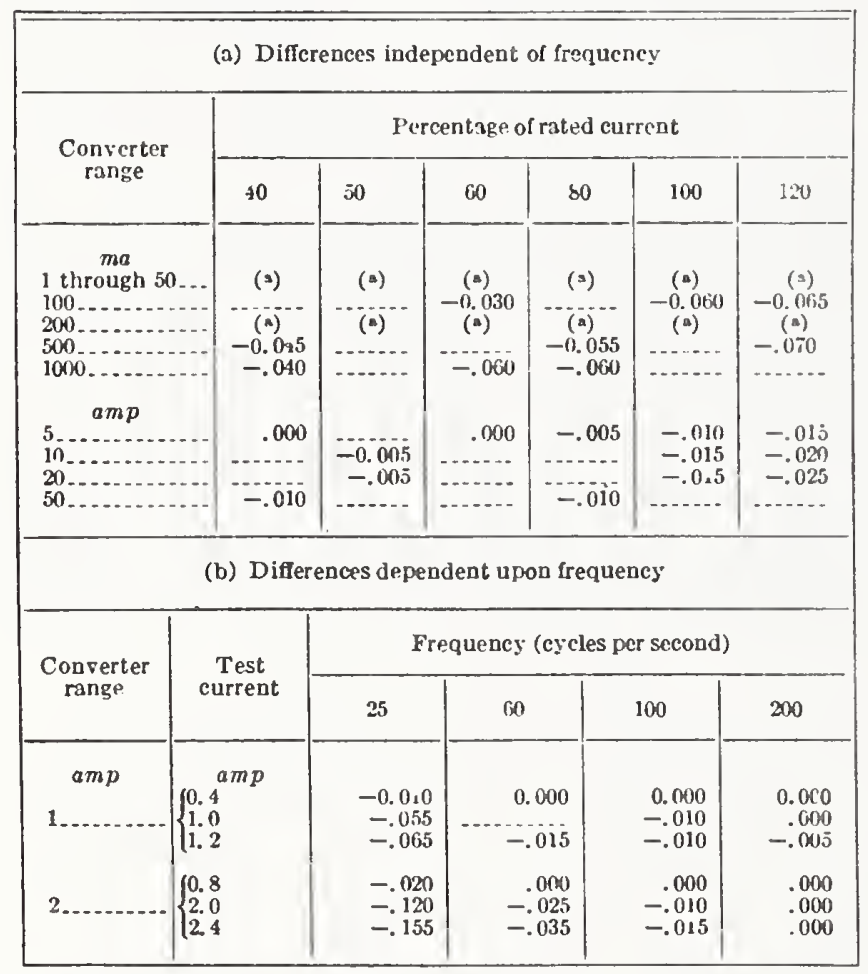

- Less than 0.01 percent throughout.

The model B voltmeter, which was developed to give a wider voltage range and greater convenience of use than the model $\AA$, was compared with the model A voltmeter at rated voltage on each range up through the 300-v range and at 400 v on the higher ranges, at selected frequencies from 30 to $20,000 \mathrm{c} / \mathrm{s}$. The results are shown in table 5 and represent directly the ac-dc difference, $\delta$, of the model $\mathrm{B}$ voltmeter. They disclosed significant but not unexpected errors for the higher voltage ranges at the higher frequencies.
TABLE 5. Kesulls of comparisnns of model $A$ and model $B$ thermal vollmelers

\begin{tabular}{|c|c|c|c|c|c|c|}
\hline \multirow{2}{*}{$\begin{array}{l}\text { Rarge of } \\
\text { model B }\end{array}$} & \multirow{2}{*}{ Voltage } & \multicolumn{5}{|c|}{ ac-de differenec of model B voltmeter } \\
\hline & & $\begin{array}{l}25 \\
\mathrm{c} / \mathrm{s}\end{array}$ & $\begin{array}{c}1,000 \\
\mathrm{c} / \mathrm{s}\end{array}$ & $\begin{array}{c}5, \mathrm{mmo} \\
\mathrm{c} / \mathrm{s}\end{array}$ & $\begin{array}{c}15,010 \\
\mathrm{c} ! \mathrm{s}\end{array}$ & $\begin{array}{c}20,0000 \\
\mathrm{c} / \mathrm{s}\end{array}$ \\
\hline $\begin{array}{l}\text { Volts } \\
3 \text { throwgh } 75 \\
150 \\
300 \ldots \\
600 \ldots \\
750 \ldots\end{array}$ & $\begin{array}{c}{ }^{v} \\
\text { Ratted } \\
400 \\
400\end{array}$ & $\begin{array}{c}\text { (a) }^{7 /} \\
+0.003\end{array}$ & $\begin{array}{c}\% \\
(=) \\
\cdots+0.011\end{array}$ & \begin{tabular}{c}
$\%$ \\
$(x)$ \\
\hdashline$\ldots$ \\
+0.014 \\
+.028
\end{tabular} & \begin{tabular}{c}
$\%$ \\
in \\
\hdashline \\
+0.044 \\
+.064
\end{tabular} & $\begin{array}{c}\% \\
(\% \\
+0.013 \\
+.02 \\
+.051 \\
+.082\end{array}$ \\
\hline
\end{tabular}

- Less than 0.01 percent. 25 to $20,000 \mathrm{c} / \mathrm{s}$ each range,.

As a result of these extensive series of tests, the transfel performance of each of this first set of thermal converters and of each of the two voltmeters was uniquely determined. Small but siguificant acde differences of both known and unsuspected causes were found. Corrections for these differences can readily be applied when these thermal converters are used as transfer standards. However, an investigation of such differences is an especially important objective of a development such as this. As a result of such an investigation, a number of factors that limit the transfer accuracy of an electrothermic instrument were discovered and evaluated, with the results given in the following sections.

\section{D-C Response}

In all measurements with a transfer standard the average of the d-c response of the standard for the two directions of current is taken as the reference upon which the a-c measurements are based. For all three types of such standards, the reversed d-c averay'e gives a better basis than does the response for one direction alone as effects that lead to small differences in response for the two directions of direct current are reduced to the second order of smallness when the average is taken.

Reversal differences may be expected in a contact thermal converter because of the flow of lieater current through the hot junction of the thermocouple. This can cause a voltage drop in the thermocouple circuit, which may lead to second-order errors because of the nonlinearities of the thermal and electric. circuits. This cause of reversal difference is minimized by the special constructions used in most high-range thermal converters, and is eliminated by the use of the small insulating bead between the heater and the thermocouple in the low-range thermal converters. Surprisingly, however, early tests of the first thermal converters purchased for the model A voltmeter showed appreciable reversal differences (up to $0.2 \%$ ) even in these insulated-heater thermal converters. It was soon realized that this was probably due to thermoclectric effects in the heater circuit. Peltier and Thomson heating of the heater can cause dissymmetry in the temperature rise of the heater, and thus cause differences in the emf of the thermocouple unless the hot junction is exactly at the 
midpoint of the heater." This supposition was verificd br an analysis of the data, which showed that the differences were larger in those thermal converters with heaters having larger thermoelectric effects. Further verification was secured by tests of special thermal converters, constructed by one of the manufacturers ${ }^{3}$ at the Bureau's request, with the hot junction of each deliberately and considerably offcenter. The reversal differences were much larger, and the signs and magnitudes were found to be roughly in agreement with calculations based on thermoelectric data for the alloys used.

Early intercomparisons of these few thermal converters indicated that no significant ac-dc differences should be expected, even with rather large reversal differences from this cause, provided that the average for the iwo directions of current is taken as the $\mathrm{d}-\mathrm{c}$ response. However, intercomparisons and tests of the much largel number of thermal converters later purchased for current measurements showed that a good proportion had measurable ac-dc differences, all of the same sign and independent of frequency. This suggested a d-c error, for which these thermoelectric efforts seemed the most likely cause. This was investigated theoretically (see appendix 2) by considering the temperature rise of a homogeneous conductor heated by an electric current and cooled solely by conduction to two relatively massive terminals; i. e. by neglecting, for simplification, radiation and convection losses and the heat abstracted by the attached thermocouple.

With only the normal joule, or resistance, heating, and with the terminals of the conductor at equal and fixed temperature, the temperature distribution along the conductor is parabolic, and the temperature rise at the midpoint is, as Groodwin showed, ${ }^{10}$ equal to

$$
\theta_{d}=\frac{V_{s}^{2}}{8 \rho k}
$$

where $V_{c}$ is the voltage drop across the conductor, and $\rho$ and $k$ are the electrical resistivity and thermal conductivity, respectively. In addition, however, there is Peltier heating or cooling at the junctions of dissimilar metals (the two junctions between the heater and its two supports) and Thomson heating along each half of the wire. Unlike joule heating, these are dependent upon the direction of the current flow and can thus cause a dissymmetry of the temperature distribution along the conductor, which reverses when the direct current through the conductor is reversed. On alternating current, even at the low frequencies in which we are interested, the reversal of current occurs so rapidly that the thermal inertia of the wire prevents any such dissymmetry, and the temperature dis-

This dissymmetry has actually been utilized to measure the Thomson effect. See [9].

'M. Rosenfleld, of the Field Electrical Instrument Co., who first showed that the reversal differences were dependent upon the type of heater material.

10 See [6]. In addition to this classic paner, Goodwin has written an excellent series of artlcles on "Thermal prohlems relating to measuring and control serv. ices", which appeared in Weston Eng. Notes, 1948-50 (Weston Electrical Instrument Corp., Newark, N. J.). tribution is unchanged by these themoelectric effects. Calculations show (see appendix 2) that with direct current the temperature rise at the midpoint of the conductor, where the hot junction of the thermocouple is attached, is unaffected by the Peltier heating, for completely symmetrical construction of a thermoelement. They show, however, that it is changed by Thomson heating, and becomes, approximately, ${ }^{11}$

$$
\theta_{\imath}=\theta_{d}\left[1-\frac{4}{3}\left(\frac{\sigma \theta_{d}}{V_{\epsilon}}\right)^{2}\right]
$$

where $\theta_{t}$ is the midpoint temperature rise in the presence of Thomson heating, in ${ }^{\circ} \mathrm{C} ; \theta_{d}$, the rise without Thomson heating; $\sigma$, the Thomson voltage coefficient in volts-degree ${ }^{-1}, V_{c}$, the voltage across the conductor in volts.

This was derived by considering $\sigma$ a constant, whereas both experimental results and the electron theory of metallic concluction indicate that $\sigma=B T$, where $B$ is a constant and $T$ is the absolnte temperature. However, a solution by a perturbation method, ${ }^{12}$ of the nonlinear equation that results when $\sigma=B T$, shows that the above results give a sufficiently close approximation for the temperature rise of about 200 deg $\mathrm{C}$ encountered in typical thermal converters. Thus eq 2 shows that an ac-clc difference, or transfer error, should be expected in a thermal converter, and enables the approximate calculation of this error. Such calculations have been made for some common metals and some of the alloys used as heaters in thermal converters, on the assumption of a $200 \mathrm{deg} C$ rise at the midpoint, and a voltage drop of $0.2 \mathrm{v}$ at rated currents. ${ }^{13}$ The results, converted to ac-dc difference for the same temperature rise, rather than temperature difference for the same current, are shown in table 6 . For the computation of these results, the Thomson coefficient $\sigma$ was considered to be equal to $B\left(\theta_{0}+2 / 3 \theta_{d}+273\right)$, where $B$ is the second derivative of the characteristic curve of emf versus temperature for a thermocouple composed of the indicated metal and lead. ${ }^{14} \quad \theta_{0}$ is the ambient temperature in $\operatorname{deg} \mathrm{C}$. The results show approximately the errors to be expected for these materials.

TABLE 6. Calculated ac-dc difference at rated current due in

\begin{tabular}{|c|c|c|}
\hline Metal & B & $\begin{array}{c}a c-d c \\
\text { difference }\end{array}$ \\
\hline $\begin{array}{l}\text { Copper } \\
\text { Platinum } \\
\text { Manganin } \\
\text { 90 Ni-10 } \mathrm{Cr}(60 \mathrm{Cu}-40 \mathrm{Ni}) \\
85 \mathrm{Pt}-15 \mathrm{lr}\end{array}$ & $\begin{aligned} & n{ }^{\circ} \mathrm{C} \\
+ & 0.8 \times 10^{-8} \\
- & 3.2 \\
- & 0.08 \\
-7.3 & \\
-3.2 & -2.1\end{aligned}$ & $\begin{array}{l}\text { Percent } \\
<0.005 \\
=.013 \\
<.005 \\
=.064 \\
-.013 \\
-.005\end{array}$ \\
\hline
\end{tabular}
Thomson effects in thermal converter heaters

11 At other points along the conductor the average temperature rise lor the two directions of direct current is also changed by Thomson heating.

12 This method was suggested hy Chester Snow.

13 From eq 1, a series of thermal converters having similar thermocouples and the same rated output emf at rated current, and having heaters that obey the Weidemann-Franz luw will all have the same tempersturo rise and the same voltage drop across the heater. The temperature rise is about $200 \mathrm{deg} C$, and the voltage drop about $0.2 \mathrm{v}$ at rated current for mast thermal converters.

14 This weighted average ahsolute temperature gives resilts in closer agreenent with those computed by the inore exact method than does the unweighted a verage, ot $1 / 20+273$. 
The results may be compared with the observed ac-de differences shown in table 4. Irom information supplied by the manufacturers, the 1-, 2-, 5-, 10-, 20-, 50-, and 200-ma thermal eonverters hare heaters of either earbon, nickel-ehromium, or platinumrhodium-ruthenium alloys. The observed ac-de differences of these converters were less than 0.01 percent. The 100-, 500- and 1,000-ma thermal converters had heaters of constantan (Advanee). 'The observed differences for these converters (at ruted current) were close to the calculated value of -0.06 pereent. The 1-and 2-amp converters, of platinumiridium, had observed differenees (from table 4,b) less than 0.005 percent, except at low frequeneies, also close to the calculated values. The 5-, 10-, 20and 50-amp converters, of a speeial platinum-3pereent-copper alloy, had differences of -0.010 to -0.015 percent. 'Thus the agreement between the computed and observed values serves as excellent verification of this source of error in transfer thermal converters. Very fortumately, the error is significantly large only for materials having unusually large thermoelectric effects, such as constantan. However, this does lead to the interesting eonclusion that for operation at a low temperature a thermal converter should have large thermoelectric effeets in the thermocouple, but that for the highest transfer accuraey it should have very small thermoeleetric effects in the heater.

Additional experimental verification of this cause of transfer error was seeured by constructing ${ }^{15}$ at the Bureau two 5-anp thermal converters differing only in that one had a heater of manganin and the other of constantan. These were 5-anp thermal converters with a snall Borax bead between the hot junction of a copper-constantan thermocouple and the center of the heater wire, to eliminate contact eflects. Careful ac-dc difference tests with the standard electrodynamic ammeter as the reference established agreement within 0.005 percent for the manganin element and indicated an ac-dc difference of -0.06 pereent for the constantan element at an emf of about $5 \mathrm{mv}$.

As a result of this work, a second set of thermal converters in the 1- to 1,000-ma range was purchased under speeifications prohibiting heaters of constantan or similar alloys. All of these eonverters were found to have ac-de differenees less than 0.01 percent at audio frequencies.

\section{Low-frequency Response}

It has long [10] been known that there is a lowfrequeney limit to the correct rms response of a thermal converter, for reasons analogous to those causing a similar low-frequency limit in all other types of transfer instruments. Below this limit the response characteristic $\mathrm{E}=f(\mathrm{I})$ is not independent of frequeney (except for symmetrieal square waves), and may be expected to be in error by an amount that is inversely proportional to the square of the frequency. $\Lambda$ s table $4, \mathrm{~b}$, shows, such an error was observed for the 1- and 2-amp thermal eonverters.

is These thermal converters were constructed by E. S. Williams.
When a constant direct rurent is applied, the heater of a thermal converter reaches a constant temperature. When an alternating current is applied (after the transient state is orer) the temperature of the heater varies cyclically about a mean value, with an amplitule that decreases with increasing frequency because of the thermal capaeity of the element. At frequencies high enough so that the crelic variations are negligible, the temperature rise of the heater is independent of frequency (provided that at very high frequencies the skin effeet and electromagnetic radiation are nogligible and that standing waves do not alter the temperature distribution). However, at lower frequencies the mean value, to which the (l-c instrument or potentiometer eonnected to the thermocouple responds, may not be the same beeause of nonlinearities in the thermal and electric eircuits. 'These nonlinearities are caused principally by radiation losses, the dependence upon temperatire of the eleetrical resistivity and the thermal eonductivity of the heater, and the corrature of the emf versus temperature eharacteristic of the thermocouple. A theoretical evaluation of these effects for the eonditions in a thermal converter, in which most of the cooling is by conduction to the heater, is difficult, but does serve as a guide in determining the approximate limiting frequeneies for an arbitrarily cliosen error and for determining whieh factors influence that error. Such an approximate analysis has been carried out by a perturbation method (see appendix 3). The results show that the average (time average) temperature rise at the midpoint of a conductor heated by a sinusoidal electrie current and cooled solely by conduction to massive terminals is approximately

$$
\theta_{a c}=\theta_{d c}\left[1+\frac{h}{2} \theta_{d c} q^{2}\right]
$$

and that the percentage ac-dc differenee of a thermal converter having the hot junction of a themocouple fastenced to the midpoint of such a heater is approximately

where

$$
\delta=-25 I I \theta_{d c}\left(\eta^{2}\right.
$$

$\theta_{a c}=$ the midpoint temperature rise in degrees eentigrade on alternating eurrent of rms value $I$ amperes

$\theta_{d c}=$ the midpoint temperature rise in degrees centigrade for the same value of direct current.

$h=\alpha-\beta-2 N T_{0}^{2} l^{2}$, where $\alpha$ is temperature eocflicient of eleetrical resistivity.

$\beta=$ the temperature coefficient of thermal conductivity of the conductor,

$N=p \zeta K / a k$.

$p$ and $a=$ the perimetel and area of the cross section of the conductor, respectively.

$\zeta=$ the emissivity.

$k=$ the Stefan-Boltzmann constant in watts-cm-2-(deg $K)^{-4}$

$k=$ the thermal eonductivity in watts$\mathrm{em}^{-1}-\mathrm{leg}^{-1}$ 
$T_{0}=$ the ambient temperature in degrees Siclvin

$q=d / / \omega l^{2}$ and is (approximately) the ratio of the erest value of the cyclic fluctuation of temperature at the midpoint to the average temperatur. rise at the midpoint

$\omega=2 \pi f$, where $f$ is the frequency of the applied current in cycles per second.

$d=$ the thermal diffusivity of the heator inatcrial in em²-sec ${ }^{-i}$.

$l=$ one-lialf the lengtli of the conductor in centimeters.

$I I=h+B / 2 A$, where $A$ and $B$ are the constants in the cmf-tcmperature characteristic for the thermocouple itself, that is, in the expression, $E=A \theta+(13 / 2) \theta^{2}$

These equations indicate the factors upon which the low-frequency error epends. 'They show that the temperature rise can corrcetly be used as a measure of the runs alternating current, at frequencies high enough so that the second term in the bracket of $\mathrm{cq} 3$ is negligible for the aceuracy desired; in other worels, at frequencies for which the heater integrates reasomably well. 'This is true even if the thermal and electrical conductivities of the heater are dependent upon temperature. As a corollary, these equations show that a thermal converter correctly inclicates rms response, except at low frequencies, cven though its response chalacteristic $E=f(I)$ is not quadratic.

'The above equations are valid only at frequen-cies for which the second term in the brackets of eq 3 is small compared to 1 .

Equation 4 may bc used to calculate approximately the frequencies for which ae-de differences may be expected to cause an error of an arbitrarily chosen value. 'This has been done for some typical materials for an error of 0.1 percent, a temperature rise of $200^{\circ} \mathrm{C}$, and a typical henter length of $0.4 \mathrm{~cm}$. The results are shown in table $7 .^{16}$

TABI.E 7. Frequencies for which the computed ac-dc difference is 0.1 percent

[TC-A signifies a thennoemule of Chromel P'and Alumel, ant 'TC-B a thermocouple of eopler and constantian, for which $13 / 2 \mathrm{~A}=-0.1 \times 10^{-3}$ and $+0.9 \times 10^{-3}$, respectively:

\begin{tabular}{|c|c|c|c|c|c|}
\hline \multirow{2}{*}{ Heater niaterial } & \multicolumn{2}{|c|}{$\begin{array}{l}\text { Apjoroximate temperature } \\
\text { eoeffiesent }\end{array}$} & \multirow{2}{*}{$\begin{array}{l}\text { 'Thernal } \\
\text { dillusivity }\end{array}$} & \multicolumn{2}{|c|}{$\begin{array}{l}\text { Frequency for } \\
\delta=0.1:\end{array}$} \\
\hline & $\begin{array}{l}\text { Eleetrical } \\
\text { resistivity }\end{array}$ & $\begin{array}{l}\text { Thermal } \\
\text { conductivity }\end{array}$ & & $1 \times C-A$ & $\mathrm{TC}-\mathrm{B}$ \\
\hline 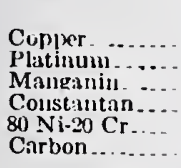 & $\begin{aligned} & \%{ }^{\circ} \mathrm{C} \\
&+ 3.9 \times 10^{-1} \\
&+ 3.5 \\
&< .1 \\
&< .1 \\
& .1 \\
&-.1\end{aligned}$ & $\begin{array}{c}\% /^{\circ} \mathrm{C} \\
-1) .2 \times 10-1 \\
+.5 \\
+2.7 \\
+2.3 \\
+3.5 \\
+.4\end{array}$ & $\begin{array}{c}\mathrm{cm}^{2}-\mathrm{sec}-1 \\
1.1 \\
.25 \\
.075 \\
.0 \mathrm{ff4} \\
.1038 \\
.2\end{array}$ & $\begin{array}{c}c / s \\
(1) \\
12 \\
3 \\
3 \\
2 \\
5\end{array}$ & $\begin{array}{c}c / s \\
70 \\
14 \\
2 \\
2 \\
2 \\
1\end{array}$ \\
\hline
\end{tabular}

- For a heater $0.4 \mathrm{rm}$ lone with a temperature rise of $200^{\circ} \mathrm{C}$.

- Estimated froin Lorenz law.

16 'The effect of radintion iosses lias been neglecterl in preparing this table. In general, this effeet is significant ouly in the very low and very high rauge In general, this effeet
thermal converters.
It is evident that the most significant factor in the cror trim of eq 4 is the length of the lieater as the error is alependent upon the fourth power of this quantity. ${ }^{17}$ It is of interest to note that the lenertis, $2 l$, of the heater of the 1 - and 2-amp thermal converters arc only 0.28 and $0.19 \mathrm{~cm}$, respectively, and that the length of cach of the hrigher-rance thermal converters is about $0.5 \mathrm{~cm}$. The value of $H$ can be determined from the departure from "square law" of the characteristic equation $E=f(I)$ of a thermal converter. For these thermal comverter's it was comsiderably less than the factor given for platinum in table 7 . 'The estimated froquencies for a $0.1-p c r c e n t$ crror, on the assumption that the diffusivity of the plat inum-iridiun alloy used in these thermal colverters is the same as that of platimum, were about one-half the observed values.

\section{High-Frequency Response}

As these thermal converters are inherently suitable at frequelleies up to $100 \mathrm{MC}$ ol more, the upperfrequency limit is set by the circuits in which they are used. The precautions indicated in section 4 are sufficient to control capacitance and leakage currents in tests of ammeters at frequencies considerably higher than the $20,000 \mathrm{c} / \mathrm{s}$ established as the present upper limit by the demand for this testing service. The actual upper-frequency limit has not been established.

The magnitude of the effective impedance sets the upper frequency limit for the model $A$ and the model I3 voltmeters. For an accuracy of 0.01 percent at $20,000 \mathrm{c} / \mathrm{s}$, a time constant of less thran $1 \times 10^{-7}$ and a skin effect less than 0.01 percent are necessary for the impedance of each step of the model $A$ and each range of the model $B$ instruments. The time constant of each decade of the model $A$ voltmeter was computed from the inductarice and direct-capacitance values furnished by the manufacturer of the resistance box used. This was less thran $5 \times 10^{-8}$ for each decade. The skin effect was also negligible for each decade. The resistance box is connected as a three-terminal resistor, with the shield connected to the grounded side of the line. For this connection the errors caused by the capacitance currents to the shield are much less than those that would occur if the shield were connected to a resistor terminal. They are significant only for the two 1,000-ohmsper-step decades. The effects of the capacitance of the resistance cards and switch studs of these decades to the shicld were computed from the manufacturer's capacitance figures of about $10 \mu \mu$ per decade by considering the capacitance as distributed along a uniform transmission line, composed of resistance elements and slıort-circuited at the end. From the usual transmission line formulas, the magnitude of

17 For a given lomiter alloy, tho low-Irequeney range can the cxteuded by using longer hesters, with propertionately greater "ros;-sectional area to give the same tenierature ise for the same current. If solit lieatiors are usel, the improved low-frequeney perforulane: will he acomponiel by iusreased error due to skin

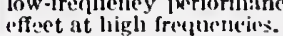


the current at the short-circuited end was computed as approximately

$$
\mid I !=\frac{V}{R}\left[1-0.0056(\omega C R)^{2}\right],
$$

where

$$
\begin{aligned}
& V=\text { the applied voltage } \\
& I=\text { the current at the short-circuited end } \\
& R=\text { the resistance in the circuit } \\
& C=\text { the total capacitance to shield of the } \\
& \omega=2 \pi f .
\end{aligned}
$$

At $20,000 \mathrm{c} / \mathrm{s}$ for a capacitance of $30 \mu \mu \mathrm{f}$ this is changed by less than 0.005 percent at $20,000 \mathrm{ohms}$, the maximum setting. The effect of the capacitance (about $10 \mu \mu f$ ) of each rather large switch blade to the shield was separately computed, and found to be less than 0.005 percent at $20,000 \mathrm{c} / \mathrm{s}$. Thus it was concluded that the errors of this voltmeter should be a maximum of 0.01 percent at the highest settings and frequencies used, and less at lower settings and frequencies. The performance at the top settings was verified by the tests outlined in section 4 .

The impedance of the model B voltmeter was similarly computed, and only the capacitance to the shield was found to be significant. As a rough but adequate approximation this may be considered the same for each step, which leads to the approximation of considering the capacitance as distributed along a nonuniform transinission line composed of resistance elements and short-circuited at the end. The effective impedance was computed by a perturbation method, ${ }^{18}$ on the rough assumption that the resistance per unit length was proportional to the square of the electrical distance from the output end. The magnitude of the current at the short-circuited end was found to be approximately

$$
|I|=\frac{V}{R}\left[1-0.0022(\omega C R)^{2}\right]
$$

From the measured capacitance of $50 \mu \mu \mathrm{f}$ and the total resistance of $100,000 \mathrm{ohms}$ for the $750-\mathrm{v}$ range, the change in current was computed as -0.09 percent at $20,000 \mathrm{c} / \mathrm{s}$, which is in very good agreement with the observed results of table 5. Moderately good agreement for the other ranges was also obtained, but the change in current was not observed to increase in proportion to the square of the frequency as indicated by eq 6 . However, the agreement is good enough to show that the ac-dc difference of this instrument is due to capacitance currents to the shield. These currents cannot easily be reduced in a shielded multirange instrument of reasonable size. (A shield is desirable to control the currents.) They could be compensated by connecting appropriate capacitors across sections of the resistors, but this seems less desirable than the procedure of applying small corrections and has not been done in the model B voltmeter. Much of the capacitance is that between the binding posts of each range (see fig. 2)

is See appandix 4. and between the high-side binding post and the top shicld. This shicld is brought as close as $2.5 \mathrm{~cm}$ to the row of lighl-side posts.

\section{Other Effects}

The intercomparisons of insulated-heater thernial converters disclosed an unsuspected ac-dc difference that was dependent upon the voltage between the heater and ground and upon the lieater current of each of these elements. This was traced to a very small leakage current flowing through the ceramic bead between the heater and thermocouple and then through the stray capacitance of the Lindeck potentiometer to ground. This current caused additional heating of the bead and thus an error as on direct current the insulation resistance of the Lindeck was much higher than the capacitive reactance, even at $20 \mathrm{c} / \mathrm{s}$. In addition, the ceramic bead of each of these thermal converters exhibited marked dielectric absorption, so that its a-c resistance was niuch less than its d-c resistance. For a typical thermal converter at rated current a leakage current of $0.7 \mu \mathrm{a}$ resulted from a difference of $50 \mathrm{v}$ between the heater and ground, resulting in a power dissipation of $35 \mu \mathrm{w}$ in the bead. This was sufficient to cause a 0.2 -percent error, which was proportional to the square of the voltage difference and was very markedly dependent upon the heater current. ${ }^{19}$ The reactance of a capacitance as small as $100 \mu \mu \mathrm{f}$ is less than the computed bead resistance, even at a frequency as low as $50 \mathrm{c} / \mathrm{s}$, so that in almost anv application this source of error makes it necessary to maintain the heater near ground potential.

\section{Other Applications}

This equipment was designed for the testing of other instruments. Portability and the ease of making other a-c measurements were not considered to be important. However, the thermal converters and voltmeter elements are readily portable and have been used, with a small portable millivolt potentiometer as the indicator, for measurements at other locations. For measurements of current or voltage to 0.01 percent, it is necessary to use a transfer method, calibrating the thermal converter or voltmeter on direct current at the time of use. For a more moderate accuracy of about 0.1 percent, it should be feasible to use a low-range thermal converter, shunting it for current measurements and adding a series resistor for voltage measurements. Only the thermal converter itself need be transferred to direct current if the resistances of the shunts and the series resistor are properly adjusted. Thus d-c sources of extended range, volt boxes, etc., should bc unnecessary. The whole apparatus could be built in a convenient case, containing a small potentiometer for the measurements of the current through. or the voltage across the thermal converter heater.

10 This was due to the marked decrease in the resistance of the ceramie bead with an increase in temperature. With a fixed voltage across the bead, the error was in some cases proportional to the tenth power of the heater current. 
A suitable low-range thermal convertcr should also make feasible an a-c potentiometer with an inherent aceuracy of 0.01 percent. The basic circuit for a polar form of such a potentiometer (to measure the magnitude but not the components or pliase angle of an altcrnating voltage) is shown in figure 6 . With the switel thrown to dircet-current tlic potentiometer is standardized in the usual manner, and the deflection of the indicator of the thermal converter is observed. The switch is then thrown to alternating current, which is adjusted for the same deflection of this indicator. The circuit is surcly not new, but a proved thermal converter makes possible a convenient transfer with accuracies comparable to those of a d-c potentiometer. The other basic limitation remains that any a-c potentiometer mcasures only the fundamental component of the unknown voltage. However for an altcrnating quantity having a harmonic content as large as 2 percent, the rms value differs from the fundamental by only 0.02 percent. Audio-frequency oscillators and amplifiers with amplitude distortion much less than this are now available. Such an oscillator could be used to supply the potentiometer directly and to supply the test circuit through an amplifier preceded by a suitable phaseshift network.

\section{Summary}

The electrothermic transfer standards used at the Bureau for the prccise measurement of voltage and current at audio frequencies, and designed primarily for the standardization of electric instruments, have been described. The cxtensive tests to establish the transfer performance of the standards have becn outlined, and the results liave bcen given. The causes of the obscrved ac-dc differences have becn outlined and the crrors cvaluated. 'The equations governing the tempcrature rise of a conductor heated by an electric current and cooled by conduction to relatively massive terminals have becn solved to establish the steady-state midpoint temperature rise on direct current with Pclticr and Thomson heating, as well as the ordinary resistance heating, in order to obtain the d-c error of a thermal convertcr. The equations have been solved to establish the average midpoint temperature rise on alternating current, in order to obtain the low-frequency crror of a thermal converter in which the elcctrical and thermal conductivitics of the heater are temperature dependent, in which some loss of heat occurs by radiation, and in which the characteristic of the thermocouple is nonlinear. Possible applications and modifications of the equipment for measurements under less stringent conditions have been suggested, and the application to an a-c potentiometer of excellent inherent accuracy has been diagrammed.

The results of this work establish the excellence of thermal converters as transfer standards of highest accuracy, comparable over wide ranges with that obtainable with other types of standards at much narrower ranges. The requirements for such standards are feasible and the manner of use to insurc such accuracy not unreasonable. The cost of the required numbcr of standards and the equipment is not prohibitive. Thus electrothermic instruments may be ranked on a par with electrodynamic and electrostatic instruments for a-c measurements of the highcst accuracy.

As a result of this work, thermal converters of negligible or small known transfer error are now available as transfer standards with which the transfer performance of other thermal converters may be evaluated at the NBS to 0.01 percent at audio frequencics.

The author acknowledges the help of Murray Blitz, who made most of the intercomparisons described in section 4 of this report and checked all the tables and equations, and of Earl Williams, who constructed all the equipment and assisted with the measurements.

\section{References}

[1] F. K. Harris, A suppressed-zero electrodyaamic voltmeter, BS J. Research 3, 445 (1929) RP105.

[2] J. H. Park and A. B. Lewis, Standard electrodynamic wattmeter and ac-dc transfer instrument, J. Research NBS 25, 545 (1940) RP1344.

[3] F. B. Silsbee, Composite-coil electrodynamic instruments, BS J. Rescarch 8, 217 (1932) RP411.

[4] C. C. Patterson, E. H. Rayner, and A. Kinnes, The use of the electrostatic method for the measurement of power, J. Inst. Elec. Engrs. (London) 51, 294 (1913).

[5] R. S. J. Spilsbury and A. Felton, The electrostatic voltmeter as a dc-ac transfer instrument, J. Inst. Elec. Engrs. (London) 89-II, 129 (1942).

[6] W. N. Goodwin, Jr., The compensated thermocouple ammeter, Trans. An. Inst. Elec. Engrs. 55, 23-33 (1936).

[7] F. L. Hermach, A precision electrothermic voltmeter for measurements between 20 and 20,000 cycles, Trans. Am. Inst. Elec. Engrs. 67, 1224 (1948).

[8] H. B. Brooks and A. W. Spinks, A multi-range potentiometer and its application to the measurement of small temperature differences, BS J. Research 9, 781 (1932) RP506.

[9] G. Borelius, Bestimmung des Thomsoneffektes aus dem thermischen Gleichgewicht in einem strōmdurchflossenen Drahte, Ann. Physik 63, 845 (1920).

[10] M. G. Lloyd, Elec. Rev. \& Western Elec. 58, 65 (Jan. 14, 1911).

\section{Appendix 1. Development of Working Equations}

By definition, the pcrcentage ac-dc difference of an instrument is the percentage difference in the quantity required to give the same response on alternating and direct current. It is

$$
\delta=100 \frac{Q_{a c}-Q_{d e}}{Q_{d e}},
$$

where $Q$ is the quantity (current, voltage, or power) that the instrument measures.

In a "straight a-c test" the dircet current or voltage is adjusted to give the same response of the transfer standard observed on alternating current. The direct current or voltage is measured with the potentiometer and its accessories. Therefore, from the above definition,

$$
Q_{a c}=Q_{d c}\left(1+\frac{\delta_{c}}{100}\right)
$$


where $O_{d c}$ is the quantity measured with the potentiometer, $\delta_{,}$is the ac-dc clifference of the transfer stanclard, and $Q_{a r}$ is the a-c value required for the observed deflection of the test instrument.

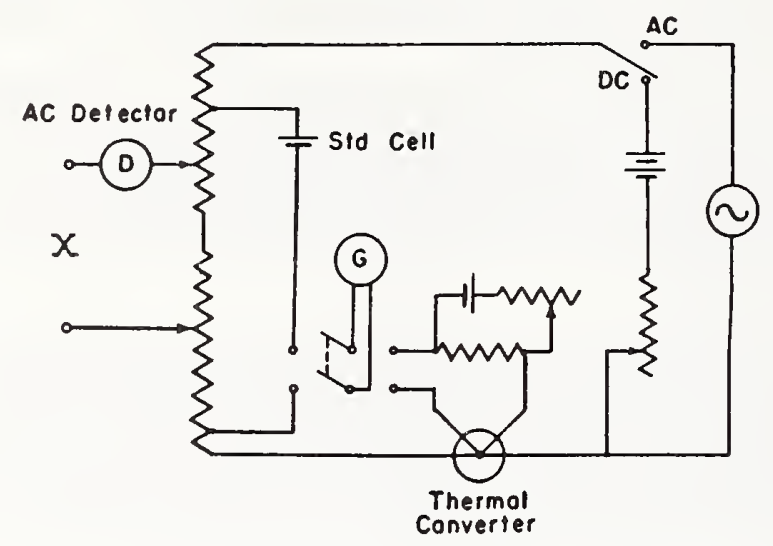

Figtre 6. Elementary form of Polar a-c potentiometer.

When a thermal converter is used for transfer tests the small percentage difference, $\delta_{t}$, between the values required for the same indication of the test instrument on alternating and direct current is determined from the resulting deflection of the Lindeck galvanometer. Therefore, for each transfer thermal converter, the sinall percentage difference in output emf resulting from a small percentage difference in heater current must be evaluated. This rolationship may be expressed as

$$
\frac{\Delta E}{E}=n \frac{\Delta I}{I}
$$

where $E$ is the output voltage, $I$ the heater current, and $n$ a number that is generally close to 2 , but may range from from 1.5 to 2.2 . The characteristic, $n$, may be evaluated on direct current by measuring with at potentiometer the sinall percentage differences in $\mathbf{E}$ resulting from small percentage differences in heater current successively set with a potentiometer at selected current levels, then computing $n$ bv the above expression and plotting $n$ versus $E$. The characteristic need not be determined with an accuracy better than 1 percent and is relatively permanent.

If the Lindeck potentiometer shown in figure 3 is used to measure $E$ and $\Delta E$ in millivolts in a transfer test, then at balance $E=V_{p},{ }^{20}$ the voltage drop) across the four-terminal resistor. If the galvanometer deflection $D$, is directly proportional to the current through the galvanometer ${ }^{21}$ and if the potentiometer voltage is unchanged during a determination,

$$
\Delta E=\frac{\Delta D R_{p}}{S}
$$

where $\Delta D$ is the difference in galvanometer deflection corresponding to a difference $\Delta E$ in the applied $\mathrm{emf}, R_{p}$ is the resistance of the galvanometer circuit, and $S$ is the current sensitivity of the galvanometer in centineters per milliampere on the scale. In the Lindeck potentiometer used in this setup, the galvanometcr sensitivity is adjusted so that numerically

$$
\frac{100 R_{p}}{S}=1
$$

These relationships may be combined with eq 9 to give

$$
100 \frac{\Delta I}{I}=\frac{\Delta D}{n} \bar{V} .
$$

20 An accuracy of 1 percent is sufficient for this ureasurcuent.

21 The use of a straight instead of cylindrical scalc introduces an error that can be shown to be less than I percent if the galvanoniter-to-scale distance is at least three times the scale length.
Therefore, for a transfer test of an ammeter with a thermal converter that has no transfer crror, the required ac-de difference of the test instrument is by ed 7 and 10

$$
\delta_{T}=100\left(\frac{I_{a c}-I_{d c}}{I_{d c}}\right)=\frac{D_{a r}-D_{d e}}{n V_{p}},
$$

where the alternating and dircet currents are: adjusted for the same response of the test instrument, and the resulting galvanomorer (leflections are 1), and 1), respectively.

If the transfor thermal converter has a percentage ae-de difference, $\delta_{s}$, at the current and freginency at which the test is made, then the ac-de clifference of the instrmment under test is, to the desired order of approximat ion,

$$
\delta r=100\left(\frac{I_{a c}-I_{d c}}{I_{d c}}\right)=\frac{D_{a c}-D_{d r}}{n V_{p}}+\delta_{s} .
$$

Similarly, for a voltmeter test,

$$
\delta_{T}=100\left(\frac{V_{a c}-V_{d c}}{V_{d c}}\right)=\frac{D_{a c}-D_{d c}}{n V_{p}}+\delta_{s},
$$

in which $\delta_{s}$ is the percentage ac-de difference of the range (or setting) of the standard voltmeter used.

In this deflection method of using the Lindeck potentiometer for rapid transfer tests, the resistance of the circuit seen l)y the galvanometer must be constant to better than 1 percent. The resistance of the thermocouple of each thermal converter has been adjusted to the same value so that the simplified formulas may always be used. The temperature coefficient of the resistance of the lindeck circuit has been computed as less than 0.1 percent per deg $\mathrm{C}$, and computations show that the resistance scen by the galvanometer is changed by less than 0.2 percent by the adjustal)le battery circuit, for all ranges of the potentiometer exeept the topmost.

\section{Appendix 2. Effect of Peltier and Thomson Heating}

The problem is to find the steady-state temperature rise of a uniform conductor of length $2 l$ and uniform cross-sectional area, $a$, carrying a constant current, $I$, and cooled solely by conduction to its terminais. The joule heating of a length $\dot{d x}_{2}$ occurs at the time rate $I^{2} \rho d x / a$ watts, where $\rho$ is the electric resistivity in ohm-cm. The difference in the heat conducted per second across the two ends of this differential clement is $a k\left(d^{2} \theta / d x^{2}\right) d x$ watts where $k$ is the thermal conductivity in watts-cm-1-(legrees ${ }^{-1}$ and $\theta$ is the temperature of the element in deg C. Jquating these and dividing by $d x$ gives the differential equation governing the temperature of the conductor

$$
a k \frac{d^{2} \theta}{d x^{2}}+\frac{I^{2} \rho}{a}=0 \text {, }
$$

which is of the form

$$
\theta^{\prime \prime}=-b \text {, }
$$

where $b=I^{2} \rho / a^{2} k$.

If the terminals remain at equal temperatures $\theta_{0}$, and if the origin is at the midpoint of the conductor, the boundary conditions are $\theta=\theta_{0}$ at $x= \pm l$. The solution, by direct integration and application of these conditions, is

$$
\theta-\theta_{0}=\frac{b}{2}\left(l^{2}-x^{2}\right)
$$

and at the midpoint

$$
\theta_{m}-\theta_{0}=\frac{b l^{2}}{2}=\theta_{d}
$$

If the terminals are of different metal than the conductor, Peltier heating and cooling (proportional to the first power of the current) may occur at the junctions of the conductor with the terminals. These will be equal and opposite in sign at the two terminals, and, if we can assume complete thermal symmetry, their effect will be to raise the tompertaure of 
one terminal by a small anount $\Delta \theta$ and to lower the other by an equal amount. The rifferential equation then has as its boumdary conflitions, $\theta=\theta_{0}+\Delta \theta$ at $x=+l$ and $\theta=\theta_{0}-\Delta \theta$ at $x=-l$. The solution is

$$
\theta-\theta_{0}=\frac{b}{2}\left(l^{2}-x^{2}\right)+\frac{\Delta \theta x}{l}
$$

and is therefore mochanged at the midpoint.22 of course,

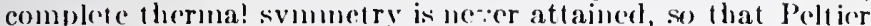
heats may calse a change in the millpoint temperature. However, for massive tominals lae temperature eloanges, $\Delta \theta$, are very small, so that l'eltier healing need not be considered significant.

Thomson heating occurs in homogencous eoneluctors having a temperature graclient in the direction of the current flow. For the onc-rlimensional ease herc considered, the Thonison heat in an clement of length $d x$ having a temperature difference $d_{y}$ i $=\sigma I d \theta$ wat $s$ where $\sigma$ is the Thomson voltage coefficient. Experiment and theory indicate that $\sigma$ is proportional to the absolute teinperature, lut, as the Themson heating is sinall compared to the jonle heating, we might, as a first approximation, consider $\sigma$ eonstant. Equation 14 then becones

$$
a k \frac{d^{2} \theta}{d x^{2}}+\frac{I^{2} \rho}{a}+\sigma I \frac{l \theta}{d x}=0,
$$

which is of the form

$$
\theta^{\prime \prime}+c \theta^{\prime}+b=0
$$

with the houndary conditions $\theta=\theta_{0}$ at $x= \pm l$, where $c=\sigma I / a k$. The steady-state solution of this eq is

$$
\theta-\theta_{0}=\frac{b l}{c}\left(\frac{1}{\tanh q}-\frac{e^{-r x}}{\sinh q}\right)-\frac{b x}{c},
$$

where $q=c l<<1$. At the midpoint, where $x=0$

$$
\theta_{\text {in }}-\theta_{o}=\frac{b l^{2}}{q}\left(\frac{\cosh q-1}{\sinh q}\right) \text {. }
$$

Expanding the hyperbolic functions gives, by neglecting higher-order terms,

$$
\theta_{m}-\theta_{0}=\theta_{\imath}=\frac{b l^{2}}{2}\left(1-\frac{q^{2}}{12}\right)=\theta_{d}\left(1-\frac{q^{2}}{12}\right) .
$$

$\mathrm{By}$ substituting the values for $b$ and $c$ and noting that $2 I \rho \dot{l} / a=V$, the vortage drop across the conductor, eq 1 and 2 of the text can be obtained. They show that Thomson heating causes a small (sceond order of smahlness) decrease in the temperature rise at the midpoint of the conductor, and can thus lead to a transfer error in a thermal converter. However, the approximation of considering $\sigma$ a constant is a drastic one, and a more accurate approach will be to consider $\sigma=B T$, where $B$ is a constant, ${ }^{23}$ and $T$ is the absolute tempcraturc. Equation 17 then becoincs

$$
a k \frac{d^{2} T}{d x^{2}}+\frac{I^{2} \rho}{a}+B I T \frac{d T}{d x}=0
$$

which is of the form

$$
T^{\prime \prime}+w T T^{\prime}+b=0
$$

with the boundary conditions $T=T_{0}$ at $x= \pm l$, where $w=B I / a k$

This is a nonlinear equation and very difficult to solve. Chester Snow showed that it eonld be rediced to a IRicatti and then to a Bessel cquation, but suggested an approximation or perturbation method to give the desired resill sis inore simply and directly. The answer is not significanly chilferent from

2. Since Peltier leating depends upon the junction temperature, a secondorder ehange in midpoint temperature may oceur.

23 $B=d^{2} E d^{\prime} T^{2}$, where $E^{\prime}$ is the einf of a thermocouple composed of the metal in

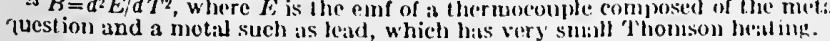

that of eq 18. The method is powerful, however, and was used later in evaluating other perturbing effects, s) that it will be outlined here. The Thomson beating, which is much sinaller than the Joulc healing, eau be eonsidered as perturbing the temperature dist ribution of the comeluctor. Ils effect can le approximated by solving the differontial equation without the perturbing term, then substituting the result, and its clerivatives as necessary, in the perturbing term only, and solving the resulling e(putation.

Without the Thomson heat term, eq 19 becomes

$$
T^{\prime \prime \prime}=-\downarrow
$$

with the boundary eomelition given, the solution of which is

$$
T_{1}-T_{0}=\frac{l}{2}\left(\ell^{2}-x^{2}\right)
$$

where the subscript 1 iurlicates the first approximation. l'lacing this value of ' $T_{1}$ and $T_{1}^{\prime}$ in the perturbing terin of 19 , reduces this to a lincar equation,

$$
T_{2}^{\prime \prime}=-b-\frac{w b^{2} x^{3}}{2}+w b x\left(T_{d}+T_{0}\right) \text {. }
$$

wherc $T_{d}=b l^{2} / 2$, the solution of which is

$$
\begin{aligned}
T_{2}-T_{0}= & \frac{b}{2}\left(l^{2}-x^{2}\right)-\frac{w b^{2} x^{5}}{40}+\frac{w b}{6}\left(T_{d}+T_{0}\right) x^{3}- \\
& w T_{d}\left(\frac{7}{30} T_{d}+\frac{T_{0}}{3}\right) x
\end{aligned}
$$

At $x=0$ this reduces to $T_{1}-T_{0}$ as only first-order terms are given, so that the process inust be repeated to find the sccondorder term indicated by eq 18 . The algebra rapidly becomes very lengthy and tedious, but the procedure is straight forward, and the resulting temperature rise at the inilpoint is found to be approximately

$T_{3}-T_{0}=T_{d}\left[1-3.4 \times 10^{-2} z_{d}^{2}-10.5 \times 10^{-2} z_{0} z_{d}-8.3 \times 10^{-2} z_{0}^{2}\right]$

where $z_{0}=w T_{0} l$, and $z_{d}=w T_{d} l$

\section{Appendix 3. Low-Frequency Error of Thermoelement}

The problem is to find the steady-state average (over an integral number of cycles) temperature rise at the center of a uniform conductor of icngth, $2 l$, and cross-scctional area, $a$, earrying a simusoirlal current and cooled chicfly by conduction to massive terminals that are at fixed and equal temperatures. Assume that the current, remains sinusoidal and that only Joule heating of the conductor occurs. If all the cooling is by conduction to the terminals, the differential equation governing the temperature of the conduetor is, under these assumptions,

$$
a k \frac{\partial^{2} \theta}{\partial x^{2}}+\frac{i^{2} \rho}{a}=a m s \frac{\partial \theta}{\partial t}
$$

with the boundary condition $\theta( \pm l, t)=0$, where

$\theta=$ temperature in deg $\mathrm{C}$

$i^{\prime}=$ thesquare of the instantaneous value of the current $=$ $I^{2}(1-\cos 2 \omega t)$

$I=$ rms value of the current

$\omega=2 \pi f$, and $f$ is the froquency of the current in cyeles pere serongl

$t=$ time in secombis

$m=$ density of the conductor in grams- $\mathrm{cm}^{-3}$

$s=$ thermal capacily of the conductor in joul's-grams $-\operatorname{dec}(:-1$. 
The procedure will be to consider $\rho$ and $k$ constant and to solve for the periodic steady-state temperature rise, then to approximate $\rho$ and $k$ as $\rho=\rho_{0}(1+\alpha \theta)$ and $k=k_{0}(1+\beta \theta)$, to introduce radiation loss, and to solve the resulting equation by the perturbation method just outlined. For the cases considered $\alpha \theta$ and $\beta \theta$ are considerably less than 1, and radiation loss is much less than the Joule heating of the conductor.

With $\alpha$ and $\beta=0$, eq 21 is the form

$$
\frac{\partial^{2} \theta}{\partial x^{2}}-g \frac{\partial \theta}{\partial t}=-b(1-\cos 2 \omega t)
$$

where $g=m s / k_{0}$. and $b=I^{2} \rho_{0} / a^{2} k_{0}$.

Let $\theta=u(x, t)+\psi(x)$. Then

$$
\frac{\partial^{2} \theta}{\partial x^{2}}=\frac{\partial^{2} u}{\partial x^{2}}+\frac{\partial^{2} \psi}{\partial x^{2}}
$$

Let $\partial^{2} \psi / \partial x^{2}=-b$. Then $\psi=-\left(b x^{2}\right) / 2+c_{1} x+c_{2}$. Applying boundary conditions gives

$$
\begin{gathered}
\theta(+l, t)=u(+l, t)+\psi(l)=0 \\
\theta(-l, l)=u(-l, t)+\psi(-l)=0 .
\end{gathered}
$$

We may take $\psi(l)=\psi(-l)=0$.

Then $\psi=b / 2\left(l^{2}-x^{2}\right)$ and eq 22 becomes

$$
\frac{\partial^{2} u}{\partial x^{3}}-g \frac{\partial u}{\partial t}=b \cos 2 \omega t
$$

with $u( \pm l, t)=0$.

From complex number theory if $u$ is harmonic in time, it can be represented by the real part of $U e^{i 2 \omega t}$, where $U$ is a cornplex function of the real variable $x$, and $j=\sqrt{-1}$. Sinilarly, $b \cos 2 \omega t$ can be represented by the real part of $b e^{i 2 \omega t}$, where $b$ is a real number. Substituitng these quantities and the appropriate derivatives in eq 23 and dividing by $e^{i 2 \omega t}$ results in

$$
\frac{d^{2} U}{d x^{2}}-j 2 \omega g U=b,
$$

with $\mathrm{U}=\mathrm{O}$ when $c= \pm l$. The solution of this is

$$
U=-\frac{b}{\mu_{2}}\left[1-\frac{\cosh \mu x}{\cosh \mu l}\right] \text {, }
$$

where $\mu=\sqrt{j 2 \omega g}$. At the midpoint

$$
U=-\frac{b}{\mu^{2}}\left[1-\frac{1}{\cosh \mu l}\right] \approx j \frac{b}{2 \omega g},
$$

as at the frequencies we are interested in $\cosh \mu l>>1$. Therefore, $u=$ real part of Ueizui= real part of

$$
\left\{\frac{j b}{2 \omega g}(\cos 2 \omega t+j \sin 2 \omega t)\right\} \approx-\frac{b}{2 \omega g} \sin 2 \omega t .
$$

So that, to this approximation, at the midpoint

$$
\theta_{m 1}=\frac{b l^{2}}{2}\left(1-\frac{1}{\omega g l^{2}} \sin 2 \omega t\right)=\theta_{d}(1-q \sin 2 \omega t),
$$

where $\theta_{d}=b l^{2} / 2$ and $q=1 / \omega g l^{2}$. Note that $q$ is the crest value of the cyclic temperature fluctuation divided by the average temperature rise at midpoint.

In general, $U=U_{a}+j U_{b}$, where $U_{a}$ and $U_{b}$ are functions of $x$, so that $u=U_{a} \cos 2 \omega t-U_{b} \sin 2 \omega t$ and

$$
\theta_{1}=\frac{b}{2}\left(l^{2}-x^{2}\right)+U_{a} \cos 2 \omega t-U_{b} \sin 2 \omega t .
$$

Thus the average value over an integral number of cycles of the temperature rise is the same as that found in appendix 2 for the same numerical value of clirect current, so that under these conditions the temperature rise of the conductor call be used as a measure of the current without ac-dc error.

We shall now consider that $\rho=\rho_{0}(1+a \theta)$ and $k=k_{0}(1+\beta \theta)$ and that some sinall fraction of the total heat is lost by radiation. If $p$ is the perimeter of the heater in centimeters, then the heat radiated per second from the surface of $a$ differential length, $d_{x}$, of the heater is

$$
d H_{r}=p \zeta K\left(T^{4}-T_{0}^{4}\right) d x,
$$

where $\zeta$ is the cmissivity of the heater material, a numerical constant, and $K$ is the Stefan-Boltzmann constant in watts$\mathrm{cm}^{-2}(\operatorname{deg} K)^{-4}$. $T$ is the absolute temperature of the element; $T_{0}$ is the absolute temperature of the surroundings. Introducing these terms in eq 22 gives, as $\beta \theta<1$,

$$
\frac{\partial^{2} \theta}{\partial x^{2}}-g(1-\beta \theta) \frac{\partial \theta}{\partial t}=-b(1-\cos 2 \omega t)(1+\eta \theta)+\frac{p \zeta K}{a k}\left(T^{4}-T_{0}^{4}\right)
$$

where $\eta=\alpha-\beta$. The perturbing terms are

$$
\begin{gathered}
+\beta g \theta \frac{\partial \theta}{\partial t} \\
-b(1-\cos 2 \omega t) \eta \theta \\
\frac{p \zeta K}{a k}\left(T^{4}-T_{0}^{4}\right) .
\end{gathered}
$$

As these perturbing terms are relatively small, the solution will differ by only a small amount from that given by eq 27 . Hence a sufficient expression for these small terms will be given by substituting the known values of the dependent variables $y$ from eq 27 , and its derivatives as necessary, in these perturbing terms. When this is done, it is found that the first of these terms reduces to functions of $x$, each inultiplied by $\sin n \omega t$ or $\cos n \omega t$, where $n=2,4$. The second reduces to similar harmonic terms plus the terms $-b[\eta P-$ $\left.\left(\eta U_{a} / 2\right)\right]$, where $P=\left(l^{2}-x^{2}\right) b / 2$. The third term becomes unnecessarily complex. It can be simplified by making the further approximation that for this term

and

$$
T-T_{0}=\theta_{r}=P(1-q \sin 2 \omega t),
$$

$$
T^{4}-T_{0}^{4}=\left(T_{0}+\theta_{r}\right)^{4}-T_{0}^{4} \approx 4 \theta_{r} T_{0}^{3}+6 \theta_{r}^{2} T_{0}^{2}, \theta_{r}<T_{0}
$$

and also that $k=k_{0}$. With these approximations, this term reduces to harmonic terms plus the terms

$$
4 N T_{0}^{3} P+6 N T_{0}^{2} P^{2}\left(1+\frac{q^{2}}{2}\right),
$$

where $N=p \zeta K / a k_{0}$.

Thus eq 28 becomes

$$
\begin{aligned}
\frac{\partial^{2} \theta}{\partial x^{2}}-g \frac{\partial \theta}{\partial t}= & -b\left(1+\eta P-\frac{\eta U_{a}}{2}\right)+2 N T_{0}^{2} P\left[2 T_{0}+3 P 1+\left(\frac{q^{2}}{2}\right)\right]+ \\
& \sum_{n=2}^{n=4}\left(F_{n} \cos n \omega t+G_{n} \sin n \omega t\right),
\end{aligned}
$$

where $F_{n}$ and $G_{n}$ are known functions of $x$ alone. We again let $\theta(x, t)=u(x, t)+\psi(x)$ and set $\partial^{2} \psi / \partial x^{2}$ equal to the terms independent of $t$ in eq 29, so that

$$
\frac{\partial^{2} u}{\partial x^{2}}-g \frac{\partial u}{\partial t}=\sum_{n=2}^{n=t}\left(F_{n} \cos n \omega t+G_{n} \sin n \omega t\right)
$$


For each frequency of the harmonic driving terms in the right-hand side of this equation, the solution of the equation that vanishes at the end points will be of the form $U_{0} \cos$ $n \omega t-U_{b} \sin n \omega t$, where $U_{a}$ and $U_{b}$ are functions of $x$. The average value, over an integral number of cycles of the lowest frequency, will therefore vanish. Since this is a linear equation, the solution is the sum of such solutions and will therefore also vanish. Thus the average value of $\theta$, which we are interested in, is equal to $\psi(x)$.

Evaluation $\psi(x)$ in the same manner as before gives for the value of $\psi$ at the midpoint where $x=0$,

$$
\psi(0)=\theta_{d}\left[1+\frac{5}{6} \eta \theta_{d}-\frac{\eta U_{a 2}(l)}{l^{2}}-\frac{5}{3} N T_{0}^{3} l^{2}-\frac{11}{5} N T_{0}^{2} \theta_{d} l^{2}\left(1+\frac{q^{2}}{2}\right)\right]
$$

where

$$
U_{a 2}(x)=\int_{0}^{x} d x_{2} \int_{0}^{x_{3}} U_{a}\left(x_{1}\right) d x_{1}
$$

and on the assumptions that $U_{a 2}(-l)=U_{a 2}(l)$ and $U_{a 2}(0)=0$.

The required expression for $U_{a}$ can be evaluated from eq 25 and 27 by noting that

$$
\mu x=\sqrt{j 2 \omega g} x=\gamma x+j \gamma x \text { and } \mu l=\mu l+j \gamma l,
$$

where $\gamma=\sqrt{\omega g}$, so that $\cosh \mu x=\cosh \gamma x \cos \gamma x+j \sinh \gamma x \sin$ $\gamma x$, and $\cosh \mu l=\cosh \gamma l \cos \gamma l+j \sinh \gamma l \sin \gamma l$. At the frequencies we are interested in $\mu l>5$, so that

$$
\cosh \mu l \approx \frac{e^{\gamma l}}{2}(\cos \gamma l+j \sin \gamma l) .
$$

Since $u=$ real part of

$U e^{i 2 \omega t}=r e\left\{\frac{j b}{2 \omega g}\left[1-\frac{\cosh \mu x}{\cosh \mu l}\right] e^{i 2 \omega t}\right\}=U_{a} \cos 2 \omega t-U_{b} \sin 2 \omega t$, it will be found that

$$
U_{a}=\frac{b}{\omega g e^{\gamma l}}[\sinh \gamma x \sin \gamma x \cos \gamma l-\cosh \gamma x \cos \gamma x \sin \gamma l] .
$$

Performing the desired integration results in

$U_{a 2}=-\frac{b}{2 \omega g \gamma^{2} e^{\gamma l}}(\cos \gamma l \cosh \gamma x \cos \gamma x+\sin \gamma l \sinh \gamma x \sin \gamma x)$.

From which

$$
U_{a 2}(l)=U_{a 2}(-l) \approx-\frac{b}{4 \omega^{2} y^{2}} \text { and } U_{a 2}(0)<<U_{a 2}(l) .
$$

Therefore, eq 31 becomes

$$
\begin{aligned}
\psi(0)=\theta_{a c}=\theta_{d}\left[1+M+\left(\frac{\eta}{2}-\frac{11}{10} N T_{0}^{2} l^{3}\right) \theta_{d} q^{2}\right]= \\
\theta_{d}\left[1+M+\frac{h}{2} \theta_{d} q^{2}\right],
\end{aligned}
$$

where $M$ is equal to the remaining terms in eq 31 and $h \approx \eta-$ $2 N T_{0}^{2} l^{2}$.

By the same procedure, eq 28 can be solved for the d-c case by considering $\partial \theta / \partial t=0$ and $i=I$, a constant of the same numerical value as the rms value of the alternating current. The temperature rise at the midpoint will be found to be

$$
\theta_{d e}=\theta_{d}[1+M] \text {. }
$$

Thus the temperature rise on direct current is the same as on alternating current when the frequency is high enough so that the third term in the brackets of eq 32 is sufficiently small compared to unity. Therefore the temperature rise at the midpoint on alternating current is

$$
\theta_{a c}=\theta_{d c}\left[1+\frac{h \theta_{d c} q^{2}}{2(1+M)}\right] \approx \theta_{d c}\left[1+\frac{h}{2} \theta_{d c} q^{2}\right]
$$

If the hot junction of the thernocouple is fastened to the midpoint of the heater, the emf of the couple inay be reprcsented by

$$
E=A \theta_{m}+\frac{B}{2} \theta_{m}^{2}
$$

where $\theta_{m}$ is the temperature of the hot junction in $\operatorname{deg} C$, and $A$ and $B$ are constants to be evaluated at the temperature of the cold junction. On direct current $\theta_{m}=\theta_{d c}$ and

$$
E=A \theta_{d c}\left[1+\frac{B}{2 A} \theta_{d c}\right] \text {. }
$$

On alternating current of the same rms value, $\theta_{m}$ may be considered to be closely enough for these purposes

$$
\theta_{m}=\theta_{d c}\left[1+\frac{h}{2} \theta_{d c} q^{2}\right](1-q \sin 2 \omega t) \text {. }
$$

The average value of emf over an integral number of cycles of alternating current is then (to the desired accuracy, and if $(B / 2 A) \theta_{d e}$ is small compared to unity)

$$
E_{A}=A \theta_{d c}\left(1+\frac{B}{2 A} \theta_{d c}\right)\left[1+\left(\frac{h}{2}+\frac{B}{4 A}\right) \theta_{d c} q^{2}\right]
$$

From these expressions

where $H=h+B / 2 A$.

$$
\frac{E_{a}-E_{d}}{E_{d}}=\frac{H}{2} \theta_{d e} q^{2}
$$

For table 7, it is desired to compute the frequency at which the ac-dc difference of a thermal converter due to this effect is equal to 0.1 percent. From eq 7 and 9 and for $n=2$ the difference in current for the same emf is one-half the difference in emf for the same current, and is opposite in sign. Therefore the percentage ac-dc difference, $\delta$, is approximately

$$
\delta=-25 H \theta_{d c} q^{2}=\frac{-25 H \theta_{d c} d^{2}}{(2 \pi f)^{2} l^{4}},
$$

where in addition to the symbols already defined, $d=1 / g=$ $k_{0} / m s$ and is the thermal diffusivity in appropriate units.

\section{Appendix 4. Effect of Shield Capacitance on the Model B Voltmeter}

We may approximate the effects of capacitance to the shield of this voltmeter by considering the voltmeter 29 consisting of a transmission line of $r_{x}=r_{0} x^{2}$ ohms per unit length, having a constant capacitance $c_{0}$ farads per unit length to the shield, witha sinusoidal voltage of rms value $I$ volts applied to the high-resistance end. The other end may be considered short-circuited, since the thermal converter, which is connected between the low-resistance end and the ground post, is of low resistance. The shield is also connected to this ground post. We wish to find the magnitude of current, $I_{0}$, at the short-circuited end. For an element of length $d x$ at a distance $x$ from this end, we have

$$
\frac{d V}{d x}=I r_{x}=I r_{0} x^{2} \text { and } \frac{d I}{d x}=j \omega c_{0} V \text {, }
$$

where $d V^{\prime}$ is the rms voltage drop across the element, and $d I$ is the rms current from the element to the shield. Differentiating the first of these and substituting the values of $I$ and $d I / d x$ in the resulting expression gives

$$
\frac{d^{2} V}{d x^{2}}-\frac{2}{x} \frac{d V}{d x}-\lambda^{2} x^{2} V=0,
$$

subject to the boundary conditions $V=0$ at $x=0$ and $V=V$. at $x=l$ and where $\lambda^{2}=j \omega c_{0} r_{0}$.

When $\lambda=0$ this becomes, with the same boundary conditions,

$$
\frac{d^{2} V}{d x^{2}}-\frac{2}{x} \frac{d V}{d x}=0
$$


the solution of which is

$$
V_{1}=\frac{V_{1} x^{3}}{l^{3}}
$$

We now put this value of $V$ in the perturbink term containing $\lambda^{2}$ in eq 36 , to get

$$
\frac{d^{2} V}{d x^{2}}-\frac{2}{x} \frac{d V}{d x}=\frac{\lambda^{2} V_{{ }^{2} x^{i}}}{l^{3}}
$$

The solution of this, with the same boundary conditions, is

$$
V_{2}=\frac{V_{a} x^{3}}{l^{3}}\left(1-\frac{\lambda^{2} l^{4}}{28}\right)+\frac{\lambda^{2} V_{n} x^{7}}{28 l^{3}}
$$

As $\lambda^{2}=j \omega c_{0} r_{0}$, we will need to repeat this process to secure: terms in $\lambda^{4}$, for we are interested in the magnitudes of $V$ and 1 . Putting the above value of $V_{2}$ in the last term of $\mathrm{ce} 36$ and solving as before gives

$V_{3}=\frac{V_{a} x^{3}}{l^{3}}\left(1-\frac{\lambda^{2 l^{4}}}{28}+8.8 \times 10^{-4} \lambda^{2} l^{4}\right)+\frac{\lambda^{2} V_{a} x^{7}}{28 l^{3}}\left(1-\frac{\lambda^{2} l^{4}}{28}\right)+\frac{\lambda^{4} V_{a} x^{11}}{2+64 l^{3}}$
From eq 35

$$
l=\frac{1}{r_{0} x^{2}} \frac{d V}{d x}
$$

Performing the indicated differentiation of eq 37 and substituting in 38, and noting that the total resistance is $R=r_{0} / 3 / 3$ leaclis to

$$
I=\frac{V_{a}}{R}\left[1-\frac{\lambda^{2 / 4}}{28}+8.8 \times 10^{-4} \lambda^{2} l^{4}+\frac{\lambda^{2} x^{4}}{12}\left(1-\frac{\lambda^{2} l^{4}}{28}\right)+\frac{\lambda^{4} x^{5}}{672}\right] .
$$

Solving for $I_{0}$ at $x=0$, and noting that the total capacitance is $C^{\prime}=c_{0} l$ gives

$$
J_{10}=\frac{V_{u}}{R}\left[1-j 0.107 \omega C R-7.9 \times 10^{-3}(\omega C R)^{2}\right] .
$$

Since the terms in the bracket are small compared with unity

$$
\left|I_{0}\right|=\frac{V_{a}}{R}\left[1-0.0022(\omega C R)^{2}\right] .
$$

Washington, August 16, 1951. 


\title{
Thermal Current Converters for Accurate AC Current
}

\section{Measurement}

\author{
EARL S. WILLIAMS
}

\begin{abstract}
A new 14-range set of thermal current converters consisting of shunted thermoelements has been constructed to measure ac-de difference and ac current from $10 \mathrm{~mA}$ to $20 \mathrm{~A}$ at 20 $\mathrm{Hz}$ to $50 \mathrm{kHz}$. The ac-dc difference corrections for all ranges can be determined relative to two ranges by a 7 -step intercomparison of certain adjacent ranges.
\end{abstract}

\section{INTRODUCTION}

\section{$\mathbf{T}$} HERMAL CURRENT converters (TCC's) consisting of ac shunts in parallel with thermoelements (TE's) are widely used as ac-dc transfer instruments to determine ac-dc difference of other instruments and to measure ac currents by comparison with known dc currents.

Shunt assemblies are sometimes incorporated into multirange instruments, but usually are individual single-range units. They are attached to a thermal voltage converter (TVC) which contains a low-voltage low-current TE. Commercial multirange TVC's usually incorporate an adjustable source of EMF and a null detector for monitoring the output of the TE.

Specific procedures and the necessary equipment have not been provided by shunt manufacturers to enable the user to make intercomparison tests between members of a set of shunts. However such tests would provide several advantages. It would be sufficient for the manufacturer or a standards laboratory to calibrate only two or three ranges. These ranges would serve as reference points for calibrating the other ranges by intercomparison. Periodic intercomparisons could then be made by the user to detect any significant changes that might occur in any shunt in the set, and redeterminations of ac-dc difference relative to other standards would not be required as often.

The new NBS set of TCC's, consisting of six shunts and two TVC's (Fig. 1) is designed so that it can be intercompared in a minimum number of steps. The TVC's have nominal inputs of 0.3 and $0.6 \mathrm{~V}$ and may be attached to any one of the shunts by a coaxial connector. This permits each shunt to be used for two ranges, as shown in Table I where current ratings, shunt resistances, and TVC currents are listed. For the purpose of calibration, intercomparisons are made between each of the six shunts or between alternate ranges. The TVC's are used without shunts for the two lowest ranges ( 10 and $20 \mathrm{~mA}$ ), and they are compared with the shunted ranges in two additional steps.

Manuscript received June 28, 1976; revised August 12, 1976.

The author is with the Electricity Division, National Bureau of Standards, Washington, DC 20234.

\section{THERMOELEMENTS AS TCC'S AND TVC'S}

TE's without shunts have been used for some years at the NBS as primary current converters. They can be very accurate ac to dc transfer devices. The short straight heater and connecting wires have minimal reactance, and an insulating bead between the heater and thermocouple prevents any appreciable interaction between the current being measured and the readout instruments. The thermocouple output is rather low ( 7 to $12 \mathrm{mV}$ ), but it can be monitored with very good precision with a modern galvanometer or microvoltmeter.

However shunted thermoelements are probably preferable as TCC's in most other calibration facilities mainly because the low current TE's used are relatively inexpensive and easy to replace if burned out-an accident that can happen quite easily. Ampere-range TE's are more expensive, and those with insulating beads are not ordinarily available. However, a few sets have heen made by one manufacturer on special arrangement.

TE's for 10 and 20 A usually have C-shaped heaters (tubular except for opening on one side) which can cause an unusual effect. The ac-dc difference is very much dependent on the location of other nearby conductors, particularly the return conductor which is usually placed along side of, and more or less parallel to, the 'TE heater. Apparently the ac current is distributed for minimum reactance and may therefore be concentrated close to, or away from, the thermocouple which is attached to the top of the heater. This nonuniform current distribution can cause an ac-dc difference change of 0.03 percent or more. Rigid copper return conductors have therefore been added to these TE's and the input terminals are both at the same end of the device. The conductors are also positioned for small ac-dc differences.

Similar TE's with straight wire heaters $(0.5$ to $5 \mathrm{~A})$ and insulation have ac-dc differences of $10 \mathrm{ppm}$ or less at 50 $\mathrm{kHz}$.

TE's for currents up to $1000 \mathrm{~mA}$ are available with insulation and vacuum bulb enclosures. They are adversely affected by Peltier and Thomson heating, which have been discussed elsewhere [1], [2]. However, skin effect is the major concern in the practical use of these TE's. The copper-coated nickel alloy widely used for passing current conductors through glass seals is sufficiently magnetic to produce a small increase in the ac impedance in the heater support stems causing additional heating with ac current but not with dc current. The effect is dependent on both 


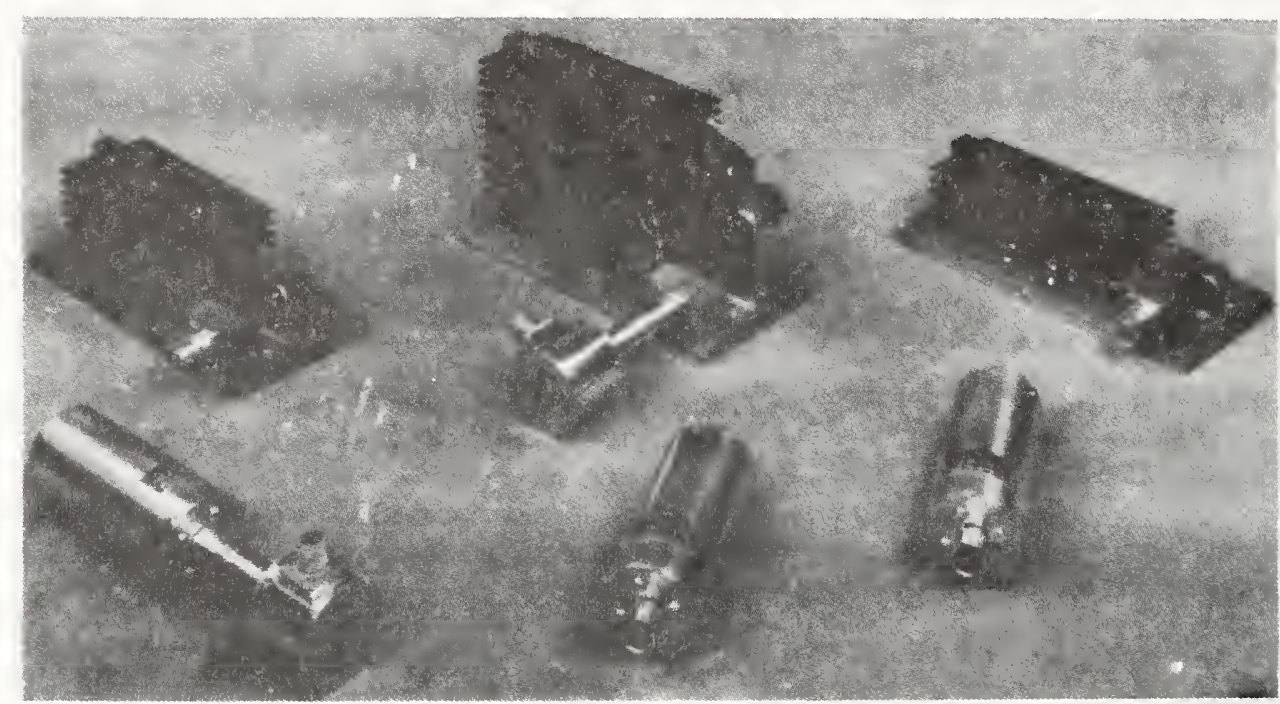

Fig. 1. Set of TCC's.

TABLE I

Thermal Current Converter Set

\begin{tabular}{|c|c|c|}
\hline \multicolumn{3}{|c|}{$\begin{array}{l}\text { Fourteen ranges are formed with six } \\
\text { shunts and two TVCs (FG and FH). }\end{array}$} \\
\hline $\begin{array}{l}\text { Current } \\
\text { Range }\end{array}$ & $\begin{array}{c}\begin{array}{c}\text { Shunt } \\
\text { Resistance }\end{array} \\
\text { (ohms) }\end{array}$ & $\begin{array}{c}\text { TVC } \\
\text { Current } \\
\text { (mA) }\end{array}$ \\
\hline 0.01 & -- & 10 (FG) \\
\hline 0.02 & -- & 20 (FH) \\
\hline $\begin{array}{l}0.03 \\
0.06\end{array}$ & 15 & $\begin{array}{l}10 \\
20\end{array}$ \\
\hline $\begin{array}{l}0.1 \\
0.2\end{array}$ & 3.33 & $\begin{array}{l}10 \\
20\end{array}$ \\
\hline $\begin{array}{l}0.3 \\
0.6\end{array}$ & 1.0 & $\begin{array}{l}10 \\
20\end{array}$ \\
\hline $\begin{array}{l}1 \\
2\end{array}$ & 0.3 & $\begin{array}{l}10 \\
20\end{array}$ \\
\hline $\begin{array}{l}3 \\
6\end{array}$ & 0.1 & $\begin{array}{l}10 \\
20\end{array}$ \\
\hline $\begin{array}{l}10 \\
20\end{array}$ & 0.03 & $\begin{array}{l}10 \\
20\end{array}$ \\
\hline
\end{tabular}

frequency and current thus complicating stepup intercomparison tests.

A few special TE's have been made with nonmagnetic $\mathrm{Ni}-\mathrm{Cr}$ quaternary alloy for both the heater and supporting stem inside the glass bulb. This not only minimizes the skin effect in the conductors near the heater, but also eliminates Peltier heating at the heater ends since the supporting conductors and the heater are of the same material.

The heating due to skin effect is usually negligible at currents less than $200 \mathrm{~mA}$ for TE's used as current converters. However, if the TE is used as a voltage converter, or as a TVC in parallel with a shunt, the additional impedance is added directly to the relatively low heater resistance. The effect is large down to at least $20 \mathrm{~mA}$ and significant to 5 or $10 \mathrm{~mA}$. At $2.5 \mathrm{~mA}$, where the heater resistance (about $400 \Omega$ ) is high relative to that of the leads, the ac-dc difference at $50 \mathrm{kHz}$ is usually less than 5 ppm.
The skin effect problem was overcome in these TVC's by using $5 \mathrm{~mA}$ and $10 \mathrm{~mA}$ TE's with platinum input wires. They were made to NBS specifications by three cooperating manufacturers. Fortunately, platinum has an expansion coefficient close to that of glass and makes a reliable seal. Although the Peltier coefficient is large, the heating effect is small at these low currents. The voltage converters designated $\mathrm{FG}$ and $\mathrm{FH}$ agree with NBS standards to $5 \mathrm{ppm}$ or better both as voltage and current converters. The differences are equally small down to well below $20 \mathrm{~Hz}$, and the corrections to a TCC are of course quite independent of the shunt at low frequency [1].

\section{TCC CONSTRUCTION, CONNECTIONS, AND GROUNDING}

The thermoelements are placed in insulating styrofoam containers and mounted in short brass tubes. The input is a GR874 coaxial connector and the thermocouple output is through a two-contact connector which has very low thermal EMF's at the contacts (Fig. 1).

The $0.3-\mathrm{V}$ TVC, designated " $\mathrm{FG}$," is a $10-\mathrm{mA} 30-\Omega \mathrm{TE}$ with no additional resistors. It also serves as a $10-\mathrm{mA}$ TCC-the lowest of the 14 ranges. The other TVC ("FH") contains a $5-\mathrm{mA}$ TE, with a heater resistance of about 90 $\Omega$, and a $33-\Omega$ resistor in series. The combination is paralleled by a second resistor of $39 \Omega$ so that FH becomes a $0.6-\mathrm{V}$ TVC and a $20-\mathrm{mA}$ TCC.

The three high current shunts are made of bifilar strips of $\mathrm{Ni}-\mathrm{Cr}$ quaternary alloy, insulated with 1-mil "Mylar" and mounted between aluminum heat sinks (Fig. 1). The shunt resistance material is gold plated at the ends, then tinned and soldered to heavy copper end pieces to ensure good current distribution. The potential taps are near the centers of the shunt strip and are brought out at right angles to the shunt by a coaxial conductor passing through one half of the heat sink. A flexible cable extends the output to a GR874 connector to which the TVC is attached. The outside of the coaxial conductor is connected to one half of the shunt, and the center conductor passes through 
a hole in that part of the shunt and the insulation to the other half where it is silver soldered.

The 10-20-A shunt resistor is 5 mils thick, $10 \mathrm{~cm}$ wide and about $34 \mathrm{~cm}$ between potential taps. After being folded into bifilar form the overall length is about $24 \mathrm{~cm}$. The resistor for the 3-6-A shunt is 2 mils thick and approximately 4 by $14 \mathrm{~cm}$ between potential taps. The 1-2-A shunt is of the same material and approximately 1.8 by $25 \mathrm{~cm}$.

Building the three shunts for the milliampere ranges with available parts or materials was an awkward problem. The required resistances were too high for bifilar strip material and yet lower than the commercial resistors ordinarily available. Tin-oxide resistors in parallel provided a satisfactory solution. The $300-600-\mathrm{mA}$ shunt $(1 \Omega)$ contains ten $10-\Omega$ resistors, and the $100-200-\mathrm{mA}$ range (3.33 $\Omega$ ) contains six $20-\Omega$ resistors. Parallel combinations of two $30-\Omega$ resistors and four $60-\Omega$ resistors were tried for the $30-60-\mathrm{mA}$ shunt, but one $15-\Omega$ resistor was used as it had the lower ac-dc difference. The resistors are mounted in 2 -in diameter brass cylinders and connected between the outer casing and a wire which joins the center contacts of both the input and output connectors. (The input connector is out of view in Fig. 1.) Where more than one resistor is used they are arranged concentrically around the center conductor.

When two shunts are connected in series for intercomparison tests care should be taken to avoid capacitive currents that might flow in parallel with one shunt after flowing through the other. Therefore a shunt casing (or heat sink) should not be at the potential of the interconnecting conductor as in Fig. 2. It is recommended that one casing be grounded and the other connected to the high (or ungrounded) input conductor.

The heat sinks for the bifilar shunts should be connected similarly. The connections shown in Fig. 3 is recommended, and it should not make an appreciable difference whether one shunt is grounded or the other. The change in ac-dc difference caused by changing the ground was about $10 \mathrm{ppm}$ at $50 \mathrm{kHz}$ on the higher ranges of these shunts. The effect was well under $10 \mathrm{ppm}$ on lower ranges and at $20 \mathrm{kHz}$.

\section{TCC CALIBRations}

Several procedures may be used for ac-dc difference measurements as described briefly in [3, section 6]. However, the TE comparator and the procedures described in [3, section 7] were used for testing these TCC's. The TE comparator affords a significant advantage where the power supplies are even slightly unstable. The precision of these tests probably could not be obtained without it. A fraction of the output emf of one TE is balanced against the emf of the second to obtain an approximate null indication on a detector. Small changes in the ratio of emfs resulting from ac-dc differences may then be measured by changes in the detector indication. The detector may be a microvoltmeter, although for these tests a galvanometer was used whose scale factor $(\mathrm{ppm} / \mathrm{mm})$ was determined for each ac-dc difference determination.

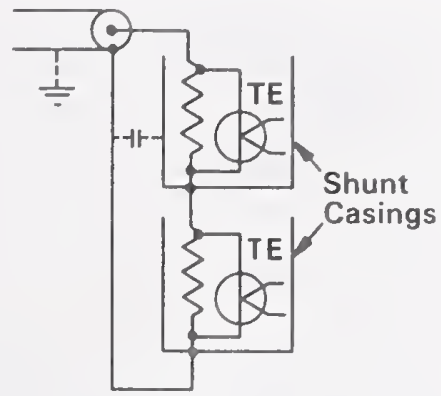

Fig. 2. TCC's in series.

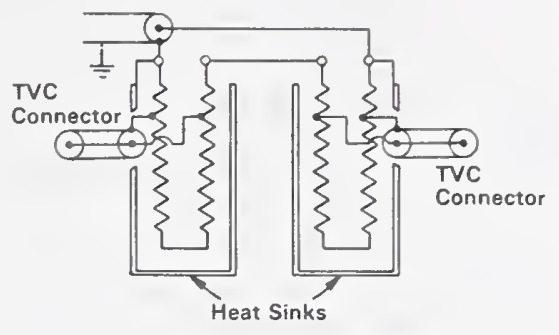

Fig. 3. TCC's in series.

TCC's (shunt and TVC combinations) are usually calibrated and used as one instrument with one correction for each frequency of interest. However the shunt and TVC corrections can be separated for all but the lowest ranges if measurements are made of the corrections to the TVCs as voltage converters. It is necessary to know these corrections if ranges are changed by changing the TVC's. Separating the shunt and TVC corrections has another advantage. Several TVC's may be used, and if one is accidentally burned out the shunts may be used with others.

A third TVC, designated "FE," was assembled using an ordinary $20-\mathrm{mA}$ TE to demonstrate the accuracy with which TVC corrections may be applied. Its correction is $+22 \mathrm{ppm}$ at $50 \mathrm{kHz}$ and is ascribed to skin effect in the heater lead-wires. Shunt tests and intercomparisons with TVC's FH and FE are discussed below.

The correction to the shunt and TVC combination $\left(\delta_{C}\right)$ is not exactly equal to the sum of the shunt correction $\left(\delta_{S}\right)$ and the TVC correction $\left(\delta_{T}\right)$. Separating these corrections is complicated because the proportion of current carried by the TVC varies with range. Hermach has developed a simple approximate formula which is accurate enough at currents of $100 \mathrm{~mA}$ or more

$$
\delta_{C}=m\left(\delta_{S}+\delta_{T}\right)
$$

where $m=\left(I-I_{T}\right) / I, I$ is the total current, and $I_{T}$ is the current through the TVC. The multiplier $m$ differs from unity by only 10 percent at 100 and $200 \mathrm{~mA}$, and its effect on $\delta_{C}$ diminishes rapidly at higher currents. The formula is more complicated at 30 and $60 \mathrm{~mA}$ and involves small reactive components of the impedance which are extremely difficult to measure. Corrections to these ranges are, therefore, determined by comparison with adjacent lower and higher ranges as explained below.

In the intercomparison tests, measurements are made 
TABLE II

Intercomparisons of TCC's (ppm)

\begin{tabular}{|lllcc|}
\hline TCC 1 & TCC $_{2}$ & $\begin{array}{l}\text { Applied } \\
\text { Current }\end{array}$ & $\frac{20 \mathrm{kHz}}{\mathrm{D}}$ & $\frac{50 \mathrm{kHz}}{\mathrm{D}}$ \\
\hline FG & FH & 0.01 & 0 & 0 \\
FG30 & FH & 0.02 & +1 & +11 \\
FG100 & FH60 & 0.06 & +10 & +23 \\
FG300 & FH200 & 0.2 & +7 & +20 \\
FG1 & FH600 & 0.6 & -7 & -33 \\
FG3 & FH2 & 2 & +12 & +22 \\
FG10 & FH6 & 6 & -24 & -54 \\
\hline
\end{tabular}

TABLE III

AC-DC Difference of TCC's (ppm)

\begin{tabular}{|c|c|c|c|c|c|}
\hline \multirow{2}{*}{ TCC } & Applied & \multicolumn{2}{|c|}{$20 \mathrm{kHz}$} & \multicolumn{2}{|c|}{$50 \mathrm{kHz}$} \\
\hline & Amperes & measured & computed & measured & computed \\
\hline $\mathrm{FG}$ & 0.01 & -1 & 0 & 0 & -2 \\
\hline $\mathrm{FH}$ & 0.02 & 0 & 0 & -2 & -2 \\
\hline FG30 & 0.03 & +3 & +1 & +10 & +9 \\
\hline $\mathrm{FH} 60$ & 0.06 & +3 & 0 & +12 & +14 \\
\hline FG100 & 0.1 & +9 & +10 & +32 & +37 \\
\hline FH200 & 0.2 & +8 & +10 & +36 & +37 \\
\hline FG 300 & 0.3 & +13 & +17 & +51 & +57 \\
\hline FH 600 & 0.6 & +19 & +17 & +53 & +57 \\
\hline $\mathrm{FGl}$ & 1 & +10 & +10 & +24 & +24 \\
\hline $\mathrm{FH} 2$ & 2 & +12 & +10 & +28 & +24 \\
\hline FG3 & 3 & +21 & +22 & +44 & +46 \\
\hline FH6 & 6 & +18 & +22 & +36 & +46 \\
\hline FG10 & 10 & +8 & -2 & +3 & -8 \\
\hline FH 20 & 16 & +5 & 2 & +2 & 8 \\
\hline
\end{tabular}

of the difference between two shunt-TVC combinations. If this difference is called " $D$ " we may write

$$
D=\delta_{C 1}-\delta_{C 2}
$$

which becomes, after substituting from (1),

$$
m_{1} \delta_{S 1}-m_{2} \delta_{S 2}=D+\left(m_{2} \delta_{T 2}-m_{1} \delta_{T 1}\right) .
$$

At 100 and $200 \mathrm{~mA}$ the effect of the multiplier $m(=0.9)$ is less than $5 \mathrm{ppm}$ for the corrections to these shunts and TVC's. Since there are other uncertainties of this magnitude the effect has been neglected. However, where the corrections are $50 \mathrm{ppm}$ or more it should be taken into account.

A 7-step intercomparison was made at 20 and $50 \mathrm{kHz}$, and the relative ac-dc differences are listed under $D$ in Table II. Measurement of ac-dc difference corrections for all ranges were also made at the same frequencies by comparison with NBS standard current converters most of which are specially made TE's. These corrections are listed under " $\delta$ measured" in Table III. In two additional columns in Table III under " $\delta$ computed" these corrections are determined from the intercomparison tests.

The base ranges serving as starting points for the computed $\delta$ 's were chosen arbitrarily. The "measured" corrections to the $20-\mathrm{mA}$ range and the 1-A range were taken as base points and the others were computed by using (2) and the data from Table II [2]. As mentioned above, the
TAB LE IV

\begin{tabular}{|c|c|c|c|c|}
\hline \multirow[b]{2}{*}{$\mathrm{TCC}_{1}$} & \multirow[b]{2}{*}{$\mathrm{TCC}_{2}$} & \multirow{2}{*}{$\begin{array}{l}\text { Applied } \\
\text { Current } \\
\text { Amperes }\end{array}$} & \multicolumn{2}{|r|}{$50 \mathrm{kHz}$} \\
\hline & & & D & $\delta_{S 1}-\delta_{S 2}$ \\
\hline FG & $\mathrm{FE}$ & 0.01 & +3 & - \\
\hline FG 30 & $\mathrm{FE}$ & 0.02 & +9 & \\
\hline FG100 & FE60 & 0.06 & +14 & \\
\hline FG 300 & FE200 & 0.2 & +8 & +30 \\
\hline $\mathrm{FGI}$ & FE600 & 0.6 & -52 & -30 \\
\hline FG3 & FE2 & 2 & 0 & +22 \\
\hline FG1O & FE6 & 6 & -64 & -42 \\
\hline
\end{tabular}

Intercomparison of TCC's with TVC Corrections (ppm)

TABLE V

\begin{tabular}{|c|c|c|c|c|c|c|}
\hline \multirow[b]{2}{*}{$\mathrm{TCC}$} & \multirow[b]{2}{*}{$\begin{array}{l}\text { Applied } \\
\text { Current } \\
\text { Amperes }\end{array}$} & \multicolumn{4}{|c|}{$50 \mathrm{kHz}$} & \multirow[b]{2}{*}{$\begin{array}{c}{ }^{\delta} \mathrm{C} \\
\text { computed }\end{array}$} \\
\hline & & ${ }^{\delta} \mathrm{C}$ & ${ }^{\delta} \mathrm{T}$ & $\begin{array}{c}\delta_{S} \\
\text { measured }\end{array}$ & $\begin{array}{c}{ }_{\mathrm{S}} \\
\text { computed }\end{array}$ & \\
\hline $\mathrm{FG}$ & 0.01 & 0 & - & 0 & -5 & \\
\hline $\mathrm{FE}$ & 0.02 & -8 & - & -8 & -8 & \\
\hline $\mathrm{FG} 30$ & 0.03 & +10 & & & & +1 \\
\hline FE60 & 0.06 & +18 & & & & +10 \\
\hline $\mathrm{FG} 100$ & 0.1 & +32 & 0 & +32 & +24 & \\
\hline FE200 & 0.2 & +48 & +22 & +26 & +24 & \\
\hline$F G 300$ & 0.3 & +42 & 0 & +42 & +54 & \\
\hline FE600 & 0.6 & +72 & +22 & +50 & +54 & \\
\hline $\mathrm{FG1}$ & 1 & +28 & 0 & +28 & +24 & \\
\hline FE2 & 2 & +53 & +22 & +31 & +24 & \\
\hline FG3 & 3 & +44 & 0 & +44 & +46 & \\
\hline FE6 & 6 & +63 & +22 & +41 & +46 & \\
\hline $\mathrm{FG} 10$ & 10 & +3 & 0 & +3 & +4 & \\
\hline FE 20 & 16 & +13 & +22 & -9 & +4 & \\
\hline
\end{tabular}

AC-DC Difference of TCC's with TVC Corrections (ppm)

relationship of the shunt and TVC corrections is complicated at 30 and $60 \mathrm{~mA}$ where the TVC's carry 33 percent of the current. Therefore, the $20-\mathrm{mA}$ TCC (FH) is used as a starting point for computing corrections to FG30 and FG.

Similar data are listed in Tables IV and V for $50 \mathrm{kHz}$, but TVC corrections are applied in these tables since FE is used. The corresponding ac-dc differences for $20 \mathrm{kHz}$ were smaller, as in Tables II and III, and are not shown. Intercomparison differences are listed under " $D$ " in Table IV, and TVC corrections from (3), with $m_{1}$ and $m_{2}$ equal to 1 , are applied. $\left(\delta_{T 2}-\delta_{T 1}=+22 \mathrm{ppm}\right)$. The remaining shunt differences are listed under " $\delta_{S 1}-\delta_{S 2}$." At 60-mA, equation (3) is not sufficiently exact, and, therefore, corrections to the $60-\mathrm{mA}$ shunt and $\mathrm{FE}$ are not separated.

Corrections to each TCC with FE were determined relative to NBS standards, and are listed under " $\delta_{C}$ " in Table $\mathrm{V}$. The corresponding values for the same shunt with FG are taken from Table III. After applying the TVC corrections ( $\delta_{T}=0$ for $\mathrm{FG}$ and $+22 \mathrm{ppm}$ for $\mathrm{FE}$ ) the remaining shunt corrections are listed under " $\delta$ measured."

Again, as in Table III, shunt corrections are computed in Table $\mathrm{V}$ for all ranges beginning, as before, with the measured correction to the $1-\mathrm{A}$ and $20-\mathrm{mA}$ ranges. The computed correction to FE60 and FG30 apply to the combination since the correction $\delta_{C}$ cannot be reliably separated at this level. 
The 20-mA range and the $1-$ and $10-\mathrm{A}$ ranges are recommended as minimum reference points to be tested relative to other standards outside the set. Intercomparison determinations would be based on the $20-\mathrm{mA}$ and $1-\mathrm{A}$ corrections, and the 10-A range would serve to verify the accuracy of the step-up tests for the highest shunt ranges. TVC corrections would also have to be determined.

The close similarity of measured corrections for the same shunt at different current levels shows that the ac-dc differences are not appreciably affected by the current. This similarity in Table III also shows that the TVC's FG and $\mathrm{FH}$, which are changed when the current is changed, have nearly the same correction. It is very unlikely that one change would offset the other on so many ranges. The similarity of measured and computed corrections in Tables III and V shows that the intercomparison tests can be made quite accurately.

\section{Using Thermal Current Converters}

Ac-dc difference measurements may be made of transfer instruments and ammeters which respond nearly equally to ac and dc currents. The TE comparator and procedures referred to earlier may be used if the test instrument has a thermocouple output voltage which can be connected to the comparator. Otherwise an indicator or readout instrument is observed which is usually a part of the device under test.

If the test instrument does not respond to direct current it may be tested by two or three procedures, but the one outlined below is recommended. Changes in detector indication are ubserved to determine the difference between the ac current and an accurately measured dc current nominally equal to it.

The suggested circuit is shown in Fig. 4, and the order of currents applied to the standard TCC is indicated (DC+, AC, DC-). The potentiometer $P$ is adjusted for an approximate null indication on the detector $D$. Detector readings are then made for each dc current after it has been adjusted to the nominal test value. The ac reading $E_{a}$ is made after the ac has been adjusted for the desired indication of the test instrument. The two readings on dc are averaged $\left(E_{d}\right)$, and the ac may then be calculated:

$$
I_{\mathrm{ac}}=I_{\mathrm{dc}}\left(1+\frac{E_{a}-E_{d}}{n E}+\delta_{S}\right)
$$

where $I_{\mathrm{dc}}$ is the dc current, $E$ is the TE EMF indicated by

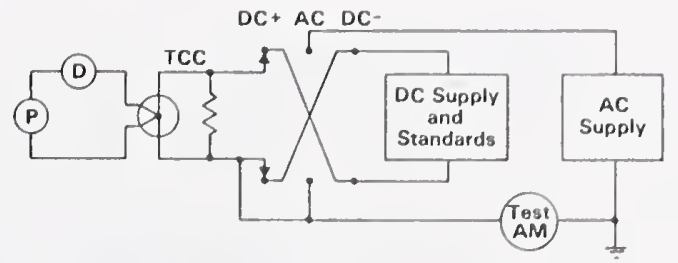

Fig. 4. Circuits for testing an ac ammeter relative to de standards.

the potentiometer $P, \delta_{S}$ is the ac-dc difference of the standard TCC and $n=\Delta E / \Delta I$. The factor $n$ is a measure of the deviation of the TE from a square-law response and is usually 1.7 to 1.9 at rated TE current, (see [1, section 9]).

\section{CONCLUSION}

It has been shown that a TCC set consisting of six shunts and two TVC's can be constructed to cover fourteen ranges from $10 \mathrm{~mA}$ to $20 \mathrm{~A}$. Intercomparison tests can be made in seven steps to assist in maintaining accurate ac-dc difference corrections. Thermoelements with platinum heater lead-wires are preferable, because the TVC corrections are negligible. However if ordinary TE's are used the TVC corrections can be accurately determined and applied. The estimated accuracies from $20 \mathrm{~Hz}$ to $50 \mathrm{kHz}$ are $50 \mathrm{ppm}$ at $6 \mathrm{~A}$ and lower and $100 \mathrm{ppm}$ at 10 and $20 \mathrm{~A}$. Measurements of ac current would require additional corrections for the $\mathrm{dc}$ standards used as reference and considerations of the precision of the instrument under test.

\section{ACKNOWLEDGMENT}

This work was supported in part by the Army Metrology and Calibration Center, Redstone, AL. Their encouragement and support are gratefully acknowledged.

\section{REFERENCES}

[1] F. L. Hermach, "Thermal converters as ac-dc transfer standards for current and voltage measurements at audio frequencies," J. Res. Nat. Bur. Stand., vol. 48, pp. 121-138, Feb. 1952.

[2] F. C. Widdis, "The theory of Peltier and Thomson effects in thermal ac-dc transfer devices," Proc. Inst. Elec. Eng., monogr. 497M, Jan. 1962.

[3] F. S. Williams, "Thermal voltage converters and comparators for very accurate ac voltage measurements," J. Res. Nat. Bur. Stand., vol. 75C, nos. 3 and 4, pp. 145-154, July-Dec. 1971.

[4] F. L. Hermach and E. S. Williams, "Thermal converters for audiofrequency voltage measurements of high accuracy," IEEE Trans. Instrum. Meas., vol. IM-15, pp. 260-268, Dec. 1966. 
Section 13

\title{
A Dual-Channel Automated Comparator for AC-DC Difference Measurements
}

\author{
EARL S. WILLIAMS AND JOSEPH R. KINARD, SENIOR MEMBER, IEEE
}

\begin{abstract}
An automated ac-dc difference calibration system is described. The system incorporates a new electronic comparator which determines ac-dc differences of thermal voltage converters (TVC's) by simultaneously measuring the difference between nearly equal ac and dc voltages with both the test and standard instruments. The comparator consists essentially of two practically identical channels each containing a digital-to-analog converter balancing circuit, an operational amplifier to amplify voltage imbalance, and an integrator circuit in which a capacitor is charged during an accurately controlled 10 -s period. The difference between the ac and dc voltages applied to the test and standard TVC's is computed from differences in capacitor voltages, and the ac-dc difference is derived from the variation between the test and standard channel indications of this voltage difference. Measurements are made in about half the time required for our manual procedures.
\end{abstract}

\section{INTRODUCTION}

A C AND DC voltages are compared using a thermal voltage converter (TVC) which responds nearly equally to ac and $\mathrm{dc}$ voltages. Those discussed here are electrothermic. Typically, they consist of ranging resistors and a thermoelement - a vacuum bulb containing a heater wire with a thermocouple at. tached to its midpoint. The output, $E$, of the thermocouple is usually $7-12 \mathrm{mV}$ with rated heater current. The TVC is

Manuscript received August 23, 1984. This work was supported by the Calibration Coordination Group of the U.S. Department of Defense.

J. R. Kinard is with the Electricity Division, National Bureau of Standards, Gaithersburg, MD 20899.

E. S. Williams (retired) was with the Electricity Division, National Bureau of Standards, Gaithersburg, MD 20899. connected alternately to an ac voltage, $V_{a}$, and to both polarities of a dc reference voltage, $V_{d} . V_{a}$ is measured relative to $V_{d}$ by observing the output EMF, $E$, of the TVC and changes in output, $E_{a}-E_{d}$, where $E_{a}$ is observed with $V_{a}$ applied, and $E_{d}$ is observed with $V_{d}$ applied. When the difference between $V_{a}$ and $V_{d}$ is small, the proportional difference can be computed from (1):

$$
\frac{\left(V_{a}-V_{d}\right)}{V_{d}}=\frac{\left(E_{a}-E_{d}\right)}{n E}+\delta
$$

The factor $n$ relates small changes in TVC heater current to corresponding changes in output EMF, $E$. It is near 2.0 (a square-law response) at very low heater currents, but is usually 1.7-1.9 at rated heater current. $\delta$ is the ac-dc difference of the TVC. It is negligible at low frequencies, but becomes significant at frequencies of $20 \mathrm{kHz}$ and higher in most TVC's.

The ac-dc difference correction for a TVC is determined by comparing it to a similar standard instrument whose correction, $\delta_{s}$, is known [1]. Ac and dc voltages are applied to both TVC's in parallel, and in most procedures, the ac and dc voltages are adjusted for equal outputs from the test TVC, i.e., $E_{a}=E_{d}$ (1). The test instrument thus indicates that $V_{a}=V_{d}$. The actual proportional difference between the ac and dc voltages is then measured by observing $E$ and changes in $E$ from the standard TVC. The correction to the test TVC, $\delta_{t}$, is computed using an equation similar to (1): 
DUAL CHANNEL COMPARATOR
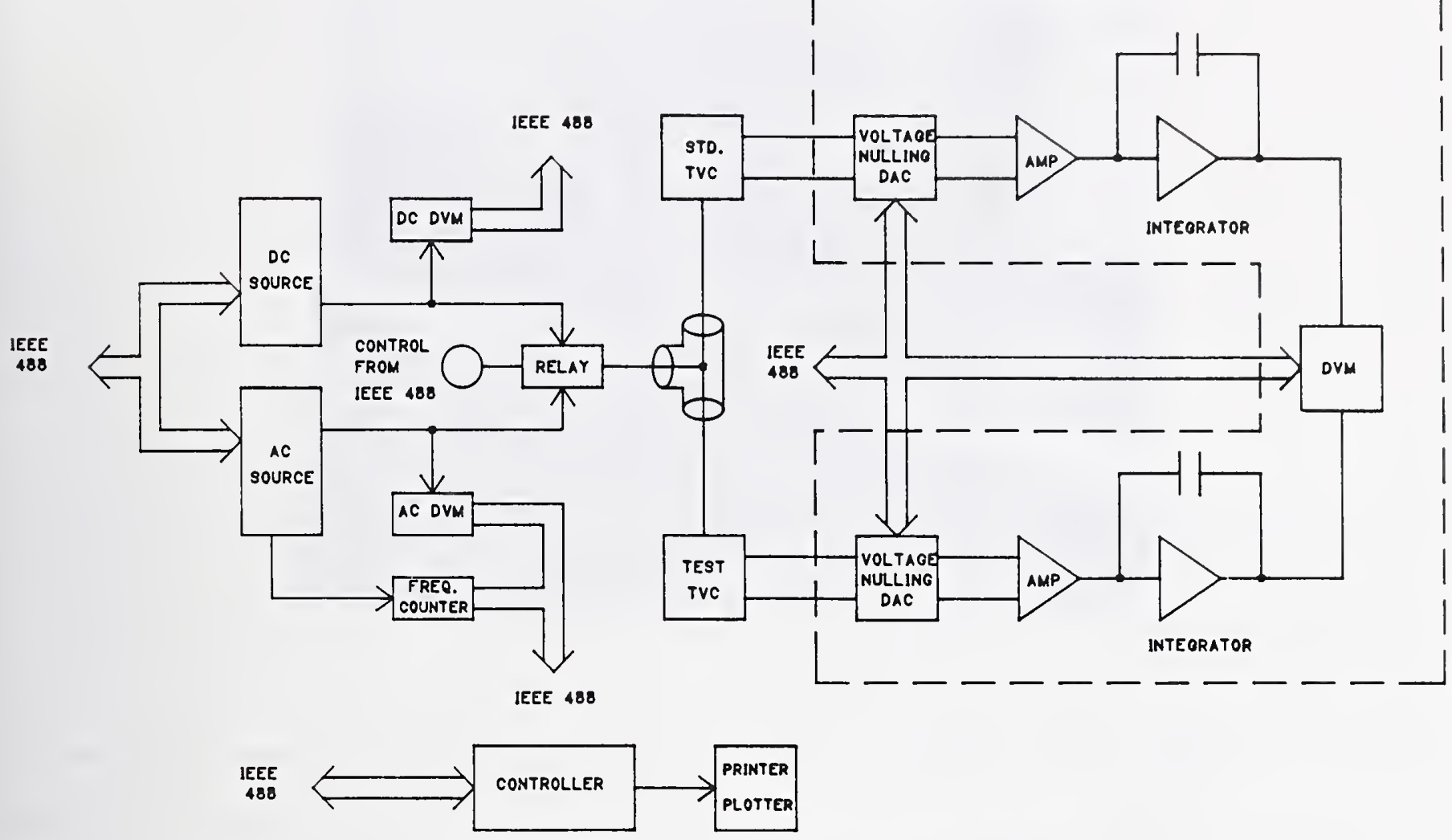

Fig. 1. Block diagram of automated ac-dc difference calibration system.

$$
\delta_{t}=\left(E_{a}-E_{d}\right) / n E+\delta_{s}
$$

where subscripts $t$ and $s$ refer to test and standard.

With a manual comparator, the precise adjust ments of ac and $\mathrm{dc}$ voltages for equal outputs from the test TVC are tedious and a skilled operator is necessary [2]. These adjustments have been made automatically but the process is slowed significantly [3]. In the alternative procedure used in the new comparator, data is collected more quickly because the ac and dc voltages are set nearly equal and not further adjusted during one determination. The ac voltage is trimmed automatically after each determination to compensate for drift and to avoid the difficulty, discussed below, of a large difference between ac and dc applied voltages. In this new approach the difference between the unequal ac and dc voltages is measured by both the test and standard TVC's, and, therefore,

$$
\frac{\left(V_{a}-V_{d}\right)}{V_{d}}=\frac{\left(E_{a s}-E_{d s}\right)}{n_{s}\left(E_{s}\right)}+\delta_{s}=\frac{\left(E_{a t}-E_{d t}\right)}{n_{t}\left(E_{t}\right)}+\delta_{t} .
$$

Eliminating the first expression and solving the remaining two for $\delta_{t}$, we have

$$
\delta_{t}=\frac{\left(E_{a s}-E_{d s}\right)}{n_{s}\left(E_{s}\right)}-\frac{\left(E_{a t}-E_{d t}\right)}{n_{t}\left(E_{t}\right)}+\delta_{s} .
$$

\section{Automated System}

The entice automated ac-dc difference calibration system is shown in block diagram form in Fig. 1. A photograph of the equipment appears in Fig. 2. The system contains program. mable ac and dc voltage sources, a high-voltage relay for switching between ac and dc, voltmeters and frequency counters, a desktop computer to provide IEEE-488 bus control, and the two-channel comparator itself. The two-channel comparator circuit is sketched in Fig. 3. It uses elements from a commercial data acquisition system (DAS) including programmable digital. to-analog converters (DAC's), programmable relays (K1 to K6 and $\mathrm{K} 9$ ), and an auto-ranging voltmeter (VM). An additional board was assembled containing the DAC balancing circuits, amplifiers A1 to A4 and their related resistors and capacitors.

The DAC's supply up to 10 volts to Lindeck-type circuits with $5-\mathrm{k} \Omega$ total circuit resistance. With only one range, the 12-bit resolution is insufficient for an appropriate null. A twopole four-position input switch allows the operator to select either a $5-$ or $10-\Omega$ resistance in the divider circuit, thus providing either a 10 - or $20-\mathrm{mV}$ range.

The measurement procedure is as follows. The operator provides ranges, voltages, frequencies, and record-keeping informa. tion to the controller interactively. The ac and dc supplies are then programmed to the appropriate values, and dc is applied to the TVC's. With the DAC set to zero and K3 closed, the standard TVC output, $E_{d s}$, is measured by the voltmeter, and a DAC voltage is computed and supplied to null the input to amplifier A1. The output of A1, with gain of 10000 , is then measured by closing relay $\mathrm{K} 2$, and the DAC signal is trimmed in several steps until the amplifier output is less than $20 \mathrm{mV}$. The same balancing procedure is followed in the test channel. When both balances have been completed, $n_{s}\left(E_{s}\right)$ and $n_{t}\left(E_{t}\right)$ are computed and printed along with other test parameters. 


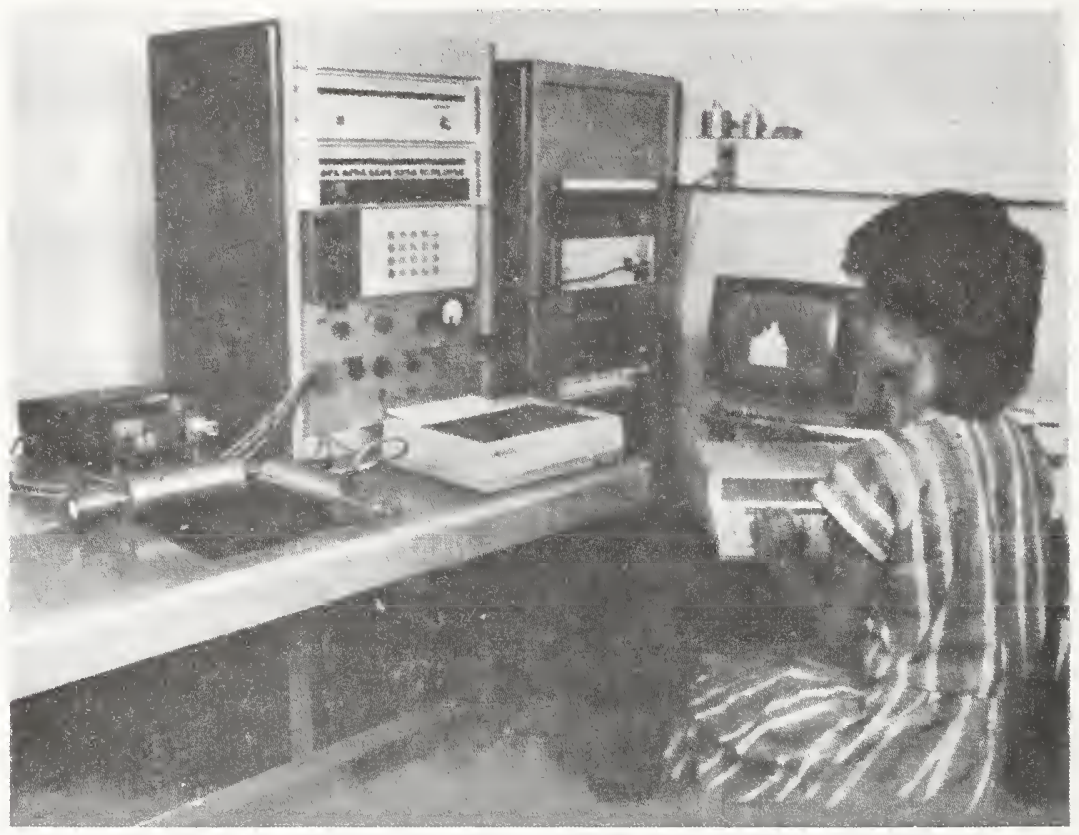

Fig. 2. Automated ac-dc difference calibration system.

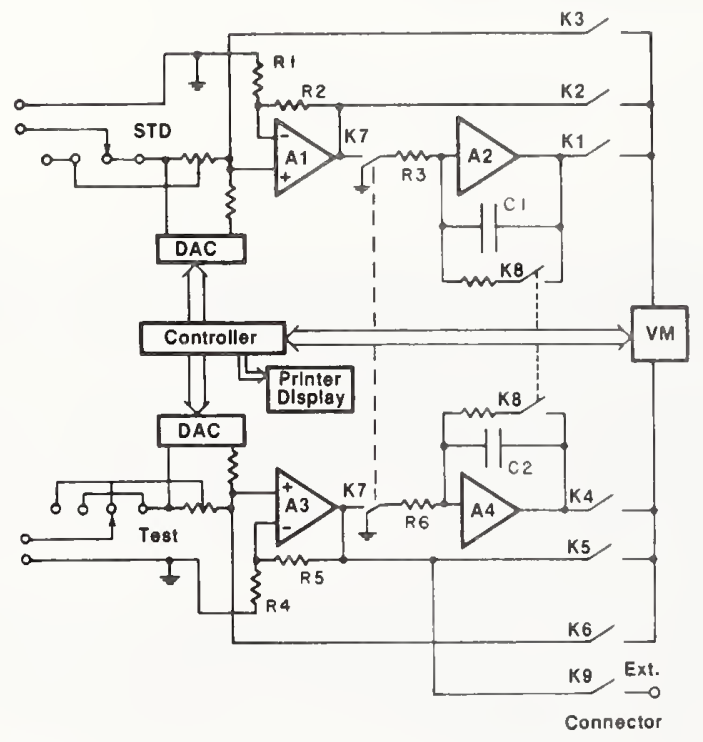

Fig. 3. Dual-channel ac-dc difference comparator.

Voltages are then applied to both TVC's in the test sequence (ac, dc+, dc- , ac), and after each one has stabilized, the relay $\mathrm{K} 7$, with a contact in each channel, is closed to charge the two capacitors during an accurately measured time interval of $10 \mathrm{~s}$. The capacitor voltages, four from each channel for a single measurement, are then measured with the voltmeter by closing relays $\mathrm{K} 1$ and $\mathrm{K} 4$ in turn, after which the capacitors are discharged by closing two-pole relay K8. Providing they are stable over the time required for one determination, amplifier of fsets cancel because the results are collected in paired sums and differences.

For the standard channel, the relation between the voltage, $e_{\mathrm{in}}$, at the input to $\mathrm{Al}$ and the voltage, $e_{\mathrm{out}}$, on the integrator capacitor is

$$
e_{\text {out }}=-\frac{1}{\mathrm{R} 3 \mathrm{C} 1} \int \frac{\mathrm{R} 1+\mathrm{R} 2}{\mathrm{R} 1} e_{\mathrm{in}} d t=-\bar{e}_{\text {in }} \times 10^{5}
$$

with $\mathrm{R} 1=10 \Omega, \mathrm{R} 2=100 \mathrm{k} \Omega, \mathrm{R} 3=1 \mathrm{M} \Omega, \mathrm{C} 1=1 \mu \mathrm{F}$, and an integration time of $10 \mathrm{~s}$. Nominally equal values of resistance and capacitance are used in the standard and test channels, so the same nominal relations apply. If the eight voltage readings are reversed in sign and set equal to $e_{2}, e_{4}, e_{6}$, and $e_{8}$ in the standard channel, and $e_{3}, e_{5}, e_{7}$, and $e_{9}$ in the test channel, then using (5), (4) may be written as

$$
\delta_{t}=\frac{5 \times\left(e_{2}-e_{4}-e_{6}+e_{8}\right)}{n_{s} E_{s} \cdot 10^{6}}-\frac{5 \times\left(e_{3}-e_{5}-e_{7}+e_{9}\right)}{n_{t} E_{t} \cdot 10^{6}}+\delta_{s} .
$$

The numerator in (6) gives twice the actual change in EMF, so the constants are adjusted appropriately. These constants are also adjusted slightly to compensate for departures from nominal in the integrators and amplifiers. The test TVC correction is computed using (6), with the appropriate ac-dc difference correction, $\delta_{s}$, determined from an equation devised to simulate the standard TVC correction curve.

Since this two-channel method requires the knowledge of $n$ for both test and standard, any uncertainty in either $n$ contributes to uncertainty in the final ac-dc difference. Should either or both terms in (4) become large due to inequality between applied ac and dc voltages, the calculation contains the difference of two large terms each involving an $n$. Since the $n$ value can only be determined to 1 or 2 percent, a constraint is added to system that the 1 st term in (4) be $50 \mathrm{ppm}$ or less to limit the uncertainty contributed by $n$ to $1 \mathrm{ppm}$, or less. With this constraint the procedure places an upper limit of about 50 ppm on the difference between the applied ac and dc voltages. Equally satisfactory results are obtained by a "two-voltmeter" 


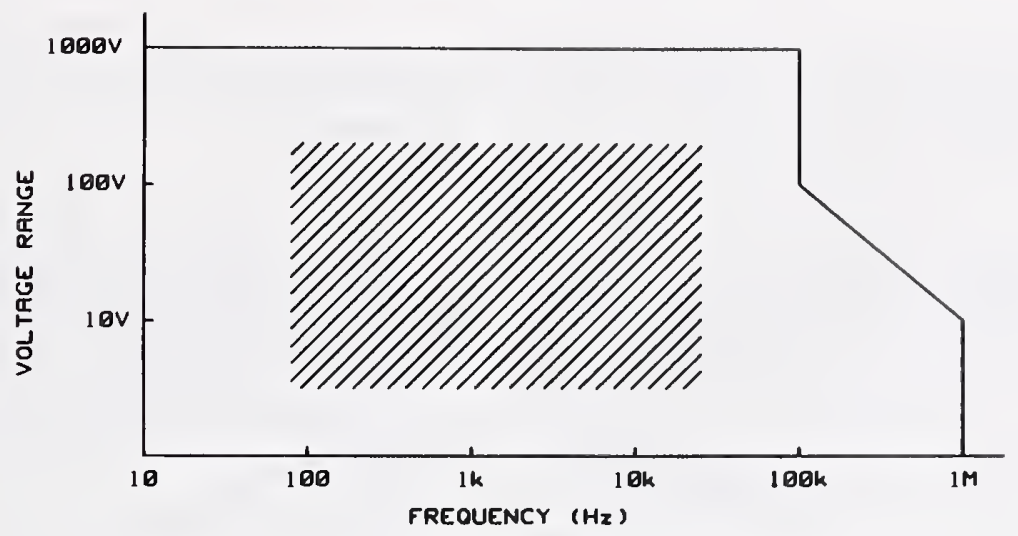

Fig. 4. Voltage and frequency limits of calibration system.

method similar to that used by Inglis in a semi-automatic system [4]. Instead of the analog integrators, an external digital voltmeter connected to relay $\mathrm{K} 9$, and the voltmeter located in the DAS, are used to measure the amplified voltage imbalances. After the DAC balances are made, relays $\mathrm{K} 2$ and $\mathrm{K} 9$ are closed and remain closed while the ac and dc voltages are applied. The voltmeters are read alternately and the two sums are accumulated. If ten readings are summed for each channel with the measurement sequence ac, $\mathrm{dc}^{+}, \mathrm{dc}^{-}, \mathrm{ac}$, and the sums set equal to $e_{2}$ through $e_{8}$ and $e_{3}$ through $e_{9}$, then $\delta_{t}$ can still be obtained from (6) with no change.

\section{Other Measurements}

Measurements of the response characteristic, $n$, and comparisons of ac and dc voltages are two types of measurements made using only one channel of the comparator. For these cases, each EMF measurement is the average of 10 voltmeter readings taken in about $10 \mathrm{~s}$.

\section{A. Measurements of $n$}

The factor $n$ is ordinarily measured at 10 percent intervals from 50 to 110 percent of rated voltage. For instruments to be calibrated at rated voltage, however, $n$ is usually determined at 80,90 , and 100 percent. In the middle voltage range, small accurate voltage steps are easily made and the stability of the system is optimum. Tests are made, therefore, most conveniently on the $100-\mathrm{V}$ range of multirange TVC's, and the resulting $n$ values are usable with any range when related to the TVC output, $E$. Low-voltage single-range TVC's are usually tested with additional resistance in series, so that $50-100 \mathrm{~V}$ may be applied. The output of the TVC is connected to the "STD" channel, and the operator enters the lowest and highest voltages applied. After the TVC has stabilized at the measurement voltage, the DAC balance is made as described above, and relay $\mathrm{K} 2$ is closed connecting the amplifier to the voltmeter. The TVC input is increased and decreased alternately by $0.1 \mathrm{~V}$, $\Delta V$, and the resulting changes in TVC output are computed from voltmeter readings.

With only dc applied, the relationship between small changes in input voltage, $\Delta V$, and changes in the output voltage, $\Delta E$, can be given by rewriting (1) as

$$
\Delta V / V=\Delta E / n E \text { or } n=(\Delta E / E) /(\Delta V / V) \text {. }
$$

As in the two-voltmeter method, 10 voltmeter readings of the output are averaged to obtain one measured value. One $\Delta E$ is calculated from measurements taken in the sequence ( $V$, $V+\Delta V, V)$. The last reading with $V$ applied from the previous set is then taken as the first reading in the next set. Four values of $n$ are computed from four values of $\Delta E$ requiring nine volt meter measurements. These data are then used to determine constants for a straight line from which the appropriate value of $n$ can then be found for any value of $E$.

\section{B. Comparison of $A C$ and DC Voltages}

$\mathrm{AC}$ voltage, such as from an ac calibrator, can be measured relative to a dc-reference voltage by a procedure similar to that used for the determination of $n$. The system applies $\mathrm{dc}+$, ac, $\mathrm{dc}^{-}$, to a TVC in that order, and its output is monitored by the "STD" channel with the DAC balance and voltmeter readings performed as described above. Computations are made using (1) with $\left(E_{a}-E_{d}\right)$ computed from three voltage readings$e_{1}, e_{2}, e_{3}-$ so that

$$
2\left(E_{a}-E_{d}\right)=-e_{1}+2 e_{2}-e_{3}
$$

where $e_{1}$ and $e_{3}$ correspond to the two directions of dc volt. age, and $e_{2}$ to the ac voltage. Again, four values of EMF difference are computed from nine voltages-each being the average of 10 voltmeter readings. When low voltages are being compared, the voltage drops along the leads from the sources to the TVC may become significant. Dedicated cables are provide with total inner and outer resistance equal for both ac and dc. These connections also permit voltage sensing of the $\mathrm{dc}$ if required.

\section{RESUltS}

A significant reduction in the time required to perform routine calibrations is achieved with this system. Four determinations, for one set of test conditions, are made and averaged in about half the time required with our manual procedures. The voltage and frequency operating ranges, shown in Fig. 4, are determined primarily by the availability and cost of a programmable ac voltage source. The region between $100 \mathrm{kHz}$ and 1 $\mathrm{MHz}$ represents a maximum volt-Hertz product of $10^{7}$. Test 
results taken over the operational region rarely differ by more than $10 \mathrm{ppm}$ from data obtained manually. The standard deviation is generally less than $5 \mathrm{ppm}$ in the middle-voltage and middle-frequency region indicated approximately by the crosshatched region in Fig. 4. For well-constructed single-range TVC's, standard deviations of $2 \mathrm{ppm}$ or less are achieved. At extreme voltages and frequencies standard deviations are usually less than $10 \mathrm{ppm}$. Although this system was built as a research tool for investigation of new thermal voltage conversion techniques and instruments, it has been used for some routine calibrations. Another system, of very similar design and dedicated to routine calibration work, is under construction. These automatic systems are expected to fulfill most of our TVC calibration requirements.

\section{ACKNOWLEDGMENT}

The authors would like to thank F. L. Hermach for his helpful suggestions.

\section{REFERENCES}

[1] F. L. Hermach and E. S. Williams, "Thermal converters for audiofrequency measurements of high accuracy," IEEE Trans. Instrum. Meas., vol. IM-15, pp. 260-268, Dec. 1966.

[2] E. S. Williams, "Thermal voltage converters and comparator for very accurate ac voltage measurements," J. Res. Nat. Bur. Stand., vol. 75C, pp. 145-154, 1971.

[3] —-, "A thermoelement comparator for automatic ac-dc difference measurements," IEEE Trans. Instrum. Meas., vol. IM-29, pp. 405409, Dec. 1980.

[4] B. D. Inglis, "Evaluation of ac-dc transfer errors for thermal converter-multiplier combinations," Metrologia, vol. 16, pp. 177$181,1980$. 
GENERAL INSTRUCTIONS FOR THE OPERATION OF NBS AUTOMATED AC-DC COMPARATOR SYSTEM II

Thomas E. Lipe

Electricity Division

National Bureau of Standards

December, 1987 


\section{General Description}

1. The new NBS automated ac-dc comparator (System II) was built in 1985-1986 to assist in the everyday calibration services of the group. It is expected that this system will eventually be responsible for the majority of the ac-dc calibrations performed in the Electricity Division.

2. The new system is composed of two racks of calibration equipment, a desktop computer which acts as the system controller, and several input/output devices. The racks are divided into an ac rack and a dc rack as follows:

From the bottom, the ac rack contains:

a) Relay box and switch panel built in-house

b) Ac voltage source (Fluke 5200A)

c) Frequency counter (Systron-Donner 6041A)

d) Ac voltmeter (Fluke 8922A) and IEEE-488 translator (Fluke 1120A)

e) Ac high-voltage power amplifier (Fluke 5215A)

The dc rack contains:

a) Dc voltage source (Fluke 5440B)

b) Data acquisition system (Hewlett-Packard 3497A)

c) Dc voltmeter (Fluke 8840A) and IEEE-488 bus optical isolator (ICS 4830B)

d) DC nanovoltmeter(s) (one or two Keithley 181 nanovoltmeters)

System control and support:

a) System contoller (Hewlett-Packard series 200/300 desktop computer)

b) Printer (Dataproducts Prism P-132 matrix printer)

c) Plotter (HewlettPackard 7475A six pen plotter)

d) Access to administrative minicomputer (DEC VAX 11/750)

e) Administrative printer (Hewlett-Packard LaserJet+ laser printer)

Future expansion:

a) Hewlett-Packard 3498A Expander Box for multi-channel calibrations 
Note that the product names in parentheses indicate the equipment supported by the system software as of August 27, 1987. Future versions of the software will have the capability to support a variety of equipment.

3. Although most of the equipment in the system was procured from commercial sources, the "guts" of the system (the relay box and several circuit boards in the data acquisition system) were built in-house. The relay box and its associated switch panel contain two sets of relays used for switching the output between ac to dc. The designation of the relays to be used depends upon the applied voltage with the crossover point at 70 volts to coincide with the use of the high voltage interlock system. The high voltage relays are coto 8407 SPST relays and the low voltage relays are potter and Brumfield KRP14DG 3PDT relays which have been modified to a single throw configuration. By using two different sets of two relays, problems such as arcing on high voltage and contact resistance on low voltage are avoided. Since there is no voltage on the output of the relays in the normally open position, there is a built-in safety factor; when the relays are open, no voltage is applied to the output connector. The function of the front panel switches are as follows:

i) OUTPUT GND-OFF-ON: This switch controls the voltage output to the units under test. In the 'GND' position, the output of the system is connected to the system ground at the ac supply. In the 'OFF' position, the output is open circuited, and in the 'ON' position, the output is connected to the units under calibration. This switch provides a second-stage safety measure, since the voltage output can be immediately disconnected from the test units, and because this switch must be activated in concert with the output on/off relay.

ii) DETECTOR SWITCH 1-2-3-4: Because the DACs which null the output EMF of the units under test have an upper limit of 10.2375 volts, they may occasionally be overranged. To prevent this, a voltage divider (in the data acquisition system) is wired in series with the output EMF. The input to the divider is controlled by this switch, and its position is determined by the output EMF of the units under test. You will be prompted by the software to set the switch to the proper position during a calibration.

iii) AC INPUT SELECT HI-OFF-LOW: when the Fluke $5200 / 5215$ ac calibrator/ amplifier is used, the ac voltage enters the relay box along separate leads for high and low voltage. This switch selects the high or low ac inputs and is set by the user before the calibration process begins. on the high setting, this switch also introduces a high-voltage oscilloscope probe in series with the ac voltmeter 
so as not to overrange it

iv) MODE SELECT AUTO-DC-AC: This switch controls the ac-dc relays and determines whether the relays are under manual or computer control. In the 'AUTO' position, both sets of ac-dc relays are controlled by the data acquisition system. When switched to 'DC', the dc high-voltage output relay is closed (output on), providing the dc output to the output connector. On 'AC' the ac relay is closed (output on), providing ac voltage to the output connector. Note that, in the present configuration, the high voltage relays are the only ones controllable from the front panel. This will be modified in future versions.

v) CONNECTIONS TO THE RELAY BOX: The connections on the front panel, from left to right are: the test and standard channel detector leads and the voltage output coaxial connector, and the ac low voltage input and sense coaxial connectors. On the back, from left to right, are the ac line connector for the relay power supply, the ac high voltage connector, the ac voltmeter coaxial connector and seven-pin control connector from the data acquisition system, the detector cable to the data acquisition system, the twin-pin connector for the high voltage shield cable, and the dc voltage input and sense connectors. The dc voltmeter lead may be plugged into either the dc input or sense connector, and a ground strap may be run from the dc input low side to the ground terminal on the ac supply.

\section{Initial Equipment Setup}

4. Turn on all the equipment in the two racks. The ac line switches for this equipment are on the front panels of the individual units. Exceptions to this instruction are the ac and dc calibrators, which should remain in standby mode instead of being shut off after use (to keep them warm), and the computer, which is turned on at the initial program load.

5. For the initial equipment setup, connect the test and standard units in parallel through a coaxial tee structure, with the voltage input at the free leg of the tee. There are several variations in the connection scheme, most notably the inclusion of a protection device in series with the units under test, and a voltage dropping resistor, also in series with the test units. The first case usually takes the form of a pair of back-to-back Zener diodes in parallel with the units under test and an expendable thermal element in series with the combination. The diode voltages are chosen to be about twice the applied voltage. When a large 
overvoltage occurs, the diodes conduct, blowing out the thermal element serving as a fuse, and protecting the test units from being overranged. In the case where the calibration voltage is less than ten volts, a series voltage-dropping resistor may be added to the circuit to force the supplies to run at a higher voltage range where they are generally more stable. The voltage drop due to the series resistor and/or the protection is accounted for in the software.

6. Connect the detector leads to the output connectors on the thermal elements. The detector connectors are polarized to accommodate NBS standards which, naturally, are of the opposite polarity to practically everyone else's. In the future, this polarity difference will be corrected for in the software; for the present, a polarity changing cable needs to be inserted in series between the TE output and the detector lead.

7. Set the switches on the relay box front panel. Initially, the output on/off switch should be set to 'OFF' (until the voltage is applied), the detector switch is irrelevent, the ac selection switch should be set to either 'HI' or 'LOW', depending on the applied voltage, and the mode switch set to 'AUTO'. If the Fluke 5215A high voltage power amplifier will be used, disconnect the input and sense leads from the Fluke $5200 \mathrm{~A}$ ac source at the front of the relay panel. This will eliminate potentially troublsome grounding problems.

8. For test voltages of 60 volts or greater, the high voltage safety shield must be set up. Since the shield is interlocked to the high voltage relays (always used at such voltages), failure to set up the shield will prevent the relays from closing. To install the shield, simply align the cutout in the end of the shield with a matching cutout in the side unit and press the coaxial phone plugs into their respective jacks.

\section{Initial Computer setup}

9. Like most desktop computers, the series 200 and 300 computers must be booted (the operating system must be loaded) from disc before use. The initial program loading utility for the new automated system includes both the bootable system and the ac-dc calibration supervisor, so that all the necessary software is loaded in the beginning of a session without operator intervention. The software is designed with the idea that, once in the calibration supervisor program, the operator need not exit the package to perform any function. This is not only a more elegant approach than having to load all the utility programs as needed, but a 
much more efficient method when confronted with the outrageously slow access time of the Model $82901 \mathrm{M}$ disc drive used on the 9816 computer system.

Note: The following instructions assume that the software being used is on either 5.25 inch or 3.5 inch flexible discs. The operating system and supervisory routines may also be on a 40 Mbyte winchester hard disc system. In the latter case, the system will boot from the winchester drive and no flexible discs will be used.

10. To load the ac-dc package, insert the flexible disc marked SYSTEM in drive 0 and the disc marked BINARY FILE LOADER in drive 1. On the 9836 computer drive 0 is the internal drive on the right hand side, drive 1 is the left; the 9816 uses external drives (models $82901 \mathrm{M}$ and 9122D) which have the opposite convention (nothing like standard ization!). After inserting the discs, turn on the computer system. For the 9836, the power switch is at the lower right edge of the cabinet; on the 9816, the power for either disc drive is on the front panel, and the power switch for the computer is in the back of the cabinet. The power switches for the series 300 computers (model 310) is on the left front of the CPU box and the left front of the monitor.

11. Upon powerup, the computer will load the BASIC 5.0 operating system, some necessary binary programs and data files, and the ac-dc calibration supervisor. If the date and time have not been set, then you will be queried to enter the correct date and time. At the completion of the boot, a command option menu will be displayed with which the user can select the operation of interest. These option are as follows:

\section{SETUP I - INSTRUMENT CALIBRATION MENU}

le;KO: FLUKE 540B: Allows calibration of Fluke 54OB multirange transfer standards. le;K1: HOLT MODEL 11: Allows calibration of Holt Model 11 coaxial transfer standards.

i) K2: HOLT MODEL 6A: Allows calibration of Holt Model $6 \mathrm{~A}$ multirange transfer standards.

ii) K3: GUILDLINE 7100: Allows calibration of Guildline 7100A multijunction transfer standards.

iii) K4: BALLANTINE 1600: Allows calibration of

iv) K5: BALLANTINE 1605: Allows calibration of Ballantine 1605A automatic multirange transfer standards.

v) K6: NBS COAXIAL TYPE: Allows calibration of coaxial single-range units similar to the NBS F7 set, such as the Julie TNB-102, Ballantine 1398A, and old Hermach-Engelhard transfer sets. 
viii) K9: MENU $\rightarrow:$ Go to setup menu II. For the instrument calibration menu listed above, pressing the SHIFT key down simultaneously with the desired function key will cause the system to calibrate an instrument using a previously defined standard calibration file. Pressing the function key alone allows the operator to input all the necessary test parameters.

\section{SETUP II - UTILITIES MENU}

i) KO: GRAPH: Uses the series 200/300 graphics capabilities to plot such relationships as the thermal element response curve, the ac-dc difference versus frequency of a thermal converter, etc.

ii) KI: SYSTEM STATUS: Displays the current system status.

iii) K2: HELP: Accesses the ac-dc supervisory help facility.

iv) K3: VAX: Boots the series 200/300 terminal emulator package to allow data communication with the Electricity Division's DEC VAX 11/750.

v) K4: STD CAL FILE: Permits the creation and modification of standard calibration files.

vi) K5: STAND. CORR.: Creates and modifies the data file containing the coefficients of the characteristic curve for each of the NBS standards, as well as their ac-dc difference corrections.

vii) K6: REPORT: Accesses the automated reporting facility.

viii) K7: AMP. BOARD: Accesses a utility to measure the amplifier gains and integration constants of the amplifier board.

ix) K8: EXIT: Exits the ac-dc package and returns processing to the BASIC 5.0 system command level.

x) K9: <- MENU: Go to setup menu $I$.

12. Upon pressing the appropriate function key, you will be instructed to insert the proper supervisory disc in drive 0 , and, in the case of the instrument calibrations, a data storage disc in drive 1 . When processing is continued, the system checks to see that the proper disc is in drive 0 , warns you if it's not, and gives you another try. If the proper disc is loaded, the system loads the appropriate supervisory program and processing resumes with that subroutine. All of the options in the setup menus, with the exception of 'SYSTEM STATUS' are separate subroutines on different discs, and are documented as such. For the instructions on a particular option, please see the appropriate section of this documentation. If the mass storage device is a Winchester drive, the data file will be placed on an appropriate volume (section of the 
disc), and the calibration master files will be read from the system volume. In this case, you will not need to change floppy discs.

13. At the end of a session, pressing the 'EXIT' key (either setup menu $I I, K 8$ ) will return processing to the BASIC 5.0 system. If you want to run the ac-dc package again, insert the BINARY FILE disc in drive 0 , and press $\mathrm{K} 5$ ('SCRATCH') on the system function key menu. Then press K6 ('LOAD " ') and enter ACDCMSTR, followed by a double quote. After the ac-dc supervisory program is loaded (the light on the drive goes out and the drive quits making noise), press the RUN key. The master program will run as described above, except that the date is already known, and the session will proceed with the initial setup menu. If the ac-dc master program is still in memory, simply insert the BINARY FILE disc in drive 0 and press the RUN key. Follow these guidlines for systems with a winchester drive, without, of course, inserting floppy discs. 
1. The system status utility (SYSTAT) may be called from any of the supervisory routines in the ac-dc software package. SYSTAT displays the present system status and allows you to modify the characteristics of the computer, serial port, and IEEE-488 bus.

2. When the package is first booted, SYSTAT is called and accesses a data file containing the last definitions of the IEEE-488 bus structure. When the status utility is initiated by a function key interrupt, the utility queries the computer system for information, and displays this along with the data read in from the IEEE-488 bus structure data file.

3. The status display is divided into three sections: the computer status, IEEE-488 bus status, and serial interface status as follows:

i) COMPUTER STATUS:

a) BASIC version: The currently loaded version of Hewlett- Packard BASIC.

b) Avaliable memory: The memory space currently available to the program.

c) Mass storage device: The present default mass storage device.

d) Printer interface: The present "PRINTER IS" device.

e) Plotter interface: The present "PLOTTER IS" device.

ii) IEEE-488 BUS STATUS: Seven lines are available to display the bus addressable instruments which the system will recognize. These are displayed along with their addresses.

iii) SERIAL STATUS:

a) Serial address: The internal address of the serial interface in the series 200 computer.

b) Baud rate: The present transmit/receive speed of the serial port.

4. In addition to the display, a function key menu is displayed at the bottom of the screen. This menu allows you to modify some of the characteristics of the system as follows:

i) KO: MODIFY COMP.: This key allows you to change the default mass storage device identifier, and the present printer and plotter interface.

ii) K1: MODIFY 488: Use this key to modify the IEEE-488 bus structure. Up to seven instruments may be defined in the system. 
ii) K2: MODIFY SERIAL: Allows you to change the serial interface select code and to modify the transmit/receive speed.

iv) K3: WRITE TO FILE: Writes out the new IEEE-488 bus information to the system status data file.

v) K8: SYSTAT EXIT: Returns processing to the calling subroutine.

5. When any of the modification keys are pressed, an arrow $(->)$ is displayed to the left of the section undergoing modification, and the system directs you to select the field to modify. This is done by pressing the SHIFT key and rotating the knob, causing the arrow to move vertically in the left hand column. When the arrow points to the field to modify, pressing the ENTER key will display a prompt into which you may type the pertinent information. When the IEEE-488 structure is modified, an additional prompt queries the operator for the address of the unit under modification. Please note that the instrument's function must be explicitly typed out: for example, a Fluke 5440A dc calibrator must have the string "DC CALIBRATOR" in the definition; the definition for a Hewlett-Packard 3497A data acquisition system must contain the string "DATA ACQUISITION SYSTEM", although it may be in lowercase text. This aids in defining variables used to output commands to the various bus instruments during a calibration. Also note that at the present time, no check is made to verify that the instruments defined by SYSTAT are actually connected to the bus. After the appropriate field is modified, the updated status is displayed and the function key menu returns. If the present IEEE-488 bus structure definitions need to be saved, pressing $\mathrm{K} 3$ (WRITE TO FILE) will save the present definitions in the file SYSTATDAT. Pressing K8 (SYSTAT EXIT) will return processing to the calling program.

6. When SYSTAT is called by a routine other than the ac-dc program supervisor (for example, the n-test routine), modifications are not permitted. The present system status is displayed, and you may press CONTINUE to exit SYSTAT and return to the calling program. 
1. Using an automated ac-dc comparator utilizing amplifiers and analog integrators entails knowing the amplifier gain factors and the time constants of the analog integrators. These quantities are usually measured by supplying a small voltage to each channel of the detector and working the relays in the Data Acquisition System to connect the voltage to be measured to a DVM. A stopwatch is used to clock the integration time of the analog integrators. From this method, it is apparant that many sources of error are present, from the instability of the voltage supply to the precision of the timing. The utility described here uses voltages supplied by an adjustable DAC to measure the gains and time constants, and the timing is controlled by a computer. In this way the error associated with the timing and sensitive adjustments to a potentiometer are avoided.

2. To enter the amplifier verification utility, press K7: 'GAIN CHECK' in the master calibration supervisory option menu. You will be instructed to insert the Utilities disc in drive 1 and press 'CONTINUE' to load the utility. If a Winchester drive is designated as the mass storage device, the utilities will be read from the system volume of the Winchester, and disc swapping is unnecessary.

3. After the amplifier verification utility is loaded, you will be instructed to short the inputs to the detector leads. This is done to check the offsets in the first-stage amplifiers and can be accomplished by inserting a shorting plug into the input connector of each detector lead. After the leads are shorted, press 'CONTINUE' to begin the verification routine.

\section{Measurement of amplifier Board Characteristics}

4. The verification routine begins by presenting a display composed of three windows. These windows and their functions are described below:

i) Message window: This window displays the current action (checking offsets, discharge voltage, etc.) being taken by the routine.

ii) Summary window: This displays a running summary of the pertinent information taken by the computer.

iii) First-stage amplifier window: This window displays data relating to the offsets and gains of the first-stage amplifier in each channel.

iv) Integrator window: This window displays the information relating to the constants associated with the analog integrators. As data is taken and 
reduced, the raw data and a summary of the data will be displayed in turn.

5. Actual checking of the amplifier board begins by measuring the offset of the first-stage amplifier in the standard channel. This is associated with the first-stage amplifiers and is generally about $0.5 \mathrm{mV}$. After getting the offset, the voltage needed to supply $1 \mathrm{mV}$ to the channel is calculated and applied to the DAC. The routine then disconnects the input to the amplifier from the DVM and connects the output of the first-stage amplifier. The system pauses to let the DVM settle and reads the output of the amplifier. The offset and measured amplifier gains are written out to the screen and the process is repeated twice more. After three determinations of this data are taken, the amplifier characteristics of the test channel are measured.

6. In order to check the analog integrators, a voltage of $1 \mathrm{~V}$ is needed at the input to the integrators. The standard channel DAC is adjusted until $1 \mathrm{~V}$ is measured at the output of the first-stage amplifier. The discharge relay is closed and the discharge voltage measured and displayed on the screen. The discharge voltage tends to be variable, but should be very small (tenths of millivolts). After reading the discharge voltage, the discharge relay is opened and the output of the first-stage amplifier connected to the integrators. Integration is timed for ten seconds and the resulting voltage read by the DVM. The integration is displayed on the DVM so you can check the integration rate. The integrator output and $1 / \mathrm{RC}$ time constant are displayed on the screen, and two more determinations are taken. The test channel is then measured in the same manner.

7. Note that, during these measurements, the status of the system is displayed in the message window.

8. After the integrator characteristics are checked, the constants for the n-test and calibration routines are calculated and displayed in the summary window.

9. Upon completion of the measurements, the previous date of board verification and the constants currently being used are presented in the message window for comparison with the new constants being displayed in the summary window. A function key menu is also presented, and the following options are allowed:

i) KO: PRINT SUMMARY: This key prints a summary of the verification measurements on the current hardcopy device.

ii) K1: SAVE CONSTANTS: Pressing this key writes the current n-test and calibration constants out to the system status data file. 

iii) K8: EXIT: Returns processing to the master
calibration supervisor.

10. If the newly measured n-test and calibration constants are to be saved in a data file, press KI: 'SAVE CONSTANTS'. You will be instructed to insert the Binary file loader disc in drive 1 and press 'CONTINUE' when ready. The new constants will be written out to SYSTATDAT, the system status data file, for use by the n-test and calibration routines. processing then returns to the option menu. With a Winchester drive, the new constants are written out to the system volume and you will not swap flexible discs.

11. Pressing K8: 'EXIT' returns processing to the command option menu of the master calibration supervisor. 
1. One of the considerable advantages of automated calibration systems is their ability to perform calibrations with instructions read from a data file on disc. For Automated System II, this is available as an option when an instrument calibration procedure is initiated.

2. The routine to create and edit a standard calibration data file is called "STDCALFILE" and is found on the Utilities disc. To access this utility, press function key $\mathrm{K} 4$ on setup menu II (Utilities) in the ac-dc program supervisor. You will then be instructed to insert the utilities disc in drive 0 and the target disc (one of the instrument calibration master discs) in drive 1. CONTINUE initiates the loading of the standard calibration file program and its execution. If a Winchester drive is designated as the mass storage device, the data will be written out to a file on he system volume of the drive and you will not swap discs.

3. Upon execution, STDCALFILE obtains a catalog of all files on the target disc with the first two characters $\mathrm{S}$; these denote standard calibration files. The catalog of these files is displayed along with a function key menu from which you may select a file operation. If more than one calibration file is found, you will be requested to type in the name of the standard calibration file to be accessed. If no files are found, the utility automatically assumes that a calibration procedure should be created and branches down to do this.

4. The top-level command option menu consists of the following options:

i) KO: CREATE FILE: This option creates a standard calibration procedure data file.

ii) KI: MODIFY FILE: This allows the user to modify an existing calibration procedure file.

iii) K2: CATALOG: Obtains a catalog of all the command procedures on the target disc.

iv) K3: DELETE FILE: Allows deletion of standard calibration files.

v) K4: NEW FILE: Deassigns the current data file and allows another data file to be edited without exiting the routine.

vi) K8: EXIT: Returns processing to the ac-dc master supervisor.

vii) K9: ABORT MODE: Used to abort the present processing mode and return control to the top-level menu. 
5. If a file is to be created (KO), the system will ask you to input a file name (up to eight characters) to be appended after the "S_", then notify you that the file will be created, and create the file on the target disc. The system then displays the standard test parameter input menu (the same one displayed during a calibration). The information that you supply will be written out to the standard calibration file. This process is repeated for all the voltage ranges to be calibrated, and is aborted by the 'ABORT MODE' function key (K9). Processing is then returned to the top-level option menu. During the data entry stage, the test parameters may be edited by using the function key menu presented after each voltage range is defined. This menu operates in the following manner:
i) K1-K6: ACCEPT PARAMETERS: These keys accept the information input to the screen menu.
ii) K3-K8: REJECT PARAMETERS: These keys reject the current input test parameters and presents a second function key menu which allows the operator to modify any field in the parameter menu.

6. If the test parameters need to be modified, the appropriate keys on the input modification function key menu are:

i) K2: STANDARD ID: Allows modifications of the NBS standard identification.

ii) K3: VOLTAGE: Allows the applied voltage to be edited.

iii) K4: TEST RANGE: Allows the test unit voltage range to be modified.

iv) K5: STANDARD RANGE: Allows editing of the standard voltage range.

v) K6: FREQUENCY: Modifies the frequencies defined for the standard calibration file.

vi) K9: ABORT MODE: Exits the command input mode and returns processing to the standard calibration file command menu. After you select the field to be modified, the input cursor will appear at the first character position of the field. You may then type in the new data, overwriting the previous, erroneous data. After the new data, are entered, the system presents the updated test parameters for you to accept or reject as before.

7. As each calibration point is accepted, the parameters are stored internally in memory and this operation is repeated until all the calibration points have been defined. After the final calibration point has been defined, press K9:'ABORT MODE' to return processing to the standard calibration routine command option menu. 
8. After K9: 'ABORT MODE' is pressed to exit the calibration point definition routine, the program once again presents the catalog of standard calibration files and the option menu. The option keys are described below.

9. If KO: 'CREATE FILE' is pressed, the program asks you to supply the necessary file name and processing continues as if no files were initially found. Again, K9: 'ABORT MODE' is used to return to the command menu.

10. To modify a standard calibration data file, press K1: 'MODIFY FILE'. The program will request the name of the standard file to be modified and will present an abbreviated listing of the file, showing the record number of each record, the applied voltage defined in that record, and the NBS standard TVC defined for that voltage. A function key menu will be displayed across the lower portion of the screen, with the following definitions:

i) KO: VIEW RECORD: Permits the display of all the information in a selected record.

ii) K1: ADD RECORD: Permits the addition of a record to the standard calibration data file.

iii) K2: CATALOG: Displays a catalog of all currently defined calibration data files on the disc.

iv) K3: MODIFY RECORD: Allows modification of a record in the data file.

v) K4: DELETE RECORD: Purges a record from the standard calibration file.

vi) K9: ABORT MODE: Returns processing to the calibration file command menu.

11. Pressing any function key in this menu which accesses a record results in an arrow $(->)$ being displayed to the left of the first record. To select the record of interest, move the arrow to that record by pressing the SHIFT key and rotating the knob clockwise to move the arrow down and counterclockwise to move the arrow up. When the arrow points to the record of interest, press the ENTER key to continue the record operation in the following manner.

12. When a record is being viewed, it is displayed in the same format that was used to input the record in the first place. After viewing the record, pressing K1-K6: 'ACCEPT PARAMETERS' returns processing to the record modification menu.

13. To add a record, press KI: 'ADD RECORD'. The usual template appears and you may enter the pertinent calibration data into the template in the normal manner. After the parameters are entered, the data is written out to file and processing is returned to the file modification menu. 
14. K2: 'CATALOG' obtains a listing of all standard calibration files on the disc, but returns processing to the master menu. To reaccess the file modification facility, K1: 'MODIFY FILE' must be pressed again.

15. To modify a record in the file, press K3: 'MODIFY RECORD'. The arrow is presented to the left of the currently defined records, and the record to be modified is chosen by using SHIFT-knob to place the arrow to the left of that record and pressing ENTER. The contents of the record are displayed in the usual fashion along with the 'ACCEPT PARAMETERS-REJECT PARAMETERS' function key menu. Pressing K1-K6: 'ACCEPT PARAMETERS' saves the record with no modification. Pressing K3-K8: 'REJECT PARAMETERS' results in a further function key menu with the following options:

i) KO: TEST NUMBER: and K1: INSTRUMENT ID: are used when defining an ac-dc difference test and are not used in this case. They are presented on this menu because the same menu is used for calibration definitions.

ii) K2: STANDARD ID: This key allows you to specify another NBS standard TVC.

iii) K3: VOLTAGE: Permits modification of the applied voltage for this determination.

iv) K4: TEST RANGE: Permits you to modify the voltage range of the test unit.

v) K5: STANDARD RANGE: Allows modification of the standard voltage range definition.

vi) K6: FREQUENCY: Permits the addition and modification of the frequencies defined for this test. Pressing any of these keys displays the block cursor at the first character position in the proper field. After you enter the data and press ENTER, the updated record will be displayed along with the function key menu. If any more modifications are required, press $\mathrm{K} 3-\mathrm{K} 8$ : 'REJECT PARAMETERS' and processing preceeds as just described. If the record update is complete, press K1-K6: 'ACCEPT PARAMETERS' to write out the updated information to the file and return to the file modification menu.

16. 'DELETE RECORD' is used to purge a particular standard test record from the file. After you press the function key, the arrow is displayed to the left of the list of records, and instructions on record deletion appear at the bottom of the screen. To delete a record, use SHIFT-knob to select the record to be deleted and press ENTER. The record is deleted from the file and the data file updated. THERE IS NO REDUNDANCY FOR THIS OPERATION. NO SECOND CHANCES ARE GIVEN IF THE WRONG RECORD IS SELECTED! 
17. Should a standard calibration file need to be deleted, press K3: 'DELETE FILE'. The program asks for the name of the calibration file to be deleted, and purges the file from the disc. AS WITH THE RECORD DELETION FACILITY, THERE IS NO REDUNDANCY FOR THIS OPERATION. The file is deleted and processing continues at the standard calibration file option menu.

18. If a new file is to be edited, press K4: 'NEW FILE'. This key closes the I/O channel to the present data file and returns processing to the catalog facility. If calibration files have been previously defined, a catalog of these files is displayed and the master menu appears. If no standard files are available, then processing continues as if the file should be created.

19. To exit the standard calibration file program, press K8: 'EXIT'. This returns processing to the ac-dc master calibration program command menu. 
1. An advantage of the new automated system is its ability to store the corrections to the standard thermal voltage and current converters in a file to be read by the system upon beginning calibration. In the past, one had to write a separate routine to accomplish the creation of these files, since the Hewlett-Packard computers are not set up to edit binary data files readily. The new software, however, allows for interactive creation and entry into such a data file without resorting to writing a separate routine.

2. To access the standard correction file utility, press K9: 'MENU'. This brings up the second-level utilities menu, which lists 'STAND. CORR' as K5. Press this key to access the standard correction file utility. You will be instructed to insert the utilities disc in drive 0 and the target disc in drive 1. After inserting these discs, press CONTINUE to load the standard correction file utility. If a Winchester drive is designated as the mass storage device, the data will be written out to the proper file on the system volume, and you will not swap discs.

3. After the subroutine loads, a function key menu will be displayed to allow you to select the operation desired. The keys are defined as follows:

i) KO: CREATE MATRIX: Assists in the creation of a new standard correction table.

ii) K1: TE REPLACEMENT: Allows the updating of the standard correction table should a thermal element of one of the standard units require replacement.

iii) K2: MODIFY V RANGE: This key allows you to change one or more ranges in the correction table; for example, if a range resistor should change value.

iv) K3: STANDARD CURVE: Allows modification of the $M$ and $N$ of one of the standards defined in the correction table.

v) K4: PRINT MATRIX: Prints the correction matrix to the screen or external hardcopy device.

vi) K5: GRAPH MATRIX: * Plots relationships such as the standard characteristic curve or the frequency-voltage response from the correction table.

vii) K6: SYSTEM STATUS: Displays the present system status.

viii) K8: EXIT: Exits the standard correction routine. Press the appropriate function key to choose the desired operation. NOTE: the options denoted by * are not operational yet (12/08/1987). 
4. Choosing KO: 'CREATE MATRIX' allows you to define a standard correction file from scratch. After the key is pressed, a heading is presented, and you are asked to enter the name of the standard correction file to be created. This file name can be up to eight characters long, to allow for the ' $C$ ' prefix which denotes a correction file.

5. After entering the name, you will be prompted to enter the number of rows (voltage ranges) and columns (cardinal frequencies) defined in the table, whereupon the program will again instruct you to insert the target disc in drive 1 (unless a winchester drive is available), and press CONTINUE. The data file will be created with the proper number of rows and columns.

6. Next, the program will prompt you for the frequencies to be defined as cardinal frequencies. The prompts will appear along the centerline of the screen together with the frequency number currently being input. Enter the frequencies in the blank provided beside the prompt. The frequency may be up to six characters long in either upper or lower case. NOTE, however, that each frequency MUST be identified as either $\mathrm{Hz}, \mathrm{kHz}$, or $\mathrm{MHz}$, so the calibration routine can select the correct frequency range. Since the program searches the frequencies entered for the letters $\mathrm{K}$ and $\mathrm{M}$ (in either upper or lower case), these suffixes are both necessary and sufficient to specify the frequency.

7. To permit information to be entered into the correction table, an input menu is displayed on the CRT with the heading STANDARD CORRECTION DEFINITION MENU. This menu is divided into the following fields:

i) Standard: The standard ID.

ii) M: The standard $M$ value.

iii) N: The value for the standard $\mathrm{N}$.

iv) Resistance (Ohms): The resistance of the series resistor in the TVC.

v) Voltage Range (V): The voltage range of the standard unit. The cardinal frequencies are listed vertically at the center of the screen with the blank fields beside them.

8. To enter the data, simply type in the information. You will be limited to the number of blanks in the fields; if this length is exceeded, only the $n$ characters represented by the blanks will be accepted. Pressing ENTER after each field will advance the cursor to the next field. Continuing in this manner, the input menu for each standard is defined in turn. Notice that, if a standard thermal element appears more than once in a correction table (for example, as part of a coaxial set), the $M$ and $N$ will be repeated in the proper fields automatically, to avoid having to type these values in again. 
9. After the correction table is filled, a routine to check the input data is run. This begins with a set of instructions to follow for checking the correction information. Pressing CONTINUE after reading these instructions begins the checking routine. After CONTINUE is pressed, the screen is cleared and the currently defined correction table displayed. Because the screen is too small to support the display of the $M$, $\mathrm{N}$, resistance, and voltage range for all the standards at once, these quantities are displayed on a single line at the bottom of the screen, along with the number of the current record. In the table itself, each standard unit is displayed vertically on the left-hand side with its defined ac-dc corrections listed horizontally to the right. The list of standards is followed by the line 'Exit to main menu' which allows you to return to the main option menu.

10. The data for the individual standards are available for editing by using the SHIFT-knob combination to move an arrow vertically up and down the left- hand column of the table. When the arrow is placed at a particular record, the $\mathrm{M}$ and $\mathrm{N}$ values, along with the resistance and voltage range of the standard unit, are displayed at the bottom of the screen. Pressing ENTER will allow you to edit the information in the record being indicated by the arrow. Choosing the option 'Exit to main menu' closes the correction file and returns processing to the main option menu.

11. When the data for a standard are to be edited, the original input menu is presented again for input. The information must be retyped (except for the $M$ and $N$ ) in the manner above. The $\mathrm{M}$ and $\mathrm{N}$ values are changed by another method described later.

12. The procedures described in steps 9-11 above are repeated until the matrix is correct. Choosing the 'Exit to main menu' option will close the correction file and return processing to the main option menu.

13. Occasionally, the ac-dc differences of one of the series resistors of a coaxial TVC set will change in value or the geometry of the resistors mounted in a multirange instrument will change, affecting the ac-dc differences of the unit. When this occurs, the correction table must be updated to reflect these changes. This is accomplished by pressing the K2: 'MODIFY $V$ RANGE' function key in the standard correction master menu. If no correction files have been defined on the target disc, then an error message is displayed and processing is returned to the master option menu. If only one file is found, it is assumed to be the file to be modified and processing proceeds at step 14 below. If more than one correction file exists on the disc, then a catalog of all the correction files on this disc is displayed, along with an arrow at the left-hand margin of the 
catalog. To choose the data file on which to operate, use SHIFT-knob to move the arrow vertically to the file to be modified and press ENTER. If none of the files defined on the current target disc are of interest, place the arrow beside the last entry in the table, labeled 'No file. Return to command menu'. This aborts the voltage range modification mode and returns processing to the option menu.

14. After selecting the file to be modified, the routine reads in the data from the disc and enters the CORRECTION TABLE CHECK MENU routine. This works as described in steps 9 and 10 above. After the desired standard ID is chosen by using SHIFT-knob, the data input menu is displayed. Since a change in the resistor of a TVC will not affect the characteristics of the thermal element itself, only the resistance (whose change may be the reason for the ac-dc difference change) and the new corrections need to be entered.

15. After the updated corrections for a particular range are entered, processing returns to the CORRECTION TABLE CHECK MENU, where another voltage range may be edited if necessary, as will probably be the case with a coaxial set. If another range needs to be modified, proceed in the same manner as in step 14. If no more changes are to be made, set the arrow to 'Exit to main menu'. The updated information will be written out to the data file, the file will be closed, and processing returned to the master command menu.

16. If the characteristics of a thermal element in a standard change, it will be necessary to update the $M$ and $\mathrm{N}$ for that unit. To do this in a previously defined data file, press K3: 'STANDARD CURVE' in the master command menu. As before, a catalog of standard correction files defined on the target disc will be presented, and the proper data file is opened by using SHIFT-knob to select the desired file.

17. After the data file to be updated is selected, the program will request the ID of the standard thermal element whose characteristics have changed. For a coaxial set, one thermal element may be used with two or more resistors, so the routine will search the correction table for each occurrence of the thermal element ID. For the NBS high frequency standards and commercial multirange units, the characteristics will be defined for one thermal element only. In any case, enter the ID of the thermal element to be modified at the prompt. You will then be prompted for its new $M$ and $\mathrm{N}$ values. After these values are entered, they are written out to disc and processing returns to the main command menu. 
18. Should a hardcopy of the correction table be required, press K4: 'PRINT MATRIX'. As usual, the program will display the catalog of all defined correction data files, and SHIFT-knob is used to pick the file to be printed. Once the file is read in from disc, the following menu is presented:

i) K1-K6: OUTPUT TO SCREEN: Directs the correction table information to the CRT.

ii) K3-K8: OUTPUT TO PRINTER: Directs the correction table information to the defined PRINTER Is device.

iii) K5: SYSTEM STATUS: Displays the present system status.

iv) K9: EXIT: Exits the 'PRINT MATRIX' routine and returns processing to the main command menu.

19. If the output should be directed to the CRT, press KI or K6. The currently defined corrections will be displayed on the $\mathrm{CRT}$, along with the instruction to press CONTINUE after viewing the matrix. CONTINUE returns the program to the 'PRINT MATRIX' menu. To direct the output to the current PRINTER IS device, press K3 or K8. This writes the output to the printer and returns processing to the 'PRINT MATRIX' menu. Pressing K9: 'EXIT' brings the program back to the master command menu.

20. To return to the ac-dc program master menu, press $\mathrm{k} 8$ : 'EXIT' in the correction file command menu. Processing will return to the master command menu and you may continue with calibrations, utilities, and other options.

21. When a thermal element is replaced (due to failure or overloading), the corrections for the standard units using that particular thermal element will be altered, particularly on the low voltage ranges where the contribution from the resistor is relatively small. In the past, a change of a thermal element meant a complete redetermination of the corrections for that thermal element. However, Hermach (1984) has devised a mathematical relationship linking the ac-dc differences of the newly replaced thermal element with its previous corrections. This process has been applied to the software for the new automated system.

22. To enter the replacement routine, press $\mathrm{K} 1$ : 'TE REPLACEMENT', in the main command option menu. This will load and execute the $\mathrm{TE}$ replacement subroutine. The subroutine begins by obtaining a catalog of all the correction files on the target disc and prompting you to select the file to be modified, as in the instructions above. You will then be prompted to enter the ID of the replaced thermal element. The program will scan the data in memory to ensure that the present correction table specifies this thermal element. If it does not, you will be given the choice of accessing a new data file or exiting the routine. 
23. To update the correction table, the thermal element must be checked both as a voltage converter and as a current converter. The program will prompt you to enter the new ac-dc differences as a voltage converter at the cardinal frequencies defined in the correction table. Next, you will be asked for the voltage range at which the thermal element was checked as a current converter and its ac-dc differences measured in this configuration. Upon entry of these data, the Hermach approach will be applied to each voltage range of the standard thermal element and a new correction table calculated.

24. The updated correction table will be written out to the target disc and will also be available to be viewed and printed as in steps 18 and 19 above. Processing then returns to the main option menu. 
1. This section covers the generic instructions for calibrating an Abbey Electronics Model TE 20 thermal transfer standard using "AC_DC_MSTR". The same calibration routine, with some minor variations, that is used to calibrate a Ballantine 1605 is used to test this instrument. It is assumed that the program is loaded and initialized per the instructions in section $I$, and that your last action was to press the 'BALL. 1605A' option on the instrument calibration menu.

2. Following the instructions on the screen, insert the flexible disc marked "BALLANTINE 1605A" in drive 0 and a calibration data disc in drive 1. The Ballantine 1605 calibration disc contains the necessary routines to do a complete calibration of this instrument. The data generated by the program will be written out to the disc in drive 1. Press CONTINUE to load the 1605 calibration supervisory program. If a Winchester drive is designated as the mass storage unit, then the data will be written out to the proper volume on the drive and no disc swapping is necessary.

3. The program will ask for the test number of the present test, and an identification of the unit under test. The program will attempt to find a previously defined data file (D_nnnnnnnn, where nnnnnnnn is the test number) for this test. If a data file has not been created, or if the one it finds is empty, the program will ask you if an n-test should be performed. If you answer with with a "Y", a data file is created, the test number and identification are written out to the file, and the n-test routine is loaded and executed. If you answer with an "N", then processing continues at the 1605 command menu, and you will be requested to supply the proper test $\mathrm{M}$ and $\mathrm{N}$ during the calibration. If a data file already exists, an option menu will be displayed. This will be covered in more detail later.

4. To calibrate an Abbey TE-20, first remove the mercury cell in the instrument case. To do this, remove the screws holding the front panel to the case. The front panel slides out of the casing, exposing the battery holder and mercury cell. Remove the cell and replace the front cover. The output EMF of the TE-20 is read through a three pin Amphenol connector which, with pins $A$ and $B$ used, is incompatable with the three pin adapter made for the Ballantine 1398A coaxial units, and which renders useless the normal method of using the analog integrators. Instead, the output EMF is measured with a nanovoltmeter, such as a Keithley 181. Use an Amphenol three pin female connector to get onto the output connector, and an appropriate cable to go to the millivolt input connector of the nanovoltmeter. Make sure the power switch of the TE-20 is on and that the 
appropriate plugin resistor is employed. For an n-test, the resistor should select the 100 volt range. Make sure the resistor is in the correct orientation, usually with the range marking reading up.

5. The n-test routine will load 11 subroutines from disc and set the default parameters. These are displayed on the CRT along with a function key menu used to modify these defaults. The options available are:

i) KO: STARTING VOLTS: The initial voltage for the n-test (default is 70 volts).

ii) K1: HIGHEST VOLTS: The final voltage for the n-test (defaults to 100 volts).

iii) K2: VOLTAGE STEP: The step between $V$ and $d V$. This is defined as either a percentage of the currently applied voltage, a percentage of the final voltage, or a flat step. The default is . $1 \%$ of the presently applied voltage.

iv) K3: VOLTAGE INC.: The increment between successive nominal levels of applied voltage (default is 5V).

v) K4: EXECUTE: Starts the n-test procedure.

vi) K5: PRINTER OFF: Disables printouts of each individual voltage step (default is printer on).

vii) K6: HEADINGS OFF: Disables headings from being printed on the hardcopy device (default is headings on).

viii) K7: DEGREE OF FIT: Allows the characteristic response equation to be fitted to any degree, although a linear fit is generally good enough and higher orders are not yet supported anyway.

ix) K8: EXIT: Returns processing to the Ballantine 1605 supervisory program.

6. If any changes to the default parameters are desired, press the function key associated with that parameter. A prompt will be displayed, and the value may be entered in the blank space at the prompt line. The option menu will then be redisplayed to request the next operation.

7. When the 'GO' key is pressed, the IEEE-488 instruments needed for an n-test (dc supply, dc voltmeter, and data acquisition system) are initialized to the required conditions.The screen will be erased, and a display, divided into five "windows", will be painted to the CRT. From the top, these windows display the following:

i) The test number, date, and test unit identification.

ii) The present voltage, output EMF, initial and final nulled output EMF, and the incremental nulled EMF.

iii) The balancing process for the standard channel DAC. Note that the 1605 output EMF is measured using an external voltmeter, such as a Keithley 181, and no balancing of the DAC is necessary. No activity will be displayed in the DAC balance window; it it a vestige of other calibration routines. 
iv) The applied voltage, output $\operatorname{EMF} \mathrm{n}$, and the standard deviation of the previous points in the n-test.

v) Finally, the fitted characteristic response equation. When the voltage is brought up on the dc calibrator, set the output switch on the front panel to the 'ON' position. The system will wait for things to settle, and during this time the output voltage may be monitored with the dc voltmeter.

8. As the n-test routine runs, the values read from the nanovoltmeter as well as the present test parameters, are displayed in the appropriate places in the realtime display. This feature is a nice advance over the older software, in which test data tended to scroll upwards and off the screen.

9. In the course of the n-test, the applied voltages are set automatically, and the data collected and reduced with no operator intervention. If a problem does occur, however, there are two procedures to avoid (or at least limit) any possible damage to the system and test units. In the first case, the computer monitors the status byte of the dc supply and its output voltage (via the dc voltmeter), before each reading. If the voltage exceeds the nominal applied voltage, or if the dc calibrator self-destructs, the computer will open the output relay to disconnect the output voltage from the test unit. For all system errors, an error message, with possible remedial action and a prompt for instructions is displayed across the bottom of the screen. Since the display line is limited to 80 characters, the message scrolls in from right to left. In most cases, you will be given a choice of continuing the measurement process or exiting the routine.

10. In the second case, for serious "total disaster" types of faults, function key K9, labeled 'HARD KILI' will immediately reset all supplies, open the output relay, and return processing to the supervisory level option menu. THIS KEY MAY BE USED AT ANY TIME IN THE PROCESSING! For faults which are not so dramatic, pressing SHIFT-K9: (K19) 'SOFT KILL' halts processing but leaves the supplies and data acquisition system unaffected. Processing continues at the n-test command menu.

11. After the n-test is complete, processing will return to the supervisory option menu for further choices by the operator. In addition, the n-test data will be written to the appropriate data file on disc (drive 1 or the appropriate winchester volume), and the results will, if desired, be printed to the current hardcopy device. automatically. 
12. If a data file for this test number already exists, you may run another n-test, or choose one of the other options. These options include the following:

i) K0: N-TEST: This option initiates an n-test as described above.

ii) K1: AC-DC DIFF.: Initiates an ac-dc calibration of the test unit.

iii) K2: SYSTEM STATUS: Displays the present system status.

iv) K3: HELP: Invokes the Ballantine 1605 help facility.

v) K8: EXIT: Exits the routine and returns to the ac-dc master program supervisor.

vi) K9: HARD KILL: THIS KEY WILL RESET THE VOLTAGE SUPPLIES AND OPEN THE OUTPUT RELAY TO DISCONNECT THE VOLTAGE FROM THE UNITS UNDER TEST.

vii) K19: SOFT KILL: Pressing the SHIFT key and K9 stops processing but leaves the supplies and data acquisition system unaffected. If the shift key is pressed simultaneously with the n-test or ac-dc difference keys (SHIFT-KO or SHIFT-KI), then the system will automatically reset the voltage supplies, open the output relay upon completion of the sequence, and return processing to the Ballantine 1605 command option menu. If the SHIFT key is not pressed, however, processing will be halted upon completion of the test sequence, the supplies will remain active, the output on, and another option menu will be displayed.

13. The next logical step is to run an ac-dc calibration, and this is initiated by pressing $\mathrm{Kl}$. Nineteen subroutines will be loaded from the supervisory disc, and several variable initializations and file assignments made. If, in the original supervisory option menu, the SHIFT key was pressed together with the 'BALLANTINE $1605^{\prime} \mathrm{key}$, the system reads the command procedure from the disc. If the 'BALLANTINE 1605' key was pressed alone, however, an input menu, requesting the standard unit, applied voltage, standard range, test range, and desired frequencies is displayed, and the parameters for the test are entered at this time. When the output voltages are set on the ac and $\mathrm{dc}$ calibrators, position the output switch to ' $\mathrm{ON}$ ' and check the output voltages on the ac and dc voltmeters.

14. If the calibration is to be made from a standard calibration file, the system will now pause to let the units under test warm up. The length of time allowed for this warmup is range-dependent and varies from five minutes at voltages of less than $60 \mathrm{~V}$ to 45 minutes for voltages of $600 \mathrm{~V}$ and more. If the test is to be done manually, a warmup menu is displayed to allow you to select the proper warmup time as follows: 
i) K1-K6: CONTINUE WITH MEASUREMENTS: starts the measurement sequence.

ii) K2-K7: START SEQUENCE: If no automatic warmup is desired, this functions in the same manner as K1-K6. If, however, the system is currently in the midst of an automatic warmup period, this action aborts the warmup and starts the sequence.

iii) K3-K8: AUTOMATIC WARMUP: When pressed, this action starts the automatic warmup period for the present voltage range.

iv) K5: SYSTEM STATUS: Displays the present system status.

v) K9: HARD KILL: ABORTS ALL PROCESSING, RESETS THE SUPPLIES, AND RETURNS PROCESSING TO THE BALLANTINE 1605 COMMAND OPTION MENU.

vi) K19: SOFT KILL: Stops processing but leaves the supplies and data acquisition system unaffected.

15. In any case, processing resumes with the system reading the EMFs from both the test and standard channel. If no output is measured, the system presents an error message and suggests corrective action. You will then be instructed as to the position in which to set the detector switch on the front panel. This action will ensure that the output EMF is divided down to a level where the standard channel DAC can work without being under- or over ranged. Press CONTINUE, when the switch is set, to continue processing.

16. After processing is continued, the program will paint a realtime display composed of the following five windows (from the top):

i) The test number and instrument identification.

ii) Present test data (the ac-dc difference and dc reversal data).

iii) A summary of the ac-dc differences for this set of four determinations.

iv) A presentation of the test parameters (standard unit, output EMFs, currently applied voltage and frequency, etc.).

v) Previous results for all frequencies at this voltage.

17. If all goes normally, the system will test all frequencies at the present applied voltage without operator intervention. The system will monitor the status bytes of the ac and dc supplies, as well as their output voltages (via the voltmeters) and will reset the system if either output voltage exceeds the present nominal voltage range. Any system faults, including output voltages out of range, equipment faults, and so forth, will be presented on the bottom of the display and scrolled from right to left. These error messages usually include several options (exit to the command 
menu, continue with the sequence) as well as suggestions for remedial action on your part. As always, function key K9: 'HARD KILL' will disconnect the output voltage from the units under test, reset the supplies, and return processing to the Ballantine 1605 command option menu. Pressing SHIFT-K9: 'SOFT KILL' will halt processing but leave the supplies and data acquistion system unaffected.

18. During the calibration sequence, the data gathered from the data acquisition system and nanovoltmeter will be displayed in realtime. At the conclusion of each frequency, the ac-dc difference results for that frequency will be sent to the currently active hardcopy device.

19. If the system is running from a standard calibration file, at the completion of each voltage range you will be prompted to reconfigure the test and standard units for the next range. Upon pressing ' $C$ ', processing will continue at the next voltage range, with the automatic warmup facility in effect.

20. When the automatic calibration test sequence is complete, or if the automatic shutdown option is in effect, at the end of the final frequency on the present range, the system will reset the supplies, open the output relay, and return processing to the Ballantine 1605 command menu. If manual shutdown has been specified, the following menu is displayed:

i) Ko: REPEAT VOLTAGE: This key repeats all frequencies for the present applied voltage.

ii) KI: REPEAT FILE: This key will force the system to repeat the previous standard calibration file.

iii) K2: INSERT NOTE: Use this key to insert a note on the hardcopy. The note may be up to 320 characters in length.

iv) K3: FREQUENCY: This key allows the definition of up to eight new frequencies to be calibrated at the currently applied voltage. The frequencies are input into the usual test parameter input menu.

v) K4: NEW VOLTAGE: This key presents the test parameter input menu and allows the definition of a new voltage range and frequencies. The supplies and data acquisition system will be reset when this option is chosen, and will be initialized to the new test parameters.

vi) K8: EXIT: Returns processing to the Ballantine 1605 command option menu.

21. In addition to the prompts and options described above, on occasion the system may need some information that has not been previously defined. The four most notable occasions are: 
i) The standard $M$ and $N$ have not been defined in a data file.

ii) No n-test was run on the test unit and therefore no test $\mathrm{M}$ and $\mathrm{N}$ have been defined.

iii) The ac-dc corrections for the standard unit have not been defined in a data file.

iv) A series voltage-dropping resistor is being used to make the ac and dc supplies operate at a higher voltage where they tend to be more stable. In any of these cases, the system will prompt you for the proper information and continue the measurement process normally.

22. Upon exiting the n-test or calibration routine and returning to the Ballantine 1605 command option menu, the function keys defined in this menu may be used to restart a test or to exit from the 1605 calibration supervisor. If the latter action is taken, the Ballantine 1605 subroutines are deleted from memory and processing is returned to the ac-dc master program option menu. From there, pressing any key will take the action described by that key, and pressing 'EXIT' will exit the program and return processing to the BASIC 5.0 system. 
1. This section covers the generic instructions for calibrating a Ballantine 1605A automated multirange thermal transfer standard using "AC_DC_MSTR". It is assumed that the program is loaded and initialized per the instructions in section $I$, and that your last action was to press the 'BALL. 1605A' option on the instrument calibration menu.

2. Following the instructions on the screen, insert the flexible disc marked "BALLANTINE 1605A" in drive 0 and a calibration data disc in drive 1. The Ballantine 1605 calibration disc contains the necessary routines to do a complete calibration of this instrument. The data generated by the program will be written out to the disc in drive 1. Press CONTINUE to load the 1605 calibration supervisory program. If a winchester drive has been designated as the mass storage device, the data file will be written to the appropriate volume, and this disc swapping is unnecessary.

3. The program will ask for the test number of the present test, and an identification of the unit under test. The program will attempt to find a previously defined data file (D_nnnnnnnn, where nnnnnnnn is the test number) for this test. If a data file has not been created, or if the one it finds is empty, the program will ask you if an n-test should be performed. If you answer with with a "Y", a data file is created, the test number and identification are written out to the file, and the n-test routine is loaded and executed. If you answer with an "N", then processing continues at the 1605 command menu, and you will be requested to supply the proper test $M$ and $N$ during the calibration. If $a$ data file already exists, an option menu will be displayed. This will be covered in more detail later.

4. To perform an n-test on a Ballantine 1605A, the instrument must be set to the 64-125 volt range, and the mode switch must be placed in the 'Manual Xfer' position. The input voltage is applied through the 'Hi Input' connector. Since the 1605 has a high output impedance, the EMF cannot be measured using the analog integrators in the normal manner. Instead, the output EMF is measured with a nanovoltmeter, such as a Keithley 181. Use a double banana-to-twin Amphenol adapter to connect the double banana output jacks to the millivolt input connector of the nanovoltmeter.

5. The n-test routine will load 11 subroutines from disc and set the default parameters. These are displayed on the CRT along with a function key menu used to modify these defaults. The options available are: 
i) Ko: STARTING VOLTS: The initial voltage for the n-test (default is 95 volts).

ii) KI: HIGHEST VOLTS: The final voltage for the n-test (defaults to 125 volts).

iii) K2: VOLTAGE STEP: The step between $V$ and $d V$. This is defined as either a percentage of the currently applied voltage, a percentage of the final voltage, or a flat step. The default is .1\% of the presently applied voltage.

iv) K3: VOLTAGE INC.: The increment between successive nominal levels of applied voltage (default is $5 \mathrm{~V}$ ).

v) K4: EXECUTE: Starts the n-test procedure.

vi) K5: PRINTER OFF: Disables printouts of each individual voltage step (default is printer on).

vii) K6: HEADINGS OFF: Disables headings from being printed on the hardcopy device (default is headings on).

viii) K7: DEGREE OF FIT: Allows the characteristic response equation to be fitted to any degree, although a linear fit is generally good enough and higher orders are not yet supported anyway.

ix) K8: EXIT: Returns processing to the Ballantine 1605 supervisory program.

6. If any changes to the default parameters are desired, press the function key associated with that parameter. A prompt will be displayed, and the value may be entered in the blank space at the prompt line. The option menu will then be redisplayed to request the next operation.

7. When the 'GO' key is pressed, the IEEE-488 instruments needed for an n-test (dc supply, dc voltmeter, and data acquisition system) are initialized to the required conditions.The screen will be erased, and a display, divided into five "windows", will be painted to the CRT. From the top, these windows display the following:

i) The test number, date, and test unit identification.

ii) The present voltage, output EMF, initial and final nulled output EMF, and the incremental nulled EMF.

iii) The balancing process for the standard channel DAC. Note that the 1605 output EMF is measured using an external voltmeter, such as a Keithley 181, and no balancing of the DAC is necessary. No activity will be displayed in the DAC balance window; it it a vestige of other calibration routines.

iv) The applied voltage, output EMF, $n$, and the standard deviation of the previous points in the n-test.

v) Finally, the fitted characteristic response equation. When the voltage is brought up on the dc calibrator, set the output switch on the front panel to the 'ON' position. The system will wait for things to settle, and during this time the output voltage may be monitored with the dc voltmeter. 
8. As the n-test routine runs, the values read from the nanovoltmeter as well as the present test parameters, are displayed in the appropriate places in the realtime display. This feature is a nice advance over the older software, in which test data tended to scroll upwards and off the screen.

9. In the course of the n-test, the applied voltages are set automatically, and the data collected and reduced with no operator intervention. If a problem does occur, however, there are two procedures to avoid (or at least limit) any possible damage to the system and test units. In the first case, the computer monitors the status byte of the dc supply and its output voltage (via the dc voltmeter), before each reading. If the voltage exceeds the nominal applied voltage, or if the dc calibrator self-destructs, the computer will open the output relay to disconnect the output voltage from the test unit. For all system errors, an error message, with possible remedial action and a prompt for instructions is displayed across the bottom of the screen. Since the display line is limited to 80 characters, the message scrolls in from right to left. In most cases, you will be given a choice of continuing the measurement process or exiting the routine.

10. In the second case, for serious "total disaster" types of faults, function key K9, labeled 'HARD KILL' will immediately reset all supplies, open the output relay, and return processing to the supervisory level option menu. THIS KEY MAY BE USED AT ANY TIME IN THE PROCESSING! For faults which are not so dramatic, pressing SHIFT-K9: (K19) 'SOFT KILL' halts processing but leaves the supplies and data acquisition system unaffected. Processing continues at the n-test command menu.

11. After the $n$-test is complete, processing will return to the supervisory option menu for further choices by the operator. In addition, the n-test data will be written to the appropriate data file on disc (drive 1 or the proper winchester volume), and the results will, if desired, be printed to the current hardcopy device. automatically.

12. If a data file for this test number already exists, you may run another n-test, or choose one of the other options. These options include the following:

i) KO: N-TEST: This option initiates an n-test as described above.

ii) KI: AC-DC DIFF.: Initiates an ac-dc calibration of the test unit.

iii) K2: SYSTEM STATUS: Displays the present system status. 
iv) K3: HELP: Invokes the Ballantine 1605 help facility.

v) K8: EXIT: Exits the routine and returns to the ac-dc master program supervisor.

vi) K9: HARD KILL: THIS KEY WILL RESET THE VOLTAGE SUPPLIES AND OPEN THE OUTPUT RELAY TO DISCONNECT THE VOLTAGE FROM THE UNITS UNDER TEST.

vii) K19: SOFT KILL: Pressing the SHIFT key and K9 stops processing but leaves the supplies and data acquisition system unaffected. If the shift key is pressed simultaneously with the n-test or ac-dc difference keys (SHIFT-Ko or SHIFT-K1), then the system will automatically reset the voltage supplies, open the output relay upon completion of the sequence, and return processing to the Ballantine 1605 command option menu. If the SHIFT key is not pressed, however, processing will be halted upon completion of the test sequence, the supplies will remain active, the output on, and another option menu will be displayed.

13. The next logical step is to run an ac-dc calibration, and this is initiated by pressing K1. Nineteen subroutines will be loaded from the supervisory disc, and several variable initializations and file assignments made. If, in the original supervisory option menu, the SHIFT key was pressed together with the 'BALLANTINE 1605' key, the system reads the command procedure from the disc. If the 'BALLANTINE 1605' key was pressed alone, however, an input menu, requesting the standard unit, applied voltage, standard range, test range, and desired frequencies is displayed, and the parameters for the test are entered at this time. When the output voltages are set on the ac and dc calibrators, position the output switch to 'ON' and check the output voltages on the ac and dc voltmeters.

14. If the calibration is to be made from a standard calibration file, the system will now pause to let the units under test warm up. The length of time allowed for this warmup is range-dependent and varies from five minutes at voltages of less than $60 \mathrm{~V}$ to 45 minutes for voltages of $600 \mathrm{~V}$ and more. If the test is to be done manually, a warmup menu is displayed to allow you to select the proper warmup time as follows:

i) K1-K6: CONTINUE WITH MEASUREMENTS: starts the measurement sequence.

ii) K2-K7: START SEQUENCE: If no automatic warmup is desired, this functions in the same manner as K1-K6. If, however, the system is currently in the midst of an automatic warmup period, this action aborts the warmup and starts the sequence.

iii) K3-K8: AUTOMATIC WARMUP: When pressed, this action starts the automatic warmup period for the present voltage range. 
iv) K5: SYSTEM STATUS: Displays the present system status.

v) K9: HARD KILL: ABORTS ALL PROCESSING, RESETS THE SUPPLIES, AND RETURNS PROCESSING TO THE BALLANTINE 1605 COMMAND OPTION MENU.

vi) K19: SOFT KILL: Stops processing but leaves the supplies and data acquisition system unaffected.

15. In any case, processing resumes with the system reading the EMFs from both the test and standard channel. If no output is measured, the system presents an error message and suggests corrective action. You will then be instructed as to the position in which to set the detector switch on the front panel. This action will ensure that the output EMF is divided down to a level where the standard channel DAC can work without being under- or over ranged. Press CONTINUE, when the switch is set, to continue processing.

16. After processing is continued, the program will paint a realtime display composed of the following five windows (from the top):

i) The test number and instrument identification.

ii) Present test data (the ac-dc difference and dc reversal data).

iii) A summary of the ac-dc differences for this set of four determinations.

iv) A presentation of the test parameters (standard unit, output EMFs, currently applied voltage and frequency, etc.).

v) Previous results for all frequencies at this voltage.

17. If all goes normally, the system will test all frequencies at the present applied voltage without operator intervention. The system will monitor the status bytes of the ac and dc supplies, as well as their output voltages (via the voltmeters) and will reset the system if either output voltage exceeds the present nominal voltage range. Any system faults, including output voltages out of range, equipment faults, and so forth, will be presented on the bottom of the display and scrolled from right to left. These error messages usually include several options (exit to the command menu, continue with the sequence) as well as suggestions for remedial action on your part. As always, function key K9: 'HARD KILL' will disconnect the output voltage from the units under test, reset the supplies, and return processing to the Ballantine 1605 command option menu. Pressing SHIFT-K9: 'SOFT KILL' will halt processing but leave the supplies and data acquistion system unaffected. 
18. During the calibration sequence, the data gathered from the data acquisition system and nanovoltmeter will be displayed in realtime. At the conclusion of each frequency, the ac-dc difference results for that frequency will be sent to the currently active hardcopy device.

19. If the system is running from a standard calibration file, at the completion of each voltage range you will be prompted to reconfigure the test and standard units for the next range. Upon pressing ' $\mathrm{C}$ ', processing will continue at the next voltage range, with the automatic warmup facility in effect.

20. When the automatic calibration test sequence is complete, or if the automatic shutdown option is in effect, at the end of the final frequency on the present range, the system will reset the supplies, open the output relay, and return processing to the Ballantine 1605 command menu. If manual shutdown has been specified, the following menu is displayed:

i) KO: REPEAT VOLTAGE: This key repeats all frequencies for the present applied voltage.

ii) K1: REPEAT FILE: This key will force the system to repeat the previous standard calibration file.

iii) K2: INSERT NOTE: Use this key to insert a note on the hardcopy. The note may be up to 320 characters in length.

iv) K3: FREQUENCY: This key allows the definition of up to eight new frequencies to be calibrated at the currently applied voltage. The frequencies are input into the usual test parameter input menu.

v) K4: NEW VOLTAGE: This key presents the test parameter input menu and allows the definition of a new voltage range and frequencies. The supplies and data acquisition system will be reset when this option is chosen, and will be initialized to the new test parameters.

vi) K8: EXIT: Returns processing to the Ballantine 1605 command option menu.

21. In addition to the prompts and options described above, on occasion the system may need some information that has not been previously defined. The four most notable occasions are:

i) The standard $\mathrm{M}$ and $\mathrm{N}$ have not been defined in a data file.

ii) No n-test was run on the test unit and therefore no test $\mathrm{M}$ and $\mathrm{N}$ have been defined.

iii) The ac-dc corrections for the standard unit have not been defined in a data file.

iv) A series voltage-dropping resistor is being used to make the ac and dc supplies operate at a higher voltage where they tend to be more stable. In any of these cases, the system will prompt you for the 
proper information and continue the measurement process normally.

22. Upon exiting the n-test or calibration routine and returning to the Ballantine 1605 command option menu, the function keys defined in this menu may be used to restart a test or to exit from the 1605 calibration supervisor. If the latter action is taken, the Ballantine 1605 subroutines are deleted from memory and processing is returned to the ac-dc master program option menu. From there, pressing any key will take the action described by that key, and pressing 'EXIT' will exit the program and return processing to the BASIC 5.0 system. 


\section{INSTRUCTIONS FOR CALIBRATING A FLUKE 540B}

1. This section covers the generic instructions for calibrating a Fluke 540B multirange thermal transfer standard using "AC_DC_MSTR". It is assumed that the program is loaded añ initialized per the instructions in section $I$, and that your last action was to press the 'FLUKE $540 \mathrm{~B}^{\prime}$ option on the instrument calibration menu.

2. Following the instructions on the screen, insert the flexible disc marked "FLUKE 540B" in drive 0 and a calibration data disc in drive 1. The Fluke $540 \mathrm{~B}$ calibration disc contains the necessary routines to do a complete calibration of this instrument. The data generated by the program will be written out to the disc in drive 1. Press CONTINUE to load the $540 \mathrm{~B}$ calibration supervisory program. If a winchester drive is designated as the mass storage device, then the calibration program will load without user intervention.

3. The program will ask for the test number of the present test, and an identification of the unit under test. The program will attempt to find a previously defined data file (D_nnnnnnnn, where nnnnnnnn is the test number) for this test. If a data file has not been created, or if the one it finds is empty, the program will ask you if an n-test should be performed. If you answer with with a "Y", a data file is created, the test number and identification are written out to the file, and the n-test routine is loaded and executed. If you answer with an "N", then processing continues at the 540B command menu, and you will be requested to supply the proper test $\mathrm{M}$ and $\mathrm{N}$ during the calibration. If a data file already exists, an option menu will be displayed. This will be covered in more detail later.

4. The n-test routine will load 11 subroutines from disc and set the default parameters. These are displayed on the CRT along with a function key menu used to modify these defaults. The options available are:

i) Ko: STARTING VOLTS: The initial voltage for the n-test (default is 60 volts).

ii) K1: HIGHEST VOLTS: The final voltage for the n-test (defaults to 100 volts).

iii) K2: VOLTAGE STEP: The step between $V$ and $d V$. This is defined as either a percentage of the currently applied voltage, a percentage of the final voltage, or a flat step. The default is . 1\% of the presently applied voltage.

iv) K3: VOLTAGE INC.: The increment between successive nominal levels of applied voltage (default is $5 \mathrm{~V}$ ).

v) K4: EXECUTE: Starts the n-test procedure.

vi) K5: PRINTER OFF: Disables printouts of each individual voltage step (default is printer on). 
vii) K6: HEADINGS OFF: Disables headings from being printed on the hardcopy device (default is headings on).

viii) K7: DEGREE OF FIT: Allows the characteristic response equation to be fitted to any degree, although a linear fit is generally good enough and higher orders are not yet supported anyway.

ix) K8: EXIT: Returns processing to the Fluke 540B supervisory program.

5. If any changes to the default parameters are desired, press the function key associated with that parameter. A prompt will be displayed, and the value may be entered in the blank space at the prompt line. The option menu will then be redisplayed to request the next operation. Connect the standard channel detector cable to the output of the TVC and set the detector switch to position 3 .

6. When the 'GO' key is pressed, the IEEE-488 instruments needed for an n-test (dc supply, dc voltmeter, and data acquisition system) are initialized to the required conditions.The screen will be erased, and a display, divided into five "windows", will be painted to the CRT. From the top, these windows display the following:

i) The test number, date, and test unit identification.

ii) The present voltage, output EMF, initial and final nulled output EMF, and the incremental nulled EMF.

iii) The balancing process for the standard channel DAC.

iv) The applied voltage, output EMF, $n$, and the standard deviation of the previous points in the n-test.

v) Finally, the fitted characteristic response equation.

7. When the voltage is brought up on the dc calibrator, set the output switch on the front panel to the 'ON' position. The system will wait for things to settle, and during this time the output voltage may be monitored with the dc voltmeter.

8. As the n-test routine runs, the values read from the data acquisition system, as well as the present test parameters, are displayed in the appropriate places in the realtime display. This feature is a nice advance over the older software, in which test data tended to scroll upwards and off the screen.

9. In the course of the n-test, the applied voltages are set automatically, the DACs balanced, and the data collected and reduced with no operator intervention. If a problem does occur, however, there are two procedures to avoid (or at least limit) any possible damage to the system and test units. In the first case, the computer 
monitors the status byte of the dc supply and its output voltage (via the dc voltmeter), before each reading. If the voltage exceeds the nominal applied voltage, or if the dc calibrator self-destructs, the computer will open the output relay to disconnect the output voltage from the test unit. For all system errors, an error message, with possible remedial action and a prompt for instructions is displayed across the bottom of the screen. Since the display line is limited to 80 characters, the message scrolls in from right to left. In most cases, you will be given a choice of continuing the measurement process or exiting the routine.

10. In the second case, for serious "total disaster" types of faults, function key K9, labeled 'HARD KILL' will immediately reset all supplies, open the output relay, and return processing to the supervisory level option menu. THIS KEY MAY BE USED AT ANY TIME IN THE PROCESSING! For faults which are not so dramatic, pressing SHIFT-K9: (K19) 'SOFT KILL' halts processing but leaves the supplies and data acquisition system unaffected. Processing continues at the n-test command menu.

11. After the n-test is complete, processing will return to the supervisory option menu for further choices by the operator. In addition, the n-test data will be written to the appropriate data file on disc (drive 1 or the appropriate winchester volume), and the results will, if desired, be printed to the current hardcopy device. automatically.

12. If a data file for this test number already exists, you may run another n-test, or choose one of the other options. These options include the following:

i) KO: N-TEST: This option initiates an n-test as described above.

ii) Kl: AC-DC DIFF.: Initiates an ac-dc calibration of the test unit.

iii) K2: SYSTEM STATUS: Displays the present system status.

iv) K3: HELP: Invokes the Fluke 540B help facility.

v) K8: EXIT: Exits the routine and returns to the ac-dc master program supervisor.

vi) K9: HARD KILL: THIS KEY WILL RESET THE VOLTAGE SUPPLIES AND OPEN THE OUTPUT RELAY TO DISCONNECT THE VOLTAGE FROM THE UNITS UNDER TEST.

vii) K19: SOFT KILL: Pressing the SHIFT key and K9 stops processing but leaves the supplies and data acquisition system unaffected. If the shift key is pressed simultaneously with the n-test or ac-dc difference keys (SHIFT-KO or SHIFT-KI), then the system will automatically reset the voltage supplies, open the output relay upon completion of the sequence, and return processing to the fluke $540 \mathrm{~B}$ command option menu. If the SHIFT key is not 
pressed, however, processing will be halted upon completion of the test sequence, the supplies will remain active, the output on, and another option menu will be displayed.

13. The next logical step is to run an ac-dc calibration, and this is initiated by pressing K1. Nineteen subroutines will be loaded from the supervisory disc, and several variable initializations and file assignments made. If, in the original supervisory option menu, the SHIFT key was pressed together with the 'FLUKE 540B' key, the system reads the command procedure from the disc. If the 'FLUKE $540 \mathrm{~B}^{\prime}$ key was pressed alone, however, an input menu, requesting the standard unit, applied voltage, standard range, test range, and desired frequencies is displayed, and the parameters for the test are entered at this time. When the output voltages are set on the ac and dc calibrators, position the output switch to 'ON' and check the output voltages on the ac and dc voltmeters.

14. If the calibration is to be made from a standard calibration file, the system will now pause to let the units under test warm up. The length of time allowed for this warmup is range-dependent and varies from five minutes at voltages of less than $60 \mathrm{~V}$ to 45 minutes for voltages of $600 \mathrm{~V}$ and more. If the test is to be done manually, a warmup menu is displayed to allow you to select the proper warmup time as follows:

i) KI-K6: CONTINUE WITH MEASUREMENTS: starts the measurement sequence.

ii) K2-K7: START SEQUENCE: If no automatic warmup is desired, this functions in the same manner as KI-K6. If, however, the system is currently in the midst of an automatic warmup period, this action aborts the warmup and starts the sequence.

iii) K3-K8: AUTOMATIC WARMUP: When pressed, this action starts the automatic warmup period for the present voltage range.

iv) K5: SYSTEM STATUS: Displays the present system status.

v) K9: HARD KILL: ABORTS ALL PROCESSING, RESETS THE SUPPLIES, AND RETURNS PROCESSING TO THE FLUKE $540 \mathrm{~B}$ COMMAND OPTION MENU.

vi) K19: SOFT KILL: Stops processing but leaves the supplies and data acquisition system unaffected.

15. In any case, processing resumes with the system reading the EMFs from both the test and standard channel. If no output is measured, the system presents an error message and suggests corrective action. You will then be instructed as to the position in which to set the detector switch on the front panel. This action will ensure that the output EMF is divided down to a level 
where the DACs can work without being under- or overranged. Press CONTINUE, when the switch is set, to continue processing.

16. After processing is continued, the program will paint a realtime display composed of the following five windows (from the top):

i) The test number and instrument identification.

ii) Present test data (the ac-dc difference and dc reversal data).

iii) A summary of the ac-dc differences for this set of four determinations.

iv) A presentation of the test parameters (standard unit, output EMFs, currently applied voltage and frequency, etc.).

v) Previous results for all frequencies at this voltage.

17. If all goes normally, the system will test all frequencies at the present applied voltage without operator intervention. The system will monitor the status bytes of the ac and dc supplies, as well as their output voltages (via the voltmeters) and will reset the system if either output voltage exceeds the present nominal voltage range. Any system faults, including output voltages out of range, equipment faults, and so forth, will be presented on the bottom of the display and scrolled from right to left. These error messages usually include several options (exit to the command menu, continue with the sequence) as well as suggestions for remedial action on your part. As always, function key K9: 'HARD KILL' will disconnect the output voltage from the units under test, reset the supplies, and return processing to the Fluke $540 \mathrm{~B}$ command option menu. Pressing SHIFT-K9: 'SOFT KILL' will halt processing but leave the supplies and data acquistion system unaffected.

18. During the calibration sequence, the data gathered from the data acquisition system will be displayed in realtime. At the conclusion of each frequency, the ac-dc difference results for that frequency will be sent to the currently active hardcopy device.

19. If the system is running from a standard calibration file, at the completion of each voltage range you will be prompted to reconfigure the test and standard units for the next range. Upon pressing ' $C$ ', processing will continue at the next voltage range, with the automatic warmup facility in effect.

20. When the automatic calibration test sequence is complete, or if the automatic shutdown option is in effect, at the end of the final frequency on the present range, the system will reset the supplies, open the 
output relay, and return processing to the Fluke $540 \mathrm{~B}$ command option menu. If manual shutdown has been specified, the following menu is displayed:

i) KO: REPEAT VOLTAGE: This key repeats all frequencies for the present applied voltage.

ii) K1: REPEAT FILE: This key will force the system to repeat the previous standard calibration file.

iii) K2: INSERT NOTE: Use this key to insert a note on the hardcopy. The note may be up to 320 characters in length.

iv) K3: FREQUENCY: This key allows the definition of up to eight new frequencies to be calibrated at the currently applied voltage. The frequencies are input into the usual test parameter input menu.

v) K4: NEW VOLTAGE: This key presents the test parameter input menu and allows the definition of a new voltage range and frequencies. The supplies and data acquisition system will be reset when this option is chosen, and will be initialized to the new test parameters.

vi) K8: EXIT: Returns processing to the Fluke 540B command option menu.

21. In addition to the prompts and options described above, on occasion the system may need some information that has not been previously defined. The four most notable occasions are:

i) The standard $M$ and $N$ have not been defined in a data file.

ii) No n-test was run on the test unit and therefore no test $\mathrm{M}$ and $\mathrm{N}$ have been defined.

iii) The ac-dc corrections for the standard unit have not been defined in a data file.

iv) A series voltage-dropping resistor is being used to make the ac and dc supplies operate at a higher voltage where they tend to be more stable. In any of these cases, the system will prompt you for the proper information and continue the measurement process normally.

22. Upon exiting the n-test or calibration routine and returning to the Fluke $540 \mathrm{~B}$ command option menu, the function keys defined in this menu may be used to restart a test or to exit from the $540 \mathrm{~B}$ calibration supervisor. If the latter action is taken, the Fluke 540B subroutines are deleted from memory and processing is returned to the ac-dc master program option menu. From there, pressing any key will take the action described by that key, and pressing 'EXIT' will exit the program and return processing to the BASIC 5.0 system. 
1. This section covers the generic instructions for calibrating a Guildline 7100A automated multirange thermal transfer standard using "AC DC MSTR". It is assumed that the program is loaded and initialized per the instructions in section $I$, and that your last action was to press the 'GUILD 7100A' option on the instrument calibration menu.

2. Following the instructions on the screen, insert the flexible disc marked "GUILDLINE 7100A" in drive 0 and a calibration data disc in drive 1. The Guildline 7100 calibration disc contains the necessary routines to do a complete calibration of this instrument. The data generated by the program will be written out to the disc in drive 1. Press CONTINUE to load the 7100 calibration supervisory program. If a winchester drive has been designated as the mass storage device, the data file will be placed in the appropriate volume and the disc swapping is unnecessary.

3. The program will ask for the test number of the present test, and an identification of the unit under test. The program will attempt to find a previously defined data file (D_nnnnnnnn, where nnnnnnnn is the test number) for this test. If a data file has not been created, or if the one it finds is empty, the program will ask you if an n-test should be performed. If you answer with with a "Y", a data file is created, the test number and identification are written out to the file, and the n-test routine is loaded and executed. If you answer with an "N", then processing continues at the 7100 command menu, and you will be requested to supply the proper test $M$ and $N$ during the calibration. If a data file already exists, an option menu will be displayed. This will be covered in more detail later.

4. Since the Guildline $7100 \mathrm{~A}$ does not require that the thermal element characteristics be known, no n-test is required. Pressing K2: AC-DC DIFF will initiate a calibration.

5. Initial setup of the Guildline $7100 \mathrm{~A}$ proceeds as follows. Connect a stable dc source to the dc input terminals on the front panel, and the ac-dc output of the comparator to the ac input terminals. Connect a Keithley 181 nanovoltmeter to the recorder output on the rear panel. Follow the setup instructions in the Guildline 7100A manual through section 3.4 .2 (G), with the following exception. The dc toggle switch must be in the "-" position if non-floating dc ballast supply is used. For the calibration sequence, the meter range switch should be set on the 1\% setting for those frequencies where the ac-dc difference of the unit is less than $10000 \mathrm{ppm}$, and at 10\% for frequencies above 
about $50 \mathrm{kHz}$. Follow the instructions in the manual to set up the high-voltage extender box if needed.

6. Nineteen subroutines will be loaded from the supervisory disc, and several variable initializations and file assignments made. If, in the original supervisory option menu, the SHIFT key was pressed together with the 'BALLANTINE 7100' key, the system reads the command procedure from the disc. If the 'BALLANTINE 7100' key was pressed alone, however, an input menu, requesting the standard unit, applied voltage, standard range, test range, and desired frequencies is displayed, and the parameters for the test are entered at this time. When the output voltages are set on the ac and dc calibrators, position the output switch to 'ON' and check the output voltages on the ac and dc voltmeters.

7. If the calibration is to be made from a standard calibration file, the system will now pause to let the units under test warm up. The length of time allowed for this warmup is range-dependent and varies from five minutes at voltages of less than $60 \mathrm{~V}$ to 45 minutes for voltages of $600 \mathrm{~V}$ and more. If the test is to be done manually, a warmup menu is displayed to allow you to select the proper warmup time as follows:

i) K1-K6: CONTINUE WITH MEASUREMENTS: starts the measurement sequence.

ii) K2-K7: START SEQUENCE: If no automatic warmup is desired, this functions in the same manner as K1-K6. If, however, the system is currently in the midst of an automatic warmup period, this action aborts the warmup and starts the sequence.

iii) K3-K8: AUTOMATIC WARMUP: When pressed, this action starts the automatic warmup period for the present voltage range.

iv) K5: SYSTEM STATUS: Displays the present system status.

v) K9: HARD KILL: ABORTS ALL PROCESSING, RESETS THE SUPPLIES, AND RETURNS PROCESSING TO THE BALLANTINE 7100 COMMAND OPTION MENU.

vi) K19: SOFT KILL: Stops processing but leaves the supplies and data acquisition system unaffected.

8. In any case, processing resumes with the system reading the EMFs from both the test and standard channel. If no output is measured, the system presents an error message and suggests corrective action. You will then be instructed as to the position in which to set the detector switch on the front panel. This action will ensure that the output EMF is divided down to a level where the standard channel DAC can work without being under- or over ranged. Press CONTINUE, when the switch is set, to continue processing. 
9. After processing is continued, the program will paint a realtime display composed of the following five windows (from the top):

i) The test number and instrument identification.

ii) Present test data (the ac-dc difference and dc reversal data).

iii) A summary of the ac-dc differences for this set of four determinations.

iv) A presentation of the test parameters (standard unit, output EMFs, currently applied voltage and frequency, etc.).

v) Previous results for all frequencies at this voltage.

10. If all goes normally, the system will test all frequencies at the present applied voltage without operator intervention. The system will monitor the status bytes of the ac and dc supplies, as well as their output voltages (via the voltmeters) and will reset the system if either output voltage exceeds the present nominal voltage range. Any system faults, including output voltages out of range, equipment faults, and so forth, will be presented on the bottom of the display and scrolled from right to left. These error messages usually include several options (exit to the command menu, continue with the sequence) as well as suggestions for remedial action on your part. As always, function key K9: 'HARD KILL' will disconnect the output voltage from the units under test, reset the supplies, and return processing to the Guildline 7100 command option menu. Pressing SHIFT-K9: 'SOFT KILL' will halt processing but leave the supplies and data acquistion system unaffected.

11. During the calibration sequence, the data gathered from the data acquisition system and nanovoltmeter will be displayed in realtime. At the conclusion of each frequency, the ac-dc difference results for that frequency will be sent to the currently active hardcopy device.

12. If the system is running from a standard calibration file, at the completion of each voltage range you will be prompted to reconfigure the test and standard units for the next range. Upon pressing ' $C$ ', processing will continue at the next voltage range, with the automatic warmup facility in effect.

13. When the automatic calibration test sequence is complete, or if the automatic shutdown option is in effect, at the end of the final frequency on the present range, the system will reset the supplies, open the output relay, and return processing to the Guildline 7100 command menu. If manual shutdown has been specified, the following menu is displayed: 
i) KO: REPEAT VOLTAGE: This key repeats all frequencies for the present applied voltage.

ii) Kl: REPEAT FILE: This key will force the system to repeat the previous standard calibration file.

iii) K2: INSERT NOTE: Use this key to insert a note on the hardcopy. The note may be up to 320 characters in length.

iv) K3: FREQUENCY: This key allows the definition of up to eight new frequencies to be calibrated at the currently applied voltage. The frequencies are input into the usual test parameter input menu.

v) K4: NEW VOLTAGE: This key presents the test parameter input menu and allows the definition of a new voltage range and frequencies. The supplies and data acquisition system will be reset when this option is chosen, and will be initialized to the new test parameters.

vi) K8: EXIT: Returns processing to the Guildline 7100 command option menu.

14. In addition to the prompts and options described above, on occasion the system may need some information that has not been previously defined. The two most notable occasions are:

i) The ac-dc corrections for the standard unit have not been defined in a data file.

ii) A series voltage-dropping resistor is being used to make the ac and dc supplies operate at a higher voltage where they tend to be more stable. In any of these cases, the system will prompt you for the proper information and continue the measurement process normally.

15. After a set of determinations has been taken with the CHANNEI REVERSE switch on the "NORM" setting, the 7100A must be calibrated again, at the same points, with the switch on the "REVERSE" setting. The ac-dc difference of the unit at each frequency is the arithmatic mean of normal and reverse points.

16. Upon exiting the calibration routine and returning to the Guildline 7100 command option menu, the function keys defined in this menu may be used to restart a test or to exit from the 7100 calibration supervisor. If the latter action is taken, the Guildline 7100 subroutines are deleted from memory and processing is returned to the ac-dc master program option menu. From there, pressing any key will take the action described by that key, and pressing 'EXIT' will exit the program and return processing to the BASIC 5.0 system. 
1. This section covers the generic instructions for calibrating a Holt Model 6A multirange thermal transfer standard using "AC_DC_MSTR". It is assumed that the program is loade $\bar{d}$ and initialized per the instructions in section $I$, and that your last action was to press the 'HOLT 6A' option on the instrument calibration menu.

2. Following the instructions on the screen, insert the flexible disc marked "HOLT MODEL 6A" in drive 0 and a calibration data disc in drive 1 . The Holt Model $6 \mathrm{~A}$ calibration disc contains the necessary routines to do a complete calibration of this instrument. The data generated by the program will be written out to the disc in drive 1 . Press CONTINUE to load the Model $6 \mathrm{~A}$ calibration supervisory program. If a winchester drive is designated as the mass storage device, the data will be written out to the appropriate volume, and you will not need to swap discs.

3. The program will ask for the test number of the present test, and an identification of the unit under test. The program will attempt to find a previously defined data file (D_nnnnnnnn, where nnnnnnnn is the test number) for this test. If a data file has not been created, or if the one it finds is empty, the program will ask you if an n-test should be performed. If you answer with with a "Y", a data file is created, the test number and identification are written out to the file, and the n-test routine is loaded and executed. If you answer with an "N", then processing continues at the Holt $6 \mathrm{~A}$ command menu, and you will be requested to supply the proper test $M$ and $N$ during the calibration. If $a$ data file already exists, an option menu will be displayed. This will be covered in more detail later.

4. To calibrate a Holt Model $6 \mathrm{~A}$ the instrument must be set to the 150 volt range, and the power switch must be placed in the 'ON' position. The input voltage is applied through the UHF input connector. Since the Model $6 \mathrm{~A}$ has a grounded output the EMF cannot be measured using the analog integrators in the normal manner. Instead, the output EMF is measured with a nanovoltmeter, such as a Keithley 181. Use a microphone-to-Amphenol adapter to connect the microphone output connector through an appropriate cable to the millivolt input connector of the nanovoltmeter. Also note that the protection button will probably need to be depressed throughout the calibration to prevent the ac-dc relay from tripping the Model $6 \mathrm{~A}$ into a standby mode.

5. The n-test routine will load 11 subroutines from disc and set the default parameters. These are displayed on the CRT along with a function key menu used to modify 
these defaults. The options available are:

i) KO: STARTING VOLTS: The initial voltage for the n-test (default is 120 volts).

ii) K1: HIGHEST VOLTS: The final voltage for the n-test (defaults to 150 volts).

iii) K2: VOLTAGE STEP: The step between $V$ and $d V$. This is defined as either a percentage of the currently applied voltage, a percentage of the final voltage, or a flat step. The default is . $1 \%$ of the presently applied voltage.

iv) K3: VOLTAGE INC.: The increment between successive nominal levels of applied voltage (default is $5 \mathrm{~V}$ ).

v) K4: EXECUTE: Starts the n-test procedure.

vi) K5: PRINTER OFF: Disables printouts of each individual voltage step (default is printer on).

vii) K6: HEADINGS OFF: Disables headings from being printed on the hardcopy device (default is headings on).

viii) K7: DEGREE OF FIT: Allows the characteristic response equation to be fitted to any degree, although a linear fit is generally good enough and higher orders are not yet supported anyway.

ix) K8: EXIT: Returns processing to the Holt Model 6A supervisory program.

6. If any changes to the default parameters are desired, press the function key associated with that parameter. A prompt will be displayed, and the value may be entered in the blank space at the prompt line. The option menu will then be redisplayed to request the next operation.

7. When the 'GO' key is pressed, the IEEE-488 instruments needed for an n-test (dc supply, dc voltmeter, and data acquisition system) are initialized to the required conditions.The screen will be erased, and a display, divided into five "windows", will be painted to the CRT. From the top, these windows display the following:

i) The test number, date, and test unit identification.

ii) The present voltage, output EMF, initial and final nulled output EMF, and the incremental nulled EMF.

iii) The balancing process for the standard channel DAC. Note that the Model $6 \mathrm{~A}$ output EMF is measured using an external voltmeter, such as a Keithley 181, and no balancing of the DAC is necessary. No activity will be displayed in the DAC balance window; it it a vestige of other calibration routines.

iv) The applied voltage, output EMF, $n$, and the standard deviation of the previous points in the n-test.

v) Finally, the fitted characteristic response equation. When the voltage is brought up on the dc calibrator, set the output switch on the front panel to the 'ON' position. The system will wait for things to settle, and during this time the output voltage may be monitored with the dc voltmeter. 
8. As the n-test routine runs, the values read from the nanovoltmeter as well as the present test parameters, are displayed in the appropriate places in the realtime display. This feature is a nice advance over the older software, in which test data tended to scroll upwards and off the screen.

9. In the course of the n-test, the applied voltages are set automatically, and the data collected and reduced with no operator intervention. If a problem does occur, however, there are two procedures to avoid (or at least limit) any possible damage to the system and test units. In the first case, the computer monitors the status byte of the dc supply and its output voltage (via the dc voltmeter), before each reading. If the voltage exceeds the nominal applied voltage, or if the dc calibrator self-destructs, the computer will open the output relay to disconnect the output voltage from the test unit. For all system errors, an error message, with possible remedial action and a prompt for instructions is displayed across the bottom of the screen. Since the display line is limited to 80 characters, the message scrolls in from right to left. In most cases, you will be given a choice of continuing the measurement process or exiting the routine.

10. In the second case, for serious "total disaster" types of faults, function key K9, labeled 'HARD KILL' will immediately reset all supplies, open the output relay, and return processing to the supervisory level option menu. THIS KEY MAY BE USED AT ANY TIME IN THE PROCESSING! For faults which are not so dramatic, pressing SHIFT-K9: (K19) 'SOFT KILL' halts processing but leaves the supplies and data acquisition system unaffected. Processing continues at the n-test command menu.

11. After the n-test is complete, processing will return to the supervisory option menu for further choices by the operator. In addition, the n-test data will be written to the appropriate data file on disc (drive 1 or the proper winchester volume), and the results will, if desired, be printed to the current hardcopy device automatically.

12. If a data file for this test number already exists, you may run another n-test, or choose one of the other options. These options include the following:

i) KO: N-TEST: This option initiates an n-test as described above.

ii) K1: AC-DC DIFF.: Initiates an ac-dc calibration of the test unit.

iii) K2: SYSTEM STATUS: Displays the present system status. 
iv) K3: HELP: Invokes the Holt Model 6A help facility.

v) K8: EXIT: Exits the routine and returns to the ac-dc master program supervisor.

vi) K9: HARD KILL: THIS KEY WILL RESET THE VOLTAGE SUPPLIES AND OPEN THE OUTPUT RELAY TO DISCONNECT THE VOLTAGE FROM THE UNITS UNDER TEST.

vii) K19: SOFT KILL: Pressing the SHIFT key and K9 stops processing but leaves the supplies and data acquisition system unaffected. If the shift key is pressed simultaneously with the n-test or ac-dc difference keys (SHIFT-Ko or SHIFT-K1), then the system will automatically reset the voltage supplies, open the output relay upon completion of the sequence, and return processing to the Holt Model 6A command option menu. If the SHIFT key is not pressed, however, processing will be halted upon completion of the test sequence, the supplies will remain active, the output on, and another option menu will be displayed.

13. The next logical step is to run an ac-dc calibration, and this is initiated by pressing $\mathrm{Kl}$. Nineteen subroutines will be loaded from the supervisory disc, and several variable initializations and file assignments made. If, in the original supervisory option menu, the SHIFT key was pressed together with the 'HOLT MODEL 6A' key, the system reads the command procedure from the disc. If the 'HOLT MODEL $6 A^{\prime}$ ' key was pressed alone, however, an input menu, requesting the standard unit, applied voltage, standard range, test range, and desired frequencies is displayed, and the parameters for the test are entered at this time. When the output voltages are set on the ac and dc calibrators, position the output switch to ' $\mathrm{ON}$ ' and check the output voltages on the ac and dc voltmeters.

14. If the calibration is to be made from a standard calibration file, the system will now pause to let the units under test warm up. The length of time allowed for this warmup is range-dependent and varies from five minutes at voltages of less than $60 \mathrm{~V}$ to 45 minutes for voltages of $600 \mathrm{~V}$ and more. If the test is to be done manually, a warmup menu is displayed to allow you to select the proper warmup time as follows:

i) K1-K6: CONTINUE WITH MEASUREMENTS: starts the measurement sequence.

ii) K2-K7: START SEQUENCE: If no automatic warmup is desired, this functions in the same manner as K1-K6. If, however, the system is currently in the midst of an automatic warmup period, this action aborts the warmup and starts the sequence.

iii) K3-K8: AUTOMATIC WARMUP: When pressed, this action starts the automatic warmup period for the present voltage range. 
iv) K5: SYSTEM STATUS: Displays the present system status.

v) K9: HARD KILL: ABORTS ALL PROCESSING, RESETS THE SUPPLIES, AND RETURNS PROCESSING TO THE HOLT MODEL 6A COMMAND OPTION MENU.

vi) K19: SOFT KILL: Stops processing but leaves the supplies and data acquisition system unaffected.

15. In any case, processing resumes with the system reading the EMFs from both the test and standard channel. If no output is measured, the system presents an error message and suggests corrective action. You will then be instructed as to the position in which to set the detector switch on the front panel. This action will ensure that the output EMF is divided down to a level where the standard channel DAC can work without being under- or over ranged. Press CONTINUE, when the switch is set, to continue processing.

16. After processing is continued, the program will paint a realtime display composed of the following five windows (from the top):

i) The test number and instrument identification.

ii) Present test data (the ac-dc difference and dc reversal data).

iii) A summary of the ac-dc differences for this set of four determinations.

iv) A presentation of the test parameters (standard unit, output EMFs, currently applied voltage and frequency, etc.).

v) Previous results for all frequencies at this voltage.

17. If all goes normally, the system will test all frequencies at the present applied voltage without operator intervention. The system will monitor the status bytes of the ac and dc supplies, as well as their output voltages (via the voltmeters) and will reset the system if either output voltage exceeds the present nominal voltage range. Any system faults, including output voltages out of range, equipment faults, and so forth, will be presented on the bottom of the display and scrolled from right to left. These error messages usually include several options (exit to the command menu, continue with the sequence) as well as suggestions for remedial action on your part. As always, function key K9: 'HARD KILL' will disconnect the output voltage from the units under test, reset the supplies, and return processing to the Holt Model $6 \mathrm{~A}$ command option menu. Pressing SHIFT-K9: 'SOFT KILL' will halt processing but leave the supplies and data acquistion system unaffected. 
18. During the calibration sequence, the data gathered from the data acquisition system and nanovoltmeter will be displayed in realtime. At the conclusion of each frequency, the ac-dc difference results for that frequency will be sent to the currently active hardcopy device.

19. If the system is running from a standard calibration file, at the completion of each voltage range you will be prompted to reconfigure the test and standard units for the next range. Upon pressing ' $C$ ', processing will continue at the next voltage range, with the automatic warmup facility in effect.

20. When the automatic calibration test sequence is complete, or if the automatic shutdown option is in effect, at the end of the final frequency on the present range, the system will reset the supplies, open the output relay, and return processing to the Holt Model 6A command menu. If manual shutdown has been specified, the following menu is displayed:

i) KO: REPEAT VOLTAGE: This key repeats all frequencies for the present applied voltage.

ii) K1: REPEAT FILE: This key will force the system to repeat the previous standard calibration file.

iii) K2: INSERT NOTE: Use this keY to insert a note on the hardcopy. The note may be up to 320 characters in length.

iv) K3: FREQUENCY: This key allows the definition of up to eight new frequencies to be calibrated at the currently applied voltage. The frequencies are input into the usual test parameter input menu.

v) K4: NEW VOLTAGE: This key presents the test parameter input menu and allows the definition of a new voltage range and frequencies. The supplies and data acquisition system will be reset when this option is chosen, and will be initialized to the new test parameters.

vi) K8: EXIT: Returns processing to the Holt Model 6A command option menu.

21. In addition to the prompts and options described above, on occasion the system may need some information that has not been previously defined. The four most notable occasions are:

i) The standard $M$ and $N$ have not been defined in a data file.

ii) No n-test was run on the test unit and therefore no test $M$ and $N$ have been defined.

iii) The ac-dc corrections for the standard unit have not been defined in a data file.

iv) A series voltage-dropping resistor is being used to make the $\dot{a}$ and dc supplies operate at a higher voltage where they tend to be more stable. In any of these cases, the system will prompt you for the 
proper information and continue the measurement process normally.

22. Upon exiting the n-test or calibration routine and returning to the Holt Model $6 \mathrm{~A}$ command option menu, the function keys defined in this menu may be used to restart a test or to exit from the Model 6A calibration supervisor. If the latter action is taken, the Holt Model 6A subroutines are deleted from memory and processing is returned to the ac-dc master program option menu. From there, pressing any key will take the action described by that key, and pressing 'EXIT' will exit the program and return processing to the BASIC 5.0 system. 
1. This section covers the generic instructions for calibrating a Holt model 11 coaxial thermal transfer standard using "AC_DC_MSTR". It is assumed that the program is loade $\bar{d}$ and initialized per the instructions in section $I$, and that your last action was to press the 'Holt Model 11' option on the instrument calibration menu.

2. Following the instructions on the screen, insert the flexible disc marked "Holt Model 11" in drive 0 and a calibration data disc in drive 1. The Holt model 11 calibration disc contains the necessary routines to do a complete calibration of this instrument. The data generated by the program will be written out to the disc in drive 1. Press conTINUE to load the Model 11 calibration supervisory program. If a winchester drive is designated as the mass storage device, then the calibration program will load without user intervention.

3. The program will ask for the test number of the present test, and an identification of the unit under test. The program will attempt to find a previously defined data file (D_nnnnnnnn, where nnnnnnnn is the test number) for this test. If a data file has not been created, or if the one it finds is empty, the program will ask you if an n-test should be performed. If you answer with with a "Y", a data file is created, the test number and identification are written out to the file, and the n-test routine is loaded and executed. If you answer with an " $N$ ", then processing continues at the Model 11 command menu, and you will be requested to supply the proper test $\mathrm{M}$ and $\mathrm{N}$ during the calibration. If $\mathrm{a}$ data file already exists, an option menu will be displayed. This will be covered in more detail later.

4. The n-test routine will load 11 subroutines from disc and set the default parameters. These are displayed on the CRT along with a function key menu used to modify these defaults. The options available are:

i) KO: STARTING VOLTS: The initial voltage for the n-test (defaults are 70, 90, and 120 volts, depending on the current rating of the thermal element under test).

ii) KI: HIGHEST VOLTS: The final voltage for the n-test (defaults to 100,120 , or 200 volts depending on the TE).

iii) K2: VOLTAGE STEP: The step between $V$ and $d V$. This is defined as either a percentage of the currently applied voltage, a percentage of the final voltage, or a flat step. The default is .1\% of the presently applied voltage.

iv) K3: VOLTAGE INC.: The increment between successive nominal levels of applied voltage (default is $5 \mathrm{~V}$ ). 
v) K4: EXECUTE: Starts the n-test procedure.

vi) K5: PRINTER OFF: Disables printouts of each individual voltage step (default is printer on).

vii) K6: HEADINGS OFF: Disables headings from being printed on the hardcopy device (default is headings on).

viii) K7: DEGREE OF FIT: Allows the characteristic response equation to be fitted to any degree, although a linear fit is generally good enough and higher orders are not yet supported anyway.

ix) K8: EXIT: Returns processing to the Holt Model 11 supervisory program.

5. If any changes to the default parameters are desired, press the function key associated with that parameter. A prompt will be displayed, and the value may be entered in the blank space at the prompt line. The option menu will then be redisplayed to request the next operation. Connect the standard channel detector cable to the output of the TVC and set the detector switch to position 3 .

6. When the 'GO' key is pressed, the IEEE-488 instruments needed for an n-test (dc supply, dc voltmeter, and data acquisition system) are initialized to the required conditions.The screen will be erased, and a display, divided into five "windows", will be painted to the CRT. From the top, these windows display the following:

i) The test number, date, and test unit identification. ii) The present voltage, output EMF, initial and final nulled output EMF, and the incremental nulled EMF.

iii) The balancing process for the standard channel DAC.

iv) The applied voltage, output EMF, $n$, and the standard deviation of the previous points in the n-test.

v) Finally, the fitted characteristic response equation.

7. When the voltage is brought up on the dc calibrator, set the output switch on the front panel to the 'ON' position. The system will wait for things to settle, and during this time the output voltage may be monitored with the dc voltmeter.

8. As the n-test routine runs, the values read from the data acquisition system, as well as the present test parameters, are displayed in the appropriate places in the realtime display. This feature is a nice advance over the older software, in which test data tended to scroll upwards and off the screen.

9. In the course of the $n$-test, the applied voltages are set automatically, the DACs balanced, and the data collected and reduced with no operator intervention. If a problem does occur, however, there are two procedures 
to avoid (or at least limit) any possible damage to the system and test units. In the first case, the computer monitors the status byte of the dc supply and its output voltage (via the dc voltmeter), before each reading. If the voltage exceeds the nominal applied voltage, or if the dc calibrator self-destructs, the computer will open the output relay to disconnect the output voltage from the test unit. For all system errors, an error message, with possible remedial action and a prompt for instructions is displayed across the bottom of the screen. Since the display line is limited to 80 characters, the message scrolls in from right to left. In most cases, you will be given a choice of continuing the measurement process or exiting the routine.

10. In the second case, for serious "total disaster" types of faults, function key K9, labeled 'HARD KILL' will immediately reset all supplies, open the output relay, and return processing to the supervisory level option menu. THIS KEY MAY BE USED AT ANY TIME IN THE PROCESSING! For faults which are not so dramatic, pressing SHIFT-K9: (K19) 'SOFT KILL' halts processing but leaves the supplies and data acquisition system unaffected. Processing continues at the n-test command menu.

11. After the n-test is complete, processing will return to the supervisory option menu for further choices by the operator. In addition, the n-test data will be written to the appropriate data file on disc (drive 1 or the appropriate winchester volume), and the results will, if desired, be printed to the current hardcopy device. automatically.

12. If a data file for this test number already exists, you may run another n-test, or choose one of the other options. These options include the following:

i) KO: N-TEST: This option initiates an n-test as described above.

ii) K1: AC-DC DIFF.: Initiates an ac-dc calibration of the test unit.

iii) K2: SYSTEM STATUS: Displays the present system status.

iv) K3: HELP: Invokes the Holt Model 11 help facility.

v) K8: EXIT: Exits the routine and returns to the ac-dc master program supervisor.

vi) K9: HARD KILL: THIS KEY WILL RESET THE VOLTAGE SUPPLIES AND OPEN THE OUTPUT RELAY TO DISCONNECT THE VOLTAGE FROM THE UNITS UNDER TEST.

vii) K19: SOFT KILL: Pressing the SHIFT key and K9 stops processing but leaves the supplies and data acquisition system unaffected. If the shift key is pressed simultaneously with the n-test or ac-dc difference keys (SHIFT-KO or SHIFT-K1), then the system will automatically reset the voltage supplies, open the output relay upon completion of 
the sequence, and return processing to the Holt Model 11 command option menu. If the SHIFT key is not pressed, however, processing will be halted upon completion of the test sequence, the supplies will remain active, the output on, and another option menu will be displayed.

13. The next logical step is to run an ac-dc calibration, and this is initiated by pressing K1. Nineteen subroutines will be loaded from the supervisory disc, and several variable initializations and file assignments made. If, in the original supervisory option menu, the SHIFT key was pressed together with the 'Holt Model 11' key, the system reads the command procedure from the disc. If the 'Holt Model 11' key was pressed alone, however, an input menu, requesting the standard unit, applied voltage, standard range, test range, and desired frequencies is displayed, and the parameters for the test are entered at this time. When the output voltages are set on the ac and dc calibrators, position the output switch to 'ON' and check the output voltages on the ac and dc voltmeters.

14. If the calibration is to be made from a standard calibration file, the system will now pause to let the units under test warm up. The length of time allowed for this warmup is range-dependent and varies from five minutes at voltages of less than $60 \mathrm{~V}$ to 45 minutes for voltages of $600 \mathrm{~V}$ and more. If the test is to be done manually, a warmup menu is displayed to allow you to select the proper warmup time as follows:

i) K1-K6: CONTINUE WITH MEASUREMENTS: starts the measurement sequence.

ii) K2-K7: START SEQUENCE: If no automatic warmup is desired, this functions in the same manner as K1-K6. If, however, the system is currently in the midst of an automatic warmup period, this action aborts the warmup and starts the sequence.

iii) K3-K8: AUTOMATIC WARMUP: When pressed, this action starts the automatic warmup period for the present voltage range.

iv) K5: SYSTEM STATUS: Displays the present system status.

v) K9: HARD KILL: ABORTS ALL PROCESSING, RESETS THE SUPPLIES, AND RETURNS PROCESSING TO THE Holt Model 11 COMMAND OPTION MENU .

vi) K19: SOFT KILL: Stops processing but leaves the supplies and data acquisition system unaffected.

15. In any case, processing resumes with the system reading the EMFs from both the test and standard channel. If no output is measured, the system presents an error message and suggests corrective action. You will then be instructed as to the position in which to set the 
detector switch on the front panel. This action will ensure that the output EMF is divided down to a level where the DACs can work without being under- or overranged. Press CONTINUE, when the switch is set, to continue processing.

16. After processing is continued, the program will paint a realtime display composed of the following five windows (from the top):

i) The test number and instrument identification.

ii) Present test data (the ac-dc difference and dc reversal data).

iii) A summary of the ac-dc differences for this set of four determinations.

iv) A presentation of the test parameters (standard unit, output EMFs, currently applied voltage and frequency, etc.).

v) Previous results for all frequencies at this voltage.

17. If all goes normally, the system will test all frequencies at the present applied voltage without operator intervention. The system will monitor the status bytes of the ac and dc supplies, as well as their output voltages (via the voltmeters) and will reset the system if either output voltage exceeds the present nominal voltage range. Any system faults, including output voltages out of range, equipment faults, and so forth, will be presented on the bottom of the display and scrolled from right to left. These error messages usually include several options (exit to the command menu, continue with the sequence) as well as suggestions for remedial action on your part. As always, function key K9: 'HARD KILL' will disconnect the output voltage from the units under test, reset the supplies, and return processing to the Holt Model 11 command option menu. Pressing SHIFT-K9: 'SOFT KILL' will halt processing but leave the supplies and data acquistion system unaffected.

18. During the calibration sequence, the data gathered from the data acquisition system will be displayed in realtime. At the conclusion of each frequency, the ac-dc difference results for that frequency will be sent to the currently active hardcopy device.

19. If the system is running from a standard calibration file, at the completion of each voltage range you will be prompted to reconfigure the test and standard units for the next range. Upon pressing ' $C$ ', processing will continue at the next voltage range, with the automatic warmup facility in effect. 
20. When the automatic calibration test sequence is complete, or if the automatic shutdown option is in effect, at the end of the final frequency on the present range, the system will reset the supplies, open the output relay, and return processing to the Holt Model 11 command option menu. If manual shutdown has been specified, the following menu is displayed:
i)
Ko:
REPEAT VOLTAGE:
This key repeats
all frequencies for the present applied voltage.

ii) K1: REPEAT FILE: This key will force the system to repeat the previous standard calibration file.

iii) K2: INSERT NOTE: Use this key to insert a note on the hardcopy. The note may be up to 320 characters in length.

iv) K3: FREQUENCY: This key allows the definition of up to eight new frequencies to be calibrated at the currently applied voltage. The frequencies are input into the usual test parameter input menu.

v) K4: NEW VOLTAGE: This key presents the test parameter input menu and allows the definition of a new voltage range and frequencies. The supplies and data acquisition system will be reset when this option is chosen, and will be initialized to the new test parameters.

vi) K8: EXIT: Returns processing to the Holt Model 11 command option menu.

21. In addition to the prompts and options described above, on occasion the system may need some information that has not been previously defined. The four most notable occasions are:

i) The standard $M$ and $N$ have not been defined in a data file.

ii) No n-test was run on the test unit and therefore no test $M$ and $N$ have been defined.

iii) The ac-dc corrections for the standard unit have not been defined in a data file.

iv) A series voltage-dropping resistor is being used to make the ac and dc supplies operate at a higher voltage where they tend to be more stable. In any of these cases, the system will prompt you for the proper information and continue the measurement process normally.

22. Upon exiting the n-test or calibration routine and returning to the Holt Model 11 command option menu, the function keys defined in this menu may be used to restart a test or to exit from the Model 11 calibration supervisor. If the latter action is taken, the Holt Model 11 subroutines are deleted from memory and processing is returned to the ac-dc master program option menu. From there, pressing any key will take the action described by that key, and pressing 'EXIT' will exit the program and return processing to the BASIC 5.0 system. 
1. This section covers the generic instructions for calibrating an NBS type coaxial thermal transfer standard (such as Julie TNB-102s, Hermach-Engelhard coaxial standards, etc.) using "AC_DC_MSTR". It is assumed that the program is loaded and iñitialized per the instructions in section $I$, and that your last action was to press the 'NBS TYPE' option on the instrument calibration menu.

2. Following the instructions on the screen, insert the flexible disc marked "NBS TYPE" in drive 0 and a calibration data disc in drive 1. The NBS type calibration disc contains the necessary routines to do a complete calibration of this instrument. The data generated by the program will be written out to the disc in drive 1 . Press CONTINUE to load the NBS type clibration supervisory program. If a winchester drive is designated as the mass storage device, then the calibration program will load without user intervention.

3. The program will ask for the test number of the present test, and an identification of the unit under test. The program will attempt to find a previously defined data file (D_nnnnnnnn, where nnnnnnnn is the test number) for this test. If a data file has not been created, or if the one it finds is empty, the program will ask you if an n-test should be performed. If you answer with with a "Y", a data file is created, the test number and identification are written out to the file, and the n-test routine is loaded and executed. If you answer with an "N", then processing continues at the NBS type command menu, and you will be requested to supply the proper test $M$ and $N$ during the calibration. If a data file already exists, an option menu will be displayed. This will be covered in more detail later.

4. The n-test routine will load 11 subroutines from disc and set the default parameters. These are displayed on the CRT along with a function key menu used to modify these defaults. The options available are:

i) KO: STARTING VOLTS: The initial voltage for the n-test (default is 70 volts or 170 volts, depending on the current rating of the thermal element under test).

ii) K1: HIGHEST VOLTS: The final voltage for the n-test (defaults to 100 volts or 200 volts, depending on the TE).

iii) K2: VOLTAGE STEP: The step between $V$ and dV. This is defined as either a percentage of the currently applied voltage, a percentage of the final voltage, or a flat step. The default is . $1 \%$ of the presently applied voltage. 
iv) K3: VOLTAGE INC.: The increment between successive nominal levels of applied voltage (default is 5V).

v) K4: EXECUTE: starts the n-test procedure.

vi) K5: PRINTER OFF: Disables printouts of each individual voltage step (default is printer on).

vii) K6: HEADINGS OFF: Disables headings from being printed on the hardcopy device (default is headings on).

viii) K7: DEGREE OF FIT: Allows the characteristic response equation to be fitted to any degree, although a linear fit is generally good enough and higher orders are not yet supported anyway.

ix) K8: EXIT: Returns processing to the NBS type supervisory program.

5. If any changes to the default parameters are desired, press the function key associated with that parameter. A prompt will be displayed, and the value may be entered in the blank space at the prompt line. The option menu will then be redisplayed to request the next operation. Connect the standard channel detector cable to the output of the TVC and set the detector switch to position 3 .

6. When the 'GO' key is pressed, the IEEE-488 instruments needed for an n-test (dc supply, dc voltmeter, and data acquisition system) are initialized to the required conditions.The screen will be erased, and a display, divided into five "windows", will be painted to the CRT. From the top, these windows display the following:

i) The test number, date, and test unit identification.

ii) The present voltage, output EMF, initial and final nulled output EMF, and the incremental nulled EMF.

iii) The balancing process for the standard channel DAC.

iv) The applied voltage, output EMF, $n$, and the standard deviation of the previous points in the n-test.

v) Finally, the fitted characteristic response equation.

7. When the voltage is brought up on the dc calibrator, set the output switch on the front panel to the 'ON' position. The system will wait for things to settle, and during this time the output voltage may be monitored with the dc voltmeter.

8. As the n-test routine runs, the values read from the data acquisition system, as well as the present test parameters, are displayed in the appropriate places in the realtime display. This feature is a nice advance over the older software, in which test data tended to scroll upwards and off the screen. 
9. In the course of the n-test, the applied voltages are set automatically, the DACs balanced, and the data collected and reduced with no operator intervention. If a problem does occur, however, there are two procedures to avoid (or at least limit) any possible damage to the system and test units. In the first case, the computer monitors the status byte of the dc supply and its output voltage (via the dc voltmeter), before each reading. If the voltage exceeds the nominal applied voltage, or if the dc calibrator self-destructs, the computer will open the output relay to disconnect the output voltage from the test unit. For all system errors, an error message, with possible remedial action and a prompt for instructions is displayed across the bottom of the screen. Since the display line is limited to 80 characters, the message scrolls in from right to left. In most cases, you will be given a choice of continuing the measurement process or exiting the routine.

10. In the second case, for serious "total disaster" types of faults, function key K9, labeled 'HARD KILL' will immediately reset all supplies, open the output relay, and return processing to the supervisory level option menu. THIS KEY MAY BE USED AT ANY TIME IN THE PROCESSING! For faults which are not so dramatic, pressing SHIFT-K9: (K19) 'SOFT KILL' halts processing but leaves the supplies and data acquisition system unaffected. Processing continues at the n-test command menu.

11. After the $n$-test is complete, processing will return to the supervisory option menu for further choices by the operator. In addition, the n-test data will be written to the appropriate data file on disc (drive 1 or the appropriate winchester volume), and the results will, if desired, be printed to the current hardcopy device. automatically.

12. If a data file for this test number already exists, you may run another n-test, or choose one of the other options. These options include the following:

i) KO: N-TEST: This option initiates an n-test as described above.

ii) KI: AC-DC DIFF.: Initiates an ac-dc calibration of the test unit.

iii) K2: SYSTEM STATUS: Displays the present system status.

iv) K3: HELP: Invokes the NBS type help facility.

v) K8: EXIT: Exits the routine and returns to the ac-dc master program supervisor.

vi) K9: HARD KILL: THIS KEY WILL RESET THE VOLTAGE SUPPLIES AND OPEN THE OUTPUT REIAY TO DISCONNECT THE VOLTAGE FROM THE UNITS UNDER TEST.

vii) K19: SOFT KILL: Pressing the SHIFT key and K9 stops processing but leaves the supplies and data acquisition system unaffected. If the shift key is 
pressed simultaneously with the n-test or ac-dc difference keys (SHIFT-Ko or SHIFT-K1), then the system will automatically reset the voltage supplies, open the output relay upon completion of the sequence, and return processing to the NBS type command option menu. If the SHIFT key is not pressed, however, processing will be halted upon completion of the test sequence, the supplies will remain active, the output on, and another option menu will be displayed.

13. The next logical step is to run an ac-dc calibration, and this is initiated by pressing Kl. Nineteen subroutines will be loaded from the supervisory disc, and several variable initializations and file assignments made. If, in the original supervisory option menu, the SHIFT key was pressed together with the 'NBS TYPE' key, the system reads the command procedure from the disc. If the 'NBS TYPE' key was pressed alone, however, an input menu, requesting the standard unit, applied voltage, standard range, test range, and desired frequencies is displayed, and the parameters for the test are entered at this time. When the output voltages are set on the ac and dc calibrators, position the output switch to 'ON' and check the output voltages on the ac and dc voltmeters.

14. If the calibration is to be made from a standard calibration file, the system will now pause to let the units under test warm up. The length of time allowed for this warmup is range-dependent and varies from five minutes at voltages of less than $60 \mathrm{~V}$ to 45 minutes for voltages of $600 \mathrm{~V}$ and more. If the test is to be done manually, a warmup menu is displayed to allow you to select the proper warmup time as follows:

i) K1-K6: CONTINUE WITH MEASUREMENTS: starts the measurement sequence.

ii) K2-K7: START SEQUENCE: If no automatic warmup is desired, this functions in the same manner as K1-K6. If, however, the system is currently in the midst of an automatic warmup period, this action aborts the warmup and starts the sequence.

iii) K3-K8: AUTOMATIC WARMUP: When pressed, this action starts the automatic warmup period for the present voltage range.

iv) K5: SYSTEM STATUS: Displays the present system status.

v) K9: HARD KILL: ABORTS ALL PROCESSING, RESETS THE SUPPLIES, AND RETURNS PROCESSING TO THE NBS TYPE COMMAND OPTION MENU.

vi) K19: SOFT KILL: Stops processing but leaves the supplies and data acquisition system unaffected. 
15. In any case, processing resumes with the system reading the EMFs from both the test and standard channel. If no output is measured, the system presents an error message and suggests corrective action. You will then be instructed as to the position in which to set the detector switch on the front panel. This action will ensure that the output EMF is divided down to a level where the DACs can work without being under- or overranged. Press CONTINUE, when the switch is set, to continue processing.

16. After processing is continued, the program will paint a realtime display composed of the following five windows (from the top):

i) The test number and instrument identification.

ii) Present test data (the ac-dc difference and dc reversal data).

iii) A summary of the ac-dc differences for this set of four determinations.

iv) A presentation of the test parameters (standard unit, output EMFs, currently applied voltage and frequency, etc.).

v) Previous results for all frequencies at this voltage.

17. If all goes normally, the system will test all frequencies at the present applied voltage without operator intervention. The system will monitor the status bytes of the ac and dc supplies, as well as their output voltages (via the voltmeters) and will reset the system if either output voltage exceeds the present nominal voltage range. Any system faults, including output voltages out of range, equipment faults, and so forth, will be presented on the bottom of the display and scrolled from right to left. These error messages usually include several options (exit to the command menu, continue with the sequence) as well as suggestions for remedial action on your part. As always, function key K9: 'HARD KILL' will disconnect the output voltage from the units under test, reset the supplies, and return processing to the NBS type command option menu. Pressing SHIFT-K9: 'SOFT KILL' will halt processing but leave the supplies and data acquistion system unaffected.

18. During the calibration sequence, the data gathered from the data acquisition system will be displayed in realtime. At the conclusion of each frequency, the ac-dc difference results for that frequency will be sent to the currently active hardcopy device.

19. If the system is running from a standard calibration file, at the completion of each voltage range you will be prompted to reconfigure the test and standard units for the next range. Upon pressing ' $C$ ', processing will 
continue at the next voltage range, with the automatic warmup facility in effect.

20. When the automatic calibration test sequence is complete, or if the automatic shutdown option is in effect, at the end of the final frequency on the present range, the system will reset the supplies, open the output relay, and return processing to the NBS type command option menu. If manual shutdown has been specified, the following menu is displayed:
i) KO: REPEAT VOLTAGE:
This key repeats
all

frequencies for the present applied voltage.

ii) K1: REPEAT FILE: This key will force the system to repeat the previous standard calibration file.

iii) K2: INSERT NOTE: Use this key to insert a note on the hardcopy. The note may be up to 320 characters in length.

iv) K3: FREQUENCY: This key allows the definition of up to eight new frequencies to be calibrated at the currently applied voltage. The frequencies are input into the usual test parameter input menu.

v) K4: NEW VOLTAGE: This key presents the test parameter input menu and allows the definition of a new voltage range and frequencies. The supplies and data acquisition system will be reset when this option is chosen, and will be initialized to the new test parameters.

vi) K8: EXIT: Returns processing to the NBS type command option menu.

21. In addition to the prompts and options described above, on occasion the system may need some information that has not been previously defined. The four most notable occasions are:

i) The standard $M$ and $N$ have not been defined in a data file.

ii) No n-test was run on the test unit and therefore no test $M$ and $N$ have been defined.

iii) The ac-dc corrections for the standard unit have not been defined in a data file.

iv) A series voltage-dropping resistor is being used to make the ac and dc supplies operate at a higher voltage where they tend to be more stable. In any of these cases, the system will prompt you for the proper information and continue the measurement process normally.

22. Upon exiting the n-test or calibration routine and returning to the NBS type command option menu, the function keys defined in this menu may be used to restart a test or to exit from the NBS type calibration supervisor. If the latter action is taken, the Holt model 11 subroutines are deleted from memory and processing is returned to the ac-dc master program 
option menu. From there, pressing any key will take the action described by that key, and pressing 'EXIT' will exit the program and return processing to the BASIC 5.0 system. 
GENERAL INSTRUCTIONS FOR PREPARING AC-DC TEST REPORTS

General Information

1. The ac-dc test report files are stored in the subdirectory [.REPORTS] of the ac-dc directory on the VAX. The reports are created and edited using EVE (Extensible VAX Editor), a text processing package supplied by Digital Equipment corp. A template for each type of transfer standard (Fluke 540B, Holt Model 11, etc.) that the Division has calibrated has been stored in the reports subdirectory using the file nomenclature 'instrument.RPT'. The extension .RPT indicates a report file and all new report files should bear this extension. Report files are generally named for the test number of the ac-dc test. In the case where no test number has been assigned to a test, the file is usually named to reflect the customer. The report file should then be renamed to the test number after one is assigned.

\section{Generating New Reports}

2. To $\log$ in to the reports subdirectory, find an unoccupied terminal connected to the VAX. Turn it on and press RETURN. The VAX will respond as follows (your replies are in upper case):

Username: ACDC REPORTS

Password: REPORTS

(note that the password is not echoed to the screen)

The message of the day will appear, followed

by a

$\$$

3. The $\$$ is the VMS prompt and indicates that the VAX is ready to receive instructions. If you need to work with an instrument template, type

\$ COPY instrument.RPT filename.RPT

This copies the instrument template into a file which you can now edit.

4. To enter EVE, type

\$ EVE filename.RPT (filename.RPT is the name of the test report file)

If the report file has not been created, EVE will create the file. If the file already exists, EVE opens the file and displays the first page of the contents on the 
screen. In either case the file is ready to be edited.

5. The EVE Users Manual is located in room A-144. The use of EVE is explained quite well and these general instructions will not go into all the gory details. A few points, however, are worth mentioning:

i) Take note of what type of terminal you're logged into. The VT240 in A-144 has a different keypad than the Microterms (which are VT100 clones). The manual provides instructions on how to use each type of terminal.

ii) In addition to the documentation in the manual, help may be obtained from EVE in two ways. On the VT240, pressing the HELP key displays a representation of the keyboard and a list of the defined control codes and function keys. Pressing DO and then typing 'HELP' on the command line will list the editting commands currently recognized by EVE. On the VT100 clones, HELP is assigned to key PF2 on the numerical keypad, and Do is assigned to PF4.

iii) There are several ways to end an EVE session. These are:

a) EXIT - This saves the text currently in the buffer and exits EVE.

b) CTRL-Z - This is the same as EXIT.

c) QUIT - This exits EVE but does not save the text currently in the buffer. If changes have been made to the text in the buffer, you will be given a chance to discontinue QUITting before EVE throws out your text.

d) CTRL-Y - This crashes EVE, wipes out the buffer, and returns processing to the VMS command level.

e) The system crashes - Who knows what happens?

6. If EVE has been exitted (EXIT or CTRL-Z) the text is written out to a file which has a version number 1 more than the version number of the file you loaded into EVE. This means that if you are totally disgusted with what you just did, the original file is not lost or written over, and can be EVEed again.

7. QUITting flushes the buffer and leaves the file as it was before the EVE session.

8. CTRL-Y and system crashes both should generate journal files. These files contain a record of every keystroke made during an EVE session. To reconstruct the file in EVE after such an event, enter EVE by typing

\section{\$ EVE/RECOVER filename.RPT}

You will be able to pick up more or less where you left off (the last few commands may not make it into the file). Note, however, that in the event of a system 
crash, the journal file may not make it to the disc. In this case, you will have to start over at instruction 4.

9. To get a hardcopy of the report, type either

\$ PRINT filename.RPT

\section{or}

\section{\$ LASER filename.RPT}

In the first case, the output will be directed to the Hewlett-Packard LaserJet printer located beside the manual comparator. This printer may be set to either cassette feed or manual single-sheet feed. See the manual for instructions on how to use these modes. The second command will send the output to the LaserJet printers in the computer room. The first 60 lines of your text will be printed on letterhead bond on the printer on the right (on top of the CPU cabinet), and the remainder of the file will be printed on plain bond on the printer on the left (atop the expander cabinet).

10. To send the hardcopy to the Printronix P-300 printer in the computer room, type

\$ PRINT/QUEUE=SYS\$PRINT filename.RPT

11. To end the session on the VAX, you may log off using any of these commands:

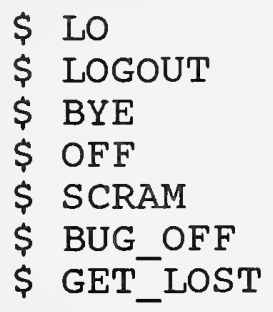

The VAX will display the time of logoff and begin ignoring you. 
INSTRUCTIONS FOR USING THE MANUAL AC-DC DIFFERENCE COMPARATOR SYSTEM E. S. Williams and C. B. Childers

\section{Contents}

I. General

II. Power Supplies and Controls

III. Console

IV. TE Comparator

V. Galvanometer Circuits and Shields

VI. AC-DC Difference Tests

VII. Grounding

VIII. Calculation of $\mathrm{nE}$

IX. Low-Frequency Tests 


\section{General}

This comparator system is used to make ac-dc difference tests of voltage and current converters plus certain other tests. The nominal range of testing is 1 to $1000 \mathrm{~V}$ from $20 \mathrm{~Hz}$ to $100 \mathrm{kHz}$ and $5 \mathrm{~mA}$ to $20 \mathrm{~A}$ from $20 \mathrm{~Hz}$ to $50 \mathrm{kHz}$; however, with certain precautions and additional equipment, tests can be made down to $2 \mathrm{~Hz}$ at low voltages and currents and up to $1 \mathrm{MHz}$ from 1 to $100 \mathrm{~V}$.

The standards are thermal voltage converters (TVC's) for voltage measurements and single-range thermoelements (TE's) for current measurements. Their ac-dc differences are determined by a series of special tests described in other parts of this publication. The corrections are generally small and are known over the full working range.

The TVC set (Model F, No. 7) consists of six series resistors and two TE's: F1 (2.5 mA, $400 \Omega)$ and F2 $(5 \mathrm{~mA}, 400 \Omega)$. Range combinations from 1 to $1000 \mathrm{~V}$ are assembled as indicated by the labels on the eight units in the set.

For calibrations at currents up to $500 \mathrm{~mA}$ the TE's that serve as thermal current converters (TCC's) are mounted on a four-pole rotary switch (in box NBS 84). These TE's are all of the insulated-bead type, and all except the 500-mA unit are in vacuum enclosures. Separate unmounted TE's are used at $1 \mathrm{~A}$ and higher currents.

Calibration tests are occasionally made for instruments with a pointer-andscale readout. All of the above tests are usually made by observing and evaluating small changes in thermal EMF's as ac and dc voltages or currents are successively applied to the test and standard instruments connected together. The equipment and procedures are described in the following sections. 


\section{Power Supplies and Controls}

The voltage and current supplies, a matching output transformer, and remote control connections are mounted in a rack which stands to the left of the operator.

Direct Voltage is supplied by a Cohu dc voltage calibrator, Model 324, which has been modified by the addition of a remote control. The six control dials are used when the local-remote switch is set at LOCAL. When the switch is turned to REMOTE, the control shown in Fig. 1 is substituted for the three lower control dials.

If another supply without fine controls is substituted for the regular one, the series-resistance control shown in Fig. 4 may be inserted in series with the output.

Direct Current is supplied by a Systron-Donner Model M5C15-50 TOV, with voltage and current controls on the front panel. The front-panel controls for current can be replaced by a 500- $\Omega$ potentiometer connected across terminals 1 and 2 on the back of the supply (see Instruction Manual, Section 2.3). Fine remote adjustment is provided by connecting $10 \Omega$ across these terminals and placing the circuit shown in Fig. 2 in parallel. One of four sensitivities may be selected by means of a lever switch on the side of the control box.

AC Voltage and Current are supplied by an Optimation oscillator, amplifier, and transformer system, Model AC-104. The output of the oscillator (10 $\mathrm{Hz}$ to $100 \mathrm{kHz}$ ) is connected to the remote coarse and fine control shown in Fig. 3. This circuit is used to adjust the input to the amplifier. The coarse control is most satisfactory when it is operated near full range: 8 to 10 turns. The oscillator gain control, therefore, should be set for an output level that is only a little higher than adequate for the desired amplifier output.

Maximum rated voltage outputs for the seven transformer taps are listed in the table below. The output is most stable at about $25 \%$ of these voltages; thus, a tap about four times higher than the test voltage is recommended. The amplifier does not perform well at the extreme frequencies. An additional tap increase to about eight times the test voltage is suggested for tests below 20 $\mathrm{Hz}$ and above $70 \mathrm{kHz}$.

The maximum current ratings for the seven taps are listed under "Current Mode" below (Table 1). Current stability is satisfactory up to at least $50 \%$ of maximum.

A small load should be kept on the transformer at all times when it is operating in the current mode. Load resistors and switches are shown at $\mathrm{E}$ in Fig. 6A. These are substituted for the current converter load by using the rotary ac-dc switch at $C$ in that figure. (See $C$ and $E$ in Section III.) 
Table 1

\section{Voltage Mode}

$16 \mathrm{~V}$

$40 \mathrm{~V}$

$80 \mathrm{~V}$

$160 \mathrm{~V}$

$400 \mathrm{~V}$

$800 \mathrm{~V}$

$1600 \mathrm{~V}$
Current Mode

$20 \mathrm{~A}$

$8 \mathrm{~A}$

$4 \mathrm{~A}$

$2 \mathrm{~A}$

$0.8 \mathrm{~A}$

$0.4 \mathrm{~A}$

$0.2 \mathrm{~A}$
Load Resistance $(\Omega)$

Short

0.5

0.5

0.5

1.5

1.5

5.0

During an intercomparison the ac and dc test voltages are adjusted to produce nearly the same response (or output) of the test TVC by using the supply controls. The final null adjustments can be made with the 10 -turn, $1000-\Omega$ helical potentiometer in the controller box. (See Fig. 4.) The 200- $\Omega$ resistor is connected in series to improve the linearity. The potentiometer is shunted by one of ten selectable resistors of different values. The minimum (nonzero) resistance is $10 \Omega$ (for low-voltage control) and the maximum is about $820 \Omega$. A suitable control sensitivity can be found by selecting an appropriate shunting resistance.

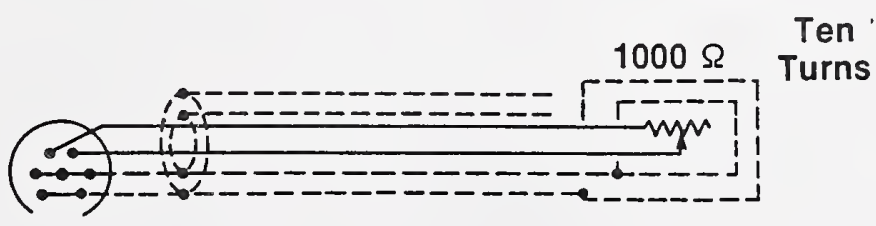

Figure 1. DV Contro1

$30 \Omega$

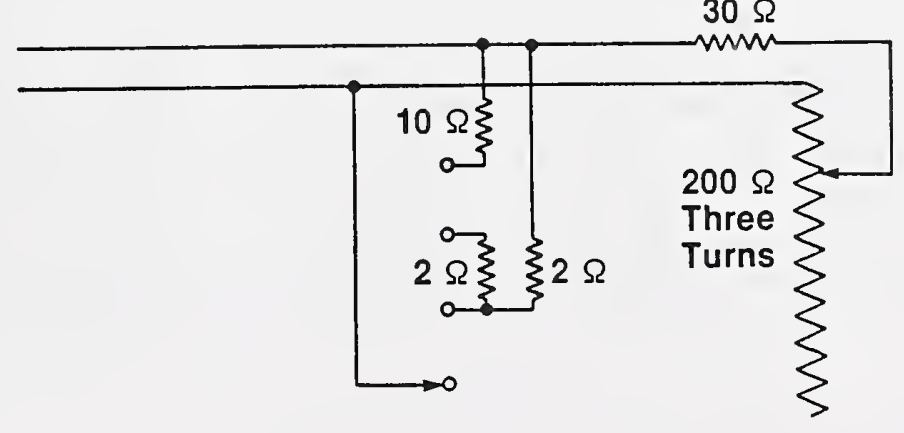

Figure 2. DC Control 


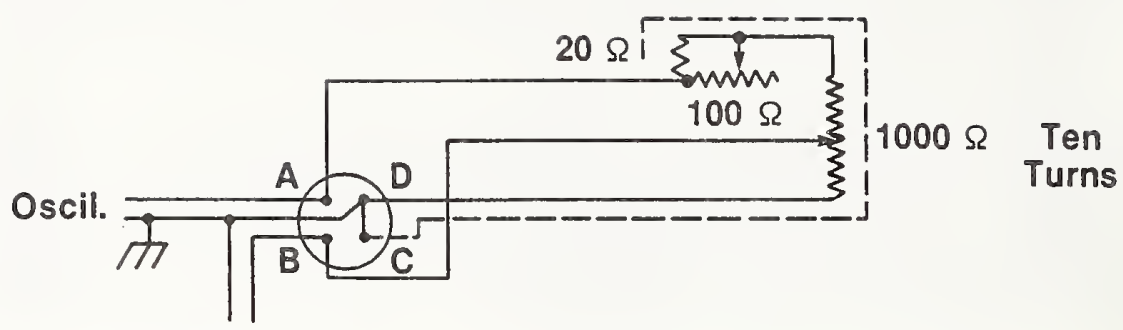

To Ampl.

Figure 3. AV-AC Control

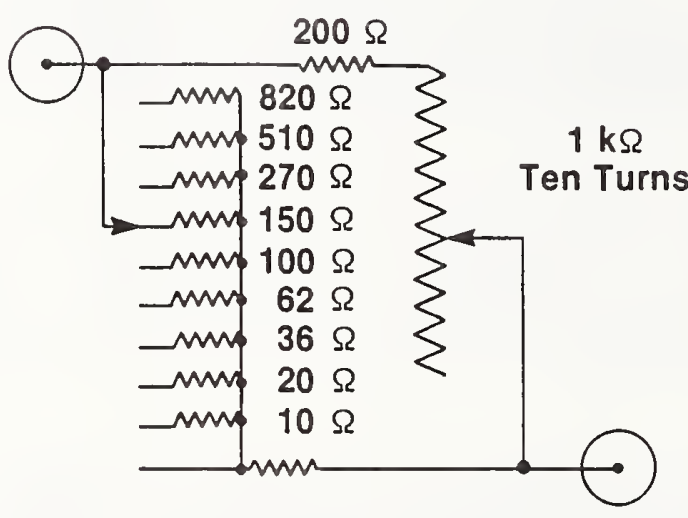

Figure 4. Series-Resistance Control 


\section{Console}

Ac power is connected to the monitoring voltmeter and ammeter and the galvanometer lamps as shown in Fig. 5. The ac-dc and av-dv test circuit diagrams are shown in Figs. $6 \mathrm{~A}$ and $6 \mathrm{~B}$.

The input to each circuit goes through a small bakelite panel on the left side of the console. Ac voltage and current are connected through separate GR 874 coaxial connectors. Dc current is carried by a twisted pair of wires extending from the power supply to the ac-dc switch and passing through a grommet in the input panel. Dc voltage is carried by a shielded pair of wires connected to the terminals on the back of the power supply inside the rack. These wires pass through a grommet in the input panel to two fuses mounted on the panel.

The signals for ac and dc voltage and current are provided through coaxial cables on the console front panel. The voltage cable (labeled "AV-DV") terminates in a GR 874 connector, and a GR 874 tee is ordinarily used to connect test and standard instruments together. The voltage circuit is completely shielded if the test instrument has a coaxial input connector and is itself shielded.

The current-carrying cable (labeled "AC-DC") has spade lugs for connecting to current converter terminals.

Certain features of these circuits are marked with the letters A through $\mathrm{J}$ on the diagram and are explained in the following paragraphs.

A. This pair of terminals (labeled "DC") lets the operator insert instruments or additional resistors in the current circuit. An ammeter or, if more exact measurements are needed (as in an n-test), a shunt box may be connected here. A resistor may be connected here also to better match the total load to the $15-\mathrm{V}$ dc supply. Additional loading is provided on the low-current ranges ( $1 \mathrm{~A}$ and lower) by a resistor added at the ammeter input terminals. The additional load requires about a $2-\mathrm{V}$ drop at rated current and is used to improve the current stability.

B. This two-pin connector makes available to the operator the voltage drop across the ammeter plus the additional resistor. This potential could be monitored during an $n$-test; however, it usually is not.

C. The ac-dc switch is a twelve-position, three-pole, enclosed silver-contact switch with no stops. Ac and dc currents are switched to the test and standard instruments in the normal test sequence: $\mathrm{AC}, \mathrm{DC}+, \mathrm{DC}-, \mathrm{AC}$. Two sequences require one complete revolution of the switch. The switch is usually turned in only one direction; however, it may be advantageous to use only one of the ac positions at high frequencies and high currents. The latter procedure keeps the total impedance more nearly constant, and less readjusting of the ac current is required. 
D. The ac-dc switch has make-before-break contacts so that as the instruments are disconnected, and while they remain disconnected, the current supply voltage is applied at the points marked "D." If a different dc supply is used that requires a continuous load, as many current-regulated supplies do, a shorting switch or a fixed load can be added at these points.

E. The Optimation ac current supply (current mode) requires a very low resistance load on the 20-A range and a small load on the other ranges. Provisions have been made for a continuous load of $5 \Omega$, and this may be shunted by $2 \Omega, 0.5 \Omega$, or a short. Loads which have been found satisfactory for each range are listed in Table 1 , Section II. The corresponding switches on the console front panel are labeled "AC load (ohms)," and they are individually labeled "1.5," "0.5," and "0." These are the approximate load resistances when that switch is closed. The ac contacts in the ac-dc switch connect these loads to the ac supply as the instrument load is disconnected and while it stays that way.

F. After the test and standard TVC's are connected to the av-dv cable, av or $d v$ is selected and $d v$ is reversed by means of relays at $F$ (Fig. 6B). The relays are powered by $24-\mathrm{V}$ dc from a rectifier supply and activated by microswitches which are closed by a cam on a stepping motor. The stepping motor is advanced by closing a foot switch momentarily.

G. The av-dv circuit is activated by closing a four-position rotary switch, which also turns on the $24-\mathrm{V}$ rectifier as well as the left-hand amber lamp to indicate that voltage is applied to the TVC's. This lamp should be on (with neither relay activated) during the preliminary adjustments, EMF measurements, and warm-up. Switch $\mathrm{G}$ can also be used to insert a voltagedropping resistance of 900 or $1800 \Omega$ in series with the TVC's. This additional resistance permits finer adjustment and promotes improved stability for the lower TVC ranges $(0.5$ to $10 \mathrm{~V})$ by allowing the ac and dc power supplies to operate at an output of $10 \mathrm{~V}$ or more. The ac-dc voltmeter (eleven ranges, 0.5 to $1000 \mathrm{~V}$ ) indicates the nominal test voltage at the av-dv cable.

Note: This voltmeter is not accurate when both the voltage and frequency are high and may read low by as much as 508 at $50 \mathrm{kHz}$.

H. A red neon light on the front panel warns that hazardous voltage is applied. It begins to glow at about $100 \mathrm{~V}$ and is brightly lit from 500 to $1000 \mathrm{~V}$.

I. A plexiglass shield is placed between the operator's position and the test and standard instruments to help prevent personal contact with the high voltage. This is of particular value for instruments having binding-post input terminals. A shock is also possible if the coaxial connectors are opened while voltage is applied. Removing the shield will create an open circuit in the av-dv output lead at relay J. Hence, the switch at $G$ should be OFF, with all three indicator lamps out, before the shield is removed.

J. This relay has been included in the above discussion. 


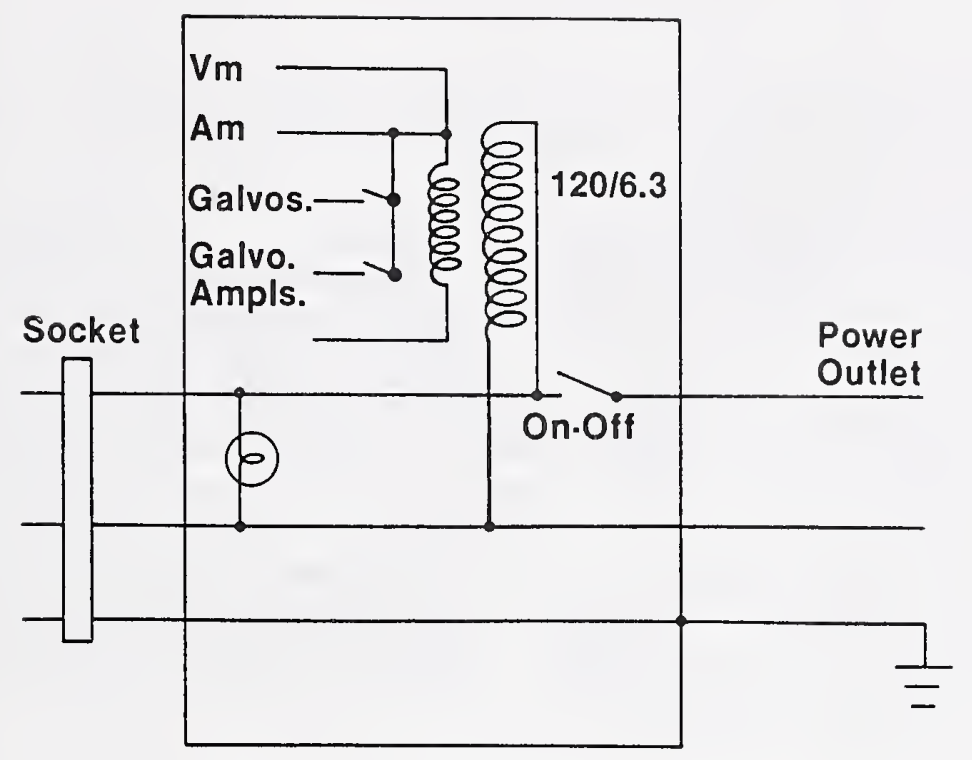

Figure 5. Console Power Switches

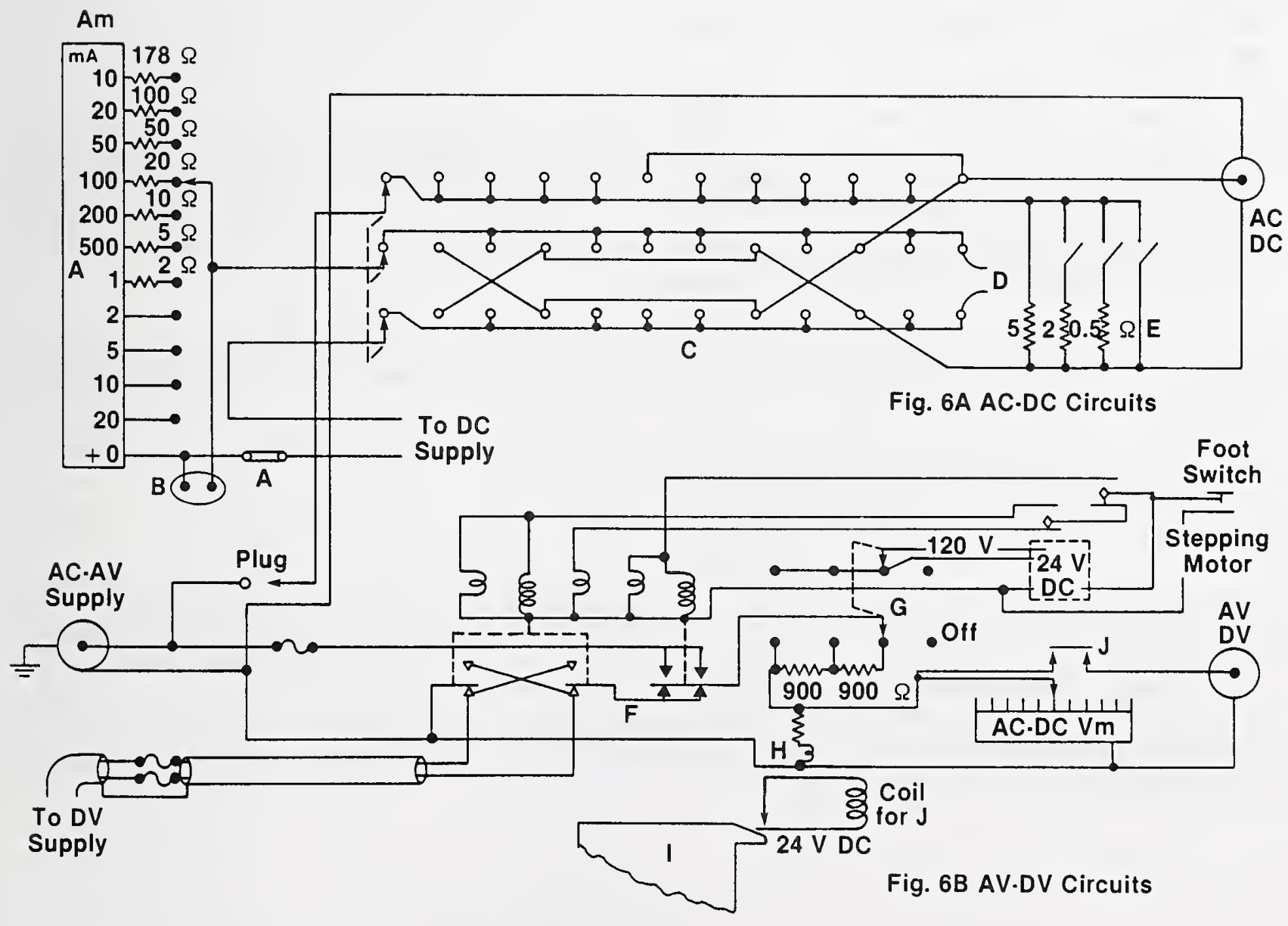

Figure 6. AC-DC and AV-DV Circuits 


\section{TE Comparator}

Various comparator circuits can be used to minimize the effect of power-supply instability. In this comparator a voltage-divider circuit is used to balance a fraction of the larger output EMF against the smaller one. The balance or null is detected by a photo-cell galvanometer. At the null the divider setting corresponds to the ratio of the two $\mathrm{EMF}^{\prime} s$. If the test EMF ( $E_{t}$ ) is held constant as ac and both polarities of dc are successively applied to the TVC's or TE's, the standard $\operatorname{EMF}\left(E_{S}\right)$ will change as a function of the ac-dc difference of the test instrument relative to the standard at the chosen frequency. The resulting imbalance in the divider will then produce a change in the detector deflection proportional to this ac-dc difference. Small fluctuations in the power-supply output produce nearly equal proportional changes in the two EMF's, and so the indication is not appreciably affected by these fluctuations. The relative immunity to instability depends on how well matched the time constants and response characteristics of the TE's are, but the stabilizing effect of the divider circuit usually affords a significant advantage. Also, this arrangement relaxes the requirement that the monitored EMF be held so precisely constant.

In this comparator the higher of the two EMF's is connected across a $1000-\Omega$ Kelvin-Varley divider ( $E_{H}$ input) and the lower EMF $\left(E_{L}\right)$ to the variable tap. The potentiometer is connected to the test instrument output $\left(E_{t}\right)$, whether $E_{L}$ or $E_{H}$, during an ac-dc difference determination. The test EMF is monitored with the potentiometer by using the set key and galvanometer \#1. Imbalance EMF's in the divider circuit are measured with the \#2 galvanometer by using the read key.

The divider switch is moved from READ to CAL to introduce a $0.5-\Omega$ change in the resistance of the divider circuit $(\Delta R / R=500 \mathrm{ppm})$, and the resulting change in indication of galvanometer \#2 is observed and used to compute the scale sensitivity $(\mathrm{K})$ in $\mathrm{ppm} / \mathrm{mm}$ or percent $/ \mathrm{cm}$. The derivation of the equations and an explanation of their practical applications are given below.* The test procedure is outlined in Section VI.

A 2- $\Omega \Delta \mathrm{R}$ can also be introduced. This resistance change and the $n$-test key can be used in making determinations of $\mathrm{n}$. (See the paper cited below in the footnote for more information.)

*This derivation is from the paper presented as section 9 of this publication (E. S. Williams, "Thermal voltage converters and comparator for very accurate ac voltage measurements," J. Res. Nat. Bur. Stand, vol. 75C, pp. 145-154, July-Dec. 1971). Figs. 6 and 8 of that paper show the circuit of the TE comparator and complement the above discussion. Fig. 9 of the same paper is a photograph of the TE comparator. 


\section{Development of Equations ${ }^{8}$}

The ac-dc difference of a TVC is defined as

$$
\delta=\frac{V_{a}-V_{d}}{V_{d}}
$$

where $V_{a}$ is the a-c voltage and $V_{d}$ the average of the two directions of dc-voltage required to produce the same output emf. In the circuit of figure 6 the a-c and $d \cdot c$ voltages are adjusted to give the same emf $E_{\imath}$, of the TVC under test, as indicated by a null on the detector $N$ with $\mathrm{k}_{1}$ closed. The same voltages are applied to the standard TVC. Then,

$$
V_{a}=V_{d}\left(1+\delta_{t}\right)=V_{d}^{\prime}\left(1+\delta_{s}\right)
$$

where $V_{d}^{\prime}$ is the d-c voltage required to produce the same output emf of the standard as $V_{a}$, and the subscripts, $t$ and $s$, refer to the test and standard instruments respectively. If $V_{d}^{\prime}-V_{d}<<1$, then, closely enough

$$
\delta_{t}-\delta_{s}=\frac{V_{d}^{\prime}-V_{d}}{V_{d}} .
$$

From the definition of $n$ given in the paper

$$
\delta_{t}-\delta_{s}=\frac{E_{s a}-E_{s d}}{n_{s} E_{s d}}
$$

With the polarities as shown in figure 6 ,

$$
N=m E_{t}-E_{s}
$$

where $N$ is the detector voltage with $\mathrm{k}_{2}$ closed and $\mathbf{k}_{\mathbf{1}}$ open, and $m$ is the divider ratio (with the detector resistance $R_{d} \gg R_{1}$ ).

Thus,

$$
\delta_{t}=\delta_{s}+\frac{N_{d}-N_{a}}{n_{s} E_{s d}}
$$

where the subscript $a$ and $d$ have the same meaning as before.

If a galvanometer is used as the detector, the current through it with $k_{2}$ closed is, by Thevenin's Theorem,

$$
I_{g b}=\frac{m E_{t}-E_{s}}{R_{e}}
$$

where $R_{e}$ is the resistance of the galvanometer circuit with $E_{l}$ and $E_{s}=0$. With $E_{t}$ and $E_{s}$ held constant and $p R_{1}$ inserted by means of $\mathrm{S}_{1}$,

$$
I_{g c}=\frac{m E_{l} /(1+p)-E_{s}}{R_{e}^{\prime}}
$$

where $R_{e}^{\prime}$ is the resistance of the galvanometer circuit.
Since $m E_{t}-E_{s}<<E_{s}$ and $p<<1$, we have to a sufficient degree of approximation,

$$
I_{g b}-I_{g c}=\frac{E_{s} p}{R_{e}}=\frac{\left(D_{b}-D_{c}\right)}{S}=\frac{D_{1}}{S}
$$

where $D_{b}$ and $D_{c}$ are the resulting galvanometer deflec. tions and $S$ is the galvanometer current sensitivity.

In the ac-dc test, with $m E_{\ell}$ constant,

$$
I_{g a}-I_{g d}=\frac{E_{8 d}-E_{s a}}{R_{e}}=\frac{D_{a}-D_{d}}{S}
$$

Thus, from (3), (8) and (9),

$$
\delta_{t}=\delta_{s}+\frac{p\left(D_{d}-D_{a}\right)}{n_{s} D_{1}}
$$

The test and standard TVCs may be interchanged if $E_{\ell}<E_{s}$. If $E_{t}$ is applied to the $\mathrm{E}_{\mathrm{L}}$ input of figure 8 and held constant, with the detector and potentiometer connected as shown in the figure, a similar analysis leads to the following equations:

$$
\delta_{\ell}=\delta_{s}+\frac{N_{d}-N_{a}}{n_{s} E_{\ell}}
$$

and

$$
\delta_{t}=\delta_{s}+\frac{p\left(D_{d}-D_{a}\right)}{n_{s} D_{1}}
$$

The characteristic $n$ is determined from

$$
n=\frac{\Delta E / E}{\Delta V / V}
$$

by applying known changes in input voltage and observing the changes in output emf with a high resistance voltmeter, as described in the text. If a galvanometer is used as the detector instead of the voltmeter its sensitivity is determined by inserting $p R_{1}$ as described. From equations similar to (8) and (9)

$$
\frac{\Delta E_{s}}{E_{s}}=\frac{p \Delta D}{D_{1}}
$$

If the thermocouple resistance of the test TVC, $R_{t c}$, is significant, $p$ in equation (7) should be replaced by $p^{\prime}$, the fraction of $R_{1}+R_{t c}$ inserted by $S_{1}{ }^{*}$ Similarly if the potentiometer resistance $R_{p}$ is significant, $p$ in equation (14) should be replaced by $p^{\prime \prime}$, the fraction of $R_{1}+R_{p}$ inseried by $\mathrm{S}_{1}$.

(Paper 75C3\&4-321)

* with a high impedance detector. With

a galvanometer the formula for $\delta_{t}$ is too complicated to be useful.

- These equations were developed by F. L. Hermach. 
These equations were derived on the assumption that only one detector would be used. Equations (5) and (11) apply if the detector is a microvoltmeter, and they differ only to the extent that either the test or standard instrument may have the higher output $\left(E_{t}\right.$ or $\left.E_{s}\right)$.

The corresponding equations [Eqs. (10) and (12)], where one calibrated galvanometer is the detector, are very similar except that EMF's do not appear directly in them. Because we use two galvanometers, we can, and do, use an earlier and more familiar sign convention in the numerator: $p\left(D_{a}-D_{d}\right) / n_{s} D_{1}$.

Deflection changes in $\mathrm{mm}$ or $\mathrm{cm}\left(\mathrm{D}_{\mathrm{a}}-\mathrm{D}_{\mathrm{d}}\right)$ are multiplied by a scale sensitivity factor $K$ to compute ac-dc differences in $\mathrm{ppm}$ or percent. It is more convenient to compute $2\left(D_{a}-D_{d}\right)$ or $2 \Delta D_{1}$ and $2 D_{1}$ from four galvanometer readings than to compute the actual deflection changes. Therefore, the value of $\mathrm{K}$ is $\mathrm{p} / \mathrm{n}_{\mathrm{s}} 2 \mathrm{D}_{1}$, where $\mathrm{p}$ is the fractional change (500 ppm or 0.05 percent) introduced in the galvanometer in the $2 \mathrm{D}_{1}$ measurement and $\mathrm{n}_{\mathrm{S}}$ is the characteristic $n$ for the standard instrument.

Thus, $\mathrm{K}=\frac{500}{2 \mathrm{nD}_{1}}=\frac{500 / \mathrm{n}}{2 \mathrm{D}_{1}} \mathrm{ppm} / \mathrm{mm}$ with $2 \mathrm{D}_{1}$ in $\mathrm{mm}$.

If $2 \mathrm{D}_{1}$ is $280 \mathrm{~mm}$ and $\mathrm{n}$ is 1.8 (as is typical), then $\mathrm{K}$ is approximately 1 . If a graph is made of $500 / n$ vs $E$, one step in the calculation is saved.

Alternatively, $K=\frac{0.05 / \mathrm{n}}{2 \mathrm{D}_{1}}$ percent $/ \mathrm{cm}$ with $2 \mathrm{D}_{1}$ in $\mathrm{cm}$.

If $2 \mathrm{D}_{1}$ is $28 \mathrm{~cm}, \mathrm{~K}$ is approximately 0.001 . 


\section{Galvanometer Circuits and Shields}

The comparator C, galvanometer amplifiers A, and secondary galvanometers $G$ are shown in Fig. 7. The shielding and shield grounds are in dashed lines, and switch $S_{1}$ is closed to connect the comparator circuit to the same ground.

Switch $S_{2}$ and resistors 2 and 3 are used to reduce the read galvanometer sensitivity.

Resistors 5 and 9 serve to insure a nominal external resistance for the galvanometer amplifiers.

The other resistors provide proper damping and sensitivity for the secondary galvanometers. They may be changed if different galvanometers are used. A list of the present values of resistance is shown beside the diagram.

To check the

read galvanometer sensitivity

$\begin{array}{ll}\mathrm{E}_{\mathrm{L}} & \text { open } \\ \mathrm{E}_{\mathrm{H}} & \text { closed } \\ \mathrm{n} \text {-test key } & \text { down } \\ \text { Divider coarse control } & 2 \mathrm{c} \\ \text { Galvo. ampl. gain } & " 6 \circ^{\prime} \text { clock" } \\ \text { Lindeck potentiometer } & 2 \mathrm{mV} \\ \text { Read galvo. light } & 30 \mathrm{~cm} \\ & \text { (use amp. zero control) } \\ \text { Read lever key } & \text { medium (pull) }\end{array}$

$\Delta D$ should be about $10 \mathrm{~cm}$ as the Lindeck potentiometer is turned on and off.

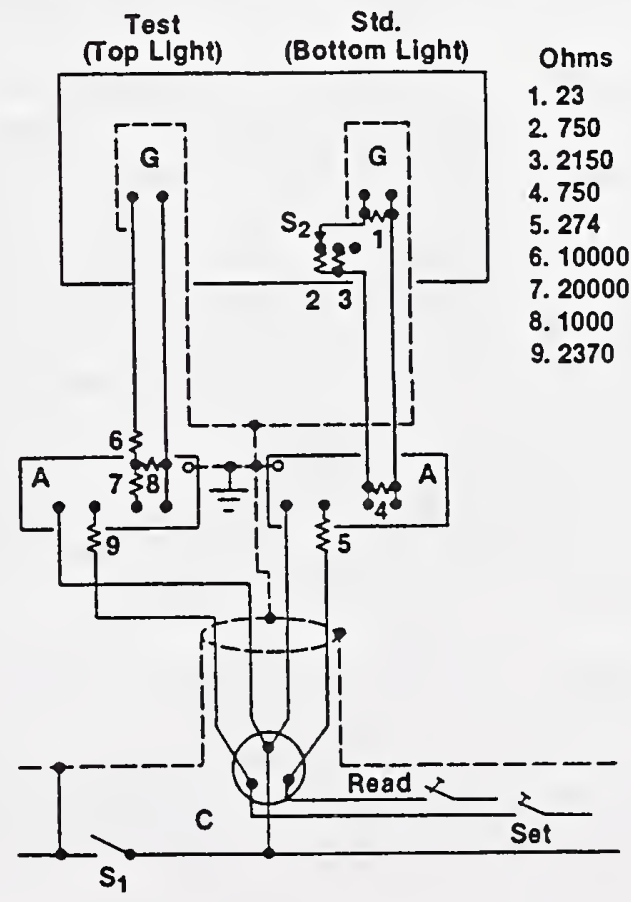

Figure 7. Galvanometer Circuits and Shields 
1. Connect the test and standard instruments to the power cables, and apply a relatively small dc voltage or current.

2. Connect the two output EMF's to the $E_{L}$ and $E_{H}$ connectors on the TE comparator, and bring up the dc input to the desired value while checking the EMF's as in 4 below.

3. Interchange the connections if necessary so that the lower EMF is connected to the $\mathrm{E}_{\mathrm{L}}$ input and the higher one to $\mathrm{E}_{\mathrm{H}}$. Be sure the output polarities are correct.

4. Measure both EMF's with the Lindeck potentiometer, using the set key and the TGL (top galvo. light). See that both EMF's are in the normal range: usually 7 to $10 \mathrm{mV}$.

5. Adjust the divider, using the read key, to set the BGL (bottom galvo. light) near midscale. (If the EMF's are not connected as in 3 above, this adjustment cannot be made. Occasionally the EMF's will be so nearly equal that the divider cannot be balanced with either connection. In this case one EMF can be lowered by inserting a 1000- $\Omega$ loading resistor between the corresponding TVC or TCC output and the comparator cable.)

6. Adjust the gain on the right-hand photo-cell galvo. amplifier so that the deflection change of the $B G L$ is about $14 \mathrm{~cm}$ when the divider switch is moved from READ to CAL with the read key closed. (If the EMF's are low, the maximum deflection change may be less than $14 \mathrm{~cm}$.)

7. Apply ac voltage or current at the test frequency, and adjust it to make the output EMF's practically equal to those obtained with dc.

8. The test parameters may now be noted or measured and entered into the computer. Apply dc for these measurements. After entering the voltage, test range, and standard, measure $E_{S}$ (EMF of the standard) as in 4 above and enter its value. It may be $E_{L}$ or $E_{H}$. Measure $E_{t}$ in the same way, but do not enter it into the computer. Leave the potentiometer set at the $E_{t}$ value for the remainder of the ac-dc difference determinations. Measure $\mathrm{D}_{1}$ as in 6 above. The BGL may be moved down scale with the fine divider control so that the deflections are to both sides of midscale. Record the down-scale deflection (e.g., $18 \mathrm{~cm}$ ), switch from READ to CAL (or vice versa), and record the up-scale deflection (e.g., $32 \mathrm{~cm}$ ). Switch back and record another down-scale reading. The computer will then display the test parameters and a computed value of $\mathrm{K}$ (in $\mathrm{ppm} / \mathrm{mm}$ ).

Reset the BGL to midscale and open the read key.

9. The computer will request the test frequency, which is entered in $\mathrm{kHz}$.

10. Two ac-dc difference determinations can now be made by entering seven galvanometer readings, as requested on the monitor, in the sequence $\mathrm{AC}$, DC, DC, AC, DC, DC, AC as follows. (These readings should be made at equal time intervals.) 
A. Apply ac voltage or current, and adjust the ac controls for a null on the TGL using the set key. (The null position may change, so it should be checked before most settings.) Hold both keys down (the read key may be locked), and read the BGL deflection while keeping the TGL at null. Enter the three-digit reading at the terminal, and unlock the read key.

B. Switch to dc and adjust the dc controls for a TGL null using the set key. Read the BGL with both keys closed. Enter the reading at the terminal, and unlock the read key.

C. Switch to reversed dc and repeat B.

D. Switch to ac and repeat A.

E. Repeat B, C, and D to complete the two determinations. The computer will then display the data and the computed values of ac-dc difference. 


\section{Grounding}

The test and standard instruments are grounded at the ac source. The switching circuit has a direct connection to ground with no switch contacts in that line. If a switch were used in the ground line, and if it opened an instant before the one in the high side opened, the TE bead could be damaged by excessive voltage.

The two cables that connect the TVC's to the comparator have insulation between the connector shell at the TVC end and the cable shield. This insulation helps to prevent the TE heater current from returning to ground by any route except through the TVC casing.

The comparator and galvanometer casings are grounded directly to the laboratory power-mains ground. Their circuits are connected to the detector case through the switch next to the detector connector. If the test TVC has one side of its EMF output grounded, as a few of them do, the grounding.switch should be opened; the comparator and detector circuits are thereby grounded at the test TVC.

The existence of an internal ground on the output of the test TVC is indicated if an ohmmeter shows continuity between the EMF output terminal and the instrument case or low input terminal. The resistance would be very low, of course, since the thermocouple itself is connected to the low input terminal in this situation.

Note: It is advisable to use a high range on the ohmmeter to minimize the current in the thermocouple circuit during this continuity check.

Current converters with binding posts for the input may be affected by the magnetic field around the terminals. Additional voltage is often induced in the thermocouple circuit, causing extra heating, when high currents are applied (5 A and higher). A coaxial lead about $10 \mathrm{~cm}$ long, therefore, should be used between the test and standard instruments at these currents. Any loop at the terminals should be minimized. When a shunted TVC is used as a standard, the shunt casing should be connected to the high or ungrounded line from the power supply.

The figure below illustrates the proper connections.

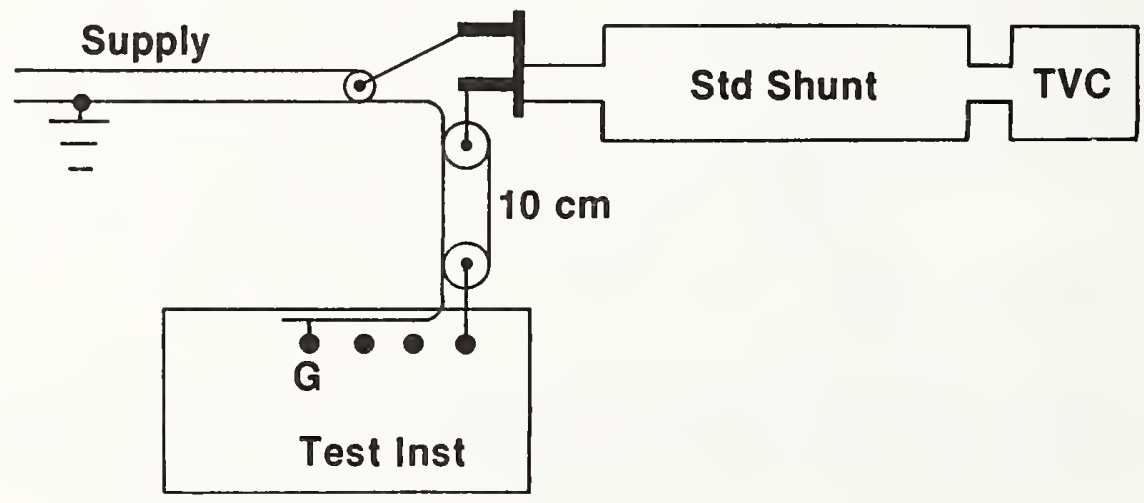

Figure 8. Shunt Connections 


\section{Calculation of $\mathrm{nE}$}

$$
\mathrm{n}=\mathrm{M}-(\mathrm{E} * \mathrm{~N})
$$

A straight line $(A-B)$ is drawn through the most-used portion of the $n$ vs $E$ plot and extended to the intercept $M$ at $E=0$. The slope $N$ is found by reading the difference between $M$ and a point on the $A-B$ line at one of the higher values of $E$ and then dividing the difference by that value of $E$. In the illustration below, $M=2.06$ and the difference is 0.29 at $E=9$. Thus, $N$ $=0.032$.

The product $\mathrm{nE}$ appears in Eqs. (3), (5), and (11) that were derived earlier in Section IV. To illustrate the use of $M$ and $N$, a sample calculation of $k^{\prime}=n E$ for $E=7$ is shown here.

$$
\begin{aligned}
K^{\prime} & =E *(M-E * N) \\
& =7 *(2.06-7 * 0.032)=12.85=n E
\end{aligned}
$$

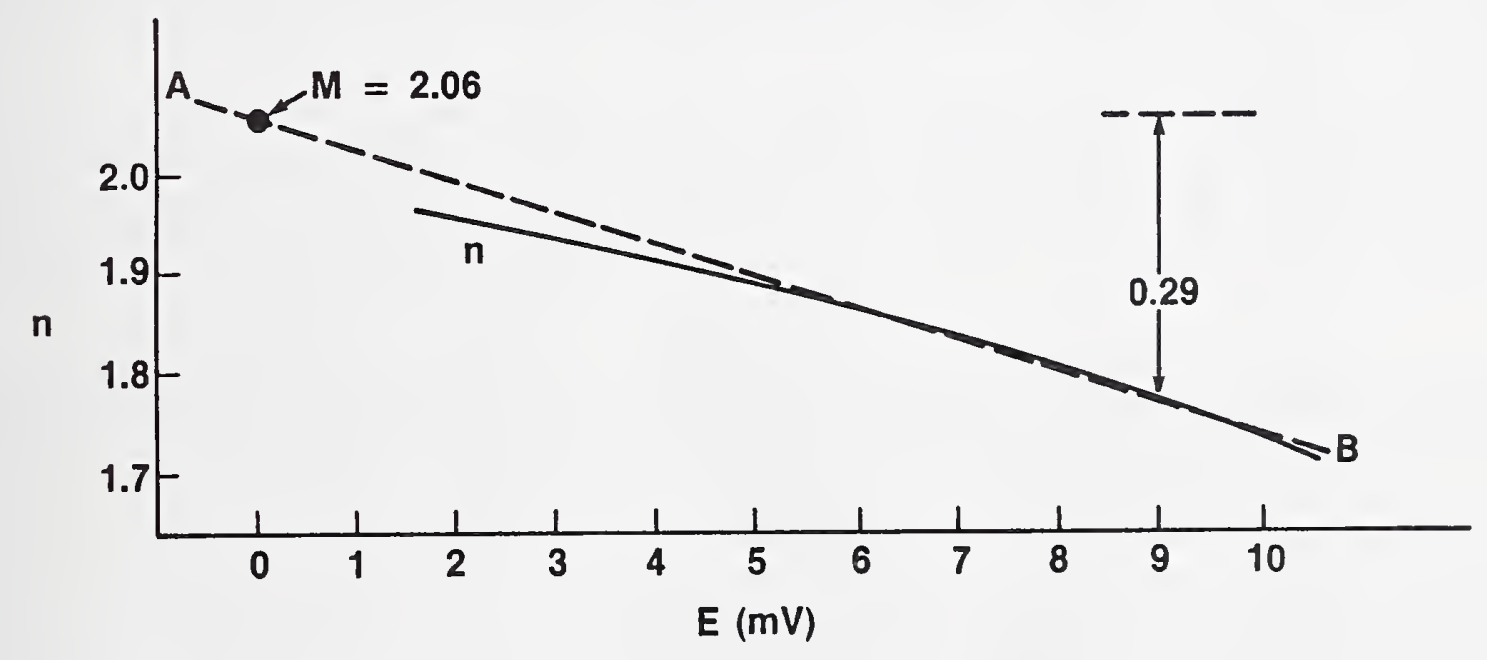

Figure 9. Plot of $\mathrm{n}$ vs $\mathrm{E}$ 


\section{Low-Frequency Tests}

Our calibration service extends down to $2 \mathrm{~Hz}$ at low voltages and low currents (not more than $50 \mathrm{~V}$ and $50 \mathrm{~mA}$ ). Most of the standard TE's have significant ac-dc differences at very low frequencies. The model $\mathrm{C}$ (MHz) TVC's with UHF thermoelements $(10,20,50$, and $100 \mathrm{~V})$ have large corrections because their very short heaters permit substantial cooling between the peaks of ac current. The standard TE's are much better, but still require corrections at $2 \mathrm{~Hz}$. Corrections for the low-mA standards vary with current rating and should increase at higher currents where the heaters are shorter. All TE's perform better at lower-than-rated current because the effect of cooling between ac peaks decreases rapidly as the heater current is lowered.

A special converter assembly has been built for low frequencies which takes advantage of the better performance at low currents. It is designated "FE" and consists of four 5-mA TE's (125 $\Omega$ each) in series. It is ordinarily operated at $2.5 \mathrm{~mA}$, so that each element has an output of about $2.5 \mathrm{mV}$. The thermocouple outputs are connected in series to give an overall output of about $10 \mathrm{mV}$.

Since the assembly has an input resistance of $500 \Omega$, it is rated at $1.25 \mathrm{~V}$ at $2.5 \mathrm{~mA}$. The table below lists rated voltages for the low-range TVC resistor sets no. 1 and no. 7 .

$\begin{array}{cccc}\text { Set No. } & \text { Rating }(\mathrm{V}) & \text { Resistance }(\Omega) & \text { Volts with FE } \\ 1 & 1 & 100 & 1.5 \\ 1 & 2 & 300 & 2.0 \\ 1 & 3 & 500 & 2.5 \\ 1 & 5 & 900 & 3.5 \\ 1 & 10 & 1900 & 6 \\ 1 & 20 & 3900 & 11 \\ 1 & 30 & 6 \mathrm{k} & 16 \\ 1 & 50 & 10 \mathrm{k} & 26 \\ 1 & 100 & 20 \mathrm{k} & 51 \\ 7 & 3-6 & 800 & 3 \\ 7 & 10-20 & 3600 & 10 \\ 7 & 30-60 & 12 \mathrm{k} & 31 \\ 7 & 100-200 & 40 \mathrm{k} & 101\end{array}$


INSTRUCTIONS FOR USING THE MJTC COMPARISON EQUIPMENT

F. L. Hermach - April 1981

(revised Nov. 1982, July 1985, April 1986, and Nov. 1986)

\section{GENERAL}

This equipment is set up in Room A144 Metrology Building to compare the ac-dc differences of multijunction and single-junction thermal converters (MJTC's and SJTC's). The standards and comparator are described in NBSIR 84-2903, the 1966 and 1976 papers included with it, and the 1986 CPEM paper [1]-[4]. You should read these papers and sections II through VII of the IR before using the equipment. The following instructions give additional information on the use of the comparator and on techniques for making comparisons. (The logbooks of Don Flach and the data books of F. L. Hermach, on which the 1976 paper on the MJTC's was based, are also in A144, along with the more recent data, math, and summary sheets on which the IR was based.)

\section{AC-DC STANDARDS}

The MJTC's are as described in the 1976 paper, covering the range from 5 to 12 $\mathrm{V}$ at 30 to $10,000 \mathrm{~Hz}$ with $0.5-\mathrm{ppm}$ accuracy. Their series resistors (for lowfrequency compensation) can be connected in parallel with the heaters to give 10- to $100-\mathrm{mA}$ standards. The MJTC's are our primary ac-dc standards, so take extreme care to avoid burning them out.

Two 5-mA thermoelements ( $\mathrm{TE}$ 's), labeled $\mathrm{FX}_{2}$ and $\mathrm{FY}_{2}$, were evaluated with the MJTC's to $10 \mathrm{kHz}$, as were the $5-$ and $10-\mathrm{V}$ thermal voltage converters (TVC's) formed from these $T E^{\prime} s$ and the $5-$ and $10-\mathrm{V}$ series resistors of the $\mathrm{F}_{1}$ set described in the 1966 paper. The ranges have been extended in frequency, current, and voltage by an involved process, which is described in the IR. These TE's (with the $F_{1}$ set of resistors) and a set of Weston TE's of very special construction (to our specifications) from $10 \mathrm{~mA}$ to $20 \mathrm{~A}$ are our reference standards. They should be used primarily to test our working standards. Their assigned ac-dc differences ( $\delta^{\prime} s$ ) and other pertinent data are filed in the ac-dc reference standards notebook in A144.

\section{COMPARISON EQUIPMENT}

Section III and figures 5 and 6 of the IR describe the basic comparison circuit. Figure A of these instructions shows the detailed wiring of the equipment, including the important shielding and grounding. Figure $B$ shows the actual wiring of the EMF comparator; $C$ the simplified schematics; $D$ the ac-dc relays; and $E$ the input connections at the tank to guard against burnout. Appendix 1 is a log of significant changes in the apparatus, which should be continued as necessary. 
Stable ac and dc supplies are vital to high precision. The Model $303 \mathrm{~B}$ Kintel dc voltage standard, RCD-9 Optimation oscillator, and PA 50 T Optimation amplifier are the same ones used in 1973-1976. The output transformer in the PA $50 \mathrm{~T}$ is useful only to $30 \mathrm{kHz}$ and has been replaced by an external KrohnHite Model MT-56R matching transformer. (Note the information on the transformer panel.) The circuits of the simple fine-control units for the ac and dc supplies are shown on their boxes. The ac-dc relays (which have an off time of less than $0.5 \mathrm{~ms}$ ) have a safe voltage limit (with resistive loads) of about $100 \mathrm{~V}$ and a safe current limit of $50 \mathrm{~mA}$ (not simultaneously). Spare relays are available for experiments. You could use higher voltages with another fast-acting switch, but you would have to take proper precautions against electric shock.

A Keithley Model 150B chopper-type dc microvoltmeter is normally used as the detector ( $N$ in figures $B$ and $C$ ), with the filter switch IN and the input resistance set at $100 \mathrm{~K}$. It is satisfactory if its amplifier is not overloaded by ac from the thermocouple outputs (which can be especially troublesome with MJTC's). Precautions and checks are given in appendix 2. The output of the detector is connected to an electronic "averager" (op-amp integrator and timer), which integrates the output for $10 \mathrm{~s}$ to reduce the effect of noise and to ease reading. Information on it is given in the ac-dc data book, vol. 1, Nov. 30, 1981.

\section{IV. n-COMPENSATION AND THE BASIC EQUATION}

The EMF comparator balances a fraction of the EMF of one thermal converter (TC) against the smaller EMF of the other. When both TC's have the same value of $\mathrm{n}$ (e.g., two MJTC's), the balance is independent of small changes in the supply voltage, which greatly increases the precision of the comparison. If, however, the two n's are different, some of this advantage is lost. Properly adjusting the added resistor $R_{r}$ in figures $B$ and $C$ restores this compensation, as described in section III-A and appendix 1 of the IR.

The basic equation for this comparator is $\Delta=\delta_{t}-\delta_{s}=\left(N_{d}-N_{a}\right) / n_{s} E_{l}$, where $t$ and $s$ refer to "test" and "standard," respectively, and $\delta_{t}$ is the ac-dc difference of the TC to which the Lindeck potentiometer is connected. ${ }^{*} \mathrm{~N}_{\mathrm{a}}$ is the average of the two detector readings with ac applied in a sequence of four ac and dc measurements, and $\mathrm{N}_{\mathrm{d}}$ is the average with the two polarities of dc applied in succession. $E_{1}$ is the lower thermal EMF. If $N$ is in nanovolts and $E_{l}$ in millivolts, $\Delta$ is in parts per million.

The "selection rules" are: 1) connect the TC with the higher EMF to $E_{h}$ on the comparator, and 2) connect the potentiometer to the TC with the higher $n$ (either one if their $\mathrm{n}^{\prime} \mathrm{s}$ are equal). The "test $\mathrm{TC} "\left(\delta_{t}\right)$ is always the one to which the potentiometer is connected, and hence is the one with the higher $n$.

\footnotetext{
*As shown in appendix 1 of the IR, this equation applies when there is no current through the detector. For greater stability the $150 \mathrm{~B}$ is generally operated with a 100-k $\Omega$ input resistance. This causes a negligible error when two SJTC's are compared in the Read-2 position of the divider, but the error can be 48 of $\Delta=\delta_{t}-\delta_{s}$ when two MJTC's are compared in the Read-1 position. However, $\Delta$ is generally only a few ppm in such tests. If it is larger than 10 $\mathrm{ppm}$, switch the $150 \mathrm{~B}$ input resistance to the OPEN position.
} 


\section{OPERATION OF THE EQUIPMENT}

\section{A. Connections}

For TVC comparisons connect the inputs of the TVC's in parallel by means of a GR 874-T connector. Lead impedances can cause serious errors at high frequencies, so the center of the tee is arbitrarily but reasonably chosen as the reference plane for a comparison. (See the math notebook, Aug. 22, 1982, for information on an unusual error caused by skin effect in the largediameter center conductor of the GR 874 connectors.) For low-voltage ranges put a higher-range $F_{1}$ resistor in series with the pair of TVC's so that the supply voltage will be about $5 \mathrm{~V}$, for greater stability of the ac and dc sources. Plug one of the Zener-diode pairs, with a voltage knee at about twice the input voltage $V_{\text {in }}$, across the input at the tank, with an expendable $\mathrm{TE}$ in series, to protect against burning out the TE's of the IVC's. See figure $E$.

The $\mathrm{KH}$ matching transformer should be used with the ac amplifier to avoid large (and slow) dc transients and errors from the dc offset voltage $V_{0}$ of many direct-coupled amplifiers. If the transformer is not used, make sure that $V_{0}$ is less than $0.1 \%$ of $V_{i n}$. (See appendix 2 of the IR re this error.) Note that the $\mathrm{KH}$ transformer has a high and a low frequency range. It is good practice to connect an oscilloscope (with floating input) across the Zenerdiode (input) posts at the tank. Use it to check the frequency (roughly) with its calibrated time scale and to look at the waveform, noise, and pickup.

Connect the outputs to the $E_{h}$ (high EMF) and $E_{l}$ (low EMF) jacks of the comparator, and check all the other connections.

For comparison of two TE's as current converters (note the 50-mA limit) connect their heaters in series. Shunt capacitance from either line (one line is grounded) to the center conductor can cause errors at high frequencies. This is best checked by repeating the test with a different lead arrangement. Bead conductance (see section $V$ of the IR) can cause errors that are best checked by repeating the test at the highest frequency with the input leads interchanged.

\section{B. Preliminary Adjustments}

Make sure that the ac and dc switches on the relay box are off and that the range switches, etc. on the supplies are properly set. Turn on the relay power switch at the back of the box. (Note that if the power switch is off, ac is always applied when the ac switch is on.) Remove any shunting resistor across $E_{1}$. Turn on the power supplies, detector (ac operation), averager, oscilloscope, etc. Set the ac-dc switch to DC, turn on the dc switch, and bring up the voltage to the desired value, checking the EMF's of the TC's on the detector with the set key down. Check $I_{d c}$ with the Weston Model 1 dc milliammeter as you bring up the voltage. Make sure it is on the right range. Make sure that the detector deflects upscale for each TC (or the signs will be wrong). Reverse polarities at the output jacks on the tank, if necessary. Then measure the EMF's of the TC's, with the potentiometer switch at $E_{h}$ and then $E_{1}$ and the divider switch at Pct'r. (Otherwise the divider loads the TC output.) Record the EMF's and other data. (See the sample data sheet.) 
Next switch the divider to Read-1 for two MJTC's (or an MJTC vs an SJTC) and to Read-2 for two SJTC's, and switch the potentiometer to the TC with the higher $n$. Set the first divider dial to 0 when in the Read-2 position. Turn on the potentiometer to an appropriate range and step down to the $1-\mu \mathrm{V}$ range of the detector, adjusting the potentiometer and the divider as needed, with the set and read keys down, respectively. Because the divider loads $E_{h}$, it may be impossible to get a read balance when the two EMF's are nearly equal. In that case connect a resistor whose resistance is approximately equal to the input resistance of the divider ( $1 \mathrm{k} \Omega$ in the Read-2 position and $10 \mathrm{k} \Omega$ in Read-1) across the $E_{l}$ terminals. Measure the new voltage $V_{l}$ and use its value in place of $E_{l}$ in the basic equation, but use the value of $n$ corresponding to $\mathrm{E}_{1}$ if this is the standard TC. (See the math notebook, Mar. 17, 1982.)

\section{C. n-Measurement}

If it is not already known, measure $n_{s}$ (after warm-up) by changing the dc input voltage by $\Delta \mathrm{V}$ and observing on the detector the change $\Delta \mathrm{E}_{\mathrm{S}}$ in the set position (with the divider switch at Pot'r). Compute $n_{S}=V \Delta E_{S} / E_{S} \Delta V$. (Set $\Delta \mathrm{V} / \mathrm{V}$ to about $5 \times 10^{-3}$.) To avoid errors from drifts, return the dc voltage to the original value, check $E_{S}$ after the same time interval, and use the average value as the reference for $\Delta \mathrm{E}_{\mathrm{S}}$. Set the same $\Delta \mathrm{V} / \mathrm{V}$ in the negative direction, and take the average of the two values of $n$. Unless the heater of the TE is of Evanohm or its equivalent, there may be special problems in defining and measuring $n$ to 18 , because of the temperature coefficient of heater resistance. (See appendix 1-D of the IR.)

\section{D. n-Compensation (not needed for two MJTC's)}

With dc applied, adjust the potentiometer until $\mathrm{N}<0.1 \mu \mathrm{V}$ (set key down) and the divider until $\mathrm{N}<0.1 \mu \mathrm{V}$ (read key down). Then increase the dc voltage by about 0.18 and adjust $R_{r}$ to give the same reading to within $5 \mathrm{ppm}$ of $\mathrm{E}_{S}$ with the read key down as before. Return the dc voltage to the original setting and check the read balance. If balance is not achieved with $\mathrm{R}_{\mathrm{r}}=\infty$ (disconnected), then the other TC probably has the larger $\mathrm{n}$. Switch the potentiometer to it (it is now the test TC) and rebalance the potentiometer and divider. Then proceed as before.

\section{E. DC Reversal Compensation}

Reverse the dc voltage and note the change in the detector reading with the read key down. If it is more than about 20 divisions on the upper scale $(1 / 5$ full scale from side to side) on the desired detector range, switch on the dc reversal compensator (RC) and adjust it to cut the change in half. The compensator is wired (see the diagram on the box) so that it may be operated in the same sequence as the main ac-dc switch. If the polarity is wrong (opposite sequence), simply rotate the switch knob $180^{\circ}$ to reverse the voltage. Reset the detector to midscale by adjusting the divider. Adjust the potentiometer (set key down) so that nearly equal and opposite deflections are obtained for the two polarities of dc voltage. 


\section{F. AC Setting}

Set the ac frequency, turn on the ac switch on the relay box, and switch to $A C$, with the RC set to AC (i.e., off). Bring up the ac voltage while monitoring the detector (set key down), adjust the voltage to make $N=0$ (midscale), and check the read balance.

\section{G. AC-DC Determinations}

Always double check to make sure that the frequency setting is correct, that the correct TC is recorded as the test TC on the data sheet, and that the correct range is set on the detector.

For a determination, switch to AC, DC, DCR, and AC. In each case, (a) first check the balance with the set key down and reset as necessary by adjusting the voltage (center the ac and split the dc to within $40 \mathrm{ppm}$ of $E_{t}$ to keep the $\Delta \mathrm{n}$ error less than $0.1 \mathrm{ppm})^{*}$; (b) then about $30 \mathrm{~s}$ after switching, hold the read key down and reset the averager to 0.0 ; (c) at $40 \mathrm{~s}$ after switching, start the averager; and (d) when its light goes out, release the read key, rotate the ac-dc and $\mathrm{RC}$ switches, and record the reading of the averager. Do not adjust the potentiometer or divider controls.

Repeat this sequence (or its inverse) three more times, with $30 \mathrm{~s}$ between the start of the last $A C$ in one sequence and the start of the first $A C$ in the next (so that a correction for nonlinear drift can be applied later if desired). If the sequence timing is off, abort the sequence. If the interval timing (between sequences) is off, record it and proceed.

\section{H. Recording and Computing Data}

See the sample data sheet for a convenient data-logging format. For each sequence, $\delta_{t}-\delta_{s}=K\left(-D_{a c 1}+D_{d c}+D_{d c r}-D_{a c 2}\right)$. The factor $K=(F / 2) /$ $\left(n_{s} E_{1}\right)$, with $E_{1}$ in $\mathrm{mV}$. $F / 2$ for the Model $150 \mathrm{~B}$ detector is 10 times the range in $\mu \mathrm{V}$ (i.e., 10 for the $1-\mu \mathrm{V}$ range). D is the reading of the averager (with its sign) in divisions.

\section{Shutting Down}

Turn off the ac and dc switches on the relay control box, reduce the ac and dc voltages, then turn off the $a c$ and dc power supplies, oscilloscope, detector, averager, potentiometer, and relay power supply (at the back of the box), and turn the reversal compensator to AC (battery off).

${ }^{*}$ If $R_{r}$ is adjusted so that $\Delta N$ in section $V-D$ is less than 5 ppm of $E_{S}$ for a 0.18 change in $V_{i n}$, then by direct proportion the error will be less than 0.1 $\mathrm{ppm}$ for a 20-ppm missetting of $V_{i n}$ in the test. This is $40 \mathrm{ppm}$ of $E_{t}$ for a nominally square-law TC (i.e., $0.4 \mu \mathrm{V}$ if $\mathrm{E}_{\mathrm{t}}=10 \mathrm{mV}$ ). 
A. Inspections

1. Check the detector battery before each use. (The 150B will not operate properly even in the ac mode if the battery is way below par.)

2. Check the various connections, especially the ground lead to the conduit under the table, if erratic readings, sensitivity of the detector to body position, etc. occur.

3. If any doubt about the RC (reversal compensator) arises, set $1000 \mathrm{div}$ on it and measure its output on the detector with the read key down. It should be about $13 \mu \mathrm{V}$ with the $\mathrm{E}_{1}$ input shorted.

\section{B. Periodic Tests (with the 150B detector)}

These tests (recommended every six months) can be made by comparing items of the measuring equipment within the setup. External standards should not be required unless discrepancies are found. Replace the $E_{1} T C$ in the tank by a shorted AN jack. No other changes are necessary. Record the results and file them in a data book for future reference.

\section{Detector vs Lindeck Potentiometer}

Zero the detector on the $10-\mathrm{mV}$ range. With the divider switch at Pot' $r$ and the potentiometer switch at $E_{1}$, adjust the potentiometer to $10.0 \mathrm{mV}$. The detector should read $-10.0 \pm 0.1 \mathrm{mV}$ with the set key down.

2. Detector vs Averager

With both keys up, adjust the zero controls on the $150 \mathrm{~B}$ to give +50.0 div (upper scale) on the 10-mV range. Operate the averager and record its reading. Readjust the zero controls to give -50.0 div, then operate the averager and record its reading as before. The total change in the averager readings should be $100 \pm 1$ div. If not, readjust $R_{5}$ on the averager. (Warning: this is a sensitive control.)

3. Interrange Tests of the Detector

These tests require careful settings but are easy to make and should suffice to check proper operation. Start as in B-1, on the 10-mV range. With the set key down, adjust the potentiometer controls to obtain a $3.0-\mathrm{mV}$ reading on the $150 \mathrm{~B}$. Switch to the $3-\mathrm{mV}$ range and check that the reading is the same after checking the zero with both keys up. Adjust the potentiometer to obtain a $1.0-\mathrm{mV}$ reading and switch to the $1-\mathrm{mV}$ range. Continue down to the $30-\mu \mathrm{V}$ range, then set the $\mathrm{RC}$ to obtain a reading of $10.0 \mu \mathrm{V}$ on the $30-\mu \mathrm{V}$ range of the $150 \mathrm{~B}$ with the read key down. Switch to the $10-\mu \mathrm{V}$ range and check. Then proceed down to the $1-\mu \mathrm{V}$ range.

4. Reversal Compensator (RC)

Check the RC against the detector, as described above in A-3. 


\section{APPENDIX 1}

Index of Changes in the MJTC Setup

1981

-...

Feb 1981

$3 / 11 / 81$

$3 / 25 / 81$

$4 / 3 / 81$

$8 / 24 / 81$

$11 / 6 / 81$

$11 / 30 / 81$

$12 / 11 / 81$

$12 / 14 / 81$

1982

- - -

$1 / 19 / 82$

$2 / 12 / 82$

$4 / 19 / 82$

$4 / 22 / 82$

$4 / 26 / 82$

$5 / 5 / 82$

$6 / 7 / 82$

$8 / 27 / 82$

$9 / 30 / 82$

$10 / 7 / 82$

$10 / 8 / 82$

$10 / 25 / 82$

$11 / 10 / 82$

MJTC comparator set up in A144 Metrology. See diagrams of $2 / 81$. Ground lead $g$ added and LO-GND link on Keithley $150 B$ opened. See diagrams of $2 / 81$.

$\mathrm{R}_{\mathrm{r}}$ circuit added and used hereafter for $\mathrm{n}$-compensation.

Grounded black output post at ac amplifier (50 V to ground with switch on dc).

Replaced this ground with $3-\mu \mathrm{F}$ capacitor.

Connected two 1000-pF disc capacitors across input leads at tank and a $1000-\mathrm{pF}$ mica capacitor from each output lead to tank for radio-frequency-interference bypass.

Op-amp timer-integrator ("averager") built and installed.

Shielded Lindeck milliammeter (by placing screen over dial) to eliminate electrostatic pickup.

DVM installed in averager in place of Triplett tester.

Started 10-s integration periods.

Changed ac supply leads to keep amplifier grounded.

Read-2 position installed (first decade of Kelvin-Varley divider switched out). All tests with two SJTC's performed in Read-2 position now, unless otherwise noted.

Detector grounding changed. See diagram on p. 2 of data sheets for this date.

Optimation amplifier replaced by Krohn-Hite Model 7500.

New relays put in ac-dc control box. Bypass capacitor for radio frequency interference placed outside tank (input leads, only).

Replaced tightly twisted pair of input leads in tank with coaxial cable. Replaced each TC output (twisted pair) with shielded twisted pair. Coaxial cable shield and each twisted pair shield grounded at tank.

Ac input leads changed. See diagram on data sheet for this date.

1000- $\Omega$ resistor (in small metal box), formerly across $E_{1}$, put in series in $E_{l}$ line with $b$ lead to form an RC filter with $2-\mu F$ capacitor across detector leads at EMF comparator. (More detail is in data book under this date.)

Series resistor for this RC filter removed due to complications in setting $R_{r}$.

Installed matching transformer in output of Krohn-Hite amplifier to avoid errors from dc offset voltage. See data sheets for $10 / 7 / 82$.

Back-to-back Zener diodes used for TE protection.

$50-\Omega$ General Radio resistor connected in series with each TE output (and mounted in tank) to avoid adding them when $\Delta \mathrm{n}$ is large and $R_{r}$ is less than $200 \Omega$, the output resistance of the potentiometer. 
Set up Optimation PA $50 \mathrm{~T}$ amplifier (modified) and Krohn-Hite MT56R transformer for the ac supply.

12/10/82 F1 TVC connectors (General Radio 874) stabilized by tinned copper wire wedges.

1983

$4 / 18 / 83$

Internal $15-\mu \mathrm{F}$ series capacitor in Krohn-Hite MT-56R transformer removed (shorted) to improve low-frequency $(20-\mathrm{Hz})$ performance.

$5 / 18 / 83$

LC network added to Cohu (Kintel) Model 303B dc voltage standard (at its terminals) to reduce transients at $50 \mathrm{~mA}$ when dc is switched off.

1985

-...

$7 / 17 / 85$

Changes made in lead connections at input binding posts of tank to accommodate Wagner ground if needed. Details are in data book for this date.

\section{APPENDIX 2}

AC Pickup in the Detector

The comparator balances out the dc EMF's of the two TE's, but may not balance out the ac EMF's, since these may not be in the same ratio or phase. They are particularly large at low frequencies, and could cause errors if they overload the detector. In addition, the 150B has peculiar "windows" of response at or near multiples of the $94-\mathrm{Hz}$ chopper frequency. To reduce these effects, the $150 \mathrm{~B}$ has a filter in its input. To reduce high-frequency effects, the comparator has a $2-\mu \mathrm{F}$ capacitor across the detector terminals.

In case of doubt, check on possible errors from these normal-mode ac effects by repeating a test with a higher range on the $150 \mathrm{~B}$, which would have less overload, or by using a different detector.

For further information, see section III-A(6) of NBSIR 84-2903. 


\section{REFERENCES}

[1] F. L. Hermach, "An investigation of the uncertainties of the NBS thermal voltage and current converters," Nat. Bur. Stand. (US), Rep. NBSIR 842903, Apr. 1985.

[2] F. L. Hermach and E. S. Williams, "Thermal converters for audio-frequency voltage measurements of high accuracy," IEEE Trans. Instrum. Meas., vol. IM-15, pp. 260-268, Dec. 1966.

[3] F. L. Hermach and D. R. Flach, "An investigation of multijunction thermal converters," IEEE Trans. Instrum. Meas., vol. IM-25, pp. 524-528, Dec. 1976.

[4] F. L. Hermach, J. R. Kinard, and J. R. Hastings, "Multijunction thermal converters as the NBS primary ac-dc transfer standards for ac current and voltage measurements," IEEE Trans. Instrum. Meas., vol. IM-36, pp. 300306, June 1987. (This paper was presented at the Conference on Precision Electromagnetic Measurements (CPEM) held at NBS (Gaithersburg) in June 1986.) 


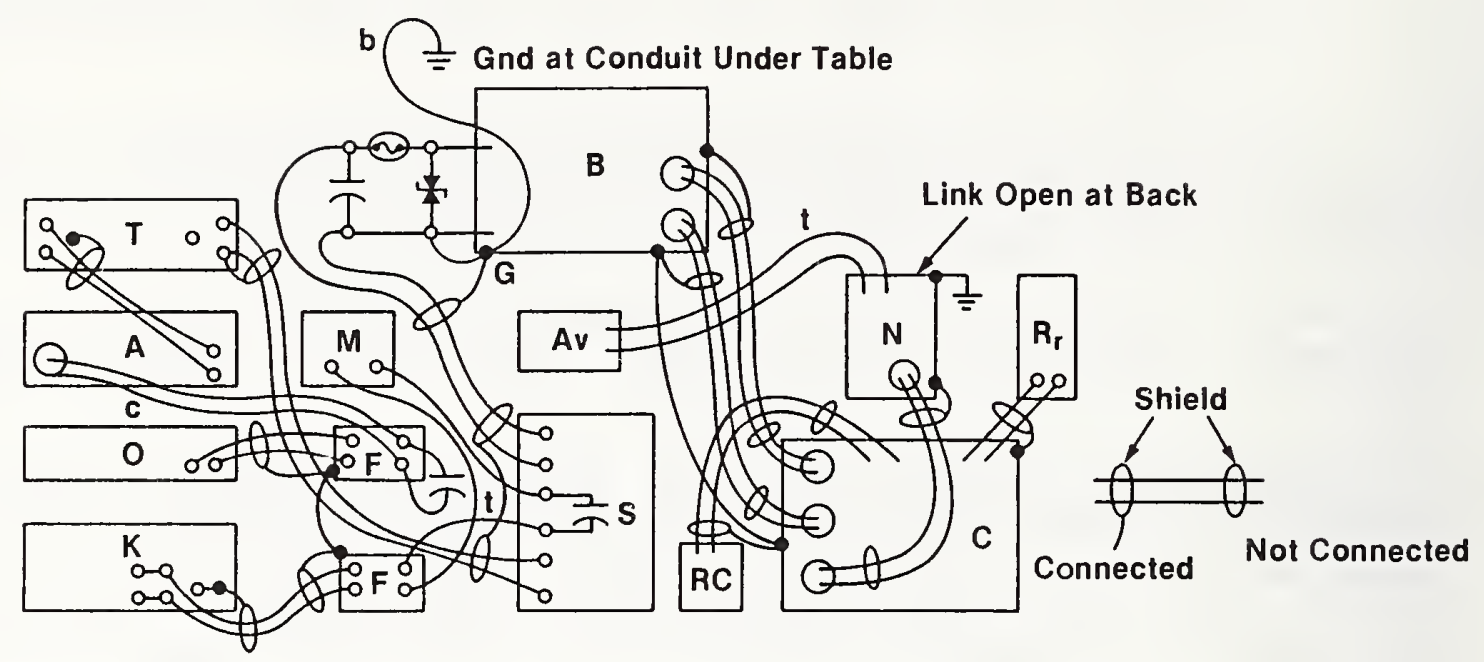

\footnotetext{
K Kintel 303B dc voltage standard

o Optimation RCD-9 oscillator

A Optimation PA $50 \mathrm{~T}$ amplifier

$T$ Krohn-Hite MT-56R matching transformer

$M$ Weston 1 dc milliammeter

S ac-dc relay box

B thermally-lagged metal tank for TC's

C EMF comparator

$\mathrm{N}$ detector (usually Keithley 150B)

$\mathrm{R}_{r}$ Leeds \& Northrup ac-dc decade resistor

RC dc reversal compensator

Av electronic "averager"

$F$ fine controls for ac and dc
}

The cases of $\mathrm{K}$ and $\mathrm{T}$ are connected to point $\mathrm{G}$ on the tank with braid (not shown). The instruments $\mathrm{K}, \mathrm{O}, \mathrm{A}$, and $\mathrm{N}$ are grounded through their line cords. All leads are two-conductor shielded cables unless marked " $t$ " for twisted pair, "c" for coaxial, or "b" for braid. Bypass capacitors for controlling radio frequency interference are shown outside the tank. Wiring, bypass capacitors, etc. inside the tank are not shown; the input to the $\mathrm{TC}^{\prime} s$ is coaxial and their output leads are two-conductor shielded cables with the shields grounded to the tank. 
A. Pot'r to $E_{1}\left(E_{1}\right.$ is "Test")

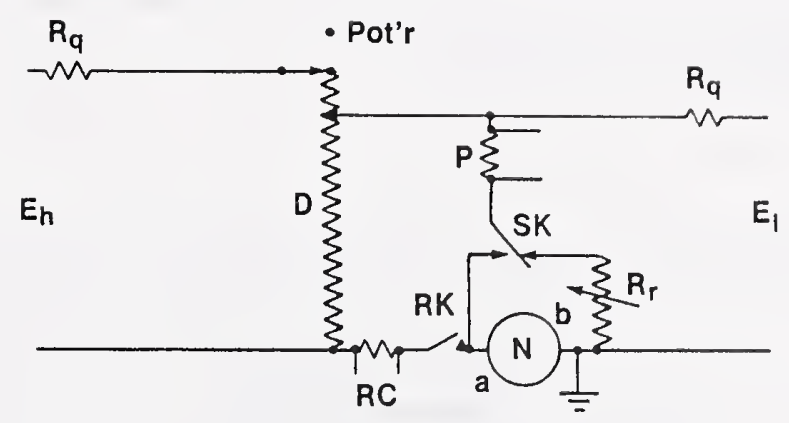

B. Pot'r to $E_{h}\left(E_{h}\right.$ is "Test")
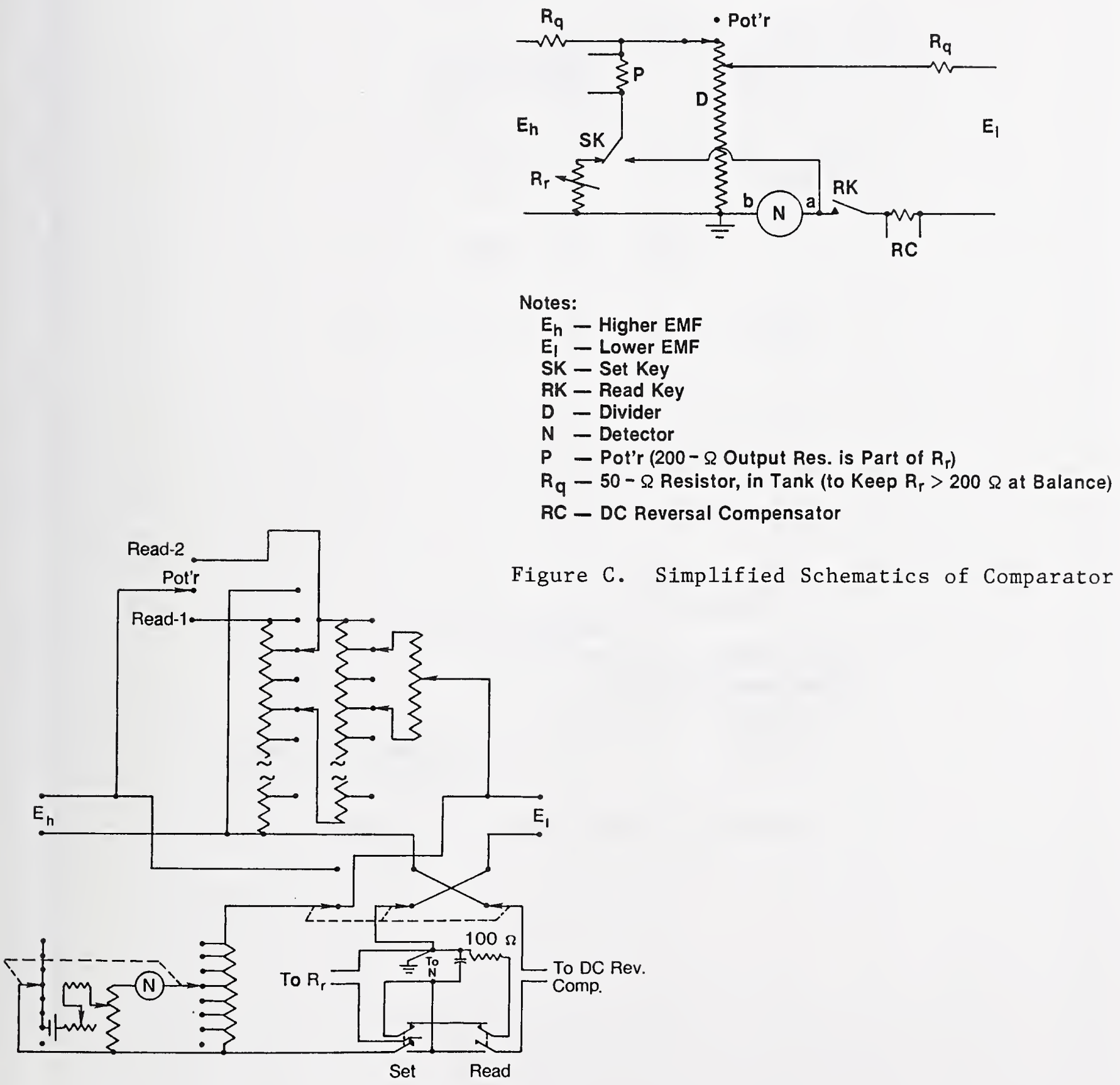

Figure B. Diagram of EMF Comparator 

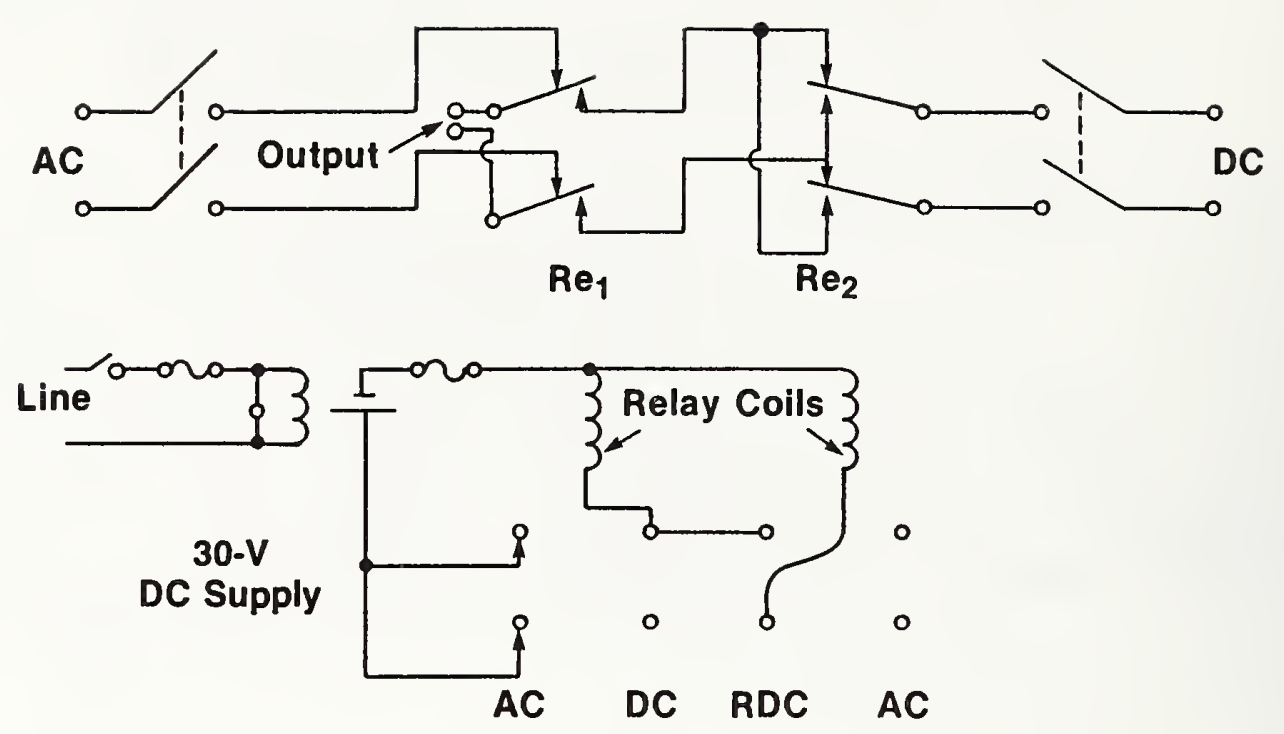

Figure D. Relay AC-DC Transfer Switch

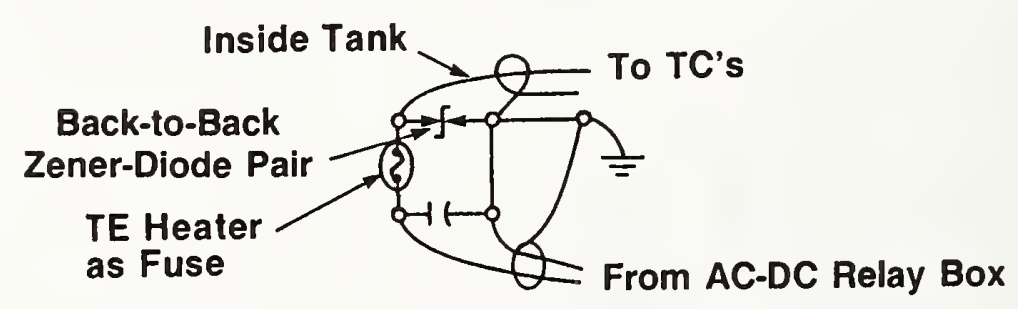

Figure E. Input Connections at Tank 
U.S. DEPARTMENT OF COMMERCE NATIONAL BUREAU OF STANDARDS

TEST NO.

DATE

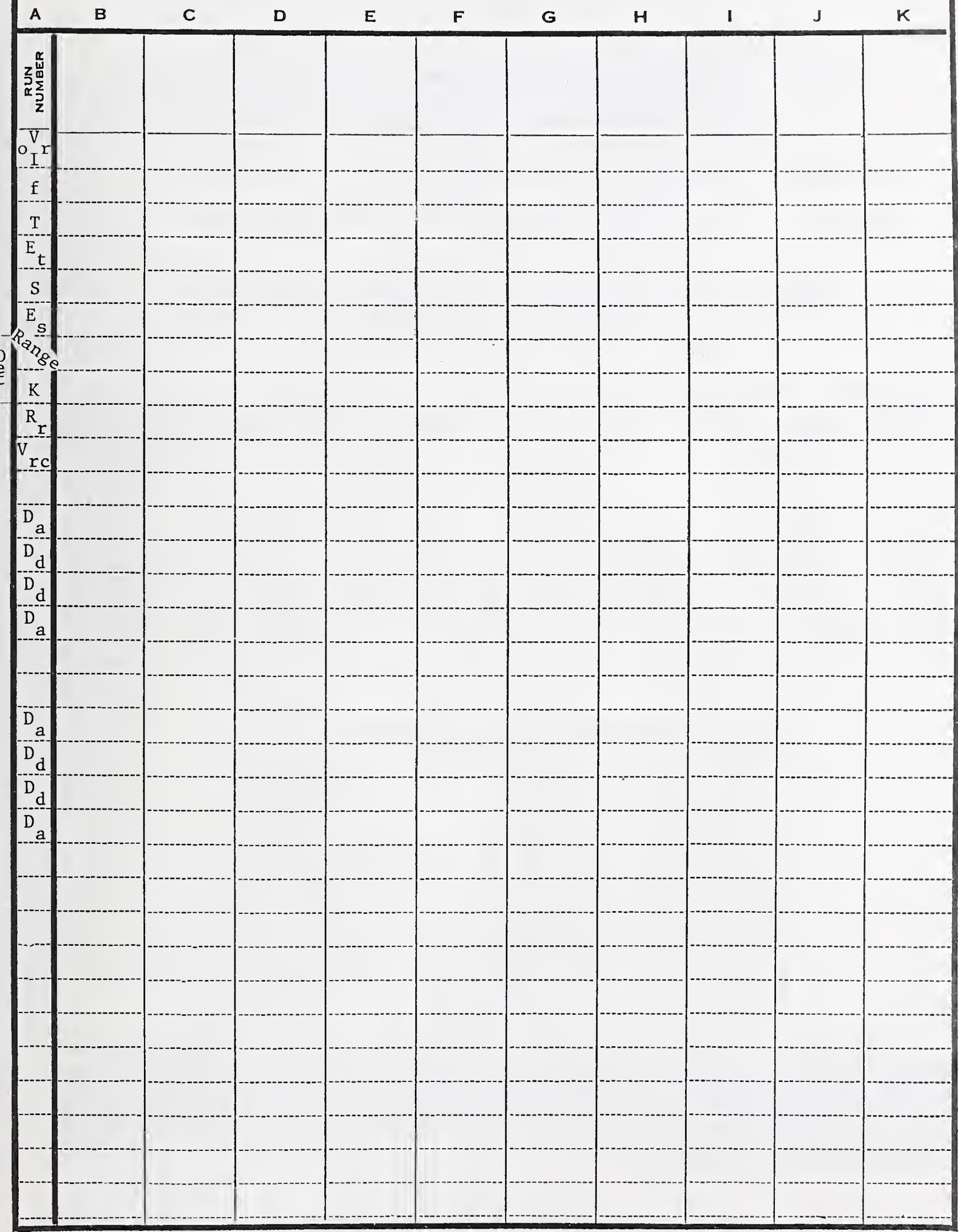




\section{SCHEDULE FOR RECALIBRATION AND PERIODIC CHECKS} OF NBS THERMAL CONVERTERS

5-10 years Recalibration of reference standards, both thermal voltage converters (TVC's) and thermal current converters (TCC's), including voltage and current build-up/build-down and frequency extension.

2-3 vears Recalibration of working standards in terms of reference standards.

Routine Checks of working standards according to attached timetables.

TABLE 1. Ranges of low-frequency, thermal voltage converter working standards, to be intercompared during routine check measurements.

Frequency $=100 \mathrm{kHz}$.

\begin{tabular}{|c|c|c|c|c|}
\hline \multirow[t]{2}{*}{ Voltage } & \multicolumn{2}{|c|}{ Week 1} & \multicolumn{2}{|r|}{ Week 3} \\
\hline & NBS F-7 & NBS S/N 302 & NBS $F-7$ & NBS S/N 54 \\
\hline $1 \mathrm{~V}$ & $1 V(F 1)$ & $1 V(T E-A)$ & $1 \vee(F 1)$ & $1 \mathrm{~V}(\mathrm{~T}-2.5)$ \\
\hline $2 \mathrm{~V}$ & $2 \mathrm{~V}(\mathrm{~F} 2)$ & $2 V(T E-B)$ & $2 V(F 2)$ & $2 \vee(T-5)$ \\
\hline $3 \mathrm{~V}$ & $3 \vee(F 1)$ & $4 \mathrm{~V}$ (TE-C) & $3 V(F 1)$ & $3 \vee(R-1, T-2.5)$ \\
\hline $10 \mathrm{~V}$ & $10 \mathrm{~V}(\mathrm{~F} 1)$ & $12 \vee(R-A, T E-C)$ & $10 \mathrm{~V}(\mathrm{~F} 1)$ & $10 \mathrm{~V}(\mathrm{R}-2, \mathrm{~T}-2.5)$ \\
\hline $20 \mathrm{~V}$ & $20 \vee(F 2)$ & $20 \mathrm{~V}(\mathrm{R}-\mathrm{B}, \mathrm{TE}-\mathrm{B})$ & $20 \vee(F 2)$ & $20 \mathrm{~V}(\mathrm{R}-2, \mathrm{~T}-5)$ \\
\hline $60 \mathrm{~V}$ & $60 \mathrm{~V}(\mathrm{~F} 2)$ & $60 \mathrm{~V}$ (R-C, TE-B) & $60 \vee(F 2)$ & $60 \mathrm{~V}(\mathrm{R}-3, \mathrm{~T}-5)$ \\
\hline $100 \mathrm{~V}$ & $100 \vee(F 1)$ & $100 \mathrm{~V}(\mathrm{R}-\mathrm{D}, \mathrm{TE}-\mathrm{A})$ & $100 \vee(F 1)$ & $100 \mathrm{~V}(\mathrm{R}-4, \mathrm{~T}-2.5)$ \\
\hline $300 \mathrm{~V}$ & $300 \vee(F 1)$ & $300 \mathrm{~V}(\mathrm{R}-\mathrm{E}, \mathrm{TE}-\mathrm{A})$ & $300 \mathrm{~V}(\mathrm{~F} 1)$ & $300 \mathrm{~V}(\mathrm{R}-5, \mathrm{~T}-2.5)$ \\
\hline $600 \mathrm{~V}$ & $1000 \mathrm{~V}(\mathrm{~F} 1)$ & $600 \mathrm{~V}$ (R-E, TE-B) & $1000 \mathrm{~V}(\mathrm{~F} 1)$ & $1000 \mathrm{~V}(\mathrm{R}-6, \mathrm{~T}-2.5)$ \\
\hline
\end{tabular}

\begin{tabular}{|c|c|c|}
\hline \multirow[t]{2}{*}{ Voltage } & \multicolumn{2}{|c|}{ Week 5} \\
\hline & NBS S/N 302 & NBS S/N 54 \\
\hline $1 \mathrm{~V}$ & $1 V(T E-A)$ & $1 \vee(T-2.5)$ \\
\hline $2 \mathrm{~V}$ & $2 \mathrm{~V}$ (TE-B) & $2 \vee(T-5)$ \\
\hline $3 \mathrm{~V}$ & $4 \mathrm{~V}$ (TE-C) & $3 \vee(R-1, T-2.5)$ \\
\hline $10 \mathrm{~V}$ & $12 \vee(R-A, T E-C)$ & $10 \vee(R-2, T-2.5)$ \\
\hline $20 \mathrm{~V}$ & $20 \mathrm{~V}(\mathrm{R}-\mathrm{B}, \mathrm{TE}-\mathrm{B})$ & $20 \vee(R-2, T-5)$ \\
\hline $60 \mathrm{~V}$ & $60 \mathrm{~V}$ (R-C, TE-B) & $60 \mathrm{~V}(\mathrm{R}-3, \mathrm{~T}-5)$ \\
\hline $100 \mathrm{~V}$ & $100 \mathrm{~V}(\mathrm{R}-\mathrm{D}, \mathrm{TE}-\mathrm{A})$ & $100 \vee(R-4, T-2.5)$ \\
\hline $300 \mathrm{~V}$ & $300 \mathrm{~V}(\mathrm{R}-\mathrm{E}, \mathrm{TE}-\mathrm{A})$ & $300 \mathrm{~V}(\mathrm{R}-5, \mathrm{~T}-2.5)$ \\
\hline $600 \mathrm{~V}$ & $600 \mathrm{~V}$ (R-E, TE-B) & $1000 \mathrm{~V}(\mathrm{R}-6, \mathrm{~T}-2.5)$ \\
\hline
\end{tabular}

The low-frequency TVC sets in Table 1 are identified as NBS F-7, NBS S/N 302, and NBS S/N 54. NBS F-7 contains two thermoelement modules labeled F1 and $F 2$ and six range resistors identified according to their voltage ranges. NBS S/N 302 contains three thermoelements labeled TE-A, TE-B, and TE-C. The range resistors are identified as $R-A$ through R-E. NBS S/N 54 contains two thermoelements labeled T-2.5 and T-5, and range resistors labeled $\mathrm{R}-1$ through $\mathrm{R}-6$. 
TABLE 2. Routine checks for high-frequency thermal voltage converter standards. Frequency $=1 \mathrm{MHz}$.

\begin{tabular}{|c|c|c|}
\hline Voltage & $\begin{array}{l}\text { Standard } \\
\text { TVC }\end{array}$ & $\begin{array}{l}\text { Check } \\
\text { Standard }\end{array}$ \\
\hline $0.5 \mathrm{~V}$ & C1P & $\mathrm{C} 0.5$ \\
\hline $1 \mathrm{~V}$ & C1P & TD0.D-8-1V \\
\hline $2 \mathrm{~V}$ & $\mathrm{C} 2 \mathrm{P}$ & TD0.D-8-3V \\
\hline $3 \mathrm{~V}$ & С3P & TD0.D-8-3V \\
\hline $5 \mathrm{~V}$ & C6P & C5 \\
\hline $10 \mathrm{~V}$ & C10 & TD0.D-8-10V \\
\hline $10 \mathrm{~V}$ & $\mathrm{C} 20$ & TDO.D-8-10V \\
\hline $50 \mathrm{~V}$ & $\mathrm{C} 50$ & TDO.D-8-50V \\
\hline $50 \mathrm{~V}$ & C100 & TD0.D-8-50V \\
\hline
\end{tabular}

The high-frequency TVC standards and check standards in Table 2 are of the coaxial single-range type. The standards are labeled with the prefix " $\mathrm{C}$ " and check standards are labeled with either "C" or "TDO".

TABLE 3. Routine checks for working-standard thermal current converters. Frequency $=50 \mathrm{kHz}$.

\begin{tabular}{rrr} 
Current & \multicolumn{1}{c}{$\begin{array}{c}\text { Standard } \\
\text { TCC }\end{array}$} & \multicolumn{1}{c}{$\begin{array}{c}\text { Check } \\
\text { Standard }\end{array}$} \\
$5 \mathrm{~mA}$ & $\mathrm{~A} 3(5 \mathrm{~mA}) \# 95$ & ESW FG $(10 \mathrm{~mA})$ \\
$10 \mathrm{~mA}$ & $\mathrm{~B} 10(10 \mathrm{~mA}) \# 57$ & ESW FG $(10 \mathrm{~mA})$ \\
$20 \mathrm{~mA}$ & $\mathrm{~F} 20(20 \mathrm{~mA}) \# 1$ & ESW $(0.03 \mathrm{FG})$ \\
$25 \mathrm{~mA}$ & W30(25 mA)\#1 & ESW $(0.03 \mathrm{FG})$ \\
$50 \mathrm{~mA}$ & $\mathrm{~F} 50 \# 2$ & ESW $(0.06 \mathrm{FH})$ \\
$100 \mathrm{~mA}$ & $\mathrm{~B} 100 \# 3$ & ESW $(0.1 \mathrm{FG})$ \\
$200 \mathrm{~mA}$ & $\mathrm{~A} 250 \# 4$ & ESW $(0.2 \mathrm{FH})$ \\
$500 \mathrm{~mA}$ & W0.5A\#0-2 & ESW $(0.6 \mathrm{FH})$ \\
$1 \mathrm{~A}$ & W1A\#7-1 & ESW (1 FG) \\
$2 \mathrm{~A}$ & W2A\#8-3 & ESW (2 FH) \\
$3 \mathrm{~A}$ & W3A\#9-2 & ESW (3 FG) \\
$5 \mathrm{~A}$ & W5A\#5-1 & ESW (6 FH) \\
$10 \mathrm{~A}$ & W10A\#0-3 & ESW (10 FG) \\
$16 \mathrm{~A}$ & W20A\#1-2 & ESW (20 FH)
\end{tabular}

The thermal current converter standards in Table 3 are all thermolements with heaters of high rated current. The check standards consist of a set of current shunts and thermoelements labeled FG and FH. 


\author{
SET OF THERMAL TRANSFER STANDARDS \\ Manufacturer's Name \\ Model T, Serial No. 54321 \\ Submitted by \\ Company's Name \\ City, State, Zip Code
}

These thermal voltage converters were tested in September 1987 to determine their ac-dc differences.

Determinations were made of the difference between the alternating voltage (of practically sine waveform) required for a given output emf and the average of the two polarities of direct voltage required for the same emf. The observed differences are generally given to the nearest five parts per million (ppm) in the table below, where a positive sign signifies that more alternating voltage was required to produce the same emf. The result at 20 volts and 20 kilohertz is rounded to the nearest part per million.

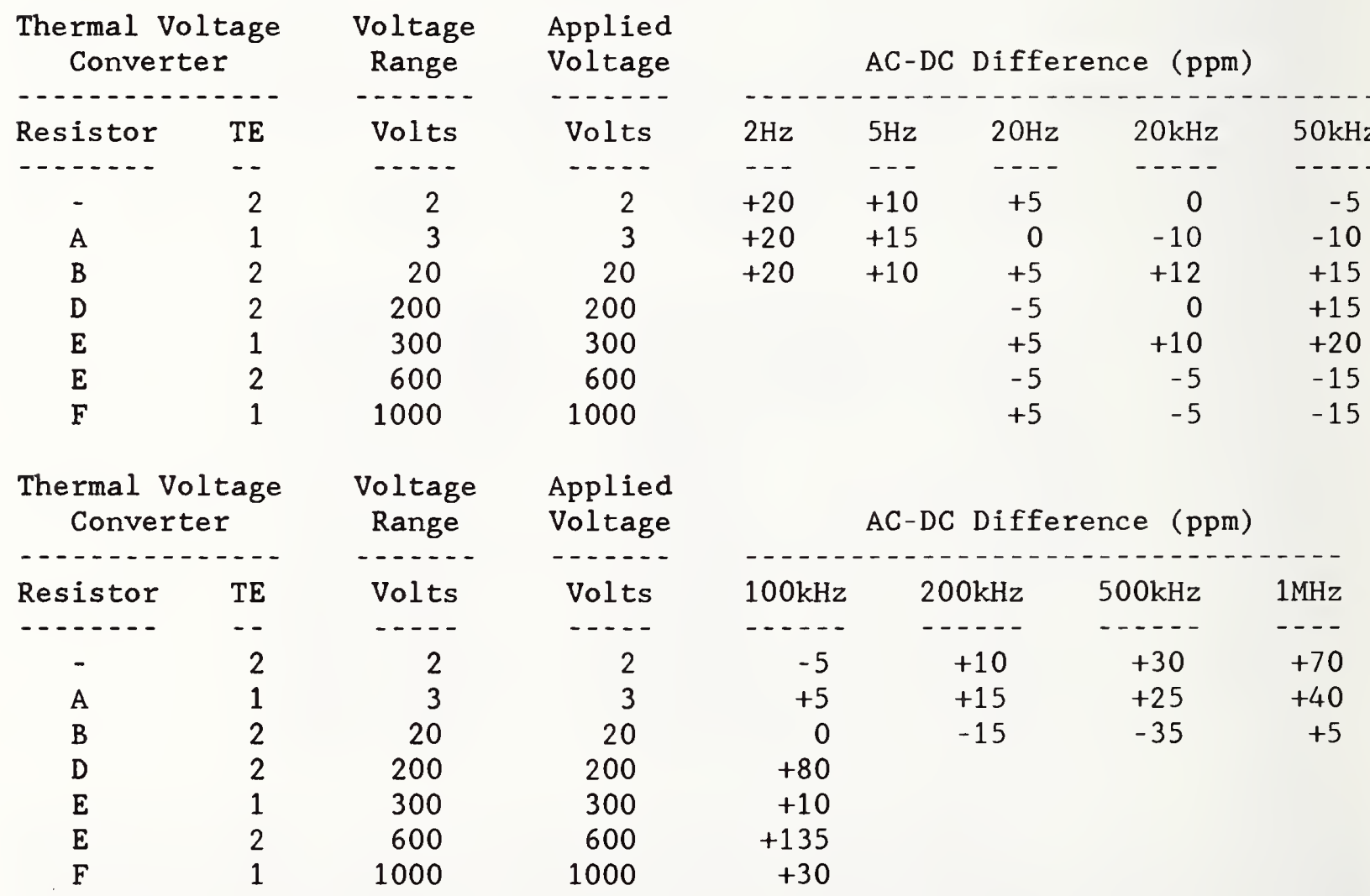


Set of Thermal Transfer Standards

Manufacturer's (brief) Name Model T, Serial No. 54321

Submitting Company's Name

City, State, Zip Code

It is very unlikely that these values of ac-dc difference are in error by more than the amounts listed in the table below. These figures include allowances for both the random and systematic uncertainties of the calibration process. No allowance has been made for the long-term stability of these standards.

\begin{tabular}{|c|c|c|c|c|c|c|c|c|c|}
\hline $\begin{array}{l}\text { Applied } \\
\text { Voltage }\end{array}$ & \multicolumn{9}{|c|}{ Uncertainty (ppm of } \\
\hline--- & & - & -1 & & $\cdots-1$ & $-\ldots$ & $\ldots$ & $\ldots$ & -- \\
\hline Volts & $2 \mathrm{~Hz}$ & $5 \mathrm{~Hz}$ & $20 \mathrm{~Hz}$ & $20 \mathrm{kHz}$ & $50 \mathrm{kHz}$ & $100 \mathrm{kHz}$ & $200 \mathrm{kHz}$ & $500 \mathrm{kHz}$ & $1 \mathrm{MHz}$ \\
\hline$\ldots$ & - - & $-\ldots$ & $\ldots$ & $\ldots-\ldots$ & $-\ldots$ & $-\ldots$ & $-\ldots$ & - . - - & - - - \\
\hline$>100$ & & & 20 & 20 & 30 & 50 & & & \\
\hline $5-100$ & 200 & 100 & 20 & 15 & 25 & 40 & 100 & 100 & 200 \\
\hline$<5$ & 200 & 100 & 20 & 20 & 30 & 50 & 100 & 100 & 200 \\
\hline
\end{tabular}

The uncertainty for the B,2 combination at 20 volts and 20 kilohertz is $10 \mathrm{ppm}$ of the applied voltage. This lower uncertainty was achieved after additional measurements were made at this test point (by prior arrangement) with different standards and a more precise comparator system. A general discussion of the uncertainties is presented in "An Investigation of the Uncertainties of the NBS Thermal Voltage and Current Converters" by F. L. Hermach (NBS Internal Report NBSIR 84-2903).

For the Director

National Measurement Laboratory

Norman B. Belecki, Physicist

Electricity Division

Center for Basic Standards

Test No.: 521/Test Number

Reference: Purchase Order Number

Date: September 30, 1987 
BIBLIOGRAPHIC DATA

1. PUBLICATION OR REPORT NO.

SHEET (See instructions)

4. TITLE AND SUBTITLE

NIST Measurement Services:

AC-DC Difference Calibrations

\section{AUTHOR(S)}

Joseph R. Kinard, John R. Hastings, Thomas E. Lipe, and Clifton B. Childers

6. PERFORMING ORGANIZATION (If joint or other than NBS, see instructions)

.

NATIONAL INSTITUTE OF STANDARDS AND TECHNOLOGY

(formerly NATIONAL BUREAU OF STANDARDS)

7. Contract/Grant No.

U.S. DEPARTMENT OF COMMERCE

GATTHERSBURG, MD 20999

8. Type of Report \& Period Covered

9. SPONSORING ORGANIZATION NAME AND COMPLETE ADDRESS (Street, City, State, ZIP)

\section{Final}

Same as item 6

10. SUPPLEMENTARY NOTES

Library of Congress Catalog Card Number: 89-600736

[ Document describes a computer program; SF-185, FIPS Software Summary, is attached.

11. ABSTRACT (A 200-word or less factual summary of most significant information. If document includes a significant bibliography or literature survey, mention it here)

This publication collects and summarizes the specialized information needed to operate the ac-dc difference laboratory and calibration service at NIST

(Gaithersburg). It also serves as a convenient reference source for the users of this calibration service and other interested people by documenting the service and its underlying background in considerable detail. It contains the following: an annotated table of contents, a topical index, and a glossary of common ac-dc acronyms; an overview of the service; selected published papers; instructions for the operation of the comparator systems; a schedule for the recalibration and periodic checks of the NIST thermal converters; and a sample report of calibration.

12. KEY WORDS (Six to twelve entries; alphabetical order: capitalize only proper names; and separate key words by semicolons) ac-dc difference; ac-dc transfer; calibration; comparator; current; measurement; standards; thermal converter; uncertainty; voltage

\section{AVAILABILITY}

X Unlimited

$\square$ For Official Distribution. Do Not Release to NTIS

X] Order From Superintendent of Documents, U.S. Government Printing Office, Washington, D.C. 20402.

Order From National Technical Information Service (NTIS), Springfield, VA. 2216 I
14. NO. OF PRINTED PAGES 308

15. Price 


\section{THE SP 250 SERIES ON NIST MEASUREMENT SERVICES*}

SP 250-1 Spectral Radiance Callbrations PB87179883

SP 250-2 Far Ultraviolet Detector Standards PB87227609

SP 250-3 Radlometric Standards in the Vacuum Uitraviolet PB87227625

SP 250-4 Fricke Doslmetry in High-Energy Electron Beams SN003-003-02816-9 \$2.75

SP 250-5 Aipha-Particle Calibrations SN003-003-02823-1 \$2.00

SP 250-6 Reguiar Spectral Transmittance PB88108550

SP 250-7 Radiance Temperature Callbrations SN003-003-02827-4 \$2.25

SP 250-8 Spectral Reflectance PB88109905

SP 250-9 Calibration of Beta-Particle-Emitting Ophthalmic Applicators SN003-003-02817-7 \$2.00

SP 250-10 Radloactivity Callbrations with the " $4 \pi$ " Gamma lonization Chamber and Other Radioactivity Calibration Capablitiles SN003-003-02824-0 \$2.25

SP 250-11 Dosimetry for High Dose Applicatlons PB88201587

SP 250-12 Neutron Personnel Dosimetry SN003-003-02811-8 $\$ 2.50$

SP 250-13 Activation Foil irradiation with Californlum Fission Sources SNO03-003-02866-5 \$2.25

SP 250-14 Activation Foll irradlatlon by Reactor Cavity Fission Sources SN003-003-02861-4 \$3.25

SP 250-15 Photometric Calibrations PB88153747

SP 250-16 Callbratlon of X-Ray and Gamma-Ray Measuring instruments SNO03-003-02862-2 \$7.00

SP 250-17 The NBS Photodetector Spectral Response Calibration Transfer Program PB88201595

SP 250-18 Neutron Source Strength Calibrations SN003-003-02863-1 \$3.25 SP 250-19 Calibration of Gamma-Ray-Emltting
Brachytherapy Sources SN003-003-02923-8

SP 250-20 Spectral Irradlance Calibratlons SN003-003-02829-1 $\$ 5.50$

SP 250-21 Calibratlon of Beta-Particle Radiation Instrumentation PB88201579

SP 250-22 Platinum Resistance Thermometer Calibrations PB88138367

SP 250-23 Liquid-in-Giass Thermometer Calibration Service SN003-003-02891-6 $\$ 6.00$

SP 250-24 Standard Cell Callbrations PB88123690

SP 250-25 Calibration Service for Inductive Voltage Dividers

SP 250-26 NBS Phase Angle Calibration Services SN003-003-02871-1 \$5.00

SP 250-27 AC-DC Difference Callbratlons

SP 250-28 Solld-State DC Voltage Standard Calibrations SN003-003-02842-8 \$2.00

SP 250-29 Traceable Frequency Calibrations SN003-003-02844-4 \$2.25

SP 250-30 GOES Sateilite Time Code Dissemination: Description and Operation PB88168760

SP 250-31 Mass Calibrations SN003-003-02919-0 \$4.00

SP 250-32 A Calibration Service for $30 \mathrm{MHz}$ Attenuation and Phase Shift SN003-003-02875-4 \$13.00

SP 250-33 A Callbration Service for Voltage Transformers and High-Voltage Capacitors SN003-003-02880-1 \$1.25

SP 250-34 High Vacuum Standard and its Use

SP 250-35 The Calibration of Thermocouples and Thermocouple Materials

*Entries containing a stock number (003-003-) and price can be purchased from the Superintendent of Documents, U.S. Government Printing Office, Washington, DC 20402. GPO will accept checks, money orders, VISA, and Mastercharge. For more infomation, or to place an order, call (202) 783-3238. Be sure to cite the stock number on all orders.

Entries contalning PB numbers can be purchased from the National Technical information Service, Springfield, VA 22161. NTIS will accept American Express in addition to the payment methods listed for GPO. For more information call (703) 487-4650; to place an order call $(800) 336-4700$. Be sure to cite the PB number on all orders.

Titles without stock numbers are in preparation. 


\section{U.S. Department of Commerce}

National Institute of Standards and Technology

(formerly National Bureau of Standards)

Gaithersburg, MD 20899

\section{Official Business}

Penalty for Private Use $\$ 300$ 